\title{
Horizontal Convective Condensation of Alternative Refrigerants within a Micro-Fin Tube
}

Mark A. Kedzierski

Building and Fire Research Laboratory

Gaithersburg, Maryland 20899

J. M . Goncalves

Escola Tecnica Federal de Santa Catarina

Sao Jose, S.C., 88.103-902, Brazil

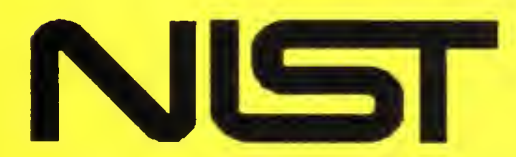

United States Department of Commerce

10logy Administration 


\section{Horizontal Convective Condensation of} Alternative Refrigerants Within a Micro-Fin Tube

Mark A. Kedzierski

J. M. Goncalves

December 1997

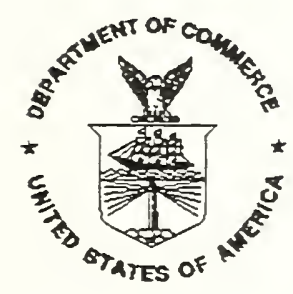

U.S. Department of Commerce

William M. Daley, Secretary

Technology Administration

Mary L. Good, Under Secretary for Technology

National Institute of Standards and Technology

Raymond G. Kammer, Director
Prepared for:

U.S. Department of Energy

Office of Building Technology

1000 Independence Avenue

Washington, DC 20585 


\section{TABLE OF CONTENTS}

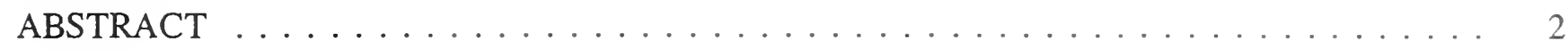

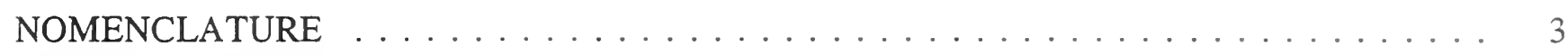

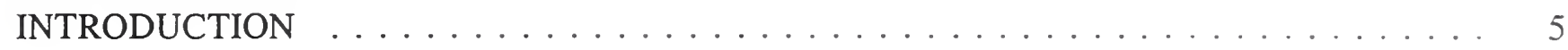

EXPERIMENTAL APPARATUS ........................... 5

HEAT TRANSFER COEFFICIENTS ..................... 7

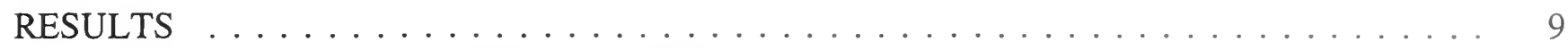

Heat Transfer (9); Pressure Drop (14)

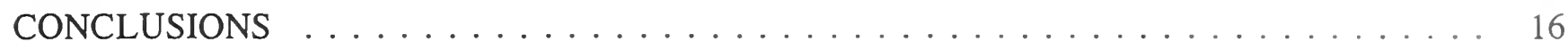

ACKNOWLEDGEMENTS ................................. 16

REFERENCES ............................... 17

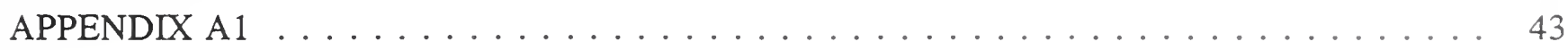

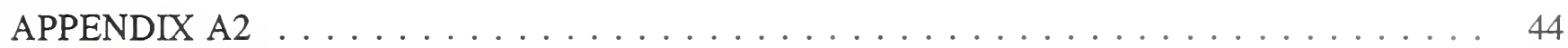

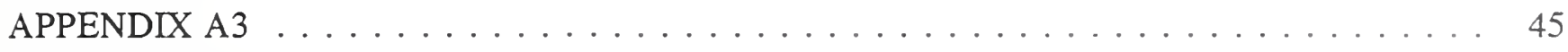

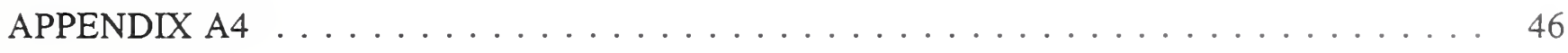

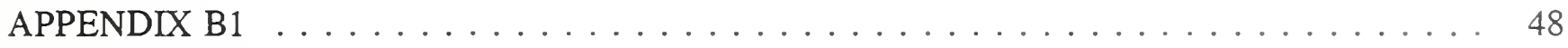

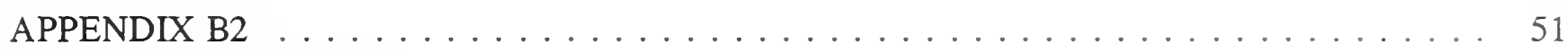

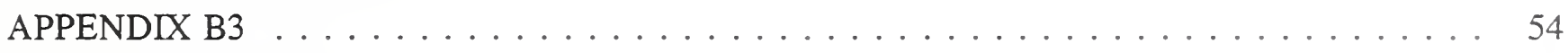

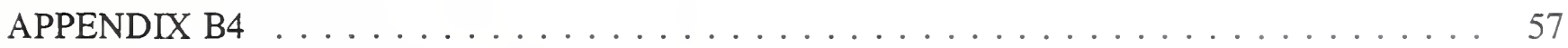

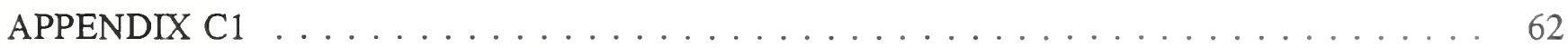

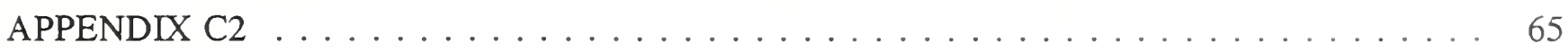

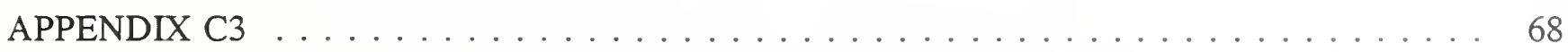

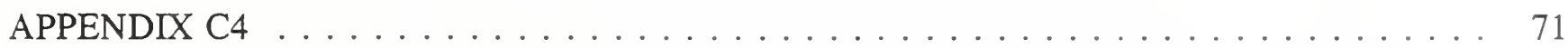


ABSTRACT

This paper presents local convective condensation measurements for four refrigerants: R134a, $\mathrm{R} 410 \mathrm{~A}\left(\mathrm{R} 32 / \mathrm{R} 125,50 / 50 \%\right.$ mass), R125, and R32 in a micro-fin tube ${ }^{1}$. Both heat transfer and pressure drop measurements are provided. The heat transfer degradation associated with R410A was shown to be relatively small and believed to be mostly due to nonlinear property effects. The measured convective condensation Nusselt numbers for all of the test refrigerants were correlated to a single expression consisting of a product of dimensionless properties. The correlation was shown to predict some existing data from the literature within acceptable limits. The correlation poorly predicted the heat transfer performance of cross-grooved, micro-fin tubes. The pressure drop measurements for the micro-fin tube were predicted satisfactorily by an existing correlation for flow boiling pressure drop in a smooth tube. Correlation of the pressure drop measurements suggested that the heat transfer enhancement was due to the fins behaving as a surface roughness.

Keywords: Enhanced heat transfer, micro-fin, refrigerant mixtures, fluid heating, condensation, pressure drop

${ }^{1}$ In order to describe materials and experimental procedures adequately, it is occasionally necessary to identify commercial products by manufacturers' name or label. In no instance does such identification imply endorsement by the National Institute of Standards and Technology, nor does it imply that the particular product or equipment is necessarily the best available for the purpose. 


\section{NOMENCLATURE}

\section{$\underline{\text { English symbols }}$}

$\mathrm{A}_{c} \quad$ cross sectional flow area inside tube $\left(\mathrm{m}^{2}\right)$

$A_{i} \quad$ actual inner surface area of tube $\left(\mathrm{m}^{2}\right)$

$c_{p} \quad$ specific heat $(\mathrm{J} / \mathrm{kg} \bullet \mathrm{K})$

$\mathrm{d}$ number of model parameters

D tube diameter $(\mathrm{m})$

$D_{e} \quad$ equivalent inner diameter of smooth tube, $\sqrt{\frac{4 A_{C}}{\pi}}(m)$

$\mathrm{D}_{\mathrm{h}} \quad$ hydraulic diameter of micro-fin tube, (m)

$\mathrm{E}_{\mathrm{h}} \quad$ heat-transfer enhancement ratio

f Fanning friction factor

g gravitational acceleration, $9.8 \mathrm{~m} / \mathrm{s}^{2}$

$\mathrm{G} \quad$ Mass velocity $\left(\mathrm{kg} / \mathrm{m}^{2} \bullet \mathrm{s}\right)$

$\mathrm{h}_{2 \phi} \quad$ local two-phase heat-transfer coefficient $\left(\mathrm{W} / \mathrm{m}^{2} \cdot \mathrm{K}\right)$

$h_{L} \quad h$ from linear fit between pure components $\left(\mathrm{W} / \mathrm{m}^{2} \cdot \mathrm{K}\right)$

i enthalpy $(\mathrm{J} / \mathrm{kg})$

$\mathrm{i}_{\mathrm{fg}} \quad$ latent heat of vaporization $(\mathrm{J} / \mathrm{kg})$

Ja refrigerant Jacob Number $\frac{i_{f g}}{c_{p_{r, 1}} \Delta T_{s}}$

$\mathrm{k}$ refrigerant thermal conductivity $(\mathrm{W} / \mathrm{m} \cdot \mathrm{K})$

L tube length (m)

$\mathrm{Nu} \quad$ local Nusselt number based on $D_{h}$

$\mathrm{N}_{\mathrm{f}} \quad$ number of fins

$\dot{\mathrm{m}}$ mass flow rate $(\mathrm{kg} / \mathrm{s})$

M molecular weight ( $\mathrm{g} / \mathrm{mole})$

$\mathrm{n}$ number of data points

$\mathrm{p} \quad$ wetted perimeter of inner micro-fin tube (m)

$\mathrm{P} \quad$ local fluid pressure $(\mathrm{Pa})$

Pr liquid refrigerant Prandtl number $\left.\frac{\mathrm{C}_{\mathrm{p}} \mu}{\mathrm{k}}\right|_{I, I}$

q" local heat flux $\left(\mathrm{W} / \mathrm{m}^{2}\right)$

Re all liquid, refrigerant Reynolds number based on $D_{h}=\frac{G D_{h}}{\mu_{r, 1}}$

$S \quad$ perimeter of one fin and channel $(\mathrm{m})$

Sv non-dimensional refrigerant specific volume $\left.\frac{v_{v}-v_{I}}{v}\right|_{I}$

$\mathrm{T}$ temperature $(\mathrm{K})$

$\mathrm{U}$ expanded relative uncertainty

$\mathrm{x}_{\mathrm{q}} \quad$ thermodynamic mass quality

$\mathrm{z} \quad$ axial distance $(\mathrm{m})$

\section{$\underline{\text { Greek symbols }}$}

$\alpha \quad$ helix angle between micro fin and tube axis

$\beta \quad$ exponent on correlation

$\Delta \mathrm{h}_{2 \phi} \quad$ heat transfer degradation $\left(\mathrm{W} / \mathrm{m}^{2} \cdot \mathrm{K}\right)$

$\Delta \mathrm{P} \quad$ pressure drop $(\mathrm{Pa})$

$\Delta \mathrm{T}_{\mathrm{s}} \quad \mathrm{T}_{\mathrm{s}}-\mathrm{T}_{\mathrm{w}}(\mathrm{K})$ 
$\Phi \quad$ two-phase number, $\frac{\Delta \mathrm{x}_{\mathrm{q}} i_{\mathrm{fg}}}{\Delta \mathrm{L} g}$

$\mu \quad$ dynamic viscosity $(\mathrm{kg} / \mathrm{m} \cdot \mathrm{s})$

$\nu$ specific volume, $\mathrm{x}_{\mathrm{q}} \nu_{\mathrm{v}}+\left(1-\mathrm{x}_{\mathrm{q}}\right) \nu_{1}\left(\mathrm{~m}^{3} / \mathrm{kg}\right)$

subscripts

b bulk condition

c critical condition

f water

i inlet, inner

1 liquid

o outside

$\mathrm{p}$ plain or smooth tube

r refrigerant

S saturated state

w heat transfer surface

v vapor 


\section{INTRODUCTION}

Most evaporators and condensers of new unitary refrigeration and air-conditioning equipment are manufactured with micro fin tubes. The micro-fin tube dominates unitary equipment design because it provides the highest heat transfer with the lowest pressure drop of the commercially available internal enhancements (Webb, 1994). Together, R134a, R22, and R22 replacements constitute by mass nearly all the refrigerants used in unitary products (Muir, 1989). Consequently, two phase heat transfer data for the micro-fin tube with R134a, R22, and R22 replacements are essential for the design of evaporators and condensers for unitary applications.

Although the study of R134a, R22 and R22 replacements in micro-fin tubes is important, the work available in the open literature is limited. For example, there are surprisingly few studies on R22 replacements and micro-fin tubes. Wang et al. (1996) present quasi-local condensation heat transfer coefficients for R22 and R407C. Also, Kaul et al. (1996) present local flow boiling heat transfer measurements for R410B and R407C. Most of the available literature focuses on the heat transfer of R134a and R22. A survey by Schlager et al. (1990), which was later updated by Eckels et al. (1992), on all the refrigerant heat transfer with micro-fin tubes, showed that of the 22 studies, five were with R113, 15 were with R22, and two examined both fluids. The survey also revealed that condensation research has been neglected relative to evaporation research. For example, 17 of the surveyed studies did not investigate condensation. With help from the Bergles et al. (1995) survey and our own search, we were able to find only ten additional papers on convective condensation in micro-fin tubes. Four of these studies were for R134a, five were for R22, and one was the Wang et al. (1996) paper on R407C. Most of the recent R134a studies were of global heat transfer measurements as done by Eckels et al. (1994). However, only four studies - Chamra and Webb (1995), Chiang (1993), Mori and Nakayama (1983), and Khanpara et al. (1986) - that present local condensation measurements in a micro-fin tube were found.

Local condensation measurements will prove most useful to the refrigeration industry. An accurate sizing of a micro-fin tubed condenser requires an accurate local heat-transfer correlation. Unfortunately, neither Ghaderi et al. (1995) nor Webb (1994) were able to find a convective condensation correlation for the micro-fin tube in the literature. The absence of a universal condensation correlation may be partly due to the many variants of the micro-fin geometry and the common practice of using the root diameter area instead of the actual surface area and the hydraulic diameter to calculate the heat transfer. A single local heat transfer correlation that can be used for different micro-fin geometries would be useful to the refrigeration industry.

Considering the shortage of convective condensation data for R22 replacements in a micro-fin tube and the absence of a heat transfer correlation, there were two main objectives of the study. The first was to characterize the convective condensation performance of two R22 alternatives in a micro-fin tube. Specifically, the local convective condensation pressure drop and heat transfer coefficient of R134a and the near-azeotropic refrigerant mixture R410A along with its pure components R32 and R125 were experimentally determined. The experimental measurements for the fluids were correlated to a single correlation as a function of non-dimensional parameters. The second objective was to fundamentally characterize the heat transfer degradation of $\mathrm{R} 410 \mathrm{~A}$ relative to the performance of the pure components. Convective condensation heat transfer coefficients for the mixtures' pure components were measured to establish a baseline for the heat transfer degradation calculations. A fundamental understanding of mixture heat transfer mechanisms can be used to refine the selection of mixture composition and/or mixtures for alternative refrigerants.

\section{EXPERIMENTAL APPARATUS}

Figure 1 shows a sketch of the experimental apparatus used to establish and measure the convective condensation. The refrigerant flow rate, pressure, and superheat were fixed at the inlet to the test section. The water flow rate and the inlet temperature were fixed to establish the overall refrigerant quality change in the test section. The water temperature drop, the tube wall temperature, the refrigerant 
temperatures, pressures, and pressure drops were measured at several axial locations along the test section. These measurements were used to calculate the local heat-transfer coefficient for the micro-fin tube.

The test section consisted of a pair of $3.34 \mathrm{~m}$ long, horizontal tubes connected by a U-bend. A fixed test pressure was maintained by balancing the refrigerant duty between the subcooler, the test section, and the evaporator. A magnetically coupled gear pump delivered the test refrigerant to the entrance of the test section with a few degrees of vapor superheat. Another magnetically coupled gear pump supplied a steady flow of water to the test section. The inlet temperature of the water loop was held constant for each test with a water chilled heat exchanger and variable electric heaters. The refrigerant and water flow rates were controlled by varying the pump speeds using frequency inverters. Redundant flow rate measurements were made with Coriolis flowmeters and with turbine flowmeters for both the refrigerant and water sides.

Figure 2 shows a cross section of the test section with a detail of the micro-fin tube geometry. The test refrigerant flowed inside a micro fin tube, while distilled water flowed either in counterflow or parallel flow to the refrigerant in the annulus that surrounded the micro-fin tube. The annulus gap was $2.2 \mathrm{~mm}$, and the micro-fin tube wall thickness was $0.3 \mathrm{~mm}$. The micro fin tube had $60,0.2 \mathrm{~mm}$ high fins with 18 degree helix angle. For this geometry, the cross sectional flow area was $60.8 \mathrm{~mm}^{2}$ giving an equivalent smooth diameter $\left(D_{e}\right)$ of $8.8 \mathrm{~mm}$. The root diameter of the micro-fin tube was $8.91 \mathrm{~mm}$. The inside-surface area per unit length of the tube was estimated to be $44.6 \mathrm{~mm}$. The hydraulic diameter $\left(\mathrm{D}_{\mathrm{h}}\right)$ of the micro-fin tube was estimated to be $5.45 \mathrm{~mm}$. The ratio of the inner surface area of the micro fin tube to the surface area of a smooth tube of the same $D_{e}$ was 1.6. The fins rifled down the axis of the tube at a helix angle of $18^{\circ}$ with respect to the tube axis.

Figure 3 provides a detailed description of the test section. The annulus was constructed by connecting a series of tubes with 14 pairs of stainless steel flanges. This construction permitted the measurement of both the outer micro-fin wall temperature and the water temperature drop as discussed in the following two paragraphs. The design also avoided abrupt discontinuities such as unheated portions of the test section and tube-wall "fins" between thermopile ends.

Figure 3 shows that thermocouple wires pass between 12 of the gasketed flange pairs to measure the refrigerant-tube wall temperature at ten locations on the top, side, and bottom of the tube wall. These locations were separated by $0.6 \mathrm{~m}$ on average, and they were located near the intersection of the shell flanges. In addition to these, thermocouples were also mounted near the middle pressure taps. The thermocouple junction was soldered to the outside surface and was sanded to a thickness of $0.5 \mathrm{~mm}$. The leads were strapped to a thin non-electrically-conducting epoxy layer on the wall for a distance of 14.3 $\mathrm{mm}$ before they passed between a pair of the shell flanges. The wall temperature was corrected for a heat flux dependent fin effect. The correction was typically $0.05 \mathrm{~K}$.

Figure 3 also shows that a chain of thermopiles was used to measure the water temperature drop between each flange location. Each thermopile consisted of ten thermocouples in series, with the ten junctions at each end evenly spaced around the circumference of the annulus. Because the upstream junctions of one thermopile and the downstream junctions of another enter the annulus at the same axial location (except at the water inlet and outlet), the junctions of the adjacent piles were alternated around the circumference. A series of teflon half-rings attached to the inner refrigerant tube centered the tube in the annulus. The half-rings were circumferentially baffled to mix the water flow. Mixing was further ensured by a high water Reynolds number (Kattan et al. 1995).

As shown in Fig. 3, six refrigerant pressure taps along the test section allowed the measurement of the upstream absolute pressure and five pressure drops along the test section. Two sets of two water pressure taps were used to measure the water pressure drop along each tube. Also, a sheathed thermocouple measured the refrigerant temperature at each end of the two refrigerant tubes, with the junction of each centered radially. Only the thermocouple at the inlet of the first tube was used in the calculations. The 
entire test section was wrapped with $5 \mathrm{~cm}$ of foam insulation to minimize heat transfer between the water and the ambient.

\section{HEAT TRANSFER COEFFICIENTS}

The convective condensation heat transfer coefficient based on the actual inner surface area $\left(h_{2 \phi}\right)$ was calculated as:

$$
\mathrm{h}_{2 \phi}=\frac{\mathrm{q}^{\prime \prime}}{\mathrm{T}_{\mathrm{r}}-\mathrm{T}_{\mathrm{w}}}
$$

where the measured wall temperatures $\left(T_{w}\right)$ were fitted to their axial position to reduce the uncertainty in the measurement. The best fits for the wall temperature for parallel flow and counterflow differed. The measured wall temperatures for counterflow were fitted to:

$$
\mathrm{T}_{\mathrm{w}}=\mathrm{A}_{0}+\mathrm{A}_{1} \mathrm{z}^{2}
$$

The measured wall temperatures for parallel flow were fitted to:

$$
T_{w}=A_{0}+A_{1} z+A_{2} z^{2}
$$

Figure 4 shows the estimated expanded uncertainty of the wall temperature fit for all the measured data as a function of thermodynamic quality. Figure 4 includes some data that was omitted from the correlation of the data as explained in the Results section. Circles and squares are used to designate counterflow and parallel flow, respectively. The uncertainty of most of the fitted wall temperatures was less than $0.5 \mathrm{~K}$. The median of the uncertainty in $T_{w}$ as shown in Table 1 was $0.35 \mathrm{~K}$.

The water temperature $\left(T_{f}\right)$ was determined from the measured temperature change obtained from each thermopile and the inlet water temperature measurement. The water temperature was regressed to the axial location of the thermopiles along the $\mathrm{z}$-coordinate. As shown for the $T_{w}$ regressions, the best fits for the water temperatures differed for parallel flow and counterflow. The water temperatures for counterflow were fitted to:

$$
T_{f}=A_{0}+A_{1} z^{2}+A_{2} z^{3}
$$

The measured water temperatures for parallel flow were fitted to:

$$
T_{f}=A_{0}+A_{1} z+A_{2} z^{2}+A_{3} z^{3}
$$

The water temperature fits, the measured water mass flow rate $\left(\dot{\mathrm{m}}_{\mathrm{f}}\right)$, and the properties of the water were used to calculate the local heat flux $\left(\mathrm{q}^{\prime \prime}\right)$ to the micro-fin tube based on the actual inner surface area:

$$
\mathrm{q}^{\prime \prime}=\frac{\mathrm{m}_{\mathrm{f}}}{\pi \mathrm{p}}\left(\mathrm{c}_{\mathrm{p}_{\mathrm{f}}} \frac{\mathrm{dT}_{\mathrm{f}}}{\mathrm{dz}}+v_{\mathrm{f}} \frac{\mathrm{dP}_{\mathrm{f}}}{\mathrm{dz}}\right)
$$

where $\mathrm{p}$ is the wetted perimeter of the inside of the micro-fin tube. The specific heat $\left(c_{p}\right)$ and the specific volume $\left(\nu_{\mathrm{f}}\right)$ of the water were calculated locally as a function of the water temperature. The local, axial water temperature gradient $\left(\mathrm{dT}_{\mathrm{f}} / \mathrm{dz}\right)$ was calculated from a derivative of either eqn. 4 or eqn. 5 depending on the corresponding flow configuration. The water pressure gradient $\left(\mathrm{dP}_{\mathrm{f}} / \mathrm{dz}\right)$ was linearly interpolated between the pressure taps to the location of the wall thermocouples. The pressure gradient term was typically less than $3 \%$ of the temperature gradient term. 
Figure 5 shows an example plot of the local heat flux as calculated from eqn. 6 versus thermodynamic quality. Counterflow and parallel flow heat flux profiles are compared for R134a at a Reynolds number of 15100 and a refrigerant pressure of $1160 \mathrm{kPa}$. The form of the counterflow and parallel flow differ, as suggested by the use of two different water temperature fits for the two flow conditions. The two flow conditions provided for a wider range of heat fluxes at a given thermodynamic quality. In this way, the sensitivity of the heat-transfer coefficient to the heat flux could be thoroughly investigated as a function of quality.

Figure 6 plots the relative uncertainty in the water temperature gradient (which is roughly equal to the uncertainty in the heat flux) versus thermodynamic quality. As shown in Fig. 6, the larger values of heat flux exhibit smaller relative uncertainties than the lower heat fluxes. For example, at low quality (low q") much of the parallel flow data exhibits relative uncertainties greater than $20 \%$. Similarly, the relative uncertainty of the counterflow data tends to increase for high quality (low q").

The equilibrium refrigerant temperature $\left(T_{\mathrm{r}}\right)$ and all other thermodynamic and transport properties were calculated with version five of REFPROP (Huber et al. 1995) with enthalpy and pressure as inputs. The enthalpy of the refrigerant vapor at the inlet of the test section was calculated from its measured temperature and pressure. The subsequent drop in refrigerant enthalpy along the test section was calculated from the local heat flux and the measured refrigerant mass flow rate. The refrigerant pressures were measured at six pressure taps along the test section. The pressure was linearly interpolated between the taps. The average $\mathrm{T}_{\mathrm{r}}$ was varied between $30^{\circ} \mathrm{C}$ and $50{ }^{\circ} \mathrm{C}$ with approximately $5 \mathrm{~K}$ of superheat at the test section inlet.

Figures 7 and 8 illustrate the results of the above-described measurement procedure for counterflow and parallel flow conditions, respectively. The difference between the appearances of the water and refrigerant temperature profiles for parallel flow and counterflow demonstrates the need for different temperature versus distance regression forms for the two flow conditions. Figures 7 and 8 also show representative results of refrigerant and water pressure profiles for counterflow and parallel flow conditions, respectively.

The local Nusselt number $(\mathrm{Nu})$ was calculated based on the actual inner surface area of the tube as:

$$
\mathrm{Nu}=\frac{h_{2 \phi} D_{h}}{k_{1}}
$$

Figure 9 shows the relative uncertainty of the Nu versus thermodynamic quality. Testing the two flow conditions also benefitted the correlation of data by providing complimentary uncertainty profiles with thermodynamic quality. For example, the uncertainty of the low quality, parallel flow Nusselt number data exhibits high uncertainties, while the low quality, counterflow data exhibits low uncertainties. Consequently, by testing with both flow conditions, much of the parallel flow data can be omitted from the correlation due to its high uncertainty, while still maintaining sufficient counterflow data in the low quality region to produce a valid correlation. Figure 9 shows that a similar, but opposite, scenario exists in the high quality region.

The cubic fit of the wall and water temperature profiles were within $\pm 0.8 \mathrm{~K}$ and $\pm 0.2 \mathrm{~K}$, respectively, of the measured temperatures. On average, the residual standard deviation of the wall and water temperature fits was $0.5 \mathrm{~K}$ and $0.1 \mathrm{~K}$, respectively. The refrigerant temperatures were obtained from pressure measurements and the REFPROP (Huber et al. 1995) equation of state.

Figure 10 provides corroboration of the present local heat flux and wall temperature measurements with the Wiegand (1945) correlation for single phase turbulent heat transfer in a smooth annulus. The local heat flux, measured wall and water temperatures were used to calculate the local water-side heat transfer coefficient for the annulus. Figure 10 shows that approximately $80 \%$ of the measured water-side heat transfer coefficients are within $\pm 25 \%$ of the Wiegand (1945) correlation. The range of the difference 
between measurements and Wiegand's (1945) correlation is nearly centered about the correlation and lies within $\pm 36 \%$ which was the range given by E. L. McMillen for his data in the "Written Discussion" section of Wiegand's (1945) paper. This provides an independent validation of the wall temperature and heat flux measurements.

Table 1 shows the expanded measurement uncertainty (U) of the various measurements along with the range of each parameter in this study. The $U$ was estimated with the law of propagation of uncertainty. All expanded measurement uncertainties are reported for a $95 \%$ confidence interval and are evaluated by statistical methods. The estimates shown in Table 1 are median values of $U$ for the correlated data.

\section{RESULTS}

The 1367 data points generated in this study for R32, R125, R410A, and R134a are tabulated in three separate appendices. Appendix A contains the pressure drop data. Appendix B contains the Nusselt and Reynolds numbers and other reduced data that were used in the correlation of the data. Appendix $C$ contains the raw data measurements including the heat flux and the wall and water temperatures and locations. The column entitled, "flow," provides a "C" or a "P" for counterflow or parallel flow, respectively. All the parameters are defined in the nomenclature.

\section{Heat Transfer}

The present heat-transfer measurements concur with the approximate magnitude of the heat transfer enhancement reported by Webb (1994). Webb (1994) states that the micro-fin tube provides anywhere from $100 \%$ to $200 \%$ improvement over smooth tube R22 condensation performance. Figure 11 shows a graph of the condensation heat-transfer enhancement ratio $\left(E_{h}\right)$,

$$
E_{h}=\frac{h_{2 \phi} A_{i}}{h_{p} \pi D_{e} L}
$$

versus Reynolds number. The heat-transfer coefficient of the plain surface $\left(h_{p}\right)$ was calculated from the smooth tube convective condensation correlation of Ackers and Rosson (1960) using the equivalent diameter $\left(D_{e}\right)$ to calculate the Reynolds and Nusselt numbers. The $h_{p}$ was calculated using the same mass velocity and fluid properties that were used for the experimental data.

The enhancement ratio shown in Fig. 11 varies from approximately 0.6 to 3.6 for the micro-fin tube. Approximately half of the enhancement factors are greater than 1.6. Enhancement ratios below 1.6 indicate that the micro-fin heat transfer coefficient based on the surface area is less than that of a smooth tube. Enhancement ratios greater than 1.6 suggest that the enhancement caused by the micro-fin surface is due to more than just the $60 \%$ surface area increase over the smooth surface with the same cross sectional flow area.

Regression of dimensionless parameters against $\mathrm{E}_{\mathrm{h}}$ was used to assist in the investigation of the mechanism that was responsible for the heat transfer enhancement. The $\mathrm{E}_{\mathrm{h}}$ was found to be primarily a function of Reynolds number and thermodynamic quality:

$$
E_{h}=9.777 \operatorname{Re}^{-0.162} x_{q}^{0.411}
$$

The relative magnitude of the exponents in eqn. 9 shows that the quality has more influence on the $E_{h}$ than does the Re. The negative exponent on the Reynolds number shows that the effectiveness of the micro-fin enhancement mechanism decreases for increasing Reynolds numbers. Conversely, eqn. 9 shows that the micro-fin enhancement mechanism is more effective for larger values of quality. The enhancement mechanism with respect to increasing quality may result from an interaction between the fins and the liquid-vapor interface of the fluid in the tube. At very high vapor qualities and very thin liquid films on the surface, the fins may be very effective at mixing the liquid-vapor interface due to their 
proximity to the liquid-vapor interface. It is also possible to obtain an additional enhancement at very high qualities from surface-tension drainage forces on the fin-tips. However, as the liquid accumulates on the surface, both the liquid-vapor interface mixing and the surface-tension effects diminish. Consequently, the $E_{h}$ is larger at higher qualities. The heat transfer enhancement with respect to the Reynolds number may result from an interaction between the fins and the turbulence in the liquid film. Smaller eddies transfer momentum more efficiently than larger eddies. Low Reynolds number flows may be enhanced more readily than high Reynolds number flows due to the reduction in the size of the turbulent eddies at the wall by the interaction of the flow with the fins. High Reynolds number flows are not enhanced as readily as low Reynolds number flows because there are fewer large eddies to be reduced at higher Reynolds numbers.

The enhancement ratio that Schlager et al. (1989) obtained for the same micro-fin tube as used in this study is presented as solid, white lines in Fig. 11. They used a global micro-fin heat transfer coefficient measurement over a 0.6 to 0.8 quality change to calculate $E_{\mathrm{h}}$. For the reference case, Schlager et al. (1989) measured smooth tube heat transfer coefficients for an $8 \mathrm{~mm}$ inner diameter tube. The solid white line shows the enhancement ratio as reported by Schlager et al. (1989) using the $8 \mathrm{~mm}$ diameter tube in its calculation. The dashed line shows the $E_{\mathrm{h}}$ after the smooth tube conductance was multiplied by $(8 / 8.8)^{0.8}$ to convert to an $E_{h}$ based on a $8.8 \mathrm{~mm}$ smooth inner diameter, i.e., $D_{e}$ for the micro-fin. The Reynolds number and quality dependence of the Schlager et al. (1989) $\mathrm{E}_{\mathrm{h}}$ roughly agrees with the present $\mathrm{E}_{\mathrm{h}}$ measurement. The slope of the Schlager et al. (1989) is consistent with the mean slope of the measurements. Considering that the Schlager et al. (1989) $\mathrm{E}_{\mathrm{h}}$ is for average heat transfer conditions, their data would be expected to lie close to the middle of the data range. The Schlager et al. (1989) enhancement ratio that was adjusted to the $8.8 \mathrm{~mm}$ diameter lies relatively close to the median of the data.

Figure 12 includes a comparison of the experimental micro-fin Nusselt numbers to the smooth tube convective condensation correlation of Ackers and Rosson (1960) using the hydraulic diameter to calculate the Reynolds and Nusselt numbers. The figure shows that use of the hydraulic diameter in a smooth tube condensation correlation predicts most of the present micro-fin data to within $+20 \%$ and $-40 \%$. The majority of the data is under-predict with the Ackers and Rosson (1960) correlation. Apparently, the smooth tube correlation does not account for the flow enhancement provided by the fins. Consequently, a new correlation is needed to account for the heat transfer enhancement due to the micro fins.

The convective condensation Nusselt numbers (Nu) were correlated following the law of Corresponding States philosophy presented by Cooper (1984). Cooper (1984) suggested that the fluid properties that govern nucleate pool boiling can be well represented by a product of the reduced pressure $\left(\mathrm{P}_{\mathrm{r}} / \mathrm{P}_{\mathrm{c}}\right)$, the acentric factor $\left(-\log _{10}\left(\mathrm{P}_{\mathrm{r}} / \mathrm{P}_{\mathrm{c}}\right)\right)$, and other dimensionless variables to various powers. The above reduced pressure terms and several other locally evaluated terms were used to correlate the 1358 (see table 2) measured local $\mathrm{Nu}$ for all condensing flow conditions and refrigerants in this study to:

$$
\mathrm{Nu}=\frac{\mathrm{h}_{2 \phi} \mathrm{D}_{\mathrm{h}}}{\mathrm{k}_{1}}=2.256 \mathrm{Re}^{\beta_{1}} \mathrm{Ja}^{\beta_{2}} \operatorname{Pr}^{\beta_{3}}\left(\frac{\mathrm{P}_{\mathrm{f}}}{\mathrm{P}_{\mathrm{c}}}\right)^{\beta_{4}}\left(-\log _{10} \frac{\mathrm{P}_{\mathrm{r}}}{\mathrm{P}_{\mathrm{c}}}\right)^{\beta_{5}} \mathrm{~Sv}^{\beta_{6}}
$$

where:

$$
\begin{aligned}
& \beta_{1}=0.303 \\
& \beta_{2}=0.232 \mathrm{x}_{\mathrm{q}} \\
& \beta_{3}=0.393 \\
& \beta_{4}=-0.578 \mathrm{x}_{\mathrm{q}}{ }^{2} \\
& \beta_{5}=-0.474 \mathrm{x}_{\mathrm{q}}{ }^{2} \\
& \beta_{6}=2.531 \mathrm{x}_{\mathrm{q}}
\end{aligned}
$$


where the Reynolds number ( $\mathrm{Re}$ ), the Jacob number (Ja), the Prandtl number (Pr), the reduced pressure $\left(\mathrm{P}_{\mathrm{r}} / \mathrm{P}_{\mathrm{c}}\right)$, the dimensionless specific volume $(\mathrm{Sv})$ and the quality $\left(\mathrm{x}_{\mathrm{q}}\right)$ are all evaluated locally. Baker's (1954) flow map for smooth tubes was used to approximately determine the flow conditions. The results showed that $79 \%, 18 \%, 2 \%$, and $1 \%$ of the data were in annular, slug, bubbly, and wavy flow, respectively.

The search for the above form of the correlation began with quadratic exponents in quality for each dimensionless variable. The quadratic exponent form was used with good results by Kedzierski and Kim (1997) to correlate several other pure refrigerants and mixtures for a wide range of qualities for both evaporative and condensing flows. The number of dimensionless variables and constants in the exponents were reduced to only those with significant influence on the residual standard deviation of the fit. For example, because R410A is a near-azeotrope, the composition difference between vapor and liquid phases had a negligible influence on the fit of the correlation. Consequently,

the composition difference was not used in the fit of the data.

Not all of the 1367 data points were used in the regression of eqn. 10. Table 2 shows the number of data points for each refrigerant that were not used in the fit. Measurements with large uncertainties, and measurements that had high influence, or high leverage on the model were all candidates for exclusion from the regression. The last column in Appendix B shows the data points that were included and excluded from the fit. The letter I signifies that the data point was included in the fit. The letters HI identifies that data point as being omitted for exhibiting high influence on the regression. The letters HL stand for omitted data points associated with high leverage. The letters HC represent high influence and high leverage data points.

The process that was used to determine which measurements were not to be included in the regression is outlined below. First, the form of eqn. 10 was determined using only those data points with an estimated expanded uncertainty less than $25 \%$ and qualities less than 1 . This process removed 686 data points, leaving no data with Nusselt numbers greater than approximately 300 in the included data set. Next, the 686 measurements that were omitted from the first regression were predicted with that same regression using the dimensionless parameters. Those excluded measurements that were predicted to within $\pm 25 \%$ of the first correlation were reintroduced into the regression data set. This process reintroduced Nusselt numbers greater than 300 to the regression data set. New coefficients were generated for eqn. 10 using the 1417 measurements of the regression data set. Measurements that were omitted from the regression with this first pass are designated with "O2" in the last column of Appendix B. The hat matrix and Cook's distance were generated for the second regression and used to determine which data had high-leverage and influence on the model, respectively. The diagonal elements of the hat matrix that were greater than approximately $2 \mathrm{~d} / \mathrm{n}$, where $\mathrm{d}$ is the number of model parameters, and $\mathrm{n}$ is the number of data points, were designated as high-leverage points (Belsley et al., 1980). The elements of the Cook array that were greater than $4 / \mathrm{n}$ were considered to be influential observations. The third stage of data filtering was performed by removing data: (1) that exhibited both high influence (i.e., $4 / n$ $>0.01$ ) and great leverage (i.e., $2 \mathrm{~d} / \mathrm{n}>0.003$ ) or (2) where the estimated uncertainty was greater than $25 \%$, and where the data had a large influence on the fit (shown as CU in last column of Appendix).

Most of the data that satisfied the last two criteria were data at or near the inlet and exit of the test section. There were nearly as many outliers at the inlet as there were for the outlet of the test section. Outliers at the inlet and outlet of the test section are consistent with the largest uncertainties in the water and wall temperature fits being located at the inlet and outlet. Also, the $\Delta \mathrm{T}_{\mathrm{s}}$ can be less than the uncertainty of the measurement at the refrigerant inlet and outlet of the test section for counterflow and parallel flow, respectively.

Figure 13 compares the measured condensation Nusselt numbers for the micro-fin tube to the Nusselt numbers predicted with equation 10 . Equation 10 correlates $95 \%$ of the pure component and nearazeotropic convective condensation Nusselt numbers to within approximately $\pm 21 \%$. The mean of the correlation has an average uncertainty of $\pm 3 \%$ over the entire range of Nusselt numbers. Only random 
trends were observed in the residual plots against each of the parameters of eqn. 10. The residual standard deviation of eqn. 10 and that for the separate fits for each fluid were nearly the same. This suggests that the scatter in the data is not caused by the different fluids.

A simpler form of eqn. 10 with a larger uncertainty is:

$$
\begin{aligned}
& \mathrm{Nu}=\frac{h_{2 \phi} D_{h}}{k_{1}}=4.94 \operatorname{Re}^{\beta_{1}} \operatorname{Pr}^{\beta_{3}}\left(\frac{P_{r}}{P_{c}}\right)^{\beta_{4}}\left(-\log _{10} \frac{P_{r}}{P_{c}}\right)^{\beta_{5}} S^{\beta_{6}} \\
& \text { where: } \\
& \beta_{1}=0.235 \\
& \beta_{3}=0.308 \\
& \beta_{4}=-1.16 \mathrm{x}_{q}^{2} \\
& \beta_{5}=-0.887 \mathrm{x}_{\mathrm{q}}^{2} \\
& \beta_{6}=2.708 \mathrm{x}_{\mathrm{q}}
\end{aligned}
$$

Equation 11 does not require an iteration procedure on the $\Delta \mathrm{T}_{\mathrm{s}}$ to evaluate the Jacob number. However, the uncertainty of fit is approximately $23.5 \%$ which is larger than that of eqn. 10 .

Figure 14 shows the heat transfer coefficient versus quality for each of the four test fluids at $\mathrm{T}_{\mathrm{r}}=40^{\circ} \mathrm{C}$, $\Delta \mathrm{T}_{\mathrm{s}}=5 \mathrm{~K}$, and $\mathrm{G}_{\mathrm{r}}=250 \mathrm{~kg} /\left(\mathrm{m}^{2} \bullet \mathrm{s}\right)$. The solid lines are predictions for the present micro-fin tube geometry which were obtained from the correlation of the data given as eqn. 10. In general, the measured condensation heat-transfer coefficient decreases for decreasing qualities. Apparently, thin liquid films and high vapor velocities at the entrance of the tube provide for high heat-transfer coefficients. As the liquid accumulates on the tube wall for decreasing quality, the heat-transfer coefficient diminishes. The refrigerant R32 exhibits the highest heat transfer performance of the four test fluids. R32 owes much of its heat transfer performance to its high thermal conductivity. As expected, the performance of the near-azeotropic mixture R410A is between that of its pure components R32 and R125. The predicted performance of $\mathrm{R} 22$ is near that of its proposed replacement R410A. The R125 exhibits lower condensation Nusselt numbers than that for R134a for the above conditions. Conversely, as shown by Kaul et al. (1996), the flow boiling performance of R125 is greater than that of R134a in the identical tube.

Over 140 figures would be required to depict the Nusselt number versus thermodynamic quality relationships for each test. Consequently, only representative plots of $\mathrm{Nu}$ versus $\mathrm{x}_{\mathrm{q}}$ are given in Figs. 15 through 18. Each figure compares counterflow to parallel flow for nominally the same Reynolds number and reduced pressure. The solid lines are predictions for the present micro-fin tube geometry which were obtained from the correlation of the data given as eqn. 10. The symbols are the measured data points. In general, parallel flow exhibits larger Nusselt numbers for the 0.3 to 0.7 quality range. Below and above this range, the Nusselt numbers for counterflow and parallel flow nearly coincide. Both the Nussult number measurements and the predictions for counterflow are concave with respect to quality. By contrast, the parallel flow Nusselt number measurements and predictions are convex with respect to quality. The difference in the Nusselt numbers in the mid-quality range may be due to the difference in the heat flux versus quality relationship between counterflow and parallel flow. The exact mechanism that is responsible for the difference between counterflow and parallel flow is not fully understood. Although the correlation predicts both the concave and convex trends with respect to quality, it, apparently, does not account for the entire difference between Nusselt numbers for the two flow conditions. Equation 10 consistently under-predicts the counterflow Nusselt numbers and over-predicts the parallel flow Nusselt numbers. 
Figure 19 compares the predictions of eqn. 4 to the local condensation heat transfer data that was available in the literature. All of the heat transfer coefficients taken from the literature were read from graphs and given based on the root-diameter area. Consequently, all of the heat transfer coefficients from the literature had to be adjusted so that they were based on the actual inner surface area of the tube in order to compare the literature data with eqn. 10. Cross-sectional schematics and tabulated dimensions of the tubes were used to calculate the surface areas and the hydraulic diameters of the micro-fin tubes from:

$$
D_{h}=\frac{4 A_{c} \cos \alpha}{N_{f} S}
$$

where $S$ is the perimeter of one fin and channel taken perpendicular to the axis of the fin, $N_{f}$ is the number of fins, $\mathrm{A}_{\mathrm{c}}$ is the cross-sectional flow area, and $\alpha$ is the helix angle of the fin.

Mori and Nakayama (1983) measured R113 quasi-local condensation heat transfer coefficients for three different micro-fin tube geometries. The Mori and Nakayama (1983) data for a $9.5 \mathrm{~mm}$ outer diameter tube with $60,0.16 \mathrm{~mm}$ high micro fins at a $20^{\circ}$ helix angle are presented as closed circles in Fig. 19. The Mori and Nakayama (1983) data presented in Fig. 19 are for a mass velocity of $160 \mathrm{~kg} / \mathrm{m}^{2} \bullet \mathrm{s}$. Most of this data lies within $\pm 20 \%$ of the predictions. Chiang (1993) graphically presented measured quasilocal condensation heat transfer coefficient for one of four micro-fin tube geometries that he tested. The local heat flux was estimated from $\mathrm{dx}_{\mathrm{q}} / \mathrm{dz}=0.09 / \mathrm{m}$ which was given by Chiang (1993). The Chiang (1993) data for R22 in a $10 \mathrm{~mm}$ outer diameter tube with $60,0.18 \mathrm{~mm}$ high micro fins at a $18^{\circ}$ helix angle and a mass velocity of $600 \mathrm{~kg} / \mathrm{m}^{2} \bullet \mathrm{s}$ are presented as closed rectangles in Fig. 19. Most of the Chiang (1993) data lies just below the $20 \%$ under-prediction line. The quasi-local condensation heat transfer measurements of Khanpara et al. (1986) for three different mass velocities $\left(223 \mathrm{~kg} / \mathrm{m}^{2} \bullet \mathrm{s}, 378\right.$ $\mathrm{kg} / \mathrm{m}^{2} \bullet \mathrm{s}$, and $570 \mathrm{~kg} / \mathrm{m}^{2} \bullet \mathrm{s}$ ) are shown as closed diamonds in Fig. 19. The local heat flux was estimated from quality change over the test section which was given as from 0.2 to 0.3 . The measured Nusselt numbers of Khanpara et al. (1986) for their intermediate mass velocity of $378 \mathrm{~kg} / \mathrm{m}^{2} \bullet$ s lie very close to those predicted by eqn. 10. However, the high-quality region of the low mass velocity data (223 $\mathrm{kg} / \mathrm{m}^{2} \bullet \mathrm{s}$ ) are over-predicted by more than $20 \%$. Similarly, nearly all of the high mass velocity (570 $\mathrm{kg} / \mathrm{m}^{2} \bullet \mathrm{s}$ ) Nusselt numbers are under-predicted by approximately $30 \%$.

The data from the literature are all within the mass velocity limits for which the correlation was developed. Also, the $e / D_{i}$ ratios for the tubes from the literature, with the exception of the Mori and Nakayama (1983) data, are approximately equivalent to the present tube (0.02). However, considering the wide range of tube diameters, helix angles, fluids, fin shapes, and estimates made for the literature data, the agreement between eqn. 10 and Nusselt numbers from the literature is to be expected. Also, it is very difficult to estimate the hydraulic diameter not knowing the exact pattern of the fins. This is especially true for the Chiang (1993) data which was for mechanically expanded micro-fin tubes. Certainly, the mechanical expansion process altered the fin profiles and increased the overall diameter of the tube. If a larger hydraulic diameter were to be used in the predictions, the Chiang (1993) agreement between the predictions and measurements would improve.

Although, eqn. 10 predicts the micro-fin data fairly well, the predictions of the cross-grooved micro-fin data of Chamra and Webb (1995) are under-predicted on average by $40 \%$. Possibly, the cross grooves provide an additional enhancement that is not accounted for by eqn. 10. The process of forming the cross groove creates a bump or a spill over into the major groove. The additional enhancement could be due to the high heat transfer that would occur on the leading edge of the bump. Equation 4 cannot account for the enhancement due to the bump. Also, the cross groove tubes were created from flat stock with a "W-shaped" fin axis then seam welded. Consequently, the main fin axis of the cross grooved tube did not rifle down the axis. Possibly, the non-spiraling fin also contributed to a difference in performance from the eqn. 10 prediction. 
Figure 20 shows the heat transfer degradation $\left(\Delta \mathrm{h}_{2 \phi}\right)$ as a function of heat flux for the R410A mixture at a mass flux of $246 \mathrm{~kg} / \mathrm{m}^{2} \mathrm{~s}$. The $\Delta \mathrm{h}_{2 \phi}$ was calculated from the correlations using the same definition as given in Kedzierski et al. (1992):

$$
\Delta h_{2 \phi}=h_{L}-h_{2 \phi}
$$

where $h_{L}$ is the heat transfer coefficient obtained from a linear interpolation of the pure components at a given composition. The heat transfer degradation is independent of heat flux and no greater than $2.5 \%$. The average difference between the vapor and liquid concentration was 0.02 mole, suggesting that the degradation was mostly due to property effects.

\section{Pressure Drop}

Pierre (1964) developed the following semi-empirical equation to predict the pressure drop for flow boiling in a horizontal smooth tube:

$$
\Delta \mathrm{P}=\left(0.0185\left(\frac{\Phi}{\operatorname{Re}}\right)^{1 / 4}+\frac{\Delta \mathrm{x}_{\mathrm{q}} \mathrm{D}_{\mathrm{h}}}{\mathrm{x}_{\mathrm{q}_{\mathrm{m}}} \Delta \mathrm{L}}\right) \frac{\Delta \mathrm{L}}{\mathrm{D}_{\mathrm{h}}} \mathrm{G}^{2} \mathrm{x}_{\mathrm{qm}} v_{\mathrm{v}}
$$

where the specific volume of the vapor $\left(\nu_{v}\right)$, the Reynolds number, the mass velocity $(\mathrm{G})$, and the twophase number ( $\Phi=\frac{\Delta \mathrm{x}_{\mathrm{q}} i_{f g}}{\Delta \mathrm{L} g}$ ) are evaluated at the average temperature of the refrigerant. The average quality over length $\Delta \mathrm{L}$ is $\mathrm{x}_{\mathrm{qm}}$. The above correlation originates from the first law of thermodynamics. The first term within the bracket represents the dimensionless friction factor. The second term is from the acceleration portion of the pressure drop. Pierre (1964) fitted his pressure drop measurements to eqn. 6 to obtain an expression for the friction factor. With the exception of the friction factor which may be germane to flow boiling, eqn. 6 applies to condensation as well as to evaporation. One might expect that pressure drops due to flow boiling (in the absence of nucleate boiling) and condensation may be governed by the same physical phenomenon. If so, then the Pierre (1964) correlation should work as well for convective condensation as it does for convective evaporation.

Figure 21 compares the micro-fin condensation pressure drop to Pierre (1964) flow boiling pressure drop correlation. The hydraulic diameter was used in the Pierre (1964) correlation to predict most of the condensation pressure drop measurements in the present micro-fin tube to within $\pm 20 \%$. Pierre recommends that eqn. 14 not be used for values of $\frac{R e L}{\Delta x_{q} i_{f g}}$ greater than 1 . Only three data points in the present data set violated this criteria. Overall, the Pierre (1964) correlation predicts the present convective condensation pressure drop measurements for the micro-fin tube acceptably well. However, the mean of the predictions lies slightly below the ideal prediction line.

Following the development of the Pierre (1964) pressure drop equation, the Fanning friction factor for the present data was calculated as:

$$
f_{r}=\frac{D_{h}}{\left(v_{0}+v_{i}\right) \Delta L}\left(\frac{\left(P_{i}-P_{o}\right)}{G^{2}}-\left(v_{o}-v_{i}\right)\right)
$$

where specific volume of the fluid $(\nu)$ was calculated from a thermodynamic quality weighted average of the liquid and vapor specific volumes. Only the two central portions of the test section (Fig. 3 ) were 
used to ensure that all of the correlated data were for two-phase flow. Appendix A shows the data that was used to calculate the friction factor from eqn. 14.

In order to center the friction factor predictions about the data, the measured friction factors were regressed to the following:

$$
f_{r}=0.00228 \operatorname{Re}^{-0.062} \Phi^{0.211}
$$

Twenty-six data points were removed from the fit because they had high influence and high leverage on the fit. The deleted data were for somewhat low pressure drop values which are associated with larger relative measurement uncertainties.

The exponent on the two-phase number given in eqn. 16, 0.211, is consistent with that given by Pierre (1964): 0.24. However, the exponent on the Reynolds number, -0.062 , is very different from that given by Pierre (1964): -0.24. The exponent on the Reynolds number of eqn. 16 is approximately equivalent to -0.06 which is the exponent that one would calculate from the transition zone of the Moody (1944) chart using the fin height $(2 \mathrm{~mm})$ for the roughness height. Consequently, the new friction factor should be more appropriate for micro-fins because it accounts for the influence of the fins on the flow.

The fact that the Reynolds number exponent is consistent with that obtained from the Moody (1944) chart suggests that the fins of a micro-fin tube act like a roughness to enhance the convective condensation heat transfer. If roughness mixing dominates the enhancement mechanism, neither swirl effects nor surfacetension drainage have much influence on the heat transfer. The lack of importance of surface-tension and swirl flow may be a consequence of the flow conditions and surface geometry. It may be possible that the roughness effect would not dominate for other flow conditions and micro-fin tubes with larger heights.

The corroboration between the present Re exponent and Moody's (1944) also suggests that the frictional pressure drop of micro-fin tubes should depend on the fin-height-to-root-tube-diameter $\left(e / D_{i}\right)$ ratio. If it is assumed that the fins act purely as a roughness, the Moody (1944) chart can be used to interpolate between the eqn. 16 friction factor and Pierre's (1964) smooth tube friction factor for a given e/ $D_{i}$ ratio as follows:

$$
f_{r}=\left(0.002275+0.00933 \exp \left(\frac{e / D_{i}}{-0.003}\right)\right) e^{\frac{-1}{4.16+532 \frac{e}{D_{i}}}} \Phi^{0.211}
$$

In the above equation, the functional forms of the leading coefficient and the Reynolds number exponent with respect to $\mathrm{e} / \mathrm{D}_{\mathrm{i}}$ were determined from the Moody (1944) chart. Also, Pierre's (1964) friction factor equation was modified for use in the interpolation. Namely, the exponent on the two-phase number of Pierre's (1964) friction factor correlation was set equal to that of eqn. 16 and the leading coefficient on Pierre's correlation was adjusted to give approximately the same results, for our data set, as the original equation.

The pressure drop equation for which eqns. 16 and 17 are valid is:

$$
\Delta P=\left(\frac{f_{r}\left(v_{0}+v_{i}\right) \Delta L}{D_{h}}+\left(v_{0}-v_{i}\right)\right) G^{2}
$$

This is essentially the same equation as given by Pierre (1964) with the exception that the specific volume 
of the liquid is not neglected. The uncertainty of the fit was reduced when the liquid specific volume was included.

\section{CONCLUSIONS}

Local convective condensation measurements for four refrigerant fluids: R134a, R410A(R32/R125, 50/50

$\%$ mass), R125, and R32 in a micro-fin tube were presented. Both heat transfer and pressure drop measurements were provided. The measured convective condensation Nusselt numbers for all of the test refrigerants were correlated to a single expression consisting of a product of dimensionless properties. The correlation was shown to predict existing condensation Nusselt numbers for micro-fins from the literature acceptably well. However, the correlation poorly predicted the Nusselt numbers for micro-fins with cross-grooves. It was speculated that bumps from the cross-grooves caused an additional enhancement that could not be accounted for by the correlation. The hydraulic diameter was used in an existing flow boiling correlation from the literature to predict most of the condensation pressure drop measurements in the present micro-fin tube to within $\pm 20 \%$.

In general, the measured condensation heat-transfer coefficient decreased with decreasing qualities. The refrigerant R32 exhibited the highest heat transfer performance due to its high thermal conductivity. As expected, the performance of the near-azeotropic mixture R410A was between that of its pure components $\mathrm{R} 32$ and R125. The heat transfer degradation associated with R410A was shown to be relatively small and believed to be mostly due to nonlinear property effects.

The enhancement ratio was shown to span from 3.6 at low $\operatorname{Re}$ to 0.6 at high $\operatorname{Re}$. It was speculated that the micro-fins enhanced the heat transfer with a combination of liquid-vapor interface mixing and turbulent mixing near the wall. The surface behaves as a roughness in the enhancement of the heat transfer. Surface-tension drainage and swirl effects are presumed to have little influence on the heat transfer. At high vapor qualities and thin liquid films on the surface, the fins act to mix the liquid-vapor interface. At lower vapor qualities and thicker liquid films on the surface, the fins have less influence on the liquid-vapor interface. The heat transfer enhancement with respect to the Reynolds number may result from an interaction between the fins and the turbulence in the liquid film. Low Reynolds number flows may be enhanced more readily than high Reynolds number flows due to the reduction in the size of the turbulent eddies at the wall by the interaction of the flow with the fins. High Reynolds number flows are not enhanced as readily as low Reynolds number flows because there are fewer large eddies to be reduced.

The condensation pressure drop for the micro-fin tube was predicted acceptably well with a modified form of the Pierre (1964) pressure drop correlation for convective evaporation. The hydraulic diameter was used and a new friction factor was developed to account for the fin effect on the flow. The influence of the fin height on the Reynolds number exponent was consist with the Moody chart, suggesting that the surface behaves like a roughness in enhancing the heat transfer.

\section{ACKNOWLEDGEMENTS}

This work was jointly funded by NIST, and the U.S. Department of Energy (project no. DE-AI0191CE23808) under Project Manager Esher Kweller. Mr. Joaquim Goncalves's NIST sabbatical was funded by CNPq of Brazil (Proj. \# 260042/95.1). The authors thank the following NIST personnel for their constructive criticism of the first draft of the manuscript: Dr. Vance Payne, Dr. Jiann Yang and Mrs. Janet Land. The authors express their appreciation to Mr. Michael Kaul, Mr. Michael Connaghan and Mr. Peter Rothfleisch for their assistance in the construction of the test rig. Furthermore, the authors extend their appreciation to Dr. E. Lagergren and Dr. S. Leigh for their valuable consultations on the uncertainty analysis. Thanks also goes to Wolverine Tube, Inc., for suppling the Turbo-A, micro-fin tube for the test section. 


\section{REFERENCES}

Ackers, W. W. and Rosson, H. F., 1960, Condensation inside a horizontal tube. Chemical Engineering Process Symposium Series, Vol. 56, No.30, pp. 145-149.

Baker, O., 1954, Simultaneous flow of oil and gas, The Oil and Gas Journal, Vol. 53, pp. 85-195.

Belsley, D. A., Kuh, E., and Welsch, R. E., 1980, Regression Diagnostics: Identifying Influential Data and Sources of Collinearity, New York: Wiley.

Bergles, A. E., Jensen, M. K., and Shome, B., 1995, "Bibliography on Enhancement of Convective Heat and Mass Transfer, " HTL-23, Rensselaer Polytechnic Institute, Troy, NY.

Chiang, R., 1993, "Heat Transfer and Pressure Drop During Evaporation and Condensation of Refrigerant-22 in $7.5 \mathrm{~mm}$ Diameter Axial and Helical Grooved Tubes, " AIChE Symposium Series No. 295, Vol. 89, pp. 205-210.

Chamra, L. M., and Webb, R. L., 1995, "Condensation and Evaporation in Micro-Fin Tubes at Equal Saturation Temperatures," J. or Enhanced Heat Transfer, Vol. 2, pp. 219-229.

Cooper, M.G., 1984, Saturation Nucleate Pool Boiling-A Simple Correlation Vol. 86, Department of Engineering Science, Oxford University, England, pp. 785-793.

Dittus, F. W. and Boelter, L. M. K., 1930, U. CA, Publ. Eng., Vol. 2, p. 443.

Eckels, S. J., Pate, M. B., and Bemisderfer, C. H., 1992, "Evaporation heat transfer coefficients for R22 in micro fin tubes of different configuration," Enhanced Heat Transfer, ASME, HTD-Vol. 202, pp. 117-126.

Eckels, S. J., Doerr, T. B., and Pate, M. B., 1994, "In-Tube Heat Transfer and Pressure Drop of R134a and Ester Lubricant Mixtures in a Smooth Tube and Micro-Fin Tube:Part II - Condensation, " $\underline{\text { ASHRAE }}$ Trans., Vol. 100, pp. 283-294.

Ghaderi, M., Salehi, M., Saeedi, M. H., 1995, Review of in-tube condensation heat transfer correlations for smooth and enhanced tubes, Advances in Enhanced Heat/Mass Transfer and Energy Efficiency ASME, HTD-Vol.320/PID-Vol.1, pp. 83-94.

Huber, M., Gallagher, J., McLinden, M., and Morrison, G., 1995, NIST Standard Reference Database 23, Version 5.0. Standard Reference Data Program, National Institute of Standards and Technology, Gaithersburg, MD.

Kattan, N., Favret, D., and Thome, J. R., 1995, "R-502 and Two Near-Azeotropic Alternatives: Part I - In Tube Flow-Boiling Tests," ASHRAE Trans., Vol. 101, Pt. 1, pp. 491-508.

Kaul, M. P., Kedzierski, M. A., and Didion, D. A., 1996, "Horizontal Flow Boiling of Alternative Refrigerants within a Fluid Heated Micro-Fin Tube," Process, Enhanced, and Multiphase Heat Transfer: A Festschrift for A. E. Bergles, Begell House, Inc., New York, pp. 167-173.

Kedzierski, M. A., and Kim, M. S., 1997, "Convective Boiling and Condensation Heat Transfer with a Twisted-Tape Insert for R12, R22, R152a, R134a, R290, R32/R134a, R32/R152a, R290/R134a, R134a/R600a," NISTIR 5905, U.S. Department of Commerce, Washington, D.C.

Khanpara, J. C., Bergles, A. E., and Pate, M. B., 1986, "Augmentation of R-113 In-Tube Condensation with Micro-Fin Tubes," Heat Transfer in Air Conditioning and Refrigeration Equipment, ASME, HTD- 
Vol. 65, pp. 21-32.

Koyiama, S., Miyara, A., Takamatsu, H., Fujii, T., 1990, Condensation heat transfer of binary refrigerant mixtures of R22 and R114 inside a horizontal tube with internal spiral grooves, International Journal of Refrigeration, Vol. 13, July, pp. 256-263.

Moody, L. F., 1944, "Friction Factors for Pipe Flow," ASME Trans., Vol. 66, pp. 671-684.

Mori, Y., and Nakayama, W., 1983, "High-Performance Mist Cooled Condensers for Geothermal Binary Cycle Plants," Heat Transfer in Energy Problems, Hemisphere, Washington, DC, pp. 211-218.

Muir, E. B., 1989, "Commercial Refrigerants and CFCs," CFCs: Today's Options- Tomorrow's Solutions, Proceedings of ASHRAE's 1989 CFC Technology Conference, pp. 81-86.

Pierre, B., 1964, "Flow Resistance with Boiling Refrigerants-Part 1," ASHRAE Journal, Vol. 6, No. 9, pp. 58-65.

Schlager, L. M., Pate, M. B., and Bergles, A. E., 1989, "Heat Transfer and Pressure Drop During Evaporation and Condensation of R22 in Horizontal Micro-Fin Tubes," Int. J. Refig., Vol. 12, pp. 6-14.

Schlager, L. M., Pate, M. B., and Bergles, A. E., 1990, "Evaporation and Condensation Heat Transfer and Pressure Drop in Horizontal, 12.7-mm Microfin Tubes with Refrigerant 22," J. Heat Transfer, Vol. 112, pp. 1041-1047.

Shah, M. M., 1979, A general correlation for heat transfer during film condensation inside pipes, International Journal of Heat and Mass Transfer, Vol. 22, pp. 547-556.

Wang, C., Kuo, C. S., Chang, Y., and Lu, D. C., 1996, "Two-Phase Flow Heat Transfer and Friction Characteristics of R-22 and R-407C," ASHRAE Transactions, Vol. 102, Pt. 1, pp. 830-838.

Webb, R. L., 1994, Principles of Enhanced Heat Transfer, Wiley Interscience.

Wiegand, J. H., 1945 "Discussion of Paper by McMillen and Larson, Trans. AIChE, Vol. 41, p. 147. 
Table 1 Median estimated $95 \%$ relative expanded uncertainties for eqn. 10 data

\begin{tabular}{|c|c|c|c|}
\hline Parameter & Minimum & Maximum & U \% \\
\hline $\mathrm{G}_{\mathrm{r}}\left[\mathrm{kg} / \mathrm{m}^{2} \bullet \mathrm{s}\right]$ & 57 & 552 & 2.0 \\
\hline $\mathrm{T}_{\mathrm{r}}[\mathrm{K}]$ & 293.0 & 323.0 & $0.1(0.3 \mathrm{~K})$ \\
\hline $\mathrm{P}[\mathrm{kPa}]$ & 600 & 2000 & 1.5 \\
\hline $\mathrm{T}_{\mathrm{w}}[\mathrm{K}]$ & 288.0 & 318.0 & $0.1(0.35 \mathrm{~K})$ \\
\hline$\dot{\mathrm{m}}_{\mathrm{f}}[\mathrm{kg} / \mathrm{s}]$ & 0.0150 & 0.0450 & 2.0 \\
\hline $\mathrm{T}_{\mathrm{f}}[\mathrm{K}]$ & 278.0 & 318.0 & 0.1 \\
\hline $\mathrm{P}_{\mathrm{f}}[\mathrm{kPa}]$ & 200 & 110 & 1.0 \\
\hline $\mathrm{q}^{\prime \prime}\left[\mathrm{kW} / \mathrm{m}^{2}\right]$ & 0.72 & 39 & 5.1 \\
\hline $\mathrm{dT}_{\mathrm{f}} / \mathrm{dz}[\mathrm{K} / \mathrm{m}]$ & 0.014 & 0.57 & 5.2 \\
\hline$\Delta \mathrm{T}_{\mathrm{s}}[\mathrm{K}]$ & 0.41 & 12.6 & $15.2(0.44 \mathrm{~K})$ \\
\hline $\mathrm{Nu}$ & 58 & 508 & 16.4 \\
\hline $\mathrm{Re}$ & 3500 & 24000 & 1.0 \\
\hline $\mathrm{Ja}$ & 6 & 256 & 16.6 \\
\hline $\operatorname{Pr}$ & 1.7 & 3.6 & 2.0 \\
\hline $\mathrm{P}_{\mathrm{r}} / \mathrm{Pc}$ & 0.22 & 0.62 & 2.0 \\
\hline Sv & 0.86 & 10.3 & 3.0 \\
\hline$x_{q}$ & 0.06 & 1.0 & 8.0 \\
\hline
\end{tabular}


Table 2 Data distribution

\begin{tabular}{|c|c|c|c|c|c|}
\hline & R134a & R410A & R125 & R32 & Total \\
\hline \# tests & 46 & 28 & 30 & 38 & 142 \\
\hline \# points & 552 & 336 & 360 & 456 & 1704 \\
\hline $\begin{array}{c}\text { \# pts used in } \\
\text { fit }\end{array}$ & 447 & 269 & 309 & 342 & 1367 \\
\hline
\end{tabular}




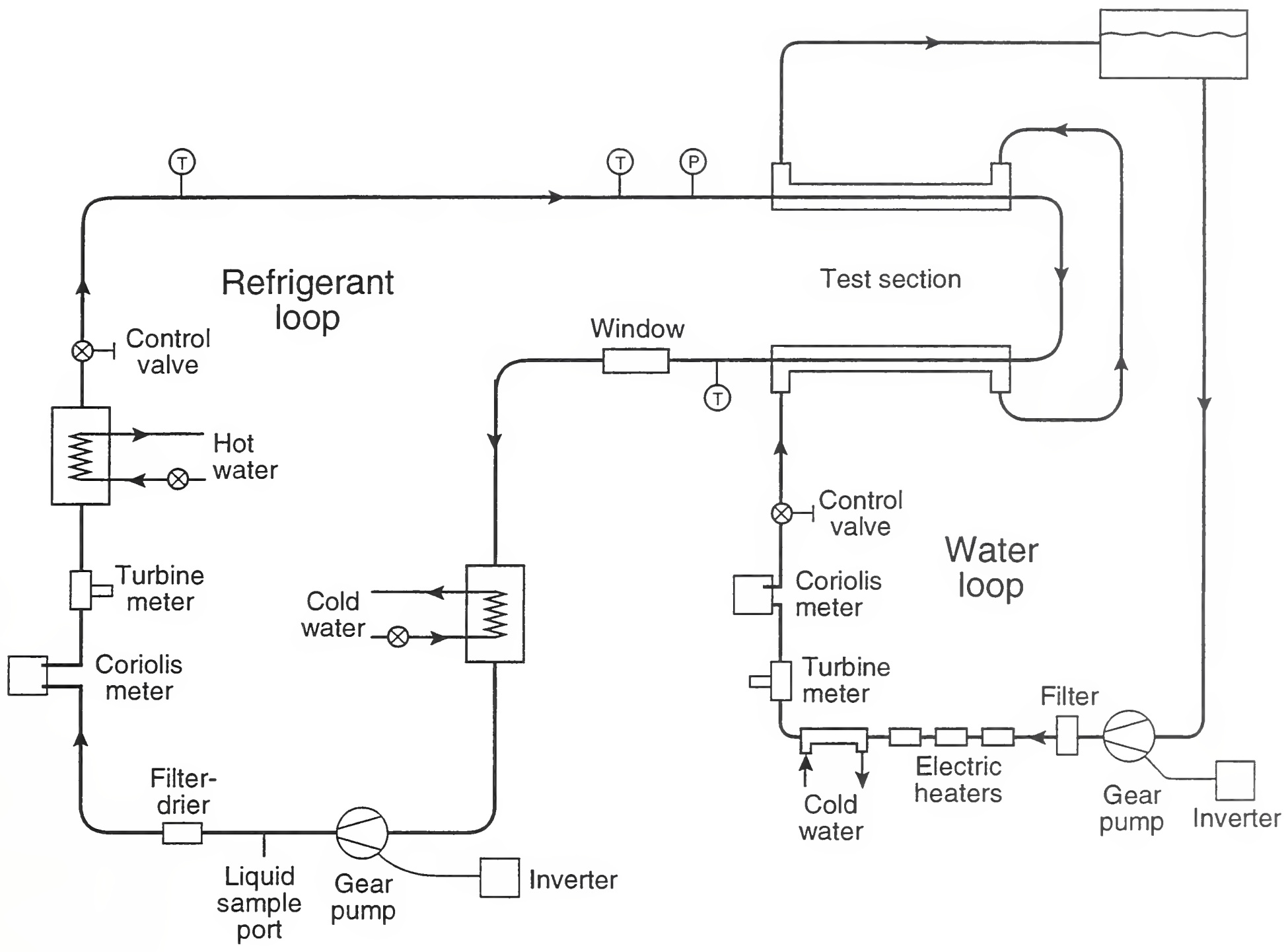

Fig. 1 Schematic of test rig 


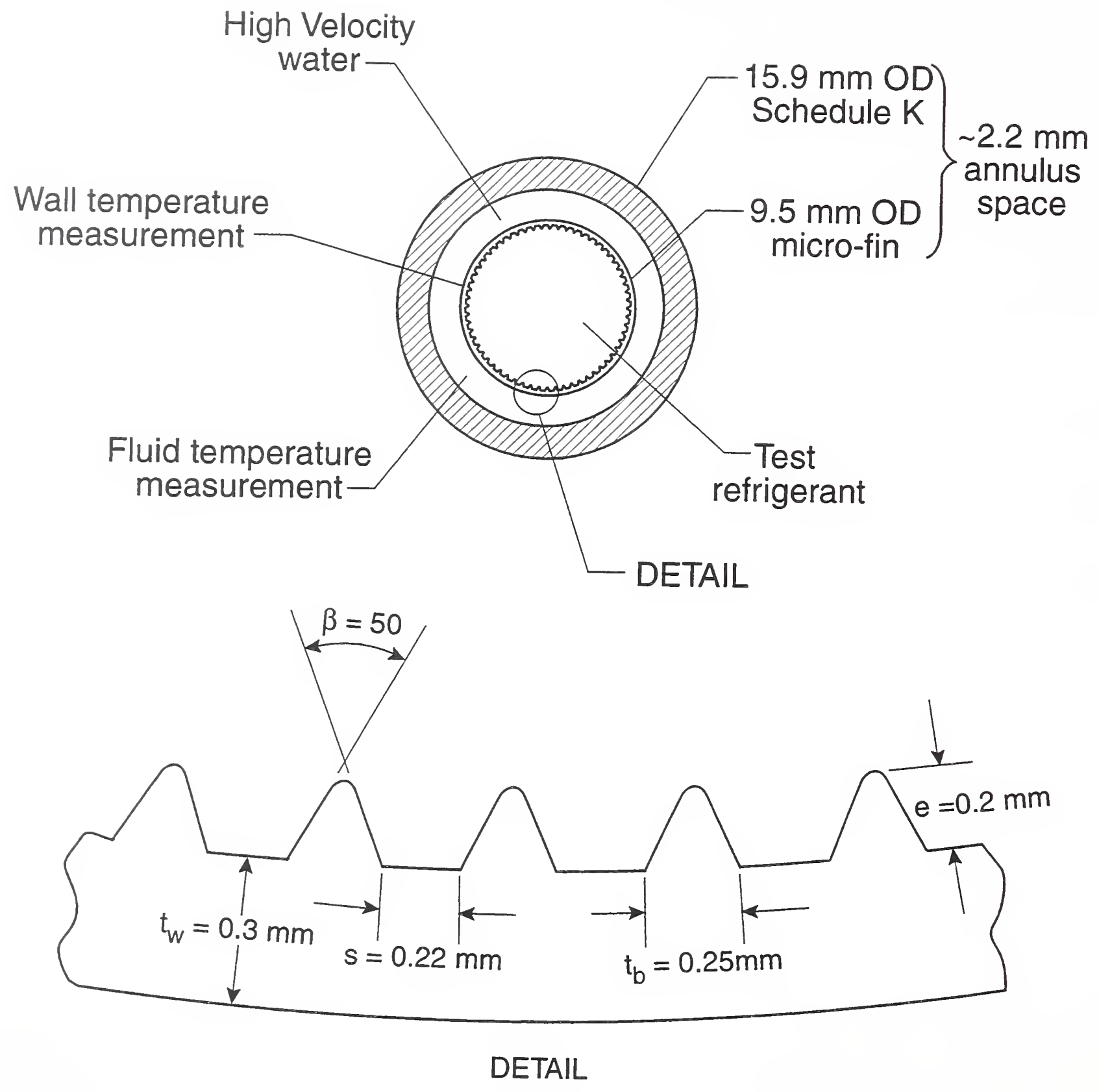

Fig. 2 Test section cross section 


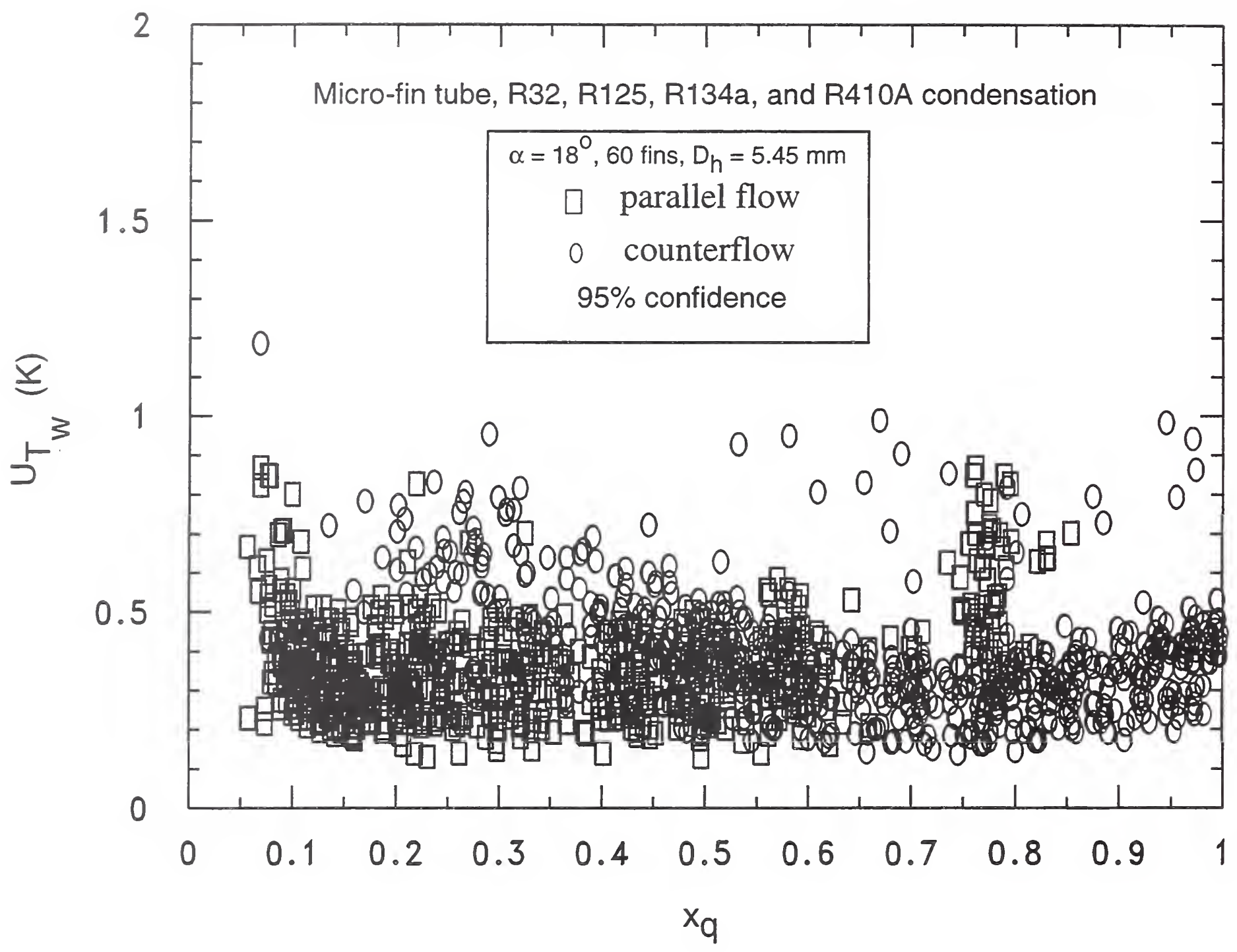

Fig. 4 Relative uncertainty of inner root surface temperature 


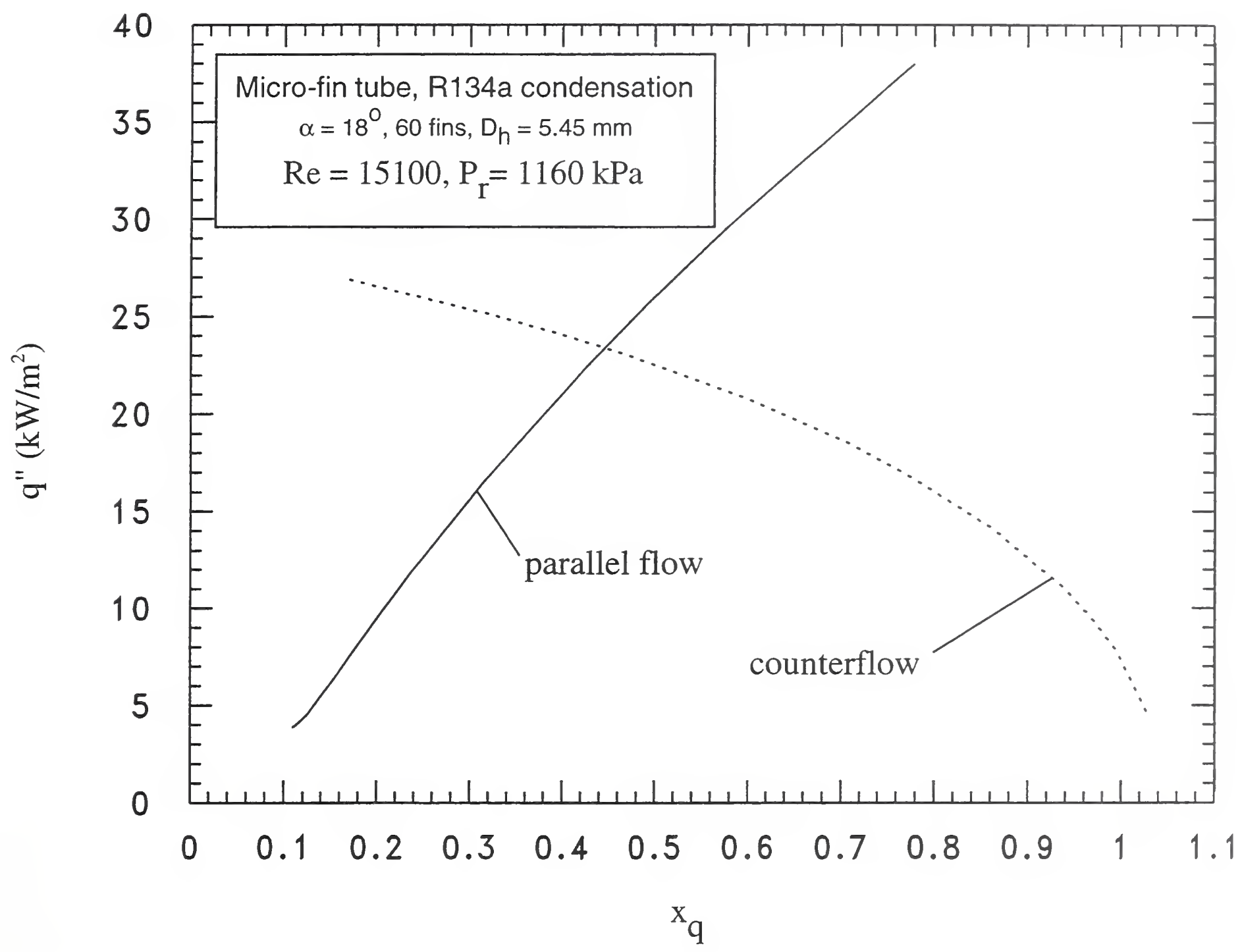

Fig. 5 Comparison of counterflow and parallel flow heat flux distributions 


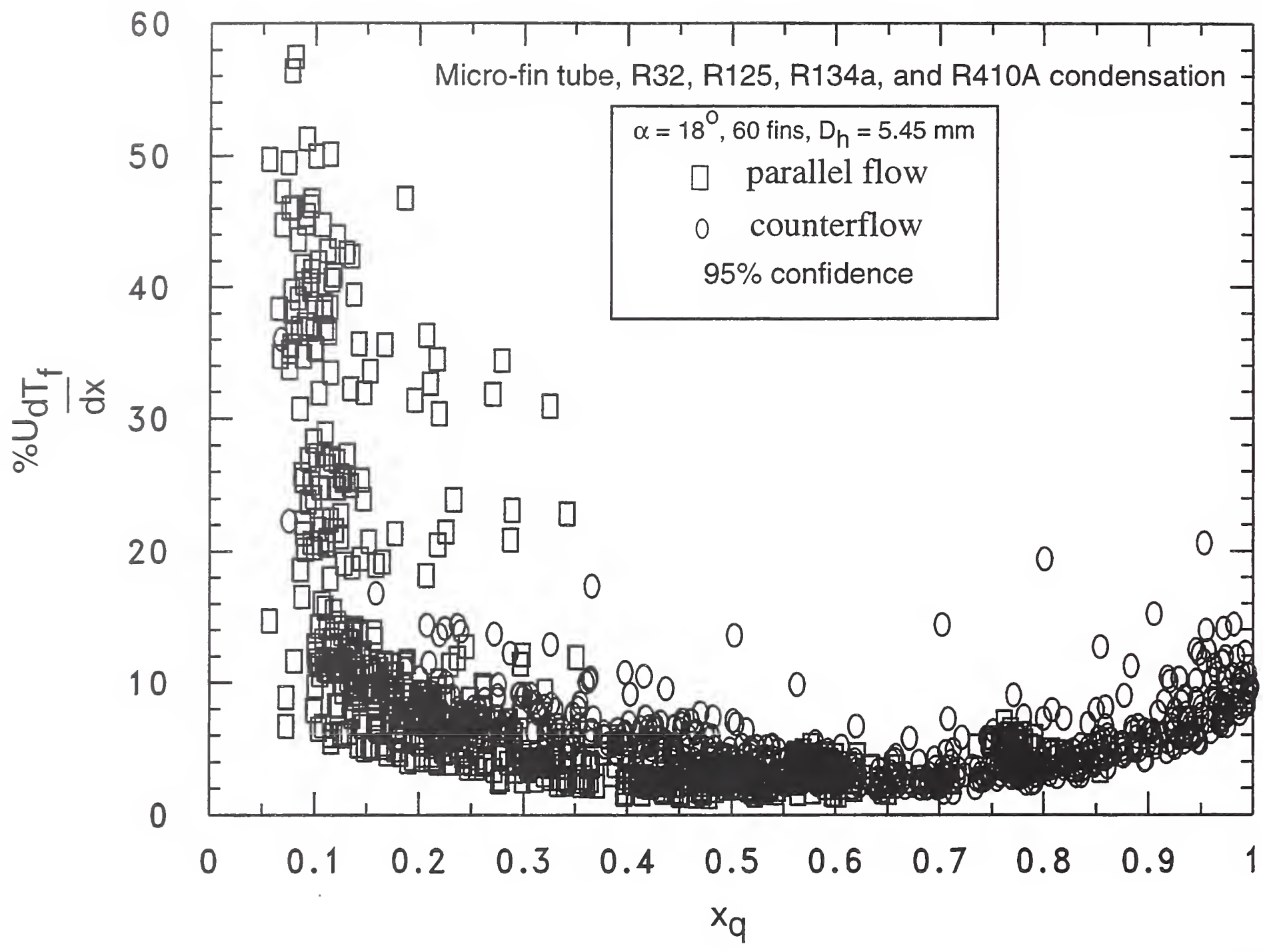

Fig. 6 Relative uncertainty of water temperature gradient with respect to quality 


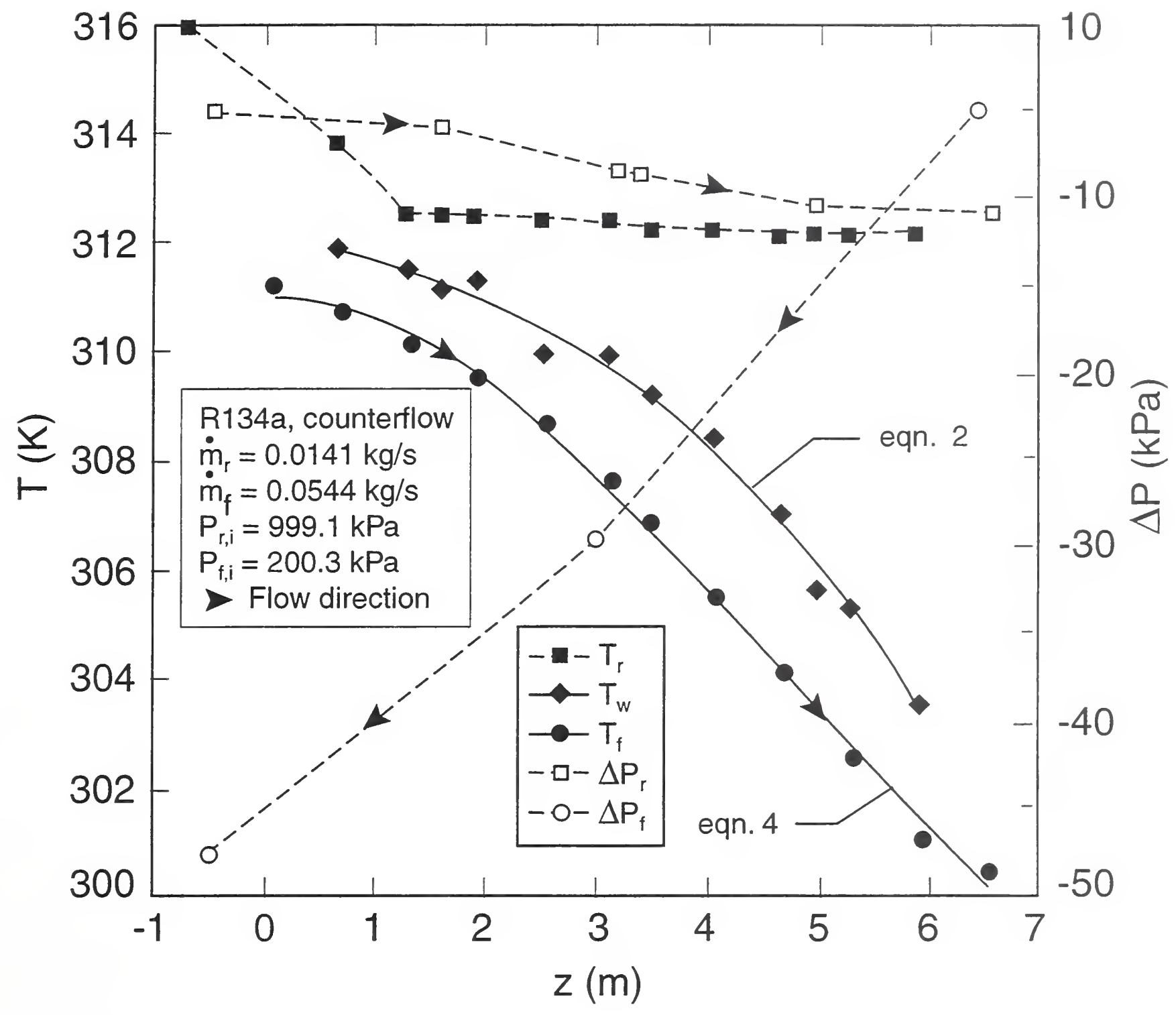

Fig. 7 Counterflow temperature and pressure profiles for a R134a test 


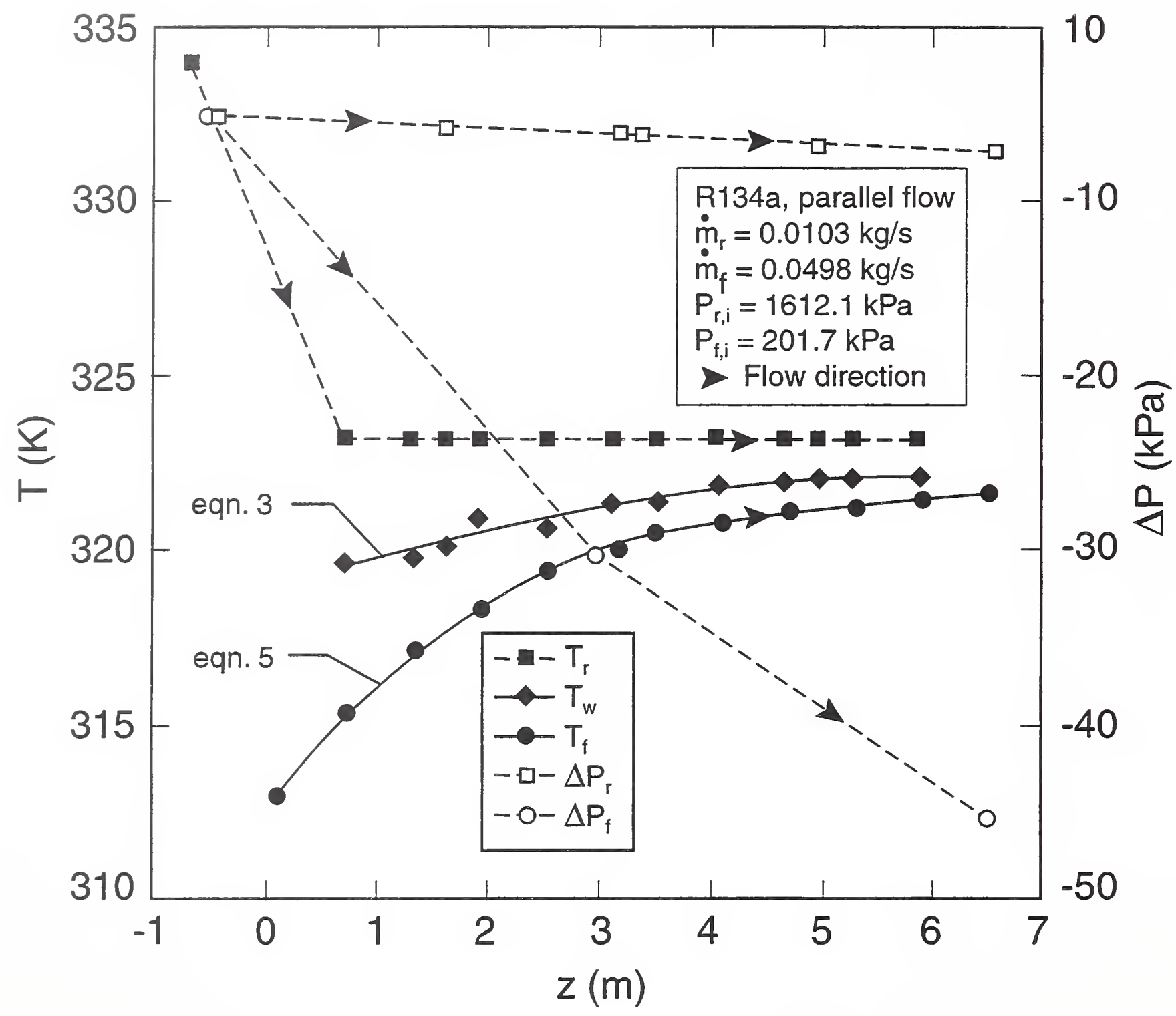

Fig. 8 Parallel flow temperature and pressure profiles for a R134a test 


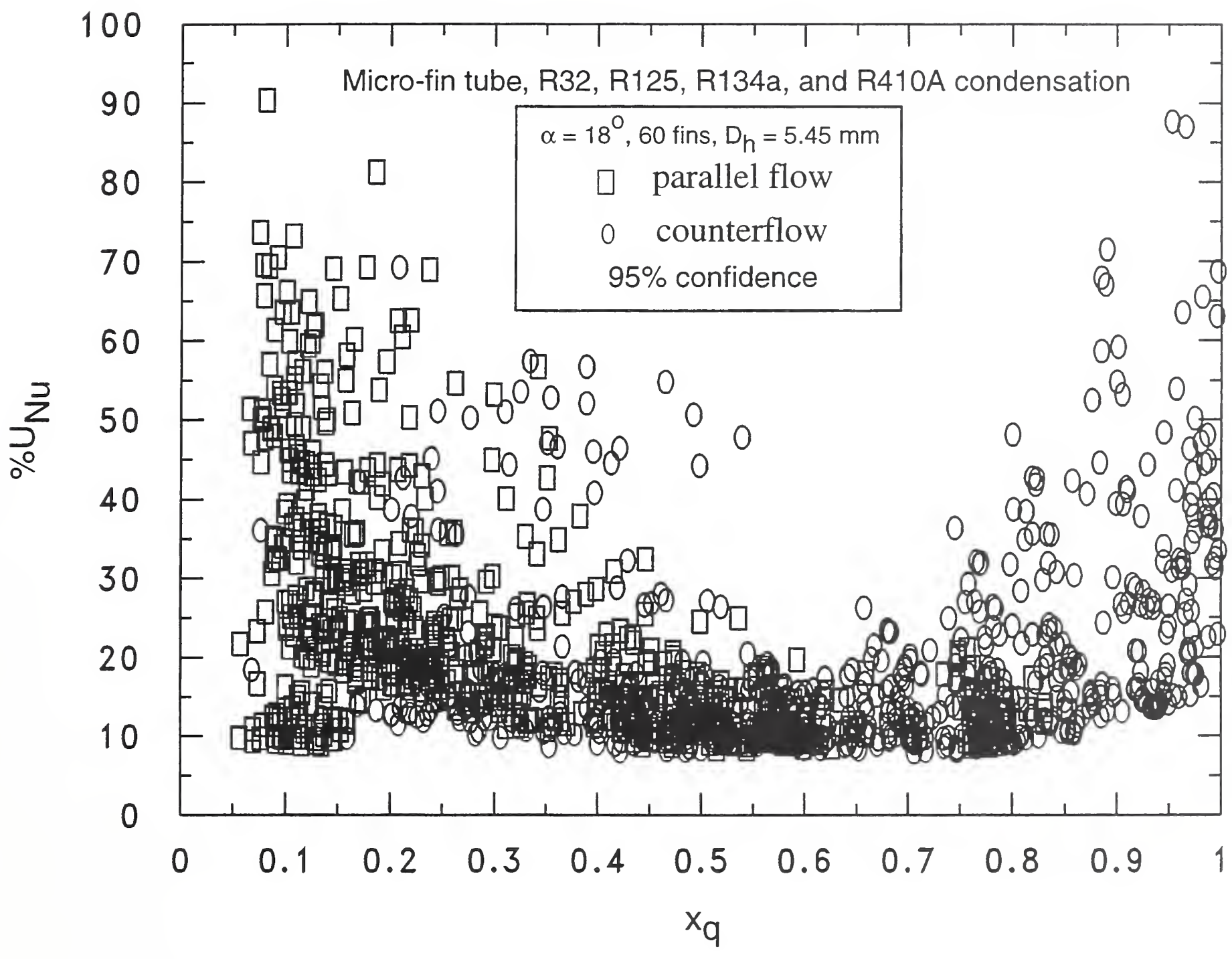

Fig. 9 Relative uncertainty of the Nusselt number with respect to the quality 


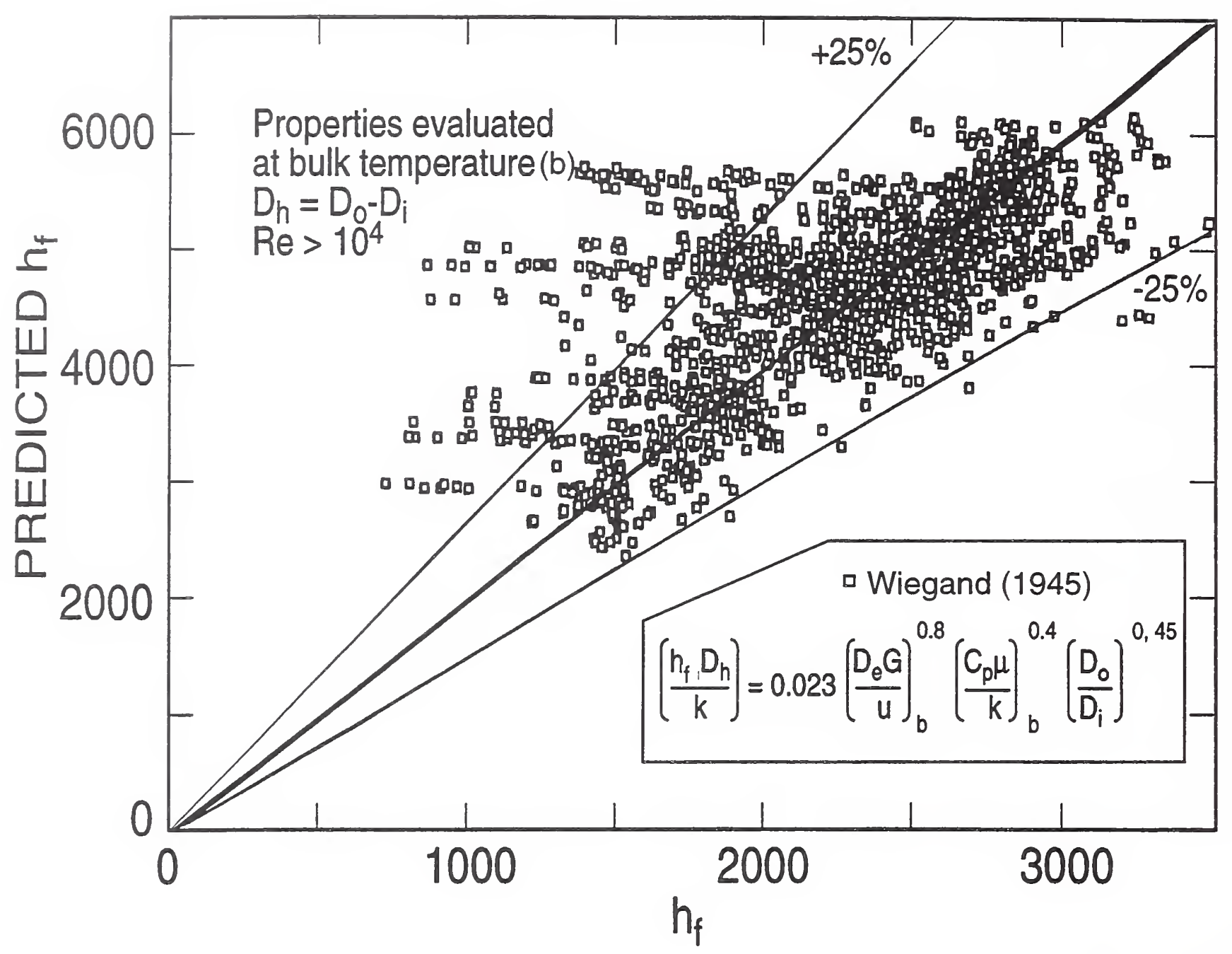

Fig. 10 Corroboration of measurement procedure 


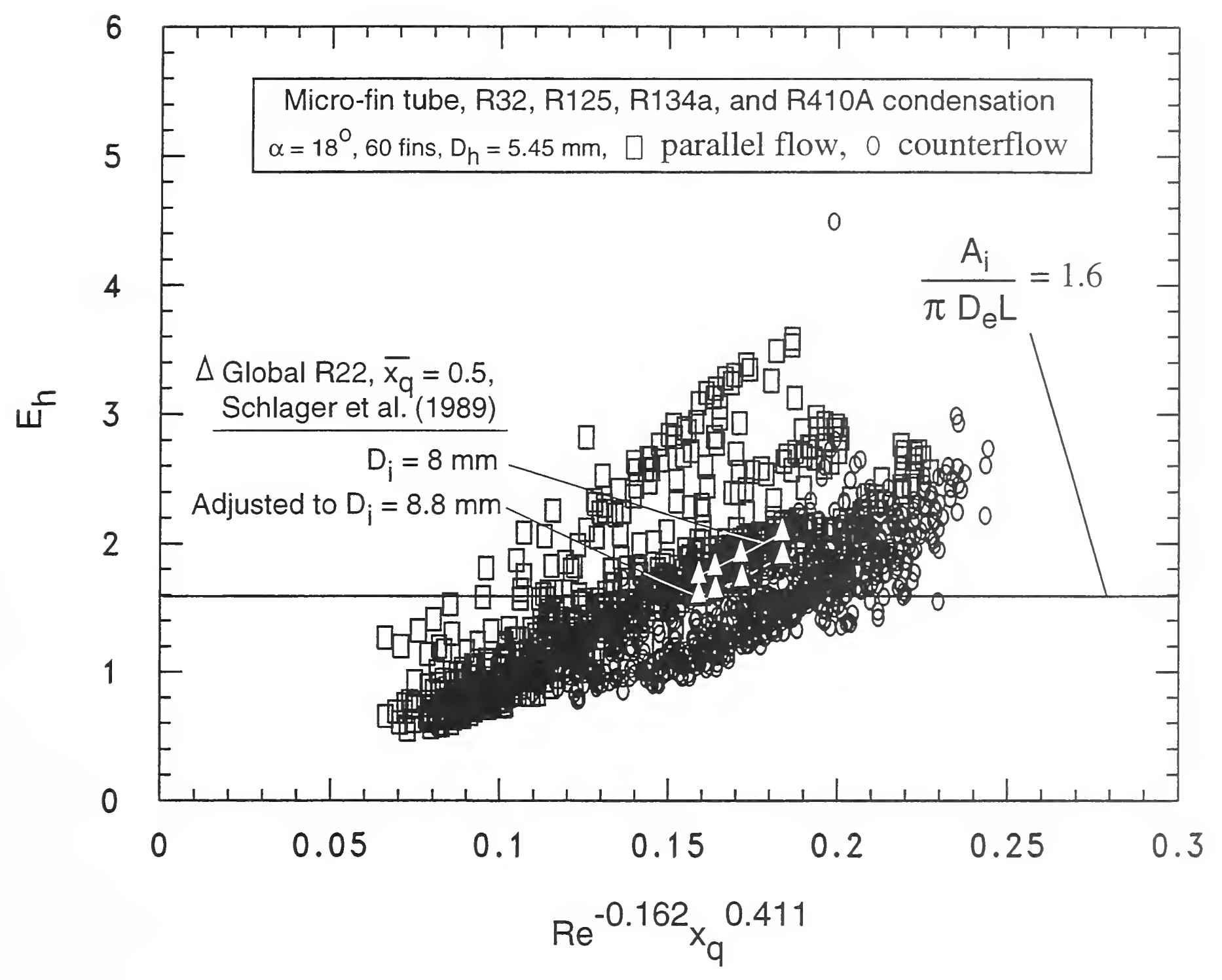

Fig. 11 Condensation heat transfer enhancement ratio for micro-fin tube 


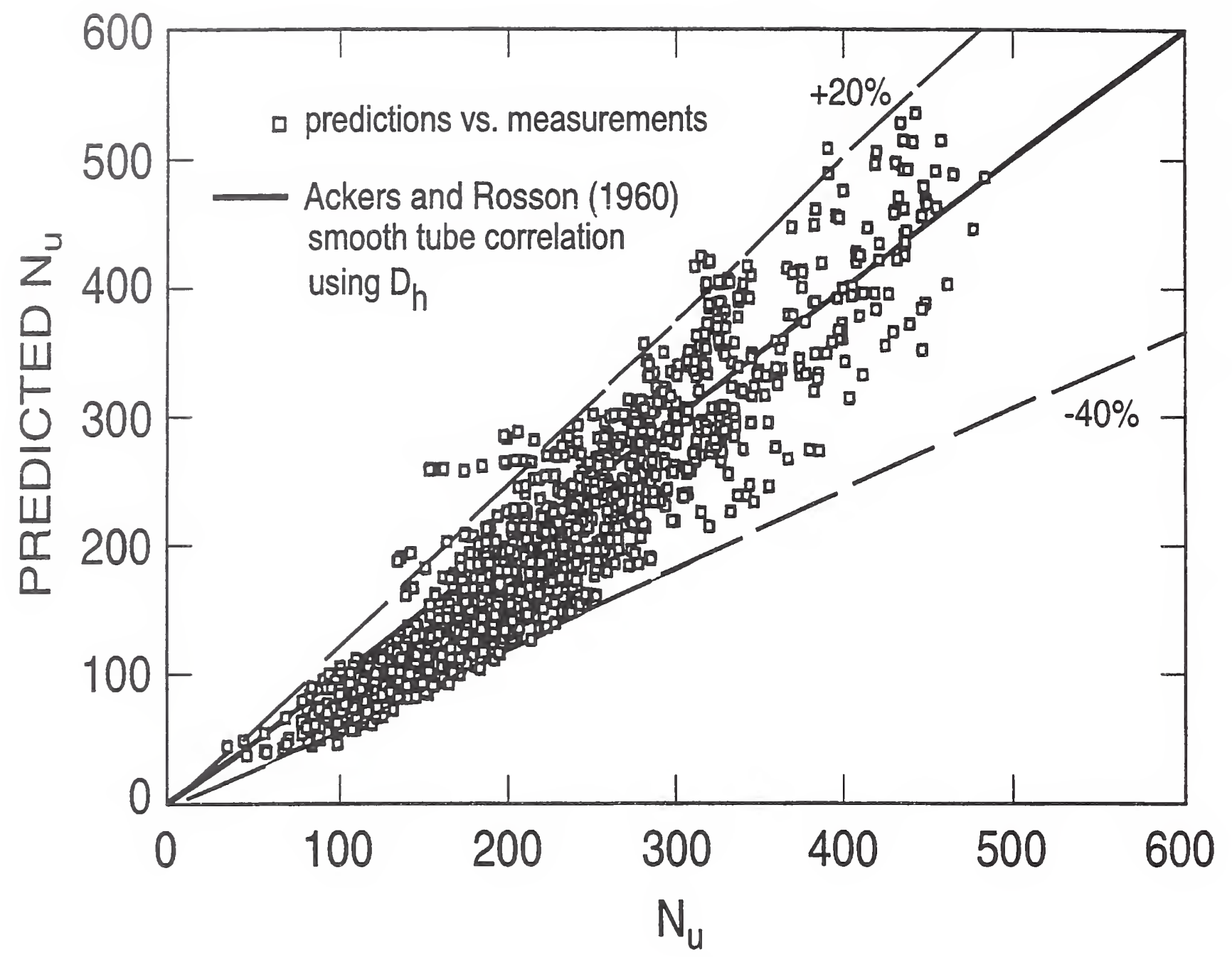

Fig. 12 Comparison of measured convective condensation Nusselt numbers to those predicted with Ackers and Rosen (1960) using $D_{h}$ 


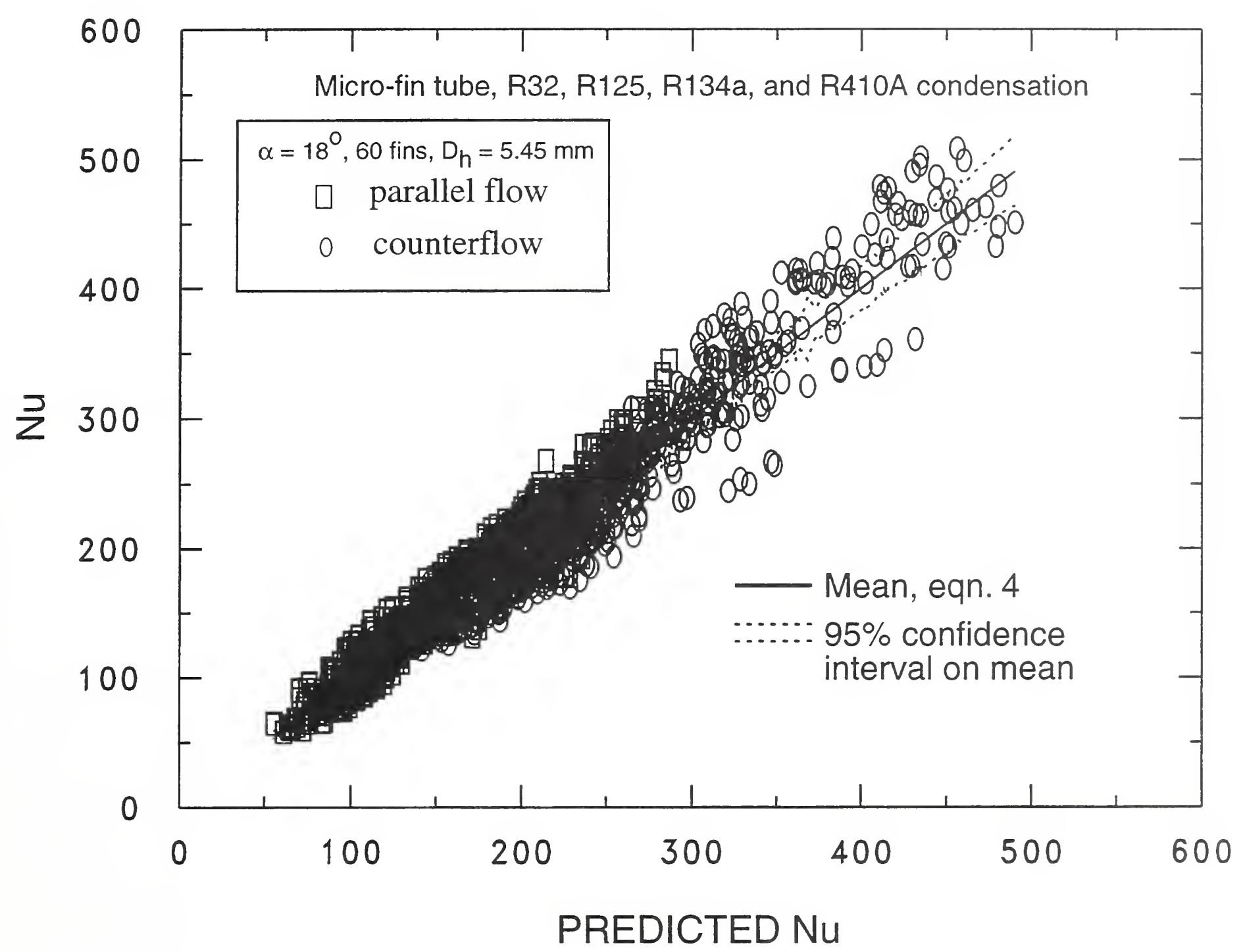

Fig. 13 Comparison of measured and predicted convective condensation Nusselt numbers 


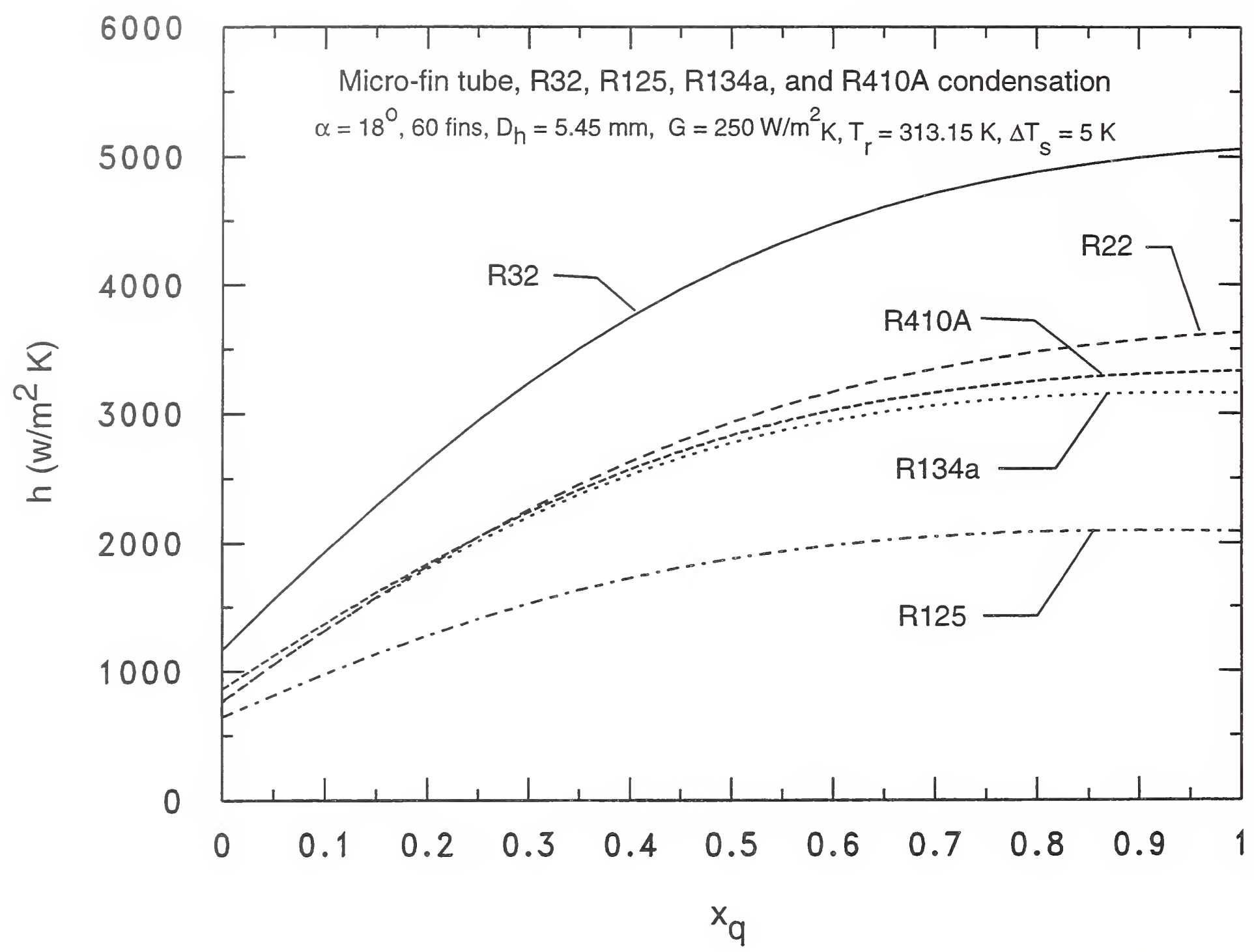

Fig. 14 Comparison of the heat-transfer coefficient for different fluids versus thermodynamic quality 


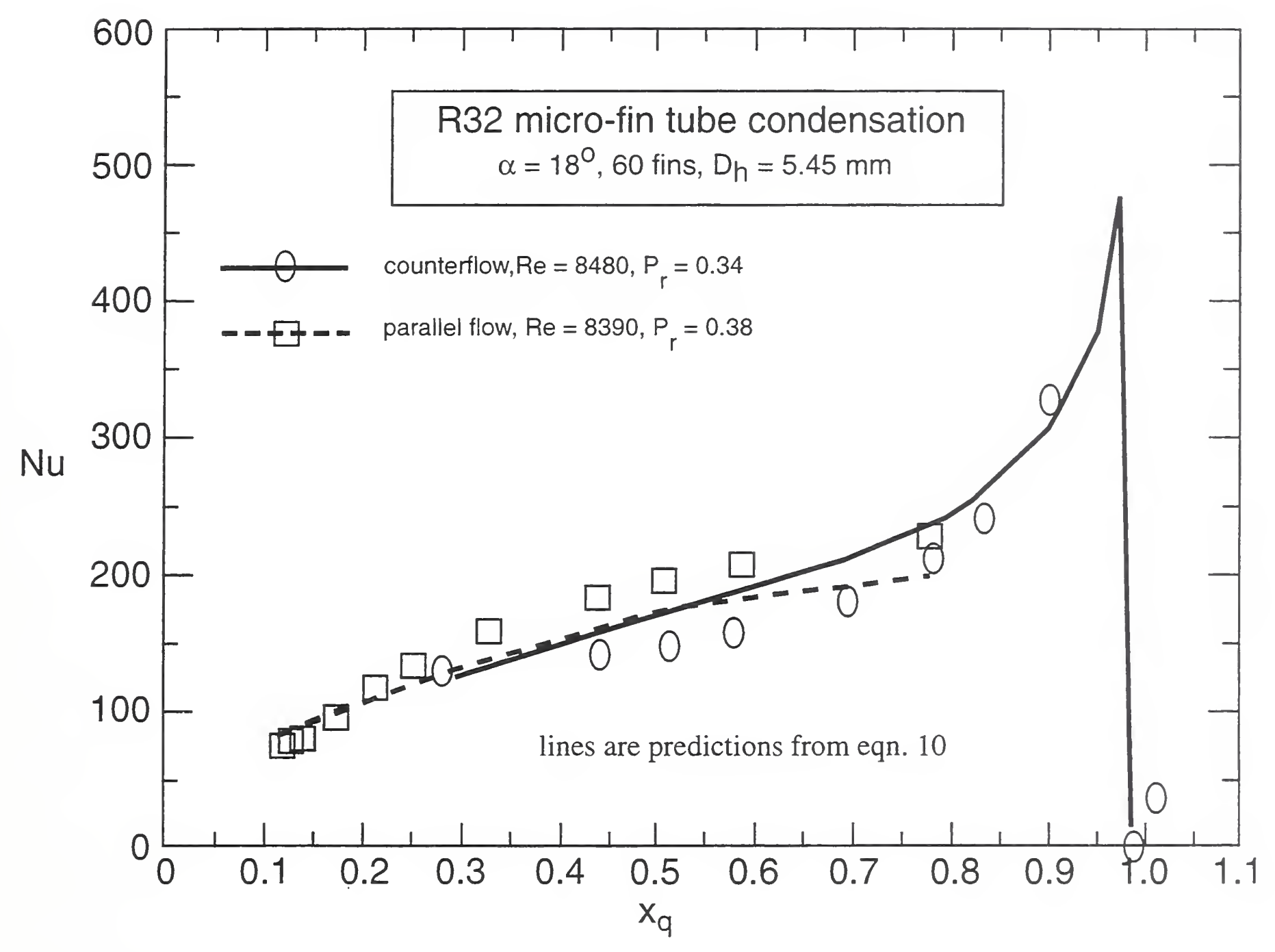

Fig. 15 Condensation Nusselt numbers versus thermodynamic quality for R32 


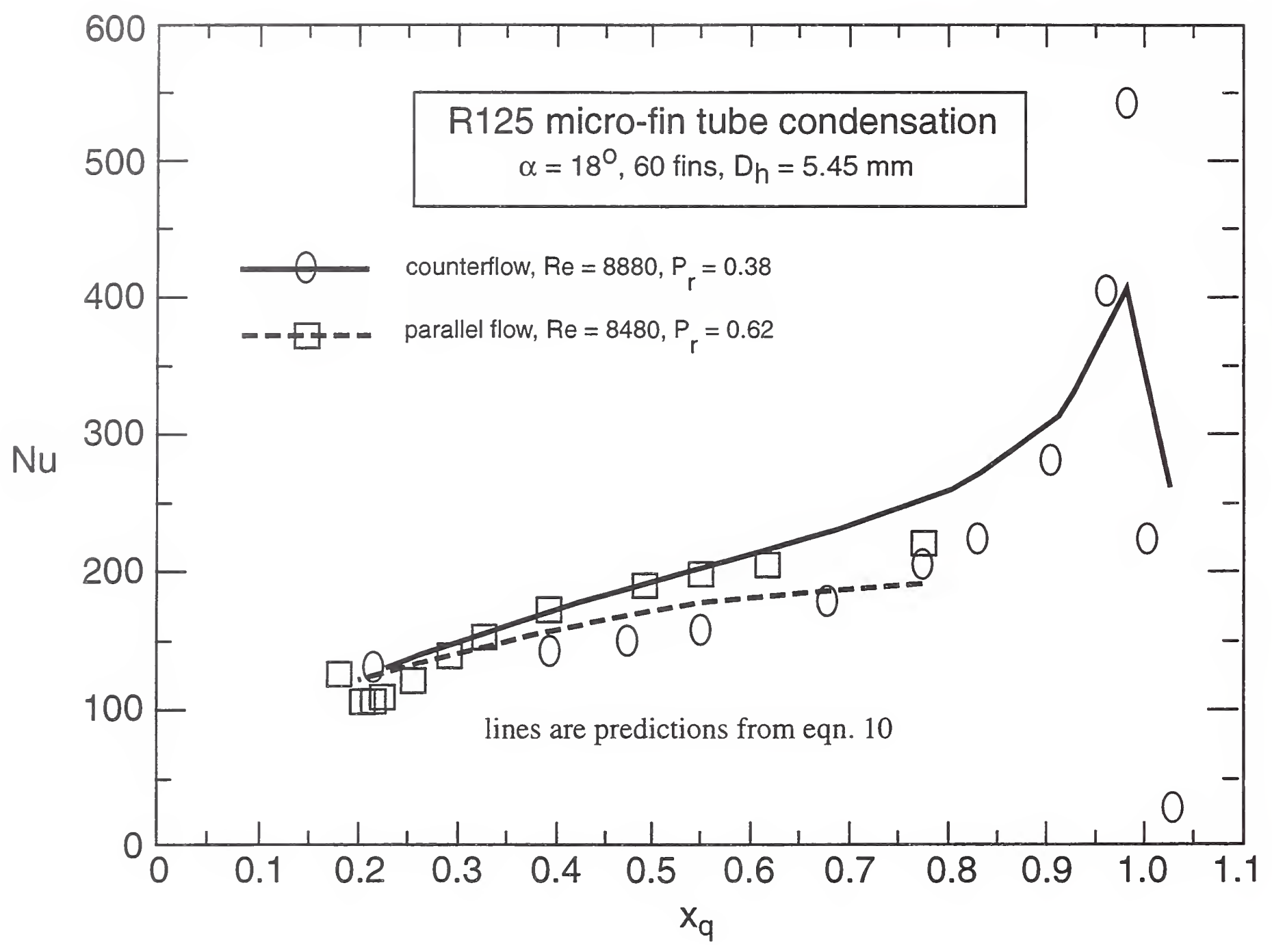

Fig 16 Condensation Nusselt numbers versus thermodynamic quality for R125 


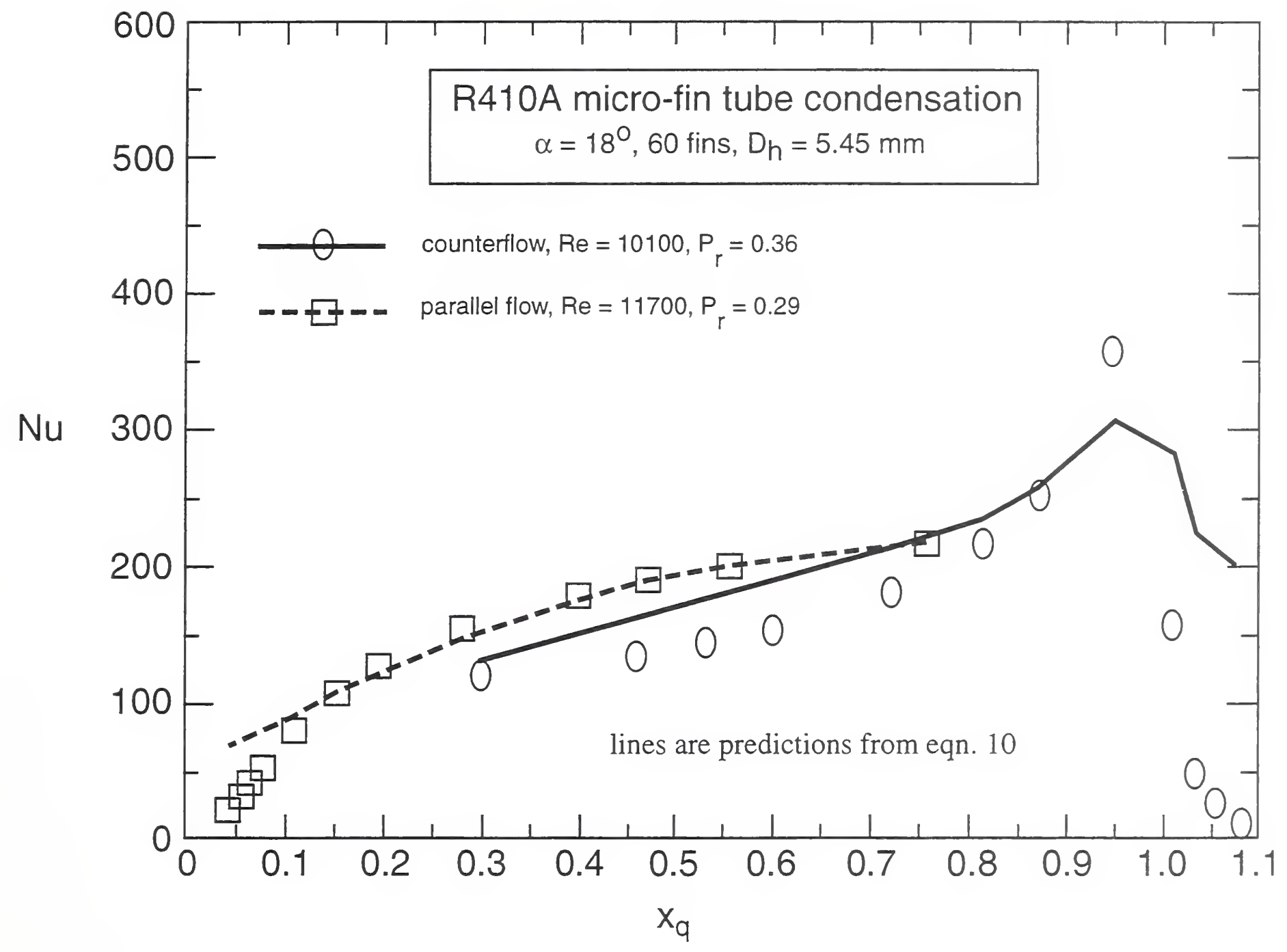

Fig. 17 Condensation Nusselt numbers versus thermodynamic quality for R410A 


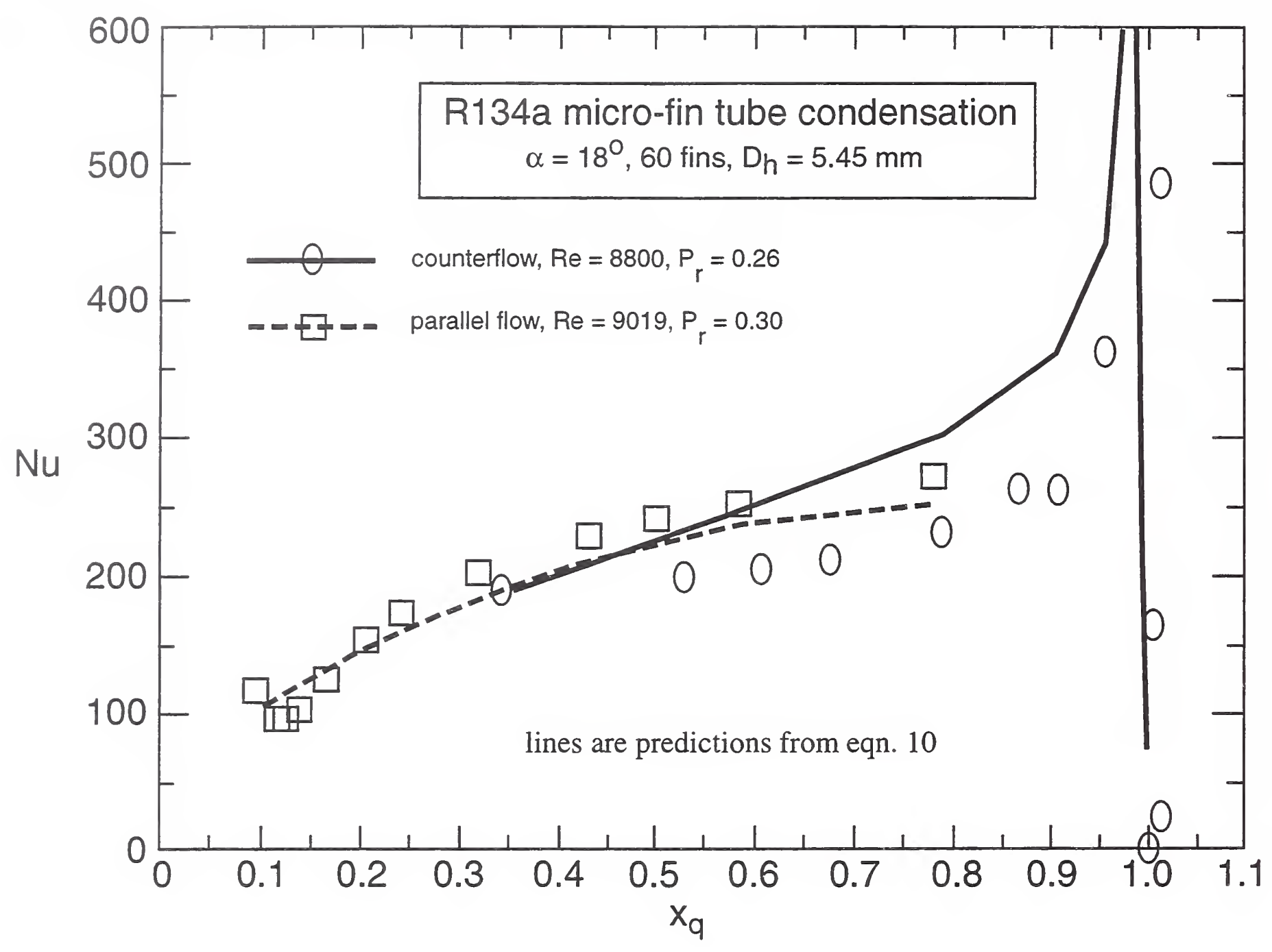

Fig. 18 Condensation Nusselt numbers versus thermodynamic quality for R134a 


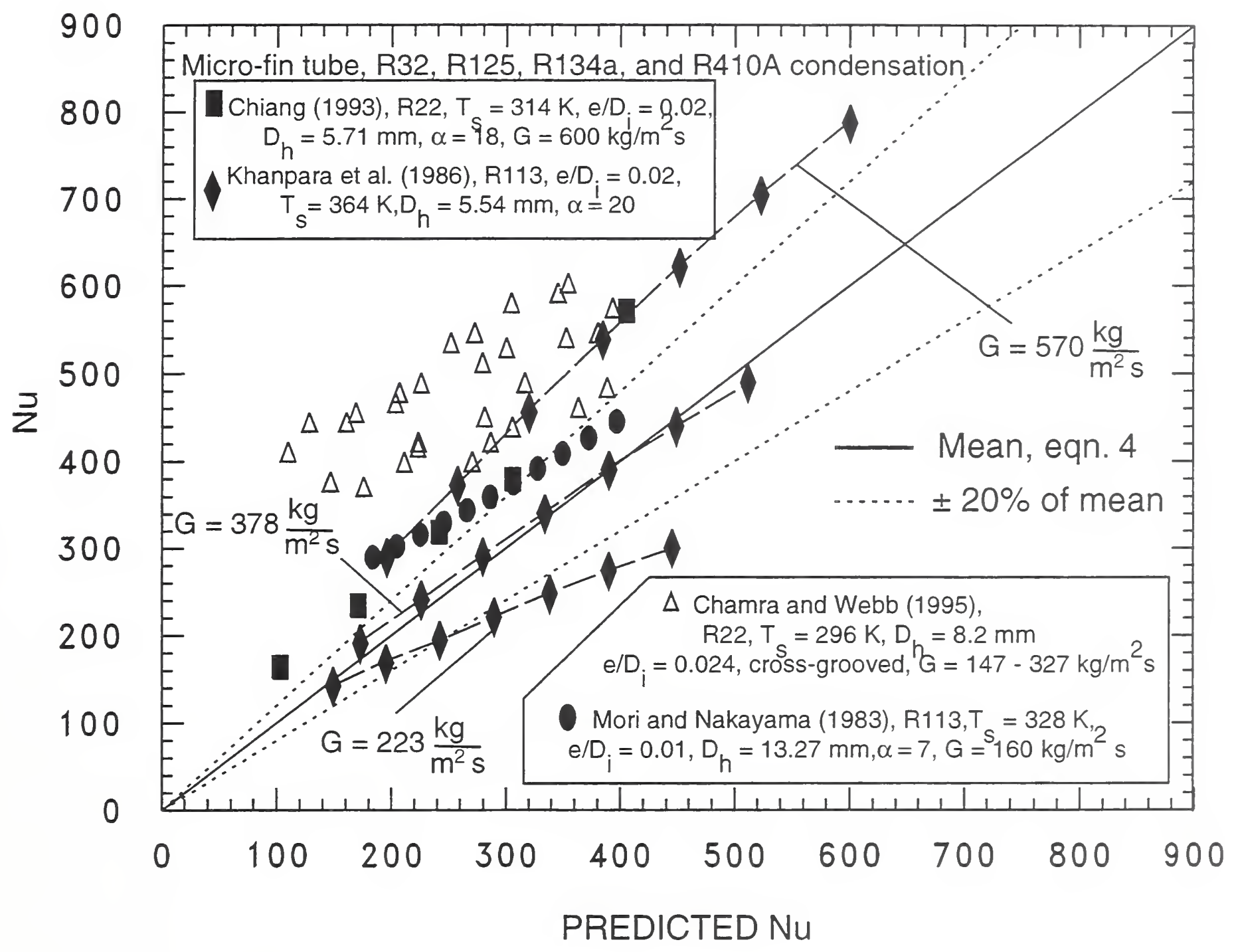

Fig. 19 Comparison of eqn. 10 to data available in the literature 


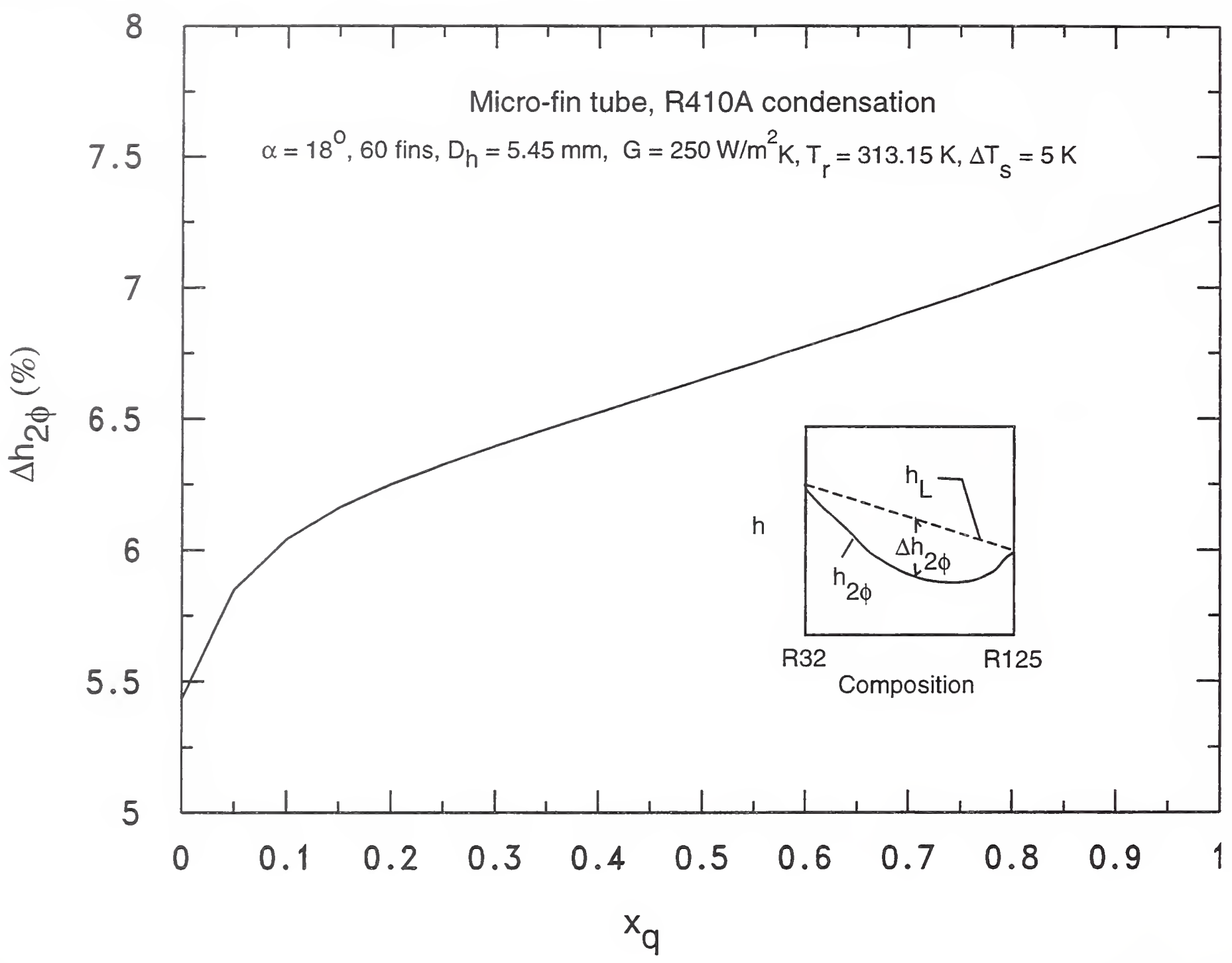

Fig. 20 Heat transfer degradation for the R410A mixture 


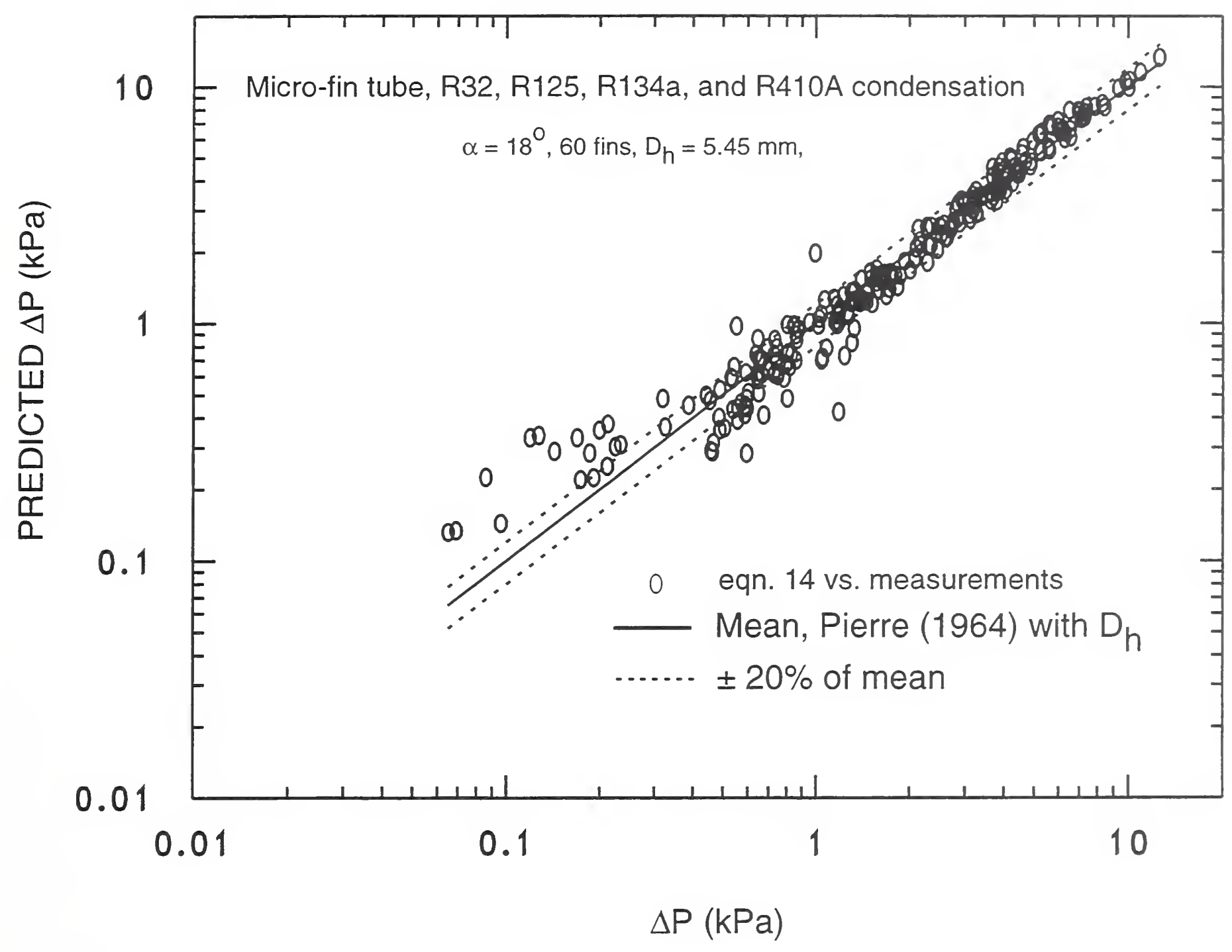

Fig. 21 Comparison of micro-fin condensation pressure drop to Pierre (1964) boiling pressure drop correlation 
APPENDIX A

CONVECTIVE CONDENSATION PRESSURE DROP DATA WITHIN A MICRO-FIN TUBE 


\section{APPENDIX A1}

\section{R32 convective condensation pressure drop data within a micro-fin tube}

(file: tapres.tbl)

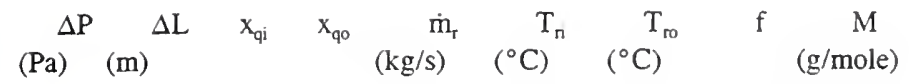

$\begin{array}{llllllllll}\text { 551. } & 1.587 & 0.485 & 0.233 & 0.01033 & 296.30 & 296.30 & 0.00483 & 52.02 & \text { I }\end{array}$

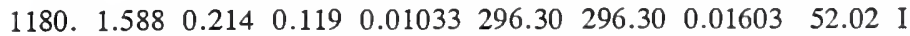
$\begin{array}{llllllllll}1184 & 1.587 & 0.487 & 0.223 & 0.01164 & 299.00 & 299.00 & 0.00804 & 52.02 & \text { I }\end{array}$ $\begin{array}{lllllllllll}\text { 454. } & 1.588 & 0.204 & 0.107 & 0.01164 & 299.00 & 299.00 & 0.00600 & 52.02 & \text { I }\end{array}$ $\begin{array}{llllllllll}650 \text {. } 1.587 & 0.494 & 0.232 & 0.00836 & 305.40 & 305.30 & 0.00958 & 52.02 & \text { I }\end{array}$

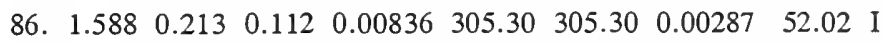

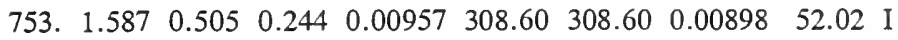
143. $\begin{array}{lllllllll}1.588 & 0.225 & 0.131 & 0.00957 & 308.60 & 308.60 & 0.00334 & 52.02 & \text { I }\end{array}$ $\begin{array}{lllllllllll}1927 . & 1.587 & 0.488 & 0.211 & 0.01632 & 304.50 & 304.40 & 0.00785 & 52.02 & \text { I }\end{array}$

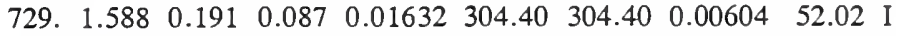
$\begin{array}{lllllllllll}2165 . & 1.587 & 0.505 & 0.230 & 0.01867 & 309.80 & 309.80 & 0.00737 & 52.02 & \text { I }\end{array}$ $\begin{array}{lllllllllll}\text { 1324. } & 1.588 & 0.210 & 0.108 & 0.01867 & 309.80 & 309.70 & 0.00798 & 52.02 & \text { I }\end{array}$ $\begin{array}{llllllllll}1638 . & 1.587 & 0.482 & 0.212 & 0.01414 & 301.20 & 301.20 & 0.00817 & 52.02 & \text { I }\end{array}$ $\begin{array}{llllllllll}666 . & 1.588 & 0.192 & 0.091 & 0.01414 & 301.20 & 301.20 & 0.00667 & 52.02 & \text { I }\end{array}$

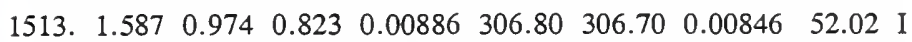
$\begin{array}{lllllllllll}\text { 805. } & 1.588 & 0.798 & 0.509 & 0.00886 & 306.70 & 306.70 & 0.00651 & 52.02 & \text { I }\end{array}$ $\begin{array}{llllllllll}1168 . & 1.587 & 0.965 & 0.820 & 0.00887 & 306.70 & 306.60 & 0.00660 & 52.02 & \text { I }\end{array}$

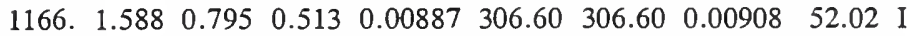

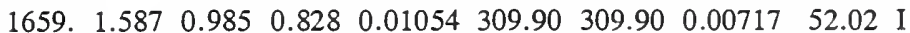

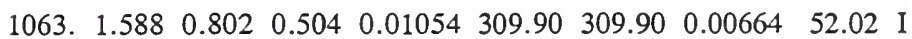

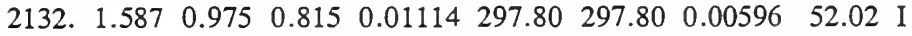

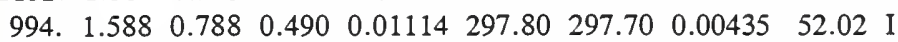

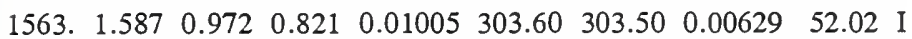

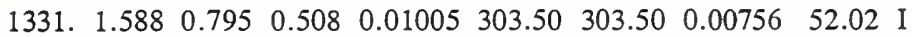
$\begin{array}{llllllllll}\text { 595. } & 1.587 & 0.963 & 0.808 & 0.00538 & 304.80 & 304.80 & 0.00868 & 52.02 & \mathrm{I}\end{array}$ 239. $1.588 \quad 0.783 \quad 0.495 \quad 0.00538 \quad 304.80 \quad 304.80 \quad 0.00526 \quad 52.02 \mathrm{HL}$ 488. $1.587 \quad 0.952 \quad 0.786 \quad 0.00489308 .60 \quad 308.60 \quad 0.00975 \quad 52.02 \quad$ I $\begin{array}{llllllllll}\text { 120. } & 1.588 & 0.759 & 0.477 & 0.00489 & 308.60 & 308.60 & 0.00387 & 52.02 & \text { HC }\end{array}$

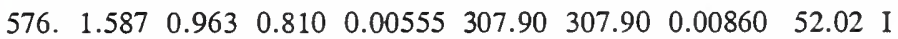

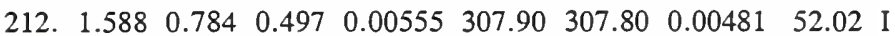
$\begin{array}{llllllllll}\text { 556. } & 1.587 & 0.961 & 0.806 & 0.00560 & 310.10 & 310.10 & 0.00874 & 52.02 & \text { I }\end{array}$

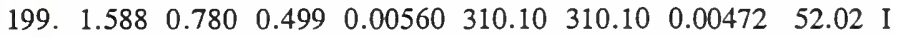
1183. $\begin{array}{lllllllll}1.588 & 0.778 & 0.483 & 0.00761 & 295.30 & 295.20 & 0.00943 & 52.02 & I\end{array}$ $\begin{array}{llllllll}\text { 1320. } 1.588 & 0.772 & 0.475 & 0.00604 & 296.10 & 296.00 & 0.01664 & 52.02 \\ \text { HC }\end{array}$

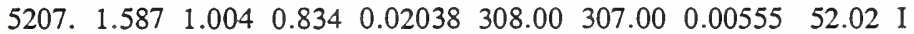

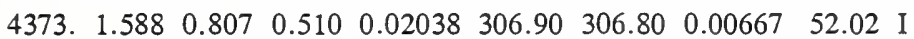

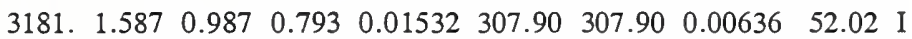

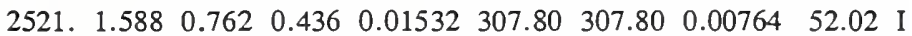
$\begin{array}{llllllllll}2854 & 1.587 & 0.987 & 0.826 & 0.01473 & 307.70 & 307.70 & 0.00599 & 52.02 & \text { I }\end{array}$

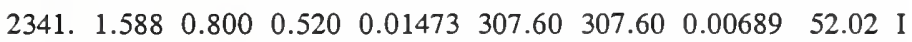

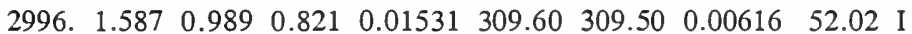
$\begin{array}{llllllllll}2498 . & 1.588 & 0.794 & 0.497 & 0.01531 & 309.50 & 309.50 & 0.00733 & 52.02 & \text { I }\end{array}$

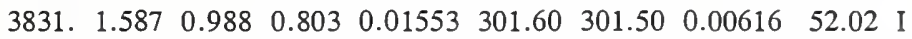

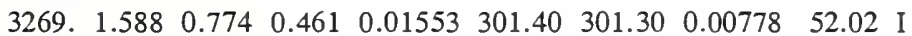

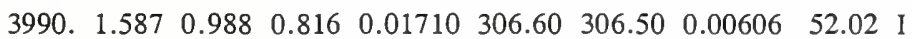

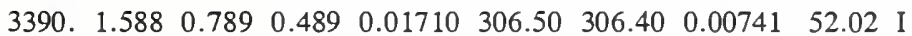
$\begin{array}{llllllllll}\text { 4351. } 1.587 & 0.948 & 0.786 & 0.01635 & 301.30 & 301.10 & 0.00639 & 52.02 & \text { I }\end{array}$

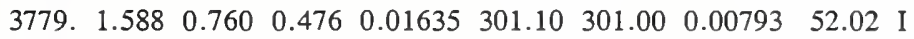
1016. $\begin{array}{llllllllll}1.587 & 0.961 & 0.783 & 0.00731 & 301.50 & 301.50 & 0.00749 & 52.02 & \text { I }\end{array}$ $\begin{array}{lllllllllll}638 . & 1.588 & 0.755 & 0.455 & 0.00731 & 301.50 & 301.50 & 0.00709 & 52.02 & \text { I }\end{array}$ 1295. $\begin{array}{llllllllll}1.587 & 0.958 & 0.752 & 0.00842 & 300.10 & 300.10 & 0.00711 & 52.02 & \text { I }\end{array}$ $\begin{array}{llllllllll}\text { 872. } 1.588 & 0.720 & 0.393 & 0.00842 & 300.10 & 300.00 & 0.00764 & 52.02 & \text { I }\end{array}$ $\begin{array}{llllllllll}864 . & 1.587 & 0.966 & 0.797 & 0.00668 & 300.60 & 300.60 & 0.00732 & 52.02 & \text { I }\end{array}$ $\begin{array}{llllllllll}\text { 542. } & 1.588 & 0.770 & 0.475 & 0.00668 & 300.60 & 300.60 & 0.00681 & 52.02 & \text { I }\end{array}$ 1371. $\begin{array}{lllllllll}1.587 & 0.486 & 0.212 & 0.01255 & 299.80 & 299.80 & 0.00831 & 52.02 & \text { I }\end{array}$

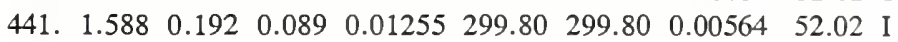

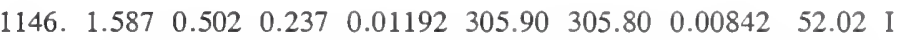
$\begin{array}{lllllllllll}387 . & 1.588 & 0.217 & 0.114 & 0.01192 & 305.80 & 305.80 & 0.00541 & 52.02 & \text { I }\end{array}$ $\begin{array}{llllllllll}1392 . & 1.587 & 0.489 & 0.218 & 0.01290 & 301.10 & 301.10 & 0.00818 & 52.02 & \text { I }\end{array}$ $\begin{array}{llllllllll}\text { 488. } & 1.588 & 0.198 & 0.096 & 0.01290 & 301.10 & 301.10 & 0.00582 & 52.02 & \text { I }\end{array}$ 1270. $\begin{array}{lllllllll}1.587 & 0.540 & 0.312 & 0.01205 & 308.70 & 308.60 & 0.00843 & 52.02 & \text { I }\end{array}$ $\begin{array}{lllllllllll}530 . & 1.588 & 0.295 & 0.207 & 0.01205 & 308.60 & 308.60 & 0.00536 & 52.02 & \text { I }\end{array}$ $\begin{array}{lllllllllll}602 . & 1.587 & 0.431 & 0.150 & 0.00828 & 296.50 & 296.50 & 0.00919 & 52.02 & \text { I }\end{array}$

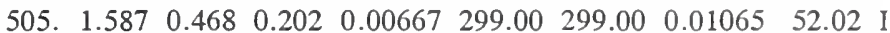
$\begin{array}{llllllllll}\text { 96. } & 1.588 & 0.183 & 0.088 & 0.00667 & 299.00 & 299.00 & 0.00455 & 52.02 & \text { I }\end{array}$

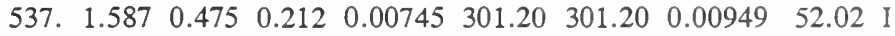

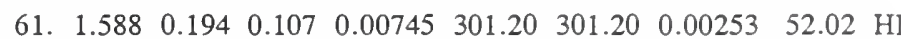

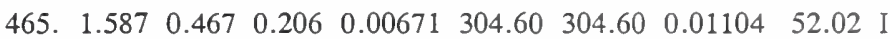

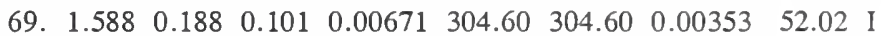

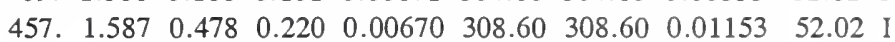

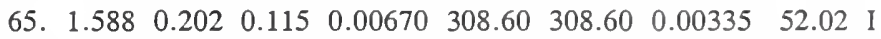

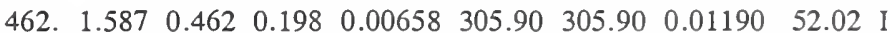

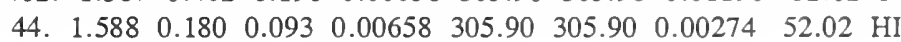




\title{
APPENDIX A2
}

\section{R125 convective condensation pressure drop data within a micro-fin tube}

\author{
(file: tapres.tbl)
}

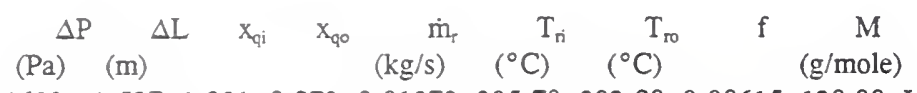
1639. $1.587 \quad 1.031 \quad 0.873 \quad 0.01379305 .70 \quad 302.80 \quad 0.00615 \quad 120.00$ I 1296. $1.588 \quad 0.847 \quad 0.5490 .01379 \quad 302.70 \quad 302.70 \quad 0.00681 \quad 120.00 \quad$ I $\begin{array}{lllllllllll}1430 & 1.587 & 1.042 & 0.894 & 0.01379 & 312.00 & 308.60 & 0.00624 & 120.00 & \text { I }\end{array}$

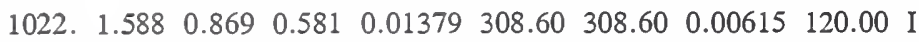

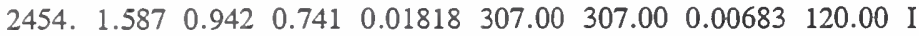
$\begin{array}{llllllllll}1562 . & 1.588 & 0.711 & 0.420 & 0.01818 & 307.00 & 306.90 & 0.00649 & 120.00 & \mathrm{I}\end{array}$

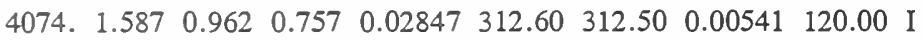
2811. $\begin{array}{lllllllll}1.588 & 0.727 & 0.412 & 0.02847 & 312.40 & 312.40 & 0.00564 & 120.00 & \text { I }\end{array}$ $\begin{array}{llllllllll}3411 . & 1.587 & 0.957 & 0.757 & 0.02787 & 316.90 & 316.80 & 0.00539 & 120.00 & \text { I }\end{array}$ 2271. $\begin{array}{llllllllll}1.588 & 0.727 & 0.418 & 0.02787 & 316.80 & 316.70 & 0.00534 & 120.00 & \text { I }\end{array}$

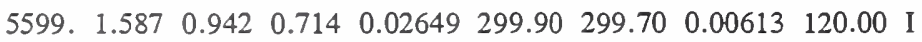

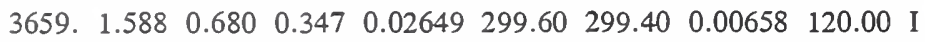

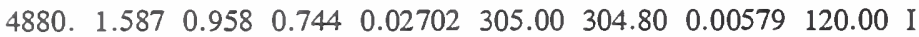
$\begin{array}{llllllllll}3256 . & 1.588 & 0.713 & 0.395 & 0.02702 & 304.80 & 304.60 & 0.00604 & 120.00 & \text { I }\end{array}$ $\begin{array}{lllllllllll}\text { 4506. } & 1.587 & 0.962 & 0.764 & 0.02726 & 308.00 & 307.80 & 0.00564 & 120.00 & \text { I }\end{array}$

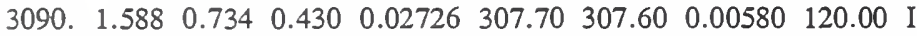

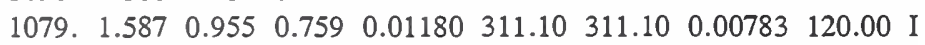

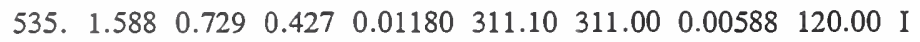

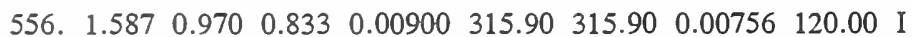

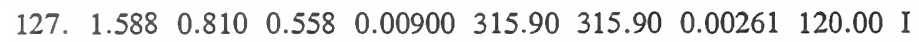
$\begin{array}{lllllllllll}\text { 675. } & 1.587 & 0.964 & 0.794 & 0.00955 & 318.10 & 318.00 & 0.00895 & 120.00 & \text { I }\end{array}$

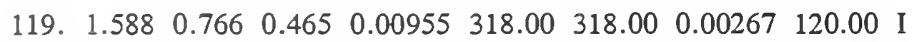
1868. $\begin{array}{llllllllll}1.587 & 0.986 & 0.820 & 0.01360 & 298.60 & 298.50 & 0.00673 & 120.00 & \text { I }\end{array}$

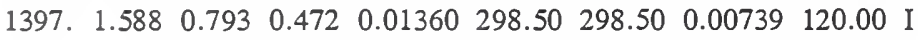
$\begin{array}{llllllllll}3871 . & 1.587 & 0.995 & 0.833 & 0.02132 & 300.40 & 300.20 & 0.00592 & 120.00 & \text { I }\end{array}$

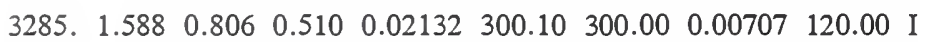
3696. $1.587 \quad 0.994 \quad 0.806 \quad 0.02178 \quad 303.70 \quad 303.60 \quad 0.00611 \quad 120.00$ I $\begin{array}{lllllllllll}2734 & 1.588 & 0.776 & 0.449 & 0.02178 & 303.50 & 303.40 & 0.00676 & 120.00 & \text { I }\end{array}$ 2281. $\begin{array}{llllllllll}1.587 & 0.962 & 0.821 & 0.01803 & 310.20 & 310.10 & 0.00661 & 120.00 & \text { I }\end{array}$ $\begin{array}{llllllllll}1600 & 1.588 & 0.797 & 0.531 & 0.01803 & 310.10 & 310.00 & 0.00630 & 120.00 & \text { I }\end{array}$

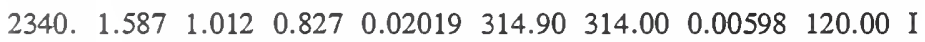

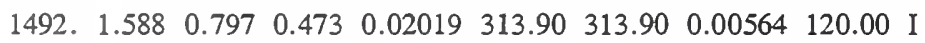
2076. $\begin{array}{llllllllll}1.587 & 1.001 & 0.840 & 0.02021 & 317.50 & 317.30 & 0.00585 & 120.00 & \text { I }\end{array}$

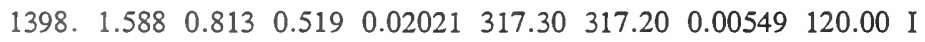

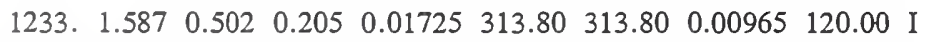

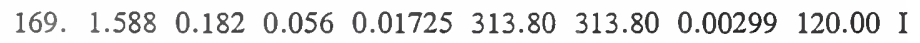

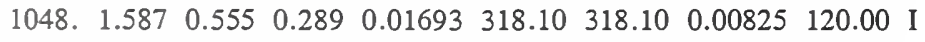
485. $1.588 \quad 0.267 \quad 0.140 \quad 0.01693 \quad 318.10 \quad 318.10 \quad 0.00596 \quad 120.00 \quad$ I $\begin{array}{llllllllll}1833 . & 1.587 & 0.515 & 0.244 & 0.02035 & 302.70 & 302.70 & 0.00766 & 120.00 & \text { I }\end{array}$ 799. $1.588 \quad 0.224 \quad 0.111 \quad 0.02035 \quad 302.60 \quad 302.60 \quad 0.00596 \quad 120.00 \quad$ I 2012. $\begin{array}{lllllllll}1.587 & 0.515 & 0.236 & 0.02110 & 299.00 & 299.00 & 0.00732 & 120.00 & \text { I }\end{array}$

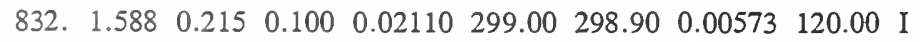

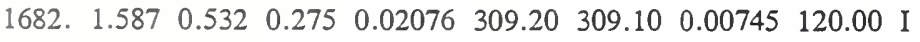

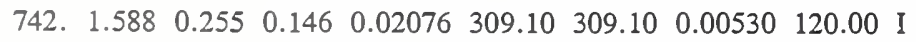

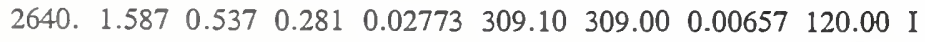

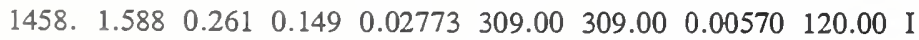
3268. $\begin{array}{llllllllll}1.587 & 0.526 & 0.261 & 0.02789 & 300.20 & 300.10 & 0.00671 & 120.00 & \text { I }\end{array}$ 1724. $\begin{array}{llllllllll}1.588 & 0.241 & 0.124 & 0.02789 & 300.00 & 300.00 & 0.00613 & 120.00 & \text { I }\end{array}$

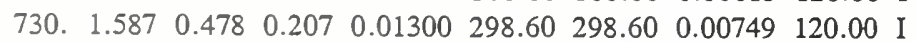
786. $\begin{array}{lllllllll}1.587 & 0.466 & 0.179 & 0.01336 & 299.90 & 299.90 & 0.00827 & 120.00 & \text { I }\end{array}$ 680. $1.588 \quad 0.159 \quad 0.061 \quad 0.01336 \quad 299.90 \quad 299.90 \quad 0.01351 \quad 120.00 \mathrm{HI}$ 805. $1.587 \quad 0.497 \quad 0.229 \quad 0.01328 \quad 311.00 \quad 310.90 \quad 0.00966 \quad 120.00 \quad$ I 714. $1.588 \quad 0.210 \quad 0.114 \quad 0.01328 \quad 310.90 \quad 310.90 \quad 0.01353 \quad 120.00 \mathrm{HC}$ $\begin{array}{lllllllllll}\text { 595. } & 1.587 & 0.549 & 0.323 & 0.01042 & 318.10 & 318.10 & 0.01162 & 120.00 & \text { I }\end{array}$ $\begin{array}{llllllllll}\text { 496. } 1.588 & 0.307 & 0.217 & 0.01042 & 318.10 & 318.10 & 0.01304 & 120.00 & \text { HC }\end{array}$

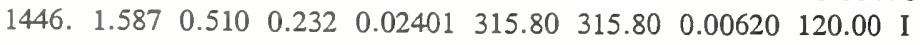

1041. $1.588 \quad 0.212 \quad 0.109 \quad 0.02401315 .80315 .80 \quad 0.00681 \quad 120.00 \mathrm{HL}$ 1803. $\begin{array}{lllllllll}1.587 & 0.538 & 0.251 & 0.02573 & 316.60 & 316.50 & 0.00650 & 120.00 & \text { I }\end{array}$ 1303. $1.588 \quad 0.229 \quad 0.107 \quad 0.02573 \quad 316.50 \quad 316.50 \quad 0.00736 \quad 120.00 \mathrm{HL}$ 


\section{APPENDIX A3}

\section{R410A convective condensation pressure drop data within a micro-fin tube}

(file: tapres.tbl)

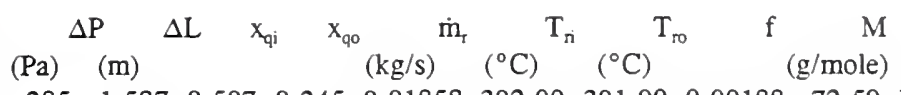

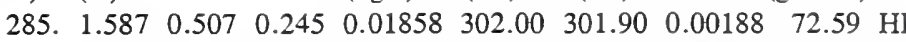

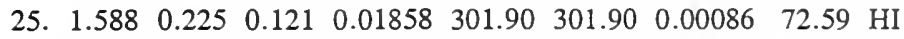

288. $\begin{array}{lllllllll}1.587 & 0.573 & 0.355 & 0.02750 & 304.50 & 304.40 & 0.00105 & 72.59 & \text { HI }\end{array}$

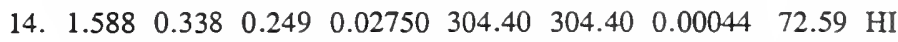

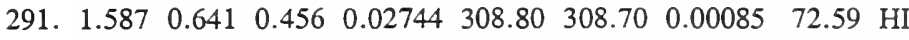

6. $\begin{array}{lllllllll}1.588 & 0.443 & 0.370 & 0.02744 & 308.70 & 308.70 & 0.00026 & 72.59 & \mathrm{HI}\end{array}$

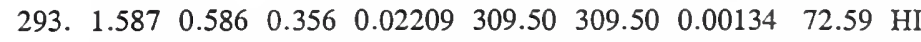

1. $\begin{array}{llllllllll}1.588 & 0.339 & 0.244 & 0.02209 & 309.50 & 309.40 & 0.00042 & 72.59 & \text { HI }\end{array}$

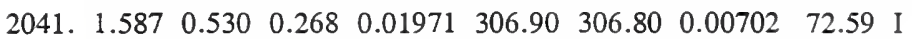

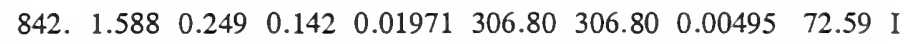
$\begin{array}{llllllllll}\text { 6000. } & 1.587 & 0.646 & 0.466 & 0.02695 & 299.40 & 299.20 & 0.00640 & 72.59 & \text { I }\end{array}$ $\begin{array}{llllllllll}\text { 4266. } 1.588 & 0.453 & 0.382 & 0.02695 & 299.10 & 298.90 & 0.00560 & 72.59 & \mathrm{I}\end{array}$

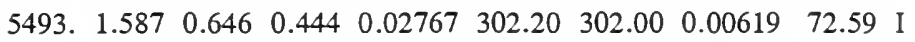

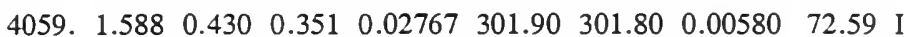

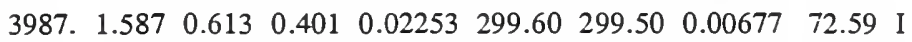

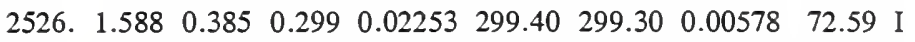

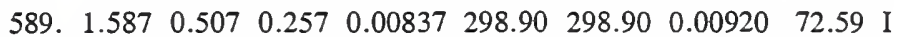

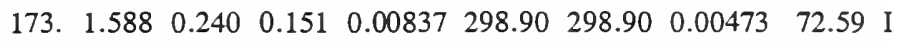

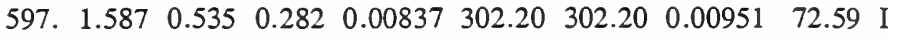
191. $\begin{array}{llllllllll}1.588 & 0.265 & 0.177 & 0.00837 & 302.20 & 302.10 & 0.00502 & 72.59 & \text { I }\end{array}$

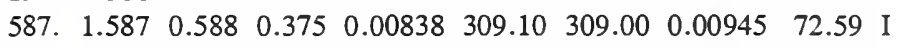
211. $\begin{array}{llllllllll}1.588 & 0.361 & 0.287 & 0.00838 & 309.00 & 309.00 & 0.00464 & 72.59 & \text { I }\end{array}$ $\begin{array}{lllllllllll}820 . & 1.587 & 0.556 & 0.321 & 0.01053 & 305.70 & 305.60 & 0.00848 & 72.59 & \mathrm{I}\end{array}$

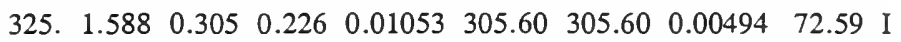

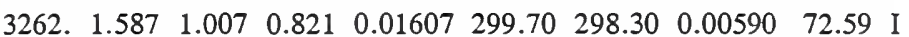
$\begin{array}{lllllllllll}2847 . & 1.588 & 0.793 & 0.492 & 0.01607 & 298.20 & 298.10 & 0.00745 & 72.59 & \text { I }\end{array}$

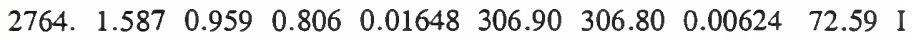

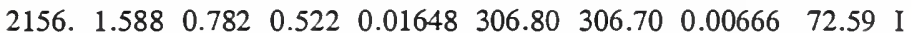

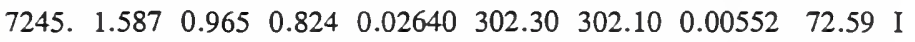

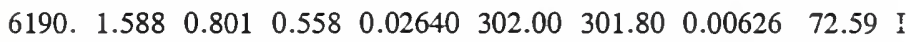

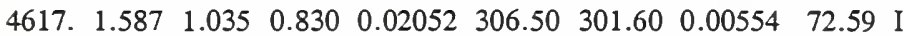

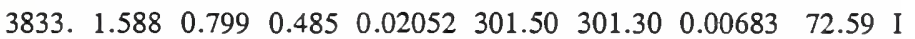

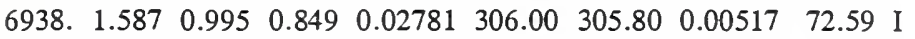

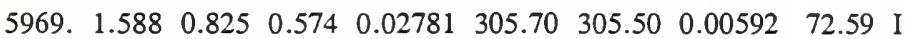

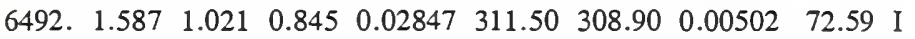
$\begin{array}{lllllllllll}5165 . & 1.588 & 0.817 & 0.526 & 0.02847 & 308.80 & 308.60 & 0.00566 & 72.59 & \text { I }\end{array}$ $\begin{array}{lllllllllll}6045 . & 1.587 & 0.968 & 0.819 & 0.02289 & 300.00 & 299.70 & 0.00573 & 72.59 & \text { I }\end{array}$ 5212. $\begin{array}{lllllllllll}1.588 & 0.795 & 0.545 & 0.02289 & 299.60 & 299.40 & 0.00664 & 72.59 & \text { I }\end{array}$

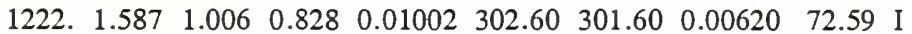

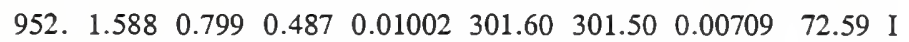
1039. $\begin{array}{lllllllllll}1.587 & 1.031 & 0.861 & 0.01014 & 312.80 & 309.20 & 0.00617 & 72.59 & \text { I }\end{array}$ 646. $\begin{array}{lllllllll}1.588 & 0.833 & 0.528 & 0.01014 & 309.20 & 309.10 & 0.00562 & 72.59 & \text { I }\end{array}$ 3678. $\begin{array}{lllllllllll}1.587 & 1.013 & 0.838 & 0.02120 & 312.50 & 310.80 & 0.00543 & 72.59 & \text { I }\end{array}$

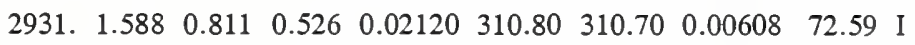
5597. $\begin{array}{llllllllll}1.587 & 1.035 & 0.842 & 0.02402 & 308.70 & 303.90 & 0.00522 & 72.59 & \text { I }\end{array}$

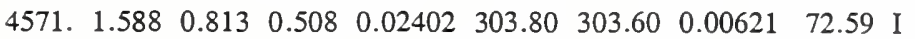

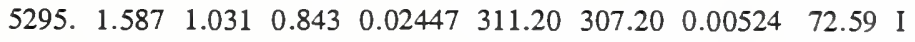

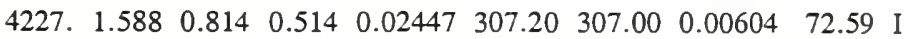

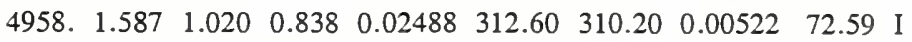

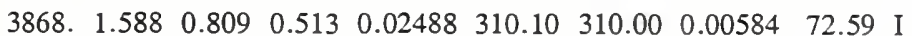
1378. $\begin{array}{lllllllllll}1.587 & 0.464 & 0.183 & 0.01557 & 300.60 & 300.50 & 0.00791 & 72.59 & \text { I }\end{array}$ $\begin{array}{lllllllllll}570 . & 1.588 & 0.163 & 0.064 & 0.01557 & 300.50 & 300.50 & 0.00685 & 72.59 & \text { I }\end{array}$ $\begin{array}{lllllllllll}2098 . & 1.587 & 0.512 & 0.253 & 0.01946 & 302.50 & 302.40 & 0.00692 & 72.59 & \mathrm{I}\end{array}$ 849. $\begin{array}{llllllllll}1.588 & 0.234 & 0.129 & 0.01946 & 302.40 & 302.30 & 0.00498 & 72.59 & \text { I }\end{array}$

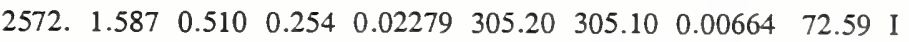
1141. $\begin{array}{lllllllll}1.588 & 0.235 & 0.132 & 0.02279 & 305.10 & 305.00 & 0.00507 & 72.59 & \text { I }\end{array}$ 


\title{
APPENDIX A4
}

\author{
R134a convective condensation pressure drop \\ data within a micro-fin tube
}

(file: tapres.tbl)

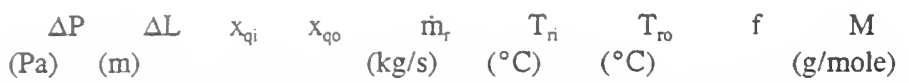

$\begin{array}{llllllllll}3687 & 1.587 & 0.999 & 0.900 & 0.01685 & 313.90 & 313.70 & 0.00458 & 102.00 & \text { I }\end{array}$

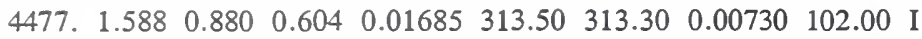
$\begin{array}{lllllllllll}3949 . & 1.587 & 1.002 & 0.890 & 0.01887 & 320.50 & 319.90 & 0.00468 & 102.00 & \text { I }\end{array}$

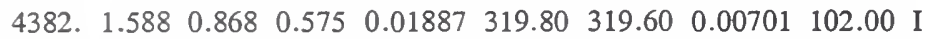
$\begin{array}{lllllllllll}\text { 4189. } & 1.587 & 1.000 & 0.869 & 0.01918 & 320.10 & 319.80 & 0.00488 & 102.00 & \text { I }\end{array}$ $\begin{array}{lllllllllll}\text { 4418. } 1.588 & 0.844 & 0.512 & 0.01918 & 319.70 & 319.50 & 0.00734 & 102.00 & \text { I }\end{array}$

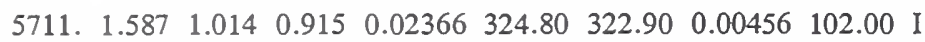

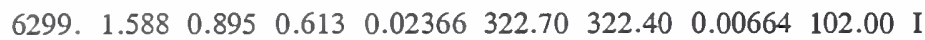
$\begin{array}{lllllllllll}5965 . & 1.587 & 1.014 & 0.908 & 0.02381 & 323.80 & 321.70 & 0.00458 & 102.00 & \text { I }\end{array}$ $\begin{array}{lllllllllll}6532 . & 1.588 & 0.887 & 0.588 & 0.02381 & 321.50 & 321.30 & 0.00677 & 102.00 & \text { I }\end{array}$

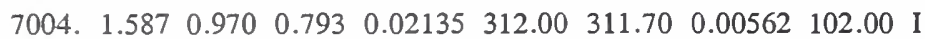

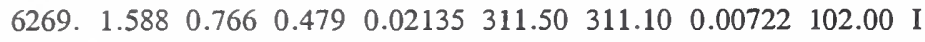

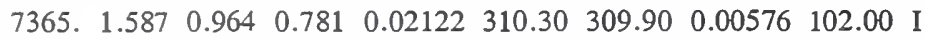
$\begin{array}{llllllllll}6553 . & 1.588 & 0.754 & 0.459 & 0.02122 & 309.70 & 309.30 & 0.00747 & 102.00 & \text { I }\end{array}$ $\begin{array}{llllllllll}9801 . & 1.587 & 1.001 & 0.840 & 0.02856 & 322.60 & 321.80 & 0.00555 & 102.00 & \text { I }\end{array}$ $\begin{array}{llllllllll}8279 & 1.588 & 0.814 & 0.528 & 0.02856 & 321.50 & 321.20 & 0.00654 & 102.00 & \text { I }\end{array}$ $\begin{array}{llllllllll}\text { 4865. } & 1.587 & 0.948 & 0.750 & 0.01642 & 312.10 & 311.90 & 0.00686 & 102.00 & \text { I }\end{array}$

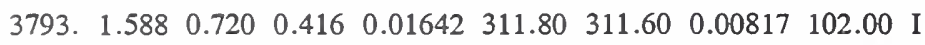
$\begin{array}{lllllllllll}\text { 3898. } & 1.587 & 0.946 & 0.751 & 0.01765 & 324.80 & 324.60 & 0.00676 & 102.00 & \text { I }\end{array}$ 2751. $\begin{array}{llllllllll}1.588 & 0.722 & 0.422 & 0.01765 & 324.60 & 324.50 & 0.00724 & 102.00 & \mathrm{I}\end{array}$

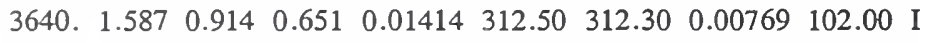

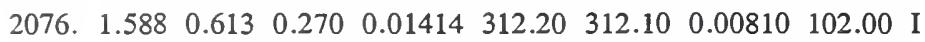

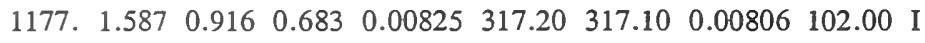
646. $\begin{array}{lllllllllll}1.588 & 0.648 & 0.298 & 0.00825 & 317.10 & 317.10 & 0.00788 & 102.00 & \text { I }\end{array}$ $\begin{array}{lllllllll}879 . & 1.587 & 1.024 & 0.930 & 0.00734 & 317.00 & 313.50 & 0.00549 & 102.00 \\ \mathrm{HL}\end{array}$

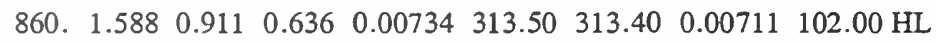
733. $1.587 \quad 1.022 \quad 0.922 \quad 0.00689316 .40 \quad 313.10 \quad 0.00517 \quad 102.00 \mathrm{HL}$ $\begin{array}{llllllllll}694 . & 1.588 & 0.901 & 0.614 & 0.00689 & 313.10 & 313.00 & 0.00664 & 102.00 \mathrm{HL}\end{array}$ $\begin{array}{lllllllllll}10950 . & 1.587 & 0.968 & 0.764 & 0.02666 & 313.90 & 313.40 & 0.00606 & 102.00 & \text { I }\end{array}$ 8421. $\begin{array}{lllllllll}1.588 & 0.734 & 0.417 & 0.02666 & 313.10 & 312.60 & 0.00715 & 102.00 & \text { I }\end{array}$ $\begin{array}{lllllllllll}12610 . & 1.587 & 0.967 & 0.778 & 0.02791 & 312.00 & 311.40 & 0.00596 & 102.00 & \text { I }\end{array}$

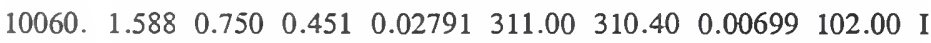
$\begin{array}{llllllllll}\text { 10100. } & 1.587 & 0.966 & 0.754 & 0.02797 & 319.60 & 319.10 & 0.00602 & 102.00 & \text { I }\end{array}$

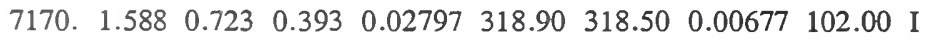

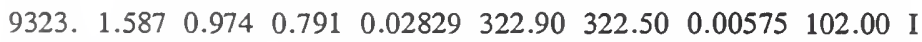
7276. $\begin{array}{lllllllll}1.588 & 0.762 & 0.461 & 0.02829 & 322.30 & 322.00 & 0.00662 & 102.00 & \text { I }\end{array}$

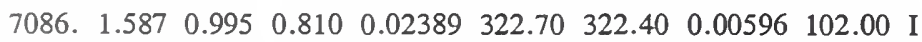
5564. $\begin{array}{llllllllll}1.588 & 0.781 & 0.478 & 0.02389 & 322.20 & 322.00 & 0.00685 & 102.00 & \text { I }\end{array}$

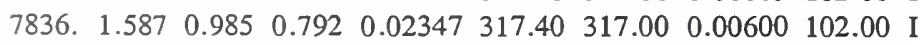
$\begin{array}{lllllllllll}6421 . & 1.588 & 0.763 & 0.454 & 0.02347 & 316.80 & 316.50 & 0.00730 & 102.00 & \text { I }\end{array}$ 7787. $\begin{array}{lllllllll}1.587 & 0.970 & 0.782 & 0.02218 & 314.00 & 313.60 & 0.00615 & 102.00 & \text { I }\end{array}$

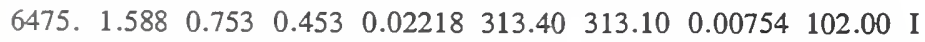

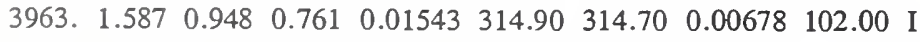
$\begin{array}{lllllllllll}3130 & 1.588 & 0.732 & 0.438 & 0.01543 & 314.60 & 314.40 & 0.00797 & 102.00 & \text { I }\end{array}$ $\begin{array}{lllllllllll}1554 . & 1.587 & 0.984 & 0.869 & 0.00994 & 315.80 & 315.70 & 0.00598 & 102.00 & \text { I }\end{array}$

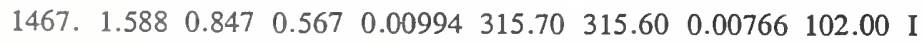
1262. $\begin{array}{llllllllll}1.587 & 0.945 & 0.755 & 0.00912 & 321.60 & 321.50 & 0.00747 & 102.00 & \text { I }\end{array}$

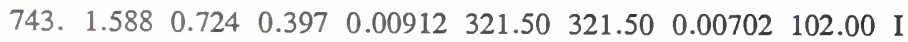

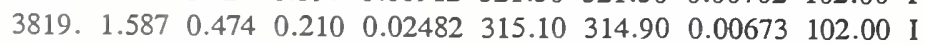

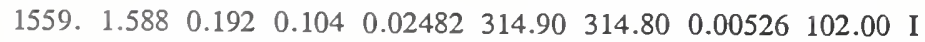

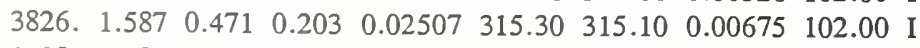
1498. $\begin{array}{llllllllll}1.588 & 0.186 & 0.096 & 0.02507 & 315.10 & 315.00 & 0.00522 & 102.00 & \text { I }\end{array}$ 1175. $\begin{array}{lllllllllll}1.587 & 0.487 & 0.231 & 0.01338 & 319.60 & 319.60 & 0.00741 & 102.00 & \text { I }\end{array}$ $\begin{array}{llllllllll}319 . & 1.588 & 0.212 & 0.121 & 0.01338 & 319.60 & 319.50 & 0.00391 & 102.00 & \text { I }\end{array}$ $\begin{array}{lllllllllll}646 . & 1.587 & 0.472 & 0.202 & 0.00993 & 319.20 & 319.20 & 0.00781 & 102.00 & \text { I }\end{array}$ 84. $\begin{array}{lllllllll}1.588 & 0.183 & 0.103 & 0.00993 & 319.20 & 319.20 & 0.00239 & 102.00 & \text { HC }\end{array}$ 702. $\begin{array}{lllllllll}1.587 & 0.492 & 0.250 & 0.00971 & 317.50 & 317.50 & 0.00763 & 102.00 & \text { I }\end{array}$
224. $1.588 \quad 0.233 \quad 0.146 \quad 0.00971 \quad 317.50 \quad 317.50 \quad 0.00432 \quad 102.00 \mathrm{HL}$

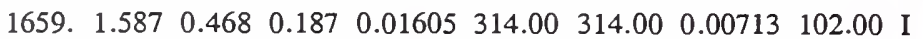
644. $\begin{array}{llllllllll}1.588 & 0.168 & 0.079 & 0.01605 & 313.90 & 313.90 & 0.00587 & 102.00 & \text { I }\end{array}$ 1595. $\begin{array}{llllllllll}1.587 & 0.501 & 0.246 & 0.01418 & 316.10 & 316.10 & 0.00783 & 102.00 & \text { I }\end{array}$ $\begin{array}{llllllllll}592 . & 1.588 & 0.227 & 0.127 & 0.01418 & 316.10 & 316.00 & 0.00545 & 102.00 & \text { I }\end{array}$ $\begin{array}{lllllllllll}4029 . & 1.587 & 0.496 & 0.220 & 0.02502 & 315.60 & 315.50 & 0.00680 & 102.00 & \text { I }\end{array}$ 1734. $\begin{array}{llllllllll}1.588 & 0.201 & 0.102 & 0.02502 & 315.40 & 315.30 & 0.00575 & 102.00 & \text { I }\end{array}$

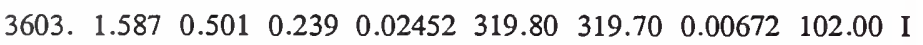
$\begin{array}{lllllllllll}\text { 1610. } & 1.588 & 0.221 & 0.124 & 0.02452 & 319.60 & 319.50 & 0.00542 & 102.00 & \text { I }\end{array}$ $\begin{array}{llllllllll}4370 . & 1.587 & 0.497 & 0.250 & 0.02802 & 321.80 & 321.70 & 0.00647 & 102.00 & \text { I }\end{array}$

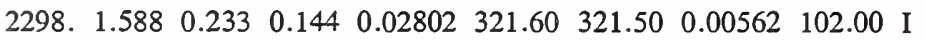

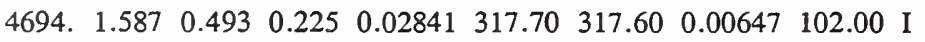
$\begin{array}{llllllllll}2104 & 1.588 & 0.207 & 0.112 & 0.02841 & 317.50 & 317.40 & 0.00541 & 102.00 & \text { I }\end{array}$ $\begin{array}{lllllllllll}5258 . & 1.587 & 0.482 & 0.214 & 0.02809 & 312.60 & 312.40 & 0.00670 & 102.00 & \text { I }\end{array}$

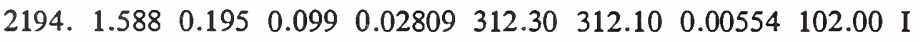
$\begin{array}{llllllllll}2886 . & 1.587 & 0.492 & 0.226 & 0.01959 & 313.80 & 313.60 & 0.00739 & 102.00 & \text { I }\end{array}$

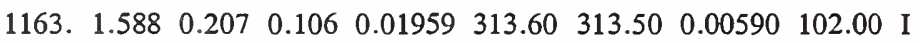

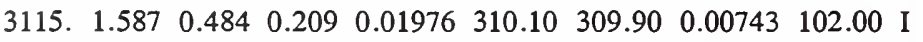

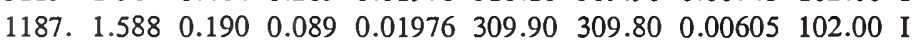
2711. $\begin{array}{llllllllll}1.587 & 0.517 & 0.275 & 0.01974 & 319.90 & 319.80 & 0.00713 & 102.00 & \text { I }\end{array}$

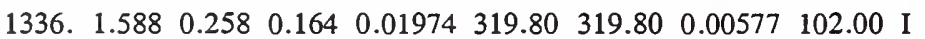

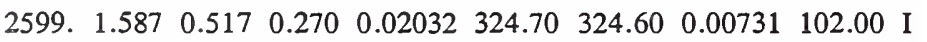

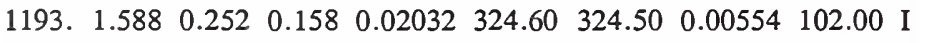

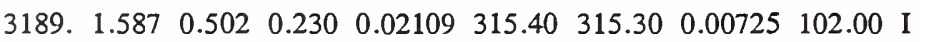
$\begin{array}{llllllllll}1328 . & 1.588 & 0.211 & 0.111 & 0.02109 & 315.30 & 315.20 & 0.00585 & 102.00 & \text { I }\end{array}$ 808. $\begin{array}{llllllllll}1.587 & 0.481 & 0.208 & 0.00985 & 310.00 & 310.00 & 0.00775 & 102.00 & \text { I }\end{array}$ $\begin{array}{llllllllll}\text { 113. } & 1.588 & 0.189 & 0.095 & 0.00985 & 310.00 & 310.00 & 0.00280 & 102.0 & \mathrm{HC}\end{array}$

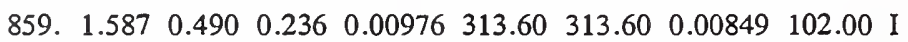

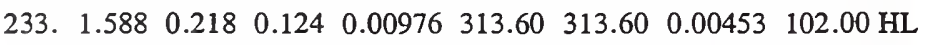
$\begin{array}{llllllllll}\text { 749. } 1.587 & 0.515 & 0.255 & 0.01002 & 323.20 & 323.20 & 0.00844 & 102.00 & \text { I }\end{array}$

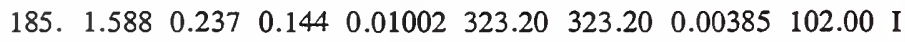
$\begin{array}{lllllllllll}1762 . & 1.587 & 0.497 & 0.234 & 0.01633 & 320.60 & 320.50 & 0.00751 & 102.00 & \text { I }\end{array}$ $\begin{array}{lllllllllll}\text { 650. } & 1.588 & 0.216 & 0.127 & 0.01633 & 320.50 & 320.50 & 0.00505 & 102.00 & \text { I }\end{array}$

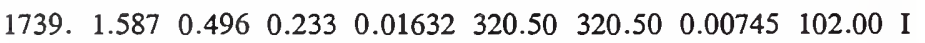

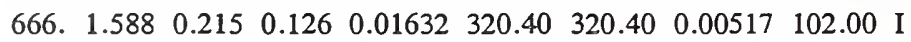


APPENDIX B

CONVECTIVE CONDENSATION HEAT TRANSFER DATA

WITHIN A MICRO-FIN TUBE 


\title{
Convective condensation of \\ R125 within a micro-fin tube
}

\author{
(file: tacond.tbl)
}

\begin{abstract}
$\begin{array}{rrr}\mathrm{Nu} & \mathrm{Re} & \mathrm{x}_{\mathrm{q}} \\ 217.7 & 7719 & 0.747\end{array}$
$\begin{array}{lll}217.7 & 7719 . & 0.747 \\ 197.6 & 7717 . & 0.566\end{array}$

$\begin{array}{lll}186.9 & 7717 . & 0.490\end{array}$

175.97717 .0 .425

$\begin{array}{lll}153.1 & 7716 . & 0.318\end{array}$

$\begin{array}{lll}130.5 & 7716 . & 0.242\end{array}$

$\begin{array}{lll}116.7 & 7715 . & 0.204\end{array}$

98.87714 .0 .164

84.5 7713. 0.133

81.47713 .0 .120

81.9 7713. 0.109

100.17713 .0 .088

217.7 8996. 0.763

$\begin{array}{lll}197.8 & 8997 . & 0.572\end{array}$

$\begin{array}{lll}187.0 & 8997 . & 0.492\end{array}$

$\begin{array}{lll}176.3 & 8996 . & 0.423\end{array}$

$\begin{array}{lll}153.4 & 8995 . & 0.312\end{array}$

130.1 8994. 0.233

115.4 8993. 0.193

95.3 8993. 0.152

$\begin{array}{lll}78.1 & 8992 . & 0.120\end{array}$

$\begin{array}{lll}73.0 & 8992 . & 0.107\end{array}$

$\begin{array}{llll}71.7 & 8992 . & 0.096\end{array}$

83.3 8991. 0.075

227.1 7022. 0.762

206.37022 .0 .577

195.27023 .0 .499

$\begin{array}{lll}184.2 & 7022 & 0.432\end{array}$

$\begin{array}{lll}161.2 & 7022 & 0.322\end{array}$

$\begin{array}{llll}138.2 & 7022 & 0.243\end{array}$

$\begin{array}{lll}123.4 & 7022 . & 0.202\end{array}$

$\begin{array}{lll}103.9 & 7022 . & 0.160\end{array}$

$\begin{array}{lll}87.0 & 7022 . & 0.126\end{array}$

81.9 7022. 0.112

80.37022 .0 .101

$\begin{array}{llll}91.5 & 7021 . & 0.079\end{array}$

230.8 8393. 0.781

$208.1 \quad 8392.0 .590$

$\begin{array}{llll}196.0 & 8392 & 0.511\end{array}$

$\begin{array}{lll}183.7 & 8392 . & 0.442\end{array}$

$\begin{array}{llll}158.2 & 8391 . & 0.332\end{array}$

132.8 8391. 0.254

$\begin{array}{llll}117.0 & 8391 . & 0.215\end{array}$

96.4 8391. 0.175

$80.0 \quad 8390.0 .144$

$\begin{array}{llll}76.0 & 8390 & 0.131\end{array}$

$\begin{array}{lll}76.5 & 8390 . & 0.121\end{array}$

$\begin{array}{llll}95.2 & 8390 . & 0.099\end{array}$

240.3 13550. 0.776

$\begin{array}{llll}218.5 & 13550 & 0.577\end{array}$

206.8 13540. 0.494

194.713540 .0 .422

$\begin{array}{lll}169.2 & 13540 . & 0.305\end{array}$

143.4 13540. 0.221

127.7 13530. 0.180

105.7 13530. 0.136

86.7 13530. 0.101

$\begin{array}{llll}81.0 & 13530 . & 0.087\end{array}$

$\begin{array}{lll}79.1 & 13530 . & 0.076\end{array}$

90.1 13530. 0.053 241.4 16650. 0.789 221.6 16640. 0.593 210.7 16640. 0.511 199.2 16640. 0.440 174.316630 .0 .324 $\begin{array}{llll}148.0 & 16630 & 0.241\end{array}$ $\begin{array}{lll}131.1 & 16630 & 0.200\end{array}$ 106.9 16630. 0.156 $\begin{array}{llll}83.9 & 16620 & 0.122\end{array}$ $\begin{array}{lll}75.4 & 16620 . & 0.109\end{array}$ $\begin{array}{lll}69.9 & 16620 . & 0.098\end{array}$

$\begin{array}{lll}70.9 & 16620 & 0.078\end{array}$

$\begin{array}{llllllll}\mathrm{Ja} & \mathrm{P} / \mathrm{P}_{\mathrm{c}} & \mathrm{T} / \mathrm{T}_{\mathrm{c}} & \mathrm{M} & \mathrm{Sv} & \mathrm{Pr} \text { flow } & \mathrm{E}_{\mathrm{t}} & \mathrm{U}_{\mathrm{No}_{\mathrm{o}}}\end{array}$ $\begin{array}{llllllllll}40.80 & 0.278 & 0.843 & 52.02 & 1.63 & 1.70 & \mathrm{P} & 1.919 & 14.20 & \mathrm{I}\end{array}$ $\begin{array}{lllllllllll}43.99 & 0.278 & 0.843 & 52.02 & 1.86 & 1.70 & P & 1.815 & 13.70 & 1\end{array}$ $\begin{array}{lllllllll}7.43 & 0.278 & 0.843 & 52.02 & 2.11 & 1.70 & \mathrm{P} & 1.708 & 14.30\end{array}$ $\begin{array}{llllllllll}55.54 & 0.278 & 0.843 & 52.02 & 2.73 & 1.70 & \mathrm{P} & 1.487 & 17.50 & \mathrm{I}\end{array}$ $\begin{array}{llllllllll}65.36 & 0.278 & 0.843 & 52.02 & 3.44 & 1.70 & P & 1.267 & 21.80 & \text { I }\end{array}$ $\begin{array}{lllllllllll}72.98 & 0.278 & 0.843 & 52.02 & 3.96 & 1.70 & \mathrm{P} & 1.134 & 24.50 & \mathrm{I}\end{array}$ $\begin{array}{lllllllllll}86.07 & 0.278 & 0.843 & 52.02 & 4.71 & 1.70 & P & 0.959 & 28.10 & 1\end{array}$ $\begin{array}{lllllllllll}04.20 & 0.278 & 0.843 & 52.02 & 5.53 & 1.70 & P & 0.821 & 33.00 & I\end{array}$ $\begin{array}{llllllllll}15.00 & 0.277 & 0.843 & 52.02 & 5.94 & 1.70 & \mathrm{P} & 0.791 & 41.30\end{array}$ $\begin{array}{llllllllll}26.10 & 0.278 & 0.843 & 52.02 & 6.36 & 1.70 & P & 0.795 & 57.20 & I\end{array}$ $\begin{array}{lllllllllll}50.90 & 0.278 & 0.843 & 52.02 & 7.36 & 1.70 & P & 0.972 & 84.30 & 02\end{array}$ $\begin{array}{llllllllll}28.69 & 0.298 & 0.851 & 52.02 & 1.23 & 1.71 & P & 2.001 & 15.30 & \mathrm{I}\end{array}$ $\begin{array}{llllllllll}33.19 & 0.298 & 0.851 & 52.02 & 1.60 & 1.71 & \mathrm{P} & 1.818 & 12.60 & \mathrm{I}\end{array}$ $\begin{array}{llllllllll}35.80 & 0.298 & 0.851 & 52.02 & 1.83 & 1.71 & \mathrm{P} & 1.720 & 12.10 & \mathrm{I}\end{array}$ $\begin{array}{llllllllll}38.72 & 0.298 & 0.851 & 52.02 & 2.10 & 1.71 & \mathrm{P} & 1.620 & 12.50 & 1\end{array}$ $\begin{array}{llllllllll}45.56 & 0.298 & 0.851 & 52.02 & 2.74 & 1.71 & \mathrm{P} & 1.411 & 15.50 & \mathrm{I}\end{array}$ $\begin{array}{lllllllllll}53.71 & 0.298 & 0.851 & 52.02 & 3.50 & 1.71 & \mathrm{P} & 1.196 & 19.50 & \mathrm{I}\end{array}$ $\begin{array}{lllllllllll}59.84 & 0.298 & 0.851 & 52.02 & 4.06 & 1.71 & \mathrm{P} & 1.061 & 21.90 & \mathrm{I}\end{array}$ $\begin{array}{llllllllll}69.68 & 0.298 & 0.851 & 52.02 & 4.88 & 1.71 & P & 0.876 & 24.90 & \text { I }\end{array}$ $\begin{array}{lllllllllll}82.14 & 0.298 & 0.851 & 52.02 & 5.78 & 1.71 & P & 0.718 & 28.50 & \text { I }\end{array}$ $\begin{array}{lllllllllll}88.90 & 0.298 & 0.851 & 52.02 & 6.23 & 1.71 & P & 0.671 & 36.70 & 1\end{array}$ $\begin{array}{lllllllllll}95.63 & 0.298 & 0.851 & 52.02 & 6.69 & 1.71 & \mathrm{P} & 0.659 & 52.60 & \mathrm{I}\end{array}$ $\begin{array}{llllllllllll}108.50 & 0.298 & 0.851 & 52.02 & 7.78 & 1.71 & \mathrm{P} & 0.766 & 73.60 \mathrm{I}\end{array}$ $\begin{array}{lllllllllll}39.01 & 0.352 & 0.869 & 52.02 & 1.21 & 1.74 & \mathrm{P} & 2.497 & 18.70 & 1\end{array}$ $\begin{array}{llllllllll}44.72 & 0.352 & 0.869 & 52.02 & 1.55 & 1.74 & \mathrm{P} & 2.679 & 15.90 & \mathrm{I}\end{array}$ $\begin{array}{llllllllll}48.03 & 0.352 & 0.869 & 52.02 & 1.77 & 1.74 & \mathrm{P} & 2.534 & 15.50 & \mathrm{I}\end{array}$ $\begin{array}{llllllllll}51.71 & 0.352 & 0.869 & 52.02 & 2.00 & 1.74 & \mathrm{P} & 2.391 & \mathrm{I} 6.10 & \mathrm{I}\end{array}$ $\begin{array}{llllllllll}60.34 & 0.352 & 0.869 & 52.02 & 2.57 & 1.74 & \mathrm{P} & 2.093 & 19.60 & \mathrm{I}\end{array}$ $\begin{array}{llllllllll}70.69 & 0.352 & 0.869 & 52.02 & 3.23 & 1.74 & P & 1.794 & 24.30 & 1\end{array}$ $\begin{array}{llllllllll}78.22 & 0.352 & 0.869 & 52.02 & 3.71 & 1.74 & \mathrm{P} & 1.602 & 27.00 & \mathrm{I}\end{array}$ $\begin{array}{lllllllllll}91.03 & 0.352 & 0.869 & 52.02 & 4.42 & 1.74 & \mathrm{P} & 1.349 & 30.90 & \mathrm{I}\end{array}$ $\begin{array}{llllllllll}08.10 & 0.352 & 0.869 & 52.02 & 5.20 & 1.74 & P & 1.130 & 35.90 & \text { I }\end{array}$ $\begin{array}{llllllllllll}117.90 & 0.352 & 0.869 & 52.02 & 5.58 & 1.74 & \mathrm{P} & 1.064 & 45.30 & \mathrm{I}\end{array}$ $\begin{array}{llllllllll}28.30 & 0.352 & 0.869 & 52.02 & 5.97 & 1.74 & \mathrm{P} & 1.043 & 66.10 & \mathrm{I}\end{array}$ $\begin{array}{llllllllll}51.20 & 0.352 & 0.869 & 52.02 & 6.84 & 1.74 & \mathrm{P} & 1.188 & 94.20 & 02\end{array}$ $\begin{array}{llllllllll}31.42 & 0.383 & 0.878 & 52.02 & 1.17 & 1.77 & \mathrm{P} & 2.375 & 17.20 & \mathrm{I}\end{array}$ $\begin{array}{llllllllll}36.18 & 0.383 & 0.878 & 52.02 & 1.50 & 1.77 & \mathrm{P} & 2.142 & 14.50 & \mathrm{I}\end{array}$ $\begin{array}{llllllllll}38.97 & 0.383 & 0.878 & 52.02 & 1.71 & 1.77 & \mathrm{P} & 2.017 & 14.00 & \mathrm{I}\end{array}$ $\begin{array}{llllllllll}42.01 & 0.383 & 0.878 & 52.02 & 1.93 & 1.77 & \mathrm{P} & 1.891 & 14.60 & \mathrm{I}\end{array}$ $\begin{array}{lllllllllll}49.14 & 0.383 & 0.878 & 52.02 & 2.46 & 1.77 & P & 1.628 & 17.80 & 1\end{array}$ $\begin{array}{lllllllllll}57.72 & 0.382 & 0.878 & 52.02 & 3.04 & 1.77 & \mathrm{P} & 1.367 & 22.30 & 1\end{array}$ $\begin{array}{llllllllll}64.08 & 0.382 & 0.878 & 52.02 & 3.44 & 1.77 & P & 1.205 & 24.90 & 1\end{array}$ $\begin{array}{llllllllll}74.75 & 0.382 & 0.878 & 52.02 & 4.00 & 1.77 & P & 0.993 & 28.50 & I\end{array}$ $\begin{array}{llllllllll}88.91 & 0.382 & 0.878 & 52.02 & 4.57 & 1.77 & P & 0.823 & 33.20 & \text { I }\end{array}$ $\begin{array}{lllllllllll}97.01 & 0.382 & 0.878 & 52.02 & 4.84 & 1.77 & P & 0.783 & 42.40 & \text { I }\end{array}$ $\begin{array}{lllllllllll}05.60 & 0.382 & 0.878 & 52.02 & 5.11 & 1.77 & P & 0.787 & 59.30 & \text { I }\end{array}$ $\begin{array}{llllllllll}24.00 & 0.382 & 0.878 & 52.02 & 5.73 & 1.77 & \mathrm{P} & 0.980 & 82.90 & \mathrm{CU}\end{array}$ $\begin{array}{llllllllll}19.86 & 0.345 & 0.867 & 52.02 & 1.19 & 1.74 & \mathrm{P} & 1.870 & 13.70 & \mathrm{I}\end{array}$ $\begin{array}{lllllllllll}22.93 & 0.344 & 0.867 & 52.02 & 1.56 & 1.74 & \mathrm{P} & 1.701 & 10.90 & \mathrm{I}\end{array}$ $\begin{array}{llllllllll}24.72 & 0.344 & 0.867 & 52.02 & 1.79 & 1.74 & \mathrm{P} & 1.609 & 10.30 & \mathrm{I}\end{array}$ $\begin{array}{llllllllll}26.64 & 0.344 & 0.866 & 52.02 & 2.05 & 1.74 & \mathrm{P} & 1.515 & 10.60 & \mathrm{I}\end{array}$ $\begin{array}{llllllllll}31.13 & 0.344 & 0.866 & 52.02 & 2.70 & 1.74 & \mathrm{P} & 1.316 & 13.20 & \mathrm{I}\end{array}$ $\begin{array}{llllllllll}36.43 & 0.344 & 0.866 & 52.02 & 3.50 & 1.74 & \mathrm{P} & 1.116 & 16.50 & \mathrm{I}\end{array}$ $\begin{array}{lllllllllll}40.55 & 0.344 & 0.866 & 52.02 & 4.09 & 1.74 & \mathrm{P} & 0.994 & 18.50\end{array}$ $\begin{array}{llllllllllll}46.94 & 0.344 & 0.866 & 52.02 & 5.00 & 1.74 & P & 0.822 & 20.80 & 1\end{array}$ $\begin{array}{llllllllll}55.06 & 0.344 & 0.866 & 52.02 & 6.04 & 1.74 & \mathrm{P} & 0.675 & 23.60 & \mathrm{I}\end{array}$ $\begin{array}{llllllllll}59.50 & 0.344 & 0.866 & 52.02 & 6.58 & 1.74 & P & 0.630 & 30.50 & I\end{array}$ $\begin{array}{llllllllll}63.97 & 0.344 & 0.866 & 52.02 & 7.14 & 1.74 & \mathrm{P} & 0.615 & 44.60 & \mathrm{I}\end{array}$ $\begin{array}{lllllllllll}72.81 & 0.344 & 0.866 & 52.02 & 8.49 & 1.74 & \mathrm{P} & 0.701 & 64.00 & \mathrm{HC}\end{array}$ $\begin{array}{llllllllll}16.12 & 0.395 & 0.882 & 52.02 & 1.15 & 1.79 & \mathrm{P} & 1.878 & 13.30 & 1\end{array}$ $\begin{array}{lllllllllll}18.72 & 0.395 & 0.882 & 52.02 & 1.49 & 1.79 & P & 1.725 & 10.50 & 1\end{array}$ $\begin{array}{lllllllllll}20.23 & 0.394 & 0.882 & 52.02 & 1.70 & 1.79 & P & 1.640 & 9.80 & 1\end{array}$ $\begin{array}{llllllllllll}21.85 & 0.394 & 0.882 & 52.02 & 1.93 & 1.79 & \mathrm{P} & 1.550 & 10.10 & 1\end{array}$ $\begin{array}{llllllllll}25.59 & 0.394 & 0.882 & 52.02 & 2.48 & 1.78 & \mathrm{P} & 1.356 & 12.60\end{array}$ $\begin{array}{lllllllllll}29.93 & 0.394 & 0.882 & 52.02 & 3.13 & 1.78 & P & 1.152 & 15.90 & I\end{array}$ $\begin{array}{llllllllllll}33.15 & 0.394 & 0.882 & 52.02 & 3.59 & 1.78 & \mathrm{P} & 1.021 & 17.80 & \mathrm{I}\end{array}$ $\begin{array}{lllllllllll}38.04 & 0.394 & 0.882 & 52.02 & 4.27 & 1.78 & P & 0.832 & 19.90\end{array}$ $\begin{array}{lllllllllll}43.78 & 0.394 & 0.882 & 52.02 & 4.99 & 1.78 & \mathrm{P} & 0.653 & 22.50 & \mathrm{I}\end{array}$ $\begin{array}{llllllllllll}46.65 & 0.394 & 0.882 & 52.02 & 5.34 & 1.78 & P & 0.587 & 31.00 & \mathrm{CU}\end{array}$ $\begin{array}{llllllllllll}49.23 & 0.394 & 0.882 & 52.02 & 5.67 & 1.78 & P & 0.544 & 50.10 & 02\end{array}$ $\begin{array}{lllllllllll}53.39 & 0.394 & 0.882 & 52.02 & 6.38 & 1.78 & P & 0.552 & 69.50 & I\end{array}$
\end{abstract}

$\begin{array}{lllllllllllll}231.4 & 11260 & 0.762 & 23.95 & 0.317 & 0.857 & 52.02 & 1.22 & 1.72 & \mathrm{P} & 1.934 & 14.40 & 1\end{array}$ $\begin{array}{lllllllllllll}209.6 & 11260 & 0.568 & 27.54 & 0.317 & 0.857 & 52.02 & 1.60 & 1.72 & P & 1.752 & 11.60 & 1\end{array}$ $\begin{array}{lllllllllllll}198.0 & 11260 & 0.487 & 29.63 & 0.317 & 0.857 & 52.02 & 1.84 & 1.72 & \mathrm{P} & 1.655 & 11.00 & 1\end{array}$ $\begin{array}{llllllllllll}186.1 & 11260 & 0.4 I 7 & 31.89 & 0.317 & 0.857 & 52.02 & 2.11 & 1.72 & \mathrm{P} & 1.556 & 11.30\end{array}$ $\begin{array}{llllllllllll}161.2 & 11250 & 0.304 & 37.14 & 0.317 & 0.857 & 52.02 & 2.77 & 1.72 & \mathrm{P} & 1.348 & 13.90\end{array}$ $\begin{array}{llllllllllllll}136.4 & 11250 & 0.222 & 43.37 & 0.316 & 0.857 & 52.02 & 3.58 & 1.72 & \mathrm{P} & 1.141 & 17.40\end{array}$ $\begin{array}{lllllllllllll}121.4 & 11250 & 0.182 & 48.17 & 0.316 & 0.857 & 52.02 & 4.18 & 1.72 & P & 1.015 & 19.50 & 1\end{array}$ $\begin{array}{lllllllllllll}100.7 & 11250 & 0.139 & 55.78 & 0.316 & 0.857 & 52.02 & 5.10 & 1.72 & P & 0.842 & 22.00\end{array}$ $\begin{array}{llllllllllllll}83.2 & 11250 & 0.105 & 65.64 & 0.316 & 0.857 & 52.02 & 6.17 & 1.72 & P & 0.696 & 25.10 & 1\end{array}$ $\begin{array}{lllllllllllllll}78.2 & 11250 & 0.091 & 71.18 & 0.316 & 0.857 & 52.02 & 6.72 & 1.72 & P & 0.654 & 32.60 & \text { I }\end{array}$ $\begin{array}{llllllllllllll}76.8 & 11250 & 0.080 & 76.86 & 0.316 & 0.857 & 52.02 & 7.29 & 1.72 & \mathrm{P} & 0.642 & 47.60 & \mathrm{I}\end{array}$ $\begin{array}{lllllllllllll}89.1 & 11250 & 0.058 & 88.67 & 0.316 & 0.857 & 52.02 & 8.68 & 1.72 & P & 0.745 & 68.30 & 02\end{array}$ $\begin{array}{lllllllllllll}31.0 & 7825 . & 1.013 & 67.15 & 0.366 & 0.879 & 52.02 & 0.92 & 1.78 & \mathrm{C} & 0.241 & 31.40 & \mathrm{O} 2\end{array}$ $\begin{array}{lllllllllllll}-977.4 & 7587 . & 0.992 & * * * * * * * & 0.365 & 0.873 & 52.02 & 0.94 & 1.76 & C & -4.971 & 435.80 & 02\end{array}$ $\begin{array}{llllllllllllll}4576.0 & 7587 . & 0.976 & 4133.00 & 0.365 & 0.873 & 52.02 & 0.96 & 1.76 & \mathrm{C} & 18.890 & * * * * * * & \text { O2 }\end{array}$ $\begin{array}{lllllllllllllll}849.1 & 7586 & 0.956 & 632.10 & 0.365 & 0.873 & 52.02 & 0.97 & 1.76 & C & 4.864 & 227.40 & 02\end{array}$ $\begin{array}{lllllllllllll}370.4 & 7585 & 0.904 & 200.80 & 0.365 & 0.873 & 52.02 & 1.03 & 1.76 & \mathrm{C} & 2.665 & 69.00 & \mathrm{O} 2\end{array}$ $\begin{array}{lllllllllllll}257.2 & 7584 & 0.837 & 108.50 & 0.365 & 0.873 & 52.02 & 1.10 & 1.76 & \mathrm{C} & 2.157 & 35.50 & \mathrm{I}\end{array}$ $\begin{array}{lllllllllllll}222.6 & 7581 & 0.784 & 81.69 & 0.365 & 0.873 & 52.02 & 1.17 & 1.76 & \mathrm{C} & 2.046 & 26.10\end{array}$ $\begin{array}{lllllllllllll}186.3 & 7581 . & 0.697 & 57.16 & 0.365 & 0.873 & 52.02 & 1.30 & 1.76 & \mathrm{C} & 1.964 & 18.50 & \mathrm{I}\end{array}$ $\begin{array}{lllllllllllll}160.4 & 7580 & 0.581 & 41.27 & 0.365 & 0.873 & 52.02 & 1.54 & 1.76 & \mathrm{C} & 1.692 & 14.70 & \mathrm{I}\end{array}$ $\begin{array}{lllllllllllll}150.7 & 7580 & 0.514 & 35.71 & 0.365 & 0.873 & 52.02 & 1.71 & 1.76 & \mathrm{C} & 1.589 & 13.90\end{array}$ $\begin{array}{llllllllllllll}142.5 & 7580 & 0.443 & 31.27 & 0.365 & 0.873 & 52.02 & 1.95 & 1.76 & \mathrm{C} & 1.503 & 13.70 & \mathrm{I}\end{array}$ $\begin{array}{lllllllllllll}129.3 & 7580 & 0.283 & 24.57 & 0.365 & 0.873 & 52.02 & 2.83 & 1.76 & \mathrm{C} & 1.364 & 13.70 & \text { I }\end{array}$ $\begin{array}{lllllllllllll}450.3 & 7622 & 1.002 & 1063.00 & 0.365 & 0.874 & 52.02 & 0.93 & 1.76 & \mathrm{C} & 2.277 & 409.80 & 02\end{array}$ $\begin{array}{llllllllllllll}-1177.0 & 7579 . & 0.982 & * * * * * * * & 0.365 & 0.873 & 52.02 & 0.95 & 1.76 & C & -5.783 & 527.70 & \mathrm{O} 2\end{array}$ $\begin{array}{lllllllllllll}2710.0 & 7579 & 0.967 & 2571.00 & 0.364 & 0.873 & 52.02 & 0.96 & 1.76 & \mathrm{C} & 12.180 & 926.80 & \mathrm{O} 2\end{array}$ $\begin{array}{lllllllllllll}760.8 & 7579 & 0.948 & 592.80 & 0.364 & 0.873 & 52.02 & 0.98 & 1.76 & C & 4.427 & 214.70 & 02\end{array}$ $\begin{array}{lllllllllllll}356.6 & 7578 & 0.898 & 200.90 & 0.364 & 0.873 & 52.02 & 1.03 & 1.76 & \mathrm{C} & 2.581 & 68.40 & \mathrm{O} 2\end{array}$ $\begin{array}{lllllllllllll}252.9 & 7577 . & 0.833 & 110.20 & 0.364 & 0.873 & 52.02 & 1.11 & 1.76 & \mathrm{C} & 2.121 & 35.50 & 1\end{array}$ $\begin{array}{lllllllllllll}221.0 & 7574 & 0.782 & 83.50 & 0.364 & 0.873 & 52.02 & 1.17 & 1.75 & \mathrm{C} & 2.024 & 26.40\end{array}$ $\begin{array}{llllllllllll}186.9 & 7573 & 0.697 & 58.77 & 0.364 & 0.873 & 52.02 & 1.30 & 1.75 & \mathrm{C} & 1.963 & 18.80\end{array}$ $\begin{array}{lllllllllllll}162.4 & 7573 . & 0.583 & 42.59 & 0.364 & 0.873 & 52.02 & 1.53 & 1.75 & \mathrm{C} & 1.705 & 15.00 & \mathrm{I}\end{array}$ $\begin{array}{llllllllllllll}153.1 & 7572 & 0.518 & 36.90 & 0.364 & 0.873 & 52.02 & 1.70 & 1.75 & \mathrm{C} & 1.608 & 14.20 & \mathrm{I}\end{array}$ $\begin{array}{llllllllllll}145.3 & 7572 & 0.448 & 32.36 & 0.364 & 0.873 & 52.02 & 1.93 & 1.75 & \mathrm{C} & 1.526 & 13.90\end{array}$ $\begin{array}{llllllllllllll}132.6 & 7572 & 0.289 & 25.47 & 0.364 & 0.873 & 52.02 & 2.78 & 1.75 & \mathrm{C} & 1.393 & 13.80 & \mathrm{I}\end{array}$ $\begin{array}{lllllllllllll}19.3 & 9972 . & 1.026 & 32.51 & 0.396 & 0.893 & 52.02 & 0.91 & 1.72 & \mathrm{C} & 0.149 & 23.70 & \mathrm{O} 2\end{array}$ $\begin{array}{llllllllllllll}361.1 & 9480 & 1.003 & 308.40 & 0.396 & 0.884 & 52.02 & 0.92 & 1.79 & C & 1.997 & 140.70 & \text { O2 }\end{array}$ $\begin{array}{lllllllllllll}2636.0 & 9412 & 0.986 & 1801.00 & 0.396 & 0.882 & 52.02 & 0.94 & 1.79 & \mathrm{C} & 11.070 & 738.90 & \mathrm{O} 2\end{array}$ $\begin{array}{lllllllllllll}774.3 & 9411 & 0.965 & 437.20 & 0.396 & 0.882 & 52.02 & 0.96 & 1.79 & \mathrm{C} & 4.185 & 185.70 & \mathrm{O} 2\end{array}$ $\begin{array}{lllllllllllll}362.8 & 9410 & 0.911 & 149.80 & 0.395 & 0.882 & 52.02 & 1.01 & 1.79 & \mathrm{C} & 2.434 & 60.00 & 02\end{array}$ $\begin{array}{lllllllllllll}255.3 & 9409 . & 0.842 & 82.35 & 0.395 & 0.882 & 52.02 & 1.09 & 1.79 & \mathrm{C} & 1.998 & 30.70 & 1\end{array}$ $\begin{array}{lllllllllllll}221.7 & 9404 & 0.788 & 62.39 & 0.395 & 0.882 & 52.02 & 1.15 & 1.79 & \mathrm{C} & 1.896 & 22.80 & \mathrm{I}\end{array}$ $\begin{array}{llllllllllll}\text { I85.9 } & 9404 & 0.698 & 43.89 & 0.395 & 0.882 & 52.02 & 1.29 & 1.79 & \mathrm{C} & 1.833 & 16.20\end{array}$ $\begin{array}{llllllllllll}160.2 & 9403 & 0.578 & 31.80 & 0.395 & 0.882 & 52.02 & 1.52 & 1.79 & . \mathrm{C} & 1.579 & 13.20\end{array}$ $\begin{array}{llllllllllll}150.4 & 9402 . & 0.510 & 27.55 & 0.395 & 0.882 & 52.02 & 1.70 & 1.79 & \mathrm{C} & 1.483 & 12.80\end{array}$ $\begin{array}{llllllllllll}142.2 & 9402 & 0.437 & 24.16 & 0.395 & 0.882 & 52.02 & 1.94 & 1.79 & \mathrm{C} & 1.402 & 13.00\end{array}$ $\begin{array}{lllllllllllll}128.9 & 9402 & 0.273 & 19.01 & 0.395 & 0.882 & 52.02 & 2.84 & 1.79 & \mathrm{C} & 1.271 & 13.50 & \mathrm{I}\end{array}$ $\begin{array}{lllllllllllll}23.7 & 8912 . & 1.018 & 42.04 & 0.289 & 0.858 & 52.02 & 0.94 & 1.72 & \mathrm{C} & 0.166 & 24.60 & \mathrm{O} 2\end{array}$

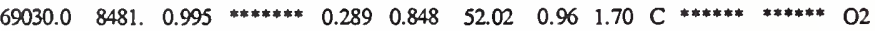
$\begin{array}{lllllllllllll}982.0 & 8481 & 0.977 & 753.40 & 0.289 & 0.848 & 52.02 & 0.97 & 1.70 & C & 4.563 & 302.70 & 02\end{array}$ $\begin{array}{lllllllllllll}554.9 & 8480 & 0.955 & 353.30 & 0.289 & 0.848 & 52.02 & 0.99 & 1.70 & \mathrm{C} & 2.968 & 139.00 & 02\end{array}$

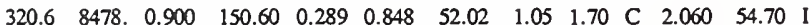
$\begin{array}{lllllllllllll}237.2 & 8476 & 0.829 & 87.71 & 0.289 & 0.848 & 52.02 & 1.14 & 1.70 & \mathrm{C} & 1.761 & 29.70 & \mathrm{I}\end{array}$ $\begin{array}{lllllllllllll}210.9 & 8469 & 0.774 & 68.41 & 0.289 & 0.847 & 52.02 & 1.21 & 1.70 & C & 1.708 & 22.50 & 1\end{array}$ $\begin{array}{lllllllllllll}177.8 & 8468 & 0.683 & 48.67 & 0.289 & 0.847 & 52.02 & 1.36 & 1.70 & \mathrm{C} & 1.658 & \text { I6.20 } & \text { I }\end{array}$ $\begin{array}{llllllllllllll}153.3 & 8468 & 0.564 & 35.53 & 0.288 & 0.847 & 52.02 & 1.63 & 1.70 & \mathrm{C} & 1.430 & 13.50 & 1\end{array}$ $\begin{array}{llllllllllllll}143.8 & 8467 . & 0.495 & 30.86 & 0.288 & 0.847 & 52.02 & 1.83 & 1.70 & \mathrm{C} & 1.342 & 13.20 & \mathrm{I}\end{array}$ $\begin{array}{llllllllllll}135.9 & 8467 & 0.423 & 27.12 & 0.288 & 0.847 & 52.02 & 2.11 & 1.70 & \mathrm{C} & 1.268 & \text { I3.40 }\end{array}$ $\begin{array}{llllllllllllll}123.0 & 8466 . & 0.261 & 21.43 & 0.288 & 0.847 & 52.02 & 3.21 & 1.70 & \mathrm{C} & 1.147 & 14.00 & \mathrm{I}\end{array}$ $\begin{array}{lllllllllllll}38.3 & 8476 . & 1.011 & 76.97 & 0.337 & 0.870 & 52.02 & 0.93 & 1.75 & \mathrm{C} & 0.267 & 36.40 & 02\end{array}$ $\begin{array}{lllllllllll}-4402.0 & 8248 . & 0.990 * * * * * * * & 0.337 & 0.864 & 52.02 & 0.95 & 1.73 & \mathrm{C} & * * * * * * * * * * * * & * 2\end{array}$ $\begin{array}{lllllllllllll}1204.0 & 8247 & 0.973 & 1010.00 & 0.337 & 0.864 & 52.02 & 0.97 & 1.73 & C & 5.773 & 391.80 & 02\end{array}$ $\begin{array}{lllllllllllll}601.5 & 8247 & 0.953 & 416.10 & 0.337 & 0.864 & 52.02 & 0.98 & 1.73 & \mathrm{C} & 3.393 & 159.40 & \mathrm{O} 2\end{array}$ $\begin{array}{llllllllllll}331.0 & 8245 & 0.901 & 167.10 & 0.337 & 0.864 & 52.02 & 1.04 & 1.73 & \mathrm{C} & 2.256 & 59.10\end{array}$ $\begin{array}{lllllllllllll}243.1 & 8244 & 0.834 & 95.67 & 0.336 & 0.864 & 52.02 & 1.12 & 1.73 & \mathrm{C} & 1.915 & 31.90\end{array}$ $\begin{array}{lllllllllllll}214.1 & 8241 & 0.782 & 73.39 & 0.336 & 0.864 & 52.02 & 1.18 & 1.73 & \mathrm{C} & 1.837 & 23.80 & 1\end{array}$ $\begin{array}{lllllllllllll}182.0 & 8240 & 0.695 & 52.26 & 0.336 & 0.864 & 52.02 & 1.32 & 1.73 & \mathrm{C} & 1.795 & 17.10\end{array}$ $\begin{array}{lllllllllllll}158.3 & 8239 & 0.579 & 38.17 & 0.336 & 0.864 & 52.02 & 1.56 & 1.73 & \mathrm{C} & 1.561 & 14.00 & \mathrm{I}\end{array}$ $\begin{array}{lllllllllllll}149.2 & 8238 & 0.513 & 33.15 & 0.336 & 0.864 & 52.02 & 1.74 & 1.73 & \mathrm{C} & 1.471 & 13.50 & \mathrm{I}\end{array}$ $\begin{array}{lllllllllllll}141.4 & 8238 & 0.442 & 29.13 & 0.336 & 0.864 & 52.02 & 1.98 & 1.73 & \mathrm{C} & 1.395 & 13.60 & \text { I }\end{array}$

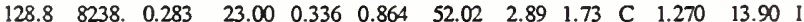
$\begin{array}{lllllllllllll}60.6 & 4528 & 1.004 & 215.30 & 0.348 & 0.870 & 52.02 & 0.94 & 1.75 & \mathrm{C} & 0.555 & 68.50 & 02\end{array}$ 
$\begin{array}{lllllllllllll}1458.0 & 4486 . & 0.981 & 2704.00 & 0.348 & 0.868 & 52.02 & 0.96 & 1.74 & \mathrm{C} & 8.966 & 764.40 & \mathrm{O} 2\end{array}$ $\begin{array}{lllllllllllll}636.9 & 4485 . & 0.964 & 941.70 & 0.348 & 0.868 & 52.02 & 0.97 & 1.74 & \mathrm{C} & 4.728 & 269.00 & \mathrm{O} 2\end{array}$ $\begin{array}{lllllllllllll}434.1 & 4485 & 0.944 & 532.20 & 0.348 & 0.868 & 52.02 & 0.99 & 1.74 & \mathrm{C} & 3.595 & 154.40 & \mathrm{O} 2\end{array}$ $\begin{array}{llllllllllllll}280.6 & 4485 & 0.890 & 253.40 & 0.348 & 0.868 & 52.02 & 1.05 & 1.74 & C & 2.735 & 71.50 & 1\end{array}$ $\begin{array}{lllllllllllll}215.9 & 4485 & 0.822 & 153.40 & 0.348 & 0.868 & 52.02 & 1.13 & 1.74 & \mathrm{C} & 2.415 & 41.80 & \text { I }\end{array}$ $\begin{array}{lllllllllllll}189.8 & 4485 & 0.769 & 118.10 & 0.348 & 0.868 & 52.02 & 1.20 & 1.74 & \mathrm{C} & 2.313 & 31.70 & 1\end{array}$ $\begin{array}{lllllllllllll}163.6 & 4485 & 0.681 & 85.80 & 0.348 & 0.868 & 52.02 & 1.34 & 1.74 & \mathrm{C} & 1.677 & 23.10 & 1\end{array}$ $\begin{array}{lllllllllllll}143.2 & 4485 & 0.566 & 63.56 & 0.348 & 0.868 & 52.02 & 1.58 & 1.74 & \mathrm{C} & 1.602 & 18.20 & 1\end{array}$ $\begin{array}{llllllllllll}135.2 & 4485 & 0.500 & 55.50 & 0.348 & 0.868 & 52.02 & 1.77 & 1.74 & \mathrm{C} & 1.587 & 16.90\end{array}$ $\begin{array}{llllllllllll}128.3 & 4484 & 0.430 & 48.99 & 0.348 & 0.868 & 52.02 & 2.02 & 1.74 & \mathrm{C} & 1.586 & 16.40\end{array}$ $\begin{array}{llllllllllllll}116.9 & 4484 & 0.274 & 38.98 & 0.348 & 0.868 & 52.02 & 2.94 & 1.74 & \mathrm{C} & 1.445 & 15.80 & 1\end{array}$ $\begin{array}{lllllllllllll}-410.2 & 4291 & 0.999 & * * * * * * * & 0.383 & 0.878 & 52.02 & 0.93 & 1.77 & C & -3.019 & 409.50 & 02\end{array}$ $\begin{array}{lllllllllllll}6792.0 & 4291 & 0.973 & * * * * * * & 0.383 & 0.878 & 52.02 & 0.95 & 1.77 & \mathrm{C} & 35.380 & * * * * * * & 02\end{array}$ $\begin{array}{lllllllllllll}1013.0 & 4291 & 0.953 & 1362.00 & 0.383 & 0.878 & 52.02 & 0.97 & 1.77 & \mathrm{C} & 7.607 & 396.90 & \mathrm{O} 2\end{array}$ $\begin{array}{lllllllllllll}589.4 & 4290 & 0.930 & 668.50 & 0.383 & 0.878 & 52.02 & 0.99 & 1.77 & \mathrm{C} & 5.076 & 196.00 & \mathrm{O} 2\end{array}$ $\begin{array}{lllllllllllllll}338.2 & 4290 & 0.872 & 292.00 & 0.383 & 0.878 & 52.02 & 1.06 & 1.77 & \mathrm{C} & 3.481 & 83.90 & \mathrm{O} 2\end{array}$ $\begin{array}{lllllllllllll}245.0 & 4290 & 0.800 & 171.70 & 0.383 & 0.878 & 52.02 & 1.14 & 1.77 & C & 2.931 & 48.10 & 1\end{array}$ $\begin{array}{lllllllllllll}208.5 & 4290 & 0.745 & 130.40 & 0.383 & 0.878 & 52.02 & 1.22 & 1.77 & \mathrm{C} & 1.987 & 36.30 & \mathrm{I}\end{array}$ $\begin{array}{lllllllllllll}173.3 & 4290 & 0.657 & 94.04 & 0.383 & 0.878 & 52.02 & 1.37 & 1.77 & \mathrm{C} & 1.791 & 26.20 & \mathrm{I}\end{array}$ $\begin{array}{llllllllllllll}146.4 & 4290 & 0.545 & 69.27 & 0.383 & 0.878 & 52.02 & 1.61 & 1.77 & \mathrm{C} & 1.652 & 20.40 & 1\end{array}$ $\begin{array}{lllllllllllll}135.9 & 4290 & 0.482 & 60.37 & 0.383 & 0.878 & 52.02 & 1.80 & 1.77 & \mathrm{C} & 1.610 & 19.40 & 1\end{array}$ $\begin{array}{llllllllllll}126.9 & 4290 & 0.416 & 53.22 & 0.383 & 0.878 & 52.02 & 2.04 & 1.77 & \mathrm{C} & 1.584 & 19.60\end{array}$ $\begin{array}{lllllllllllll}112.1 & 4290 & 0.272 & 42.24 & 0.383 & 0.878 & 52.02 & 2.88 & 1.77 & C & 1.400 & 20.10 & 1\end{array}$ $\begin{array}{lllllllllllll}78.8 & 4861 . & 1.004 & 260.60 & 0.376 & 0.878 & 52.02 & 0.93 & 1.77 & \mathrm{C} & 0.686 & 85.70 & \mathrm{O} 2\end{array}$ $\begin{array}{lllllllllllll}985.6 & 4819 & 0.965 & 1350.00 & 0.376 & 0.876 & 52.02 & 0.96 & 1.77 & C & 6.754 & 421.70 & 02\end{array}$ $\begin{array}{lllllllllllll}538.1 & 4819 & 0.944 & 610.70 & 0.376 & 0.876 & 52.02 & 0.98 & 1.77 & \mathrm{C} & 4.277 & 186.60 & \mathrm{O} 2\end{array}$ $\begin{array}{llllllllllllll}306.8 & 4819 & 0.891 & 256.00 & 0.376 & 0.876 & 52.02 & 1.04 & 1.77 & \mathrm{C} & 2.925 & 74.90 & \mathrm{HC}\end{array}$ $\begin{array}{llllllllllll}226.9 & 4818 & 0.823 & 148.60 & 0.376 & 0.876 & 52.02 & 1.12 & 1.77 & C & 2.505 & 42.50\end{array}$ $\begin{array}{lllllllllllllll}196.9 & 4818 & 0.770 & 112.80 & 0.376 & 0.876 & 52.02 & 1.19 & 1.77 & \mathrm{C} & 2.377 & 31.90 & 1\end{array}$ $\begin{array}{llllllllllll}167.8 & 4818 & 0.683 & 80.92 & 0.375 & 0.876 & 52.02 & 1.32 & 1.77 & \mathrm{C} & 2.318 & 23.10\end{array}$ $\begin{array}{lllllllllllll}145.9 & 4818 & 0.568 & 59.42 & 0.375 & 0.876 & 52.02 & 1.56 & 1.77 & \mathrm{C} & 1.639 & 17.90 & 1\end{array}$ $\begin{array}{llllllllllllll}137.3 & 4818 & 0.502 & 51.72 & 0.375 & 0.876 & 52.02 & 1.74 & 1.77 & \mathrm{C} & 1.613 & 16.70 & \mathrm{I}\end{array}$ $\begin{array}{llllllllllll}130.2 & 4818 & 0.432 & 45.55 & 0.375 & 0.876 & 52.02 & 1.98 & 1.77 & \mathrm{C} & 1.609 & 16.20\end{array}$ $\begin{array}{lllllllllllll}118.3 & 4818 & 0.276 & 36.11 & 0.375 & 0.876 & 52.02 & 2.87 & 1.77 & \mathrm{C} & 1.463 & 15.60 & 1\end{array}$ $\begin{array}{lllllllllllll}85.0 & 5051 . & 1.004 & 252.10 & 0.398 & 0.884 & 52.02 & 0.92 & 1.80 & \mathrm{C} & 0.742 & 88.90 & \mathrm{O} 2\end{array}$

$\begin{array}{llllllllllllllll}3973.0 & 5009 . & 0.980 & 6229.00 & 0.398 & 0.883 & 52.02 & 0.94 & 1.79 & \mathrm{C} & 20.820 & * * * * * * & \mathrm{O} 2\end{array}$ $\begin{array}{lllllllllllll}853.7 & 5009 & 0.963 & 1073.00 & 0.398 & 0.883 & 52.02 & 0.96 & 1.79 & \mathrm{C} & 6.076 & 343.00 & \mathrm{O} 2\end{array}$ $\begin{array}{lllllllllllll}517.3 & 5009 & 0.942 & 542.60 & 0.398 & 0.883 & 52.02 & 0.98 & 1.79 & \mathrm{C} & 4.176 & 168.90 & \mathrm{O} 2\end{array}$ $\begin{array}{lllllllllllllll}308.4 & 5008 & 0.888 & 241.00 & 0.398 & 0.883 & 52.02 & 1.03 & 1.79 & \mathrm{C} & 2.973 & 73.50 & \mathrm{HC}\end{array}$

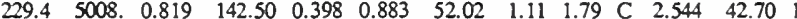
$\begin{array}{lllllllllllll}198.7 & 5008 & 0.766 & 108.70 & 0.398 & 0.883 & 52.02 & 1.18 & 1.79 & \mathrm{C} & 2.410 & 32.10 & 1\end{array}$ $\begin{array}{llllllllllllll}168.5 & 5008 & 0.680 & 78.48 & 0.398 & 0.883 & 52.02 & 1.32 & 1.79 & \mathrm{C} & 2.340 & 23.40 & 1\end{array}$ $\begin{array}{lllllllllllll}145.4 & 5008 & 0.568 & 57.87 & 0.398 & 0.883 & 52.02 & 1.54 & 1.79 & \mathrm{C} & 1.633 & 18.40 & 1\end{array}$ $\begin{array}{llllllllllllll}136.4 & 5008 & 0.504 & 50.45 & 0.398 & 0.883 & 52.02 & 1.71 & 1.79 & \mathrm{C} & 1.601 & 17.70 & \text { I }\end{array}$ $\begin{array}{llllllllllllll}128.7 & 5008 & 0.437 & 44.48 & 0.398 & 0.883 & 52.02 & 1.94 & 1.79 & C & 1.591 & 17.90 & 1\end{array}$ $\begin{array}{llllllllllllll}116.0 & 5008 & 0.287 & 35.32 & 0.398 & 0.883 & 52.02 & 2.73 & 1.79 & \mathrm{C} & 1.434 & 18.50 & 1\end{array}$ $\begin{array}{lllllllllllll}45.8 & 5712 . & 1.007 & 127.80 & 0.270 & 0.844 & 52.02 & 0.95 & 1.70 & \mathrm{C} & 0.353 & 46.20 & \mathrm{O} 2\end{array}$

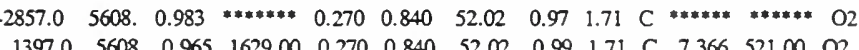

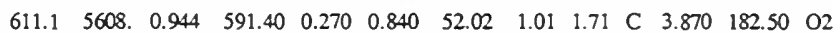
$\begin{array}{lllllllllllll}317.4 & 5608 & 0.889 & 226.50 & 0.270 & 0.840 & 52.02 & 1.07 & 1.71 & \mathrm{C} & 2.455 & 67.00 & 1\end{array}$ $\begin{array}{lllllllllllll}228.5 & 5608 & 0.819 & 128.40 & 0.270 & 0.840 & 52.02 & 1.16 & 1.71 & \mathrm{C} & 2.057 & 35.60 & 1\end{array}$ $\begin{array}{llllllllllllll}201.6 & 5606 . & 0.764 & 99.26 & 0.270 & 0.840 & 52.02 & 1.23 & 1.71 & \mathrm{C} & 1.980 & 27.00 & 1\end{array}$ $\begin{array}{llllllllllllll}170.3 & 5605 & 0.674 & 70.82 & 0.270 & 0.840 & 52.02 & 1.39 & 1.71 & C & 1.921 & 19.50 & \text { I }\end{array}$ $\begin{array}{lllllllllllll}147.0 & 5605 & 0.556 & 51.78 & 0.270 & 0.840 & 52.02 & 1.66 & 1.71 & \mathrm{C} & 1.989 & 15.70 & 1\end{array}$ $\begin{array}{llllllllllll}138.0 & 5604 & 0.488 & 45.00 & 0.270 & 0.840 & 52.02 & 1.87 & 1.71 & \mathrm{C} & 2.085 & 15.00\end{array}$ $\begin{array}{lllllllllllllll}130.2 & 5604 & 0.417 & 39.51 & 0.270 & 0.840 & 52.02 & 2.16 & 1.71 & \mathrm{C} & 1.968 & 14.80 & \text { I }\end{array}$ $\begin{array}{llllllllllllll}117.6 & 5604 & 0.256 & 31.17 & 0.270 & 0.840 & 52.02 & 3.30 & 1.71 & \mathrm{C} & 1.777 & 14.90 & \mathrm{I}\end{array}$ $\begin{array}{lllllllllllll}45.6 & 4564 & 1.005 & 154.00 & 0.276 & 0.846 & 52.02 & 0.95 & 1.70 & \mathrm{C} & 0.400 & 47.30 & 02\end{array}$ $\begin{array}{lllllllllllllll}3716.0 & 4499 & 0.981 & 6597.00 & 0.276 & 0.843 & 52.02 & 0.97 & 1.71 & C & 17.940 & * * * * * * & O 2\end{array}$ $\begin{array}{lllllllllllll}848.8 & 4499 & 0.963 & 1205.00 & 0.276 & 0.843 & 52.02 & 0.99 & 1.71 & C & 5.510 & 339.00 & 02\end{array}$ $\begin{array}{llllllllllllll}498.3 & 4499 & 0.941 & 588.60 & 0.276 & 0.843 & 52.02 & 1.01 & 1.71 & \mathrm{C} & 3.699 & 159.30 & \mathrm{O} 2\end{array}$ $\begin{array}{lllllllllllll}293.6 & 4499 & 0.885 & 257.00 & 0.276 & 0.843 & 52.02 & 1.07 & 1.71 & \mathrm{C} & 2.606 & 67.90 & 1\end{array}$ $\begin{array}{llllllllllllll}219.0 & 4500 & 0.813 & 151.60 & 0.276 & 0.843 & 52.02 & 1.16 & 1.71 & \mathrm{C} & 2.245 & 38.40 & 1\end{array}$ $\begin{array}{lllllllllllll}193.0 & 4499 & 0.758 & 117.40 & 0.276 & 0.843 & 52.02 & 1.24 & 1.71 & \mathrm{C} & 2.168 & 29.20 & 1\end{array}$ $\begin{array}{llllllllllllll}165.8 & 4499 & 0.667 & 85.48 & 0.276 & 0.843 & 52.02 & 1.40 & 1.71 & \mathrm{C} & 2.142 & 21.40 & 1\end{array}$ $\begin{array}{llllllllllllll}144.5 & 4498 & 0.548 & 63.37 & 0.276 & 0.843 & 52.02 & 1.68 & 1.71 & \mathrm{C} & 1.589 & 17.10 & \mathrm{I}\end{array}$ $\begin{array}{llllllllllllll}136.1 & 4498 & 0.481 & 55.34 & 0.276 & 0.843 & 52.02 & 1.89 & 1.71 & \mathrm{C} & 1.576 & 16.00 & 1\end{array}$ $\begin{array}{lllllllllllll}128.5 & 4498 & 0.409 & 48.73 & 0.276 & 0.843 & 52.02 & 2.19 & 1.71 & \mathrm{C} & 1.566 & 15.50 & 1\end{array}$ $\begin{array}{lllllllllllll}116.1 & 4498 & 0.249 & 38.60 & 0.276 & 0.843 & 52.02 & 3.37 & 1.71 & \mathrm{C} & 1.415 & 15.00 & 1\end{array}$ $\begin{array}{lllllllllllll}20.1 & 19950 & 1.052 & 13.73 & 0.369 & 0.899 & 52.02 & 0.89 & 1.81 & \mathrm{C} & 0.245 & 15.10 & \mathrm{O} 2\end{array}$ $\begin{array}{lllllllllllll}73.2 & 18610 & 1.026 & 27.77 & 0.369 & 0.886 & 52.02 & 0.91 & 1.81 & \mathrm{C} & 0.892 & 19.40 & 02\end{array}$ $\begin{array}{lllllllllllll}263.2 & 17760 & 1.006 & 84.90 & 0.369 & 0.877 & 52.02 & 0.93 & 1.77 & \mathrm{C} & 3.207 & 48.30 & \mathrm{O} 2\end{array}$ $\begin{array}{lllllllllllll}536.5 & 17520 & 0.982 & 147.30 & 0.368 & 0.874 & 52.02 & 0.95 & 1.76 & \mathrm{C} & 6.538 & 79.10 & \mathrm{O} 2\end{array}$ $\begin{array}{llllllllllllll}368.7 & 17510 & 0.923 & 76.48 & 0.368 & 0.874 & 52.02 & 1.01 & 1.76 & \mathrm{C} & 4.493 & 37.80 & 1\end{array}$ $\begin{array}{llllllllllllll}285.8 & 17510 & 0.848 & 47.74 & 0.368 & 0.874 & 52.02 & 1.09 & 1.76 & C & 1.564 & 21.60 & 1\end{array}$ $\begin{array}{llllllllllllll}260.5 & 17470 & 0.792 & 38.79 & 0.366 & 0.873 & 52.02 & 1.16 & 1.76 & \mathrm{C} & 1.426 & 17.00 & \text { I }\end{array}$ $\begin{array}{lllllllllllll}219.6 & 17460 & 0.700 & 28.14 & 0.366 & 0.873 & 52.02 & 1.30 & 1.76 & \mathrm{C} & 1.202 & 12.50\end{array}$ $\begin{array}{llllllllllllll}187.8 & 17460 & 0.581 & 20.81 & 0.366 & 0.873 & 52.02 & 1.53 & 1.76 & \mathrm{C} & 1.028 & 10.80 & 1\end{array}$ $\begin{array}{lllllllllllll}175.2 & 17450 & 0.515 & 18.16 & 0.366 & 0.873 & 52.02 & 1.71 & 1.76 & \mathrm{C} & 0.959 & 10.80 & 1\end{array}$ $\begin{array}{llllllllllll}164.3 & 17450 & 0.445 & 16.01 & 0.366 & 0.873 & 52.02 & 1.94 & 1.76 & \mathrm{C} & 0.899 & 11.30\end{array}$ $\begin{array}{lllllllllllll}146.3 & 17450 & 0.290 & 12.70 & 0.365 & 0.873 & 52.02 & 2.77 & 1.76 & \mathrm{C} & 0.801 & 12.20 & 1\end{array}$ $\begin{array}{lllllllllllll}21.6 & 14810 & 1.044 & 16.72 & 0.377 & 0.897 & 52.02 & 0.90 & 1.78 & \mathrm{C} & 0.135 & 16.10 & \mathrm{O} 2\end{array}$ $\begin{array}{lllllllllllll}127.9 & 13710 . & 1.013 & 55.89 & 0.376 & 0.882 & 52.02 & 0.92 & 1.79 & \mathrm{C} & 0.712 & 30.20 & \mathrm{O} 2\end{array}$ $\begin{array}{lllllllllllll}772.2 & 13320 & 0.990 & 282.80 & 0.376 & 0.876 & 52.02 & 0.94 & 1.77 & \mathrm{C} & 3.433 & 136.50 & \mathrm{O} 2\end{array}$ $\begin{array}{lllllllllllll}558.6 & 13320 & 0.962 & 173.10 & 0.376 & 0.876 & 52.02 & 0.97 & 1.77 & C & 2.744 & 81.00 & 02\end{array}$ $\begin{array}{lllllllllllllll}367.2 & 13310 & 0.894 & 87.22 & 0.376 & 0.876 & 52.02 & 1.03 & 1.77 & C & 2.127 & 37.80 & \text { O2 }\end{array}$ $\begin{array}{llllllllllll}277.6 & 13310 & 0.809 & 53.86 & 0.376 & 0.876 & 52.02 & 1.13 & 1.77 & \mathrm{C} & 1.863 & 21.70\end{array}$ $\begin{array}{llllllllllll}246.2 & 13290 & 0.746 & 42.90 & 0.375 & 0.876 & 52.02 & 1.22 & 1.77 & \mathrm{C} & 1.652 & 16.80\end{array}$ $\begin{array}{lllllllllllll}205.1 & 13290 & 0.643 & 31.16 & 0.375 & 0.876 & 52.02 & 1.40 & 1.77 & \mathrm{C} & 1.376 & 12.40 & 1\end{array}$ $\begin{array}{lllllllllllll}173.1 & 13290 & 0.514 & 23.07 & 0.375 & 0.876 & 52.02 & 1.70 & 1.76 & \mathrm{C} & 1.161 & 10.50\end{array}$ $\begin{array}{llllllllllllll}160.3 & 13290 & 0.441 & 20.14 & 0.375 & 0.876 & 52.02 & 1.94 & 1.76 & \mathrm{C} & 1.076 & 10.70\end{array}$ $\begin{array}{lllllllllllll}149.4 & 13290 & 0.366 & 17.76 & 0.375 & 0.876 & 52.02 & 2.28 & 1.76 & \mathrm{C} & 1.003 & 11.50 & 1\end{array}$ $\begin{array}{llllllllllll}131.3 & 13280 & 0.202 & 14.11 & 0.375 & 0.876 & 52.02 & 3.64 & 1.76 & \mathrm{C} & 0.881 & 12.70\end{array}$ $\begin{array}{lllllllllllll}18.9 & 13800 & 1.033 & 23.17 & 0.375 & 0.891 & 52.02 & 0.91 & 1.51 & \mathrm{C} & 0.126 & 17.90 & \mathrm{O} 2\end{array}$ $\begin{array}{lllllllllllll}158.3 & 12990 & 1.008 & 90.88 & 0.375 & 0.879 & 52.02 & 0.93 & 1.78 & \mathrm{C} & 0.847 & 42.30 & 02\end{array}$ $\begin{array}{lllllllllllll}718.9 & 12770 & 0.989 & 339.40 & 0.374 & 0.876 & 52.02 & 0.94 & 1.76 & \mathrm{C} & 3.186 & 147.30 & \mathrm{O} 2\end{array}$ $\begin{array}{lllllllllllll}521.7 & 12770 & 0.967 & 207.00 & 0.374 & 0.876 & 52.02 & 0.96 & 1.76 & \mathrm{C} & 2.548 & 87.10 & 02\end{array}$ $\begin{array}{lllllllllllll}346.5 & 12760 & 0.910 & 103.90 & 0.374 & 0.876 & 52.02 & 1.02 & 1.76 & \mathrm{C} & 1.976 & 41.00 & 1\end{array}$ $\begin{array}{lllllllllllll}265.1 & 12760 & 0.840 & 64.08 & 0.374 & 0.876 & 52.02 & 1.10 & 1.76 & \mathrm{C} & 1.729 & 23.50 & 1\end{array}$ $\begin{array}{lllllllllllll}237.2 & 12750 & 0.786 & 51.05 & 0.373 & 0.876 & 52.02 & 1.16 & 1.76 & \mathrm{C} & 1.679 & 18.30\end{array}$ $\begin{array}{llllllllllll}199.9 & 12740 & 0.700 & 37.05 & 0.373 & 0.875 & 52.02 & 1.30 & 1.76 & \mathrm{C} & 1.415 & 13.50\end{array}$ $\begin{array}{lllllllllllll}170.8 & 12740 & 0.588 & 27.41 & 0.373 & 0.875 & 52.02 & 1.51 & 1.76 & \mathrm{C} & 1.209 & 11.30 & 1\end{array}$ $\begin{array}{lllllllllllll}159.3 & 12740 & 0.525 & 23.92 & 0.373 & 0.875 & 52.02 & 1.67 & 1.76 & \mathrm{C} & 1.128 & 11.20 & 1\end{array}$ $\begin{array}{lllllllllllll}149.4 & 12740 & 0.459 & 21.10 & 0.373 & 0.875 & 52.02 & 1.88 & 1.76 & \mathrm{C} & 1.058 & 11.70 & 1\end{array}$ $\begin{array}{llllllllllllll}133.1 & 12740 & 0.314 & 16.75 & 0.373 & 0.875 & 52.02 & 2.59 & 1.76 & \mathrm{C} & 0.942 & 12.60\end{array}$ $\begin{array}{lllllllllllll}22.3 & 14800 & 1.037 & 20.91 & 0.393 & 0.897 & 52.02 & 0.90 & 1.78 & \mathrm{C} & 0.138 & 16.30 & \mathrm{O} 2\end{array}$ $\begin{array}{lllllllllllll}135.3 & 13920 & 1.011 & 68.98 & 0.393 & 0.886 & 52.02 & 0.92 & 1.80 & \mathrm{C} & 0.733 & 34.20 & \mathrm{O} 2\end{array}$ $\begin{array}{lllllllllllll}803.5 & 13610 & 0.991 & 338.50 & 0.393 & 0.881 & 52.02 & 0.94 & 1.78 & \mathrm{C} & 3.471 & 153.70 & 02\end{array}$ $\begin{array}{lllllllllllll}568.5 & 13610 & 0.968 & 200.90 & 0.392 & 0.881 & 52.02 & 0.96 & 1.78 & \mathrm{C} & 2.720 & 88.30 & 02\end{array}$ $\begin{array}{lllllllllllll}371.4 & 13600 & 0.909 & 98.80 & 0.392 & 0.881 & 52.02 & 1.01 & 1.78 & \mathrm{C} & 2.086 & 40.40 & \mathrm{O} 2\end{array}$ $\begin{array}{llllllllllll}283.0 & 13600 & 0.835 & 60.45 & 0.392 & 0.881 & 52.02 & 1.09 & 1.78 & \mathrm{C} & 1.823 & 23.30\end{array}$ $\begin{array}{llllllllllll}253.1 & 13590 & 0.779 & 48.03 & 0.391 & 0.881 & 52.02 & 1.17 & 1.78 & \mathrm{C} & 1.777 & 18.00\end{array}$ $\begin{array}{lllllllllllll}213.5 & 13580 & 0.687 & 34.77 & 0.391 & 0.881 & 52.02 & 1.31 & 1.78 & \mathrm{C} & 1.499 & 13.20\end{array}$ $\begin{array}{llllllllllll}182.9 & 13580 & 0.569 & 25.69 & 0.391 & 0.881 & 52.02 & 1.55 & 1.78 & \mathrm{C} & 1.284 & 11.00\end{array}$ $\begin{array}{llllllllllll}170.8 & 13580 & 0.502 & 22.40 & 0.391 & 0.881 & 52.02 & 1.72 & 1.78 & \mathrm{C} & 1.199 & 10.80\end{array}$ $\begin{array}{llllllllllll}160.4 & 13580 & 0.432 & 19.75 & 0.391 & 0.881 & 52.02 & 1.96 & 1.78 & C & 1.126 & 11.10\end{array}$ $\begin{array}{lllllllllllll}143.2 & 13580 & 0.277 & 15.67 & 0.391 & 0.881 & 52.02 & 2.82 & 1.78 & \mathrm{C} & 1.006 & 11.80 & 1\end{array}$ $\begin{array}{lllllllllllll}19.5 & 13890 & 1.042 & 16.85 & 0.320 & 0.881 & 52.02 & 0.91 & 1.80 & \mathrm{C} & 0.137 & 14.60 & \mathrm{O} 2\end{array}$ $\begin{array}{lllllllllllll}108.1 & 12810 & 1.012 & 54.99 & 0.320 & 0.865 & 52.02 & 0.94 & 1.74 & \mathrm{C} & 0.594 & 28.20 & \mathrm{O} 2\end{array}$ $\begin{array}{lllllllllllll}682.0 & 12420 & 0.990 & 290.20 & 0.320 & 0.858 & 52.02 & 0.95 & 1.72 & \mathrm{C} & 2.966 & 134.90 & 02\end{array}$ $\begin{array}{lllllllllllll}513.9 & 12410 & 0.964 & 184.80 & 0.320 & 0.858 & 52.02 & 0.98 & 1.72 & \mathrm{C} & 2.454 & 82.00 & 02\end{array}$ $\begin{array}{lllllllllllll}349.1 & 12410 & 0.899 & 96.01 & 0.319 & 0.858 & 52.02 & 1.04 & 1.72 & C & 1.948 & 39.40 & 1\end{array}$ $\begin{array}{lllllllllllll}267.6 & 12410 & 0.819 & 59.95 & 0.319 & 0.858 & 52.02 & 1.14 & 1.72 & \mathrm{C} & 1.719 & 22.80\end{array}$ $\begin{array}{llllllllllll}241.0 & 12390 & 0.758 & 48.45 & 0.318 & 0.858 & 52.02 & 1.23 & 1.72 & \mathrm{C} & 1.692 & 17.80\end{array}$ $\begin{array}{llllllllllll}201.3 & 12380 & 0.660 & 35.21 & 0.318 & 0.858 & 52.02 & 1.39 & 1.72 & \mathrm{C} & 1.413 & 13.20\end{array}$ $\begin{array}{lllllllllllll}170.3 & 12380 & 0.536 & 26.07 & 0.318 & 0.858 & 52.02 & 1.68 & 1.72 & \mathrm{C} & 1.195 & 11.20 & 1\end{array}$ $\begin{array}{llllllllllll}157.9 & 12380 & 0.467 & 22.76 & 0.318 & 0.858 & 52.02 & 1.91 & 1.72 & \mathrm{C} & 1.109 & 11.20\end{array}$ $\begin{array}{llllllllllll}147.3 & 12370 & 0.394 & 20.06 & 0.318 & 0.858 & 52.02 & 2.21 & 1.72 & \mathrm{C} & 1.034 & 11.70\end{array}$ $\begin{array}{lllllllllllll}129.7 & 12370 & 0.236 & 15.92 & 0.317 & 0.858 & 52.02 & 3.40 & 1.72 & \mathrm{C} & 0.910 & 12.70 & 1\end{array}$ $\begin{array}{lllllllllllll}24.8 & 15980 & 1.037 & 20.30 & 0.365 & 0.890 & 52.02 & 0.91 & 1.84 & \mathrm{C} & 0.174 & 16.20 & \mathrm{O} 2\end{array}$ $\begin{array}{lllllllllllll}147.6 & 14950 & 1.010 & 69.48 & 0.364 & 0.877 & 52.02 & 0.93 & 1.77 & \mathrm{C} & 0.742 & 36.40 & \mathrm{O} 2\end{array}$ $\begin{array}{lllllllllllll}742.5 & 14610 & 0.990 & 288.90 & 0.364 & 0.873 & 52.02 & 0.94 & 1.76 & C & 3.055 & 140.80 & 02\end{array}$ $\begin{array}{lllllllllllll}547.2 & 14610 & 0.966 & 178.90 & 0.364 & 0.873 & 52.02 & 0.97 & 1.76 & \mathrm{C} & 2.476 & 84.70 & 02\end{array}$ $\begin{array}{lllllllllllll}367.8 & 14610 & 0.906 & 90.79 & 0.364 & 0.873 & 52.02 & 1.02 & 1.75 & \mathrm{C} & 1.947 & 39.30 & 1\end{array}$ $\begin{array}{lllllllllllll}282.7 & 14600 & 0.831 & 56.19 & 0.364 & 0.873 & 52.02 & 1.11 & 1.75 & \mathrm{C} & 1.714 & 22.60 & 1\end{array}$ $\begin{array}{llllllllllll}255.4 & 14580 & 0.774 & 45.21 & 0.363 & 0.872 & 52.02 & 1.19 & 1.75 & \mathrm{C} & 1.549 & 17.60\end{array}$ $\begin{array}{llllllllllll}215.1 & 14580 & 0.681 & 32.77 & 0.362 & 0.872 & 52.02 & 1.33 & 1.75 & \mathrm{C} & 1.305 & 13.00\end{array}$ $\begin{array}{lllllllllllll}183.9 & 14570 & 0.561 & 24.22 & 0.362 & 0.872 & 52.02 & 1.59 & 1.75 & \mathrm{C} & 1.115 & 10.90\end{array}$

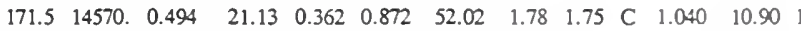
$\begin{array}{llllllllllll}160.9 & 14570 & 0.423 & 18.62 & 0.362 & 0.872 & 52.02 & 2.03 & 1.75 & \mathrm{C} & 0.976 & 11.30\end{array}$

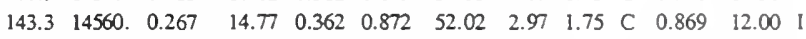
$\begin{array}{lllllllllllllll}8069.0 & 13030 & 0.994 & 8962.00 & 0.317 & 0.858 & 52.02 & 0.95 & 1.72 & \mathrm{C} & 19.110 & * * * * * * & 02\end{array}$ $\begin{array}{lllllllllllll}829.2 & 13020 & 0.968 & 489.10 & 0.317 & 0.857 & 52.02 & 0.97 & 1.72 & \mathrm{C} & 3.241 & 218.00 & 02\end{array}$ $\begin{array}{lllllllllllll}599.7 & 13020 & 0.950 & 285.40 & 0.317 & 0.857 & 52.02 & 0.99 & 1.72 & \mathrm{C} & 2.600 & 126.10 & 02\end{array}$ $\begin{array}{lllllllllllll}478.6 & 13020 & 0.928 & 191.10 & 0.317 & 0.857 & 52.02 & 1.02 & 1.72 & \mathrm{C} & 2.252 & 80.60 & \mathrm{O} 2\end{array}$ $\begin{array}{lllllllllllll}344.5 & 13010 & 0.871 & 103.60 & 0.317 & 0.857 & 52.02 & 1.08 & 1.72 & \mathrm{C} & 1.874 & 40.60\end{array}$ $\begin{array}{lllllllllllll}272.4 & 13010 & 0.800 & 65.79 & 0.316 & 0.857 & 52.02 & 1.17 & 1.72 & \mathrm{C} & 1.692 & 23.90\end{array}$ $\begin{array}{lllllllllllll}251.4 & 12980 & 0.746 & 53.99 & 0.315 & 0.857 & 52.02 & 1.24 & 1.72 & \mathrm{C} & 1.561 & 19.00 & 1\end{array}$ $\begin{array}{llllllllllll}213.4 & 12980 & 0.658 & 39.36 & 0.315 & 0.857 & 52.02 & 1.40 & 1.72 & \mathrm{C} & 1.325 & 14.00\end{array}$ $\begin{array}{lllllllllllll}183.5 & 12970 & 0.545 & 29.19 & 0.315 & 0.857 & 52.02 & 1.66 & 1.72 & \mathrm{C} & 1.140 & 11.90 & 1\end{array}$ $\begin{array}{lllllllllllll}171.6 & 12970 & 0.481 & 25.49 & 0.315 & 0.857 & 52.02 & 1.86 & 1.72 & \mathrm{C} & 1.066 & 11.80 & 1\end{array}$ $\begin{array}{llllllllllll}161.2 & 12970 & 0.413 & 22.48 & 0.315 & 0.857 & 52.02 & 2.13 & 1.72 & \mathrm{C} & 1.001 & 12.10\end{array}$ $\begin{array}{lllllllllllll}144.0 & 12970 & 0.265 & 17.85 & 0.314 & 0.857 & 52.02 & 3.11 & 1.72 & \mathrm{C} & 0.895 & 12.80 & 1\end{array}$ $\begin{array}{lllllllllllll}27.9 & 6030 & 1.013 & 58.41 & 0.319 & 0.865 & 52.02 & 0.93 & 1.74 & \mathrm{C} & 0.250 & 20.60 & \mathrm{O} 2\end{array}$ $\begin{array}{lllllllllllll}855.1 & 5838 & 0.984 & 992.40 & 0.319 & 0.858 & 52.02 & 0.96 & 1.72 & \mathrm{C} & 5.032 & 288.30 & \mathrm{O} 2\end{array}$ $\begin{array}{lllllllllllll}569.2 & 5838 & 0.963 & 536.70 & 0.319 & 0.858 & 52.02 & 0.98 & 1.72 & \mathrm{C} & 3.770 & 158.60 & \mathrm{O} 2\end{array}$ $\begin{array}{lllllllllllll}442.2 & 5838 & 0.938 & 352.30 & 0.319 & 0.858 & 52.02 & 1.00 & 1.72 & \mathrm{C} & 3.191 & 102.30 & \mathrm{O} 2\end{array}$ $\begin{array}{lllllllllllll}309.5 & 5837 & 0.876 & 188.50 & 0.319 & 0.858 & 52.02 & 1.07 & 1.72 & \mathrm{C} & 2.596 & 52.40 & 1\end{array}$ $\begin{array}{lllllllllllll}240.5 & 5836 & 0.798 & 119.40 & 0.319 & 0.858 & 52.02 & 1.17 & 1.72 & \mathrm{C} & 2.318 & 31.70 & 1\end{array}$ $\begin{array}{lllllllllllll}212.4 & 5835 & 0.739 & 94.39 & 0.319 & 0.858 & 52.02 & 1.25 & 1.72 & \mathrm{C} & 2.240 & 24.90 & \text { L }\end{array}$ $\begin{array}{llllllllllllll}179.5 & 5835 & 0.646 & 69.44 & 0.319 & 0.858 & 52.02 & 1.42 & 1.72 & \mathrm{C} & 2.188 & 18.30 & 1\end{array}$ $\begin{array}{lllllllllllll}153.2 & 5834 & 0.526 & 51.87 & 0.319 & 0.858 & 52.02 & 1.71 & 1.72 & \mathrm{C} & 2.248 & 14.70 & \text { I }\end{array}$ $\begin{array}{lllllllllllll}142.5 & 5834 & 0.460 & 45.43 & 0.319 & 0.858 & 52.02 & 1.93 & 1.72 & C & 2.092 & 13.80 & \text { I }\end{array}$ $\begin{array}{lllllllllllll}133.3 & 5834 & 0.390 & 40.20 & 0.319 & 0.858 & 52.02 & 2.23 & 1.72 & \mathrm{C} & 1.957 & 13.50 & \mathrm{I}\end{array}$ $\begin{array}{llllllllllll}118.0 & 5834 & 0.239 & 32.08 & 0.319 & 0.858 & 52.02 & 3.37 & 1.72 & \mathrm{C} & 1.731 & 13.30\end{array}$ $\begin{array}{lllllllllllll}23.6 & 6976 & 1.021 & 34.78 & 0.308 & 0.865 & 52.02 & 0.93 & 1.74 & \mathrm{C} & 0.204 & 16.70 & \mathrm{O} 2\end{array}$ $\begin{array}{lllllllllllll}\pi 7.9 & 6605 . & 0.986 & 661.60 & 0.308 & 0.854 & 52.02 & 0.96 & 1.71 & \mathrm{C} & 4.446 & 215.80 & \mathrm{O} 2\end{array}$ $\begin{array}{lllllllllllll}552.0 & 6605 & 0.960 & 385.50 & 0.308 & 0.854 & 52.02 & 0.98 & 1.71 & \mathrm{C} & 3.512 & 123.20 & 02\end{array}$ $\begin{array}{lllllllllllll}434.5 & 6605 & 0.931 & 259.10 & 0.308 & 0.854 & 52.02 & 1.01 & 1.71 & \mathrm{C} & 3.018 & 81.60 & \mathrm{O} 2\end{array}$ $\begin{array}{lllllllllllll}303.9 & 6604 & 0.857 & 141.50 & 0.307 & 0.854 & 52.02 & 1.10 & 1.71 & C & 2.461 & 42.30 & \text { ! }\end{array}$ $\begin{array}{llllllllllll}233.2 & 6603 & 0.769 & 90.40 & 0.307 & 0.854 & 52.02 & 1.21 & 1.71 & \mathrm{C} & 2.190 & 25.70\end{array}$ $\begin{array}{lllllllllllll}204.4 & 6600 & 0.702 & 71.99 & 0.307 & 0.854 & 52.02 & 1.32 & 1.71 & C & 2.121 & 20.10\end{array}$ $\begin{array}{lllllllllllll}169.7 & 6600 & 0.598 & 53.10 & 0.307 & 0.854 & 52.02 & 1.53 & 1.71 & \mathrm{C} & 2.058 & 15.00 & 1\end{array}$ $\begin{array}{lllllllllllll}141.6 & 6599 & 0.469 & 39.73 & 0.307 & 0.854 & 52.02 & 1.91 & 1.71 & \mathrm{C} & 1.718 & 12.40 & 1\end{array}$ 
$\begin{array}{lllllllllllll}130.2 & 6599 & 0.398 & 34.82 & 0.307 & 0.854 & 52.02 & 2.21 & 1.71 & \mathrm{C} & 1.580 & 12.20 & \mathrm{I}\end{array}$ $\begin{array}{lllllllllllll}120.4 & 6599 & 0.325 & 30.82 & 0.307 & 0.854 & 52.02 & 2.63 & 1.71 & \mathrm{C} & 1.460 & 12.80\end{array}$ $\begin{array}{lllllllllllll}103.9 & 6598 & 0.169 & 24.62 & 0.307 & 0.854 & 52.02 & 4.46 & 1.71 & \mathrm{C} & 1.260 & 13.90 & \mathrm{I}\end{array}$ $\begin{array}{lllllllllllll}22.5 & 5458 . & 1.013 & 57.17 & 0.312 & 0.862 & 52.02 & 0.94 & 1.73 & C & 0.214 & 23.30 & 02\end{array}$ $\begin{array}{lllllllllllll}1054.0 & 5276 & 0.987 & 1470.00 & 0.312 & 0.856 & 52.02 & 0.96 & 1.71 & C & 6.147 & 460.70 & 02\end{array}$ $\begin{array}{lllllllllllll}599.3 & 5276 & 0.967 & 675.00 & 0.312 & 0.856 & 52.02 & 0.98 & 1.71 & \mathrm{C} & 4.032 & 203.60 & 02\end{array}$ $\begin{array}{lllllllllllll}438.0 & 5276 & 0.944 & 414.10 & 0.311 & 0.856 & 52.02 & 1.00 & 1.71 & \mathrm{C} & 3.251 & 120.80 & 02\end{array}$ $\begin{array}{lllllllllllll}293.4 & 5275 . & 0.885 & 209.30 & 0.311 & 0.856 & 52.02 & 1.06 & 1.71 & \mathrm{C} & 2.544 & 58.60 & \mathrm{I}\end{array}$ $\begin{array}{lllllllllllll}225.7 & 5275 & 0.812 & 129.50 & 0.311 & 0.856 & 52.02 & 1.15 & 1.71 & \mathrm{C} & 2.251 & 34.90\end{array}$ $\begin{array}{llllllllllll}198.9 & 5274 & 0.755 & 101.40 & 0.311 & 0.855 & 52.02 & 1.23 & 1.71 & \mathrm{C} & 2.169 & 26.90\end{array}$ $\begin{array}{llllllllllll}169.0 & 5274 & 0.664 & 74.04 & 0.311 & 0.855 & 52.02 & 1.39 & 1.71 & C & 2.119 & 19.70\end{array}$ $\begin{array}{llllllllllll}145.4 & 5273 & 0.546 & 55.03 & 0.311 & 0.855 & 52.02 & 1.66 & 1.71 & \mathrm{C} & 2.183 & 15.80\end{array}$ $\begin{array}{lllllllllllll}135.9 & 5273 & 0.480 & 48.11 & 0.311 & 0.855 & 52.02 & 1.87 & 1.71 & \mathrm{C} & 1.582 & 15.00 & \mathrm{I}\end{array}$ $\begin{array}{llllllllllll}127.8 & 5273 & 0.410 & 42.50 & 0.311 & 0.855 & 52.02 & 2.14 & 1.71 & \mathrm{C} & 1.487 & 15.00\end{array}$ $\begin{array}{llllllllllll}114.3 & 5273 & 0.257 & 33.85 & 0.311 & 0.855 & 52.02 & 3.19 & 1.71 & \mathrm{C} & 1.331 & 15.10\end{array}$ $\begin{array}{lllllllllllll}231.1 & 9810 & 0.772 & 27.02 & 0.305 & 0.853 & 52.02 & 1.21 & 1.71 & \mathrm{P} & 2.031 & 15.50 & \mathrm{I}\end{array}$ $\begin{array}{lllllllllllll}208.9 & 9808 & 0.575 & 31.05 & 0.305 & 0.853 & 52.02 & 1.59 & 1.71 & \mathrm{P} & 1.836 & 12.50\end{array}$ $\begin{array}{llllllllllll}197.1 & 9807 & 0.492 & 33.40 & 0.305 & 0.853 & 52.02 & 1.83 & 1.71 & \mathrm{P} & 1.732 & 11.90\end{array}$ $\begin{array}{llllllllllll}185.0 & 9806 & 0.421 & 35.93 & 0.305 & 0.853 & 52.02 & 2.10 & 1.71 & \mathrm{P} & 1.626 & 12.20\end{array}$ $\begin{array}{lllllllllllll}159.9 & 9805 & 0.306 & 41.82 & 0.305 & 0.853 & 52.02 & 2.78 & 1.71 & \mathrm{P} & 1.405 & 15.10 & \mathrm{I}\end{array}$ $\begin{array}{lllllllllllll}134.8 & 9803 & 0.223 & 48.79 & 0.305 & 0.853 & 52.02 & 3.60 & 1.71 & \mathrm{P} & 1.185 & 18.80 & 1\end{array}$ $\begin{array}{lllllllllllll}119.7 & 9802 & 0.182 & 54.13 & 0.305 & 0.853 & 52.02 & 4.23 & 1.71 & \mathrm{P} & 1.052 & 21.00\end{array}$ $\begin{array}{lllllllllllll}99.0 & 9801 & 0.138 & 62.55 & 0.305 & 0.853 & 52.02 & 5.19 & 1.71 & \mathrm{P} & 0.870 & 23.70 & \mathrm{I}\end{array}$ $\begin{array}{llllllllllllll}81.9 & 9801 . & 0.104 & 73.40 & 0.305 & 0.853 & 52.02 & 6.31 & 1.71 & \mathrm{P} & 0.719 & 26.90 & 1\end{array}$ $\begin{array}{lllllllllllll}77.1 & 9800 & 0.090 & 79.44 & 0.305 & 0.853 & 52.02 & 6.90 & 1.71 & \mathrm{P} & 0.678 & 34.80 & \mathrm{I}\end{array}$ $\begin{array}{lllllllllllll}76.3 & 9800 & 0.078 & 85.66 & 0.305 & 0.853 & 52.02 & 7.51 & 1.71 & \mathrm{P} & 0.670 & 50.10 & \mathrm{I}\end{array}$ $\begin{array}{lllllllllllllll}89.7 & 9800 & 0.056 & 98.47 & 0.305 & 0.853 & 52.02 & 9.07 & 1.71 & P & 0.788 & 71.30 & 02\end{array}$ $\begin{array}{lllllllllllll}228.0 & 10090 & 0.773 & 26.85 & 0.357 & 0.871 & 52.02 & 1.19 & 1.75 & \mathrm{P} & 2.089 & 15.20\end{array}$ $\begin{array}{llllllllllll}208.5 & 10080 & 0.586 & 31.02 & 0.357 & 0.871 & 52.02 & 1.53 & 1.75 & \mathrm{P} & 1.910 & 12.60\end{array}$ $\begin{array}{lllllllllllll}198.0 & 10080 & 0.507 & 33.44 & 0.357 & 0.871 & 52.02 & 1.74 & 1.75 & \mathrm{P} & 1.814 & 12.00\end{array}$ $\begin{array}{llllllllllll}187.2 & 10080 & 0.439 & 36.08 & 0.357 & 0.871 & 52.02 & 1.97 & 1.75 & \mathrm{P} & 1.715 & 12.40\end{array}$ $\begin{array}{llllllllllll}164.4 & 10080 & 0.328 & 42.23 & 0.357 & 0.870 & 52.02 & 2.52 & 1.75 & \mathrm{P} & 1.506 & 15.30\end{array}$ $\begin{array}{lllllllllllll}141.1 & 10080 & 0.248 & 49.52 & 0.357 & 0.870 & 52.02 & 3.17 & 1.75 & P & 1.293 & 19.20\end{array}$ $\begin{array}{llllllllllll}126.6 & 10080 & 0.207 & 54.99 & 0.357 & 0.870 & 52.02 & 3.64 & 1.75 & \mathrm{P} & 1.160 & 21.40\end{array}$ $\begin{array}{lllllllllllll}106.5 & 10080 & 0.163 & 63.75 & 0.357 & 0.870 & 52.02 & 4.32 & 1.75 & \mathrm{P} & 0.976 & 24.30\end{array}$ $\begin{array}{lllllllllllll}88.9 & 10080 & 0.129 & 74.90 & 0.357 & 0.870 & 52.02 & 5.08 & 1.75 & \mathrm{P} & 0.815 & 27.80\end{array}$ $\begin{array}{llllllllllll}83.5 & 10080 & 0.115 & 80.99 & 0.357 & 0.870 & 52.02 & 5.46 & 1.75 & \mathrm{P} & 0.765 & 36.00\end{array}$ $\begin{array}{llllllllllll}81.3 & 10080 & 0.103 & 87.13 & 0.357 & 0.870 & 52.02 & 5.84 & 1.75 & \mathrm{P} & 0.745 & 53.40\end{array}$

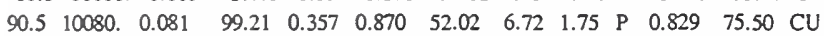
$\begin{array}{llllllllllll}229.0 & 10260 & 0.772 & 25.74 & 0.316 & 0.857 & 52.02 & 1.20 & 1.72 & \mathrm{P} & 1.992 & 15.00\end{array}$ $\begin{array}{llllllllllll}207.7 & 10250 & 0.577 & 29.68 & 0.316 & 0.857 & 52.02 & 1.58 & 1.72 & \mathrm{P} & 1.807 & 12.20\end{array}$ $\begin{array}{lllllllllllll}196.3 & 10250 & 0.495 & 31.97 & 0.316 & 0.857 & 52.02 & 1.81 & 1.72 & \mathrm{P} & 1.708 & 11.60 & 1\end{array}$ $\begin{array}{llllllllllllll}184.6 & 10250 & 0.425 & 34.45 & 0.316 & 0.857 & 52.02 & 2.07 & 1.72 & \mathrm{P} & 1.606 & 11.90 & 1\end{array}$ $\begin{array}{lllllllllllll}160.0 & 10250 & 0.310 & 40.23 & 0.316 & 0.857 & 52.02 & 2.72 & 1.72 & \mathrm{P} & 1.392 & 14.70\end{array}$ $\begin{array}{llllllllllll}135.4 & 10250 & 0.228 & 47.07 & 0.316 & 0.857 & 52.02 & 3.50 & 1.72 & \mathrm{P} & 1.178 & 18.40\end{array}$ $\begin{array}{lllllllllllll}120.3 & 10250 & 0.187 & 52.26 & 0.316 & 0.857 & 52.02 & 4.09 & 1.72 & \mathrm{P} & 1.047 & 20.60 & \mathrm{I}\end{array}$ $\begin{array}{lllllllllllllll}99.7 & 10250 & 0.144 & 60.49 & 0.316 & 0.857 & 52.02 & 4.97 & 1.72 & \mathrm{P} & 0.868 & 23.30 & 1\end{array}$ $\begin{array}{lllllllllllll}82.5 & 10250 & 0.110 & 70.98 & 0.316 & 0.857 & 52.02 & 5.98 & 1.72 & \mathrm{P} & 0.718 & 26.50\end{array}$ $\begin{array}{lllllllllllll}77.7 & 10250 & 0.097 & 76.76 & 0.316 & 0.857 & 52.02 & 6.50 & 1.72 & \mathrm{P} & 0.676 & 34.00 & 1\end{array}$ $\begin{array}{lllllllllllll}76.7 & 10250 & 0.085 & 82.61 & 0.316 & 0.857 & 52.02 & 7.04 & 1.72 & P & 0.667 & 48.80\end{array}$ $\begin{array}{lllllllllllll}89.6 & 10250 . & 0.062 & 94.33 & 0.316 & 0.857 & 52.02 & 8.38 & 1.72 & \mathrm{P} & 0.779 & 69.40 & \mathrm{O} 2\end{array}$ $\begin{array}{lllllllllllll}245.7 & 10580 & 0.777 & 30.87 & 0.384 & 0.879 & 52.02 & 1.17 & 1.77 & \mathrm{P} & 2.181 & 15.50\end{array}$ $\begin{array}{llllllllllll}226.5 & 10580 & 0.613 & 36.20 & 0.383 & 0.879 & 52.02 & 1.45 & 1.77 & \mathrm{P} & 2.011 & 13.60\end{array}$ $\begin{array}{llllllllllll}216.1 & 10580 & 0.545 & 39.40 & 0.383 & 0.879 & 52.02 & 1.61 & 1.77 & \mathrm{P} & 1.919 & 13.40\end{array}$ $\begin{array}{llllllllllllll}205.5 & 10580 & 0.486 & 42.94 & 0.383 & 0.879 & 52.02 & 1.78 & 1.77 & \mathrm{P} & 1.825 & 14.10 & \mathrm{I}\end{array}$ $\begin{array}{lllllllllllll}183.2 & 10570 . & 0.389 & 51.50 & 0.383 & 0.879 & 52.02 & 2.15 & 1.77 & P & 1.626 & I 7.60\end{array}$ $\begin{array}{llllllllllll}160.7 & 10570 & 0.321 & 62.27 & 0.383 & 0.878 & 52.02 & 2.53 & 1.77 & \mathrm{P} & 1.427 & 22.30\end{array}$ $\begin{array}{lllllllllllll}147.5 & 10570 & 0.286 & 71.01 & 0.383 & 0.878 & 52.02 & 2.77 & 1.77 & \mathrm{P} & 1.310 & 25.50 & \mathrm{I}\end{array}$ $\begin{array}{lllllllllllllll}129.5 & 10570 & 0.249 & 85.80 & 0.383 & 0.878 & 52.02 & 3.08 & 1.77 & \mathrm{P} & 1.150 & 30.00 & \mathrm{I}\end{array}$ $\begin{array}{llllllllllllll}117.2 & 10570 & 0.219 & 106.70 & 0.383 & 0.878 & 52.02 & 3.39 & 1.77 & \mathrm{P} & 1.040 & 36.00 & 1\end{array}$ $\begin{array}{llllllllllllll}116.7 & 10570 & 0.207 & 119.30 & 0.383 & 0.878 & 52.02 & 3.54 & 1.77 & \mathrm{P} & 1.036 & 43.70 & 1\end{array}$ $\begin{array}{lllllllllllllll}122.2 & 10570 & 0.196 & 133.10 & 0.383 & 0.878 & 52.02 & 3.68 & 1.77 & \mathrm{P} & 1.085 & 57.30 & 1\end{array}$ $\begin{array}{lllllllllllll}159.5 & 10570 & 0.175 & 164.10 & 0.383 & 0.878 & 52.02 & 3.99 & 1.77 & \mathrm{P} & 1.416 & 87.60 & 02\end{array}$ $\begin{array}{llllllllllll}202.8 & 6200 & 0.735 & 35.26 & 0.280 & 0.844 & 52.02 & 1.28 & 1.70 & \mathrm{P} & 2.308 & 17.80\end{array}$ $\begin{array}{llllllllllllll}184.8 & 6200 & 0.524 & 41.16 & 0.280 & 0.844 & 52.02 & 1.75 & 1.70 & \mathrm{P} & 2.567 & 14.80 & \mathrm{I}\end{array}$ $\begin{array}{llllllllllll}174.2 & 6200 & 0.437 & 44.45 & 0.280 & 0.844 & 52.02 & 2.06 & 1.70 & \mathrm{P} & 2.419 & 14.30\end{array}$ $\begin{array}{llllllllllllll}162.5 & 6200 & 0.362 & 47.90 & 0.280 & 0.844 & 52.02 & 2.43 & 1.70 & \mathrm{P} & 2.257 & 14.90\end{array}$ $\begin{array}{lllllllllllllll}135.9 & 6199 & 0.243 & 55.22 & 0.280 & 0.844 & 52.02 & 3.43 & 1.70 & \mathrm{P} & 1.887 & 18.00 & \text { I }\end{array}$ $\begin{array}{llllllllllll}106.4 & 6199 & 0.160 & 62.38 & 0.279 & 0.844 & 52.02 & 4.80 & 1.70 & P & 1.477 & 21.70\end{array}$ $\begin{array}{lllllllllllll}86.7 & 6199 & 0.120 & 66.49 & 0.279 & 0.844 & 52.02 & 5.92 & 1.70 & \mathrm{P} & 1.204 & 23.50 & \mathrm{I}\end{array}$ $\begin{array}{llllllllllllll}60.4 & 6196 . & 0.081 & 72.27 & 0.279 & 0.844 & 52.02 & 7.69 & 1.70 & \mathrm{P} & 0.839 & 25.70 & \text { I }\end{array}$ $\begin{array}{llllllllllllll}35.7 & 6193 & 0.055 & 76.28 & 0.279 & 0.844 & 52.02 & 9.61 & 1.70 & \mathrm{P} & 0.496 & 29.20 & \mathrm{O} 2\end{array}$ $\begin{array}{lllllllllllll}26.4 & 6192 & 0.047 & 77.01 & 0.279 & 0.844 & 52.02 & 10.43 & 1.70 & \mathrm{P} & 0.366 & 53.40 & \mathrm{O} 2\end{array}$ $\begin{array}{lllllllllllll}19.7 & 6192 & 0.041 & 75.91 & 0.279 & 0.844 & 52.02 & 11.14 & 1.70 & \mathrm{P} & 0.273 & 107.70 & \mathrm{O} 2\end{array}$ $\begin{array}{lllllllllllll}15.1 & 6193 & 0.032 & 71.14 & 0.279 & 0.844 & 52.02 & 12.41 & 1.70 & P & 0.210 & 115.60 & 02\end{array}$ $\begin{array}{llllllllllll}208.1 & 5161 . & 0.748 & 47.19 & 0.299 & 0.851 & 52.02 & 1.25 & 1.71 & \mathrm{P} & 2.597 & 20.50\end{array}$ $\begin{array}{lllllllllllll}189.2 & 5161 & 0.554 & 54.70 & 0.299 & 0.851 & 52.02 & 1.65 & 1.71 & \mathrm{P} & 2.810 & 17.80\end{array}$ $\begin{array}{lllllllllllllll}178.9 & 5161 & 0.473 & 59.06 & 0.299 & 0.851 & 52.02 & 1.90 & 1.71 & \mathrm{P} & 2.019 & 17.50 & 1\end{array}$ $\begin{array}{lllllllllllll}168.4 & 5161 . & 0.404 & 63.84 & 0.299 & 0.851 & 52.02 & 2.19 & 1.71 & \mathrm{P} & 1.933 & 18.40\end{array}$ $\begin{array}{lllllllllllll}145.7 & 5161 & 0.291 & 74.96 & 0.299 & 0.851 & 52.02 & 2.90 & 1.71 & \mathrm{P} & 1.673 & 22.30\end{array}$ $\begin{array}{llllllllllllll}122.2 & 5160 & 0.212 & 88.02 & 0.299 & 0.851 & 52.02 & 3.77 & 1.71 & \mathrm{P} & 1.403 & 27.50 & \text { I }\end{array}$ $\begin{array}{llllllllllllll}106.5 & 5161, & 0.172 & 96.94 & 0.299 & 0.851 & 52.02 & 4.43 & 1.71 & \mathrm{P} & 1.223 & 30.50\end{array}$ $\begin{array}{lllllllllllll}85.6 & 5161 . & 0.132 & 111.80 & 0.299 & 0.851 & 52.02 & 5.41 & 1.71 & \mathrm{P} & 0.983 & 34.40 & 1\end{array}$ $\begin{array}{llllllllllllll}66.9 & 5161 . & 0.101 & 129.80 & 0.299 & 0.851 & 52.02 & 6.49 & 1.71 & \mathrm{P} & 0.768 & 39.20 & 1\end{array}$ $\begin{array}{llllllllllllll}60.7 & 5161 . & 0.089 & 139.10 & 0.299 & 0.851 & 52.02 & 7.03 & 1.71 & \mathrm{P} & 0.697 & 48.00 & 1\end{array}$ $\begin{array}{llllllllllll}57.8 & 5161 . & 0.079 & 148.10 & 0.299 & 0.851 & 52.02 & 7.57 & 1.71 & \mathrm{P} & 0.664 & 65.60\end{array}$ $\begin{array}{lllllllllllll}64.3 & 5160 & 0.060 & 164.00 & 0.299 & 0.851 & 52.02 & 8.82 & 1.71 & \mathrm{P} & 0.738 & 89.00 & \mathrm{HC}\end{array}$ $\begin{array}{lllllllllllll}206.0 & 5921 . & 0.758 & 39.73 & 0.316 & 0.857 & 52.02 & 1.23 & 1.72 & \text { P } & 2.441 & 18.50 & \text { I }\end{array}$ $\begin{array}{llllllllllllll}188.2 & 5921 . & 0.561 & 46.68 & 0.316 & 0.857 & 52.02 & 1.62 & 1.72 & \mathrm{P} & 2.657 & 16.10 & \text { I }\end{array}$ $\begin{array}{llllllllllllll}178.4 & 5921 . & 0.480 & 50.79 & 0.316 & 0.857 & 52.02 & 1.86 & 1.72 & \mathrm{P} & 2.012 & 15.80 & 1\end{array}$ $\begin{array}{llllllllllllll}168.1 & 5921 & 0.411 & 55.32 & 0.316 & 0.857 & 52.02 & 2.14 & 1.72 & \mathrm{P} & 1.896 & 16.70 & \mathrm{I}\end{array}$ $\begin{array}{lllllllllllll}145.7 & 5920 & 0.299 & 66.06 & 0.316 & 0.857 & 52.02 & 2.80 & 1.72 & \mathrm{P} & 1.644 & 20.80 & \mathrm{I}\end{array}$ $\begin{array}{lllllllllllll}122.0 & 5920 & 0.222 & 79.01 & 0.316 & 0.857 & 52.02 & 3.58 & 1.72 & \text { P } & 1.376 & 26.10 & \text { I }\end{array}$ $\begin{array}{lllllllllllll}106.0 & 5920 & 0.185 & 88.26 & 0.316 & 0.857 & 52.02 & 4.13 & 1.72 & \mathrm{P} & 1.196 & 29.40\end{array}$ $\begin{array}{lllllllllllll}84.5 & 5920 & 0.146 & 103.70 & 0.316 & 0.857 & 52.02 & 4.91 & 1.72 & \mathrm{P} & 0.953 & 34.00 & \mathrm{I}\end{array}$ $\begin{array}{lllllllllllll}65.6 & 5920 & 0.118 & 122.60 & 0.316 & 0.857 & 52.02 & 5.69 & 1.72 & \mathrm{P} & 0.740 & 39.60 & 1\end{array}$ $\begin{array}{lllllllllllll}59.9 & 5920 & 0.108 & 132.30 & 0.316 & 0.857 & 52.02 & 6.06 & 1.72 & \mathrm{P} & 0.676 & 50.40 & \mathrm{Cl}\end{array}$ $\begin{array}{lllllllllllll}58.2 & 5920 & 0.099 & 141.60 & 0.316 & 0.857 & 52.02 & 6.41 & 1.72 & \mathrm{P} & 0.657 & 68.90 & \mathrm{CU}\end{array}$ $\begin{array}{llllllllllllll}70.0 & 5920 & 0.081 & 157.10 & 0.316 & 0.857 & 52.02 & 7.23 & 1.72 & \mathrm{P} & 0.790 & 90.30 & \mathrm{I}\end{array}$

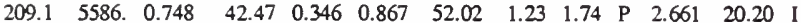
$\begin{array}{lllllllllllll}190.1 & 5586 . & 0.553 & 49.64 & 0.346 & 0.867 & 52.02 & 1.62 & 1.74 & \mathrm{P} & 2.889 & 17.60 & \mathrm{I}\end{array}$ $\begin{array}{lllllllllllllll}179.7 & 5586 . & 0.472 & 53.89 & 0.346 & 0.867 & 52.02 & 1.86 & 1.74 & \mathrm{P} & 2.054 & 17.40 & \mathrm{I}\end{array}$ $\begin{array}{lllllllllllll}169.0 & 5585 & 0.403 & 58.58 & 0.346 & 0.867 & 52.02 & 2.13 & 1.74 & \mathrm{P} & 1.932 & 18.30 & \mathrm{I}\end{array}$ $\begin{array}{lllllllllllll}146.0 & 5585 & 0.293 & 69.74 & 0.346 & 0.867 & 52.02 & 2.79 & 1.74 & \mathrm{P} & 1.669 & 22.60 & \text { I }\end{array}$ $\begin{array}{llllllllllllll}122.2 & 5585 & 0.216 & 83.33 & 0.346 & 0.867 & 52.02 & 3.56 & 1.74 & \mathrm{P} & 1.397 & 28.30 & \text { I }\end{array}$ $\begin{array}{llllllllllllll}106.3 & 5585 & 0.178 & 93.11 & 0.346 & 0.867 & 52.02 & 4.11 & 1.74 & \mathrm{P} & 1.216 & 31.80 & \text { I }\end{array}$ $\begin{array}{llllllllllllll}85.4 & 5585 . & 0.140 & 110.00 & 0.346 & 0.867 & 52.02 & 4.87 & 1.74 & \mathrm{P} & 0.977 & 36.70 & \mathrm{I}\end{array}$ $\begin{array}{llllllllllllll}67.6 & 5585 . & 0.112 & 131.90 & 0.346 & 0.867 & 52.02 & 5.64 & 1.74 & \mathrm{P} & 0.773 & 43.40 & \mathrm{I}\end{array}$ $\begin{array}{lllllllllllll}62.8 & 5585 . & 0.102 & 143.90 & 0.346 & 0.867 & 52.02 & 6.00 & 1.74 & \mathrm{P} & 0.718 & 53.40 & 1\end{array}$ $\begin{array}{lllllllllllll}62.2 & 5585 . & 0.092 & 156.20 & 0.346 & 0.867 & 52.02 & 6.35 & 1.74 & \mathrm{P} & 0.711 & 70.50 & \mathrm{I}\end{array}$ $\begin{array}{lllllllllllll}78.0 & 5585 . & 0.074 & 179.80 & 0.346 & 0.867 & 52.02 & 7.17 & 1.74 & \mathrm{P} & 0.892 & 97.80 & \mathrm{O} 2\end{array}$ $\begin{array}{lllllllllllll}217.1 & 5878 . & 0.757 & 41.47 & 0.383 & 0.878 & 52.02 & 1.20 & 1.77 & \mathrm{P} & 2.762 & 20.90 & \mathrm{I}\end{array}$ $\begin{array}{lllllllllllll}197.3 & 5878 & 0.563 & 48.48 & 0.383 & 0.878 & 52.02 & 1.57 & 1.77 & \mathrm{P} & 2.980 & 18.20 & \mathrm{I}\end{array}$ $\begin{array}{lllllllllllll}186.5 & 5878 & 0.483 & 52.64 & 0.383 & 0.878 & 52.02 & 1.79 & 1.77 & \mathrm{P} & 2.122 & 18.10 & 1\end{array}$ $\begin{array}{llllllllllllll}175.4 & 5877 & 0.415 & 57.23 & 0.383 & 0.878 & 52.02 & 2.04 & 1.77 & \mathrm{P} & 1.996 & 19.10 & \mathrm{I}\end{array}$ $\begin{array}{lllllllllllll}151.7 & 5877 & 0.305 & 68.21 & 0.383 & 0.878 & 52.02 & 2.63 & 1.77 & \mathrm{P} & 1.726 & 23.60 & \mathrm{I}\end{array}$ $\begin{array}{llllllllllllll}127.2 & 5877 & 0.229 & 81.70 & 0.383 & 0.878 & 52.02 & 3.29 & 1.77 & \mathrm{P} & 1.448 & 29.60 & 1\end{array}$ $\begin{array}{llllllllllllll}111.0 & 5877 & 0.192 & 91.52 & 0.383 & 0.878 & 52.02 & 3.74 & 1.77 & \mathrm{P} & 1.264 & 33.30 & \mathrm{I}\end{array}$ $\begin{array}{lllllllllllll}89.9 & 5877 . & 0.154 & 108.70 & 0.383 & 0.878 & 52.02 & 4.35 & 1.77 & \mathrm{P} & 1.023 & 38.50 & \mathrm{I}\end{array}$ $\begin{array}{lllllllllllll}72.3 & 5877 . & 0.126 & 131.50 & 0.383 & 0.878 & 52.02 & 4.96 & 1.77 & \mathrm{P} & 0.822 & 45.90 & \mathrm{I}\end{array}$ $\begin{array}{lllllllllllll}67.9 & 5877 . & 0.116 & 144.40 & 0.383 & 0.878 & 52.02 & 5.23 & 1.77 & \mathrm{P} & 0.772 & 56.10 & \text { I }\end{array}$ $\begin{array}{lllllllllllll}68.1 & 5877 & 0.107 & 157.90 & 0.383 & 0.878 & 52.02 & 5.50 & 1.77 & \mathrm{P} & 0.775 & 73.20 & \mathrm{I}\end{array}$ $\begin{array}{lllllllllllll}87.8 & 5877 . & 0.088 & 185.20 & 0.383 & 0.878 & 52.02 & 6.12 & 1.77 & \mathrm{P} & 1.000 & 103.70 & \mathrm{O} 2\end{array}$ $\begin{array}{lllllllllllll}209.8 & 5572 . & 0.749 & 41.58 & 0.358 & 0.871 & 52.02 & 1.22 & 1.75 & \mathrm{P} & 2.710 & 19.90 & \mathrm{I}\end{array}$ $\begin{array}{llllllllllllll}190.6 & 5572 & 0.549 & 48.71 & 0.358 & 0.871 & 52.02 & 1.62 & 1.75 & \mathrm{P} & 2.150 & 17.40 & \mathrm{I}\end{array}$ $\begin{array}{llllllllllllll}\mathrm{I} 80.0 & 5572 & 0.467 & 52.93 & 0.358 & 0.871 & 52.02 & 1.87 & 1.75 & \mathrm{P} & 2.067 & 17.30 & \mathrm{I}\end{array}$ $\begin{array}{lllllllllllllll}169.1 & 5572 . & 0.397 & 57.60 & 0.358 & 0.871 & 52.02 & 2.15 & 1.75 & \mathrm{P} & 1.942 & 18.30 & \mathrm{I}\end{array}$ $\begin{array}{lllllllllllll}145.8 & 5572 . & 0.285 & 68.71 & 0.358 & 0.871 & 52.02 & 2.83 & 1.75 & \mathrm{P} & 1.674 & 22.60 & 02\end{array}$ $\begin{array}{lllllllllllll}121.6 & 5572 & 0.207 & 82.27 & 0.358 & 0.87 \mathrm{I} & 52.02 & 3.63 & 1.75 & \mathrm{P} & \mathrm{I} .396 & 28.30 & \mathrm{I}\end{array}$ $\begin{array}{llllllllllllll}105.5 & 5572 & 0.170 & 92.03 & 0.358 & 0.871 & 52.02 & 4.20 & 1.75 & \mathrm{P} & 1.2 \mathrm{I} 1 & 31.70 & \mathrm{I}\end{array}$ $\begin{array}{llllllllllllll}84.4 & 5572 . & 0.132 & 108.80 & 0.358 & 0.871 & 52.02 & 4.99 & 1.75 & \mathrm{P} & 0.969 & 37.00 & \text { I }\end{array}$ $\begin{array}{llllllllllllll}66.9 & 5572 . & 0.104 & 130.60 & 0.358 & 0.871 & 52.02 & 5.80 & 1.75 & \mathrm{P} & 0.768 & 43.50 & \mathrm{I}\end{array}$ $\begin{array}{lllllllllllll}62.4 & 5572 & 0.094 & 142.40 & 0.358 & 0.871 & 52.02 & 6.18 & 1.75 & \mathrm{P} & 0.717 & 53.30 & \mathrm{I}\end{array}$ $\begin{array}{llllllllllllll}62.5 & 5572 . & 0.084 & 154.40 & 0.358 & 0.871 & 52.02 & 6.55 & 1.75 & \mathrm{P} & 0.718 & 69.30 & \mathrm{I}\end{array}$ $\begin{array}{lllllllllllll}80.7 & 5572 . & 0.066 & 177.10 & 0.358 & 0.871 & 52.02 & 7.46 & 1.75 & \mathrm{P} & 0.927 & 95.20 & 02\end{array}$ 
Convective condensation of

R125 within a micro-fin tube

(file: tacond.tbl)

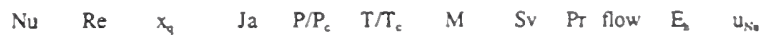

$\begin{array}{lllllllllllll}12.0 & 10210 & 1.073 & 10.54 & 0.428 & 0.913 & 120.00 & 0.86 & 3.34 & \mathrm{C} & 0.091 & 20.20 & 02\end{array}$ $\begin{array}{llllllllllllll}32.4 & 9826 . & 1.050 & 15.02 & 0.428 & 0.906 & 120.00 & 0.87 & 3.30 & \mathrm{C} & 0.242 & 18.10 & \mathrm{O} 2\end{array}$ $\begin{array}{llllllllllllll}58.0 & 9562 . & 1.033 & 21.72 & 0.428 & 0.901 & 120.00 & 0.89 & 3.28 & \mathrm{C} & 0.422 & 18.00 & \mathrm{O} 2\end{array}$ $\begin{array}{lllllllllllll}146.6 & 9265 . & 1.012 & 46.11 & 0.428 & 0.896 & 120.00 & 0.90 & 3.27 & \mathrm{C} & 0.981 & 28.80 & 02\end{array}$ $\begin{array}{llllllllllllll}314.6 & 9109 & 0.957 & 72.92 & 0.428 & 0.892 & 120.00 & 0.95 & 3.26 & C & 2.058 & 41.00 & 1\end{array}$ $\begin{array}{lllllllllllll}248.2 & 9107 & 0.887 & 44.98 & 0.428 & 0.892 & 120.00 & 1.01 & 3.26 & \mathrm{C} & 1.853 & 24.30 & \text { I }\end{array}$ $\begin{array}{lllllllllllll}225.4 & 9101 & 0.833 & 35.67 & 0.427 & 0.892 & 120.00 & 1.07 & 3.26 & \mathrm{C} & 1.820 & 18.90\end{array}$ $\begin{array}{llllllllllll}195.2 & 9100 & 0.743 & 25.93 & 0.427 & 0.892 & 120.00 & 1.19 & 3.26 & \mathrm{C} & 1.795 & 13.80\end{array}$ $\begin{array}{lllllllllllll}171.8 & 9098 & 0.623 & 19.20 & 0.427 & 0.892 & 120.00 & 1.39 & 3.26 & \mathrm{C} & 1.579 & 11.20 & 1\end{array}$ $\begin{array}{llllllllllll}162.5 & 9097 & 0.554 & 16.76 & 0.427 & 0.892 & : 20.00 & 1.53 & 3.26 & \mathrm{C} & 1.494 & 11.00\end{array}$ $\begin{array}{llllllllllllll}154.5 & 9097 & 0.481 & 14.79 & 0.427 & 0.892 & 120.00 & 1.73 & 3.26 & \mathrm{C} & 1.420 & 11.30 & 1\end{array}$ $\begin{array}{llllllllllll}141.3 & 9096 . & 0.317 & 11.75 & 0.427 & 0.892 & 120.00 & 2.41 & 3.26 & \mathrm{C} & 1.299 & 11.90\end{array}$ $\begin{array}{lllllllllllll}11.1 & 11120 & 1.080 & 9.55 & 0.496 & 0.929 & 120.00 & 0.84 & 3.47 & \mathrm{C} & 0.084 & 23.60 & \mathrm{O} 2\end{array}$ $\begin{array}{lllllllllllll}28.2 & 10740 & 1.059 & 12.80 & 0.496 & 0.924 & 120.00 & 0.85 & 3.40 & \mathrm{C} & 0.215 & 20.20 & \mathrm{O} 2\end{array}$ $\begin{array}{lllllllllllll}46.2 & 10470 . & 1.043 & 16.92 & 0.495 & 0.920 & 120.00 & 0.86 & 3.36 & \mathrm{C} & 0.348 & 18.10 & \mathrm{O} 2\end{array}$ $\begin{array}{lllllllllllll}88.5 & 10180 & 1.024 & 27.17 & 0.495 & 0.915 & 120.00 & 0.87 & 3.32 & \mathrm{C} & 0.640 & 20.30 & \mathrm{O} 2\end{array}$ $\begin{array}{llllllllllll}313.8 & 9858 . & 0.973 & 71.04 & 0.495 & 0.910 & 120.00 & 0.91 & 3.29 & \mathrm{C} & 2.068 & 43.30\end{array}$ $\begin{array}{llllllllllllll}249.4 & 9856 . & 0.907 & 43.71 & 0.495 & 0.910 & 120.00 & 0.97 & 3.29 & \mathrm{C} & 1.868 & 25.70 & 1\end{array}$ $\begin{array}{llllllllllll}226.4 & 9852 & 0.855 & 34.42 & 0.495 & 0.909 & 120.00 & 1.02 & 3.29 & \mathrm{C} & 1.834 & 19.80\end{array}$ $\begin{array}{lllllllllllll}197.8 & 9850 & 0.768 & 25.03 & 0.495 & 0.909 & 120.00 & 1.12 & 3.29 & \mathrm{C} & 1.819 & 14.50 & 1\end{array}$ $\begin{array}{llllllllllll}175.6 & 9849 & 0.653 & 18.55 & 0.494 & 0.909 & 120.00 & 1.29 & 3.29 & \mathrm{C} & 1.615 & 11.70\end{array}$ $\begin{array}{llllllllllll}166.9 & 9849 & 0.586 & 16.20 & 0.494 & 0.909 & 120.00 & 1.41 & 3.29 & \mathrm{C} & 1.534 & 11.40\end{array}$ $\begin{array}{lllllllllllll}159.4 & 9848 & 0.514 & 14.30 & 0.494 & 0.909 & 120.00 & 1.57 & 3.29 & \mathrm{C} & 1.465 & 11.80\end{array}$ $\begin{array}{lllllllllllll}146.9 & 9847 & 0.353 & 11.37 & 0.494 & 0.909 & 120.00 & 2.10 & 3.29 & \mathrm{C} & 1.351 & 12.30 & 1\end{array}$ $\begin{array}{lllllllllllll}115.7 & 12850 & 1.007 & 45.76 & 0.477 & 0.907 & 120.00 & 0.89 & 3.28 & \mathrm{C} & 0.658 & 30.40 & \mathrm{O} 2\end{array}$ $\begin{array}{llllllllllll}283.8 & 12720 & 0.971 & 63.49 & 0.476 & 0.905 & 120.00 & 0.92 & 3.28 & C & 1.584 & 39.20\end{array}$ $\begin{array}{lllllllllllllll}300.8 & 12720 & 0.945 & 55.97 & 0.476 & 0.905 & 120.00 & 0.94 & 3.28 & \mathrm{C} & 1.742 & 34.00 & 1\end{array}$ $\begin{array}{lllllllllllll}304.9 & 12720 & 0.915 & 49.10 & 0.476 & 0.905 & 120.00 & 0.97 & 3.28 & C & 1.845 & 29.20 & 1\end{array}$ $\begin{array}{lllllllllllll}289.0 & 12710 & 0.842 & 37.38 & 0.476 & 0.905 & 120.00 & 1.04 & 3.28 & \mathrm{C} & 1.934 & 21.50 & 1\end{array}$ $\begin{array}{llllllllllllll}259.3 & 12710 & 0.757 & 28.77 & 0.476 & 0.905 & 120.00 & 1.15 & 3.28 & C & 1.947 & 16.00 & 1\end{array}$ $\begin{array}{lllllllllllll}243.3 & 12700 & 0.695 & 25.04 & 0.475 & 0.905 & 120.00 & 1.23 & 3.28 & \mathrm{C} & 1.827 & 13.70 & 1\end{array}$ $\begin{array}{llllllllllll}212.1 & 12700 & 0.600 & 20.02 & 0.475 & 0.904 & 120.00 & 1.40 & 3.28 & \mathrm{C} & 1.593 & 10.90\end{array}$ $\begin{array}{lllllllllllll}181.1 & 12690 & 0.486 & 15.93 & 0.474 & 0.904 & 120.00 & 1.66 & 3.28 & \mathrm{C} & 1.360 & 9.70\end{array}$ $\begin{array}{lllllllllllll}167.0 & 12690 & 0.425 & 14.29 & 0.474 & 0.904 & 120.00 & 1.85 & 3.28 & \mathrm{C} & 1.254 & 10.60 & 1\end{array}$ $\begin{array}{llllllllllll}154.0 & 12690 & 0.364 & 12.89 & 0.474 & 0.904 & 120.00 & 2.09 & 3.28 & \mathrm{C} & 1.156 & 12.80\end{array}$ $\begin{array}{lllllllllllll}130.6 & 12690 & 0.237 & 10.62 & 0.474 & 0.904 & 120.00 & 2.84 & 3.28 & \mathrm{C} & 0.981 & 16.20 & \mathrm{I}\end{array}$ $\begin{array}{lllllllllllll}83.2 & 22210 . & 1.026 & 19.43 & 0.546 & 0.927 & 120.00 & 0.86 & 3.39 & \mathrm{C} & 0.625 & 21.50 & 02\end{array}$ $\begin{array}{lllllllllllll}301.5 & 21490 & 0.990 & 40.01 & 0.546 & 0.921 & 120.00 & 0.88 & 3.34 & \mathrm{C} & 1.345 & 36.70 & \text { I }\end{array}$ $\begin{array}{lllllllllllll}318.9 & 21480 & 0.964 & 34.91 & 0.546 & 0.921 & 120.00 & 0.90 & 3.33 & \mathrm{C} & 1.482 & 31.20 & \mathrm{I}\end{array}$ $\begin{array}{lllllllllllll}322.8 & 21480 & 0.935 & 30.31 & 0.545 & 0.921 & 120.00 & 0.93 & 3.33 & \mathrm{C} & 1.568 & 26.20 & \mathrm{I}\end{array}$ $\begin{array}{llllllllllllll}306.5 & 21470 & 0.861 & 22.69 & 0.545 & 0.921 & 120.00 & 0.99 & 3.33 & C & 1.650 & 18.50 & 1\end{array}$ $\begin{array}{llllllllllll}277.3 & 21460 & 0.774 & 17.25 & 0.545 & 0.921 & 120.00 & 1.09 & 3.33 & \mathrm{C} & 1.492 & 13.20\end{array}$ $\begin{array}{lllllllllllll}263.6 & 21420 & 0.710 & 15.04 & 0.543 & 0.921 & 120.00 & 1.17 & 3.33 & \mathrm{C} & 1.419 & 11.20 & \mathrm{I}\end{array}$ $\begin{array}{lllllllllllll}233.3 & 21420 & 0.609 & 11.93 & 0.543 & 0.921 & 120.00 & 1.33 & 3.33 & \mathrm{C} & 1.255 & 8.90 & 1\end{array}$ $\begin{array}{lllllllllllll}203.5 & 21410 & 0.485 & 9.43 & 0.542 & 0.921 & 120.00 & 1.59 & 3.33 & \mathrm{C} & 1.095 & 8.10 & \mathrm{I}\end{array}$ $\begin{array}{llllllllllllll}190.0 & 21410 & 0.417 & 8.44 & 0.542 & 0.921 & 120.00 & 1.78 & 3.33 & \mathrm{C} & 1.022 & 8.80 & \mathrm{I}\end{array}$ $\begin{array}{llllllllllll}177.6 & 21400 & 0.348 & 7.60 & 0.542 & 0.920 & 120.00 & 2.03 & 3.33 & \mathrm{C} & 0.956 & 10.40\end{array}$ $\begin{array}{llllllllllllll}155.5 & 21400 & 0.201 & 6.24 & 0.542 & 0.920 & 120.00 & 2.90 & 3.33 & \mathrm{C} & 0.837 & 12.60\end{array}$ $\begin{array}{lllllllllllll}100.8 & 22830 & 1.020 & 22.66 & 0.605 & 0.937 & 120.00 & 0.84 & 3.49 & \mathrm{C} & 0.482 & 25.90 & \mathrm{O} 2\end{array}$ $\begin{array}{lllllllllllll}302.3 & 22310 & 0.985 & 38.46 & 0.604 & 0.934 & 120.00 & 0.86 & 3.43 & C & 1.390 & 37.80 & 1\end{array}$ $\begin{array}{lllllllllllll}319.9 & 22310 & 0.960 & 33.56 & 0.604 & 0.934 & 120.00 & 0.88 & 3.43 & C & 1.530 & 32.30 & 1\end{array}$ $\begin{array}{lllllllllllll}323.9 & 22300 & 0.931 & 29.13 & 0.604 & 0.934 & 120.00 & 0.90 & 3.43 & \mathrm{C} & 1.622 & 27.20 & \mathrm{I}\end{array}$ $\begin{array}{lllllllllllll}307.8 & 22290 & 0.859 & 21.81 & 0.604 & 0.934 & 120.00 & 0.97 & 3.43 & C & 1.541 & 19.20 & \text { I }\end{array}$ $\begin{array}{llllllllllll}278.9 & 22290 & 0.773 & 16.59 & 0.603 & 0.934 & 120.00 & 1.05 & 3.43 & \mathrm{C} & 1.396 & 13.90\end{array}$ $\begin{array}{lllllllllllll}264.2 & 22260 & 0.711 & 14.39 & 0.602 & 0.933 & 120.00 & 1.13 & 3.43 & \mathrm{C} & 1.323 & 11.70 & \mathrm{I}\end{array}$ $\begin{array}{lllllllllllll}234.4 & 22250 & 0.612 & 11.44 & 0.602 & 0.933 & 120.00 & 1.27 & 3.43 & \mathrm{C} & 1.173 & 9.30 & \mathrm{I}\end{array}$ 205.022250 .0 .489 $191.622250 . \quad 0.423$ 179.422240 .0 .355 157.422240 .0 .210 $\begin{array}{lll}103.0 & 17250 . & 1.016\end{array}$ $\begin{array}{llll}327.6 & 16830 & 0.974\end{array}$ 342.3 I 6820 . 0.945 $\begin{array}{llll}342.4 & 16820 & 0.911\end{array}$ $\begin{array}{llll}317.0 & 16800 & 0.828\end{array}$ 279.516790 .0 .731 $\begin{array}{llll}265.6 & 16740 & 0.662\end{array}$ $228.2 \quad 16730.0 .554$ 192.8 16730. 0.422 $\begin{array}{lllll}177.1 & 16720 & 0.353\end{array}$ $\begin{array}{lll}162.8 & 16720 & 0.282\end{array}$
$137.5 \quad 16720.0 .135$ 77.419070 .1 .025 $\begin{array}{lll}310.2 & 18390.0 .987\end{array}$ $\begin{array}{lll}327.2 & 18390 & 0.960\end{array}$ $\begin{array}{llll}330.1 & 18380 & 0.929\end{array}$ $\begin{array}{lll}310.6 & 18370 . & 0.852\end{array}$ $\begin{array}{llll}278.1 & 18360 & 0.761\end{array}$ 264.918320 .0 .696 $\begin{array}{llll}231.0 & 18310 & 0.593\end{array}$ $198.2 \quad 18300,0.467$ $\begin{array}{llll}183.5 & 18300 & 0.400\end{array}$ $\begin{array}{llll}170.0 & 18300 & 0.331\end{array}$ $\begin{array}{lll}146.1 & 18290 & 0.187\end{array}$ $\begin{array}{lll}78.5 & 19950 . & 1.024\end{array}$ $\begin{array}{lll}306.4 & 19310 . & 0.989\end{array}$ $\begin{array}{llll}324.2 & 19310 & 0.964\end{array}$ 328.1 19300. 0.93 311.2 19290. 0.864 $\begin{array}{lll}281.0 & 19280 & 0.779\end{array}$ $\begin{array}{llll}268.7 & 19240 & 0.71\end{array}$ $236.8 \quad 19240.0 .620$ 205.7 19230. 0.500 191.7 19230. 0.435 $\begin{array}{lll}178.8 & 19220, & 0.368\end{array}$ $\begin{array}{lll}156.0 & 19220.0 .226\end{array}$ 61.68899 .1 .016 294.18720 .0 .982 $296.5 \quad 8720 . \quad 0.95$ 288.4 8719. 0.929 257.7 8718. 0.859 224.1 8717. 0.775 $\begin{array}{lll}204.8 & 8715 . & 0.713\end{array}$ $\begin{array}{llll}178.5 & 8715 . & 0.616\end{array}$ $\begin{array}{lll}153.8 & 8714 & 0.497\end{array}$ 143.0 8714. 0.432 133.2 8714. 0.365 $115.98714,0.225$ 75.07170 .1 .007 $\begin{array}{llll}356.6 & 7109 . & 0.987\end{array}$ $\begin{array}{llllllllllllll} & 0.59 & 0.931 & 120.00 & 0.88 & 3.40 & \mathrm{C} & 2.453 & 120.10 & 02\end{array}$ $\begin{array}{lllllllllllll}287.0 & 7108 & 0.953 & 127.00 & 0.590 & 0.931 & 120.00 & 0.89 & 3.40 & C & 2.347 & 87.60\end{array}$ $\begin{array}{lllllllllllll}236.7 & 7108 . & 0.905 & 77.57 & 0.590 & 0.931 & 120.00 & 0.93 & 3.40 & \mathrm{C} & 2.176 & 53.10 & \text { I }\end{array}$ $\begin{array}{lllllllllllll}201.8 & 7108 . & 0.845 & 52.27 & 0.590 & 0.931 & 120.00 & 0.99 & 3.40 & \mathrm{C} & 2.077 & 35.00 & \mathrm{HC}\end{array}$ $\begin{array}{llllllllllllll}184.5 & 7107 & 0.798 & 41.97 & 0.590 & 0.931 & 120.00 & 1.03 & 3.40 & \mathrm{C} & 2.046 & 27.70 & \mathrm{HC}\end{array}$ $\begin{array}{llllllllllllll}164.5 & 7107 & 0.721 & 31.69 & 0.590 & 0.931 & 120.00 & 1.12 & 3.40 & C & 2.043 & 20.90 & \text { I }\end{array}$ $\begin{array}{lllllllllllll}147.4 & 7107 & 0.620 & 24.12 & 0.590 & 0.931 & 120.00 & 1.27 & 3.40 & \mathrm{C} & 1.511 & 17.30 & \text { I }\end{array}$ $\begin{array}{lllllllllllll}140.2 & 7107 & 0.563 & 21.28 & 0.590 & 0.931 & 120.00 & 1.37 & 3.40 & \mathrm{C} & 1.437 & 17.60 & \mathrm{I}\end{array}$ $\begin{array}{llllllllllllll}134.0 & 7107 & 0.502 & 18.95 & 0.590 & 0.931 & 120.00 & 1.49 & 3.40 & \mathrm{C} & 1.373 & 19.30 & \text { I }\end{array}$ $\begin{array}{lllllllllllll}123.2 & 7107 & 0.366 & 15.28 & 0.590 & 0.931 & 120.00 & 1.87 & 3.40 & \mathrm{C} & 1.263 & 21.40 & \mathrm{I}\end{array}$ $\begin{array}{lllllllllllll}75.0 & 7873 & 1.012 & 65.03 & 0.621 & 0.939 & 120.00 & 0.84 & 3.51 & C & 0.625 & 61.40 & 02\end{array}$ $\begin{array}{lllllllllllll}435.6 & 768 . & 0.985 & 202.90 & 0.621 & 0.937 & 120.00 & 0.85 & 3.47 & \mathrm{C} & 3.109 & 169.70 & 02\end{array}$ $\begin{array}{lllllllllllll}360.8 & 7768 & 0.966 & 135.50 & 0.621 & 0.937 & 120.00 & 0.87 & 3.47 & \mathrm{C} & 2.791 & 111.70 & \mathrm{O}_{2}\end{array}$ $\begin{array}{lllllllllllll}309.1 & 767 . & 0.942 & 97.33 & 0.621 & 0.937 & 120.00 & 0.89 & 3.47 & \mathrm{C} & 2.568 & 79.10 & \mathrm{HC}\end{array}$ $\begin{array}{lllllllllllll}240.1 & 7767 & 0.883 & 56.83 & 0.621 & 0.937 & 120.00 & 0.93 & 3.47 & \mathrm{C} & 2.280 & 44.60 \mathrm{I}\end{array}$ $\begin{array}{lllllllllllll}197.3 & 7766 & 0.808 & 37.45 & 0.621 & 0.937 & 120.00 & 1.00 & 3.47 & \mathrm{C} & 2.133 & 28.40 & \text { I }\end{array}$ $\begin{array}{lllllllllllll}177.2 & 7766 . & 0.751 & 29.79 & 0.621 & 0.937 & 120.00 & 1.07 & 3.47 & \mathrm{C} & 2.091 & 22.20 & \mathrm{I}\end{array}$ $\begin{array}{lllllllllllll}154.8 & 7766 & 0.658 & 22.30 & 0.621 & 0.937 & 120.00 & 1.18 & 3.47 & \mathrm{C} & 2.093 & 16.70 & \mathrm{I}\end{array}$ $\begin{array}{llllllllllllll}136.0 & 7766 . & 0.538 & 16.87 & 0.621 & 0.937 & 120.00 & 1.38 & 3.47 & \mathrm{C} & 1.840 & 13.90 & \mathrm{I}\end{array}$ $\begin{array}{llllllllllllll}128.2 & 7766 . & 0.470 & 14.85 & 0.621 & 0.937 & 120.00 & 1.52 & 3.47 & \mathrm{C} & 1.735 & 14.20 & \mathrm{I}\end{array}$ $\begin{array}{lllllllllllll}121.5 & 7766 & 0.398 & 13.19 & 0.621 & 0.937 & 120.00 & 1.71 & 3.47 & \mathrm{C} & 1.644 & 15.60 & \mathrm{I}\end{array}$ $\begin{array}{lllllllllllll}110.1 & 7766 & 0.240 & 10.60 & 0.620 & 0.937 & 120.00 & 2.34 & 3.47 & \mathrm{C} & 1.489 & 17.50 & \mathrm{I}\end{array}$ $\begin{array}{llllllllllllll}28.6 & 8879 & 1.029 & 27.73 & 0.384 & 0.889 & 120.00 & 0.90 & 3.27 & \mathrm{C} & 0.201 & 29.70 & 02\end{array}$ $\begin{array}{lllllllllllll}224.7 & 8559 . & 1.005 & 112.40 & 0.384 & 0.882 & 120.00 & 0.92 & 3.27 & \mathrm{C} & 1.310 & 80.40 & 02\end{array}$ $\begin{array}{lllllllllllll}543.9 & 8491 . & 0.987 & 215.50 & 0.384 & 0.880 & 120.00 & 0.93 & 3.27 & \mathrm{C} & 2.897 & 146.80 & \mathrm{O} 2\end{array}$ $\begin{array}{lllllllllllll}405.8 & 8490 & 0.966 & 132.30 & 0.384 & 0.880 & 120.00 & 0.95 & 3.27 & \mathrm{C} & 2.382 & 86.90 & \mathrm{I}\end{array}$ $\begin{array}{lllllllllllll}282.5 & 8487 . & 0.909 & 66.75 & 0.384 & 0.880 & 120.00 & 1.01 & 3.27 & \mathrm{C} & 1.936 & 41.40 & \mathrm{I}\end{array}$ $\begin{array}{llllllllllllll}225.2 & 8485 & 0.835 & 41.24 & 0.383 & 0.880 & 120.00 & 1.09 & 3.27 & \mathrm{C} & 1.768 & 24.00 & \text { I }\end{array}$ $\begin{array}{llllllllllllll}205.9 & 8478 & 0.778 & 32.75 & 0.383 & 0.880 & 120.00 & 1.16 & 3.27 & \mathrm{C} & 1.761 & 18.60 & \text { I }\end{array}$ $\begin{array}{lllllllllllll}179.6 & 8476 & 0.681 & 23.81 & 0.383 & 0.880 & 120.00 & 1.31 & 3.27 & \mathrm{C} & 1.771 & 13.60 & \mathrm{I}\end{array}$ $\begin{array}{lllllllllllll}159.2 & 8475 & 0.552 & 17.63 & 0.382 & 0.880 & 120.00 & 1.57 & 3.27 & \mathrm{C} & 1.569 & 11.50 & 1\end{array}$ $\begin{array}{llllllllllllll}151.1 & 8474 & 0.477 & 15.39 & 0.382 & 0.880 & 120.00 & 1.78 & 3.27 & \mathrm{C} & 1.490 & 11.40 & \text { I }\end{array}$ $\begin{array}{llllllllllll}144.1 & 8473 & 0.398 & 13.58 & 0.382 & 0.880 & 120.00 & 2.08 & 3.27 & \mathrm{C} & 1.421 & 12.00\end{array}$ $\begin{array}{lllllllllllll}132.7 & 8473 & 0.219 & 10.79 & 0.382 & 0.879 & 120.00 & 3.31 & 3.27 & \mathrm{C} & 1.308 & 12.80 & \text { I }\end{array}$ 
$\begin{array}{lllllllllllll}32.4 & 14500 & 1.040 & 18.13 & 0.402 & 0.897 & 120.00 & 0.89 & 3.28 & \mathrm{C} & 0.177 & 19.20 & \mathrm{O} 2\end{array}$ $\begin{array}{lllllllllllll}124.8 & 13950 & 1.015 & 37.33 & 0.402 & 0.889 & 120.00 & 0.91 & 3.27 & \mathrm{C} & 0.639 & 28.70 & \mathrm{O} 2\end{array}$ $\begin{array}{lllllllllllll}408.2 & 13630 & 0.997 & 98.62 & 0.402 & 0.885 & 120.00 & 0.92 & 3.27 & C & 1.834 & 68.70 & 1\end{array}$ $\begin{array}{lllllllllllll}369.3 & 13630 & 0.975 & 74.38 & 0.402 & 0.885 & 120.00 & 0.94 & 3.27 & \mathrm{C} & 1.767 & 50.10 & \mathrm{I}\end{array}$ $\begin{array}{lllllllllllllll}305.0 & 13620 & 0.918 & 45.67 & 0.401 & 0.885 & 120.00 & 0.99 & 3.27 & C & 1.645 & 28.70 & \text { I }\end{array}$ $\begin{array}{lllllllllllll}259.0 & 13610 & 0.846 & 30.77 & 0.401 & 0.885 & 120.00 & 1.07 & 3.27 & \mathrm{C} & 1.578 & 18.10 & 1\end{array}$ $\begin{array}{llllllllllllll}246.3 & 13580 & 0.792 & 25.82 & 0.399 & 0.884 & 120.00 & 1.14 & 3.27 & \mathrm{C} & 1.611 & 14.80 & \mathrm{I}\end{array}$ $\begin{array}{lllllllllllll}216.3 & 13580 & 0.701 & 19.26 & 0.399 & 0.884 & 120.00 & 1.27 & 3.27 & \mathrm{C} & 1.414 & 11.10 & 1\end{array}$ $\begin{array}{llllllllllllll}191.3 & 13570 & 0.583 & 14.52 & 0.398 & 0.884 & 120.00 & 1.49 & 3.27 & \mathrm{C} & 1.251 & 9.50 & \text { I }\end{array}$ $\begin{array}{lllllllllllll}181.0 & 13570 & 0.515 & 12.76 & 0.398 & 0.884 & 120.00 & 1.66 & 3.27 & \mathrm{C} & 1.184 & 9.50 & \mathrm{I}\end{array}$ $\begin{array}{llllllllllllll}171.9 & 13570 & 0.444 & 11.31 & 0.398 & 0.884 & 120.00 & 1.88 & 3.27 & \mathrm{C} & 1.124 & 9.90 & 1\end{array}$ $\begin{array}{lllllllllllll}156.5 & 13570 & 0.285 & 9.05 & 0.398 & 0.884 & 120.00 & 2.68 & 3.27 & \mathrm{C} & 1.024 & 10.60 & 1\end{array}$ $\begin{array}{llllllllllllll}35.5 & 15650 & 1.047 & 15.13 & 0.438 & 0.908 & 120.00 & 0.87 & 3.30 & \mathrm{C} & 0.197 & 19.80 & \mathrm{O} 2\end{array}$ $\begin{array}{llllllllllllll}133.4 & 14950 & 1.018 & 31.17 & 0.438 & 0.900 & 120.00 & 0.89 & 3.27 & \mathrm{C} & 0.696 & 28.60 & 02\end{array}$ $\begin{array}{lllllllllllll}406.4 & 14570 . & 0.996 & 77.63 & 0.438 & 0.895 & 120.00 & 0.91 & 3.26 & \mathrm{C} & 1.892 & 63.00 \mathrm{I}\end{array}$ $\begin{array}{lllllllllllll}366.1 & 14560 & 0.970 & 58.78 & 0.438 & 0.895 & 120.00 & 0.93 & 3.26 & \mathrm{C} & 1.819 & 46.30 & \text { I }\end{array}$ $\begin{array}{lllllllllllll}299.0 & 14560 & 0.904 & 36.31 & 0.437 & 0.895 & 120.00 & 0.99 & 3.26 & \mathrm{C} & 1.687 & 26.60 & 1\end{array}$ $\begin{array}{llllllllllllll}250.9 & 14550 & 0.822 & 24.56 & 0.437 & 0.895 & 120.00 & 1.08 & 3.26 & \mathrm{C} & 1.609 & 16.80 & 1\end{array}$ $\begin{array}{lllllllllllll}233.8 & 14530 & 0.761 & 20.39 & 0.436 & 0.894 & 120.00 & 1.16 & 3.26 & \mathrm{C} & 1.499 & 13.50 & 1\end{array}$ $\begin{array}{lllllllllllll}203.5 & 14520 & 0.659 & 15.29 & 0.435 & 0.894 & 120.00 & 1.32 & 3.26 & \mathrm{C} & 1.305 & 10.20 & \mathrm{I}\end{array}$ $\begin{array}{lllllllllllllll}178.0 & 14520 & 0.528 & 11.57 & 0.435 & 0.894 & 120.00 & 1.59 & 3.26 & \mathrm{C} & 1.141 & 8.90 & \mathrm{I}\end{array}$ $\begin{array}{lllllllllllll}167.4 & 14510 & 0.455 & 10.18 & 0.435 & 0.894 & 120.00 & 1.80 & 3.26 & C & 1.073 & 9.30 & 1\end{array}$ $\begin{array}{lllllllllllll}158.1 & 14510 & 0.378 & 9.04 & 0.435 & 0.894 & 120.00 & 2.09 & 3.26 & \mathrm{C} & 1.013 & 10.20 & \mathrm{I}\end{array}$ $\begin{array}{lllllllllllll}142.3 & 14510 & 0.208 & 7.25 & 0.435 & 0.894 & 120.00 & 3.24 & 3.26 & \mathrm{C} & 0.912 & 11.40 \mathrm{I}\end{array}$ $\begin{array}{lllllllllllll}278.4 & 13170 & 1.000 & 197.70 & 0.515 & 0.914 & 120.00 & 0.88 & 3.30 & \mathrm{C} & 1.275 & 143.20 & \mathrm{O} 2\end{array}$ $\begin{array}{lllllllllllll}324.6 & 13170 & 0.979 & 118.90 & 0.514 & 0.914 & 120.00 & 0.90 & 3.30 & \mathrm{C} & 1.641 & 83.50 & \mathrm{HC}\end{array}$ $\begin{array}{lllllllllllll}314.6 & 13160 & 0.963 & 91.68 & 0.514 & 0.914 & 120.00 & 0.91 & 3.30 & \mathrm{C} & 1.679 & 63.60 & \mathrm{I}\end{array}$ $\begin{array}{lllllllllllll}299.2 & 13160 & 0.945 & 72.14 & 0.514 & 0.914 & 120.00 & 0.93 & 3.30 & \mathrm{C} & 1.684 & 48.40 & 1\end{array}$ $\begin{array}{llllllllllllll}264.5 & 13160 & 0.896 & 46.77 & 0.514 & 0.914 & 120.00 & 0.97 & 3.30 & \mathrm{C} & 1.659 & 30.10 & \mathrm{I}\end{array}$ $\begin{array}{llllllllllllll}234.6 & 13160 & 0.833 & 32.48 & 0.514 & 0.914 & 120.00 & 1.04 & 3.30 & \mathrm{C} & 1.642 & 20.00 & \text { I }\end{array}$ $\begin{array}{lllllllllllll}222.8 & 13150 & 0.785 & 26.97 & 0.513 & 0.914 & 120.00 & 1.09 & 3.30 & \mathrm{C} & 1.673 & 16.30 & \mathrm{I}\end{array}$ $\begin{array}{llllllllllllll}201.6 & 13140 & 0.704 & 20.51 & 0.513 & 0.914 & 120.00 & 1.20 & 3.30 & \mathrm{C} & 1.514 & 12.40 & \text { I }\end{array}$ $\begin{array}{lllllllllllll}183.0 & 13140 & 0.597 & 15.69 & 0.513 & 0.914 & 120.00 & 1.37 & 3.30 & \mathrm{C} & 1.374 & 10.40 & \text { I }\end{array}$ $\begin{array}{llllllllllllll}175.1 & 13140 & 0.536 & 13.86 & 0.513 & 0.914 & 120.00 & 1.50 & 3.30 & \mathrm{C} & 1.315 & 10.20 & \mathbf{I}\end{array}$ $\begin{array}{lllllllllllll}168.0 & 13140 & 0.470 & 12.35 & 0.513 & 0.914 & 120.00 & 1.66 & 3.30 & \mathrm{C} & 1.262 & 10.50 & \mathrm{I}\end{array}$ $\begin{array}{lllllllllllll}155.9 & 13140 & 0.324 & 9.96 & 0.512 & 0.914 & 120.00 & 2.20 & 3.30 & \mathrm{C} & 1.170 & 11.00 & \mathrm{I}\end{array}$ $\begin{array}{lllllllllllll}29.9 & 16960 & 1.064 & 11.34 & 0.564 & 0.938 & 120.00 & 0.83 & 3.57 & C & 0.179 & 20.30 & 02\end{array}$ $\begin{array}{lllllllllllll}86.9 & 16240 & 1.035 & 18.45 & 0.564 & 0.932 & 120.00 & 0.84 & 3.45 & \mathrm{C} & 0.514 & 22.20 & \mathrm{O} 2\end{array}$ $\begin{array}{lllllllllllll}181.4 & 15790 & 1.014 & 31.87 & 0.564 & 0.928 & 120.00 & 0.86 & 3.39 & \mathrm{C} & 1.023 & 30.50 & \mathrm{O} 2\end{array}$ $\begin{array}{llllllllllllll}342.8 & 15530 & 0.988 & 51.17 & 0.564 & 0.925 & 120.00 & 0.87 & 3.36 & C & 1.853 & 44.70 & 1\end{array}$ $\begin{array}{lllllllllllll}284.8 & 15520 & 0.923 & 32.06 & 0.564 & 0.925 & 120.00 & 0.93 & 3.36 & \mathrm{C} & 1.739 & 26.50 & \text { I }\end{array}$ $\begin{array}{llllllllllllll}241.7 & 15520 & 0.842 & 21.87 & 0.563 & 0.925 & 120.00 & 1.00 & 3.36 & C & 1.674 & 17.10 & \text { I }\end{array}$ $\begin{array}{llllllllllllll}223.2 & 15510 & 0.781 & 17.95 & 0.563 & 0.925 & 120.00 & 1.07 & 3.36 & \mathrm{C} & 1.546 & 13.70 & \mathrm{I}\end{array}$ $\begin{array}{llllllllllllll}196.3 & 15510 & 0.681 & 13.57 & 0.563 & 0.925 & 120.00 & 1.20 & 3.36 & \mathrm{C} & 1.360 & 10.40 & \mathrm{I}\end{array}$ $\begin{array}{llllllllllllll}173.3 & 15500 & 0.551 & 10.34 & 0.562 & 0.925 & 120.00 & 1.42 & 3.36 & \mathrm{C} & 1.200 & 9.00 & 1\end{array}$ $\begin{array}{llllllllllll}163.6 & 15500 & 0.478 & 9.12 & 0.562 & 0.925 & 120.00 & 1.58 & 3.36 & \mathrm{C} & 1.133 & 9.40 \mathrm{I}\end{array}$ $\begin{array}{lllllllllllllll}155.1 & 15500 & 0.402 & 8.12 & 0.562 & 0.925 & 120.00 & 1.80 & 3.36 & \mathrm{C} & 1.074 & 10.50 & 1\end{array}$ $\begin{array}{llllllllllllll}140.5 & 15500 & 0.232 & 6.54 & 0.562 & 0.925 & 120.00 & 2.58 & 3.36 & C & 0.973 & 11.90 & 1\end{array}$ $\begin{array}{lllllllllllll}37.2 & 17240 & 1.046 & 16.09 & 0.611 & 0.943 & 120.00 & 0.82 & 3.62 & \mathrm{C} & 0.220 & 25.10 & \mathrm{O} 2\end{array}$ $\begin{array}{llllllllllllll}118.1 & 16690 & 1.021 & 27.84 & 0.611 & 0.939 & 120.00 & 0.83 & 3.51 & \mathrm{C} & 0.673 & 30.30 & 02\end{array}$ $\begin{array}{lllllllllllll}289.3 & 16330 & 1.003 & 55.79 & 0.610 & 0.936 & 120.00 & 0.84 & 3.45 & \mathrm{C} & 1.525 & 51.10 & \mathrm{O} 2\end{array}$ $\begin{array}{lllllllllllll}331.2 & 16270 & 0.981 & 53.39 & 0.610 & 0.935 & 120.00 & 0.86 & 3.44 & \mathrm{C} & 1.794 & 47.20 & \mathrm{I}\end{array}$ $\begin{array}{llllllllllllll}280.9 & 16270 & 0.925 & 33.68 & 0.610 & 0.935 & 120.00 & 0.90 & 3.44 & C & 1.708 & 28.30 & \text { I }\end{array}$ $\begin{array}{lllllllllllll}242.5 & 16270 & 0.854 & 23.07 & 0.610 & 0.935 & 120.00 & 0.97 & 3.44 & \mathrm{C} & 1.657 & 18.50 & \mathrm{I}\end{array}$ $\begin{array}{lllllllllllll}226.0 & 16260 & 0.799 & 18.95 & 0.609 & 0.935 & 120.00 & 1.02 & 3.44 & \mathrm{C} & 1.544 & 14.90 & \mathrm{I}\end{array}$ $\begin{array}{lllllllllllll}201.5 & 16250 & 0.709 & 14.35 & 0.609 & 0.935 & 120.00 & 1.12 & 3.44 & \mathrm{C} & 1.377 & 11.30 & \mathrm{I}\end{array}$ $\begin{array}{lllllllllllll}180.3 & 16250 & 0.591 & 10.95 & 0.609 & 0.935 & 120.00 & 1.30 & 3.44 & C & 1.232 & 9.70 & 1\end{array}$ $\begin{array}{lllllllllllll}171.3 & 16250 & 0.524 & 9.66 & 0.609 & 0.935 & 120.00 & 1.42 & 3.44 & \mathrm{C} & 1.171 & 9.90 & \mathrm{I}\end{array}$ $\begin{array}{lllllllllllll}163.5 & 16250 & 0.453 & 8.61 & 0.609 & 0.935 & 120.00 & 1.58 & 3.44 & C & 1.117 & 10.90 & 1\end{array}$ $\begin{array}{llllllllllllll}149.9 & 16250 & 0.296 & 6.94 & 0.609 & 0.935 & 120.00 & 2.10 & 3.44 & \mathrm{C} & 1.025 & 12.10 & 1\end{array}$ $\begin{array}{lllllllllllll}232.4 & 13230 & 0.786 & 10.60 & 0.561 & 0.925 & 120.00 & 1.06 & 3.36 & \mathrm{P} & 2.114 & 10.50 & 1\end{array}$ $\begin{array}{lllllllllllll}217.9 & 13230 & 0.592 & 12.20 & 0.561 & 0.925 & 120.00 & 1.34 & 3.36 & \mathrm{P} & 1.982 & 9.20 & 1\end{array}$ $\begin{array}{lllllllllllll}209.4 & 13230 & 0.508 & 13.09 & 0.561 & 0.925 & \mathrm{I} 20.00 & 1.51 & 3.36 & \mathrm{P} & 1.905 & 9.00 \mathrm{I}\end{array}$ $\begin{array}{lllllllllllll}200.1 & 13230 & 0.434 & 14.04 & 0.561 & 0.925 & 120.00 & 1.70 & 3.36 & P & 1.821 & 9.40 & 1\end{array}$ $\begin{array}{llllllllllllll}178.9 & 13230 & 0.311 & 16.10 & 0.561 & 0.925 & 120.00 & 2.15 & 3.36 & \mathrm{P} & 1.627 & 11.10 & 1\end{array}$ $\begin{array}{lllllllllllllll}154.7 & 13230 & 0.218 & 18.26 & 0.561 & 0.925 & 120.00 & 2.69 & 3.36 & \mathrm{P} & 1.408 & 13.20 & 1\end{array}$ $\begin{array}{llllllllllllll}138.2 & 13220 & 0.170 & 19.70 & 0.561 & 0.925 & 120.00 & 3.09 & 3.35 & \mathrm{P} & 1.257 & 14.30 & 1\end{array}$ $\begin{array}{lllllllllllll}112.5 & 13220 & 0.116 & 21.55 & 0.561 & 0.925 & 120.00 & 3.70 & 3.35 & \mathrm{P} & 1.023 & 15.50 & \mathrm{I}\end{array}$ $\begin{array}{lllllllllllllll}84.6 & 13220 & 0.073 & 23.23 & 0.561 & 0.925 & 120.00 & 4.40 & 3.35 & \mathrm{P} & 0.770 & 16.60 & \mathrm{I}\end{array}$ $\begin{array}{lllllllllllll}71.8 & 13220 & 0.057 & 23.83 & 0.561 & 0.925 & 120.00 & 4.74 & 3.35 & P & 0.653 & 21.70 & 1\end{array}$ $\begin{array}{llllllllllllll}60.7 & 13220 & 0.044 & 24.22 & 0.561 & 0.925 & 120.00 & 5.06 & 3.35 & \mathrm{P} & 0.552 & 41.00 & \mathrm{O} 2\end{array}$ $\begin{array}{llllllllllllll}44.1 & 13220 & 0.023 & 24.32 & 0.561 & 0.925 & 120.00 & 5.66 & 3.35 & \mathrm{P} & 0.401 & 72.40 & \mathrm{O} 2\end{array}$ $\begin{array}{lllllllllllllllll}232.9 & 13790 & 0.806 & 11.22 & 0.622 & 0.938 & 120.00 & 1.01 & 3.47 & \mathrm{P} & 2.104 & 11.50 & \mathrm{HC}\end{array}$ $\begin{array}{llllllllllll}219.2 & 13790 & 0.634 & 12.91 & 0.622 & 0.938 & 120.00 & 1.22 & 3.47 & \mathrm{P} & 1.980 & 10.40\end{array}$ $\begin{array}{lllllllllllll}211.6 & 13790 & 0.561 & 13.87 & 0.622 & 0.937 & 120.00 & 1.34 & 3.47 & P & 1.911 & 10.30 & 1\end{array}$ $\begin{array}{llllllllllllll}203.7 & 13780 & 0.495 & 14.91 & 0.622 & 0.937 & 120.00 & 1.46 & 3.47 & \mathrm{P} & 1.840 & 10.70 & 1\end{array}$ $\begin{array}{llllllllllll}186.4 & 13780 & 0.385 & 17.27 & 0.621 & 0.937 & 120.00 & 1.75 & 3.47 & \mathrm{P} & 1.683 & 12.80\end{array}$ $\begin{array}{llllllllllllll}167.9 & 13780 & 0.300 & 19.96 & 0.621 & 0.937 & 120.00 & 2.05 & 3.47 & \mathrm{P} & 1.517 & 15.30 & 1\end{array}$ $\begin{array}{lllllllllllllll}155.9 & 13780 & 0.255 & 21.88 & 0.621 & 0.937 & 120.00 & 2.26 & 3.47 & \mathrm{P} & 1.408 & 16.90 & 1\end{array}$ $\begin{array}{lllllllllllllll}138.1 & 13780 & 0.204 & 24.78 & 0.621 & 0.937 & 120.00 & 2.55 & 3.47 & \mathrm{P} & 1.247 & 18.80 & 1\end{array}$ $\begin{array}{llllllllllllll}119.9 & 13780 . & 0.160 & 28.14 & 0.621 & 0.937 & 120.00 & 2.88 & 3.47 & \mathrm{P} & 1.083 & 21.00 & 1\end{array}$ $\begin{array}{llllllllllllll}112.2 & 13780 & 0.141 & 29.79 & 0.621 & 0.937 & 120.00 & 3.04 & 3.47 & \mathrm{P} & 1.014 & 24.60 & 1\end{array}$ $\begin{array}{lllllllllllllll}106.1 & 13780 & 0.125 & 31.32 & 0.621 & 0.937 & 120.00 & 3.20 & 3.47 & \mathrm{P} & 0.959 & 34.80 & 1\end{array}$ $\begin{array}{lllllllllllll}100.3 & 13780 & 0.097 & 33.85 & 0.621 & 0.937 & 120.00 & 3.52 & 3.47 & \mathrm{P} & 0.906 & 51.70 & 1\end{array}$ $\begin{array}{llllllllllllll}246.3 & 13440 & 0.784 & 11.87 & 0.427 & 0.892 & 120.00 & 1.13 & 3.26 & \mathrm{P} & 1.923 & 10.70 & 1\end{array}$ $\begin{array}{llllllllllllll}233.7 & 13430 & 0.599 & 14.05 & 0.427 & 0.892 & 120.00 & 1.44 & 3.26 & \mathrm{P} & 1.824 & 9.30 & 1\end{array}$ $\begin{array}{lllllllllllll}226.3 & 13430 & 0.521 & 15.33 & 0.427 & 0.892 & 120.00 & 1.62 & 3.26 & \mathrm{P} & 1.766 & 9.10 & 1\end{array}$ $\begin{array}{llllllllllllll}218.3 & 13430 & 0.452 & 16.73 & 0.427 & 0.892 & 120.00 & 1.82 & 3.26 & P & 1.703 & 9.60 & 1\end{array}$ $\begin{array}{lllllllllllll}199.7 & 13430 & 0.339 & 20.01 & 0.427 & 0.892 & 120.00 & 2.29 & 3.26 & \mathrm{P} & 1.558 & 11.90 & \mathrm{I}\end{array}$ $\begin{array}{llllllllllllll}178.4 & 13420 & 0.255 & 23.87 & 0.426 & 0.892 & 120.00 & 2.84 & 3.26 & \mathrm{P} & 1.393 & 14.90 & \mathrm{I}\end{array}$ $\begin{array}{lllllllllllll}164.6 & 13420 & 0.213 & 26.84 & 0.426 & 0.892 & 120.00 & 3.22 & 3.26 & \mathrm{P} & 1.285 & 16.80 & \mathrm{I}\end{array}$ $\begin{array}{llllllllllllll}141.6 & 13420 & 0.166 & 31.11 & 0.426 & 0.892 & 120.00 & 3.80 & 3.26 & \mathrm{P} & 1.105 & 18.90 & \mathrm{I}\end{array}$ $\begin{array}{llllllllllllll}117.3 & 13420 & 0.128 & 35.80 & 0.426 & 0.892 & 120.00 & 4.44 & 3.26 & \mathrm{P} & 0.915 & 21.10 & 1\end{array}$ $\begin{array}{lllllllllllllll}107.0 & 13410 & 0.112 & 37.91 & 0.426 & 0.892 & 120.00 & 4.77 & 3.26 & \mathrm{P} & 0.835 & 25.00 & 1\end{array}$ $\begin{array}{lllllllllllll}99.1 & 13410 & 0.099 & 39.61 & 0.426 & 0.892 & 120.00 & 5.08 & 3.26 & \mathrm{P} & 0.774 & 34.80 & \mathrm{I}\end{array}$ $\begin{array}{lllllllllllllll}92.9 & 13410 & 0.076 & 41.46 & 0.426 & 0.892 & 120.00 & 5.76 & 3.26 & \mathrm{P} & 0.725 & 49.90 & 1\end{array}$ $\begin{array}{lllllllllllllll}249.7 & 13260 & 0.791 & 11.84 & 0.389 & 0.881 & 120.00 & 1.14 & 3.27 & \mathrm{P} & 1.870 & 11.00 & 1\end{array}$ $\begin{array}{lllllllllllll}236.2 & 13250 & 0.601 & 13.96 & 0.388 & 0.881 & 120.00 & 1.46 & 3.27 & \mathrm{P} & 1.769 & 9.30 & \mathrm{I}\end{array}$ $\begin{array}{llllllllllllll}228.4 & 13250 & 0.520 & 15.20 & 0.388 & 0.881 & 120.00 & 1.65 & 3.27 & \mathrm{P} & 1.710 & 9.00 & \mathrm{I}\end{array}$ $\begin{array}{llllllllllllll}219.7 & 13250 & 0.450 & 16.55 & 0.388 & 0.881 & 120.00 & 1.87 & 3.27 & \mathrm{P} & 1.645 & 9.50 & 1\end{array}$ $\begin{array}{lllllllllllll}199.8 & 13250 & 0.333 & 19.67 & 0.388 & 0.881 & 120.00 & 2.39 & 3.27 & \mathrm{P} & 1.496 & 11.80 & \mathrm{I}\end{array}$ $\begin{array}{llllllllllllll}176.9 & 13240 & 0.247 & 23.28 & 0.388 & 0.881 & 120.00 & 3.02 & 3.27 & \mathrm{P} & 1.325 & 14.60 & 1\end{array}$ $\begin{array}{lllllllllllllll}162.1 & 13240 & 0.203 & 26.06 & 0.387 & 0.881 & 120.00 & 3.47 & 3.27 & \mathrm{P} & 1.214 & 16.40 & 1\end{array}$ $\begin{array}{llllllllllllll}137.1 & 13230 & 0.155 & 29.89 & 0.387 & 0.881 & 120.00 & 4.17 & 3.27 & \mathrm{P} & 1.027 & 18.20 & \mathrm{I}\end{array}$ $\begin{array}{lllllllllllll}110.5 & 13230 & 0.116 & 33.97 & 0.387 & 0.881 & 120.00 & 4.97 & 3.27 & \mathrm{P} & 0.828 & 20.10 & \mathrm{I}\end{array}$ $\begin{array}{llllllllllllll}99.0 & 13230 & 0.101 & 35.74 & 0.387 & 0.881 & 120.00 & 5.38 & 3.27 & \mathrm{P} & 0.741 & 24.20 & \mathrm{I}\end{array}$ $\begin{array}{lllllllllllllll}89.8 & 13230 & 0.088 & 37.13 & 0.387 & 0.881 & 120.00 & 5.78 & 3.27 & \mathrm{P} & 0.673 & 35.10 & \mathrm{I}\end{array}$ $\begin{array}{lllllllllllll}80.6 & 13230 & 0.066 & 38.49 & 0.387 & 0.881 & 120.00 & 6.63 & 3.27 & \mathrm{P} & 0.604 & 51.30 & \mathrm{I}\end{array}$ $\begin{array}{llllllllllllll}245.5 & 14950 & 0.785 & 11.22 & 0.502 & 0.911 & 120.00 & 1.10 & 3.29 & \mathrm{P} & 1.839 & 10.60 & 1\end{array}$ $\begin{array}{llllllllllllll}232.9 & 14950 & 0.611 & 13.23 & 0.502 & 0.911 & 120.00 & 1.36 & 3.29 & \mathrm{P} & 1.744 & 9.30 & \mathrm{I}\end{array}$ $\begin{array}{llllllllllllll}225.6 & 14950 & 0.537 & 14.42 & 0.502 & 0.911 & 120.00 & 1.51 & 3.29 & \mathrm{P} & 1.689 & 9.20 & \text { I }\end{array}$ $\begin{array}{llllllllllllll}217.8 & 14950 & 0.473 & 15.72 & 0.502 & 0.911 & 120.00 & 1.67 & 3.29 & \mathrm{P} & 1.631 & 9.70 & 1\end{array}$ $\begin{array}{lllllllllllll}200.0 & 14940 & 0.365 & 18.78 & 0.502 & 0.911 & 120.00 & 2.03 & 3.29 & \mathrm{P} & 1.497 & 12.00 & \mathrm{I}\end{array}$ $\begin{array}{llllllllllllll}180.0 & 14940 & 0.285 & 22.41 & 0.501 & 0.911 & 120.00 & 2.43 & 3.29 & \mathrm{P} & 1.348 & 15.00 & 1\end{array}$ $\begin{array}{llllllllllllll}167.2 & 14930 & 0.245 & 25.24 & 0.501 & 0.911 & 120.00 & 2.70 & 3.29 & \mathrm{P} & 1.252 & 16.90 & 1\end{array}$ $\begin{array}{llllllllllllll}146.3 & 14930 & 0.199 & 29.42 & 0.501 & 0.911 & 120.00 & 3.07 & 3.29 & \mathrm{P} & 1.095 & 19.20 & 1\end{array}$ $\begin{array}{lllllllllllll}124.4 & 14930 & 0.162 & 34.24 & 0.501 & 0.911 & 120.00 & 3.47 & 3.29 & \mathrm{P} & 0.932 & 21.80 & \mathrm{I}\end{array}$ $\begin{array}{llllllllllllll}115.3 & 14930 & 0.147 & 36.55 & 0.501 & 0.911 & 120.00 & 3.67 & 3.29 & \mathrm{P} & 0.864 & 25.90 & \mathrm{I}\end{array}$ $\begin{array}{llllllllllllll}108.7 & 14930 & 0.134 & 38.58 & 0.501 & 0.911 & 120.00 & 3.85 & 3.29 & \mathrm{P} & 0.814 & 36.00 & \mathrm{I}\end{array}$ $\begin{array}{lllllllllllllll}104.7 & 14930 & 0.110 & 41.34 & 0.501 & 0.911 & 120.00 & 4.23 & 3.29 & \mathrm{P} & 0.784 & 51.80 & \mathrm{I}\end{array}$ $\begin{array}{lllllllllllll}278.8 & 19970 & 0.788 & 9.62 & 0.502 & 0.911 & 120.00 & 1.09 & 3.29 & \mathrm{P} & 2.087 & 10.80 & \mathrm{HC}\end{array}$ $\begin{array}{lllllllllllll}263.1 & 19960 & 0.615 & 11.29 & 0.501 & 0.911 & 120.00 & 1.35 & 3.29 & \mathrm{P} & 1.970 & 9.10 & 1\end{array}$ $\begin{array}{lllllllllllllll}254.3 & 19960 & 0.542 & 12.27 & 0.501 & 0.911 & 120.00 & 1.50 & 3.29 & \mathrm{P} & 1.904 & 8.80 & \mathrm{I}\end{array}$ $\begin{array}{llllllllllllll}244.9 & 19950 & 0.478 & 13.33 & 0.501 & 0.911 & 120.00 & 1.66 & 3.29 & \mathrm{P} & 1.834 & 9.20 & \mathrm{I}\end{array}$ $\begin{array}{llllllllllllll}224.1 & 19950 & 0.371 & 15.82 & 0.501 & 0.911 & 120.00 & 2.01 & 3.29 & \mathrm{P} & 1.678 & 11.50 & \mathrm{I}\end{array}$ $\begin{array}{lllllllllllllll}201.6 & 19940 & 0.291 & 18.76 & 0.501 & 0.911 & 120.00 & 2.40 & 3.29 & \mathrm{P} & 1.510 & 14.20 & 1\end{array}$ $\begin{array}{lllllllllllll}189.2 & 19920 & 0.250 & 21.22 & 0.500 & 0.911 & 120.00 & 2.66 & 3.29 & \mathrm{P} & 1.417 & 16.10 & \mathrm{I}\end{array}$ $\begin{array}{llllllllllllll}167.3 & 19920 & 0.204 & 24.66 & 0.500 & 0.911 & 120.00 & 3.03 & 3.29 & \mathrm{P} & 1.253 & 18.10 & 1\end{array}$ $\begin{array}{lllllllllllllll}146.1 & 19920 & 0.166 & 28.73 & 0.500 & 0.911 & 120.00 & 3.43 & 3.29 & \mathrm{P} & 1.094 & 20.30 & \mathrm{I}\end{array}$ $\begin{array}{lllllllllllll}138.0 & 19920 & 0.150 & 30.73 & 0.499 & 0.911 & 120.00 & 3.63 & 3.29 & \mathrm{P} & 1.034 & 23.70 & \mathrm{I}\end{array}$ $\begin{array}{lllllllllllll}133.0 & 19920 . & 0.136 & 32.55 & 0.499 & 0.911 & 120.00 & 3.83 & 3.29 & \mathrm{P} & 0.996 & 31.40 & \mathrm{I}\end{array}$ $\begin{array}{lllllllllllllll}134.0 & 19910 & 0.110 & 35.30 & 0.499 & 0.911 & 120.00 & 4.24 & 3.29 & \mathrm{P} & 1.004 & 46.60 & \mathrm{I}\end{array}$ $\begin{array}{lllllllllllll}289.5 & 17810 & 0.785 & 10.97 & 0.401 & 0.885 & 120.00 & 1.14 & 3.27 & \mathrm{P} & 2.168 & 11.50 & \mathrm{I}\end{array}$ $\begin{array}{lllllllllllllll}270.6 & 17800 & 0.608 & 12.71 & 0.400 & 0.885 & 120.00 & 1.44 & 3.27 & P & 2.026 & 9.40 & I\end{array}$ $\begin{array}{llllllllllllll}260.2 & 17790 & 0.532 & 13.72 & 0.400 & 0.885 & 120.00 & 1.61 & 3.27 & \mathrm{P} & 1.949 & 9.00 & \mathrm{I}\end{array}$ $\begin{array}{lllllllllllll}249.2 & 17790 & 0.465 & 14.81 & 0.400 & 0.885 & 120.00 & 1.80 & 3.27 & \mathrm{P} & 1.866 & 9.30 & \mathrm{I}\end{array}$ $\begin{array}{lllllllllllll}225.3 & 17780 & 0.355 & 17.31 & 0.400 & 0.884 & 120.00 & 2.26 & 3.27 & \mathrm{P} & 1.687 & 11.40 & \mathrm{I}\end{array}$ $\begin{array}{llllllllllllll}200.3 & 17770 & 0.272 & 20.23 & 0.399 & 0.884 & 120.00 & 2.78 & 3.27 & \mathrm{P} & 1.500 & 14.10 & \mathrm{I}\end{array}$ $\begin{array}{llllllllllllll}187.4 & 17750 & 0.230 & 22.81 & 0.398 & 0.884 & 120.00 & 3.15 & 3.27 & \mathrm{P} & 1.403 & 15.80 & \text { I }\end{array}$

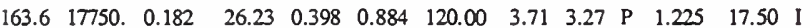
$\begin{array}{lllllllllllll}140.4 & 17740 & 0.142 & 30.36 & 0.398 & 0.884 & 120.00 & 4.35 & 3.27 & \mathrm{P} & 1.052 & 19.50 & 1\end{array}$ $\begin{array}{llllllllllllll}131.4 & 17740 & 0.125 & 32.50 & 0.398 & 0.884 & 120.00 & 4.68 & 3.27 & \mathrm{P} & 0.984 & 23.10 & \mathrm{I}\end{array}$ $\begin{array}{lllllllllllll}125.2 & 17740 & 0.111 & 34.52 & 0.398 & 0.884 & 120.00 & 5.02 & 3.27 & \mathrm{P} & 0.938 & 31.90 & \mathrm{I}\end{array}$ $\begin{array}{lllllllllllll}123.8 & 17740 & 0.086 & 38.05 & 0.398 & 0.884 & 120.00 & 5.75 & 3.27 & \mathrm{P} & 0.927 & 49.00 & 1\end{array}$ $\begin{array}{llllllllllllllll}224.9 & 8127 & 0.759 & 16.99 & 0.384 & 0.880 & 120.00 & 1.19 & 3.27 & \mathrm{P} & 2.252 & 12.60 & \mathrm{I}\end{array}$ $\begin{array}{lllllllllllll}211.9 & 8126 & 0.565 & 20.22 & 0.384 & 0.880 & 120.00 & 1.54 & 3.27 & P & 2.122 & 11.30 & \text { I }\end{array}$ $\begin{array}{lllllllllllll}204.2 & 8126 & 0.484 & 22.14 & 0.384 & 0.880 & 120.00 & 1.76 & 3.27 & \mathrm{P} & 2.045 & 11.40 & \mathrm{I}\end{array}$ $\begin{array}{llllllllllllll}195.8 & 8125 & 0.414 & 24.26 & 0.384 & 0.880 & 120.00 & 2.01 & 3.27 & \mathrm{P} & 1.961 & 12.10 & \mathrm{I}\end{array}$ $\begin{array}{lllllllllllll}176.2 & 8124 & 0.299 & 29.23 & 0.384 & 0.880 & 120.00 & 2.61 & 3.27 & \mathrm{P} & 1.764 & 15.10 & \mathrm{I}\end{array}$ $\begin{array}{lllllllllllll}153.3 & 8123 & 0.217 & 35.08 & 0.384 & 0.880 & 120.00 & 3.33 & 3.27 & P & 1.536 & 18.80 & 1\end{array}$ $\begin{array}{llllllllllllll}137.0 & 8123 & 0.177 & 39.29 & 0.384 & 0.880 & 120.00 & 3.84 & 3.27 & \mathrm{P} & 1.372 & 21.20 & 1\end{array}$ $\begin{array}{llllllllllllll}111.7 & 8123 & 0.133 & 45.45 & 0.384 & 0.880 & 120.00 & 4.61 & 3.27 & P & 1.119 & 24.00 & 1\end{array}$ $\begin{array}{llllllllllllll}85.3 & 8124 & 0.100 & 51.85 & 0.384 & 0.880 & 120.00 & 5.43 & 3.27 & \mathrm{P} & 0.854 & 26.90 & \mathrm{I}\end{array}$ $\begin{array}{lllllllllllllll}74.4 & 8124 & 0.088 & 54.47 & 0.384 & 0.880 & 120.00 & 5.82 & 3.27 & \mathrm{P} & 0.745 & 32.20 & 1\end{array}$ $\begin{array}{llllllllllllll}66.6 & 8124 & 0.078 & 56.56 & 0.384 & 0.880 & 120.00 & 6.19 & 3.27 & \mathrm{P} & 0.667 & 45.40 \mathrm{CU}\end{array}$ $\begin{array}{lllllllllllll}61.8 & 8123 & 0.060 & 58.19 & 0.384 & 0.880 & 120.00 & 6.96 & 3.27 & \mathrm{P} & 0.619 & 60.70 & \mathrm{CU}\end{array}$ $\begin{array}{llllllllllllll}223.1 & 8489 & 0.764 & 15.18 & 0.397 & 0.884 & 120.00 & 1.17 & 3.27 & \mathrm{P} & 2.234 & 12.00 & \mathrm{I}\end{array}$ $\begin{array}{lllllllllllllll}210.2 & 8488 & 0.558 & 18.09 & 0.397 & 0.884 & 120.00 & 1.55 & 3.27 & \mathrm{P} & 2.105 & 10.60 & \mathrm{I}\end{array}$ $\begin{array}{lllllllllllll}202.2 & 8488 & 0.472 & 19.79 & 0.397 & 0.884 & 120.00 & 1.79 & 3.27 & \mathrm{P} & 2.025 & 10.60 & \mathrm{I}\end{array}$ $\begin{array}{llllllllllllll}193.2 & 8487 & 0.397 & 21.64 & 0.397 & 0.884 & 120.00 & 2.06 & 3.27 & \mathrm{P} & 1.935 & 11.30 & \mathrm{I}\end{array}$ $\begin{array}{lllllllllllllll}171.5 & 8486 & 0.276 & 25.87 & 0.397 & 0.884 & 120.00 & 2.75 & 3.27 & \mathrm{P} & 1.717 & 14.00 & \mathrm{I}\end{array}$ $\begin{array}{lllllllllllll}145.1 & 8485 & 0.190 & 30.56 & 0.397 & 0.884 & 120.00 & 3.60 & 3.27 & \mathrm{P} & 1.453 & 17.30 & \text { I }\end{array}$ $\begin{array}{llllllllllllll}125.9 & 8485 & 0.148 & 33.69 & 0.397 & 0.884 & 120.00 & 4.24 & 3.27 & P & 1.261 & 19.10 & \text { I }\end{array}$ $\begin{array}{lllllllllllllll}96.7 & 8484 & 0.104 & 37.94 & 0.397 & 0.884 & 120.00 & 5.21 & 3.27 & \mathrm{P} & 0.968 & 21.20 & \mathrm{I}\end{array}$ $\begin{array}{llllllllllllll}65.8 & 8483 & 0.073 & 41.54 & 0.397 & 0.884 & 120.00 & 6.24 & 3.27 & \mathrm{P} & 0.659 & 23.30 & 1\end{array}$ $\begin{array}{lllllllllllll}52.8 & 8483 & 0.062 & 42.58 & 0.397 & 0.884 & 120.00 & 6.70 & 3.27 & \mathrm{P} & 0.528 & 31.00 & \mathrm{O} 2\end{array}$ $\begin{array}{lllllllllllllll}42.7 & 8483 & 0.053 & 42.95 & 0.397 & 0.884 & 120.00 & 7.11 & 3.27 & \mathrm{P} & 0.428 & 54.40 & \mathrm{O} 2\end{array}$ $\begin{array}{lllllllllllll}32.9 & 8482 & 0.040 & 41.67 & 0.397 & 0.884 & 120.00 & 7.86 & 3.27 & \mathrm{P} & 0.329 & 71.50 & \mathrm{O} 2\end{array}$ $\begin{array}{llllllllllllll}227.1 & 9794 & 0.780 & 13.86 & 0.524 & 0.916 & 120.00 & 1.09 & 3.31 & P & 2.347 & 11.80 & 1\end{array}$ $\begin{array}{llllllllllllll}212.6 & 9794 & 0.585 & 16.54 & 0.524 & 0.916 & 120.00 & 1.39 & 3.31 & P & 2.197 & 11.00 & 1\end{array}$ $\begin{array}{llllllllllllll}204.2 & 9793 & 0.503 & 18.14 & 0.524 & 0.916 & 120.00 & 1.56 & 3.31 & \mathrm{P} & 2.110 & 11.10 & 1\end{array}$ $\begin{array}{llllllllllllll}195.1 & 9793 & 0.433 & 19.91 & 0.524 & 0.916 & 120.00 & 1.76 & 3.31 & \mathrm{P} & 2.016 & 11.90 & \mathrm{I}\end{array}$ $\begin{array}{llllllllllllllll}174.3 & 9792 & 0.320 & 24.12 & 0.524 & 0.916 & 120.00 & 2.19 & 3.31 & \mathrm{P} & 1.802 & 14.90 & \mathrm{I}\end{array}$ $\begin{array}{lllllllllllllll}150.9 & 9791 . & 0.239 & 29.16 & 0.524 & 0.916 & 120.00 & 2.66 & 3.31 & \mathrm{P} & 1.559 & 18.70 & 1\end{array}$ $\begin{array}{lllllllllllll}134.7 & 9790 & 0.200 & 32.86 & 0.524 & 0.916 & 120.00 & 2.97 & 3.31 & \mathrm{P} & 1.392 & 21.20 & 1\end{array}$ $\begin{array}{lllllllllllllll}111.2 & 9790 & 0.159 & 38.58 & 0.524 & 0.916 & 120.00 & 3.39 & 3.31 & P & 1.149 & 24.50 & 1\end{array}$ 
$\begin{array}{lll}88.6 & 9789 & 0.127\end{array}$ $\begin{array}{lll}80.8 & 9788 . & 0.115\end{array}$ $\begin{array}{lll}76.6 & 9788 . & 0.104\end{array}$

81.4 9787. 0.084 $221.6 \quad 8482 . \quad 0.782$ 207.0 8481. 0.621 198.9 8481. 0.554 190.5 8481. 0.496 $\begin{array}{llll}172.2 & 8480 & 0.401\end{array}$ $\begin{array}{llll}153.0 & 8480 & 0.332\end{array}$ $\begin{array}{llll}139.9 & 8480 & 0.298\end{array}$ 122.9 8480. 0.261 109.3 8479. 0.230 $\begin{array}{lll}106.4 & 8479 . & 0.218\end{array}$ $\begin{array}{llll}107.6 & 8479 . & 0.207\end{array}$ 126.4 8478. 0.186 250.1 18940. 0.800 236.218940 .0 .599 228.1 18930. 0.515 $219.2 \quad 18930.0 .443$ 198.7 18930. 0.326 $\begin{array}{llll}175.0 & 18930 & 0.242\end{array}$ $\begin{array}{llll}159.2 & 18920 & 0.201\end{array}$ $\begin{array}{lll}134.5 & 18920 . & 0.158\end{array}$ $\begin{array}{lll}110.4 & 18920 & 0.123\end{array}$ $101.8 \quad 18920.0 .110$ $\begin{array}{lll}97.0 & 18920 . & 0.099\end{array}$ $\begin{array}{lll}102.1 & 18920 . & 0.077\end{array}$ $\begin{array}{lll}267.4 & 20510 . & 0.820\end{array}$ 252.8 20510. 0.626 244.4 20510. 0.544 235.320500 .0 .472 $214.8 \quad 20500 . \quad 0.352$ $\begin{array}{llll}191.9 & 20500 & 0.263\end{array}$ $\begin{array}{llll}177.4 & 20490 \text {. } & 0.218\end{array}$ $\begin{array}{lll}53.9 & 20490 & 0.167\end{array}$ $\begin{array}{lll}129.9 & 20480 . & 0.125\end{array}$ $\begin{array}{lll}120.0 & 20480 \text {. } & 0.108\end{array}$ $\begin{array}{llll}112.6 & 20480 \text {. } & 0.094\end{array}$ $\begin{array}{lll}107.8 & 20480 . & 0.067\end{array}$ $\begin{array}{llllllllll}44.90 & 0.524 & 0.916 & 120.00 & 3.80 & 3.31 & P & 0.916 & 28.10 & 1\end{array}$ $\begin{array}{llllllllll}4.68 & 0.524 & 0.916 & 120.00 & 3.99 & 3.31 & \mathrm{P} & 0.835 & 33.90 & \text { I }\end{array}$ $\begin{array}{lllllllllll}49.84 & 0.524 & 0.916 & 120.00 & 4.16 & 3.31 & \mathrm{P} & 0.791 & 44.90 & \mathrm{I}\end{array}$ $\begin{array}{llllllllll}51.74 & 0.524 & 0.916 & 120.00 & 4.55 & 3.31 & \mathrm{P} & 0.841 & 57.00 & \mathrm{I}\end{array}$ $\begin{array}{lllllllllll}18.18 & 0.622 & 0.938 & 120.00 & 1.03 & 3.47 & \mathrm{P} & 2.602 & 15.70 & \mathrm{HC}\end{array}$ $\begin{array}{llllllllll}21.51 & 0.622 & 0.938 & 120.00 & 1.24 & 3.47 & \mathrm{P} & 2.431 & 15.60 & 1\end{array}$ $\begin{array}{llllllllll}23.50 & 0.622 & 0.938 & 120.00 & 1.35 & 3.47 & \mathrm{P} & 2.336 & 16.10 & \text { I }\end{array}$ $\begin{array}{llllllllll}25.73 & 0.622 & 0.937 & 120.00 & 1.46 & 3.47 & \mathrm{P} & 2.237 & 17.30 & \mathrm{I}\end{array}$ $\begin{array}{llllllllll}31.08 & 0.622 & 0.937 & 120.00 & 1.70 & 3.47 & P & 2.023 & 21.30 & \text { I }\end{array}$ $\begin{array}{llllllllll}37.71 & 0.622 & 0.937 & 120.00 & 1.92 & 3.47 & \mathrm{P} & 1.796 & 26.60 & 1\end{array}$ $\begin{array}{llllllllll}42.60 & 0.622 & 0.937 & 120.00 & 2.06 & 3.47 & \mathrm{P} & 1.643 & 30.20 & \text { I }\end{array}$ $\begin{array}{llllllllll}51.06 & 0.621 & 0.937 & 120.00 & 2.23 & 3.47 & \mathrm{P} & 1.444 & 35.80 & \text { I }\end{array}$ $\begin{array}{llllllllll}61.98 & 0.621 & 0.937 & 120.00 & 2.39 & 3.47 & \mathrm{P} & 1.283 & 42.90 & \mathrm{I}\end{array}$ $\begin{array}{llllllllll}67.86 & 0.621 & 0.937 & 120.00 & 2.46 & 3.47 & \mathrm{P} & 1.249 & 50.20 & \mathrm{I}\end{array}$ $\begin{array}{lllllllllll}73.63 & 0.621 & 0.937 & 120.00 & 2.53 & 3.47 & \mathrm{P} & 1.264 & 62.40 & \mathrm{I}\end{array}$ $\begin{array}{llllllllll}83.71 & 0.621 & 0.937 & 120.00 & 2.68 & 3.47 & \mathrm{P} & 1.484 & 81.20 & \mathrm{I}\end{array}$ $\begin{array}{lllllllllll}7.48 & 0.589 & 0.931 & 120.00 & 1.03 & 3.40 & \mathrm{P} & 2.937 & 9.80 & \mathrm{HC}\end{array}$ $\begin{array}{llllllllll}8.99 & 0.589 & 0.931 & 120.00 & 1.30 & 3.40 & \mathrm{P} & 2.774 & 8.40 & \mathrm{I}\end{array}$ $\begin{array}{lllllllllll}9.90 & 0.589 & 0.931 & 120.00 & 1.46 & 3.40 & \mathrm{P} & 2.679 & 8.30 & \mathrm{I}\end{array}$ $\begin{array}{lllllllllll}10.91 & 0.589 & 0.931 & 120.00 & 1.63 & 3.40 & \mathrm{P} & 2.574 & 8.80 & \mathrm{I}\end{array}$ $\begin{array}{llllllllll}13.33 & 0.589 & 0.931 & 120.00 & 2.02 & 3.40 & \mathrm{P} & 2.333 & 11.20 & \mathrm{I}\end{array}$ $\begin{array}{llllllllllll}16.28 & 0.589 & 0.931 & 120.00 & 2.43 & 3.40 & \mathrm{P} & 2.056 & 14.50 & \mathrm{I}\end{array}$ $\begin{array}{lllllllllll}18.57 & 0.588 & 0.931 & 120.00 & 2.70 & 3.40 & \mathrm{P} & 1.869 & 16.60 & 1\end{array}$ $\begin{array}{llllllllll}22.09 & 0.588 & 0.931 & 120.00 & 3.07 & 3.40 & \mathrm{P} & 1.580 & 19.20 & 1\end{array}$ $\begin{array}{llllllllll}26.06 & 0.588 & 0.931 & 120.00 & 3.43 & 3.40 & \mathrm{P} & 1.296 & 22.10 & \text { I }\end{array}$ $\begin{array}{llllllllll}27.84 & 0.588 & 0.931 & 120.00 & 3.59 & 3.40 & \mathrm{P} & 1.195 & 27.50\end{array}$ $\begin{array}{llllllllll}29.23 & 0.588 & 0.931 & 120.00 & 3.75 & 3.40 & \mathrm{P} & 1.139 & 38.40 & \mathrm{I}\end{array}$ $\begin{array}{lllllllllll}30.43 & 0.588 & 0.931 & 120.00 & 4.08 & 3.40 & P & 1.199 & 51.10 & \text { I }\end{array}$ $\begin{array}{lllllllllll}7.68 & 0.600 & 0.933 & 120.00 & 1.00 & 3.42 & \mathrm{P} & 3.140 & 10.10 & \mathrm{HC}\end{array}$ $\begin{array}{lllllllllll}9.03 & 0.600 & 0.933 & 120.00 & 1.25 & 3.42 & \mathrm{P} & 2.968 & 8.50 & \mathrm{I}\end{array}$ $\begin{array}{llllllllll}9.83 & 0.600 & 0.933 & 120.00 & 1.39 & 3.42 & \mathrm{P} & 2.870 & 8.30 \mathrm{I}\end{array}$ $\begin{array}{lllllllllll}10.69 & 0.600 & 0.933 & 120.00 & 1.55 & 3.42 & P & 2.764 & 8.70 & 1\end{array}$ $\begin{array}{llllllllll}2.70 & 0.599 & 0.933 & 120.00 & 1.90 & 3.42 & \mathrm{P} & 2.523 & 10.80 & \mathrm{I}\end{array}$ $\begin{array}{lllllllllll}15.04 & 0.599 & 0.933 & 120.00 & 2.29 & 3.42 & \mathrm{P} & 2.253 & 13.40 & 1\end{array}$ $\begin{array}{llllllllll}16.86 & 0.599 & 0.933 & 120.00 & 2.55 & 3.42 & \mathrm{P} & 2.083 & 15.10 & \mathrm{I}\end{array}$ $\begin{array}{lllllllllll}19.47 & 0.599 & 0.933 & 120.00 & 2.93 & 3.42 & \mathrm{P} & 1.807 & 16.90 & \mathrm{I}\end{array}$ $\begin{array}{lllllllllll}22.39 & 0.599 & 0.933 & 120.00 & 3.34 & 3.42 & \mathrm{P} & 1.525 & 18.90 & \mathrm{I}\end{array}$ $\begin{array}{lllllllllll}23.72 & 0.598 & 0.933 & 120.00 & 3.54 & 3.42 & \mathrm{P} & 1.409 & 22.80 & 1\end{array}$ $\begin{array}{lllllllllll}24.81 & 0.598 & 0.933 & 120.00 & 3.73 & 3.42 & P & 1.322 & 32.40 & I\end{array}$ $\begin{array}{llllllllll}26.12 & 0.598 & 0.933 & 120.00 & 4.14 & 3.42 & P & 1.266 & 47.00 & \text { I }\end{array}$ 


\section{$\mathrm{Nu} \operatorname{Re} x_{9}$ \\ 234.3 14240. 0.775 217.4 14250. 0.590 $\begin{array}{lll}208.0 & 14250 & 0.513\end{array}$ 198.1 14250. 0.446 $\begin{array}{lll}176.4 & 14260 . & 0.336\end{array}$ $\begin{array}{llll}152.9 & 14260 & 0.255\end{array}$ $\begin{array}{lll}136.6 & 14270 . & 0.215\end{array}$ 114.2 14270. 0.171 $\begin{array}{llll}92.4 & 14270 . & 0.136\end{array}$ $\begin{array}{lll}83.9 & 14270 & 0.122\end{array}$ $\begin{array}{lll}78.1 & 14270 . & 0.110\end{array}$ $\begin{array}{llll}76.1 & 14270 & 0.089\end{array}$ 277.621810 .0 .795 260.021820 .0 .642 $250.321830,0.578$ 240.321830 .0 .522 218.5 21840. 0.431 $\begin{array}{lll}195.5 & 21840 . & 0.364\end{array}$ 179.421850 .0 .330 $\begin{array}{lll}158.0 & 21850 . & 0.292\end{array}$} $\begin{array}{lll}138.321860 . & 0.262\end{array}$ 131.921860 .0 .250 $\begin{array}{llll}129.3 & 21860 & 0.239\end{array}$ $\begin{array}{lll}139.5 & 21860 . & 0.219\end{array}$ $297.123100 . \quad 0.830$ $\begin{array}{lll}281.2 & 23110 . & 0.699\end{array}$ 272.2 23120. 0.645 $\begin{array}{lll}262.8 & 23120 . & 0.597\end{array}$ 241.8 23120. 0.520 218.623130 .0 .464 201.623130 .0 .435 $\begin{array}{lll}178.6 & 23140 . & 0.405\end{array}$ $\begin{array}{llll}57.0 & 23140 . & 0.380\end{array}$ $\begin{array}{lll}150.1 & 23140 . & 0.370\end{array}$ $\begin{array}{lll}147.7 & 23140 . & 0.362\end{array}$ $\begin{array}{llll}161.4 & 23140 . & 0.346\end{array}$ $\begin{array}{lll}266.1 & 18790 & 0.821\end{array}$ $\begin{array}{llll}249.5 & 18800 & 0.659\end{array}$ 240.4 18810. 0.591 $231.0 \quad 18810.0 .532$ 210.7 18810. 0.436 $\begin{array}{lll}189.2 & 18820 & 0.365\end{array}$ $\begin{array}{lll}174.4 & 18820 . & 0.330\end{array}$ $\begin{array}{lll}155.1 & 18830 & 0.290\end{array}$ $\begin{array}{llll}138.5 & 18830 & 0.258\end{array}$ $\begin{array}{lll}134.0 & 18830 & 0.245\end{array}$ 133.6 18830. 0.233 $\begin{array}{llll}149.0 & 18830 & 0.211\end{array}$ 244.7 16170. 0.796 229.3 16180. 0.612 220.7 16180. 0.535 $\begin{array}{llll}211.6 & 16180 & 0.469\end{array}$ $\begin{array}{llll}191.4 & 16190 . & 0.359\end{array}$ $\begin{array}{lll}169.3 & 16190 . & 0.279\end{array}$ $\begin{array}{lll}155.3 & 16180 . & 0.238\end{array}$ $\begin{array}{lll}133.6 & 16180 . & 0.194\end{array}$ 112.4 16190. 0.158 104.2 16190. 0.143 99.0 16190. 0.131 99.4 16190. 0.108 300.9 19930. 0.831 284.7 19930. 0.703 276.019920 .0 .650 266.6 19920. 0.603 246.4 19910. 0.528 225.019900 .0 .473 $226.6 \quad 19850.0 .446$ 207.9 19850. 0.416 193.2 19840. 0.392 191.2 19840. 0.383
$\begin{array}{llllllll}\mathrm{Ja} & \mathrm{P} / \mathrm{P}_{\mathrm{c}} & \mathrm{T} / \mathrm{T}_{\mathrm{c}} & \mathrm{M} & \mathrm{S} & \mathrm{Pr} \text { flow } & \mathrm{E}_{\mathrm{q}} & \mathrm{u}_{\mathrm{Na}}\end{array}$

$\begin{array}{llllllllll}16.07 & 0.302 & 0.844 & 72.59 & 1.18 & 2.20 & \mathrm{P} & 2.751 & 12.10 & \mathrm{I}\end{array}$ $\begin{array}{llllllllll}18.85 & 0.302 & 0.843 & 72.59 & \text { I.51 } & 2.20 & \mathrm{P} & 2.553 & 9.90 & \mathrm{I}\end{array}$ $\begin{array}{lllllllllll}20.45 & 0.302 & 0.843 & 72.59 & 1.71 & 2.19 & \mathrm{P} & 2.442 & 9.40 & 1\end{array}$ $\begin{array}{llllllllll}22.19 & 0.302 & 0.843 & 72.59 & 1.93 & 2.19 & \mathrm{P} & 2.326 & 9.80 & \mathrm{I}\end{array}$ $\begin{array}{lllllllllll}26.17 & 0.302 & 0.843 & 72.59 & 2.45 & 2.19 & \mathrm{P} & 2.071 & 12.10 & \mathrm{I}\end{array}$

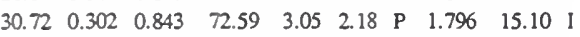
$\begin{array}{llllllllll}33.77 & 0.302 & 0.843 & 72.59 & 3.48 & 2.18 & \mathrm{P} & 1.604 & 16.70 \mathrm{I}\end{array}$ $\begin{array}{llllllllll}38.61 & 0.302 & 0.843 & 72.59 & 4.11 & 2.18 & \mathrm{P} & 1.342 & 18.40 & \mathrm{I}\end{array}$ $\begin{array}{llllllllll}43.94 & 0.302 & 0.843 & 72.59 & 4.81 & 2.18 & \mathrm{P} & 1.085 & 20.30 & \mathrm{I}\end{array}$ $\begin{array}{llllllllll}46.37 & 0.302 & 0.843 & 72.59 & 5.15 & 2.18 & P & 0.985 & 25.30 & 1\end{array}$ $\begin{array}{llllllllll}48.49 & 0.302 & 0.843 & 72.59 & 5.49 & 2.18 & P & 0.917 & 37.50 & \text { I }\end{array}$ $\begin{array}{llllllllll}51.38 & 0.302 & 0.843 & 72.59 & 6.22 & 2.18 & \mathrm{P} & 0.894 & 54.00 & \mathrm{I}\end{array}$ $\begin{array}{llllllllll}14.95 & 0.322 & 0.851 & 72.59 & 1.14 & 2.22 & \mathrm{P} & 3.260 & 13.10 & \mathrm{I}\end{array}$ $\begin{array}{llllllllll}17.66 & 0.322 & 0.850 & 72.59 & 1.39 & 2.22 & \mathrm{P} & 3.053 & 10.60 & \mathrm{I}\end{array}$ $\begin{array}{llllllllll}19.26 & 0.322 & 0.850 & 72.59 & 1.52 & 2.21 & \mathrm{P} & 2.940 & 10.20 \mathrm{I}\end{array}$ $\begin{array}{llllllllll}21.03 & 0.322 & 0.850 & 72.59 & 1.66 & 2.21 & \mathrm{P} & 2.822 & 10.60 & \mathrm{I}\end{array}$ $\begin{array}{llllllllll}25.25 & 0.322 & 0.850 & 72.59 & 1.96 & 2.21 & \mathrm{P} & 2.566 & 13.40 & 1\end{array}$ $\begin{array}{lllllllllll}30.38 & 0.322 & 0.850 & 72.59 & 2.26 & 2.20 & \mathrm{P} & 2.295 & 17.10 & \mathrm{I}\end{array}$ $\begin{array}{llllllllll}34.09 & 0.322 & 0.850 & 72.59 & 2.44 & 2.20 & \mathrm{P} & 2.107 & 19.10 & \mathrm{I}\end{array}$ $\begin{array}{llllllllll}40.47 & 0.322 & 0.850 & 72.59 & 2.69 & 2.20 & \mathrm{P} & 1.855 & 21.70 & \mathrm{I}\end{array}$ $\begin{array}{llllllllll}8.62 & 0.322 & 0.850 & 72.59 & 2.93 & 2.20 & \mathrm{P} & 1.624 & 24.80 & \mathrm{I}\end{array}$ $\begin{array}{llllllllll}53.02 & 0.322 & 0.850 & 72.59 & 3.04 & 2.20 & \mathrm{P} & 1.549 & 29.70 & \mathrm{CU}\end{array}$ $\begin{array}{llllllllll}57.38 & 0.322 & 0.850 & 72.59 & 3.14 & 2.20 & \mathrm{P} & 1.519 & 39.70 & \mathrm{CU}\end{array}$ $\begin{array}{lllllllllll}65.27 & 0.322 & 0.850 & 72.59 & 3.35 & 2.20 & \mathrm{P} & 1.638 & 62.50 & \mathrm{I}\end{array}$ $\begin{array}{llllllllll}17.28 & 0.359 & 0.863 & 72.59 & 1.08 & 2.27 & \mathrm{P} & 3.489 & 13.10 & \mathrm{I}\end{array}$ $\begin{array}{llllllllll}20.72 & 0.359 & 0.862 & 72.59 & 1.26 & 2.26 & \mathrm{P} & 3.302 & 11.20 & \mathrm{I}\end{array}$ $\begin{array}{llllllllll}22.79 & 0.359 & 0.862 & 72.59 & 1.35 & 2.26 & \mathrm{P} & 3.197 & 11.00 & 1\end{array}$ $\begin{array}{llllllllll}25.11 & 0.359 & 0.862 & 72.59 & 1.45 & 2.26 & \mathrm{P} & 3.086 & 11.70 & \mathrm{I}\end{array}$ $\begin{array}{lllllllllll}30.75 & 0.359 & 0.862 & 72.59 & 1.63 & 2.25 & \mathrm{P} & 2.839 & 14.90 & \mathrm{I}\end{array}$ $\begin{array}{llllllllll}37.83 & 0.359 & 0.862 & 72.59 & 1.80 & 2.25 & \mathrm{P} & 2.567 & 19.20 & \mathrm{I}\end{array}$ $\begin{array}{llllllllll}43.03 & 0.359 & 0.862 & 72.59 & 1.89 & 2.25 & \mathrm{P} & 2.367 & 21.80 & \mathrm{I}\end{array}$ $\begin{array}{lllllllllll}52.16 & 0.359 & 0.862 & 72.59 & 2.01 & 2.25 & \mathrm{P} & 2.097 & 25.40 & \mathrm{CU}\end{array}$ $\begin{array}{lllllllllll}63.96 & 0.359 & 0.862 & 72.59 & 2.11 & 2.25 & \mathrm{P} & 1.844 & 30.10 & \mathrm{O} 2\end{array}$ $\begin{array}{llllllllll}70.25 & 0.359 & 0.862 & 72.59 & 2.16 & 2.25 & \mathrm{P} & 1.763 & 35.50 & \mathrm{O} 2\end{array}$ $\begin{array}{lllllllllll}76.28 & 0.359 & 0.862 & 72.59 & 2.20 & 2.25 & \mathrm{P} & 1.735 & 46.00 & \mathrm{O} 2\end{array}$ $\begin{array}{lllllllllll}86.09 & 0.359 & 0.862 & 72.59 & 2.28 & 2.25 & \mathrm{P} & 1.895 & 69.60 & \mathrm{CU}\end{array}$ $\begin{array}{llllllllll}15.35 & 0.366 & 0.865 & 72.59 & 1.09 & 2.28 & \mathrm{P} & 3.125 & 12.00 & \mathrm{I}\end{array}$ $\begin{array}{llllllllll}18.19 & 0.366 & 0.864 & 72.59 & 1.32 & 2.27 & \mathrm{P} & 2.930 & 10.00 & \mathrm{I}\end{array}$ $\begin{array}{llllllllll}19.88 & 0.366 & 0.864 & 72.59 & 1.45 & 2.27 & \mathrm{P} & 2.823 & 9.70 & \mathrm{I}\end{array}$ $\begin{array}{llllllllll}21.75 & 0.366 & 0.864 & 72.59 & 1.59 & 2.26 & P & 2.713 & 10.20 & \text { I }\end{array}$ $\begin{array}{llllllllll}26.22 & 0.365 & 0.864 & 72.59 & 1.88 & 2.26 & P & 2.474 & 12.90 & \text { I }\end{array}$ $\begin{array}{llllllllll}31.71 & 0.365 & 0.864 & 72.59 & 2.17 & 2.26 & P & 2.222 & 16.40 & \text { I }\end{array}$ $\begin{array}{llllllllll}35.70 & 0.366 & 0.864 & 72.59 & 2.35 & 2.25 & \mathrm{P} & 2.048 & 18.50 & \text { I }\end{array}$ $\begin{array}{llllllllll}42.62 & 0.366 & 0.864 & 72.59 & 2.59 & 2.25 & \mathrm{P} & 1.822 & 21.30 & \mathrm{I}\end{array}$ $\begin{array}{llllllllll}51.57 & 0.366 & 0.864 & 72.59 & 2.83 & 2.25 & \mathrm{P} & 1.627 & 24.70 & \mathrm{I}\end{array}$ $\begin{array}{llllllllll}56.45 & 0.366 & 0.864 & 72.59 & 2.94 & 2.25 & \mathrm{P} & 1.574 & 29.70 & 1\end{array}$ $\begin{array}{llllllllll}61.26 & 0.366 & 0.864 & 72.59 & 3.04 & 2.25 & \mathrm{P} & 1.569 & 40.00 & 1\end{array}$ $\begin{array}{llllllllll}70.07 & 0.366 & 0.864 & 72.59 & 3.26 & 2.25 & \mathrm{P} & 1.750 & 60.40 & 1\end{array}$ $\begin{array}{llllllllll}14.62 & 0.343 & 0.857 & 72.59 & 1.13 & 2.24 & \mathrm{P} & 2.874 & 11.70 & \mathrm{I}\end{array}$ $\begin{array}{lllllllllll}7.30 & 0.343 & 0.857 & 72.59 & 1.43 & 2.24 & \mathrm{P} & 2.693 & 9.60 \mathrm{I}\end{array}$ $\begin{array}{llllllllll}18.88 & 0.343 & 0.857 & 72.59 & 1.61 & 2.23 & \mathrm{P} & 2.592 & 9.20 \mathrm{I}\end{array}$ $\begin{array}{llllllllll}20.60 & 0.343 & 0.857 & 72.59 & 1.80 & 2.23 & \mathrm{P} & 2.485 & 9.60 & 1\end{array}$ $\begin{array}{llllllllllll}24.61 & 0.342 & 0.857 & 72.59 & 2.24 & 2.23 & \mathrm{P} & 2.247 & 12.10 & \mathrm{I}\end{array}$ $\begin{array}{llllllllll}29.35 & 0.342 & 0.857 & 72.59 & 2.74 & 2.22 & \mathrm{P} & 1.989 & 15.20 & \mathrm{I}\end{array}$ $\begin{array}{llllllllll}32.98 & 0.342 & 0.857 & 72.59 & 3.08 & 2.22 & \mathrm{P} & 1.824 & 17.20 & \text { I }\end{array}$ $\begin{array}{lllllllllll}38.39 & 0.342 & 0.857 & 72.59 & 3.57 & 2.22 & \mathrm{P} & 1.569 & 19.30 & \mathrm{I}\end{array}$ $\begin{array}{llllllllll}44.67 & 0.342 & 0.857 & 72.59 & 4.10 & 2.22 & \mathrm{P} & 1.319 & 21.60 & \text { I }\end{array}$ $\begin{array}{llllllllll}47.71 & 0.342 & 0.857 & 72.59 & 4.36 & 2.22 & \mathrm{P} & 1.224 & 26.60 & \mathrm{I}\end{array}$ $\begin{array}{llllllllll}50.42 & 0.342 & 0.857 & 72.59 & 4.61 & 2.22 & \mathrm{P} & 1.162 & 37.90 & 1\end{array}$ $\begin{array}{lllllllllll}54.21 & 0.342 & 0.857 & 72.59 & 5.15 & 2.22 & \mathrm{P} & 1.167 & 55.20 & 1\end{array}$ $\begin{array}{llllllllllll}21.47 & 0.283 & 0.837 & 72.59 & 1.11 & 2.19 & \mathrm{P} & 3.534 & 13.90 & \mathrm{I}\end{array}$ $\begin{array}{llllllllllll}25.71 & 0.283 & 0.836 & 72.59 & 1.30 & 2.19 & \mathrm{P} & 3.344 & 11.80 & \mathrm{I}\end{array}$ $\begin{array}{lllllllllll}28.30 & 0.282 & 0.836 & 72.59 & 1.40 & 2.18 & \mathrm{P} & 3.241 & 11.70 & \mathrm{I}\end{array}$ $\begin{array}{llllllllllll}31.18 & 0.282 & 0.836 & 72.59 & 1.49 & 2.18 & \mathrm{P} & 3.131 & 12.40 & 1\end{array}$ $\begin{array}{llllllllllll}38.31 & 0.282 & 0.836 & 72.59 & 1.69 & 2.18 & P & 2.893 & 15.80 & \text { I }\end{array}$ $\begin{array}{llllllllllll}47.55 & 0.281 & 0.836 & 72.59 & 1.86 & 2.18 & \mathrm{P} & 2.642 & 20.60 & \mathrm{I}\end{array}$ $\begin{array}{lllllllllll}59.28 & 0.280 & 0.835 & 72.59 & 1.96 & 2.17 & \mathrm{P} & 2.660 & 25.40 & \mathrm{I}\end{array}$ $\begin{array}{llllllllllll}74.59 & 0.280 & 0.835 & 72.59 & 2.08 & 2.17 & \mathrm{P} & 2.442 & 30.80 & \mathrm{I}\end{array}$ $\begin{array}{llllllllllll}97.51 & 0.280 & 0.835 & 72.59 & 2.19 & 2.17 & \mathrm{P} & 2.269 & 38.50 & \mathrm{CU}\end{array}$ $\begin{array}{lllllllllll}111.90 & 0.279 & 0.835 & 72.59 & 2.23 & 2.17 & \mathrm{P} & 2.246 & 46.80 & \mathrm{HC}\end{array}$

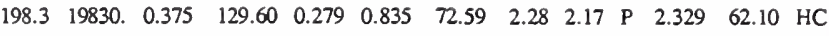
$\begin{array}{lllllllllllll}248.8 & 19810 & 0.360 & 173.00 & 0.279 & 0.835 & 72.59 & 2.35 & 2.17 & \mathrm{P} & 2.921 & 111.60 & \mathrm{O} 2\end{array}$ $\begin{array}{lllllllllllll}305.3 & 21280 & 0.854 & 18.02 & 0.305 & 0.844 & 72.59 & 1.08 & 2.21 & \mathrm{P} & 3.585 & 13.20 & 1\end{array}$ $\begin{array}{llllllllllll}288.3 & 21280 & 0.711 & 21.57 & 0.304 & 0.844 & 72.59 & 1.28 & 2.20 & \mathrm{P} & 3.386 & 11.10\end{array}$ $\begin{array}{llllllllllllll}279.1 & 21270 & 0.651 & 23.74 & 0.304 & 0.844 & 72.59 & 1.38 & 2.20 & \mathrm{P} & 3.278 & 10.80 & \mathrm{I}\end{array}$ $\begin{array}{lllllllllllll}269.4 & 21270 & 0.599 & 26.15 & 0.304 & 0.844 & 72.59 & 1.49 & 2.20 & \mathrm{P} & 3.164 & 11.50 \mathrm{I}\end{array}$ $\begin{array}{llllllllllll}248.5 & 21260 & 0.514 & 32.11 & 0.303 & 0.844 & 72.59 & 1.70 & 2.19 & \mathrm{P} & 2.919 & 14.70\end{array}$ $\begin{array}{lllllllllllll}226.7 & 21260 & 0.452 & 39.83 & 0.303 & 0.844 & 72.59 & 1.90 & 2.19 & \mathrm{P} & 2.662 & 19.10 & \text { I }\end{array}$ $\begin{array}{lllllllllllllll}224.0 & 21210 & 0.422 & 48.71 & 0.302 & 0.843 & 72.59 & 2.02 & 2.19 & \mathrm{P} & 2.630 & 23.30 & \mathrm{I}\end{array}$ $\begin{array}{lllllllllllll}205.5 & 21210 & 0.389 & 61.18 & 0.302 & 0.843 & 72.59 & 2.17 & 2.19 & \mathrm{P} & 2.413 & 27.90 & 1\end{array}$ $\begin{array}{llllllllllll}191.6 & 21200 & 0.362 & 79.89 & 0.301 & 0.843 & 72.59 & 2.30 & 2.19 & \mathrm{P} & 2.250 & 34.80\end{array}$ $\begin{array}{lllllllllllll}190.6 & 21200 & 0.351 & 91.73 & 0.301 & 0.843 & 72.59 & 2.36 & 2.19 & \mathrm{P} & 2.238 & 42.60 & \mathrm{I}\end{array}$ $\begin{array}{lllllllllllll}196.3 & 21190 & 0.342 & 104.90 & 0.301 & 0.843 & 72.59 & 2.41 & 2.18 & \mathrm{P} & 2.305 & 56.60 & 1\end{array}$ $\begin{array}{lllllllllllll}240.2 & 21180 & 0.325 & 134.70 & 0.301 & 0.843 & 72.59 & 2.52 & 2.18 & \mathrm{P} & 2.821 & 99.00\end{array}$ $\begin{array}{lllllllllllll}280.4 & 16710 & 0.830 & 20.44 & 0.285 & 0.837 & 72.59 & 1.12 & 2.19 & \mathrm{P} & 1.693 & 14.00 \mathrm{I}\end{array}$ $\begin{array}{lllllllllllll}262.8 & 16710 & 0.680 & 24.20 & 0.284 & 0.837 & 72.59 & 1.34 & 2.19 & \mathrm{P} & 1.586 & 11.70 & 1\end{array}$ $\begin{array}{llllllllllllll}253.4 & 16710 & 0.618 & 26.46 & 0.284 & 0.837 & 72.59 & 1.46 & 2.18 & \mathrm{P} & 1.529 & 11.40 & \mathrm{I}\end{array}$ $\begin{array}{llllllllllllll}243.6 & 16710 & 0.564 & 28.96 & 0.284 & 0.837 & 72.59 & 1.59 & 2.18 & \mathrm{P} & 1.471 & 12.00 & \mathrm{I}\end{array}$ $\begin{array}{llllllllllllll}223.1 & 16710 & 0.474 & 35.07 & 0.284 & 0.837 & 72.59 & 1.85 & 2.18 & \mathrm{P} & 1.347 & 15.10 & \mathrm{I}\end{array}$ $\begin{array}{llllllllllllll}202.4 & 16710 & 0.409 & 42.85 & 0.283 & 0.837 & 72.59 & 2.10 & 2.18 & \mathrm{P} & 1.222 & 19.40 & \mathrm{I}\end{array}$ $\begin{array}{lllllllllllll}195.8 & 16690 & 0.377 & 50.83 & 0.283 & 0.836 & 72.59 & 2.26 & 2.17 & \mathrm{P} & 1.182 & 22.80 & \mathrm{I}\end{array}$ $\begin{array}{llllllllllllll}178.8 & 16690 & 0.341 & 62.52 & 0.283 & 0.836 & 72.59 & 2.46 & 2.17 & \mathrm{P} & 1.079 & 27.00 & \mathrm{I}\end{array}$ $\begin{array}{lllllllllllll}166.7 & 16680 & 0.311 & 79.72 & 0.282 & 0.836 & 72.59 & 2.65 & 2.17 & \mathrm{P} & 1.006 & 33.00 & 1\end{array}$

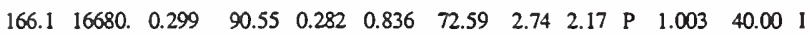
$\begin{array}{lllllllllllll}171.3 & 16680 & 0.289 & 102.60 & 0.282 & 0.836 & 72.59 & 2.82 & 2.17 & \mathrm{P} & 1.034 & 53.10 & \mathrm{I}\end{array}$ $\begin{array}{llllllllllll}210.0 & 16680 & 0.270 & 131.30 & 0.282 & 0.836 & 72.59 & 2.98 & 2.17 & \mathrm{P} & 1.267 & 91.70\end{array}$ $\begin{array}{llllllllllllll}213.2 & 6149 . & 0.776 & 33.46 & 0.279 & 0.835 & 72.59 & 1.19 & 2.19 & \mathrm{P} & 2.394 & 15.50 & \mathrm{I}\end{array}$ $\begin{array}{llllllllllllll}197.4 & 6153 & 0.590 & 39.94 & 0.279 & 0.835 & 72.59 & 1.53 & 2.18 & \mathrm{P} & 2.589 & 14.30 & \text { I }\end{array}$ $\begin{array}{llllllllllllll}188.8 & 6155 . & 0.513 & 43.84 & 0.279 & 0.835 & 72.59 & 1.73 & 2.18 & \mathrm{P} & 2.680 & 14.50 & \mathrm{I}\end{array}$ $\begin{array}{lllllllllllllll}179.9 & 6156 & 0.447 & 48.22 & 0.279 & 0.835 & 72.59 & 1.95 & 2.17 & \mathrm{P} & 2.554 & 15.60 & \mathrm{I}\end{array}$ $\begin{array}{lllllllllllllll}160.7 & 6158 & 0.341 & 58.90 & 0.279 & 0.835 & 72.59 & 2.46 & 2.17 & \mathrm{P} & 2.281 & 19.50 & \mathrm{I}\end{array}$ $\begin{array}{llllllllllllll}140.6 & 6159 & 0.267 & 72.50 & 0.279 & 0.835 & 72.59 & 3.02 & 2.17 & \mathrm{P} & 1.995 & 25.00 & \mathrm{I}\end{array}$ $\begin{array}{llllllllllllll}126.8 & 6161 & 0.230 & 82.81 & 0.279 & 0.835 & 72.59 & 3.39 & 2.17 & \mathrm{P} & 1.799 & 28.50 & \mathrm{I}\end{array}$ $\begin{array}{llllllllllllll}108.8 & 6162 & 0.192 & 101.40 & 0.279 & 0.835 & 72.59 & 3.89 & 2.16 & \mathrm{P} & 1.544 & 34.10 & \mathrm{I}\end{array}$ $\begin{array}{llllllllllllll}94.3 & 6162 & 0.163 & 127.50 & 0.279 & 0.835 & 72.59 & 4.39 & 2.16 & \mathrm{P} & 1.338 & 41.90 & 1\end{array}$ $\begin{array}{lllllllllllll}91.6 & 6162 & 0.152 & 143.00 & 0.279 & 0.835 & 72.59 & 4.62 & 2.16 & \mathrm{P} & 1.301 & 50.80 & \mathrm{I}\end{array}$ $\begin{array}{lllllllllllll}94.0 & 6162 & 0.142 & 159.40 & 0.279 & 0.835 & 72.59 & 4.84 & 2.16 & \mathrm{P} & 1.334 & 65.20 & \mathrm{I}\end{array}$ $\begin{array}{lllllllllllll}121.2 & 6163 & 0.123 & 194.10 & 0.279 & 0.835 & 72.59 & 5.35 & 2.16 & P & 1.720 & 94.80 & 02\end{array}$ $\begin{array}{llllllllllllll}222.0 & 6434 & 0.808 & 32.42 & 0.304 & 0.844 & 72.59 & 1.13 & 2.21 & \mathrm{P} & 2.451 & 15.70 & \mathrm{I}\end{array}$ $\begin{array}{lllllllllllll}205.2 & 6439 & 0.618 & 38.76 & 0.304 & 0.844 & 72.59 & 1.45 & 2.20 & \mathrm{P} & 2.625 & 14.50 & \mathrm{I}\end{array}$ $\begin{array}{llllllllllllll}196.1 & 6440 & 0.540 & 42.58 & 0.304 & 0.844 & 72.59 & 1.63 & 2.20 & \mathrm{P} & 2.709 & 14.80 & \mathrm{I}\end{array}$ $\begin{array}{llllllllllllll}186.7 & 6442 & 0.473 & 46.89 & 0.304 & 0.844 & 72.59 & 1.83 & 2.19 & \mathrm{P} & 2.579 & 15.80 & \mathrm{I}\end{array}$ $\begin{array}{llllllllllllll}166.5 & 6444 & 0.366 & 57.46 & 0.304 & 0.844 & 72.59 & 2.28 & 2.19 & \mathrm{P} & 2.300 & 19.90 & \mathrm{I}\end{array}$ $\begin{array}{llllllllllllll}145.5 & 6445 & 0.292 & 71.07 & 0.304 & 0.844 & 72.59 & 2.74 & 2.19 & \mathrm{P} & 2.011 & 25.50 & \mathrm{I}\end{array}$ $\begin{array}{lllllllllllll}131.3 & 6446 & 0.255 & 81.52 & 0.304 & 0.844 & 72.59 & 3.05 & 2.18 & \mathrm{P} & 1.814 & 29.50 & \mathrm{I}\end{array}$ $\begin{array}{llllllllllllll}113.2 & 6447 & 0.218 & 100.80 & 0.304 & 0.844 & 72.59 & 3.44 & 2.18 & \mathrm{P} & 1.564 & 35.60 & \mathrm{I}\end{array}$ $\begin{array}{lllllllllllll}99.9 & 6448 & 0.189 & 128.80 & 0.304 & 0.844 & 72.59 & 3.82 & 2.18 & \mathrm{P} & 1.380 & 44.20 & 1\end{array}$ $\begin{array}{lllllllllllll}98.8 & 6448 & 0.177 & \mathrm{I} 45.90 & 0.304 & 0.844 & 72.59 & 4.00 & 2.18 & \mathrm{P} & 1.366 & 53.70 & \mathrm{I}\end{array}$ $\begin{array}{lllllllllllllll}104.1 & 6448 & 0.167 & 165.00 & 0.304 & 0.844 & 72.59 & 4.17 & 2.18 & \mathrm{P} & 1.438 & 69.20 & \mathrm{I}\end{array}$ $\begin{array}{llllllllllllll}143.5 & 6448 . & 0.147 & 208.10 & 0.304 & 0.844 & 72.59 & 4.54 & 2.18 & \mathrm{P} & 1.982 & 104.10 & 02\end{array}$ $\begin{array}{lllllllllllll}240.0 & 7088 & 0.819 & 36.65 & 0.362 & 0.863 & 72.59 & 1.09 & 2.27 & \mathrm{P} & 2.584 & 17.70 & 1\end{array}$ $\begin{array}{lllllllllllll}224.3 & 7091 . & 0.659 & 44.30 & 0.362 & 0.863 & 72.59 & 1.33 & 2.26 & \mathrm{P} & 2.708 & 17.30 & \mathrm{I}\end{array}$ $\begin{array}{lllllllllllll}215.7 & 7092 & 0.593 & 49.01 & 0.362 & 0.863 & 72.59 & 1.45 & 2.26 & \mathrm{P} & 2.745 & 18.00 & \mathrm{I}\end{array}$ $\begin{array}{lllllllllllll}206.8 & 7093 . & 0.536 & 54.37 & 0.362 & 0.863 & 72.59 & 1.58 & 2.26 & \mathrm{P} & 2.632 & 19.70 & \mathrm{I}\end{array}$ $\begin{array}{lllllllllllll}187.7 & 7095 & 0.446 & 67.89 & 0.362 & 0.863 & 72.59 & 1.85 & 2.25 & \mathrm{P} & 2.388 & 24.90\end{array}$ $\begin{array}{lllllllllllll}167.6 & 7096 & 0.383 & 86.05 & 0.362 & 0.863 & 72.59 & 2.09 & 2.25 & \mathrm{P} & 2.133 & 32.40 \mathrm{I}\end{array}$ $\begin{array}{llllllllllllll}153.8 & 7097 & 0.353 & 100.60 & 0.362 & 0.863 & 72.59 & 2.24 & 2.25 & \mathrm{P} & 1.958 & 37.80 & \mathrm{I}\end{array}$ $\begin{array}{lllllllllllll}137.0 & 7098 & 0.321 & 128.70 & 0.362 & 0.863 & 72.59 & 2.41 & 2.25 & \mathrm{P} & 1.743 & 47.60 & \mathrm{I}\end{array}$ $\begin{array}{llllllllllllll}127.1 & 7098 & 0.297 & 173.20 & 0.362 & 0.863 & 72.59 & 2.56 & 2.25 & \mathrm{P} & 1.618 & 62.50 & \mathrm{I}\end{array}$ $\begin{array}{lllllllllllllll}130.1 & 7098 & 0.287 & 202.80 & 0.362 & 0.863 & 72.59 & 2.62 & 2.25 & \mathrm{P} & 1.656 & 75.70 & \mathrm{I}\end{array}$ $\begin{array}{llllllllllllll}142.8 & 7098 . & 0.279 & 238.00 & 0.362 & 0.863 & 72.59 & 2.68 & 2.25 & \mathrm{P} & 1.817 & 95.60 & 1\end{array}$ $\begin{array}{lllllllllllll}217.1 & 7098 & 0.262 & 327.30 & 0.362 & 0.863 & 72.59 & 2.81 & 2.25 & \mathrm{P} & 2.763 & 158.40 & \mathrm{O} 2\end{array}$ $\begin{array}{lllllllllllll}229.4 & 8491 . & 0.814 & 26.53 & 0.332 & 0.854 & 72.59 & 1.11 & 2.23 & \mathrm{P} & 2.245 & 14.10 & \mathrm{I}\end{array}$ $\begin{array}{lllllllllllll}214.5 & 8496 & 0.635 & 32.23 & 0.332 & 0.854 & 72.59 & 1.39 & 2.23 & \mathrm{P} & 2.099 & 13.10 \mathrm{I}\end{array}$ $\begin{array}{lllllllllllll}206.2 & 8497 & 0.561 & 35.73 & 0.332 & 0.854 & 72.59 & 1.55 & 2.22 & \mathrm{P} & 2.018 & 13.40 & \mathrm{I}\end{array}$ $\begin{array}{llllllllllllll}197.6 & 8499 & 0.499 & 39.71 & 0.332 & 0.854 & 72.59 & 1.72 & 2.22 & \mathrm{P} & 1.934 & 14.50 \mathrm{I}\end{array}$ $\begin{array}{llllllllllllll}178.5 & 8501 & 0.399 & 49.68 & 0.332 & 0.854 & 72.59 & 2.07 & 2.22 & \mathrm{P} & 1.747 & 18.60 & \mathrm{I}\end{array}$ $\begin{array}{llllllllllllll}158.0 & 8502 . & 0.330 & 62.84 & 0.332 & 0.854 & 72.59 & 2.42 & 2.21 & \mathrm{P} & 1.546 & 24.50 & \mathrm{I}\end{array}$ $\begin{array}{llllllllllllll}144.1 & 8503 & 0.297 & 73.46 & 0.332 & 0.854 & 72.59 & 2.63 & 2.21 & \mathrm{P} & 1.410 & 28.60 & \mathrm{I}\end{array}$ $\begin{array}{llllllllllllll}125.3 & 8504 & 0.262 & 92.74 & 0.332 & 0.854 & 72.59 & 2.90 & 2.21 & \mathrm{P} & 1.227 & 35.30 & \mathrm{I}\end{array}$ $\begin{array}{lllllllllllll}111.6 & 8504 & 0.237 & 120.50 & 0.332 & 0.854 & 72.59 & 3.13 & 2.21 & \mathrm{P} & 1.092 & 44.90 & \mathrm{I}\end{array}$ $\begin{array}{llllllllllllll}111.1 & 8504 & 0.226 & 137.10 & 0.332 & 0.854 & 72.59 & 3.23 & 2.21 & \mathrm{P} & 1.088 & 54.50 & \text { I }\end{array}$ 
$\begin{array}{lllllllllllll}117.9 & 8504 & 0.217 & 154.40 & 0.332 & 0.854 & 72.59 & 3.33 & 2.21 & \mathrm{P} & 1.154 & 68.90 & \mathrm{I}\end{array}$ $\begin{array}{lllllllllllll}162.6 & 8505 . & 0.199 & 187.50 & 0.332 & 0.854 & 72.59 & 3.55 & 2.21 & \mathrm{P} & 1.591 & 101.00 & 02\end{array}$ $\begin{array}{lllllllllllll}16.3 & 13580 & 1.064 & 11.94 & 0.275 & 0.860 & 72.59 & 0.89 & 2.30 & \mathrm{C} & 0.159 & 11.70 & \mathrm{O} 2\end{array}$ $\begin{array}{lllllllllllll}53.5 & 12580 . & 1.032 & 23.06 & 0.275 & 0.847 & 72.59 & 0.92 & 2.23 & \mathrm{C} & 0.316 & 13.50 & \mathrm{O} 2\end{array}$ $\begin{array}{lllllllllllll}168.3 & 11940 . & 1.009 & 61.80 & 0.275 & 0.837 & 72.59 & 0.93 & 2.20 & \mathrm{C} & 0.897 & 27.30 & \mathrm{O} 2\end{array}$ $\begin{array}{lllllllllllll}473.7 & 11700 & 0.983 & 144.10 & 0.275 & 0.833 & 72.59 & 0.96 & 2.19 & \mathrm{C} & 2.305 & 61.70 & 02\end{array}$ $\begin{array}{llllllllllll}346.7 & 11700 & 0.917 & 81.55 & 0.275 & 0.833 & 72.59 & 1.02 & 2.19 & \mathrm{C} & 1.943 & 32.90\end{array}$ $\begin{array}{lllllllllllll}272.5 & 11700 & 0.837 & 52.89 & 0.274 & 0.833 & 72.59 & 1.11 & 2.19 & \mathrm{C} & 1.743 & 20.00\end{array}$ $\begin{array}{llllllllllll}247.0 & 11680 & 0.777 & 43.34 & 0.274 & 0.833 & 72.59 & 1.19 & 2.19 & \mathrm{C} & 1.715 & 15.90\end{array}$ $\begin{array}{llllllllllll}206.8 & 11690 & 0.682 & 31.98 & 0.273 & 0.833 & 72.59 & 1.34 & 2.18 & \mathrm{C} & 1.436 & 11.90\end{array}$ $\begin{array}{llllllllllll}174.4 & 11690 & 0.563 & 23.94 & 0.273 & 0.833 & 72.59 & 1.60 & 2.18 & \mathrm{C} & 1.21 \mathrm{I} & 10.00\end{array}$ $\begin{array}{llllllllllll}161.2 & 11690 & 0.497 & 20.99 & 0.273 & 0.833 & 72.59 & 1.79 & 2.17 & \mathrm{C} & 1.120 & 9.90\end{array}$ $\begin{array}{llllllllllll}149.7 & 11690 & 0.429 & 18.56 & 0.273 & 0.833 & 72.59 & 2.03 & 2.17 & \mathrm{C} & 1.039 & 10.30\end{array}$ $\begin{array}{llllllllllllll}130.4 & 11700 & 0.283 & 14.81 & 0.273 & 0.833 & 72.59 & 2.90 & 2.16 & \mathrm{C} & 0.906 & 11.20 & 1\end{array}$ $\begin{array}{lllllllllllll}220.4 & 13580 & 1.003 & 199.50 & 0.342 & 0.858 & 72.59 & 0.92 & 2.26 & \mathrm{C} & 0.977 & 88.10 & \mathrm{O} 2\end{array}$ $\begin{array}{lllllllllllll}535.0 & 13500 & 0.978 & 249.40 & 0.342 & 0.857 & 72.59 & 0.94 & 2.25 & \mathrm{C} & 2.363 & 110.60 & 02\end{array}$ $\begin{array}{lllllllllllll}467.1 & 13500 & 0.961 & 176.40 & 0.342 & 0.857 & 72.59 & 0.95 & 2.25 & \mathrm{C} & 2.214 & 77.40 & \mathrm{O} 2\end{array}$ $\begin{array}{lllllllllllll}412.1 & 13500 & 0.939 & 131.10 & 0.342 & 0.857 & 72.59 & 0.97 & 2.25 & \mathrm{C} & 2.083 & 55.70 & \mathrm{HC}\end{array}$ $\begin{array}{lllllllllllll}328 . \mathrm{I} & 13500 & 0.886 & 79.35 & 0.342 & 0.857 & 72.59 & 1.03 & 2.25 & \mathrm{C} & 1.875 & 32.20\end{array}$ $\begin{array}{lllllllllllll}271.1 & 13500 & 0.819 & 53.17 & 0.342 & 0.857 & 72.59 & 1.10 & 2.24 & \mathrm{C} & 1.746 & 20.50\end{array}$ $\begin{array}{lllllllllllll}249.6 & 13490 & 0.769 & 43.75 & 0.341 & 0.857 & 72.59 & 1.17 & 2.24 & C & 1.608 & 16.50\end{array}$ $\begin{array}{llllllllllll}215.6 & 13490 & 0.688 & 32.77 & 0.341 & 0.857 & 72.59 & 1.29 & 2.24 & \mathrm{C} & 1.389 & 12.50\end{array}$ $\begin{array}{llllllllllll}187.1 & 13490 & 0.584 & 24.80 & 0.341 & 0.857 & 72.59 & 1.49 & 2.23 & \mathrm{C} & 1.205 & 10.30\end{array}$ $\begin{array}{llllllllllll}175.3 & 13490 & 0.527 & 21.83 & 0.341 & 0.857 & 72.59 & 1.63 & 2.23 & \mathrm{C} & \mathrm{I} .129 & 10.00\end{array}$ $\begin{array}{llllllllllll}164.9 & 13490 & 0.466 & 19.40 & 0.341 & 0.857 & 72.59 & 1.81 & 2.23 & \mathrm{C} & 1.063 & 10.30\end{array}$ $\begin{array}{llllllllllllll}147.4 & 13500 & 0.334 & 15.59 & 0.341 & 0.856 & 72.59 & 2.38 & 2.22 & \mathrm{C} & 0.949 & 10.80 & \mathrm{I}\end{array}$ $\begin{array}{lllllllllllll}129.8 & 20580 & 1.006 & 85.14 & 0.305 & 0.847 & 72.59 & 0.93 & 2.22 & \mathrm{C} & 0.836 & 43.80 & \mathrm{O} 2\end{array}$ $\begin{array}{lllllllllllll}434.8 & 20310 & 0.984 & 147.70 & 0.305 & 0.845 & 72.59 & 0.95 & 2.22 & \mathrm{C} & 2.801 & 75.30 & \text { I }\end{array}$ $\begin{array}{llllllllllll}423.5 & 20300 & 0.967 & 116.50 & 0.305 & 0.844 & 72.59 & 0.96 & 2.21 & \mathrm{C} & 2.728 & 57.30\end{array}$ $\begin{array}{llllllllllll}402.1 & 20290 & 0.947 & 93.13 & 0.304 & 0.844 & 72.59 & 0.98 & 2.21 & \mathrm{C} & 2.591 & 44.40\end{array}$ $\begin{array}{llllllllllll}349.2 & 20280 & 0.897 & 61.41 & 0.304 & 0.844 & 72.59 & 1.03 & 2.21 & C & 2.250 & 27.20\end{array}$ $\begin{array}{llllllllllll}301.6 & 20270 & 0.836 & 42.95 & 0.304 & 0.844 & 72.59 & 1.10 & 2.21 & \mathrm{C} & 1.943 & 17.80\end{array}$ $\begin{array}{llllllllllll}294.8 & 20210 & 0.789 & 37.61 & 0.302 & 0.843 & 72.59 & 1.16 & 2.20 & \mathrm{C} & 1.899 & 15.10\end{array}$ $\begin{array}{llllllllllll}256.2 & 20200 & 0.713 & 28.31 & 0.301 & 0.843 & 72.59 & 1.27 & 2.20 & \mathrm{C} & 1.651 & 11.40\end{array}$ $\begin{array}{llllllllllll}223.3 & 20190 & 0.617 & 21.49 & 0.301 & 0.843 & 72.59 & 1.45 & 2.20 & \mathrm{C} & 1.439 & 9.70\end{array}$ $\begin{array}{lllllllllllll}209.6 & 20190 & 0.563 & 18.94 & 0.301 & 0.843 & 72.59 & 1.58 & 2.19 & \mathrm{C} & 1.350 & 9.60 \mathrm{I}\end{array}$ $\begin{array}{lllllllllllll}197.3 & 20190 & 0.506 & 16.81 & 0.301 & 0.843 & 72.59 & 1.73 & 2.19 & \mathrm{C} & 1.271 & 9.90 & \text { I }\end{array}$ $\begin{array}{llllllllllll}176.5 & 20190 & 0.382 & 13.50 & 0.300 & 0.843 & 72.59 & 2.20 & 2.19 & \mathrm{C} & 1.137 & 10.40\end{array}$ $\begin{array}{lllllllllllll}17.1 & 19820 & 1.099 & 7.12 & 0.300 & 0.882 & 72.59 & 0.86 & 2.50 & \mathrm{C} & 0.110 & 11.90 & 02\end{array}$ $\begin{array}{lllllllllllll}44.0 & 18000 & 1.063 & 11.53 & 0.300 & 0.868 & 72.59 & 0.89 & 2.33 & C & 0.284 & 11.50 & 02\end{array}$ $\begin{array}{lllllllllllll}80.6 & 16940 & 1.037 & 18.56 & 0.300 & 0.857 & 72.59 & 0.91 & 2.27 & \mathrm{C} & 0.519 & 12.50 & 02\end{array}$ $\begin{array}{lllllllllllll}256.7 & 15870 & 1.007 & 53.64 & 0.300 & 0.845 & 72.59 & 0.93 & 2.22 & \mathrm{C} & 1.654 & 26.40 & \mathrm{O} 2\end{array}$ $\begin{array}{lllllllllllll}364.3 & 15630 & 0.934 & 58.05 & 0.299 & 0.843 & 72.59 & 0.99 & 2.21 & \mathrm{C} & 1.821 & 27.10 & 1\end{array}$ $\begin{array}{lllllllllllll}293.4 & 15630 & 0.847 & 39.20 & 0.299 & 0.842 & 72.59 & 1.09 & 2.20 & \mathrm{C} & 1.670 & 17.00 & 1\end{array}$ $\begin{array}{lllllllllllll}268.0 & 15600 & 0.783 & 32.77 & 0.298 & 0.842 & 72.59 & 1.17 & 2.20 & \mathrm{C} & 1.525 & 13.80\end{array}$

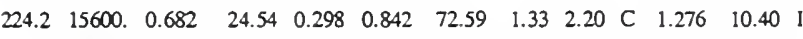
$\begin{array}{lllllllllllll}187.6 & 15600 & 0.558 & 18.57 & 0.298 & 0.842 & 72.59 & 1.59 & 2.19 & \mathrm{C} & 1.067 & 9.10 & \text { I }\end{array}$ $\begin{array}{lllllllllllll}172.4 & 15600 & 0.490 & 16.34 & 0.297 & 0.842 & 72.59 & 1.78 & 2.19 & \mathrm{C} & 0.981 & 9.40\end{array}$ $\begin{array}{llllllllllll}159.1 & 15600 & 0.421 & 14.51 & 0.297 & 0.842 & 72.59 & 2.03 & 2.19 & \mathrm{C} & 0.906 & 10.50 \text { I }\end{array}$ $\begin{array}{llllllllllll}136.7 & 15610 & 0.275 & 11.65 & 0.297 & 0.842 & 72.59 & 2.90 & 2.18 & \mathrm{C} & 0.778 & 12.10\end{array}$ $\begin{array}{lllllllllllll}37.6 & 24290 & 1.038 & 20.13 & 0.335 & 0.868 & 72.59 & 0.90 & 2.32 & \mathrm{C} & 0.214 & 16.40 & \mathrm{O} 2\end{array}$ $\begin{array}{lllllllllllll}144.6 & 23130 & 1.014 & 43.32 & 0.335 & 0.860 & 72.59 & 0.91 & 2.27 & \mathrm{C} & 0.823 & 25.20 & \mathrm{O} 2\end{array}$ $\begin{array}{lllllllllllll}473.9 & 22500 & 0.997 & 112.80 & 0.335 & 0.855 & 72.59 & 0.92 & 2.24 & \mathrm{C} & 2.697 & 61.30 & \mathrm{HC}\end{array}$ $\begin{array}{llllllllllll}441.0 & 22490 & 0.976 & 88.30 & 0.334 & 0.855 & 72.59 & 0.94 & 2.24 & \mathrm{C} & 2.510 & 46.30\end{array}$ $\begin{array}{lllllllllllll}373.7 & 22480 & 0.925 & 56.73 & 0.334 & 0.855 & 72.59 & 0.99 & 2.24 & \mathrm{C} & 2.126 & 27.60 & \mathrm{I}\end{array}$ $\begin{array}{lllllllllllll}318.8 & 22470 & 0.861 & 39.14 & 0.334 & 0.854 & 72.59 & 1.06 & 2.24 & \mathrm{C} & 1.814 & 17.70 & \text { I }\end{array}$ $\begin{array}{llllllllllll}307.6 & 22410 & 0.813 & 33.82 & 0.332 & 0.854 & 72.59 & 1.12 & 2.23 & \mathrm{C} & 1.751 & 14.80\end{array}$ $\begin{array}{lllllllllllll}266.9 & 22400 & 0.734 & 25.37 & 0.331 & 0.854 & 72.59 & 1.22 & 2.23 & C & 1.519 & 11.20 & \text { I }\end{array}$ $\begin{array}{lllllllllllll}232.4 & 22390 & 0.634 & 19.22 & 0.331 & 0.853 & 72.59 & 1.39 & 2.22 & \mathrm{C} & 1.323 & 9.60 & \mathrm{I}\end{array}$ $\begin{array}{llllllllllll}218.1 & 22390 & 0.578 & 16.92 & 0.331 & 0.853 & 72.59 & 1.51 & 2.22 & \mathrm{C} & 1.241 & 9.60\end{array}$ $\begin{array}{llllllllllll}205.4 & 22390 & 0.519 & 15.02 & 0.331 & 0.853 & 72.59 & 1.66 & 2.22 & \mathrm{C} & 1.169 & 10.10\end{array}$ $\begin{array}{lllllllllllllll}183.9 & 22390 & 0.391 & 12.06 & 0.330 & 0.853 & 72.59 & 2.11 & 2.21 & \mathrm{C} & 1.046 & 10.90 & \text { I }\end{array}$ $\begin{array}{lllllllllllll}28.8 & 27960 & 1.073 & 10.20 & 0.362 & 0.888 & 72.59 & 0.86 & 2.52 & \mathrm{C} & 0.164 & 15.10 & 02\end{array}$ $\begin{array}{lllllllllllll}78.5 & 26180 . & 1.044 & 16.51 & 0.361 & 0.878 & 72.59 & 0.88 & 2.39 & \mathrm{C} & 0.447 & 15.60 & \mathrm{O} 2\end{array}$ $\begin{array}{lllllllllllll}152.1 & 25070 & 1.023 & 27.39 & 0.361 & 0.871 & 72.59 & 0.90 & 2.33 & \mathrm{C} & 0.866 & 19.50 & 02\end{array}$ $\begin{array}{lllllllllllll}477.4 & 24030 & 0.997 & 72.63 & 0.361 & 0.863 & 72.59 & 0.92 & 2.28 & \mathrm{C} & 2.716 & 45.50 & \mathrm{O} 2\end{array}$ $\begin{array}{lllllllllllll}388.1 & 24020 & 0.935 & 45.35 & 0.361 & 0.863 & 72.59 & 0.97 & 2.27 & \mathrm{C} & 2.209 & 26.30 & \text { I }\end{array}$ $\begin{array}{lllllllllllll}322.4 & 24010 & 0.859 & 30.86 & 0.360 & 0.863 & 72.59 & 1.05 & 2.27 & \mathrm{C} & 1.835 & 16.60 & \text { I }\end{array}$ $\begin{array}{lllllllllllll}301.8 & 23950 & 0.802 & 26.06 & 0.359 & 0.862 & 72.59 & 1.12 & 2.26 & \mathrm{C} & 1.717 & 13.50 & \mathrm{I}\end{array}$ $\begin{array}{llllllllllll}258.6 & 23950 & 0.711 & 19.54 & 0.358 & 0.862 & 72.59 & 1.24 & 2.26 & \mathrm{C} & 1.472 & 10.20\end{array}$ $\begin{array}{lllllllllllll}222.4 & 23940 & 0.595 & 14.80 & 0.358 & 0.862 & 72.59 & 1.45 & 2.26 & \mathrm{C} & 1.266 & 9.00 & \mathrm{I}\end{array}$ $\begin{array}{llllllllllllll}207.4 & 23940 & 0.531 & 13.03 & 0.358 & 0.862 & 72.59 & 1.60 & 2.25 & \mathrm{C} & 1.180 & 9.30 & 1\end{array}$ $\begin{array}{llllllllllll}194.2 & 23940 & 0.465 & 11.58 & 0.358 & 0.862 & 72.59 & 1.79 & 2.25 & \mathrm{C} & \mathrm{I} .105 & 10.30\end{array}$ $\begin{array}{lllllllllllll}171.7 & 23950 & 0.320 & 9.30 & 0.357 & 0.862 & 72.59 & 2.42 & 2.24 & \mathrm{C} & 0.977 & 11.60 & 1\end{array}$ $\begin{array}{lllllllllllll}77.7 & 17470 & 1.012 & 56.30 & 0.287 & 0.843 & 72.59 & 0.93 & 2.21 & \mathrm{C} & 0.442 & 28.30 & 02\end{array}$ $\begin{array}{lllllllllllll}459.1 & 17030 & 0.987 & 175.20 & 0.286 & 0.838 & 72.59 & 0.95 & 2.20 & \mathrm{C} & 2.612 & 84.00 & 1\end{array}$ $\begin{array}{lllllllllllll}437.5 & 17030 & 0.970 & 135.60 & 0.286 & 0.838 & 72.59 & 0.96 & 2.20 & \mathrm{C} & 2.490 & 62.90\end{array}$ $\begin{array}{lllllllllllll}408.8 & 17020 & 0.949 & 107.10 & 0.286 & 0.838 & 72.59 & 0.98 & 2.20 & \mathrm{C} & 2.326 & 48.00 & 1\end{array}$ $\begin{array}{lllllllllllll}346.9 & 17010 & 0.896 & 69.52 & 0.286 & 0.838 & 72.59 & 1.04 & 2.20 & \mathrm{C} & 1.603 & 29.10 & \text { I }\end{array}$ $\begin{array}{llllllllllll}294.8 & 17010 & 0.831 & 48.20 & 0.285 & 0.837 & 72.59 & 1.11 & 2.19 & \text { C } & \text { I.523 } & 18.90\end{array}$ $\begin{array}{lllllllllllll}284.3 & 16960 & 0.783 & 41.81 & 0.284 & 0.837 & 72.59 & 1.18 & 2.19 & C & 1.469 & 15.90 & \text { I }\end{array}$ $\begin{array}{llllllllllll}244.9 & 16960 & 0.704 & 31.39 & 0.283 & 0.837 & 72.59 & 1.30 & 2.19 & C & 1.266 & 12.00\end{array}$ $\begin{array}{llllllllllll}211.6 & 16950 & 0.605 & 23.79 & 0.283 & 0.836 & 72.59 & 1.49 & 2.18 & \mathrm{C} & \mathrm{I} .093 & 10.20\end{array}$

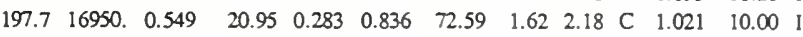
$\begin{array}{lllllllllllll}185.3 & 16950 & 0.492 & 18.59 & 0.283 & 0.836 & 72.59 & 1.79 & 2.18 & \mathrm{C} & 0.958 & 10.30 & \mathrm{I}\end{array}$ $\begin{array}{lllllllllllll}164.4 & 16950 & 0.366 & 14.92 & 0.283 & 0.836 & 72.59 & 2.31 & 2.17 & \mathrm{C} & 0.849 & 10.80 & \mathrm{I}\end{array}$ $\begin{array}{lllllllllllll}11.1 & 8638 & 1.056 & 14.44 & 0.299 & 0.865 & 72.59 & 0.89 & 2.31 & \mathrm{C} & 0.091 & 18.00 & \mathrm{O} 2\end{array}$ $\begin{array}{lllllllllllll}39.4 & 8099 & 1.028 & 28.97 & 0.299 & 0.853 & 72.59 & 0.91 & 2.25 & \mathrm{C} & 0.312 & 19.20 & \mathrm{O} 2\end{array}$ $\begin{array}{lllllllllllll}155.1 & 7747 . & 1.008 & 95.35 & 0.299 & 0.845 & 72.59 & 0.93 & 2.22 & \mathrm{C} & 1.065 & 43.30 & 02\end{array}$ $\begin{array}{lllllllllllll}598.9 & 7627 & 0.983 & 299.40 & 0.299 & 0.843 & 72.59 & 0.95 & 2.21 & \mathrm{C} & 3.549 & 133.30 & 02\end{array}$ $\begin{array}{llllllllllllll}325.8 & 7628 & 0.921 & 122.30 & 0.299 & 0.842 & 72.59 & 1.01 & 2.21 & \mathrm{C} & 2.345 & 50.60\end{array}$

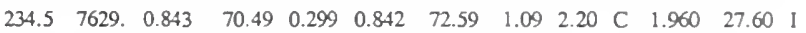
$\begin{array}{llllllllllll}203.0 & 7628 & 0.784 & 54.07 & 0.299 & 0.842 & 72.59 & 1.17 & 2.20 & \mathrm{C} & 1.860 & 20.70\end{array}$ $\begin{array}{llllllllllll}169.2 & 7630 & 0.688 & 38.67 & 0.299 & 0.842 & 72.59 & 1.32 & 2.20 & \mathrm{C} & 1.792 & 14.90\end{array}$ $\begin{array}{lllllllllllll}143.9 & 7633 & 0.563 & 28.36 & 0.299 & 0.842 & 72.59 & 1.58 & 2.19 & \mathrm{C} & 1.524 & 12.30 & \text { I }\end{array}$

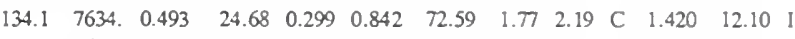
$\begin{array}{llllllllllll}125.8 & 7636 & 0.419 & 21.74 & 0.299 & 0.842 & 72.59 & 2.04 & 2.19 & \mathrm{C} & 1.332 & 12.50\end{array}$ $\begin{array}{lllllllllllll}1.12 .1 & 7640 & 0.257 & 17.24 & 0.299 & 0.842 & 72.59 & 3.05 & 2.18 & \mathrm{C} & \mathrm{I} .187 & 13.30 & \mathrm{I}\end{array}$ $\begin{array}{lllllllllllll}9.2 & 10120 & 1.079 & 10.04 & 0.363 & 0.890 & 72.59 & 0.86 & 2.56 & \mathrm{C} & 0.074 & 20.40 & \mathrm{O} 2\end{array}$ $\begin{array}{lllllllllllll}24.9 & 9518 . & 1.053 & 15.70 & 0.363 & 0.881 & 72.59 & 0.88 & 2.43 & \mathrm{C} & 0.204 & 18.20 & \mathrm{O} 2\end{array}$ $\begin{array}{lllllllllllll}47.0 & 9137 & 1.033 & 24.97 & 0.363 & 0.874 & 72.59 & 0.89 & 2.36 & C & 0.376 & 17.80 & 02\end{array}$ $\begin{array}{lllllllllllll}155.8 & 8733 & 1.009 & 72.47 & 0.363 & 0.867 & 72.59 & 0.91 & 2.30 & \mathrm{C} & 1.112 & 34.60 & \mathrm{O} 2\end{array}$ $\begin{array}{lllllllllllll}357.2 & 8584 & 0.950 & 120.60 & 0.363 & 0.864 & 72.59 & 0.96 & 2.28 & \mathrm{C} & 2.514 & 53.90 & \mathrm{CU}\end{array}$ $\begin{array}{lllllllllllll}251.8 & 8586 & 0.875 & 67.68 & 0.363 & 0.864 & 72.59 & 1.03 & 2.27 & \mathrm{C} & 2.065 & 28.80 & \mathrm{I}\end{array}$ $\begin{array}{llllllllllllll}216.3 & 8585 & 0.818 & 51.29 & 0.362 & 0.864 & 72.59 & 1.09 & 2.27 & \mathrm{C} & 1.940 & 21.40 & \text { I }\end{array}$ $\begin{array}{lllllllllllll}\text { I80.1 } & 8587 & 0.724 & 36.44 & 0.362 & 0.864 & 72.59 & 1.22 & 2.27 & \mathrm{C} & 1.854 & 15.30 & \text { I }\end{array}$ $\begin{array}{lllllllllllll}153.4 & 8590 & 0.602 & 26.62 & 0.362 & 0.863 & 72.59 & 1.43 & 2.26 & \text { C } & \text { I. } 579 & 12.40 & \text { I }\end{array}$ $\begin{array}{lllllllllllll}143.1 & 8592 & 0.533 & 23.14 & 0.362 & 0.863 & 72.59 & 1.59 & 2.26 & \mathrm{C} & 1.473 & 12.30 & \text { I }\end{array}$ $\begin{array}{lllllllllllll}134.5 & 8593 & 0.461 & 20.36 & 0.362 & 0.863 & 72.59 & 1.80 & 2.26 & \mathrm{C} & 1.384 & 12.80 & \text { I }\end{array}$ $\begin{array}{lllllllllllll}120.3 & 8597 & 0.301 & 16.13 & 0.362 & 0.863 & 72.59 & 2.53 & 2.25 & \mathrm{C} & 1.238 & 13.70 & \text { I }\end{array}$ $\begin{array}{lllllllllllll}25.1 & 20940 . & 1.066 & 11.64 & 0.379 & 0.890 & 72.59 & 0.86 & 2.53 & \mathrm{C} & 0.259 & 14.40 & 02\end{array}$ $\begin{array}{lllllllllllll}74.0 & 19680 & 1.037 & 20.37 & 0.378 & 0.880 & 72.59 & 0.88 & 2.40 & \mathrm{C} & 0.762 & 15.50 & \mathrm{O} 2\end{array}$ $\begin{array}{lllllllllllll}167.2 & 18890 & 1.015 & 39.36 & 0.378 & 0.873 & 72.59 & 0.90 & 2.34 & \mathrm{C} & 1.721 & 22.30 & 02\end{array}$ $\begin{array}{lllllllllllll}466.1 & 18380 & 0.990 & 91.64 & 0.378 & 0.869 & 72.59 & 0.92 & 2.30 & \mathrm{C} & 2.044 & 48.30 & \mathrm{O} 2\end{array}$ $\begin{array}{lllllllllllll}371.0 & 18380 & 0.928 & 56.32 & 0.378 & 0.868 & 72.59 & 0.97 & 2.30 & \mathrm{C} & 1.843 & 27.90 & 1\end{array}$ $\begin{array}{lllllllllllll}304.3 & 18370 & 0.853 & 38.04 & 0.378 & 0.868 & 72.59 & 1.05 & 2.30 & \mathrm{C} & 1.709 & 17.80 & \mathrm{I}\end{array}$ $\begin{array}{llllllllllllll}278.6 & 18350 & 0.796 & 31.45 & 0.377 & 0.868 & 72.59 & 1.11 & 2.29 & \mathrm{C} & 1.565 & 14.30 & \mathrm{I}\end{array}$ $\begin{array}{llllllllllllll}238.0 & 18350 & 0.706 & 23.64 & 0.377 & 0.868 & 72.59 & 1.24 & 2.29 & \mathrm{C} & 1.337 & 10.80 & \text { I }\end{array}$ $\begin{array}{lllllllllllll}203.9 & 18360 & 0.593 & 17.94 & 0.377 & 0.868 & 72.59 & 1.44 & 2.28 & \mathrm{C} & 1.145 & 9.30 & \mathrm{I}\end{array}$ $\begin{array}{llllllllllll}189.6 & 18360 & 0.531 & 15.81 & 0.376 & 0.868 & 72.59 & 1.58 & 2.28 & \mathrm{C} & 1.065 & 9.50\end{array}$ $\begin{array}{lllllllllllll}177.2 & 18360 & 0.466 & 14.05 & 0.376 & 0.868 & 72.59 & 1.76 & 2.28 & \mathrm{C} & 0.995 & 10.30 & \text { I }\end{array}$ $\begin{array}{lllllllllllll}156.0 & 18360 & 0.327 & 11.31 & 0.376 & 0.868 & 72.59 & 2.34 & 2.27 & \mathrm{C} & 0.876 & 11.60 & \mathrm{I}\end{array}$ $\begin{array}{lllllllllllll}20.1 & 23540 . & 1.095 & 7.50 & 0.319 & 0.885 & 72.59 & 0.86 & 2.53 & \mathrm{C} & 0.113 & 13.20 & 02\end{array}$ $\begin{array}{lllllllllllll}51.0 & 21600 & 1.061 & 11.82 & 0.319 & 0.872 & 72.59 & 0.88 & 2.36 & \mathrm{C} & 0.286 & 12.70 & \mathrm{O} 2\end{array}$ $\begin{array}{lllllllllllll}90.4 & 20450 . & 1.037 & 18.30 & 0.319 & 0.863 & 72.59 & 0.90 & 2.29 & \mathrm{C} & 0.507 & 13.60 & \mathrm{O} 2\end{array}$

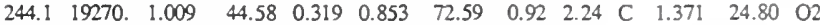
$\begin{array}{lllllllllllll}377.2 & 18910 & 0.941 & 52.26 & 0.318 & 0.849 & 72.59 & 0.98 & 2.22 & \mathrm{C} & 2.119 & 27.20 & \mathrm{I}\end{array}$ $\begin{array}{llllllllllllll}306.9 & 18900 & 0.858 & 35.34 & 0.318 & 0.849 & 72.59 & 1.07 & 2.22 & \mathrm{C} & 1.592 & 17.00 & \mathrm{I}\end{array}$ $\begin{array}{lllllllllllll}283.6 & 18860 & 0.797 & 29.72 & 0.316 & 0.848 & 72.59 & 1.14 & 2.22 & \mathrm{C} & 1.471 & 13.80 & \mathrm{I}\end{array}$ $\begin{array}{lllllllllllll}239.1 & 18860 & 0.700 & 22.23 & 0.316 & 0.848 & 72.59 & 1.29 & 2.21 & \mathrm{C} & 1.240 & 10.50 & \mathrm{I}\end{array}$ $\begin{array}{lllllllllllll}201.9 & 18860 & 0.579 & 16.81 & 0.316 & 0.848 & 72.59 & 1.52 & 2.21 & \mathrm{C} & 1.048 & 9.20 & 1\end{array}$ $\begin{array}{llllllllllll}186.6 & 18850 & 0.513 & 14.80 & 0.316 & 0.848 & 72.59 & 1.69 & 2.20 & \mathrm{C} & 0.968 & 9.60\end{array}$ $\begin{array}{lllllllllllll}173.1 & 18860 & 0.445 & 13.13 & 0.316 & 0.848 & 72.59 & 1.92 & 2.20 & \mathrm{C} & 0.898 & 10.60 & \mathrm{I}\end{array}$ $\begin{array}{lllllllllllll}150.4 & 18860 & 0.299 & 10.54 & 0.315 & 0.848 & 72.59 & 2.66 & 2.20 & \mathrm{C} & 0.780 & 12.20 & \mathrm{I}\end{array}$ $\begin{array}{lllllllllllll}22.3 & 24520 & 1.089 & 8.09 & 0.347 & 0.890 & 72.59 & 0.86 & 2.58 & \mathrm{C} & 0.116 & 14.10 & 02\end{array}$ $\begin{array}{lllllllllllll}57.5 & 22660 & 1.057 & 12.85 & 0.347 & 0.878 & 72.59 & 0.88 & 2.41 & \mathrm{C} & 0.298 & 13.70 & 02\end{array}$ $\begin{array}{lllllllllllll}103.5 & 21550 & 1.034 & 20.13 & 0.346 & 0.870 & 72.59 & 0.90 & 2.33 & C & 0.537 & 15.00 & 02\end{array}$ $\begin{array}{lllllllllllll}294.0 & 20420 . & 1.006 & 51.40 & 0.346 & 0.861 & 72.59 & 0.92 & 2.27 & \mathrm{C} & 1.525 & 29.10 & 02\end{array}$

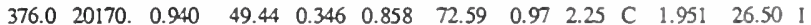
$\begin{array}{lllllllllllll}308.5 & 20160 & 0.859 & 33.59 & 0.346 & 0.858 & 72.59 & \text { I.06 } & 2.25 & \mathrm{C} & 1.597 & 16.80 & \text { I }\end{array}$ $\begin{array}{lllllllllllll}284.7 & 20120 & 0.799 & 28.16 & 0.344 & 0.858 & 72.59 & 1.13 & 2.25 & \mathrm{C} & 1.474 & 13.60 & \text { I }\end{array}$ $\begin{array}{lllllllllllll}241.7 & 20120 & 0.704 & 21.14 & 0.344 & 0.858 & 72.59 & 1.26 & 2.24 & \mathrm{C} & 1.252 & 10.30 & 1\end{array}$ $\begin{array}{lllllllllllll}205.5 & 20120 & 0.585 & 16.03 & 0.344 & 0.858 & 72.59 & 1.49 & 2.24 & \mathrm{C} & 1.064 & 9.10 & \mathrm{I}\end{array}$ $\begin{array}{lllllllllllllll}190.5 & 20120 & 0.519 & 14.13 & 0.344 & 0.857 & 72.59 & 1.65 & 2.23 & \mathrm{C} & 0.986 & 9.50 & \mathrm{I}\end{array}$ $\begin{array}{llllllllllll}177.3 & 20120 & 0.452 & 12.55 & 0.344 & 0.857 & 72.59 & 1.85 & 2.23 & \mathrm{C} & 0.918 & 10.60\end{array}$ $\begin{array}{llllllllllll}154.9 & 20130 & 0.307 & 10.09 & 0.343 & 0.857 & 72.59 & 2.54 & 2.22 & \mathrm{C} & 0.802 & 12.30\end{array}$ $\begin{array}{lllllllllllll}26.8 & 24930 & 1.075 & 9.96 & 0.373 & 0.891 & 72.59 & 0.86 & 2.57 & \mathrm{C} & 0.139 & 14.60 & \mathrm{O} 2\end{array}$ $\begin{array}{lllllllllllll}74.1 & 23280 & 1.044 & 16.55 & 0.373 & 0.881 & 72.59 & 0.88 & 2.42 & \mathrm{C} & 0.384 & 15.30 & 02\end{array}$ $\begin{array}{lllllllllllll}147.7 & 22270 & 1.022 & 28.41 & 0.373 & 0.874 & 72.59 & 0.90 & 2.35 & \mathrm{C} & 0.765 & 19.40 & 02\end{array}$ $\begin{array}{llllllllllllll}473.7 & 21390 & 0.996 & 77.18 & 0.373 & 0.867 & 72.59 & 0.91 & 2.29 & C & 2.453 & 46.60 & 02\end{array}$ $\begin{array}{lllllllllllll}379.7 & 21380 & 0.932 & 47.78 & 0.372 & 0.867 & 72.59 & 0.97 & 2.29 & \mathrm{C} & 1.966 & 26.70 & \mathrm{I}\end{array}$ $\begin{array}{llllllllllll}312.4 & 21380 & 0.853 & 32.38 & 0.372 & 0.867 & 72.59 & 1.05 & 2.29 & \mathrm{C} & 1.618 & 16.90 \mathrm{I}\end{array}$ $\begin{array}{lllllllllllll}288.0 & 21340 & 0.794 & 27.00 & 0.371 & 0.866 & 72.59 & 1.12 & 2.28 & \mathrm{C} & 1.49 \mathrm{I} & 13.60 & \mathrm{I}\end{array}$ $\begin{array}{llllllllllll}245.9 & 21340 & 0.701 & 20.28 & 0.371 & 0.866 & 72.59 & 1.25 & 2.28 & \mathrm{C} & 1.273 & 10.30\end{array}$ $\begin{array}{llllllllllll}210.4 & 21340 & 0.583 & 15.38 & 0.370 & 0.866 & 72.59 & 1.47 & 2.27 & \mathrm{C} & 1.089 & 9.00\end{array}$ $\begin{array}{lllllllllllll}195.7 & 21340 & 0.518 & 13.55 & 0.370 & 0.866 & 72.59 & 1.62 & 2.27 & \mathrm{C} & 1.013 & 9.40 & 1\end{array}$ $\begin{array}{lllllllllllll}182.8 & 21340 & 0.451 & 12.05 & 0.370 & 0.866 & 72.59 & 1.82 & 2.27 & \mathrm{C} & 0.946 & 10.40\end{array}$ $\begin{array}{lllllllllllll}160.8 & 21350 & 0.306 & 9.69 & 0.370 & 0.866 & 72.59 & 2.47 & 2.26 & \mathrm{C} & 0.832 & 11.80 & 1\end{array}$ $\begin{array}{lllllllllllll}219.7 & 11710 & 0.756 & 16.86 & 0.292 & 0.840 & 72.59 & 1.21 & 2.20 & \mathrm{P} & 1.867 & 11.90\end{array}$ $\begin{array}{lllllllllllll}202.4 & 11720 & 0.554 & 19.68 & 0.292 & 0.840 & 72.59 & 1.60 & 2.19 & P & 1.720 & 9.70 & \text { I }\end{array}$ $\begin{array}{llllllllllll}192.3 & 11720 & 0.470 & 21.24 & 0.292 & 0.840 & 72.59 & 1.85 & 2.18 & P & 1.634 & 9.30\end{array}$ $\begin{array}{lllllllllllll}181.2 & 11720 & 0.398 & 22.86 & 0.292 & 0.840 & 72.59 & 2.14 & 2.18 & P & 1.540 & 9.60 & 1\end{array}$ $\begin{array}{lllllllllllll}155.7 & 11730 & 0.279 & 26.31 & 0.292 & 0.839 & 72.59 & 2.88 & 2.18 & P & 1.324 & 11.60 & 1\end{array}$ $\begin{array}{lllllllllllll}127.3 & 11730 & 0.194 & 29.73 & 0.292 & 0.839 & 72.59 & 3.8 \mathrm{I} & 2.17 & \mathrm{P} & 1.082 & 13.90 & 1\end{array}$ $\begin{array}{llllllllllll}107.9 & 11730 & 0.152 & 31.76 & 0.291 & 0.839 & 72.59 & 4.54 & 2.17 & P & 0.917 & 15.00\end{array}$ $\begin{array}{lllllllllllllll}80.0 & 11730 & 0.108 & 34.13 & 0.291 & 0.839 & 72.59 & 5.67 & 2.17 & P & 0.680 & 16.00 & I\end{array}$ $\begin{array}{lllllllllllll}52.3 & 11730 & 0.076 & 35.73 & 0.291 & 0.839 & 72.59 & 6.94 & 2.17 & \mathrm{P} & 0.444 & 17.20 & \mathrm{O} 2\end{array}$ $\begin{array}{lllllllllllll}40.8 & 11730 & 0.065 & 35.99 & 0.291 & 0.839 & 72.59 & 7.52 & 2.17 & \mathrm{P} & 0.347 & 27.30 & \mathrm{O} 2\end{array}$ $\begin{array}{lllllllllllll}31.7 & 11730 & 0.056 & 35.85 & 0.291 & 0.839 & 72.59 & 8.04 & 2.17 & P & 0.269 & 61.90 & 02\end{array}$ $\begin{array}{lllllllllllll}21.1 & 11730 & 0.043 & 34.45 & 0.291 & 0.839 & 72.59 & 8.99 & 2.17 & \mathrm{P} & 0.179 & 93.40 & \mathrm{O} 2\end{array}$ $\begin{array}{lllllllllllll}237.1 & 15020 & 0.775 & 15.74 & 0.307 & 0.845 & 72.59 & 1.18 & 2.21 & \mathrm{P} & 2.015 & 12.00 & \text { I }\end{array}$ $\begin{array}{lllllllllllll}220.4 & 15030 & 0.594 & 18.43 & 0.306 & 0.845 & 72.59 & 1.50 & 2.20 & \mathrm{P} & 1.873 & 9.80 & \mathrm{I}\end{array}$ $\begin{array}{lllllllllllll}211.0 & 15030 & 0.518 & 19.99 & 0.306 & 0.845 & 72.59 & 1.69 & 2.20 & P & 1.794 & 9.40 & 1\end{array}$ $\begin{array}{llllllllllll}201.2 & 15030 & 0.452 & 21.65 & 0.306 & 0.845 & 72.59 & 1.90 & 2.19 & \mathrm{P} & 1.710 & 9.70\end{array}$ $\begin{array}{lllllllllllll}179.4 & 15030 & 0.343 & 25.46 & 0.306 & 0.845 & 72.59 & 2.40 & 2.19 & P & 1.525 & 12.00 & \text { I }\end{array}$ $\begin{array}{llllllllllllll}156.0 & 15030 & 0.263 & 29.79 & 0.306 & 0.845 & 72.59 & 2.97 & 2.19 & \mathrm{P} & 1.325 & 14.90 & \mathrm{I}\end{array}$ 
$\begin{array}{llllllllllllll}141.0 & 15030 & 0.223 & 33.04 & 0.306 & 0.845 & 72.59 & 3.37 & 2.18 & \mathrm{P} & 1.199 & 16.50 & \mathrm{I}\end{array}$ $\begin{array}{lllllllllllll}118.1 & 15030 & 0.179 & 37.64 & 0.306 & 0.845 & 72.59 & 3.96 & 2.18 & \mathrm{P} & 1.004 & 18.20\end{array}$ $\begin{array}{lllllllllllll}95.3 & 15030 & 0.144 & 42.73 & 0.306 & 0.845 & 72.59 & 4.60 & 2.18 & \mathrm{P} & 0.810 & 20.00 & \mathrm{I}\end{array}$ $\begin{array}{llllllllllllll}86.0 & 15030 & 0.130 & 45.12 & 0.306 & 0.844 & 72.59 & 4.92 & 2.18 & \mathrm{P} & 0.731 & 24.70 & \mathrm{I}\end{array}$ $\begin{array}{lllllllllllll}79.3 & 15030 & 0.118 & 47.10 & 0.306 & 0.844 & 72.59 & 5.22 & 2.18 & \mathrm{P} & 0.674 & 36.80 & \mathrm{O} 2\end{array}$ $\begin{array}{lllllllllllll}74.9 & 15030 & 0.098 & 49.89 & 0.306 & 0.844 & 72.59 & 5.86 & 2.18 & \mathrm{P} & 0.636 & 54.00 & \mathrm{CU}\end{array}$ $\begin{array}{lllllllllllll}248.3 & 18270 & 0.770 & 13.59 & 0.329 & 0.853 & 72.59 & 1.17 & 2.23 & \mathrm{P} & 2.110 & 11.90 & 1\end{array}$ $\begin{array}{lllllllllllll}231.4 & 18270 & 0.591 & 15.96 & 0.328 & 0.852 & 72.59 & 1.49 & 2.22 & \mathrm{P} & 1.967 & 9.60 & \mathrm{I}\end{array}$ $\begin{array}{llllllllllll}222.1 & 18270 & 0.516 & 17.34 & 0.328 & 0.852 & 72.59 & 1.67 & 2.22 & \mathrm{P} & 1.887 & 9.10\end{array}$ $\begin{array}{lllllllllllll}212.1 & 18270 & 0.450 & 18.83 & 0.328 & 0.852 & 72.59 & 1.88 & 2.21 & \mathrm{P} & 1.803 & 9.40 & 1\end{array}$ $\begin{array}{llllllllllllll}190.3 & 18280 & 0.343 & 22.26 & 0.328 & 0.852 & 72.59 & 2.36 & 2.21 & \mathrm{P} & 1.617 & 11.70 & \mathrm{I}\end{array}$ $\begin{array}{lllllllllllll}166.7 & 18280 & 0.264 & 26.25 & 0.328 & 0.852 & 72.59 & 2.89 & 2.21 & \mathrm{P} & 1.416 & 14.70 & 1\end{array}$ $\begin{array}{lllllllllllll}151.8 & 18270 & 0.225 & 29.33 & 0.328 & 0.852 & 72.59 & 3.27 & 2.20 & \mathrm{P} & 1.290 & 16.40 & 1\end{array}$ $\begin{array}{lllllllllllll}128.7 & 18270 & 0.181 & 33.77 & 0.327 & 0.852 & 72.59 & 3.81 & 2.20 & \mathrm{P} & 1.094 & 18.20 & \mathrm{I}\end{array}$ $\begin{array}{llllllllllllll}105.6 & 18270 & 0.146 & 38.85 & 0.327 & 0.852 & 72.59 & 4.40 & 2.20 & \mathrm{P} & 0.898 & 20.10 & \mathrm{I}\end{array}$ $\begin{array}{lllllllllllll}96.4 & 18270 & 0.133 & 41.30 & 0.327 & 0.852 & 72.59 & 4.69 & 2.20 & \mathrm{P} & 0.819 & 24.80 & \mathrm{I}\end{array}$ $\begin{array}{lllllllllllllll}89.8 & 18270 & 0.121 & 43.47 & 0.327 & 0.852 & 72.59 & 4.96 & 2.20 & \mathrm{P} & 0.764 & 36.50 & \mathrm{I}\end{array}$ $\begin{array}{lllllllllllll}86.8 & 18270 & 0.100 & 46.63 & 0.327 & 0.852 & 72.59 & 5.54 & 2.20 & \mathrm{P} & 0.737 & 54.20 & \mathrm{I}\end{array}$ 


\title{
Convective condensation of \\ R134a within a micro-fin tube
}

\author{
(file: tacond.tbl)
}

\begin{abstract}
$\begin{array}{lllllllllll}\text { Nu } & \operatorname{Re} & x_{q} & J a & P / P_{c} & T / T_{c} & M & \text { Sv } & \operatorname{Pr} \text { flow } & E_{a} & U_{\mathrm{su}}\end{array}$
\end{abstract}
$\begin{array}{lllllllllllll}22.5 & 8805 & 1.014 & 86.66 & 0.255 & 0.844 & 102.00 & 0.94 & 3.61 & C & 0.108 & 213.60 & 02\end{array}$ $\begin{array}{llllllllllllll}161.9 & 8686 . & 1.007 & 198.80 & 0.255 & 0.841 & 102.00 & 0.95 & 3.61 & C & 0.685 & 172.90 & 02\end{array}$ $\begin{array}{lllllllllllll}-805.7 & 8581 . & 1.000 & -668.00 & 0.255 & 0.839 & 102.00 & 0.96 & 3.62 & C & -2.824 & 532.80 & 02\end{array}$ $\begin{array}{lllllllllllllll}1486.0 & 8578 & 0.990 & 889.10 & 0.255 & 0.839 & 102.00 & 0.96 & 3.62 & C & 4.999 & 680.00 & 02\end{array}$ $\begin{array}{lllllllllllll}360.9 & 8573 & 0.959 & 127.40 & 0.254 & 0.838 & 102.00 & 0.99 & 3.62 & \mathrm{C} & 1.714 & 87.20 & \text { HC }\end{array}$ $\begin{array}{lllllllllllll}261.3 & 8567 . & 0.911 & 61.32 & 0.254 & 0.838 & 102.00 & 1.04 & 3.62 & \mathrm{C} & 1.452 & 38.30 & \mathrm{CU}\end{array}$ $\begin{array}{lllllllllllll}261.8 & 8538 & 0.870 & 49.37 & 0.252 & 0.838 & 102.00 & 1.09 & 3.62 & \mathrm{C} & 1.555 & 29.40 & \mathrm{CU}\end{array}$ $\begin{array}{llllllllllllll}229.4 & 8532 & 0.792 & 32.65 & 0.252 & 0.837 & 102.00 & 1.19 & 3.62 & \mathrm{C} & 1.552 & 19.90 & 1\end{array}$ $\begin{array}{lllllllllllll}209.8 & 8526 . & 0.679 & 22.74 & 0.251 & 0.837 & 102.00 & 1.38 & 3.62 & \mathrm{C} & 1.668 & 16.20 & 1\end{array}$ $\begin{array}{lllllllllllll}203.0 & 8522 & 0.609 & 19.42 & 0.251 & 0.837 & 102.00 & 1.53 & 3.62 & C & 1.614 & 15.70 & 1\end{array}$ $\begin{array}{lllllllllllllll}197.3 & 8521 & 0.532 & 16.81 & 0.251 & 0.837 & 102.00 & 1.73 & 3.62 & C & 1.569 & 15.70 & 1\end{array}$ $\begin{array}{lllllllllllllll}188.8 & 8517 & 0.347 & 12.97 & 0.251 & 0.837 & 102.00 & 2.54 & 3.62 & C & 1.501 & 15.90 & 1\end{array}$ $\begin{array}{lllllllllllll}23.3 & 10780 & 1.022 & 46.91 & 0.300 & 0.863 & 102.00 & 0.93 & 3.59 & C & 0.114 & 128.00 & 02\end{array}$ $\begin{array}{llllllllllllll}105.5 & 10590 & 1.012 & 81.82 & 0.300 & 0.860 & 102.00 & 0.94 & 3.59 & C & 0.483 & 102.80 & 02\end{array}$ $\begin{array}{lllllllllllllll}533.0 & 10420 & 1.003 & 294.40 & 0.300 & 0.856 & 102.00 & 0.94 & 3.60 & C & 2.007 & 291.70 & 02\end{array}$ $\begin{array}{lllllllllllll}955.3 & 10360 & 0.991 & 396.20 & 0.300 & 0.855 & 102.00 & 0.95 & 3.60 & \mathrm{C} & 3.466 & 377.30 & 02\end{array}$ $\begin{array}{lllllllllllll}339.7 & 10350 & 0.955 & 87.57 & 0.299 & 0.855 & 102.00 & 0.99 & 3.60 & \mathrm{C} & 1.625 & 74.90 & 1\end{array}$ $\begin{array}{lllllllllllll}249.9 & 10340 & 0.901 & 44.31 & 0.299 & 0.855 & 102.00 & 1.04 & 3.60 & \mathrm{C} & 1.391 & 34.50 & \mathrm{CU}\end{array}$ $\begin{array}{lllllllllllll}241.6 & 10310 & 0.856 & 35.00 & 0.297 & 0.854 & 102.00 & 1.10 & 3.60 & \mathrm{C} & 1.448 & 26.10 & \mathrm{CU}\end{array}$ $\begin{array}{lllllllllllll}212.4 & 10300 & 0.773 & 23.65 & 0.296 & 0.854 & 102.00 & 1.20 & 3.60 & \mathrm{C} & 1.456 & 17.90 & 1\end{array}$ $\begin{array}{llllllllllllll}193.7 & 10300 & 0.654 & 16.70 & 0.296 & 0.854 & 102.00 & 1.41 & 3.60 & C & 1.328 & 15.10 & 1\end{array}$ $\begin{array}{llllllllllll}187.2 & 10290 & 0.581 & 14.33 & 0.296 & 0.854 & 102.00 & 1.57 & 3.60 & \mathrm{C} & 1.283 & 15.10\end{array}$ $\begin{array}{llllllllllll}181.6 & 10290 & 0.501 & 12.46 & 0.296 & 0.854 & 102.00 & 1.79 & 3.60 & \mathrm{C} & 1.244 & 15.60\end{array}$ $\begin{array}{llllllllllllll}173.1 & 10290 & 0.311 & 9.68 & 0.296 & 0.854 & 102.00 & 2.72 & 3.60 & \mathrm{C} & 1.186 & 16.40 & 1\end{array}$ $\begin{array}{lllllllllllll}29.4 & 10980 & 1.023 & 47.44 & 0.300 & 0.863 & 102.00 & 0.93 & 3.59 & C & 0.142 & 111.60 & 02\end{array}$ $\begin{array}{lllllllllllll}154.1 & 10740 & 1.011 & 98.85 & 0.300 & 0.859 & 102.00 & 0.94 & 3.59 & \mathrm{C} & 0.676 & 132.70 & 02\end{array}$ $\begin{array}{lllllllllll}-7559.0 & 10540 & 1.001 & * * * * * * * & 0.299 & 0.855 & 102.00 & 0.94 & 3.60 & C * * * * * * * * * * * * & 02\end{array}$ $\begin{array}{lllllllllllll}1018.0 & 10520 & 0.987 & 355.10 & 0.299 & 0.855 & 102.00 & 0.96 & 3.60 & C & 3.734 & 410.40 & 02\end{array}$ $\begin{array}{lllllllllllll}337.6 & 10510 & 0.945 & 74.07 & 0.299 & 0.855 & 102.00 & 1.00 & 3.60 & \mathrm{C} & 1.655 & 76.60\end{array}$ $\begin{array}{llllllllllllll}244.6 & 10510 & 0.883 & 37.21 & 0.298 & 0.855 & 102.00 & 1.06 & 3.60 & \mathrm{C} & 1.408 & 34.80 & \mathrm{CU}\end{array}$ $\begin{array}{lllllllllllllll}232.6 & 10470 & 0.831 & 29.02 & 0.296 & 0.854 & 102.00 & 1.13 & 3.60 & \mathrm{C} & 1.452 & 25.90 & \mathrm{CU}\end{array}$ $\begin{array}{lllllllllllll}203.9 & 10460 & 0.736 & 19.64 & 0.296 & 0.854 & 102.00 & 1.26 & 3.60 & \mathrm{C} & 1.472 & 17.80 & 1\end{array}$

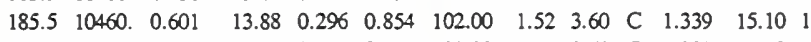
$\begin{array}{lllllllllllll}179.0 & 10450 & 0.518 & 11.92 & 0.295 & 0.854 & 102.00 & 1.74 & 3.60 & \mathrm{C} & 1.292 & 15.10 & 1\end{array}$ $\begin{array}{lllllllllllll}173.5 & 10450 & 0.427 & 10.37 & 0.295 & 0.854 & 102.00 & 2.07 & 3.60 & C & 1.252 & 15.60\end{array}$ $\begin{array}{lllllllllllll}165.1 & 10450 & 0.213 & 8.06 & 0.295 & 0.854 & 102.00 & 3.72 & 3.60 & C & 1.191 & 16.30 & 1\end{array}$ $\begin{array}{lllllllllllllll}12.8 & 14150 & 1.027 & 36.92 & 0.324 & 0.873 & 102.00 & 0.92 & 3.59 & \mathrm{C} & 0.056 & 612.70 & 02\end{array}$ $\begin{array}{lllllllllllll}52.2 & 13980 & 1.021 & 43.33 & 0.324 & 0.870 & 102.00 & 0.92 & 3.59 & \mathrm{C} & 0.226 & 96.30 & 02\end{array}$ $\begin{array}{llllllllllllll}103.2 & 13820 & 1.014 & 56.56 & 0.324 & 0.868 & 102.00 & 0.93 & 3.59 & \mathrm{C} & 0.433 & 72.30 & 02\end{array}$ $\begin{array}{lllllllllllll}278.7 & 13580 & 1.005 & 109.30 & 0.323 & 0.865 & 102.00 & 0.94 & 3.59 & \mathrm{C} & 1.070 & 116.10 & 02\end{array}$ $\begin{array}{llllllllllll}341.0 & 13460 & 0.974 & 77.50 & 0.323 & 0.863 & 102.00 & 0.96 & 3.59 & C & 1.423 & 74.00\end{array}$ $\begin{array}{lllllllllllll}264.4 & 13450 & 0.926 & 39.47 & 0.322 & 0.863 & 102.00 & 1.01 & 3.59 & \mathrm{C} & 1.279 & 34.00 \mathrm{CU}\end{array}$

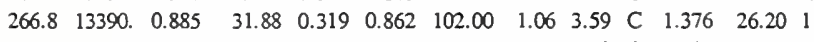
$\begin{array}{lllllllllllll}239.3 & 13380 & 0.806 & 21.43 & 0.319 & 0.862 & 102.00 & 1.15 & 3.59 & \mathrm{C} & 1.405 & 17.90 & 1\end{array}$ $\begin{array}{lllllllllllll}222.2 & 13370 & 0.690 & 15.08 & 0.318 & 0.861 & 102.00 & 1.33 & 3.59 & \mathrm{C} & 1.305 & 15.00 & 1\end{array}$ $\begin{array}{llllllllllllll}216.3 & 13360 & 0.619 & 12.93 & 0.318 & 0.861 & 102.00 & 1.47 & 3.59 & C & 1.270 & 14.80 & 1\end{array}$ $\begin{array}{lllllllllllll}211.2 & 13360 & 0.539 & 11.22 & 0.318 & 0.861 & 102.00 & 1.66 & 3.59 & C & 1.240 & 15.00 & 1\end{array}$ $\begin{array}{lllllllllllll}203.6 & 13350 & 0.348 & 8.70 & 0.318 & 0.861 & 102.00 & 2.44 & 3.59 & \mathrm{C} & 1.196 & 15.40 & 1\end{array}$ $\begin{array}{lllllllllllll}14.4 & 14120 & 1.030 & 33.67 & 0.315 & 0.871 & 102.00 & 0.92 & 3.59 & C & 0.063 & 284.10 & 02\end{array}$ $\begin{array}{lllllllllllllll}55.4 & 13930 & 1.023 & 40.70 & 0.315 & 0.868 & 102.00 & 0.92 & 3.59 & C & 0.239 & 86.60 & 02\end{array}$ $\begin{array}{lllllllllllllll}110.6 & 13740 & 1.015 & 54.72 & 0.315 & 0.865 & 102.00 & 0.93 & 3.59 & \mathrm{C} & 0.462 & 72.90 & 02\end{array}$ $\begin{array}{lllllllllllll}322.9 & 13480 & 1.004 & 115.80 & 0.314 & 0.862 & 102.00 & 0.94 & 3.59 & \mathrm{C} & 1.220 & 131.30 & 02\end{array}$

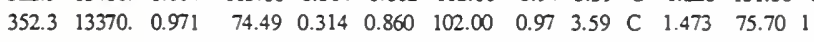
$\begin{array}{lllllllllllll}266.7 & 13350 & 0.919 & 37.42 & 0.313 & 0.860 & 102.00 & 1.02 & 3.59 & \mathrm{C} & 1.298 & 34.40 & \mathrm{CU}\end{array}$ $\begin{array}{lllllllllllll}267.1 & 13290 & 0.875 & 30.15 & 0.310 & 0.859 & 102.00 & 1.07 & 3.59 & \mathrm{C} & 1.391 & 26.30 & \text { I }\end{array}$ $\begin{array}{llllllllllllll}237.4 & 13280 & 0.792 & 20.20 & 0.310 & 0.859 & 102.00 & 1.17 & 3.59 & C & 1.413 & 18.00 & 1\end{array}$ $\begin{array}{llllllllllll}218.9 & 13270 & 0.669 & 14.18 & 0.309 & 0.858 & 102.00 & 1.37 & 3.59 & C & 1.303 & 15.00\end{array}$ $\begin{array}{llllllllllll}212.6 & 13260 & 0.594 & 12.15 & 0.309 & 0.858 & 102.00 & 1.53 & 3.59 & C & 1.265 & 14.80\end{array}$ $\begin{array}{lllllllllllllll}207.1 & 13260 & 0.510 & 10.53 & 0.309 & 0.858 & 102.00 & 1.76 & 3.59 & C & 1.233 & 15.00 & \text { l }\end{array}$ $\begin{array}{lllllllllllll}199.0 & 13250 & 0.310 & 8.16 & 0.308 & 0.858 & 102.00 & 2.71 & 3.59 & \mathrm{C} & 1.184 & 15.50 & 1\end{array}$ $\begin{array}{lllllllllllll}52.6 & 11110 & 1.023 & 27.83 & 0.244 & 0.843 & 102.00 & 0.94 & 3.61 & C & 0.253 & 20.00 & 02\end{array}$ $\begin{array}{lllllllllllll}432.6 & 10640 & 0.993 & 127.20 & 0.243 & 0.834 & 102.00 & 0.96 & 3.62 & \mathrm{C} & 1.706 & 64.40 & 1\end{array}$ $\begin{array}{lllllllllllllll}432.4 & 10630 & 0.972 & 104.00 & 0.243 & 0.834 & 102.00 & 0.98 & 3.62 & C & 1.787 & 51.00 & 1\end{array}$ $\begin{array}{llllllllllll}417.3 & 10630 & 0.946 & 85.38 & 0.242 & 0.834 & 102.00 & 1.01 & 3.62 & C & 1.815 & 40.60 \text { । }\end{array}$ $\begin{array}{llllllllllllll}366.3 & 10620 & 0.884 & 58.08 & 0.242 & 0.833 & 102.00 & 1.08 & 3.62 & C & 1.779 & 25.80\end{array}$ $\begin{array}{llllllllllllll}314.6 & 10600 & 0.807 & 41.20 & 0.241 & 0.833 & 102.00 & 1.18 & 3.63 & C & 1.718 & 17.20 & \text { I }\end{array}$ $\begin{array}{lllllllllllll}326.6 & 10540 & 0.752 & 38.89 & 0.238 & 0.832 & 102.00 & 1.26 & 3.63 & C & 1.883 & 15.60 & 1\end{array}$

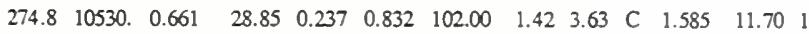
$\begin{array}{lllllllllllll}232.1 & 10520 & 0.546 & 21.63 & 0.237 & 0.831 & 102.00 & 1.70 & 3.63 & C & 1.338 & 10.10 & 1\end{array}$

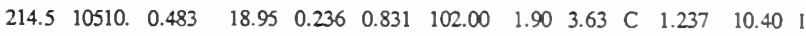

$\begin{array}{lllllllllllll}198.6 & 10510 & 0.418 & 16.71 & 0.236 & 0.831 & 102.00 & 2.17 & 3.63 & C & 1.146 & 11.50 & 1\end{array}$ $\begin{array}{lllllllllllll}172.2 & 10510 & 0.276 & 13.26 & 0.236 & 0.831 & 102.00 & 3.13 & 3.63 & C & 0.993 & 13.10 & \text { I }\end{array}$ $\begin{array}{lllllllllllll}60.5 & 10770 & 1.019 & 31.48 & 0.233 & 0.837 & 102.00 & 0.94 & 3.62 & \mathrm{C} & 0.287 & 19.20 & 02\end{array}$ $\begin{array}{lllllllllllll}450.9 & 10360 & 0.989 & 130.40 & 0.232 & 0.829 & 102.00 & 0.97 & 3.63 & C & 1.773 & 63.00 & 1\end{array}$ $\begin{array}{lllllllllllll}450.1 & 10360 & 0.966 & 106.60 & 0.232 & 0.829 & 102.00 & 0.99 & 3.63 & C & 1.858 & 50.20 & 1\end{array}$ $\begin{array}{lllllllllllll}434.0 & 10350 & 0.940 & 87.58 & 0.231 & 0.829 & 102.00 & 1.02 & 3.63 & C & 1.884 & 39.90 & 1\end{array}$ $\begin{array}{lllllllllllll}379.6 & 10340 & 0.875 & 59.53 & 0.231 & 0.829 & 102.00 & 1.09 & 3.63 & \text { C } & 1.844 & 25.40 & \text { I }\end{array}$ $\begin{array}{lllllllllllll}324.7 & 10320 & 0.796 & 42.17 & 0.230 & 0.828 & 102.00 & 1.19 & 3.63 & \mathrm{C} & 1.781 & 16.90 & \text { I }\end{array}$ $\begin{array}{lllllllllllll}340.5 & 10260 & 0.739 & 40.31 & 0.227 & 0.827 & 102.00 & 1.28 & 3.64 & C & 1.972 & 15.50 & 1\end{array}$ $\begin{array}{lllllllllllll}284.3 & 10250 & 0.645 & 29.75 & 0.226 & 0.827 & 102.00 & 1.46 & 3.64 & C & 1.647 & 11.60 & \text { I }\end{array}$ $\begin{array}{lllllllllllll}238.4 & 10240 & 0.528 & 22.21 & 0.225 & 0.826 & 102.00 & 1.76 & 3.64 & C & 1.381 & 9.80 & 1\end{array}$ $\begin{array}{lllllllllllll}219.7 & 10230 & 0.464 & 19.44 & 0.225 & 0.826 & 102.00 & 1.99 & 3.64 & \mathrm{C} & 1.272 & 9.90 & 1\end{array}$ $\begin{array}{lllllllllllll}202.7 & 10230 & 0.397 & 17.11 & 0.225 & 0.826 & 102.00 & 2.29 & 3.64 & C & 1.174 & 10.60 & \text { I }\end{array}$ $\begin{array}{lllllllllllll}174.8 & 10220 & 0.252 & 13.54 & 0.225 & 0.826 & 102.00 & 3.42 & 3.64 & \mathrm{C} & 1.012 & 11.80 & 1\end{array}$ $\begin{array}{lllllllllllll}36.0 & 17530 & 1.047 & 14.75 & 0.317 & 0.877 & 102.00 & 0.90 & 3.60 & \mathrm{C} & 0.209 & 18.00 & 02\end{array}$ $\begin{array}{llllllllllllll}119.4 & 16720 & 1.022 & 26.59 & 0.317 & 0.868 & 102.00 & 0.92 & 3.59 & C & 0.692 & 20.70 & 02\end{array}$ $\begin{array}{lllllllllllllll}347.9 & 16180 & 1.003 & 63.38 & 0.316 & 0.862 & 102.00 & 0.94 & 3.59 & C & 2.015 & 38.80 & 02\end{array}$ $\begin{array}{lllllllllllll}457.0 & 16070 & 0.981 & 70.00 & 0.316 & 0.861 & 102.00 & 0.96 & 3.59 & \mathrm{C} & 2.647 & 40.80 & 1\end{array}$ $\begin{array}{lllllllllllll}403.3 & 16050 & 0.924 & 46.52 & 0.315 & 0.860 & 102.00 & 1.01 & 3.59 & \text { C } & 1.673 & 25.20 & \text { I }\end{array}$ $\begin{array}{llllllllllllll}351.4 & 16030 & 0.853 & 32.54 & 0.314 & 0.860 & 102.00 & 1.09 & 3.59 & C & 1.630 & 16.30 & 1\end{array}$ $\begin{array}{lllllllllllll}362.9 & 15930 & 0.800 & 30.04 & 0.310 & 0.859 & 102.00 & 1.16 & 3.59 & \text { C } & 1.683 & 14.40 & \text { I }\end{array}$ $\begin{array}{lllllllllllll}312.5 & 15910 & 0.712 & 22.21 & 0.309 & 0.858 & 102.00 & 1.30 & 3.59 & \mathrm{C} & 1.450 & 10.80 & 1\end{array}$ $\begin{array}{llllllllllllll}271.2 & 15890 & 0.597 & 16.61 & 0.308 & 0.858 & 102.00 & 1.52 & 3.59 & C & 1.258 & 9.30 & 1\end{array}$ $\begin{array}{lllllllllllll}254.4 & 15880 & 0.533 & 14.55 & 0.308 & 0.858 & 102.00 & 1.69 & 3.59 & \mathrm{C} & 1.180 & 9.60 & 1\end{array}$ $\begin{array}{lllllllllllll}239.2 & 15880 & 0.465 & 12.83 & 0.308 & 0.858 & 102.00 & 1.91 & 3.59 & \mathrm{C} & 1.109 & 10.30 & 1\end{array}$ $\begin{array}{lllllllllllll}213.9 & 15870 & 0.314 & 10.18 & 0.307 & 0.858 & 102.00 & 2.68 & 3.59 & C & 0.992 & 11.50 & 1\end{array}$ $\begin{array}{lllllllllllll}95.5 & 8341 . & 1.009 & 56.33 & 0.244 & 0.838 & 102.00 & 0.95 & 3.62 & C & 0.502 & 25.00 & 02\end{array}$ $\begin{array}{llllllllllllllll}415.1 & 8193 & 0.975 & 136.80 & 0.243 & 0.834 & 102.00 & 0.98 & 3.62 & C & 1.950 & 54.70 & 1\end{array}$ $\begin{array}{lllllllllllll}416.9 & 8190 & 0.950 & 113.30 & 0.243 & 0.834 & 102.00 & 1.01 & 3.62 & C & 2.055 & 44.30 & 1\end{array}$ $\begin{array}{lllllllllllll}404.2 & 8187 . & 0.921 & 94.34 & 0.243 & 0.834 & 102.00 & 1.04 & 3.62 & C & 2.097 & 35.90 & 1\end{array}$ $\begin{array}{lllllllllllll}356.5 & 8180 & 0.850 & 65.65 & 0.243 & 0.834 & 102.00 & 1.12 & 3.62 & \mathrm{C} & 2.074 & 23.90 & \text { I }\end{array}$ $\begin{array}{lllllllllllll}305.2 & 8173 & 0.765 & 47.30 & 0.242 & 0.833 & 102.00 & 1.24 & 3.62 & C & 2.012 & 16.50 & 1\end{array}$ $\begin{array}{llllllllllllll}297.3 & 8148 & 0.704 & 42.28 & 0.240 & 0.833 & 102.00 & 1.34 & 3.63 & C & 2.110 & 14.40 & 1\end{array}$ $\begin{array}{lllllllllllll}251.0 & 8144 & 0.606 & 32.06 & 0.240 & 0.833 & 102.00 & 1.54 & 3.63 & C & 1.782 & 11.00 & 1\end{array}$ $\begin{array}{lllllllllllll}210.8 & 8138 . & 0.486 & 24.45 & 0.240 & 0.833 & 102.00 & 1.89 & 3.63 & \mathrm{C} & 1.496 & 9.30 & \mathrm{I}\end{array}$ $\begin{array}{llllllllllllll}193.9 & 8136 & 0.420 & 21.57 & 0.240 & 0.832 & 102.00 & 2.16 & 3.63 & C & 1.376 & 9.40 & \text { I }\end{array}$ $\begin{array}{lllllllllllll}178.6 & 8134 & 0.354 & 19.14 & 0.240 & 0.832 & 102.00 & 2.52 & 3.63 & \mathrm{C} & 1.267 & 10.40 & 1\end{array}$ $\begin{array}{lllllllllllll}152.5 & 8132 & 0.212 & 15.35 & 0.239 & 0.832 & 102.00 & 3.92 & 3.63 & \mathrm{C} & 1.082 & 11.90 & 1\end{array}$ $\begin{array}{llllllllllllll}148.9 & 10380 & 1.006 & 72.68 & 0.338 & 0.870 & 102.00 & 0.93 & 3.59 & C & 0.746 & 36.40 & 02\end{array}$ $\begin{array}{lllllllllllll}426.0 & 10280 & 0.972 & 115.70 & 0.338 & 0.868 & 102.00 & 0.96 & 3.59 & \mathrm{C} & 2.034 & 52.70 & 1\end{array}$ $\begin{array}{lllllllllllll}423.3 & 10270 & 0.948 & 94.85 & 0.338 & 0.868 & 102.00 & 0.98 & 3.59 & C & 2.124 & 42.60 & 1\end{array}$ $\begin{array}{lllllllllllll}407.1 & 10270 & 0.920 & 78.21 & 0.337 & 0.868 & 102.00 & 1.01 & 3.59 & C & 2.155 & 34.50 & 1\end{array}$ $\begin{array}{lllllllllllll}356.4 & 10270 & 0.850 & 53.92 & 0.337 & 0.868 & 102.00 & 1.09 & 3.59 & \mathrm{C} & 2.116 & 23.00 & 1\end{array}$ $\begin{array}{lllllllllllll}304.9 & 10260 & 0.767 & 38.75 & 0.337 & 0.867 & 102.00 & 1.20 & 3.59 & \text { C } & 2.046 & 16.00 & \text { I }\end{array}$ $\begin{array}{lllllllllllllll}286.9 & 10240 & 0.706 & 33.38 & 0.335 & 0.867 & 102.00 & 1.29 & 3.59 & \mathrm{C} & 2.088 & 13.40 & 1\end{array}$ $\begin{array}{llllllllllllll}245.3 & 10240 & 0.610 & 25.58 & 0.335 & 0.867 & 102.00 & 1.48 & 3.59 & C & 1.785 & 10.30 & 1\end{array}$ $\begin{array}{lllllllllllll}208.3 & 10230 & 0.491 & 19.67 & 0.335 & 0.867 & 102.00 & 1.79 & 3.59 & \mathrm{C} & 1.516 & 9.10 & \mathrm{I}\end{array}$ $\begin{array}{lllllllllllll}192.4 & 10230 & 0.427 & 17.41 & 0.335 & 0.867 & 102.00 & 2.03 & 3.59 & \mathrm{C} & 1.400 & 10.10 & 1\end{array}$ $\begin{array}{lllllllllllll}178.2 & 10230 & 0.361 & 15.52 & 0.335 & 0.867 & 102.00 & 2.34 & 3.59 & \mathrm{C} & 1.297 & 12.30 & 1\end{array}$ $\begin{array}{lllllllllllll}153.7 & 10230 & 0.220 & 12.53 & 0.335 & 0.867 & 102.00 & 3.48 & 3.59 & \mathrm{C} & 1.119 & 15.20 & \text { l }\end{array}$ $\begin{array}{llllllllllllll}188.4 & 7138 & 1.004 & 85.94 & 0.246 & 0.837 & 102.00 & 0.95 & 3.62 & C & 1.031 & 36.00 & 02\end{array}$ $\begin{array}{lllllllllllllll}449.3 & 7080 & 0.953 & 118.30 & 0.246 & 0.835 & 102.00 & 1.00 & 3.62 & C & 2.428 & 46.50 & 1\end{array}$ $\begin{array}{lllllllllllll}438.9 & 7079 . & 0.918 & 97.32 & 0.245 & 0.835 & 102.00 & 1.04 & 3.62 & C & 2.514 & 37.80 & 1\end{array}$ $\begin{array}{lllllllllllll}414.5 & 7076 & 0.877 & 80.52 & 0.245 & 0.835 & 102.00 & 1.09 & 3.62 & \mathrm{C} & 2.525 & 30.90 & \text { I }\end{array}$ $\begin{array}{llllllllllllll}347.5 & 7072 & 0.781 & 55.77 & 0.245 & 0.835 & 102.00 & 1.21 & 3.62 & C & 2.433 & 20.80 & 1\end{array}$ $\begin{array}{lllllllllllll}282.9 & 7068 & 0.671 & 40.18 & 0.245 & 0.835 & 102.00 & 1.40 & 3.62 & \mathrm{C} & 2.313 & 14.60 & \mathrm{I}\end{array}$ $\begin{array}{llllllllllllll}256.3 & 7054 & 0.593 & 34.55 & 0.244 & 0.834 & 102.00 & 1.57 & 3.62 & \mathrm{C} & 2.331 & 12.30 & 1\end{array}$ $\begin{array}{llllllllllllll}206.4 & 7052 & 0.477 & 26.47 & 0.243 & 0.834 & 102.00 & 1.92 & 3.62 & \mathrm{C} & 1.878 & 9.50 & 1\end{array}$ $\begin{array}{lllllllllllll}162.4 & 7049 & 0.344 & 20.35 & 0.243 & 0.834 & 102.00 & 2.58 & 3.62 & \mathrm{C} & 1.478 & 8.80\end{array}$ $\begin{array}{lllllllllllll}143.7 & 7048 & 0.275 & 18.01 & 0.243 & 0.834 & 102.00 & 3.13 & 3.62 & C & 1.307 & 11.00 & 1\end{array}$ $\begin{array}{lllllllllllll}127.0 & 7048 & 0.208 & 16.06 & 0.243 & 0.834 & 102.00 & 3.96 & 3.62 & C & 1.155 & 15.70 & 1\end{array}$ $\begin{array}{llllllllllllll}98.3 & 7047 . & 0.076 & 12.97 & 0.243 & 0.834 & 102.00 & 8.31 & 3.62 & \mathrm{C} & 0.894 & 23.10 & 1\end{array}$ $\begin{array}{lllllllllllll}.229 .3 & 4374 & 0.990 & -214.20 & 0.278 & 0.847 & 102.00 & 0.96 & 3.60 & C & -1.592 & 188.00 & 02\end{array}$ $\begin{array}{lllllllllllll}-1231.0 & 4374 & 0.948 & -642.80 & 0.278 & 0.847 & 102.00 & 1.00 & 3.60 & C & -7.310 & 537.80 & 02\end{array}$ $\begin{array}{lllllllllllll}3116.0 & 4374 & 0.919 & 1347.00 & 0.278 & 0.847 & 102.00 & 1.03 & 3.60 & \text { C } & 16.700 & \cdots \cdots \cdots & 02\end{array}$ $\begin{array}{lllllllllllll}77.2 & 4373 & 0.885 & 289.30 & 0.278 & 0.847 & 102.00 & 1.07 & 3.60 & \mathrm{C} & 5.532 & 221.90 & 02\end{array}$ $\begin{array}{lllllllllllll}327.3 & 4372 . & 0.801 & 96.74 & 0.278 & 0.847 & 102.00 & 1.17 & 3.60 & \mathrm{C} & 2.988 & 69.20 & 1\end{array}$ $\begin{array}{llllllllllllll}211.3 & 4372 & 0.702 & 52.89 & 0.278 & 0.847 & 102.00 & 1.33 & 3.60 & \mathrm{C} & 2.333 & 36.00 & 1\end{array}$ $\begin{array}{lllllllllllll}172.6 & 4370 & 0.629 & 39.69 & 0.278 & 0.847 & 102.00 & 1.47 & 3.60 & \mathrm{C} & 1.576 & 26.60 & \mathrm{CU}\end{array}$ $\begin{array}{lllllllllllll}134.3 & 4370 & 0.516 & 27.93 & 0.278 & 0.847 & 102.00 & 1.76 & 3.60 & \mathrm{C} & 1.355 & 19.30 & 02\end{array}$ $\begin{array}{lllllllllllllll}106.3 & 4369 & 0.378 & 20.25 & 0.278 & 0.847 & 102.00 & 2.32 & 3.60 & \mathrm{C} & 1.205 & 18.10 & 02\end{array}$ $\begin{array}{lllllllllllll}95.6 & 4369 & 0.304 & 17.54 & 0.278 & 0.847 & 102.00 & 2.81 & 3.60 & \mathrm{C} & 1.159 & 22.30 & \mathrm{O} 2\end{array}$ 
$\begin{array}{lllllllllllll}86.5 & 4369 & 0.228 & 15.39 & 0.278 & 0.847 & 102.00 & 3.57 & 3.60 & \mathrm{C} & 1.050 & 29.50 & \mathrm{O} 2\end{array}$ 71.8 4369. 0.068 $\begin{array}{lllllllllll}12.12 & 0.277 & 0.847 & 102.00 & 8.30 & 3.60 & \mathrm{C} & 0.871 & 38.70 & \mathrm{I}\end{array}$ $\begin{array}{lll}1.8 & 3983 . & 1.036\end{array}$ 7.83940 .1 .031 14.7 3893. 1.024 $31.13828 . \quad 1.016$ $294.6 \quad 3718,0.987$ 219.8 3718. 0.941 203.3 3717. 0.900 $\begin{array}{llll}185.1 & 3716 & 0.824\end{array}$ $\begin{array}{lll}175.4 & 3716 . & 0.712\end{array}$ $\begin{array}{lll}171.7 & 3715 & 0.642\end{array}$ $\begin{array}{llll}168.7 & 3715 & 0.564\end{array}$ 164.3 3715. 0.379 $2.1 \quad 3730 . \quad 1.037$ $8.5 \quad 3680,1.030$ 16.23631 .1 .023 37.1 3564. 1.013 $293.0 \quad 3476.0 .98$ $\begin{array}{lll}216.8 & 3475 & 0.933\end{array}$ $\begin{array}{lll}198.2 & 3475 . & 0.890\end{array}$ $\begin{array}{lll}180.8 & 3474, & 0.810\end{array}$ 169.7 3474, 0.693 165.9 3474. 0.620 $\begin{array}{llll}162.8 & 3474, & 0.539\end{array}$ $\begin{array}{llll}158.1 & 3473 & 0.347\end{array}$ 59.2 14420. 1.03 $\begin{array}{lll}463.3 & 13590 \text {. } & 0.995\end{array}$ $\begin{array}{llll}468.5 & 13580 & 0.970\end{array}$ 457.2 13570. 0.940 $\begin{array}{lll}405.5 & 13550 & 0.867\end{array}$ $\begin{array}{lll}347.8 & 13520, & 0.780\end{array}$ $\begin{array}{lll}359.4 & 13420 & 0.717\end{array}$ 298.5 13400. 0.616 $\begin{array}{lll}248.0 & 13380 & 0.490\end{array}$ 227.2 13370. 0.422 208.5 13370. 0.351 177.4 13360. 0.201 65.8 14600. 1.02 447.7 13910. 0.992 460.6 13900. 0.969 $\begin{array}{llll}456.4 & 13880 & 0.942\end{array}$ $\begin{array}{llll}413.5 & 13850 & 0.874\end{array}$ $\begin{array}{llll}359.0 & 13820 & 0.792\end{array}$ $\begin{array}{llll}390.1 & 13700 & 0.735\end{array}$ $\begin{array}{lll}322.1 & 13670 . & 0.639\end{array}$ 266.8 13650. 0.521 $\begin{array}{llll}244.5 & 13640 & 0.456\end{array}$ $224.2 \quad 13630.0 .389$ $\begin{array}{lll}190.8 & 13620 & 0.245\end{array}$ 68.5 16170. 1.031 508.3 15270. 0.995 $\begin{array}{llll}501.3 & 15260 & 0.968\end{array}$ $\begin{array}{llll}479.1 & 15250 & 0.938\end{array}$ $\begin{array}{llll}413.7 & 15220 & 0.862\end{array}$ $\begin{array}{llll}350.0 & 15200 & 0.77\end{array}$ $\begin{array}{lll}346.1 & 15110 . & 0.706\end{array}$ 289.0 15090. 0.600 241.3 15070. 0.470 221.6 15070. 0.399 203.9 15060. 0.32 $\begin{array}{lll}174.2 & 15060 . & 0.170\end{array}$ 66.5 16910. 1.028 $\begin{array}{llll}499.0 & 16080 & 0.998\end{array}$ $\begin{array}{llll}495.5 & 16080 & 0.976\end{array}$ 477.3 16060. 0.950 $\begin{array}{llll}419.1 & 16040 & 0.885\end{array}$ $\begin{array}{lll}361.0 & 16020 \text {. } 0.805\end{array}$ $\begin{array}{llll}363.4 & 15930 & 0.747\end{array}$ $\begin{array}{llll}309.2 & 15920 & 0.652\end{array}$ $\begin{array}{llll}263.8 & 15900 & 0.532\end{array}$ $\begin{array}{llll}245.0 & 15890 & 0.466\end{array}$ $228.1 \quad 15890.0 .396$ $\begin{array}{llll}199.8 & 15880 & 0.246\end{array}$ 35.4 14840. 1.051 131.8 14010. 1.020 $\begin{array}{llll}490.8 & 13540 & 0.997\end{array}$ 467.2 13530. 0.97 $\begin{array}{llll}404.0 & 13520 & 0.905\end{array}$ $\begin{array}{llll}344.9 & 13500 & 0.82\end{array}$ $\begin{array}{llll}338.7 & 13450 \text {. } 0.766\end{array}$ 288.7 13440. 0.670 $\begin{array}{llll}246.4 & 13430 . & 0.549\end{array}$ $228.8 \quad 13420.0 .483$ 213.1 13420. 0.41 $\begin{array}{llll}186.6 & 13410 . & 0.263\end{array}$ $38.6 \quad 13530, \quad 1.044$ $\begin{array}{lll}185.8 & 12720 \text {. } 1.011\end{array}$

$\begin{array}{llllllllllll}22.12 & 0.253 & 0.852 & 102.00 & 0.93 & 3.60 & \mathrm{C} & 0.017 & * * * * * * & \mathrm{O} 2\end{array}$ $\begin{array}{llllllllll}25.57 & 0.252 & 0.850 & 102.00 & 0.93 & 3.60 & \mathrm{C} & 0.076 & 80.90 & \mathrm{O} 2\end{array}$ $\begin{array}{llllllllll}31.23 & 0.252 & 0.847 & 102.00 & 0.94 & 3.61 & \mathrm{C} & 0.141 & 36.40 & \mathrm{O} 2\end{array}$ $\begin{array}{llllllllll}46.75 & 0.252 & 0.844 & 102.00 & 0.94 & 3.61 & \mathrm{C} & 0.286 & 27.90 & \mathrm{O} 2\end{array}$ $\begin{array}{llllllllll}254.60 & 0.252 & 0.838 & 102.00 & 0.97 & 3.62 & \mathrm{C} & 2.126 & 108.90 & 02\end{array}$ $\begin{array}{llllllllll}23.50 & 0.252 & 0.838 & 102.00 & 1.01 & 3.62 & \mathrm{C} & 1.850 & 49.80 & \mathrm{O} 2\end{array}$ $\begin{array}{lllllllllll}90.40 & 0.252 & 0.837 & 102.00 & 1.06 & 3.62 & \mathrm{C} & 1.856 & 35.60 & \mathrm{O} 2\end{array}$ $\begin{array}{llllllllll}61.69 & 0.252 & 0.837 & 102.00 & 1.15 & 3.62 & \mathrm{C} & 1.923 & 24.60 & \mathrm{O} 2\end{array}$ $\begin{array}{llllllllll}43.83 & 0.252 & 0.837 & 102.00 & 1.32 & 3.62 & \mathrm{C} & 2.117 & 19.50 & \mathrm{I}\end{array}$ $\begin{array}{llllllllll}37.71 & 0.252 & 0.837 & 102.00 & 1.45 & 3.62 & \mathrm{C} & 1.607 & 18.40 & 1\end{array}$ $\begin{array}{llllllllll}32.88 & 0.252 & 0.837 & 102.00 & 1.64 & 3.62 & \mathrm{C} & 1.657 & 17.90 & \mathrm{I}\end{array}$ $\begin{array}{llllllllll}25.67 & 0.252 & 0.837 & 102.00 & 2.35 & 3.62 & \mathrm{C} & 1.822 & 17.60 & 1\end{array}$ $\begin{array}{llllllllll}21.60 & 0.250 & 0.851 & 102.00 & 0.93 & 3.60 & \mathrm{C} & 0.022 & 321.30 & 02\end{array}$ $\begin{array}{llllllllll}25.86 & 0.250 & 0.848 & 102.00 & 0.93 & 3.60 & \mathrm{C} & 0.087 & 64.70 & \mathrm{O} 2\end{array}$ $\begin{array}{lllllllllll}32.84 & 0.250 & 0.846 & 102.00 & 0.94 & 3.61 & C & 0.161 & 33.50 & O 2\end{array}$ $\begin{array}{llllllllll}54.13 & 0.250 & 0.842 & 102.00 & 0.94 & 3.61 & \mathrm{C} & 0.348 & 29.70 & \mathrm{O} 2\end{array}$ $\begin{array}{llllllllll}251.40 & 0.250 & 0.836 & 102.00 & 0.97 & 3.62 & \mathrm{C} & 2.214 & 107.90 & \mathrm{O} 2\end{array}$ $\begin{array}{lllllllllll}122.60 & 0.250 & 0.836 & 102.00 & 1.02 & 3.62 & \mathrm{C} & 1.911 & 48.90 & \mathrm{O} 2\end{array}$ $\begin{array}{llllllllll}89.27 & 0.249 & 0.836 & 102.00 & 1.07 & 3.62 & \mathrm{C} & 1.903 & 34.80 & \mathrm{O} 2\end{array}$ $\begin{array}{lllllllllll}1.13 & 0.249 & 0.836 & 102.00 & 1.17 & 3.62 & \mathrm{C} & 1.970 & 24.10 & \mathrm{O} 2\end{array}$ $\begin{array}{llllllllll}43.53 & 0.249 & 0.836 & 102.00 & 1.36 & 3.62 & \mathrm{C} & 1.549 & 19.10 & 1\end{array}$ $\begin{array}{llllllllll}37.48 & 0.249 & 0.836 & 102.00 & 1.50 & 3.62 & \mathrm{C} & 1.587 & 18.00 & 1\end{array}$ $\begin{array}{llllllllll}32.70 & 0.249 & 0.836 & 102.00 & 1.71 & 3.62 & \mathrm{C} & 1.636 & 17.60\end{array}$ $\begin{array}{llllllllll}25.56 & 0.249 & 0.836 & 102.00 & 2.55 & 3.62 & \mathrm{C} & 1.807 & 17.30 & \mathrm{I}\end{array}$ $\begin{array}{llllllllll}20.22 & 0.256 & 0.851 & 102.00 & 0.93 & 3.60 & \mathrm{C} & 0.677 & 14.40 & \mathrm{O} 2\end{array}$ $\begin{array}{llllllllll}89.75 & 0.256 & 0.839 & 102.00 & 0.96 & 3.62 & \mathrm{C} & 5.296 & 47.80\end{array}$ $\begin{array}{lllllllllll}74.80 & 0.255 & 0.839 & 102.00 & 0.98 & 3.62 & \mathrm{C} & 5.356 & 38.70 & \text { I }\end{array}$ $\begin{array}{llllllllll}62.56 & 0.255 & 0.838 & 102.00 & 1.01 & 3.62 & \mathrm{C} & 1.823 & 31.20 & \mathrm{I}\end{array}$ $\begin{array}{llllllllll}43.66 & 0.254 & 0.838 & 102.00 & 1.09 & 3.62 & \mathrm{C} & 1.813 & 20.30\end{array}$ $\begin{array}{llllllllll}31.41 & 0.253 & 0.838 & 102.00 & 1.21 & 3.62 & \mathrm{C} & 1.762 & 13.60 & 1\end{array}$ $\begin{array}{llllllllll}29.88 & 0.248 & 0.836 & 102.00 & 1.31 & 3.62 & \mathrm{C} & 1.820 & 12.40 & \mathrm{I}\end{array}$ $\begin{array}{llllllllll}22.20 & 0.248 & 0.836 & 102.00 & 1.51 & 3.62 & \mathrm{C} & 1.512 & 9.40 & \mathrm{I}\end{array}$ $\begin{array}{lllllllll}16.67 & 0.247 & 0.835 & 102.00 & 1.87 & 3.62 & \mathrm{C} & 1.256 & 8.10\end{array}$ $\begin{array}{llllllllll}14.62 & 0.246 & 0.835 & 102.00 & 2.14 & 3.62 & \mathrm{C} & 1.151 & 8.30 \mathrm{I}\end{array}$ $\begin{array}{llllllllll}12.89 & 0.246 & 0.835 & 102.00 & 2.53 & 3.62 & \mathrm{C} & 1.056 & 9.20 \mathrm{I}\end{array}$ $\begin{array}{llllllllll}10.23 & 0.246 & 0.835 & 102.00 & 4.07 & 3.62 & \mathrm{C} & 0.899 & 10.50 & \mathrm{I}\end{array}$ $\begin{array}{lllllllllll}24.02 & 0.244 & 0.844 & 102.00 & 0.94 & 3.6 \mathrm{I} & \mathrm{C} & 0.333 & 15.60 & \mathrm{O} 2\end{array}$ $\begin{array}{llllllllll}91.79 & 0.243 & 0.834 & 102.00 & 0.96 & 3.62 & \mathrm{C} & 2.268 & 47.00\end{array}$ $\begin{array}{llllllllll}7.65 & 0.243 & 0.834 & 102.00 & 0.99 & 3.62 & \mathrm{C} & 2.333 & 38.60\end{array}$ $\begin{array}{llllllllll}65.81 & 0.242 & 0.833 & 102.00 & 1.01 & 3.62 & \mathrm{C} & 2.312 & 31.80 & 1\end{array}$ $\begin{array}{llllllllll}46.69 & 0.241 & 0.833 & 102.00 & 1.09 & 3.63 & \mathrm{C} & 1.754 & 20.90\end{array}$ $\begin{array}{lllllllll}33.85 & 0.240 & 0.832 & 102.00 & 1.20 & 3.63 & \mathrm{C} & 1.714 & 14.10\end{array}$ $\begin{array}{lllllllll}33.81 & 0.235 & 0.830 & 102.00 & 1.29 & 3.63 & \mathrm{C} & 1.863 & 13.50\end{array}$ $\begin{array}{lllllllll}24.86 & 0.234 & 0.830 & 102.00 & 1.47 & 3.63 & \mathrm{C} & 1.538 & 10.10\end{array}$ $\begin{array}{llllllllll}18.51 & 0.233 & 0.830 & 102.00 & 1.78 & 3.63 & \mathrm{C} & 1.274 & 8.50 & \mathrm{I}\end{array}$ $\begin{array}{llllllllll}16.18 & 0.232 & 0.829 & 102.00 & 2.01 & 3.63 & \mathrm{C} & 1.167 & 8.70 & \mathrm{I}\end{array}$ $\begin{array}{llllllllll}14.21 & 0.232 & 0.829 & 102.00 & 2.32 & 3.63 & \mathrm{C} & 1.070 & 9.30 & \mathrm{I}\end{array}$ $\begin{array}{lllllllll}1.20 & 0.232 & 0.829 & 102.00 & 3.49 & 3.63 & \mathrm{C} & 0.911 & 10.40\end{array}$ $\begin{array}{llllllllll}20.19 & 0.297 & 0.865 & 102.00 & 0.92 & 3.59 & \mathrm{C} & 0.327 & 16.30 & \mathrm{O} 2\end{array}$ $\begin{array}{llllllllll}85.01 & 0.296 & 0.854 & 102.00 & 0.95 & 3.60 & \mathrm{C} & 2.428 & 52.00 & \mathrm{I}\end{array}$ $\begin{array}{lllllllll}69.08 & 0.296 & 0.854 & 102.00 & 0.98 & 3.60 & \mathrm{C} & 2.394 & 40.90\end{array}$ $\begin{array}{lllllllll}56.58 & 0.295 & 0.854 & 102.00 & 1.01 & 3.60 & \mathrm{C} & 1.916 & 32.50\end{array}$ $\begin{array}{lllllllll}38.44 & 0.294 & 0.853 & 102.00 & 1.09 & 3.60 & \mathrm{C} & 1.869 & 20.50\end{array}$ $\begin{array}{lllllllll}27.27 & 0.294 & 0.853 & 102.00 & 1.21 & 3.60 & \mathrm{C} & 1.581 & 13.50\end{array}$ $\begin{array}{llllllllll}4.79 & 0.290 & 0.852 & 102.00 & 1.31 & 3.60 & \mathrm{C} & 1.563 & 11.80\end{array}$

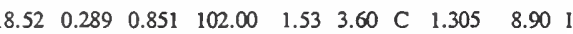
$\begin{array}{llllllllll}13.97 & 0.288 & 0.851 & 102.00 & 1.91 & 3.60 & \mathrm{C} & 1.090 & 7.80 & \mathrm{I}\end{array}$ $\begin{array}{llllllllll}12.27 & 0.288 & 0.851 & 102.00 & 2.21 & 3.60 & \mathrm{C} & 1.001 & 8.30 & \mathrm{I}\end{array}$ $\begin{array}{llllllllll}10.85 & 0.288 & 0.851 & 102.00 & 2.63 & 3.60 & \mathrm{C} & 0.921 & 9.40 & \mathrm{I}\end{array}$ $\begin{array}{llllllllll}8.65 & 0.288 & 0.851 & 102.00 & 4.46 & 3.60 & \mathrm{C} & 0.787 & 11.10 & \mathrm{I}\end{array}$ $\begin{array}{llllllllll}22.83 & 0.323 & 0.873 & 102.00 & 0.92 & 3.59 & \mathrm{C} & 0.300 & 17.10 & \mathrm{O} 2\end{array}$ $\begin{array}{llllllllll}95.29 & 0.322 & 0.863 & 102.00 & 0.94 & 3.59 & \mathrm{C} & 2.254 & 53.50 & \mathrm{I}\end{array}$ $\begin{array}{llllllllll}77.27 & 0.322 & 0.863 & 102.00 & 0.96 & 3.59 & \mathrm{C} & 2.238 & 42.20 & \mathrm{I}\end{array}$ $\begin{array}{llllllllll}63.19 & 0.321 & 0.863 & 102.00 & 0.99 & 3.59 & \mathrm{C} & 2.156 & 33.40\end{array}$ $\begin{array}{lllllllll}42.82 & 0.321 & 0.862 & 102.00 & 1.06 & 3.59 & \mathrm{C} & 1.829 & 21.20\end{array}$ $\begin{array}{lllllllll}30.34 & 0.320 & 0.862 & 102.00 & 1.15 & 3.59 & \mathrm{C} & 1.575 & 14.10\end{array}$ $\begin{array}{lllllllll}27.70 & 0.316 & 0.861 & 102.00 & 1.24 & 3.59 & \mathrm{C} & 1.586 & 12.40\end{array}$ $\begin{array}{llllllllll}20.68 & 0.315 & 0.860 & 102.00 & 1.40 & 3.59 & \mathrm{C} & 1.349 & 9.30\end{array}$ $\begin{array}{llllllllll}15.59 & 0.315 & 0.860 & 102.00 & 1.68 & 3.59 & \mathrm{C} & 1.151 & 8.00 \mathrm{I}\end{array}$ $\begin{array}{llllllllll}13.69 & 0.314 & 0.860 & 102.00 & 1.90 & 3.59 & \mathrm{C} & 1.069 & 8.20 & \mathrm{I}\end{array}$ $\begin{array}{lllllllllll}9.11 & 0.314 & 0.860 & 102.00 & 2.19 & 3.59 & \mathrm{C} & 0.996 & 9.00 & 1\end{array}$ $\begin{array}{llllllllll}9.65 & 0.314 & 0.860 & 102.00 & 3.25 & 3.59 & \mathrm{C} & 0.872 & 10.20 & \mathrm{I}\end{array}$ $\begin{array}{lllllllllll}13.89 & 0.321 & 0.880 & 102.00 & 0.90 & 3.60 & \mathrm{C} & 0.154 & 14.70 & \mathrm{O} 2\end{array}$ $\begin{array}{lllllllllll}29.04 & 0.320 & 0.869 & 102.00 & 0.92 & 3.59 & \mathrm{C} & 0.606 & 18.60 & \mathrm{O} 2\end{array}$ $\begin{array}{llllllllll}89.69 & 0.320 & 0.862 & 102.00 & 0.94 & 3.59 & \mathrm{C} & 1.954 & 46.00 & \mathrm{I}\end{array}$ $\begin{array}{lllllllll}72.55 & 0.320 & 0.862 & 102.00 & 0.97 & 3.59 & \mathrm{C} & 1.962 & 36.10\end{array}$ $\begin{array}{lllllllll}48.50 & 0.319 & 0.862 & 102.00 & 1.03 & 3.59 & \mathrm{C} & 1.90 \mathrm{I} & 22.90\end{array}$ $\begin{array}{llllllllll}34.13 & 0.318 & 0.862 & 102.00 & 1.13 & 3.59 & \mathrm{C} & 1.830 & 15.10\end{array}$ $\begin{array}{lllllllll}30.37 & 0.316 & 0.861 & 102.00 & 1.21 & 3.59 & \mathrm{C} & 1.797 & 13.10\end{array}$ $\begin{array}{llllllllll}22.76 & 0.315 & 0.860 & 102.00 & 1.37 & 3.59 & \mathrm{C} & 1.531 & 9.90 & \mathrm{I}\end{array}$ $\begin{array}{llllllllll}17.20 & 0.315 & 0.860 & 102.00 & 1.64 & 3.59 & \mathrm{C} & 1.307 & 8.50 & \mathrm{I}\end{array}$ $\begin{array}{llllllllll}15.13 & 0.315 & 0.860 & 102.00 & 1.84 & 3.59 & \mathrm{C} & 1.214 & 8.80 & \mathrm{I}\end{array}$ $\begin{array}{llllllllll}13.40 & 0.314 & 0.860 & 102.00 & 2.11 & 3.59 & \mathrm{C} & 1.131 & 9.90 & \mathrm{I}\end{array}$ $\begin{array}{llllllllll}10.71 & 0.314 & 0.860 & 102.00 & 3.08 & 3.59 & \mathrm{C} & 0.990 & 11.30 & 1\end{array}$ $\begin{array}{llllllllll}5.56 & 0.280 & 0.864 & 102.00 & 0.91 & 3.60 & \mathrm{C} & 0.205 & 12.90 & \mathrm{O} 2\end{array}$ $\begin{array}{llllllllll}42.21 & 0.280 & 0.852 & 102.00 & 0.94 & 3.60 & \mathrm{C} & 0.816 & 22.60 & \mathrm{O} 2\end{array}$ $\begin{array}{llllllllllll}486.8 & 12460 & 0.988 & 91.43 & 0.279 & 0.848 & 102.00 & 0.96 & 3.60 & \mathrm{C} & 1.941 & 44.50\end{array}$ $\begin{array}{llll}466.0 & 12450 . & 0.960\end{array}$ 404.4 12440. 0.891 $344.4 \quad 12420,0.808$ 344.4 12360. 0.747 $\begin{array}{llll}290.2 & 12350 \text {. } & 0.649\end{array}$ $244.8 \quad 12340.0 .526$ 226.1 12330. 0.459 209.2 12330. 0.389 $\begin{array}{llll}181.0 & 12320 & 0.239\end{array}$ $\begin{array}{llll}51.6 & 11920 . & 1.027\end{array}$ $479.5 \quad 11310$. 0.995 476.6 11310. 0.972 459.3 11300. 0.945 $401.6 \quad 11290.0 .878$ $\begin{array}{llll}342.8 & 11270 & 0.797\end{array}$ 348.3 11210. 0.738 $\begin{array}{llll}292.0 & \text { I1 } & 000 . & 0.642\end{array}$

$245.1 \quad 11190,0.523$ $225.8 \quad 11180.0 .457$ $\begin{array}{llll}208.4 & 11180 & 0.390\end{array}$ $\begin{array}{lll}179.4 & 11170 . & 0.245\end{array}$ $\begin{array}{lll}134.7 & 8032 . & 1.005\end{array}$ $461.8 \quad 7950 . \quad 0.973$ $\begin{array}{llll}453.5 & 7948 \quad 0.950\end{array}$ $\begin{array}{llll}432.4 & 7946 & 0.923\end{array}$ 373.47940 .0 .856 $\begin{array}{llll}316.9 & 7935 . & 0.776\end{array}$

$305.1 \quad 7917,0.717$ 258.5 7913. 0.623 218.3 7909. 0.507 $201.5 \quad 7907,0.443$ 186.4 7906. 0.378 160.8 7904. 0.238 $\begin{array}{lllllllllllllllll}5175 . & 0.985 & 6130.00 & 0.268 & 0.844 & 102.00 & 0.97 & 3.61 & \mathrm{C} & 21.110 & * * 4 * * & 02\end{array}$ $\begin{array}{lllllllllllll}721.1 & 5175 . & 0.972 & 563.90 & 0.268 & 0.844 & 102.00 & 0.98 & 3.61 & \mathrm{C} & 3.787 & 264.00 & 02\end{array}$ $\begin{array}{lllllllllllll}336.2 & 5173 . & 0.934 & 170.10 & 0.268 & 0.844 & 102.00 & 1.02 & 3.61 & \mathrm{C} & 2.216 & 75.60 & 1\end{array}$ $\begin{array}{lllllllllllll}253.5 & 5172 & 0.881 & 90.73 & 0.268 & 0.844 & 102.00 & 1.08 & 3.61 & \mathrm{C} & 1.932 & 37.60 & \mathrm{CU}\end{array}$ $\begin{array}{lllllllllllll}235.1 & 5168 . & 0.836 & 69.44 & 0.267 & 0.843 & 102.00 & 1.13 & 3.61 & \mathrm{C} & 1.941 & 28.00 & \mathrm{CU}\end{array}$ $\begin{array}{lllllllllllll}208.6 & 5167 . & 0.755 & 48.15 & 0.267 & 0.843 & 102.00 & 1.24 & 3.61 & \mathrm{C} & 1.953 & 19.70 & 1\end{array}$ $\begin{array}{lllllllllllll}190.6 & 5166 . & 0.641 & 34.55 & 0.267 & 0.843 & 102.00 & 1.45 & 3.61 & C & 2.104 & 15.80\end{array}$ $\begin{array}{llllllllllll}184.0 & 5165 . & 0.573 & 29.82 & 0.267 & 0.843 & 102.00 & 1.61 & 3.61 & \mathrm{C} & 2.253 & 15.10\end{array}$ $\begin{array}{llllllllllll}178.5 & 5165 & 0.498 & 26.06 & 0.267 & 0.843 & 102.00 & 1.83 & 3.61 & \mathrm{C} & 1.767 & 14.90\end{array}$ $\begin{array}{lllllllllllllll}169.8 & 5164 & 0.321 & 20.40 & 0.267 & 0.843 & 102.00 & 2.70 & 3.61 & \mathrm{C} & 1.681 & 15.00 & \mathrm{I}\end{array}$ $\begin{array}{llllllllllllll}-1452.0 & 5099 . & 1.000 & * * * * * * * & 0.311 & 0.859 & 102.00 & 0.94 & 3.59 & \mathrm{C} & -6.747 & 831.50 & \mathrm{O} 2\end{array}$ $\begin{array}{lllllllllllll}1218.0 & 5096 & 0.970 & 729.70 & 0.311 & 0.859 & 102.00 & 0.97 & 3.59 & \mathrm{C} & 6.612 & 344.80 & 02\end{array}$ $\begin{array}{llllllllllllll}667.7 & 5096 & 0.948 & 324.20 & 0.311 & 0.859 & 102.00 & 0.99 & 3.59 & \mathrm{C} & 4.217 & 152.90 & \mathrm{O} 2\end{array}$ $\begin{array}{lllllllllllll}478.5 & 5096 . & 0.921 & 195.80 & 0.311 & 0.859 & 102.00 & 1.02 & 3.59 & \mathrm{C} & 3.350 & 91.00 & 02\end{array}$ $\begin{array}{lllllllllllll}313.9 & 5095 . & 0.854 & 97.69 & 0.311 & 0.859 & 102.00 & 1.09 & 3.59 & \mathrm{C} & 2.591 & 44.20\end{array}$ $\begin{array}{lllllllllllll}238.3 & 5094 & 0.771 & 60.10 & 0.311 & 0.859 & 102.00 & 1.20 & 3.59 & \mathrm{C} & 2.289 & 26.10\end{array}$ $\begin{array}{lllllllllllll}209.2 & 5092 & 0.708 & 47.06 & 0.311 & 0.859 & 102.00 & 1.30 & 3.59 & C & 2.215 & 20.10\end{array}$ $\begin{array}{lllllllllllll}175.9 & 5091 & 0.606 & 34.25 & 0.311 & 0.859 & 102.00 & 1.50 & 3.59 & \mathrm{C} & 2.174 & 14.70\end{array}$ $\begin{array}{lllllllllllll}149.8 & 5091 . & 0.476 & 25.39 & 0.311 & 0.859 & 102.00 & 1.86 & 3.59 & \mathrm{C} & 1.536 & 12.70\end{array}$ $\begin{array}{lllllllllllll}139.5 & 5090 & 0.403 & 22.18 & 0.311 & 0.859 & 102.00 & 2.16 & 3.59 & \mathrm{C} & 1.514 & 13.90\end{array}$ $\begin{array}{llllllllllll}130.6 & 5090 & 0.326 & 19.57 & 0.310 & 0.859 & 102.00 & 2.59 & 3.59 & \mathrm{C} & 1.417 & 16.40\end{array}$ $\begin{array}{lllllllllllll}115.8 & 5090 & 0.159 & 15.57 & 0.310 & 0.859 & 102.00 & 4.56 & 3.59 & \mathrm{C} & 1.257 & 19.50 & 1\end{array}$ $\begin{array}{llllllllllll}312.1 & 12840 & 0.760 & 13.00 & 0.264 & 0.842 & 102.00 & 1.24 & 3.61 & \mathrm{P} & 1.962 & 12.00\end{array}$ $\begin{array}{lllllllllllll}285.2 & 12830 & 0.562 & 15.32 & 0.263 & 0.842 & 102.00 & 1.64 & 3.61 & \mathrm{P} & 1.793 & 9.70 & 1\end{array}$ $\begin{array}{lllllllllllll}270.4 & 12820 & 0.480 & 16.71 & 0.263 & 0.842 & 102.00 & 1.89 & 3.61 & P & 1.700 & 9.00 & 1\end{array}$ $\begin{array}{llllllllllll}254.4 & 12820 & 0.409 & 18.19 & 0.263 & 0.842 & 102.00 & 2.18 & 3.61 & \mathrm{P} & 1.599 & 9.30\end{array}$ $\begin{array}{llllllllllll}219.6 & 12810 & 0.297 & 21.69 & 0.262 & 0.842 & 102.00 & 2.89 & 3.61 & \mathrm{P} & 1.380 & 11.90\end{array}$ $\begin{array}{llllllllllll}183.4 & 12800 & 0.219 & 25.90 & 0.262 & 0.841 & 102.00 & 3.73 & 3.61 & \mathrm{P} & 1.153 & 15.60\end{array}$ $\begin{array}{lllllllllllll}163.3 & 12780 & 0.183 & 29.68 & 0.261 & 0.841 & 102.00 & 4.32 & 3.61 & P & 1.026 & 18.00\end{array}$ $\begin{array}{lllllllllllll}130.6 & 12780 & 0.145 & 34.81 & 0.261 & 0.841 & 102.00 & 5.19 & 3.61 & P & 0.821 & 20.90\end{array}$ $\begin{array}{lllllllllllll}103.1 & 12780 & 0.116 & 41.09 & 0.261 & 0.841 & 102.00 & 6.09 & 3.61 & P & 0.648 & 24.30\end{array}$ $\begin{array}{lllllllllllllll}95.8 & 12780 & 0.105 & 44.33 & 0.261 & 0.841 & 102.00 & 6.53 & 3.61 & P & 0.602 & 33.80 & 1\end{array}$ $\begin{array}{llllllllllllll}94.7 & 12770 & 0.095 & 47.33 & 0.261 & 0.841 & 102.00 & 6.96 & 3.61 & P & 0.595 & 48.90\end{array}$ $\begin{array}{lllllllllllll}116.7 & 12770 & 0.076 & 52.25 & 0.261 & 0.841 & 102.00 & 8.04 & 3.61 & \mathrm{P} & 0.734 & 63.60\end{array}$ $\begin{array}{lllllllllllll}313.3 & 13000 & 0.762 & 12.73 & 0.266 & 0.843 & 102.00 & 1.23 & 3.61 & P & 1.970 & 12.00\end{array}$ $\begin{array}{lllllllllllll}286.3 & 12990 & 0.560 & 15.00 & 0.265 & 0.842 & 102.00 & 1.64 & 3.61 & P & 1.800 & 9.60\end{array}$ $\begin{array}{lllllllllllll}271.5 & 12980 & 0.477 & 16.35 & 0.265 & 0.842 & 102.00 & 1.90 & 3.61 & P & 1.707 & 9.00\end{array}$ $\begin{array}{lllllllllllllll}255.3 & 12980 & 0.406 & 17.78 & 0.264 & 0.842 & 102.00 & 2.20 & 3.61 & \mathrm{P} & 1.605 & 9.30 & \mathrm{I}\end{array}$ $\begin{array}{llllllllllll}220.1 & 12970 & 0.292 & 21.16 & 0.264 & 0.842 & 102.00 & 2.93 & 3.61 & \mathrm{P} & 1.384 & 11.90\end{array}$ $\begin{array}{llllllllllll}183.4 & 12960 & 0.213 & 25.20 & 0.264 & 0.842 & 102.00 & 3.82 & 3.61 & \mathrm{P} & 1.153 & 15.40\end{array}$ $\begin{array}{lllllllllllll}162.8 & 12940 & 0.176 & 28.79 & 0.263 & 0.842 & 102.00 & 4.45 & 3.61 & \mathrm{P} & 1.023 & 17.80\end{array}$ $\begin{array}{llllllllllllll}129.4 & 12940 & 0.137 & 33.61 & 0.263 & 0.842 & 102.00 & 5.39 & 3.61 & \mathrm{P} & 0.813 & 20.60 & \mathrm{I}\end{array}$ $\begin{array}{llllllllllll}100.8 & 12940 & 0.108 & 39.45 & 0.263 & 0.842 & 102.00 & 6.38 & 3.61 & P & 0.634 & 23.90\end{array}$ $\begin{array}{lllllllllllll}92.6 & 12940 & 0.097 & 42.42 & 0.263 & 0.842 & 102.00 & 6.86 & 3.61 & P & 0.582 & 33.60\end{array}$ $\begin{array}{lllllllllllllll}90.5 & 12940 & 0.088 & 45.14 & 0.263 & 0.842 & 102.00 & 7.35 & 3.61 & P & 0.569 & 49.40 & 1\end{array}$ $\begin{array}{lllllllllllll}109.4 & 12930 & 0.069 & 49.50 & 0.262 & 0.842 & 102.00 & 8.53 & 3.61 & \mathrm{P} & 0.688 & 63.50\end{array}$ $\begin{array}{llllllllllll}265.1 & 7303 & 0.760 & 20.62 & 0.296 & 0.854 & 102.00 & 1.22 & 3.60 & P & 2.362 & 12.30\end{array}$ $\begin{array}{llllllllllllll}244.7 & 7302 & 0.571 & 24.35 & 0.296 & 0.854 & 102.00 & 1.59 & 3.60 & P & 2.180 & 10.80 & 1\end{array}$ $\begin{array}{lllllllllllll}233.2 & 7301 & 0.493 & 26.56 & 0.296 & 0.854 & 102.00 & 1.82 & 3.60 & P & 2.077 & 10.60 & 1\end{array}$ $\begin{array}{llllllllllll}220.9 & 7300 & 0.426 & 28.96 & 0.296 & 0.854 & 102.00 & 2.07 & 3.60 & P & 1.968 & 11.10\end{array}$ $\begin{array}{llllllllllllll}193.7 & 7299 & 0.317 & 34.62 & 0.296 & 0.854 & 102.00 & 2.68 & 3.60 & P & 1.726 & 13.90 & 1\end{array}$ $\begin{array}{lllllllllllll}164.6 & 7297 & 0.240 & 41.33 & 0.296 & 0.854 & 102.00 & 3.37 & 3.60 & P & 1.466 & 17.70 & \text { I }\end{array}$ 
$\begin{array}{lllllllllllll}145 . I & 7297 . & 0.203 & 46.18 & 0.296 & 0.854 & 102.00 & 3.86 & 3.60 & \mathrm{P} & 1.293 & 20.10 & \text { I }\end{array}$ $\begin{array}{llllllllllll}117.9 & 7297 & 0.164 & 53.70 & 0.296 & 0.854 & 102.00 & 4.54 & 3.60 & \mathrm{P} & 1.050 & 23.10\end{array}$ $\begin{array}{lllllllllllll}93.6 & 7296 . & 0.134 & 62.30 & 0.296 & 0.854 & 102.00 & 5.26 & 3.60 & P & 0.834 & 26.70\end{array}$ $\begin{array}{llllllllllll}86.1 & 7296 & 0.122 & 66.37 & 0.296 & 0.854 & 102.00 & 5.60 & 3.60 & \mathrm{P} & 0.767 & 35.40\end{array}$ $\begin{array}{lllllllllllll}83.1 & 7296 . & 0.112 & 69.90 & 0.296 & 0.854 & 102.00 & 5.93 & 3.60 & \mathrm{P} & 0.740 & 51.50 & \mathrm{I}\end{array}$ $\begin{array}{llllllllllllll}93.7 & 7295 . & 0.092 & 74.61 & 0.296 & 0.854 & 102.00 & 6.72 & 3.60 & \mathrm{P} & 0.835 & 64.90 & \mathrm{I}\end{array}$ $\begin{array}{lllllllllllll}252.3 & 5393 & 0.766 & 24.58 & 0.293 & 0.853 & 102.00 & 1.22 & 3.60 & \mathrm{P} & 2.650 & 14.10 & \text { I }\end{array}$ $\begin{array}{lllllllllllll}233.8 & 5392 & 0.562 & 29.25 & 0.293 & 0.853 & 102.00 & 1.62 & 3.60 & P & 2.932 & 12.60 & 1\end{array}$ $\begin{array}{lllllllllllll}222.5 & 5392 & 0.478 & 31.98 & 0.293 & 0.853 & 102.00 & 1.87 & 3.60 & P & 2.153 & 12.40 & I\end{array}$ $\begin{array}{lllllllllllll}209.9 & 5392 . & 0.406 & 34.90 & 0.293 & 0.853 & 102.00 & 2.17 & 3.60 & \mathrm{P} & 2.031 & 13.10 & 1\end{array}$ $\begin{array}{lllllllllllll}180.1 & 5391 . & 0.291 & 41.49 & 0.293 & 0.853 & 102.00 & 2.89 & 3.60 & \mathrm{P} & 1.742 & 16.40 & 1\end{array}$ $\begin{array}{lllllllllllllll}145.3 & 539 I & 0.21 I & 48.59 & 0.293 & 0.853 & 102.00 & 3.75 & 3.60 & \mathrm{P} & \mathrm{I} .406 & 20.50 & \mathrm{I}\end{array}$ $\begin{array}{llllllllllllll}120.0 & 5392 & 0.174 & 52.67 & 0.293 & 0.853 & 102.00 & 4.36 & 3.60 & \mathrm{P} & 1.161 & 22.70 & \mathrm{I}\end{array}$ $\begin{array}{llllllllllllll}84.8 & 5392 & 0.137 & 58.28 & 0.293 & 0.853 & 102.00 & 5.20 & 3.60 & \mathrm{P} & 0.821 & 26.10 & 1\end{array}$ $\begin{array}{lllllllllllll}51.2 & 5392 & 0.112 & 62.37 & 0.293 & 0.853 & 102.00 & 5.97 & 3.60 & \mathrm{P} & 0.495 & 31.30 & \mathrm{O} 2\end{array}$ $\begin{array}{lllllllllllll}38.6 & 5391 & 0.104 & 63.19 & 0.293 & 0.853 & 102.00 & 6.27 & 3.60 & \mathrm{P} & 0.373 & 59.40 & \mathrm{O} 2\end{array}$ $\begin{array}{lllllllllllll}30.2 & 5391 & 0.098 & 63.13 & 0.293 & 0.853 & 102.00 & 6.51 & 3.60 & \mathrm{P} & 0.292 & 111.20 & 02\end{array}$ $\begin{array}{lllllllllllll}26.1 & 5391 & 0.088 & 60.30 & 0.293 & 0.853 & 102.00 & 6.94 & 3.60 & P & 0.253 & 115.90 & 02\end{array}$ $\begin{array}{lllllllllllll}258.9 & 5168 & 0.751 & 29.84 & 0.281 & 0.848 & 102.00 & 1.24 & 3.60 & P & 2.697 & 14.50 & \text { I }\end{array}$ $\begin{array}{llllllllllll}237.6 & 5167 & 0.572 & 35.15 & 0.281 & 0.848 & 102.00 & 1.60 & 3.60 & \mathrm{P} & 2.88 \mathrm{I} & 13.40\end{array}$ $\begin{array}{lllllllllllll}225.9 & 5167 & 0.497 & 38.33 & 0.281 & 0.848 & 102.00 & 1.82 & 3.60 & \mathrm{P} & 2.107 & 13.50 & \mathrm{I}\end{array}$ $\begin{array}{llllllllllllll}213.7 & 5167 & 0.434 & 41.83 & 0.281 & 0.848 & 102.00 & 2.06 & 3.60 & \mathrm{P} & 2.023 & 14.40 & \text { I }\end{array}$ \begin{tabular}{lllllllllllll|}
187.6 & 5166. & 0.331 & 50.23 & 0.281 & 0.848 & 102.00 & 2.60 & 3.60 & $\mathrm{P}$ & 1.776 & 17.80 & I
\end{tabular} $\begin{array}{lllllllllllll}160.7 & 5165 . & 0.259 & 60.63 & 0.280 & 0.848 & 102.00 & 3.21 & 3.60 & \mathrm{P} & 1.52 \mathrm{I} & 22.50\end{array}$ $\begin{array}{lllllllllllllll}142.4 & 5166 . & 0.224 & 68.04 & 0.281 & 0.848 & 102.00 & 3.62 & 3.60 & \mathrm{P} & 1.348 & 25.60 & \mathrm{I}\end{array}$ $\begin{array}{lllllllllllll}119.4 & 5166 . & 0.187 & 81.14 & 0.281 & 0.848 & 102.00 & 4.17 & 3.60 & \mathrm{P} & 1.130 & 30.10 & \mathrm{I}\end{array}$ $\begin{array}{llllllllllllll}101.6 & 5166 & 0.158 & 98.35 & 0.281 & 0.848 & 102.00 & 4.74 & 3.60 & \mathrm{P} & 0.962 & 36.00 & \text { I }\end{array}$ $\begin{array}{llllllllllllll}98.5 & 5 \mathrm{I} 65 . & 0.147 & \mathrm{I} 07.90 & 0.281 & 0.848 & 102.00 & 5.01 & 3.60 & \mathrm{P} & 0.932 & 44.30 & \mathrm{I}\end{array}$ $\begin{array}{lllllllllllll}101.2 & 5165 & 0.137 & 117.70 & 0.280 & 0.848 & 102.00 & 5.28 & 3.60 & \mathrm{P} & 0.958 & 58.10 & 1\end{array}$ $\begin{array}{lllllllllllll}130.2 & 5165 & 0.116 & 136.60 & 0.280 & 0.848 & 102.00 & 5.92 & 3.60 & \mathrm{P} & 1.232 & 76.90 & \mathrm{O} 2\end{array}$ $\begin{array}{lllllllllllll}266.5 & 8193 & 0.768 & 16.76 & 0.256 & 0.839 & 102.00 & 1.23 & 3.61 & \mathrm{P} & 2.132 & 12.10 & \text { I }\end{array}$ $\begin{array}{llllllllllllll}247.4 & 8189 & 0.56 \mathrm{I} & 19.86 & 0.256 & 0.839 & 102.00 & 1.65 & 3.61 & \mathrm{P} & 1.978 & 10.10 & \text { I }\end{array}$ $\begin{array}{lllllllllllll}235.9 & 8186 & 0.474 & 21.67 & 0.256 & 0.839 & 102.00 & 1.92 & 3.62 & \mathrm{P} & 1.887 & 9.50 & \mathrm{I}\end{array}$ $\begin{array}{lllllllllllll}222.9 & 8185 & 0.400 & 23.58 & 0.256 & 0.839 & 102.00 & 2.24 & 3.62 & \mathrm{P} & 1.783 & 9.90 & \mathrm{I}\end{array}$ $\begin{array}{lllllllllllll}192.4 & 8183 & 0.281 & 27.87 & 0.255 & 0.839 & 102.00 & 3.05 & 3.62 & \mathrm{P} & 1.539 & 12.50 & \mathrm{I}\end{array}$ $\begin{array}{lllllllllllll}157.4 & 8181 & 0.198 & 32.52 & 0.255 & 0.839 & 102.00 & 4.09 & 3.62 & \mathrm{P} & 1.259 & 16.00 & \mathrm{I}\end{array}$ $\begin{array}{llllllllllllll}133.5 & 8179 & 0.158 & 35.65 & 0.255 & 0.839 & 102.00 & 4.88 & 3.62 & \mathrm{P} & 1.067 & 18.00 & 1\end{array}$ $\begin{array}{lllllllllllllll}97.7 & 8178 . & 0.118 & 39.57 & 0.255 & 0.839 & 102.00 & 6.09 & 3.62 & \mathrm{P} & 0.782 & 20.80 & \mathrm{I}\end{array}$ $\begin{array}{lllllllllllll}62.7 & 8177 & 0.089 & 42.75 & 0.255 & 0.839 & 102.00 & 7.35 & 3.62 & \mathrm{P} & 0.502 & 24.90 & \mathrm{O} 2\end{array}$ $\begin{array}{lllllllllllll}49.1 & 8177 . & 0.080 & 43.62 & 0.255 & 0.839 & 102.00 & 7.90 & 3.62 & P & 0.393 & 47.60 & 02\end{array}$ $\begin{array}{llllllllllllll}39.5 & 8177 & 0.073 & 43.84 & 0.255 & 0.839 & 102.00 & 8.38 & 3.62 & \mathrm{P} & 0.316 & 94.80 & \mathrm{O} 2\end{array}$ $\begin{array}{lllllllllllll}32.9 & 8176 . & 0.061 & 42.46 & 0.255 & 0.839 & 102.00 & 9.29 & 3.62 & P & 0.263 & 110.30 & 02\end{array}$ $\begin{array}{lllllllllllll}278.3 & 7421 & 0.765 & 22.09 & 0.271 & 0.845 & 102.00 & 1.23 & 3.61 & P & 2.327 & 11.90 & \text { I }\end{array}$ $\begin{array}{llllllllllllll}255.6 & 7418 & 0.582 & 25.75 & 0.271 & 0.845 & 102.00 & 1.58 & 3.61 & \mathrm{P} & 2.137 & 10.50 & \mathrm{I}\end{array}$ $\begin{array}{lllllllllllll}243.2 & 7417 & 0.506 & 27.90 & 0.270 & 0.845 & 102.00 & 1.80 & 3.61 & P & 2.034 & 10.20 & 1\end{array}$ $\begin{array}{llllllllllll}230.2 & 7416 & 0.440 & 30.2 \mathrm{I} & 0.270 & 0.845 & 102.00 & 2.04 & 3.6 \mathrm{I} & \mathrm{P} & 1.925 & 10.70\end{array}$ $\begin{array}{lllllllllllll}202.6 & 7414 & 0.333 & 35.63 & 0.270 & 0.844 & 102.00 & 2.6 \mathrm{I} & 3.6 \mathrm{I} & \mathrm{P} & 1.694 & 13.20 & \mathrm{I}\end{array}$ $\begin{array}{lllllllllllll}174.5 & 7412 & 0.256 & 42.07 & 0.270 & 0.844 & 102.00 & 3.27 & 3.6 \mathrm{I} & \mathrm{P} & 1.459 & 16.50 & \mathrm{I}\end{array}$ $\begin{array}{lllllllllllll}157.5 & 7410 & 0.217 & 47.02 & 0.270 & 0.844 & 102.00 & 3.74 & 3.61 & P & 1.317 & 18.70\end{array}$ $\begin{array}{llllllllllllll}133.4 & 7410 & 0.175 & 54.51 & 0.270 & 0.844 & 102.00 & 4.44 & 3.61 & P & 1.115 & 21.40 & I\end{array}$ $\begin{array}{llllllllllllll}113.7 & 7409 & 0.141 & 63.68 & 0.270 & 0.844 & 102.00 & 5.22 & 3.61 & P & 0.951 & 24.40 & \text { I }\end{array}$ $\begin{array}{lllllllllllllll}108.7 & 7409 & 0.128 & 68.44 & 0.270 & 0.844 & 102.00 & 5.62 & 3.61 & P & 0.909 & 30.80\end{array}$ $\begin{array}{llllllllllllllll}108.3 & 7408 & 0.115 & 72.97 & 0.270 & 0.844 & 102.00 & 6.04 & 3.61 & P & 0.906 & 43.20 & 1\end{array}$ $\begin{array}{lllllllllllllll}125.1 & 7408 . & 0.091 & 80.91 & 0.270 & 0.844 & 102.00 & 7.07 & 3.61 & P & 1.046 & 57.70 & 02\end{array}$ $\begin{array}{lllllllllllll}329.0 & 13030 & 0.790 & 13.26 & 0.268 & 0.844 & 102.00 & 1.19 & 3.61 & \mathrm{P} & 2.005 & 12.00 & \mathrm{HC}\end{array}$ $\begin{array}{llllllllllllll}299.2 & 13020 & 0.586 & 15.44 & 0.267 & 0.843 & 102.00 & 1.57 & 3.61 & \mathrm{P} & 1.823 & 9.60 \mathrm{I}\end{array}$ $\begin{array}{lllllllllllllll}283.1 & 13010 & 0.502 & 16.73 & 0.267 & 0.843 & 102.00 & 1.81 & 3.61 & P & 1.725 & 8.90 & 1\end{array}$ $\begin{array}{lllllllllllll}265.7 & 13010 & 0.429 & 18.08 & 0.267 & 0.843 & 102.00 & 2.09 & 3.61 & \mathrm{P} & 1.619 & 9.20 & \mathrm{I}\end{array}$ $\begin{array}{lllllllllllll}229.0 & 13000 & 0.312 & 21.22 & 0.266 & 0.843 & 102.00 & 2.76 & 3.61 & \mathrm{P} & 1.395 & 11.50 & \mathrm{I}\end{array}$ $\begin{array}{llllllllllllll}191.7 & 12990 & 0.230 & 24.93 & 0.266 & 0.843 & 102.00 & 3.58 & 3.61 & \mathrm{P} & 1.168 & 14.70 & \mathrm{I}\end{array}$ $\begin{array}{lllllllllllll}171.9 & 12970 . & 0.190 & 28.3 I & 0.265 & 0.843 & 102.00 & 4.18 & 3.61 & \mathrm{P} & 1.047 & 16.90 & \mathrm{I}\end{array}$ $\begin{array}{lllllllllllll}139.6 & 12970 & 0.148 & 32.70 & 0.265 & 0.843 & 102.00 & 5.08 & 3.61 & P & 0.851 & 19.20 & 1\end{array}$ $\begin{array}{llllllllllllll}112.7 & 12960 & 0.115 & 38.08 & 0.265 & 0.842 & 102.00 & 6.08 & 3.61 & P & 0.687 & 21.90 & 1\end{array}$ $\begin{array}{llllllllllllll}105.3 & 12960 & 0.103 & 40.89 & 0.265 & 0.842 & 102.00 & 6.60 & 3.61 & P & 0.642 & 30.00\end{array}$ $\begin{array}{lllllllllllllll}103.6 & 12960 & 0.091 & 43.57 & 0.265 & 0.842 & 102.00 & 7.13 & 3.61 & P & 0.632 & 44.60\end{array}$ $\begin{array}{lllllllllllllll}122.5 & 12960 & 0.069 & 48.33 & 0.265 & 0.842 & 102.00 & 8.46 & 3.61 & \mathrm{P} & 0.746 & 59.90 & \mathrm{I}\end{array}$ $\begin{array}{lllllllllllllll}319.9 & 13430 & 0.778 & 13.36 & 0.298 & 0.855 & 102.00 & 1.20 & 3.60 & \mathrm{P} & 2.016 & 11.40 & 1\end{array}$ $\begin{array}{llllllllllllll}293.6 & 13420 & 0.586 & 15.66 & 0.298 & 0.854 & 102.00 & 1.55 & 3.60 & \mathrm{P} & 1.850 & 9.30 & \mathrm{I}\end{array}$ $\begin{array}{lllllllllllll}279.3 & 13410 & 0.507 & 17.03 & 0.298 & 0.854 & 102.00 & 1.77 & 3.60 & \mathrm{P} & 1.760 & 8.70 & \mathrm{I}\end{array}$ $\begin{array}{llllllllllllll}263.9 & 13410 . & 0.438 & 18.49 & 0.297 & 0.854 & 102.00 & 2.02 & 3.60 & \mathrm{P} & 1.663 & 9.00 & \text { I }\end{array}$ $\begin{array}{lllllllllllll}230.8 & 13400 & 0.328 & 21.91 & 0.297 & 0.854 & 102.00 & 2.60 & 3.60 & \mathrm{P} & 1.454 & 11.30\end{array}$ $\begin{array}{lllllllllllll}196.7 & 13400 & 0.249 & 26.00 & 0.297 & 0.854 & 102.00 & 3.27 & 3.60 & \mathrm{P} & 1.240 & 14.60 & \mathrm{I}\end{array}$ $\begin{array}{lllllllllllll}178.5 & 13380 & 0.211 & 29.67 & 0.296 & 0.854 & 102.00 & 3.74 & 3.60 & \mathrm{P} & 1.125 & 16.90 & 1\end{array}$

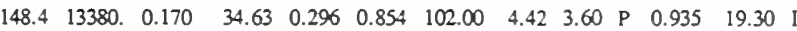
$\begin{array}{lllllllllllll}123.1 & 13370 & 0.138 & 40.76 & 0.296 & 0.854 & 102.00 & 5.15 & 3.60 & \mathrm{P} & 0.776 & 22.10 & 1\end{array}$ $\begin{array}{llllllllllllll}116.3 & 13370 & 0.125 & 43.95 & 0.296 & 0.854 & 102.00 & 5.52 & 3.60 & \mathrm{P} & 0.733 & 29.90 & 1\end{array}$ $\begin{array}{lllllllllllllll}115.1 & 13370 & 0.113 & 46.97 & 0.296 & 0.854 & 102.00 & 5.88 & 3.60 & \mathrm{P} & 0.726 & 44.30 & \text { I }\end{array}$ $\begin{array}{lllllllllllll}134.8 & 13370 & 0.091 & 52.17 & 0.295 & 0.854 & 102.00 & 6.76 & 3.60 & \mathrm{P} & 0.850 & 59.80 & 1\end{array}$ $\begin{array}{llllllllllllll}333.9 & 15740 & 0.761 & 12.44 & 0.314 & 0.860 & 102.00 & 1.22 & 3.59 & \mathrm{P} & 2.104 & 11.30 & \mathrm{I}\end{array}$ $\begin{array}{llllllllllllll}307.4 & 15720 & 0.578 & 14.70 & 0.314 & 0.860 & 102.00 & 1.57 & 3.59 & \mathrm{P} & 1.937 & 9.20 & 1\end{array}$ $\begin{array}{lllllllllllll}293.0 & 15720 & 0.502 & 16.06 & 0.313 & 0.860 & 102.00 & 1.78 & 3.59 & \mathrm{P} & 1.846 & 8.60 & 1\end{array}$ $\begin{array}{lllllllllllllll}277.4 & 15710 & 0.437 & 17.52 & 0.313 & 0.860 & 102.00 & 2.01 & 3.59 & \mathrm{P} & 1.748 & 9.00 & 1\end{array}$ $\begin{array}{lllllllllllll}243.9 & 15700 & 0.333 & 21.02 & 0.313 & 0.860 & 102.00 & 2.54 & 3.59 & \mathrm{P} & 1.537 & 11.50 & 1\end{array}$ $\begin{array}{lllllllllllll}209.4 & 15690 & 0.259 & 25.34 & 0.312 & 0.859 & 102.00 & 3.13 & 3.59 & \mathrm{P} & 1.320 & 14.90\end{array}$ $\begin{array}{lllllllllllll}193.0 & 15660 & 0.224 & 29.55 & 0.311 & 0.859 & 102.00 & 3.52 & 3.59 & \mathrm{P} & 1.216 & 17.50\end{array}$ $\begin{array}{lllllllllllllll}163.1 & 15660 & 0.186 & 35.26 & 0.311 & 0.859 & 102.00 & 4.06 & 3.59 & \mathrm{P} & 1.028 & 20.30 & 1\end{array}$ $\begin{array}{lllllllllllllll}139.8 & 15650 & 0.156 & 42.69 & 0.311 & 0.859 & 102.00 & 4.62 & 3.59 & \mathrm{P} & 0.881 & 23.70\end{array}$

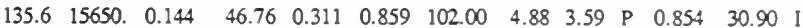

$\begin{array}{lllllllllllll}138.6 & 15650 & 0.134 & 50.73 & 0.311 & 0.859 & 102.00 & 5.15 & 3.59 & \mathrm{P} & 0.874 & 43.40 & \mathrm{I}\end{array}$ 174.215650 .0 .112 $\begin{array}{lll}344.5 & 15180 . & 0.779\end{array}$ 313.9 15170. 0.580 $297.415160,0.498$ 279.615150 .0 .428 241.8 15140. 0.315 203.415130 .0 .235 $\begin{array}{llll}184.3 & 15100 & 0.197\end{array}$ $\begin{array}{llll}151.0 & 15100.0 .156\end{array}$ $\begin{array}{lllll}123.9 & 15090 . & 0.125\end{array}$ $\begin{array}{llll}117.1 & 15090 & 0.112\end{array}$ $\begin{array}{lll}117.0 & 15090 & 0.10\end{array}$ $\begin{array}{llll}142.8 & 15080 & 0.080\end{array}$ $\begin{array}{lll}344 . I & 14120 . & 0.768\end{array}$ $\begin{array}{llll}312.3 & 14100 . & 0.570\end{array}$ $\begin{array}{llll}295.2 & 14080 & 0.488\end{array}$ $\begin{array}{llll}276.8 & 14080 & 0.417\end{array}$ 237.9 14060. 0.30 198.9 I4050. 0.223 $\begin{array}{llll}180.3 & 14020 & 0.186\end{array}$ 146.514020 .0 .14 118.7 14010. 0.113 111.2 I40I0. 0.100 109.9 [4010. 0.089 I31.4 I4000. 0.068 307.0 9968. 0.769 279.59961 .0 .578 264.7 9957. 0.498 249.19955 .0 .429 216.29950 .0 .316 183.3 9945. 0.236 $\begin{array}{lll}165.0 & 9937,0.196\end{array}$ $136.89935,0.154$ 113.29933 .0 .120 $\begin{array}{lll}106.5 & 9932 . & 0.106\end{array}$ $\begin{array}{llll}104.8 & 9932.0 .095\end{array}$ 120.49930 .0 .07 $\begin{array}{lll}305.0 & 9625 . & 0.772\end{array}$ $\begin{array}{lll}276.7 & 9616 . & 0.573\end{array}$ 261.5 9611. 0.490 245.3 9609. 0.418 $\begin{array}{lll}211.0 & 9603 . & 0.303\end{array}$ 176.69598 .0 .220 157.3 9589. 0.179 27.4 9587. 0.136 10I.6 9585. 0.102 $\begin{array}{llll}93.6 & 9584 . & 0.089\end{array}$ $90.29584,0.078$ $100.6 \quad 9583,0.056$ 297.6 I0830. 0.768 $274.6 \quad 10820.0 .595$ $262.1 \quad 10820.0 .522$ 248.9 10820. 0.460 220.610810 .0 .358 $\begin{array}{lll}191.8 & 10810, & 0.285\end{array}$ $\begin{array}{llll}176.7 & 10800 & 0.24\end{array}$ $\begin{array}{lll}151.8 & 10800 & 0.209\end{array}$ $\begin{array}{llll}132.2 & 10800 & 0.178\end{array}$ $\begin{array}{llll}127.9 & 10790 & 0.16\end{array}$ $\begin{array}{lll}129.2 & 10790 & 0.153\end{array}$ $\begin{array}{llll}153.5 & 10790 & 0.130\end{array}$ 297.4 11820. 0.778 275.1 11820. 0.59 $\begin{array}{lll}262.8 & 11810 & 0.522\end{array}$ $\begin{array}{llll}249.8 & \text { I1810. } & 0.458\end{array}$ 221.711810 .0 .353 $\begin{array}{llll}192.6 & 11800 & 0.279\end{array}$ 176.5 I1790. 0.242 151.3 I1790. 0.20 $\begin{array}{lll}31.7 & 11790,0.172\end{array}$ $\begin{array}{llll}128.0 & 11790 & 0.159\end{array}$ $\begin{array}{llll}130.5 & \text { I1790. } & 0.14\end{array}$ $\begin{array}{lll}158.8 & 11790 . & 0.12\end{array}$ $\begin{array}{llll}311.9 & 10960 & 0.787\end{array}$ 283.7 10950. 0.59 $\begin{array}{lllll}268.5 & 10940 & 0.507\end{array}$ $\begin{array}{lll}252.5 & \text { I0940 } & 0.437\end{array}$ 218.5 10930. 0.32 $\begin{array}{lll}184.5 & 10930 . & 0.24\end{array}$ $\begin{array}{lll}166.1 & 10920, & 0.20\end{array}$ 137.1 I0920. 0.158 113.4 10910. 0.125 $\begin{array}{lll}107.3 & 10910 . & 0.112\end{array}$ 106.6 10910. 0.100 126.4 10910. 0.076 255.4 4793. 0.768 $\begin{array}{lll}237.4 & 4792 & 0.569\end{array}$

$\begin{array}{llllllllllllll} & 96.310 & 0.859 & 102.00 & 5.79 & 3.59 & \mathrm{P} & 1.097 & 61.00 & 02\end{array}$ $\begin{array}{lllllllllll}12.25 & 0.283 & 0.849 & 102.00 & 1.20 & 3.60 & \mathrm{P} & 2.171 & 11.90 & \mathrm{HC}\end{array}$ $\begin{array}{llllllllll}14.31 & 0.283 & 0.849 & 102.00 & 1.58 & 3.60 & \mathrm{P} & 1.978 & 9.50 & \text { I }\end{array}$ $\begin{array}{llllllllll}5.54 & 0.282 & 0.849 & 102.00 & 1.81 & 3.60 & \mathrm{P} & 1.874 & 8.80 & 1\end{array}$ $\begin{array}{llllllllll}16.84 & 0.282 & 0.849 & 102.00 & 2.08 & 3.60 & \mathrm{P} & 1.762 & 9.10 & 1\end{array}$ $\begin{array}{llllllllll}19.88 & 0.282 & 0.849 & 102.00 & 2.72 & 3.60 & P & 1.523 & 11.50 & 1\end{array}$ $\begin{array}{lllllllllll}3.54 & 0.281 & 0.849 & 102.00 & 3.48 & 3.60 & \mathrm{P} & 1.282 & 14.80 & \mathrm{I}\end{array}$ $\begin{array}{llllllllll}27.07 & 0.280 & 0.848 & 102.00 & 4.01 & 3.60 & \mathrm{P} & 1.161 & 17.10 & \mathrm{I}\end{array}$ $\begin{array}{llllllllll}31.62 & 0.280 & 0.848 & 102.00 & 4.79 & 3.60 & \mathrm{P} & 0.952 & 19.60 & \mathrm{I}\end{array}$ $\begin{array}{llllllllll}37.39 & 0.280 & 0.848 & 102.00 & 5.64 & 3.60 & \mathrm{P} & 0.781 & 22.40 & \mathrm{I}\end{array}$ $\begin{array}{llllllllll}40.50 & 0.280 & 0.848 & 102.00 & 6.06 & 3.60 & P & 0.738 & 30.20 & \text { I }\end{array}$ $\begin{array}{lllllllllll}43.54 & 0.279 & 0.848 & 102.00 & 6.49 & 3.60 & \mathrm{P} & 0.737 & 44.00 & \mathrm{I}\end{array}$ $\begin{array}{llllllllll}49.20 & 0.279 & 0.848 & 102.00 & 7.55 & 3.60 & \mathrm{P} & 0.900 & 60.50 & \mathrm{CU}\end{array}$ $\begin{array}{lllllllllll}13.09 & 0.248 & 0.836 & 102.00 & 1.23 & 3.62 & \mathrm{P} & 2.168 & 12.40 & \mathrm{HC}\end{array}$ $\begin{array}{llllllllll}15.22 & 0.247 & 0.835 & 102.00 & 1.63 & 3.62 & \mathrm{P} & 1.968 & 9.80 & \mathrm{I}\end{array}$ $\begin{array}{lllllllll}16.48 & 0.246 & 0.835 & 102.00 & 1.88 & 3.62 & \mathrm{P} & 1.860 & 9.10\end{array}$ $\begin{array}{llllllllll}17.79 & 0.246 & 0.835 & 102.00 & 2.16 & 3.62 & \mathrm{P} & 1.744 & 9.30 & 1\end{array}$ $\begin{array}{llllllllll}20.83 & 0.245 & 0.835 & 102.00 & 2.87 & 3.62 & P & 1.499 & 11.70\end{array}$ $\begin{array}{lllllllll}24.46 & 0.245 & 0.835 & 102.00 & 3.73 & 3.62 & \mathrm{P} & 1.253 & 14.90\end{array}$ $\begin{array}{llllllllll}28.10 & 0.244 & 0.834 & 102.00 & 4.35 & 3.62 & \mathrm{P} & 1.136 & 17.20 & \mathrm{I}\end{array}$ $\begin{array}{llllllllll}32.51 & 0.244 & 0.834 & 102.00 & 5.31 & 3.62 & \mathrm{P} & 0.923 & 19.40\end{array}$ $\begin{array}{llllllllll}38.03 & 0.243 & 0.834 & 102.00 & 6.38 & 3.62 & \mathrm{P} & 0.748 & 22.10 & \mathrm{I}\end{array}$ $\begin{array}{llllllllll}41.00 & 0.243 & 0.834 & 102.00 & 6.93 & 3.62 & \mathrm{P} & 0.701 & 29.70 & \text { I }\end{array}$ $\begin{array}{lllllllllll}43.89 & 0.243 & 0.834 & 102.00 & 7.49 & 3.62 & \mathrm{P} & 0.692 & 43.40 & \mathrm{I}\end{array}$ $\begin{array}{llllllllll}49.35 & 0.243 & 0.834 & 102.00 & 8.93 & 3.62 & P & 0.828 & 59.90 & C U\end{array}$ $\begin{array}{lllllllll}17.18 & 0.255 & 0.839 & 102.00 & 1.23 & 3.62 & \mathrm{P} & 2.132 & 12.10\end{array}$ $\begin{array}{llllllllll}19.89 & 0.254 & 0.838 & 102.00 & 1.60 & 3.62 & \mathrm{P} & 1.941 & 10.00\end{array}$ $\begin{array}{llllllllll}21.48 & 0.254 & 0.838 & 102.00 & 1.84 & 3.62 & P & 1.838 & 9.40 & I\end{array}$ $\begin{array}{llllllllll}23.15 & 0.254 & 0.838 & 102.00 & 2.10 & 3.62 & P & 1.730 & 9.70 & 1\end{array}$ $\begin{array}{lllllllll}27.04 & 0.254 & 0.838 & 102.00 & 2.76 & 3.62 & P & 1.502 & 12.00\end{array}$ $\begin{array}{lllllllll}31.64 & 0.253 & 0.838 & 102.00 & 3.54 & 3.62 & P & 1.273 & 15.10\end{array}$ $\begin{array}{llllllllll}35.56 & 0.253 & 0.838 & 102.00 & 4.12 & 3.62 & \mathrm{P} & 1.146 & 17.20 & 1\end{array}$ $\begin{array}{llllllllll}40.96 & 0.253 & 0.838 & 102.00 & 5.00 & 3.62 & P & 0.950 & 19.50 & 1\end{array}$ $\begin{array}{llllllllll}47.66 & 0.253 & 0.838 & 102.00 & 6.02 & 3.62 & P & 0.786 & 22.20\end{array}$ $\begin{array}{llllllllll}51.25 & 0.253 & 0.838 & 102.00 & 6.55 & 3.62 & \mathrm{P} & 0.740 & 29.70\end{array}$ $\begin{array}{lllllllll}54.73 & 0.253 & 0.838 & 102.00 & 7.10 & 3.62 & \mathrm{P} & 0.728 & 44.40\end{array}$ $\begin{array}{lllllllllll}61.27 & 0.253 & 0.838 & 102.00 & 8.48 & 3.62 & \mathrm{P} & 0.836 & 60.40 & \mathrm{CU}\end{array}$ $\begin{array}{llllllllll}16.95 & 0.231 & 0.829 & 102.00 & 1.23 & 3.63 & \mathrm{P} & 2.084 & 12.20 & 1\end{array}$ $\begin{array}{llllllllll}19.58 & 0.230 & 0.829 & 102.00 & 1.63 & 3.63 & \mathrm{P} & 1.891 & 9.90\end{array}$ $\begin{array}{lllllllllll}21.11 & 0.230 & 0.828 & 102.00 & 1.88 & 3.63 & P & 1.787 & 9.30 & 1\end{array}$ $\begin{array}{llllllllll}22.7 \mathrm{I} & 0.230 & 0.828 & 102.00 & 2.18 & 3.63 & \mathrm{P} & 1.676 & 9.50 & \mathrm{I}\end{array}$ $\begin{array}{lllllllll}26.39 & 0.229 & 0.828 & 102.00 & 2.91 & 3.63 & \mathrm{P} & 1.442 & 11.80\end{array}$ $\begin{array}{lllllllll}30.66 & 0.229 & 0.828 & 102.00 & 3.83 & 3.63 & \mathrm{P} & 1.207 & 14.90\end{array}$ $\begin{array}{llllllllll}34.29 & 0.229 & 0.828 & 102.00 & 4.54 & 3.64 & \mathrm{P} & 1.075 & 16.90\end{array}$ $\begin{array}{lllllllll}39.06 & 0.229 & 0.828 & 102.00 & 5.65 & 3.64 & \mathrm{P} & 0.870 & 19.00\end{array}$ $\begin{array}{llllllllll}44.78 & 0.228 & 0.828 & 102.00 & 6.98 & 3.64 & P & 0.695 & 21.50\end{array}$ $\begin{array}{lllllllllll}47.73 & 0.228 & 0.828 & 102.00 & 7.68 & 3.64 & P & 0.640 & 29.80 & I\end{array}$ $\begin{array}{lllllllllll}50.47 & 0.228 & 0.828 & 102.00 & 8.43 & 3.64 & P & 0.616 & 46.10\end{array}$ $\begin{array}{lllllllllll}55.32 & 0.228 & 0.828 & 102.00 & 10.33 & 3.64 & P & 0.688 & 61.30 & \text { I }\end{array}$ $\begin{array}{llllllllll}17.05 & 0.299 & 0.855 & 102.00 & 1.21 & 3.60 & P & 2.100 & 11.40 & 1\end{array}$ $\begin{array}{lllllllll}20.00 & 0.299 & 0.855 & 102.00 & 1.53 & 3.60 & P & 1.938 & 9.70\end{array}$ $\begin{array}{lllllllll}21.75 & 0.299 & 0.855 & 102.00 & 1.73 & 3.60 & P & 1.850 & 9.30\end{array}$ $\begin{array}{lllllllllll}23.64 & 0.299 & 0.855 & 102.00 & 1.93 & 3.60 & P & 1.757 & 9.70 & I\end{array}$ $\begin{array}{lllllllll}28.13 & 0.298 & 0.855 & 102.00 & 2.41 & 3.60 & \mathrm{P} & 1.557 & 12.10\end{array}$ $\begin{array}{lllllllll}33.58 & 0.298 & 0.855 & 102.00 & 2.92 & 3.60 & \mathrm{P} & 1.354 & 15.50\end{array}$ $\begin{array}{llllllllll}38.39 & 0.298 & 0.854 & 102.00 & 3.27 & 3.60 & P & 1.247 & 17.80\end{array}$ $\begin{array}{lllllllll}45.27 & 0.297 & 0.854 & 102.00 & 3.76 & 3.60 & P & 1.072 & 20.60\end{array}$ $\begin{array}{llllllllll}54.09 & 0.297 & 0.854 & 102.00 & 4.27 & 3.60 & \mathrm{P} & 0.933 & 23.80\end{array}$ $\begin{array}{llllllllll}58.90 & 0.297 & 0.854 & 102.00 & 4.51 & 3.60 & P & 0.903 & 30.60\end{array}$ $\begin{array}{lllllllll}63.6 \mathrm{I} & 0.297 & 0.854 & 102.00 & 4.76 & 3.60 & \mathrm{P} & 0.9 \mathrm{I} 2 & 43.60\end{array}$ $\begin{array}{llllllllll}72.34 & 0.297 & 0.854 & 102.00 & 5.34 & 3.60 & \mathrm{P} & 1.084 & 60.10\end{array}$ $\begin{array}{llllllllll}14.99 & 0.337 & 0.868 & 102.00 & 1.18 & 3.59 & P & 2.110 & 10.70\end{array}$ $\begin{array}{llllllllll}17.71 & 0.337 & 0.868 & 102.00 & 1.50 & 3.59 & P & 1.952 & 9.10 & 1\end{array}$ $\begin{array}{llllllllll}19.34 & 0.337 & 0.867 & 102.00 & 1.70 & 3.59 & \mathrm{P} & \mathrm{I} .865 & 8.80\end{array}$ $\begin{array}{lllllllll}21.12 & 0.337 & 0.867 & 102.00 & 1.90 & 3.59 & P & 1.773 & 9.20\end{array}$ $\begin{array}{llllllllll}25.37 & 0.336 & 0.867 & 102.00 & 2.38 & 3.59 & P & 1.574 & 11.60 & 1\end{array}$ $\begin{array}{lllllllll}30.59 & 0.336 & 0.867 & 102.00 & 2.89 & 3.59 & P & I .367 & 15.00\end{array}$ $\begin{array}{llllllllll}35.06 & 0.336 & 0.867 & 102.00 & 3.23 & 3.59 & P & I .253 & 17.40\end{array}$ $\begin{array}{llllllllll}41.66 & 0.336 & 0.867 & 102.00 & 3.70 & 3.59 & P & 1.074 & 20.20\end{array}$ $\begin{array}{llllllllll}50.02 & 0.336 & 0.867 & 102.00 & 4.19 & 3.59 & P & 0.935 & 23.60\end{array}$ $\begin{array}{lllllllll}54.46 & 0.335 & 0.867 & 102.00 & 4.43 & 3.59 & \mathrm{P} & 0.909 & 30.10\end{array}$ $\begin{array}{llllllllll}58.72 & 0.335 & 0.867 & 102.00 & 4.67 & 3.59 & P & 0.926 & 42.10\end{array}$ $\begin{array}{llllllllll}66.03 & 0.335 & 0.867 & 102.00 & 5.23 & 3.59 & \mathrm{P} & 1.127 & 57.00 & \mathrm{O} 2\end{array}$ $\begin{array}{lllllllll}15.40 & 0.266 & 0.843 & 102.00 & 1.20 & 3.61 & \mathrm{P} & 2.082 & 11.70\end{array}$ $\begin{array}{lllllllllll}17.88 & 0.266 & 0.843 & 102.00 & 1.57 & 3.61 & P & 1.894 & 9.60 & 1\end{array}$ $\begin{array}{lllllllll}19.35 & 0.266 & 0.843 & 102.00 & 1.80 & 3.61 & P & 1.793 & 9.00\end{array}$ $\begin{array}{llllllllll}20.90 & 0.265 & 0.843 & 102.00 & 2.06 & 3.61 & P & 1.685 & 9.20\end{array}$ $\begin{array}{lllllllll}24.50 & 0.265 & 0.843 & 102.00 & 2.69 & 3.61 & P & 1.459 & 11.50\end{array}$ $\begin{array}{llllllllll}28.78 & 0.265 & 0.842 & 102.00 & 3.45 & 3.61 & \mathrm{P} & 1.231 & 14.70 & 1\end{array}$ $\begin{array}{llllllllll}32.55 & 0.264 & 0.842 & 102.00 & 4.00 & 3.61 & P & 1.109 & 16.80\end{array}$ $\begin{array}{lllllllll}37.64 & 0.264 & 0.842 & 102.00 & 4.83 & 3.61 & P & 0.915 & 19.10\end{array}$ $\begin{array}{llllllllll}43.98 & 0.264 & 0.842 & 102.00 & 5.76 & 3.61 & P & 0.757 & 21.80 & 1\end{array}$ $\begin{array}{llllllllll}47.35 & 0.264 & 0.842 & 10200 & 6.23 & 3.61 & P & 0.716 & 29.20\end{array}$ $\begin{array}{lllllllllll}50.63 & 0.264 & 0.842 & 102.00 & 6.73 & 3.61 & \mathrm{P} & 0.712 & 43.10 & 1\end{array}$ $\begin{array}{lllllllllll}56.72 & 0.264 & 0.842 & 102.00 & 7.98 & 3.61 & P & 0.844 & 58.10 & C U\end{array}$ $\begin{array}{lllllllllll}28.55 & 0.230 & 0.828 & 102.00 & 1.24 & 3.63 & P & 2.558 & 14.90 & 1\end{array}$ $\begin{array}{lllllllllll}33.79 & 0.230 & 0.828 & 102.00 & \text { I.64 } & 3.63 & \text { P } & 2.813 & 13.30 & \text { I }\end{array}$ 
\begin{tabular}{llllllllllll|}
226.8 & 4792 & 0.486 & 36.88 & 0.230 & 0.828 & 102.00 & 1.90 & 3.63 & $\mathrm{P}$ & 2.115 & 13.10
\end{tabular} \begin{tabular}{lllllllllllll|}
215.2 & 4791. & 0.415 & 40.21 & 0.230 & 0.828 & 102.00 & 2.19 & 3.63 & $\mathrm{P}$ & 2.044 & 13.80
\end{tabular} $\begin{array}{lllllllllllll}188.6 & 4791 . & 0.300 & 47.88 & 0.230 & 0.828 & 102.00 & 2.93 & 3.63 & P & 1.792 & 17.10 & \text { I }\end{array}$ $\begin{array}{lllllllllllllll}158.6 & 4790 & 0.219 & 56.62 & 0.229 & 0.828 & 102.00 & 3.85 & 3.63 & \mathrm{P} & 1.507 & 21.50 & \mathrm{I}\end{array}$ $\begin{array}{llllllllllllll}137.0 & 4790 & 0.179 & 62.19 & 0.230 & 0.828 & 102.00 & 4.55 & 3.63 & \mathrm{P} & 1.301 & 23.80 & \text { I }\end{array}$ $\begin{array}{llllllllllllll}106.5 & 4790 & 0.138 & 70.51 & 0.230 & 0.828 & 102.00 & 5.60 & 3.63 & \mathrm{P} & 1.012 & 27.20 & \mathrm{I}\end{array}$ $\begin{array}{lllllllllllll}76.9 & 4790 & 0.107 & 78.55 & 0.230 & 0.828 & 102.00 & 6.75 & 3.63 & \mathrm{P} & 0.730 & 30.80 & \mathrm{I}\end{array}$ $\begin{array}{llllllllllllll}65.6 & 4790 & 0.096 & 81.58 & 0.230 & 0.828 & 102.00 & 7.29 & 3.63 & \mathrm{P} & 0.623 & 43.80 & \mathrm{O} 2\end{array}$ $\begin{array}{lllllllllllll}58.2 & 4790 & 0.087 & 83.72 & 0.229 & 0.828 & 102.00 & 7.82 & 3.63 & \mathrm{P} & 0.553 & 71.30 & 02\end{array}$ $\begin{array}{lllllllllllll}56.2 & 4790 & 0.070 & 84.53 & 0.229 & 0.828 & 102.00 & 8.97 & 3.63 & P & 0.533 & 88.10 & 02\end{array}$ $\begin{array}{lllllllllllll}263.2 & 4956 . & 0.759 & 30.44 & 0.253 & 0.838 & 102.00 & 1.24 & 3.62 & \mathrm{P} & 2.667 & 15.00 & \mathrm{I}\end{array}$ $\begin{array}{lllllllllllll}242.0 & 4955 & 0.573 & 35.77 & 0.253 & 0.838 & 102.00 & 1.61 & 3.62 & \mathrm{P} & 2.881 & 13.60 & \mathrm{I}\end{array}$ $\begin{array}{llllllllllllll}230.3 & 4955 & 0.496 & 38.95 & 0.253 & 0.838 & 102.00 & 1.84 & 3.62 & \mathrm{P} & 2.133 & 13.60 & 1\end{array}$ $\begin{array}{lllllllllllllll}218.1 & 4955 & 0.429 & 42.44 & 0.253 & 0.838 & 102.00 & 2.10 & 3.62 & \mathrm{P} & 2.049 & 14.40 & \mathrm{I}\end{array}$

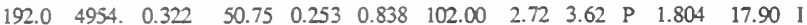
$\begin{array}{lllllllllllll}165.0 & 4953 . & 0.246 & 60.91 & 0.253 & 0.838 & 102.00 & 3.43 & 3.62 & \mathrm{P} & 1.550 & 22.40 & 1\end{array}$ $\begin{array}{llllllllllllll}146.5 & 4954 & 0.208 & 68.02 & 0.253 & 0.838 & 102.00 & 3.93 & 3.62 & \mathrm{P} & 1.376 & 25.20 & \mathrm{I}\end{array}$ $\begin{array}{llllllllllllll}122.8 & 4953 & 0.168 & 80.25 & 0.253 & 0.838 & 102.00 & 4.66 & 3.62 & \mathrm{P} & 1.154 & 29.30 & \mathrm{I}\end{array}$ $\begin{array}{lllllllllllll}103.8 & 4953 . & 0.137 & 95.75 & 0.253 & 0.838 & 102.00 & 5.45 & 3.62 & \mathrm{P} & 0.975 & 34.40 & 1\end{array}$ $\begin{array}{lllllllllllll}99.4 & 4953 . & 0.125 & 104.00 & 0.253 & 0.838 & 102.00 & 5.84 & 3.62 & \mathrm{P} & 0.934 & 42.10 & \mathrm{I}\end{array}$ $\begin{array}{lllllllllllll}100.4 & 4953 & 0.114 & 112.20 & 0.253 & 0.838 & 102.00 & 6.24 & 3.62 & \mathrm{P} & 0.943 & 56.00 & \mathrm{I}\end{array}$ $\begin{array}{lllllllllllll}122.6 & 4953 . & 0.092 & 127.00 & 0.253 & 0.838 & 102.00 & 7.24 & 3.62 & \mathrm{P} & 1.152 & 74.50 & 02\end{array}$ $\begin{array}{lllllllllllll}265.6 & 5720 & 0.792 & 25.95 & 0.325 & 0.864 & 102.00 & 1.17 & 3.59 & \mathrm{P} & 2.713 & 14.00 & \mathrm{I}\end{array}$ $\begin{array}{lllllllllllll}243.4 & 5719 & 0.600 & 30.50 & 0.325 & 0.864 & 102.00 & 1.51 & 3.59 & \mathrm{P} & 2.911 & 12.80 & \mathrm{I}\end{array}$

$\begin{array}{lllllllllllll}231.1 & 5719 . & 0.520 & 33.18 & 0.325 & 0.864 & 102.00 & 1.71 & 3.59 & \mathrm{P} & 2.187 & 12.70 & \text { I }\end{array}$ $\begin{array}{llllllllllllll}218.2 & 5718 & 0.452 & 36.13 & 0.325 & 0.864 & 102.00 & 1.94 & 3.59 & \mathrm{P} & 2.065 & 13.50 & \mathrm{I}\end{array}$ $\begin{array}{llllllllllllll}190.2 & 5718 & 0.342 & 43.07 & 0.325 & 0.864 & 102.00 & 2.46 & 3.59 & \mathrm{P} & 1.800 & 16.70 & 1\end{array}$ $\begin{array}{lllllllllllll}160.9 & 5717 & 0.265 & 51.36 & 0.325 & 0.864 & 102.00 & 3.04 & 3.59 & \mathrm{P} & 1.523 & 20.90 & 1\end{array}$ $\begin{array}{lllllllllllll}141.0 & 5718 & 0.227 & 57.10 & 0.325 & 0.864 & 102.00 & 3.44 & 3.59 & \mathrm{P} & 1.335 & 23.50 & \mathrm{I}\end{array}$ $\begin{array}{lllllllllllllll}115.1 & 5718 & 0.187 & 66.58 & 0.325 & 0.864 & 102.00 & 3.98 & 3.59 & \mathrm{P} & 1.089 & 27.40 & \mathrm{I}\end{array}$ $\begin{array}{lllllllllllll}93.3 & 5717 & 0.157 & 77.86 & 0.325 & 0.864 & 102.00 & 4.52 & 3.59 & \mathrm{P} & 0.883 & 31.80 & 1\end{array}$ $\begin{array}{lllllllllllllll}87.5 & 5717 & 0.145 & 83.46 & 0.325 & 0.864 & 102.00 & 4.78 & 3.59 & P & 0.828 & 40.20 & \text { I }\end{array}$ $\begin{array}{llllllllllllllll}86.7 & 5717 . & 0.135 & 88.63 & 0.325 & 0.864 & 102.00 & 5.03 & 3.59 & \mathrm{P} & 0.820 & 54.90 & \mathrm{I}\end{array}$ $\begin{array}{lllllllllllll}103.4 & 5717 & 0.114 & 96.64 & 0.325 & 0.864 & 102.00 & 5.63 & 3.59 & \mathrm{P} & 0.978 & 69.00 & \mathrm{I}\end{array}$ $\begin{array}{lllllllllllll}274.6 & 9019 & 0.782 & 16.47 & 0.304 & 0.857 & 102.00 & 1.19 & 3.59 & \mathrm{P} & 2.190 & 11.40 & \text { I }\end{array}$ $\begin{array}{llllllllllllll}254.3 & 9016 . & 0.584 & 19.67 & 0.304 & 0.856 & 102.00 & 1.56 & 3.59 & \mathrm{P} & 2.028 & 9.80 & \mathrm{I}\end{array}$ $\begin{array}{lllllllllllll}242.9 & 9014 & 0.502 & 21.61 & 0.303 & 0.856 & 102.00 & 1.78 & 3.59 & \mathrm{P} & 1.936 & 9.40 & \mathrm{I}\end{array}$ $\begin{array}{lllllllllllll}230.4 & 9013 & 0.432 & 23.73 & 0.303 & 0.856 & 102.00 & 2.04 & 3.59 & \mathrm{P} & 1.837 & 9.90 & \mathrm{I}\end{array}$ $\begin{array}{lllllllllllllll}202.8 & 9010 & 0.321 & 28.84 & 0.303 & 0.856 & 102.00 & 2.63 & 3.59 & \mathrm{P} & 1.617 & 12.80 & \mathrm{I}\end{array}$ $\begin{array}{lllllllllllll}172.7 & 9008 & 0.244 & 35.08 & 0.303 & 0.856 & 102.00 & 3.31 & 3.59 & \mathrm{P} & 1.377 & 16.70 & \mathrm{I}\end{array}$ $\begin{array}{lllllllllllll}153.3 & 9006 & 0.207 & 39.93 & 0.303 & 0.856 & 102.00 & 3.77 & 3.59 & \mathrm{P} & 1.223 & 19.30 & \mathrm{I}\end{array}$ $\begin{array}{llllllllllllll}125.2 & 9005 & 0.168 & 47.36 & 0.303 & 0.856 & 102.00 & 4.42 & 3.59 & \mathrm{P} & 0.998 & 22.70 & \mathrm{I}\end{array}$ $\begin{array}{lllllllllllll}101.4 & 9004 & 0.139 & 56.09 & 0.303 & 0.856 & 102.00 & 5.07 & 3.59 & \mathrm{P} & 0.809 & 26.60 & \mathrm{I}\end{array}$ $\begin{array}{lllllllllllllll}95.4 & 9003 & 0.128 & 60.24 & 0.303 & 0.856 & 102.00 & 5.38 & 3.59 & \mathrm{P} & 0.761 & 35.80 & \mathrm{I}\end{array}$ $\begin{array}{lllllllllllllll}95.2 & 9003 . & 0.118 & 63.74 & 0.303 & 0.856 & 102.00 & 5.69 & 3.59 & \mathrm{P} & 0.759 & 49.90 & \mathrm{I}\end{array}$ $\begin{array}{lllllllllllllll}115.8 & 9003 & 0.097 & 67.95 & 0.303 & 0.856 & 102.00 & 6.45 & 3.59 & \mathrm{P} & 0.924 & 62.00 & \mathrm{I}\end{array}$ $\begin{array}{lllllllllllll}274.7 & 9009 & 0.781 & 16.46 & 0.303 & 0.856 & 102.00 & 1.19 & 3.59 & \mathrm{P} & 2.190 & 11.30 & \mathrm{I}\end{array}$ $\begin{array}{llllllllllllll}254.2 & 9005 & 0.583 & 19.66 & 0.303 & 0.856 & 102.00 & 1.56 & 3.59 & \mathrm{P} & 2.027 & 9.70 & \mathrm{I}\end{array}$ $\begin{array}{llllllllllllll}242.7 & 9004 & 0.502 & 21.58 & 0.303 & 0.856 & 102.00 & 1.79 & 3.59 & \mathrm{P} & 1.935 & 9.40 & 1\end{array}$ $\begin{array}{lllllllllllll}230.2 & 9002 . & 0.432 & 23.70 & 0.303 & 0.856 & 102.00 & 2.04 & 3.59 & \mathrm{P} & 1.835 & 9.80 & \mathrm{I}\end{array}$ $\begin{array}{lllllllllllll}202.4 & 9000 & 0.320 & 28.78 & 0.303 & 0.856 & 102.00 & 2.64 & 3.59 & \mathrm{P} & 1.614 & 12.70 & \mathrm{I}\end{array}$ $\begin{array}{lllllllllllllll}172.3 & 8998 . & 0.243 & 34.99 & 0.303 & 0.856 & 102.00 & 3.32 & 3.59 & \mathrm{P} & 1.374 & 16.60 & \mathrm{I}\end{array}$ $\begin{array}{lllllllllllll}153.1 & 8995 & 0.206 & 39.86 & 0.302 & 0.856 & 102.00 & 3.79 & 3.60 & \mathrm{P} & 1.221 & 19.10 & \mathrm{I}\end{array}$ $\begin{array}{lllllllllllllll}125.0 & 8994 & 0.167 & 47.30 & 0.302 & 0.856 & 102.00 & 4.44 & 3.60 & \mathrm{P} & 0.997 & 22.60 & \mathrm{I}\end{array}$ $\begin{array}{lllllllllllllll}101.4 & 8993 & 0.138 & 56.09 & 0.302 & 0.856 & 102.00 & 5.10 & 3.60 & \mathrm{P} & 0.809 & 26.50 & \mathrm{I}\end{array}$

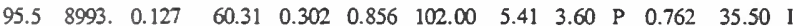
$\begin{array}{llllllllllllll}95.5 & 8993 . & 0.116 & 63.94 & 0.302 & 0.856 & 102.00 & 5.73 & 3.60 & \mathrm{P} & 0.761 & 49.50\end{array}$ $\begin{array}{llllllllllllll}116.8 & 8992 . & 0.096 & 68.50 & 0.302 & 0.856 & 102.00 & 6.50 & 3.60 & \mathrm{P} & 0.931 & 61.80 & 1\end{array}$ 
APPENDIX C

CONVECTIVE CONDENSATION RAW HEAT TRANSFER

AND PRESSURE DROP DATA WITHIN A MICRO-FIN TUBE 


\author{
Convective condensation of \\ R32 within a micro-fin tube
}

(file: taraw.tbl)

\begin{tabular}{|c|c|c|c|c|c|c|c|c|c|c|c|}
\hline $\begin{array}{c}q^{n} \\
\left.W / m^{2}\right)\end{array}$ & $\begin{array}{l}\Delta \mathrm{T}_{1} \\
(\mathrm{~K})\end{array}$ & $x_{q}$ & $\begin{array}{c}\dot{m}_{\mathrm{T}} \\
(\mathrm{kg} / \mathrm{s})\end{array}$ & $\begin{array}{c}\mathrm{P}_{r} \\
\text { (Pa) }\end{array}$ & $\begin{array}{c}T_{r} \\
\left({ }^{\circ} \mathrm{C}\right)\end{array}$ & $\begin{array}{c}z_{r} \\
(\mathrm{~m})\end{array}$ & $\begin{array}{c}T_{r} \\
\left({ }^{\circ} \mathrm{C}\right)\end{array}$ & $\begin{array}{r}z \\
(\mathrm{~m})\end{array}$ & $\begin{array}{l}\mathrm{T}_{\bar{\nu}} \\
\left({ }^{\circ} \mathrm{C}\right)\end{array}$ & $\underset{(\mathrm{g} / \mathrm{mole})}{\mathrm{M}}$ & \\
\hline & 4.072 & 0.747 & & & & & 9.186 & & & & \\
\hline 275. & & .566 & & & & & & & & & \\
\hline 5161. & 1 & & & & & & & & & & \\
\hline 3235. & 038 & 425 & & & 23.167 & 1.964 & & & 0.732 & & \\
\hline 838. & 595 & 318 & & & & & & & & & \\
\hline 124. & & & & & & & & & & & \\
\hline & & & & & & & & & & & \\
\hline 097. & & & & & & & & & & & \\
\hline 897. & & & & & & & & 4.688 & & & \\
\hline 527. & & & & & & & & 4.997 & & & \\
\hline 319. & & & & & 23.128 & & & 5.3 & & & \\
\hline 370. & & & & & & & & & & & \\
\hline & & & & & & & & & & & \\
\hline & & & & & & & & & & & \\
\hline & & & & & & & & & & & \\
\hline 365 . & & & & & & 1.964 & & & & & \\
\hline & & & & & & 2.577 & & & & & \\
\hline & & & & & & & & & & & \\
\hline & & & & & & & & & & & \\
\hline 9 & & & & & & 03 & & 4.078 & & & \\
\hline 210. & & & & & & & & 4.688 & & & \\
\hline 775 & & & & & & & & 997 & & & \\
\hline 531 & & & & & & & & & & & \\
\hline 593. & & & & & & & & & & & \\
\hline & & & & & & & & & & & \\
\hline & & & & & & & & & & & \\
\hline & & & & & & & & & & & \\
\hline & & & & & & 964 & & 1 & & & \\
\hline & & & & & & & & & & & \\
\hline & & & & & & & & & & & \\
\hline & & & & & & & & & & & \\
\hline & & & & & & & & & & & \\
\hline & & & & & & & & & & & \\
\hline & & & & & & & & & & & \\
\hline 05. & & & & & & & & & & & \\
\hline & & & & & & & & & & & \\
\hline & & & & & & & & & & & \\
\hline & & & & & & & & & & & \\
\hline & & & & & & & & & & & \\
\hline & & & & & & & & & & & \\
\hline & & & & & & & & & & & \\
\hline & & & & & & & & & & & 5 \\
\hline & & & & & & & & & & & $P$ \\
\hline & & & & & & & & & & & \\
\hline & & & & & & & & & & & \\
\hline & & & & & & & & & & & \\
\hline & & & & & & & & & & & \\
\hline & & & & & & & & & & & $=$ \\
\hline & & & & & & & & & & & \\
\hline & & & & & & & & & & & \\
\hline & & & & & & & & & & & \\
\hline & & & & & & & & & & & \\
\hline & & & & & & & & & & & \\
\hline & & & & & & & & & & & \\
\hline & & & & & & & & & & & $P$ \\
\hline & & & & & & & & 778 & & & $P$ \\
\hline & & & & & & & & 4.688 & & & \\
\hline & & & & & & & & & & & \\
\hline & & & & & & & & & & & $P$ \\
\hline & & & & & & & & & & & \\
\hline & & & & & & & & & & & \\
\hline & & & & & & & 45 & 1. & & & \\
\hline & & & & & & & & & & & \\
\hline & & & & & & 1.964 & & & & & \\
\hline & & & & & & & & 2.55 & & & \\
\hline & & & & & & 3.174 & & & & & \\
\hline & & 200 & & & & 3.5 & & 3.530 & & & \\
\hline & 3.096 & & & & & & & & & & \\
\hline & & & & & & & & & & & \\
\hline & & 0.109 & 0.01867 & & & & & & & & \\
\hline
\end{tabular}

$\begin{array}{llllllllllll}3626 . & 2.393 & 0.098 & 0.01867 & 2281270 & 36.582 & 5.938 & 32.846 & 5.301 & 34.210 & 52.02 & \mathrm{P}\end{array}$

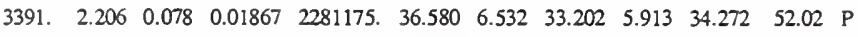
$\begin{array}{lllllllllllll}30885 . & 5.631 & 0.762 & 0.01414 & 1836843 & 28.109 & 0.125 & 10.043 & 0.712 & 22.689 & 52.02 & \mathrm{P}\end{array}$ $\begin{array}{lllllllllllll}24339 & 4.899 & 0.568 & 0.01414 & 1835993 & 28.091 & 0.738 & 14.336 & 1.325 & 22.845 & 52.02 & \mathrm{P}\end{array}$ $\begin{array}{llllllllllllll}21374 . & 4.554 & 0.487 & 0.01414 & 1835565 & 28.083 & 1.352 & 17.354 & 1.634 & 23.222 & 52.02 & P\end{array}$ $\begin{array}{llllllllllllll}18670 . & 4.232 & 0.417 & 0.01414 & 1835242 & 28.076 & 1.964 & 19.766 & 1.939 & 24.690 & 52.02 & \mathrm{P}\end{array}$ $\begin{array}{llllllllllllll}13894 & 3.634 & 0.304 & 0.01414 & 1834611 & 28.063 & 2.577 & 21.637 & 2.550 & 23.803 & 52.02 & P\end{array}$ $\begin{array}{llllllllllllll}10069 & 3.112 & 0.222 & 0.01414 & 1833994 & 28.050 & 3.174 & 22.761 & 3.148 & 24.982 & 52.02 & \mathrm{P}\end{array}$ $\begin{array}{llllllllllll}8069 . & 2.803 & 0.182 & 0.01414 & 1833230 & 28.034 & 3.504 & 23.482 & 3.530 & 25.131 & 52.02 & P\end{array}$ $\begin{array}{lllllllllllll}5780 . & 2.421 & 0.139 & 0.01414 & 1833000 & 28.029 & 4.103 & 24.255 & 4.078 & 25.947 & 52.02 & \mathrm{P}\end{array}$ $\begin{array}{llllllllllllll}4060 . & 2.057 & 0.105 & 0.01414 & 1832744 & 28.024 & 4.714 & 24.854 & 4.688 & 25.974 & 52.02 & \mathrm{P}\end{array}$ $\begin{array}{lllllllllllll}3518 . & 1.897 & 0.091 & 0.01414 & 1832615 & 28.021 & 5.325 & 25.376 & 4.997 & 26.129 & 52.02 & \mathrm{P}\end{array}$ $\begin{array}{lllllllllllll}3203 . & 1.757 & 0.080 & 0.01414 & 1832542 & 28.020 & 5.938 & 25.696 & 5.301 & 26.322 & 52.02 & P\end{array}$ $\begin{array}{llllllllllllll}3221 . & 1.523 & 0.058 & 0.01414 & 1832403 & 28.017 & 6.532 & 25.913 & 5.913 & 26.364 & 52.02 & P\end{array}$ $\begin{array}{lllllllllllll}\text { 1243. } & 1.834 & 1.013 & 0.00886 & 2118251 . & 35.768 & 0.125 & 33.262 & 0.712 & 33.727 & 52.02 & \mathrm{C}\end{array}$ $\begin{array}{lllllllllllll}2433 & -0.111 & 0.992 & 0.00886 & 2117952 & 33.626 & 0.738 & 32.965 & 1.325 & 33.459 & 52.02 & \mathrm{C}\end{array}$ $\begin{array}{lllllllllllll}\text { 3072. } & 0.030 & 0.976 & 0.00886 & 2117801 & 33.623 & 1.352 & 32.695 & 1.634 & 33.322 & 52.02 & \mathrm{C}\end{array}$ $\begin{array}{llllllllllllll}3728 & 0.196 & 0.956 & 0.00886 & 2117523 & 33.618 & 1.964 & 32.342 & 1.939 & 33.410 & 52.02 & \mathrm{C}\end{array}$ $\begin{array}{llllllllllllll}5121 . & 0.618 & 0.904 & 0.00886 & 2116940 & 33.607 & 2.577 & 31.754 & 2.550 & 32.672 & 52.02 & \mathrm{C}\end{array}$ $\begin{array}{llllllllllllll}6582 . & 1.143 & 0.837 & 0.00886 & 2116370 & 33.597 & 3.174 & 31.082 & 3.148 & 32.964 & 52.02 & \mathrm{C}\end{array}$ $\begin{array}{lllllllllllllll}7570 . & 1.519 & 0.784 & 0.00886 & 2114927 & 33.570 & 3.504 & 30.447 & 3.530 & 32.436 & 52.02 & C\end{array}$ $\begin{array}{lllllllllllllll}9055 . & 2.171 & 0.697 & 0.00886 & 2114650 & 33.565 & 4.103 & 29.357 & 4.078 & 31.977 & 52.02 & \mathrm{C}\end{array}$ $\begin{array}{llllllllllllll}10804 . & 3.007 & 0.581 & 0.00886 & 2114341 . & 33.559 & 4.714 & 27.951 & 4.688 & 30.912 & 52.02 & C\end{array}$ $\begin{array}{lllllllllllll}11729 . & 3.475 & 0.514 & 0.00886 & 2114184 & 33.556 & 5.325 & 26.166 & 4.997 & 29.808 & 52.02 & C\end{array}$ $\begin{array}{llllllllllll}12666 . & 3.968 & 0.443 & 0.00886 & 2114189 & 33.556 & 5.938 & 24.265 & 5.301 & 29.665 & 52.02 & C\end{array}$ $\begin{array}{lllllllllllll}14628 . & 5.050 & 0.283 & 0.00886 & 2114225 & 33.557 & 6.532 & 22.435 & 5.913 & 27.953 & 52.02 & \mathrm{C}\end{array}$ $\begin{array}{llllllllllllll}1172 . & 0.117 & 1.002 & 0.00887 & 2112634 & 33.916 & 0.125 & 33.167 & 0.712 & 33.586 & 52.02 & \mathrm{C}\end{array}$ $\begin{array}{lllllllllllll}2314 & -0.088 & 0.982 & 0.00887 & 2112325 & 33.521 & 0.738 & 32.885 & 1.325 & 33.335 & 52.02 & \mathrm{C}\end{array}$ $\begin{array}{lllllllllllll}2933 & 0.048 & 0.967 & 0.00887 & 2112169 & 33.519 & 1.352 & 32.628 & 1.634 & 33.210 & 52.02 & \mathrm{C}\end{array}$ $\begin{array}{llllllllllllll}3572 . & 0.209 & 0.948 & 0.00887 & 2111951 . & 33.514 & 1.964 & 32.290 & 1.939 & 33.289 & 52.02 & \mathrm{C}\end{array}$ $\begin{array}{llllllllllllll}4940 . & 0.618 & 0.898 & 0.00887 & 2111501 & 33.506 & 2.577 & 31.728 & 2.550 & 32.583 & 52.02 & \mathrm{C}\end{array}$ $\begin{array}{lllllllllllll}6388 . & 1.127 & 0.833 & 0.00887 & 2111061 & 33.498 & 3.174 & 31.075 & 3.148 & 32.880 & 52.02 & \mathrm{C}\end{array}$ $\begin{array}{llllllllllllll}\text { 7373. } & 1.488 & 0.782 & 0.00887 & 2109616 & 33.471 & 3.504 & 30.449 & 3.530 & 32.359 & 52.02 & C\end{array}$ $\begin{array}{llllllllllllll}8863 . & 2.115 & 0.697 & 0.00887 & 2109214 & 33.463 & 4.103 & 29.389 & 4.078 & 31.914 & 52.02 & C\end{array}$ $\begin{array}{lllllllllllll}10626 . & 2.918 & 0.583 & 0.00887 & 2108766 . & 33.455 & 4.714 & 28.012 & 4.688 & 30.893 & 52.02 & \mathrm{C}\end{array}$ $\begin{array}{lllllllllllll}11564 . & 3.369 & 0.518 & 0.00887 & 2108539 & 33.451 & 5.325 & 26.268 & 4.997 & 29.800 & 52.02 & C\end{array}$ $\begin{array}{llllllllllllll}12515 . & 3.842 & 0.448 & 0.00887 & 2108421 & 33.449 & 5.938 & 24.377 & 5.301 & 29.694 & 52.02 & C\end{array}$ $\begin{array}{llllllllllllll}14516 . & 4.881 & 0.289 & 0.00887 & 2108199 & 33.444 & 6.532 & 22.541 & 5.913 & 28.018 & 52.02 & C\end{array}$ $\begin{array}{lllllllllllll}\text { 1515. } & 3.547 & 1.026 & 0.01054 & 2293237 & 40.679 & 0.125 & 36.239 & 0.712 & 36.883 & 52.02 & C\end{array}$ $\begin{array}{lllllllllllllll}2949 . & 0.380 & 1.003 & 0.01054 & 2292875 & 37.273 & 0.738 & 35.837 & 1.325 & 36.598 & 52.02 & \mathrm{C}\end{array}$ $\begin{array}{lllllllllllll}3714 . & 0.065 & 0.986 & 0.01054 & 2292692 & 36.782 & 1.352 & 35.506 & 1.634 & 36.393 & 52.02 & \mathrm{C}\end{array}$ $\begin{array}{lllllllllllll}4496 . & 0.269 & 0.965 & 0.01054 & 2292385 & 36.777 & 1.964 & 35.072 & 1.939 & 36.490 & 52.02 & \mathrm{C}\end{array}$ $\begin{array}{llllllllllllll}6150 . & 0.784 & 0.911 & 0.01054 & 2291746 . & 36.766 & 2.577 & 34.362 & 2.550 & 35.597 & 52.02 & \mathrm{C}\end{array}$ $\begin{array}{llllllllllll}\text { 7874. } & 1.426 & 0.842 & 0.01054 & 2291122 & 36.755 & 3.174 & 33.568 & 3.148 & 35.924 & 52.02 & \mathrm{C}\end{array}$ $\begin{array}{llllllllllllll}9034 & 1.884 & 0.788 & 0.01054 & 2289261 & 36.722 & 3.504 & 32.869 & 3.530 & 35.295 & 52.02 & \mathrm{C}\end{array}$ $\begin{array}{llllllllllllll}10773 . & 2.678 & 0.698 & 0.01054 & 2288894 & 36.716 & 4.103 & 31.545 & 4.078 & 34.692 & 52.02 & \mathrm{C}\end{array}$ $\begin{array}{lllllllllllll}12811 . & 3.695 & 0.578 & 0.01054 & 22884866 & 36.709 & 4.714 & 29.894 & 4.688 & 33.458 & 52.02 & C\end{array}$ $\begin{array}{llllllllllllll}13887 . & 4.266 & 0.510 & 0.01054 & 2288279 & 36.705 & 5.325 & 27.742 & 4.997 & 32.098 & 52.02 & C\end{array}$ $\begin{array}{llllllllllllll}14973 . & 4.866 & 0.437 & 0.01054 & 2288240 & 36.704 & 5.938 & 25.478 & 5.301 & 31.957 & 52.02 & \mathrm{C}\end{array}$ $\begin{array}{llllllllllllll}17243 & 6.182 & 0.273 & 0.01054 & 2288186 & 36.703 & 6.532 & 23.402 & 5.913 & 29.863 & 52.02 & \mathrm{C}\end{array}$ $\begin{array}{llllllllllllll}1871 . & 3.341 & 1.018 & 0.01114 & 1677273 & 28.279 & 0.125 & 24.107 & 0.712 & 24.579 & 52.02 & \mathrm{C}\end{array}$ $\begin{array}{llllllllllllll}3603 & 0.002 & 0.995 & 0.01114 & 1677259 & 24.687 & 0.738 & 23.791 & 1.325 & 24.373 & 52.02 & \mathrm{C}\end{array}$ $\begin{array}{llllllllllllll}4517 . & 0.188 & 0.977 & 0.01114 & 1677252 & 24.687 & 1.352 & 23.377 & 1.634 & 24.169 & 52.02 & C\end{array}$ $\begin{array}{llllllllllllll}5444 . & 0.400 & 0.955 & 0.01114 & 1676875 & 24.679 & 1.964 & 22.884 & 1.939 & 24.258 & 52.02 & \mathrm{C}\end{array}$ $\begin{array}{lllllllllllll}7383 & 0.939 & 0.900 & 0.01114 & 1676053 & 24.661 & 2.577 & 22.122 & 2.550 & 23.219 & 52.02 & C\end{array}$ $\begin{array}{lllllllllllllll}9381 . & 1.612 & 0.829 & 0.01114 & 1675250 & 24.643 & 3.174 & 21.170 & 3.148 & 23.827 & 52.02 & C\end{array}$ $\begin{array}{llllllllllllll}10712 . & 2.069 & 0.774 & 0.01114 & 1672468 \text {. } & 24.581 & 3.504 & 20.112 & 3.530 & 23.186 & 52.02 & \mathrm{C}\end{array}$ $\begin{array}{lllllllllllll}12693 . & 2.908 & 0.683 & 0.01114 & 1672124 & 24.573 & 4.103 & 18.649 & 4.078 & 22.342 & 52.02 & C\end{array}$ $\begin{array}{lllllllllllll}14995 . & 3.984 & 0.564 & 0.01114 & 1671743 & 24.565 & 4.714 & 16.688 & 4.688 & 21.020 & 52.02 & C\end{array}$ $\begin{array}{lllllllllllll}16202 . & 4.587 & 0.495 & 0.01114 & 1671549 & 24.561 & 5.325 & 14.299 & 4.997 & 19.474 & 52.02 & \mathrm{C}\end{array}$ 17417. $\quad \begin{array}{llllllllllll}5.219 & 0.423 & 0.01114 & 1671390 & 24.557 & 5.938 & 11.741 & 5.301 & 19.361 & 52.02 & C\end{array}$ $\begin{array}{lllllllllllll}19944 . & 6.605 & 0.261 & 0.01114 & 1671074 & 24.550 & 6.532 & 9.337 & 5.913 & 17.361 & 52.02 & \mathrm{C}\end{array}$ $\begin{array}{lllllllllllll}1462 . & 1.686 & 1.011 & 0.01005 & 1951837 & 32.365 & 0.125 & 29.913 & 0.712 & 30.370 & 52.02 & C\end{array}$ $\begin{array}{lllllllllllll}2854 . & -0.028 & 0.990 & 0.01005 & 1951585 & 30.433 & 0.738 & 29.592 & 1.325 & 30.182 & 52.02 & \mathrm{C}\end{array}$ $\begin{array}{lllllllllllll}3599 & 0.129 & 0.973 & 0.01005 & 1951457 & 30.431 & 1.352 & 29.269 & 1.634 & 29.990 & 52.02 & \mathrm{C}\end{array}$ $\begin{array}{lllllllllllllll}4362 . & 0.313 & 0.953 & 0.01005 & 1951172 & 30.425 & 1.964 & 28.864 & 1.939 & 30.117 & 52.02 & \mathrm{C}\end{array}$ $\begin{array}{lllllllllllllll}5981 . & 0.781 & 0.901 & 0.01005 & 1950570 . & 30.413 & 2.577 & 28.222 & 2.550 & 29.260 & 52.02 & \mathrm{C}\end{array}$ $\begin{array}{lllllllllllll}\text { 7673. } & 1.363 & 0.834 & 0.01005 & 1949981 & 30.401 & 3.174 & 27.449 & 3.148 & 29.698 & 52.02 & \mathrm{C}\end{array}$ $\begin{array}{llllllllllllll}8814 & 1.778 & 0.782 & 0.01005 & 1948406 & 30.370 & 3.504 & 26.690 & 3.530 & 29.080 & 52.02 & \mathrm{C}\end{array}$ $\begin{array}{llllllllllllll}10529 . & 2.497 & 0.695 & 0.01005 & 1947946 & 30.361 & 4.103 & 25.450 & 4.078 & 28.457 & 52.02 & C\end{array}$ $\begin{array}{lllllllllllll}\text { 12543. } & 3.420 & 0.579 & 0.01005 & 1947435 & 30.351 & 4.714 & 23.842 & 4.688 & 27.332 & 52.02 & \mathrm{C}\end{array}$ $\begin{array}{llllllllllllll}13608 . & 3.937 & 0.513 & 0.01005 & 1947176 & 30.346 & 5.325 & 21.798 & 4.997 & 26.055 & 52.02 & C\end{array}$ 


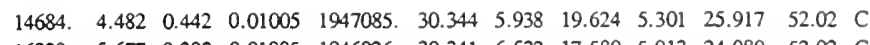
$\begin{array}{lllllllllllllll}16938 . & 5.677 & 0.283 & 0.01005 & 1946926 & 30.341 & 6.532 & 17.589 & 5.913 & 24.089 & 52.02 & C\end{array}$ $\begin{array}{llllllllllllll}815 & 0.593 & 1.004 & 0.00538 & 2016100 & 32.361 & 0.125 & 31.317 & 0.712 & 31.645 & 52.02 & \mathrm{C}\end{array}$ $\begin{array}{lllllllllllll}1575 . & 0.047 & 0.981 & 0.00538 & 2015991 . & 31.693 & 0.738 & 31.080 & 1.325 & 31.510 & 52.02 & \mathrm{C}\end{array}$ $\begin{array}{lllllllllllll}1976 . & 0.136 & 0.964 & 0.00538 & 2015936 & 31.692 & 1.352 & 30.886 & 1.634 & 31.395 & 52.02 & \mathrm{C}\end{array}$ $\begin{array}{lllllllllllll}2383 . & 0.240 & 0.944 & 0.00538 & 2015827 & 31.690 & 1.964 & 30.633 & 1.939 & 31.453 & 52.02 & C\end{array}$ $\begin{array}{llllllllllll}3235 . & 0.505 & 0.890 & 0.00538 & 2015598 & 31.685 & 2.577 & 30.265 & 2.550 & 30.917 & 52.02 & \mathrm{C}\end{array}$ $\begin{array}{lllllllllllll}4113 . & 0.834 & 0.822 & 0.00538 & 2015374 & 31.681 & 3.174 & 29.869 & 3.148 & 31.218 & 52.02 & \mathrm{C}\end{array}$ 4699. $\begin{array}{lllllllllll}1.083 & 0.769 & 0.00538 & 2015337 & 31.680 & 3.504 & 29.514 & 3.530 & 30.816 & 52.02 & \mathrm{C}\end{array}$ $\begin{array}{llllllllllllll}5571 & 1.491 & 0.681 & 0.00538 & 2015254 & 31.679 & 4.103 & 28.833 & 4.078 & 30.606 & 52.02 & \mathrm{C}\end{array}$ $\begin{array}{lllllllllllllll}6585 & 2.012 & 0.566 & 0.00538 & 2015162 . & 31.677 & 4.714 & 27.991 & 4.688 & 29.817 & 52.02 & \mathrm{C}\end{array}$ $\begin{array}{lllllllllllll}7116 . & 2.304 & 0.500 & 0.00538 & 2015116 & 31.676 & 5.325 & 26.900 & 4.997 & 29.121 & 52.02 & \mathrm{C}\end{array}$ $\begin{array}{llllllllllllll}7651 . & 2.610 & 0.430 & 0.00538 & 2015077 . & 31.675 & 5.938 & 25.742 & 5.301 & 29.061 & 52.02 & C\end{array}$ $\begin{array}{lllllllllllll}8763 . & 3.281 & 0.274 & 0.00538 & 2015000 & 31.674 & 6.532 & 24.674 & 5.913 & 28.166 & 52.02 & \mathrm{C}\end{array}$ $\begin{array}{lllllllllllll}\text { 853. } & -0.095 & 0.999 & 0.00489 & 2218042 & 35.457 & 0.125 & 35.034 & 0.712 & 35.547 & 52.02 & \mathrm{C}\end{array}$ $\begin{array}{llllllllllllll}1590 & 0.011 & 0.973 & 0.00489 & 2217956 & 35.456 & 0.738 & 34.741 & 1.325 & 35.313 & 52.02 & \mathrm{C}\end{array}$ $\begin{array}{lllllllllllll}1961 . & 0.088 & 0.953 & 0.00489 & 2217912 & 35.455 & 1.352 & 34.546 & 1.634 & 35.200 & 52.02 & \mathrm{C}\end{array}$ $\begin{array}{lllllllllllll}2325 & 0.180 & 0.930 & 0.00489 & 2217823 & 35.453 & 1.964 & 34.283 & 1.939 & 35.248 & 52.02 & \mathrm{C}\end{array}$ $\begin{array}{lllllllllllll}3054 . & 0.412 & 0.872 & 0.00489 & 2217635 . & 35.450 & 2.577 & 33.900 & 2.550 & 34.790 & 52.02 & \mathrm{C}\end{array}$

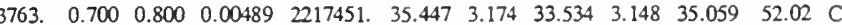
$\begin{array}{lllllllllllll}4215 . & 0.921 & 0.745 & 0.00489 & 2217599 & 35.449 & 3.504 & 33.349 & 3.530 & 34.686 & 52.02 & C\end{array}$

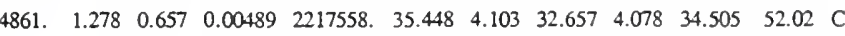

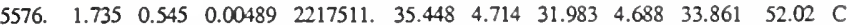
$\begin{array}{lllllllllllll}5937 . & 1.991 & 0.482 & 0.00489 & 2217488 & 35.447 & 5.325 & 30.933 & 4.997 & 33.261 & 52.02 & C\end{array}$ $\begin{array}{lllllllllllllll}6291 . & 2.259 & 0.416 & 0.00489 & 2217455 & 35.447 & 5.938 & 30.021 & 5.301 & 33.229 & 52.02 & C\end{array}$ $\begin{array}{lllllllllllll}7002 . & 2.845 & 0.272 & 0.00489 & 2217387 & 35.445 & 6.532 & 29.258 & 5.913 & 32.377 & 52.02 & \mathrm{C}\end{array}$ $\begin{array}{llllllllllllll}808 . & 0.466 & 1.004 & 0.00555 & 2176792 & 35.304 & 0.125 & 34.394 & 0.712 & 34.779 & 52.02 & \mathrm{C}\end{array}$ $\begin{array}{lllllllllllll}\text { 1565. } & -0.002 & 0.981 & 0.00555 & 2176679 & 34.708 & 0.738 & 34.144 & 1.325 & 34.555 & 52.02 & C\end{array}$ $\begin{array}{llllllllllllll}1965 . & 0.090 & 0.965 & 0.00555 & 2176622 & 34.707 & 1.352 & 33.953 & 1.634 & 34.427 & 52.02 & C\end{array}$ $\begin{array}{llllllllllllll}2373 . & 0.199 & 0.944 & 0.00555 & 2176516 & 34.705 & 1.964 & 33.703 & 1.939 & 34.487 & 52.02 & \mathrm{C}\end{array}$ $\begin{array}{lllllllllllllll}3228 & 0.476 & 0.891 & 0.00555 & 2176294 & 34.701 & 2.577 & 33.340 & 2.550 & 33.954 & 52.02 & C\end{array}$ $\begin{array}{lllllllllllll}4113 & 0.819 & 0.823 & 0.00555 & 2176077 & 34.697 & 3.174 & 32.942 & 3.148 & 34.252 & 52.02 & \mathrm{C}\end{array}$ $\begin{array}{llllllllllll}4704 . & 1.080 & 0.770 & 0.00555 & 2176038 & 34.697 & 3.504 & 32.578 & 3.530 & 33.825 & 52.02 & \mathrm{C}\end{array}$ $\begin{array}{lllllllllllllll}5586 . & 1.505 & 0.683 & 0.00555 & 2175965 & 34.695 & 4.103 & 31.891 & 4.078 & 33.622 & 52.02 & C\end{array}$ $\begin{array}{lllllllllllll}6613 . & 2.049 & 0.568 & 0.00555 & 2175883 & 34.694 & 4.714 & 31.031 & 4.688 & 32.814 & 52.02 & \mathrm{C}\end{array}$ $\begin{array}{lllllllllllll}7153 & 2.354 & 0.502 & 0.00555 & 2175842 & 34.693 & 5.325 & 29.956 & 4.997 & 32.088 & 52.02 & C\end{array}$

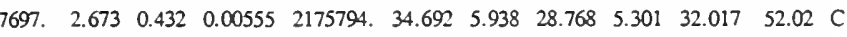
$\begin{array}{llllllllllllll}8829 . & 3.372 & 0.276 & 0.00555 & 2175697 & 34.690 & 6.532 & 27.684 & 5.913 & 31.082 & 52.02 & \mathrm{C}\end{array}$ $\begin{array}{llllllllllllll}843 & 0.463 & 1.004 & 0.00560 & 2304611 & 37.558 & 0.125 & 36.487 & 0.712 & 37.055 & 52.02 & C\end{array}$ $\begin{array}{llllllllllllll}1609 . & 0.019 & 0.980 & 0.00560 & 2304507 & 36.989 & 0.738 & 36.169 & 1.325 & 36.816 & 52.02 & \mathrm{C}\end{array}$ $\begin{array}{llllllllllll}2007 . & 0.109 & 0.963 & 0.00560 & 2304455 & 36.988 & 1.352 & 35.970 & 1.634 & 36.695 & 52.02 & \mathrm{C}\end{array}$ $\begin{array}{llllllllllllll}2406 & 0.216 & 0.942 & 0.00560 & 2304353 & 36.986 & 1.964 & 35.695 & 1.939 & 36.745 & 52.02 & \mathrm{C}\end{array}$ $\begin{array}{llllllllllllll}3229 & 0.485 & 0.888 & 0.00560 & 2304139 & 36.983 & 2.577 & 35.305 & 2.550 & 36.222 & 52.02 & \mathrm{C}\end{array}$ $\begin{array}{llllllllllllll}4063 . & 0.821 & 0.819 & 0.00560 & 2303930 & 36.979 & 3.174 & 34.922 & 3.148 & 36.520 & 52.02 & \mathrm{C}\end{array}$ $\begin{array}{llllllllllllllll}4611 . & 1.076 & 0.766 & 0.00560 & 2303958 . & 36.980 & 3.504 & 34.691 & 3.530 & 36.107 & 52.02 & \mathrm{C}\end{array}$ $\begin{array}{lllllllllllll}5418 . & 1.490 & 0.680 & 0.00560 & 2303889 & 36.978 & 4.103 & 33.953 & 4.078 & 35.905 & 52.02 & C\end{array}$ $\begin{array}{llllllllllllll}6342 . & 2.021 & 0.568 & 0.00560 & 2303813 & 36.977 & 4.714 & 33.169 & 4.688 & 35.117 & 52.02 & \mathrm{C}\end{array}$ $\begin{array}{llllllllllllll}6822 & 2.319 & 0.504 & 0.00560 & 2303774 & 36.976 & 5.325 & 32.005 & 4.997 & 34.406 & 52.02 & \mathrm{C}\end{array}$ 7302. $\begin{array}{llllllllllll}2.630 & 0.437 & 0.00560 & 2303720 & 36.975 & 5.938 & 30.895 & 5.301 & 34.375 & 52.02 & \mathrm{C}\end{array}$ $\begin{array}{llllllllllllll}8290 . & 3.311 & 0.287 & 0.00560 & 2303608 & 36.973 & 6.532 & 29.976 & 5.913 & 33.419 & 52.02 & \mathrm{C}\end{array}$ 1290. $\quad \begin{array}{llllllllllll}1.140 & 1.007 & 0.00761 & 1566603 & 23.508 & 0.125 & 21.824 & 0.712 & 22.110 & 52.02 & C\end{array}$ $\begin{array}{llllllllllllll}2486 & -0.035 & 0.983 & 0.00761 & 1566004 & 22.154 & 0.738 & 21.636 & 1.325 & 21.929 & 52.02 & \mathrm{C}\end{array}$ $\begin{array}{lllllllllllll}3117 . & 0.090 & 0.965 & 0.00761 & 1565703 & 22.147 & 1.352 & 21.350 & 1.634 & 21.810 & 52.02 & \mathrm{C}\end{array}$ $\begin{array}{llllllllllllll}3756 . & 0.247 & 0.944 & 0.00761 & 1565730 & 22.148 & 1.964 & 21.016 & 1.939 & 21.902 & 52.02 & C\end{array}$ $\begin{array}{lllllllllllll}5092 . & 0.644 & 0.889 & 0.00761 & 1565842 . & 22.150 & 2.577 & 20.491 & 2.550 & 21.161 & 52.02 & C\end{array}$ $\begin{array}{lllllllllllll}6467 . & 1.137 & 0.819 & 0.00761 & 1565951 & 22.153 & 3.174 & 19.826 & 3.148 & 21.574 & 52.02 & C\end{array}$ 7384. $\begin{array}{llllllllllllll}1.471 & 0.764 & 0.00761 & 1564516 & 22.119 & 3.504 & 19.049 & 3.530 & 21.066 & 52.02 & C\end{array}$ $\begin{array}{llllllllllllll}\text { 8746. } & 2.062 & 0.674 & 0.00761 & 1564108 . & 22.110 & 4.103 & 18.067 & 4.078 & 20.627 & 52.02 & C\end{array}$ $\begin{array}{lllllllllllll}10328 & 2.820 & 0.556 & 0.00761 & 1563654 & 22.099 & 4.714 & 16.696 & 4.688 & 19.610 & 52.02 & C\end{array}$ $\begin{array}{llllllllllllll}11157 . & 3.245 & 0.488 & 0.00761 & 1563424 & 22.094 & 5.325 & 15.088 & 4.997 & 18.534 & 52.02 & C\end{array}$ $\begin{array}{llllllllllllll}11991 . & 3.696 & 0.417 & 0.00761 & 1563437 & 22.094 & 5.938 & 13.343 & 5.301 & 18.415 & 52.02 & \mathrm{C}\end{array}$ $\begin{array}{lllllllllllll}13724 & 4.685 & 0.256 & 0.00761 & 1563502 & 22.095 & 6.532 & 11.625 & 5.913 & 16.929 & 52.02 & \mathrm{C}\end{array}$ $\begin{array}{lllllllllllll}1052 . & 0.937 & 1.005 & 0.00604 & 1599801 & 23.983 & 0.125 & 22.554 & 0.712 & 22.882 & 52.02 & C\end{array}$ $\begin{array}{lllllllllllll}2019 . & 0.022 & 0.981 & 0.00604 & 1599366 & 22.927 & 0.738 & 22.361 & 1.325 & 22.740 & 52.02 & \mathrm{C}\end{array}$ $\begin{array}{lllllllllllllll}2524 & 0.120 & 0.963 & 0.00604 & 1599146 & 22.922 & 1.352 & 22.120 & 1.634 & 22.604 & 52.02 & C\end{array}$ $\begin{array}{lllllllllllllll}3034 & 0.246 & 0.941 & 0.00604 & 1599280 & 22.926 & 1.964 & 21.828 & 1.939 & 22.701 & 52.02 & \mathrm{C}\end{array}$ 4094. $\begin{array}{llllllllllll}0.562 & 0.885 & 0.00604 & 1599611 & 22.933 & 2.577 & 21.379 & 2.550 & 22.073 & 52.02 & C\end{array}$ $\begin{array}{llllllllllllll}5177 & 0.953 & 0.813 & 0.00604 & 1599934 & 22.941 & 3.174 & 20.855 & 3.148 & 22.410 & 52.02 & \mathrm{C}\end{array}$ $\begin{array}{llllllllllllll}5893 . & 1.231 & 0.758 & 0.00604 & 1599505 . & 22.931 & 3.504 & 20.314 & 3.530 & 21.994 & 52.02 & \mathrm{C}\end{array}$ $\begin{array}{lllllllllllll}6954 . & 1.691 & 0.667 & 0.00604 & 1599049 & 22.920 & 4.103 & 19.505 & 4.078 & 21.721 & 52.02 & C\end{array}$ $\begin{array}{lllllllllllllll}8178 . & 2.282 & 0.548 & 0.00604 & 1598543 & 22.909 & 4.714 & 18.459 & 4.688 & 20.852 & 52.02 & C\end{array}$ $\begin{array}{lllllllllllllll}8817 . & 2.613 & 0.481 & 0.00604 & 1598286 & 22.903 & 5.325 & 17.134 & 4.997 & 19.949 & 52.02 & \mathrm{C}\end{array}$ $\begin{array}{lllllllllllllll}9457 . & 2.967 & 0.409 & 0.00604 & 1598359 & 22.904 & 5.938 & 15.751 & 5.301 & 19.881 & 52.02 & C\end{array}$ $\begin{array}{lllllllllllll}0783 & 3.745 & 0.249 & 0.00604 & 1598559 & 22.909 & 6.532 & 14.425 & 5.913 & 18.931 & 52.02 & \mathrm{C}\end{array}$ $\begin{array}{llllllllllllll}3617 . & 8.560 & 1.052 & 0.02038 & 2138438 . & 42.634 & 0.125 & 33.039 & 0.712 & 33.890 & 52.02 & C\end{array}$ $\begin{array}{lllllllllllll}6792 . & 4.370 & 1.026 & 0.02038 & 2136986 & 38.074 & 0.738 & 32.336 & 1.325 & 33.354 & 52.02 & \mathrm{C}\end{array}$ 8410. $\quad \begin{array}{lllllllllllll}1.448 & 1.006 & 0.02038 & 2136255 & 34.881 & 1.352 & 31.664 & 1.634 & 33.000 & 52.02 & \mathrm{C}\end{array}$ $\begin{array}{lllllllllllll}10015 . & 0.837 & 0.982 & 0.02038 & 2135278 & 33.948 & 1.964 & 30.852 & 1.939 & 33.145 & 52.02 & \mathrm{C}\end{array}$ $\begin{array}{llllllllllll}\text { 13271. } & 1.613 & 0.923 & 0.02038 & 2133272 & 33.911 & 2.577 & 29.673 & 2.550 & 31.382 & 52.02 & C\end{array}$ $\begin{array}{llllllllllll}16495 . & 2.586 & 0.848 & 0.02038 & 2131311 & 33.874 & 3.174 & 28.265 & 3.148 & 32.311 & 52.02 & C\end{array}$ $\begin{array}{llllllllllllll}18581 . & 3.191 & 0.792 & 0.02038 & 2123135 & 33.723 & 3.504 & 26.904 & 3.530 & 31.210 & 52.02 & \mathrm{C}\end{array}$ $\begin{array}{llllllllllllll}21603 . & 4.399 & 0.700 & 0.02038 & 2121625 & 33.695 & 4.103 & 24.827 & 4.078 & 30.122 & 52.02 & C\end{array}$ $\begin{array}{lllllllllllll}25003 . & 5.951 & 0.581 & 0.02038 & 2119946 . & 33.663 & 4.714 & 22.172 & 4.688 & 28.302 & 52.02 & \mathrm{C}\end{array}$ $\begin{array}{llllllllllllll}26743 . & 6.822 & 0.515 & 0.02038 & 2119096 & 33.648 & 5.325 & 18.975 & 4.997 & 26.085 & 52.02 & \mathrm{C}\end{array}$ $\begin{array}{llllllllllll}28467 . & 7.742 & 0.445 & 0.02038 & 2118675 & 33.640 & 5.938 & 15.478 & 5.301 & 25.998 & 52.02 & \mathrm{C}\end{array}$ $\begin{array}{llllllllllllll}31972 . & 9.761 & 0.290 & 0.02038 & 2117894 & 33.625 & 6.532 & 12.257 & 5.913 & 23.236 & 52.02 & C\end{array}$ $\begin{array}{lllllllllllll}3225 & 7.008 & 1.044 & 0.01532 & 2182092 & 41.926 & 0.125 & 33.925 & 0.712 & 34.746 & 52.02 & \mathrm{C}\end{array}$ $\begin{array}{lllllllllllll}5969 . & 2.161 & 1.013 & 0.01532 & 2181269 & 36.754 & 0.738 & 33.326 & 1.325 & 34.292 & 52.02 & \mathrm{C}\end{array}$ $\begin{array}{llllllllllll}7336 & 0.430 & 0.990 & 0.01532 & 2180854 & 34.784 & 1.352 & 32.750 & 1.634 & 33.983 & 52.02 & C\end{array}$ $\begin{array}{lllllllllllll}8672 . & 0.702 & 0.962 & 0.01532 & 2180260 & 34.774 & 1.964 & 32.044 & 1.939 & 34.113 & 52.02 & C\end{array}$ $\begin{array}{llllllllllll}11324 & 1.395 & 0.894 & 0.01532 & 2179035 & 34.751 & 2.577 & 31.012 & 2.550 & 32.658 & 52.02 & C\end{array}$ $\begin{array}{llllllllllll}13872 & 2.259 & 0.809 & 0.01532 & 2177837 & 34.729 & 3.174 & 29.819 & 3.148 & 33.387 & 52.02 & C\end{array}$ $\begin{array}{llllllllllllll}15483 . & 2.841 & 0.746 & 0.01532 & 2173255 & 34.646 & 3.504 & 28.762 & 3.530 & 32.355 & 52.02 & C\end{array}$ $\begin{array}{lllllllllllll}17762 . & 3.911 & 0.643 & 0.01532 & 2172384 & 34.630 & 4.103 & 26.998 & 4.078 & 31.285 & 52.02 & \mathrm{C}\end{array}$ $\begin{array}{lllllllllllll}20255 . & 5.285 & 0.514 & 0.01532 & 2171416 . & 34.612 & 4.714 & 24.845 & 4.688 & 29.797 & 52.02 & C\end{array}$ $\begin{array}{llllllllllll}21502 . & 6.055 & 0.441 & 0.01532 & 2170926 & 34.603 & 5.325 & 22.149 & 4.997 & 27.934 & 52.02 & C\end{array}$ 22719. $\begin{array}{lllllllllllll}6.866 & 0.366 & 0.01532 & 2170692 & 34.599 & 5.938 & 19.381 & 5.301 & 27.840 & 52.02 & \mathrm{C}\end{array}$ $\begin{array}{lllllllllllll}25136 . & 8.645 & 0.202 & 0.01532 & 2170259 & 34.591 & 6.532 & 17.056 & 5.913 & 25.450 & 52.02 & C\end{array}$ $\begin{array}{lllllllllllll}2463 & 5.146 & 1.033 & 0.01473 & 2171324 & 39.853 & 0.125 & 33.500 & 0.712 & 34.555 & 52.02 & C\end{array}$ $\begin{array}{lllllllllllll}\text { 4624. } & 1.337 & 1.008 & 0.01473 & 2170573 & 35.769 & 0.738 & 32.937 & 1.325 & 34.169 & 52.02 & C\end{array}$ $\begin{array}{llllllllllll}5723 . & 0.359 & 0.989 & 0.01473 & 2170195 & 34.590 & 1.352 & 32.487 & 1.634 & 33.905 & 52.02 & C\end{array}$ $\begin{array}{lllllllllllll}6811 . & 0.589 & 0.967 & 0.01473 & 2169662 & 34.580 & 1.964 & 31.900 & 1.939 & 34.026 & 52.02 & C\end{array}$ $\begin{array}{lllllllllllll}9015 . & 1.174 & 0.910 & 0.01473 & 2168562 & 34.560 & 2.577 & 31.045 & 2.550 & 32.787 & 52.02 & C\end{array}$ $\begin{array}{lllllllllllll}11191 . & 1.905 & 0.840 & 0.01473 & 2167487 & 34.540 & 3.174 & 30.090 & 3.148 & 33.415 & 52.02 & C\end{array}$

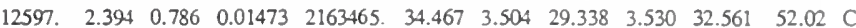
$\begin{array}{llllllllllllll}14629 . & 3.299 & 0.700 & 0.01473 & 2162656 & 34.452 & 4.103 & 27.863 & 4.078 & 31.664 & 52.02 & C\end{array}$ $\begin{array}{llllllllllllll}16909 . & 4.461 & 0.588 & 0.01473 & 2161758 . & 34.435 & 4.714 & 26.087 & 4.688 & 30.377 & 52.02 & C\end{array}$ $\begin{array}{lllllllllllll}18074 & 5.112 & 0.525 & 0.01473 & 2161303 & 34.427 & 5.325 & 23.845 & 4.997 & 28.785 & 52.02 & C\end{array}$ $\begin{array}{llllllllllllll}19227 . & 5.798 & 0.459 & 0.01473 & 2161072 & 34.423 & 5.938 & 21.463 & 5.301 & 28.712 & 52.02 & C\end{array}$ $\begin{array}{lllllllllllll}21566 . & 7.303 & 0.314 & 0.01473 & 2160642 & 34.415 & 6.532 & 19.331 & 5.913 & 26.677 & 52.02 & C\end{array}$ $\begin{array}{llllllllllll}2601 & 5.483 & 1.037 & 0.01531 & 2276256 & 42.102 & 0.125 & 35.719 & 0.712 & 36.465 & 52.02 \mathrm{C}\end{array}$ $\begin{array}{llllllllllll}4903 . & 1.702 & 1.011 & 0.01531 & 2275469 & 38.035 & 0.738 & 35.195 & 1.325 & 36.062 & 52.02 & C\end{array}$ $\begin{array}{llllllllllllll}6080 & 0.349 & 0.991 & 0.01531 & 2275072 & 36.473 & 1.352 & 34.703 & 1.634 & 35.795 & 52.02 & \mathrm{C}\end{array}$ $\begin{array}{llllllllllll}7250 . & 0.588 & 0.968 & 0.01531 & 2274512 & 36.463 & 1.964 & 34.107 & 1.939 & 35.892 & 52.02 & \mathrm{C}\end{array}$ $\begin{array}{lllllllllllll}9634 . & 1.195 & 0.909 & 0.01531 & 2273358 & 36.442 & 2.577 & 33.219 & 2.550 & 34.643 & 52.02 & \mathrm{C}\end{array}$ $\begin{array}{llllllllllll}12007 . & 1.954 & 0.835 & 0.01531 & 2272230 & 36.422 & 3.174 & 32.187 & 3.148 & 35.286 & 52.02 & \mathrm{C}\end{array}$ $\begin{array}{lllllllllllll}13547 . & 2.463 & 0.779 & 0.01531 & 2267965 & 36.347 & 3.504 & 31.270 & 3.530 & 34.390 & 52.02 & C\end{array}$ $\begin{array}{lllllllllllll}15787 & 3.403 & 0.687 & 0.01531 & 2267102 & 36.332 & 4.103 & 29.713 & 4.078 & 33.422 & 52.02 & C\end{array}$ $\begin{array}{lllllllllllll}\text { 18317. } & 4.608 & 0.569 & 0.01531 & 2266144 & 36.315 & 4.714 & 27.784 & 4.688 & 32.125 & 52.02 & C\end{array}$ $\begin{array}{lllllllllllll}19617 . & 5.285 & 0.502 & 0.01531 & 2265658 & 36.306 & 5.325 & 25.404 & 4.997 & 30.484 & 52.02 & C\end{array}$ $\begin{array}{llllllllllll}20906 . & 5.996 & 0.432 & 0.01531 & 2265394 & 36.302 & 5.938 & 22.816 & 5.301 & 30.396 & 52.02 & C\end{array}$ $\begin{array}{lllllllllllllll}23536 . & 7.558 & 0.277 & 0.01531 & 2264898 & 36.293 & 6.532 & 20.435 & 5.913 & 28.288 & 52.02 & C\end{array}$ $\begin{array}{lllllllllllll}3265 . & 7.754 & 1.042 & 0.01553 & 1854116 & 36.282 & 0.125 & 27.571 & 0.712 & 28.277 & 52.02 & \mathrm{C}\end{array}$ $\begin{array}{llllllllllll}6058 . & 2.430 & 1.012 & 0.01553 & 1853143 & 30.637 & 0.738 & 27.046 & 1.325 & 27.920 & 52.02 & \mathrm{C}\end{array}$ $\begin{array}{lllllllllllll}\text { 7454. } & 0.463 & 0.990 & 0.01553 & 1852653 & 28.436 & 1.352 & 26.450 & 1.634 & 27.620 & 52.02 & \mathrm{C}\end{array}$ $\begin{array}{llllllllllllll}8822 & 0.727 & 0.964 & 0.01553 & 1851938 & 28.421 & 1.964 & 25.763 & 1.939 & 27.747 & 52.02 & \mathrm{C}\end{array}$ $\begin{array}{lllllllllllllll}11546 . & 1.399 & 0.899 & 0.01553 & 1850462 & 28.391 & 2.577 & 24.739 & 2.550 & 26.221 & 52.02 & \mathrm{C}\end{array}$ $\begin{array}{llllllllllllll}14179 . & 2.242 & 0.819 & 0.01553 & 1849019 & 28.361 & 3.174 & 23.509 & 3.148 & 27.065 & 52.02 & C\end{array}$ $\begin{array}{lllllllllllll}15849 . & 2.778 & 0.758 & 0.01553 & 1843336 & 28.243 & 3.504 & 22.291 & 3.530 & 26.136 & 52.02 & \mathrm{C}\end{array}$ $\begin{array}{llllllllllllll}18224 & 3.824 & 0.660 & 0.01553 & 1842207 & 28.220 & 4.103 & 20.535 & 4.078 & 25.009 & 52.02 & C\end{array}$ $\begin{array}{lllllllllllll}20834 . & 5.167 & 0.536 & 0.01553 & 1840952 & 28.194 & 4.714 & 18.288 & 4.688 & 23.511 & 52.02 & C\end{array}$ $\begin{array}{lllllllllllll}22147 . & 5.920 & 0.467 & 0.01553 & 1840316 & 28.181 & 5.325 & 15.636 & 4.997 & 21.554 & 52.02 & \mathrm{C}\end{array}$ $\begin{array}{llllllllllllll}23430 . & 6.716 & 0.394 & 0.01553 & 1840023 & 28.175 & 5.938 & 12.795 & 5.301 & 21.543 & 52.02 & C\end{array}$ $\begin{array}{lllllllllllll}25995 . & 8.463 & 0.236 & 0.01553 & 1839485 & 28.164 & 6.532 & 10.269 & 5.913 & 19.217 & 52.02 & \mathrm{C}\end{array}$ $\begin{array}{lllllllllllll}3076 . & 5.960 & 1.037 & 0.01710 & 2112909 & 39.587 & 0.125 & 32.628 & 0.712 & 33.443 & 52.02 & \mathrm{C}\end{array}$ $\begin{array}{lllllllllllllll}5778 . & 1.779 & 1.010 & 0.01710 & 2111823 & 35.086 & 0.738 & 32.070 & 1.325 & 33.001 & 52.02 & \mathrm{C}\end{array}$ $\begin{array}{llllllllllll}7154 & 0.430 & 0.990 & 0.01710 & 2111275 & 33.502 & 1.352 & 31.494 & 1.634 & 32.704 & 52.02 & \mathrm{C}\end{array}$ $\begin{array}{lllllllllllllll}8519 . & 0.695 & 0.966 & 0.01710 & 2110528 & 33.488 & 1.964 & 30.807 & 1.939 & 32.829 & 52.02 & \mathrm{C}\end{array}$ $\begin{array}{lllllllllllll}11289 & 1.369 & 0.906 & 0.01710 & 2108990 & 33.459 & 2.577 & 29.787 & 2.550 & 31.345 & 52.02 & \mathrm{C}\end{array}$ $\begin{array}{llllllllllll}14031 . & 2.213 & 0.831 & 0.01710 & 2107488 & 33.431 & 3.174 & 28.589 & 3.148 & 32.143 & 52.02 & C\end{array}$ $\begin{array}{lllllllllllll}15804 . & 2.755 & 0.774 & 0.01710 & 2101506 & 33.319 & 3.504 & 27.466 & 3.530 & 31.168 & 52.02 & C\end{array}$ $\begin{array}{lllllllllllll}18373 . & 3.802 & 0.681 & 0.01710 & 2100335 & 33.297 & 4.103 & 25.685 & 4.078 & 30.108 & 52.02 & \mathrm{C}\end{array}$ $\begin{array}{llllllllllllll}21262 . & 5.147 & 0.561 & 0.01710 & 2099034 & 33.273 & 4.714 & 23.442 & 4.688 & 28.618 & 52.02 & C\end{array}$ $\begin{array}{llllllllllllll}22741 . & 5.901 & 0.494 & 0.01710 & 2098374 & 33.261 & 5.325 & 20.707 & 4.997 & 26.706 & 52.02 & C\end{array}$ $\begin{array}{llllllllllllll}24205 & 6.697 & 0.423 & 0.01710 & 2098035 & 33.254 & 5.938 & 17.754 & 5.301 & 26.643 & 52.02 & C\end{array}$ $\begin{array}{llllllllllllll}27181 . & 8.443 & 0.267 & 0.01710 & 2097400 & 33.242 & 6.532 & 15.034 & 5.913 & 24.301 & 52.02 & \mathrm{C}\end{array}$ $\begin{array}{lllllllllllll}2875 & 0.015 & 0.994 & 0.01635 & 1839027 & 28.154 & 0.125 & 27.709 & 0.712 & 27.846 & 52.02 & C\end{array}$ $\begin{array}{lllllllllllll}5417 . & 0.276 & 0.968 & 0.01635 & 1837733 & 28.128 & 0.738 & 27.403 & 1.325 & 27.573 & 52.02 & \mathrm{C}\end{array}$ $\begin{array}{llllllllllllll}6717 . & 0.473 & 0.950 & 0.01635 & 1837080 & 28.114 & 1.352 & 26.877 & 1.634 & 27.309 & 52.02 & \mathrm{C}\end{array}$ $\begin{array}{llllllllllllll}8011 . & 0.706 & 0.928 & 0.01635 & 1836261 & 28.097 & 1.964 & 26.302 & 1.939 & 27.463 & 52.02 & \mathrm{C}\end{array}$

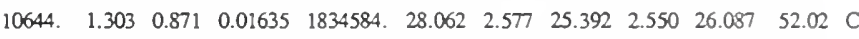

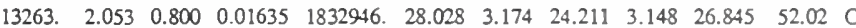

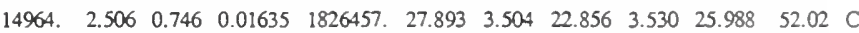
$\begin{array}{lllllllllllll}17437 . & 3.439 & 0.658 & 0.01635 & 1825152 & 27.866 & 4.103 & 21.283 & 4.078 & 25.099 & 52.02 & C\end{array}$ $\begin{array}{lllllllllllll}20231 . & 4.639 & 0.545 & 0.01635 & 1823701 & 27.836 & 4.714 & 19.058 & 4.688 & 23.646 & 52.02 & C\end{array}$ $\begin{array}{lllllllllllll}21666 . & 5.312 & 0.481 & 0.01635 & 1822966 & 27.821 & 5.325 & 16.655 & 4.997 & 21.839 & 52.02 & C\end{array}$ $\begin{array}{llllllllllllll}23090 & 6.025 & 0.413 & 0.01635 & 1822590 & 27.813 & 5.938 & 13.834 & 5.301 & 21.817 & 52.02 & C\end{array}$ $\begin{array}{llllllllllllll}25996 . & 7.590 & 0.265 & 0.01635 & 1821888 & 27.798 & 6.532 & 11.120 & 5.913 & 19.761 & 52.02 & C\end{array}$ $\begin{array}{lllllllllllll}1472 . & 2.289 & 1.013 & 0.00731 & 1850022 & 30.693 & 0.125 & 27.940 & 0.712 & 28.280 & 52.02 & C\end{array}$ $\begin{array}{llllllllllllllll}2736 & 0.135 & 0.984 & 0.00731 & 1850198 & 28.385 & 0.738 & 27.691 & 1.325 & 28.101 & 52.02 & C\end{array}$ $\begin{array}{lllllllllllll}3367 . & 0.250 & 0.963 & 0.00731 & 1850286 & 28.387 & 1.352 & 27.427 & 1.634 & 27.942 & 52.02 & C\end{array}$ $\begin{array}{llllllllllllll}3985 & 0.381 & 0.938 & 0.00731 & 1850114 & 28.383 & 1.964 & 27.109 & 1.939 & 28.050 & 52.02 & \mathrm{C}\end{array}$ $\begin{array}{lllllllllllllll}5215 . & 0.713 & 0.876 & 0.00731 & 1849722 & 28.375 & 2.577 & 26.621 & 2.550 & 27.330 & 52.02 & \mathrm{C}\end{array}$ $\begin{array}{llllllllllllll}6400 . & 1.126 & 0.798 & 0.00731 & 1849340 & 28.367 & 3.174 & 26.059 & 3.148 & 27.654 & 52.02 & C\end{array}$ $\begin{array}{llllllllllllll}7151 . & 1.424 & 0.739 & 0.00731 & 1848483 & 28.350 & 3.504 & 25.541 & 3.530 & 27.156 & 52.02 & C\end{array}$ $\begin{array}{llllllllllllll}8217 . & 1.936 & 0.646 & 0.00731 & 1848262 & 28.345 & 4.103 & 24.739 & 4.078 & 26.861 & 52.02 & C\end{array}$ $\begin{array}{lllllllllllllll}9387 . & 2.591 & 0.526 & 0.00731 & 1848017 & 28.340 & 4.714 & 23.717 & 4.688 & 25.968 & 52.02 & \mathrm{C}\end{array}$ $\begin{array}{lllllllllllll}9974 . & 2.959 & 0.460 & 0.00731 & 1847893 & 28.338 & 5.325 & 22.527 & 4.997 & 25.075 & 52.02 & \mathrm{C}\end{array}$ $\begin{array}{llllllllllllll}10547 . & 3.345 & 0.390 & 0.00731 & 1847816 & 28.336 & 5.938 & 21.272 & 5.301 & 24.972 & 52.02 & C\end{array}$ $\begin{array}{lllllllllllllll}11690 . & 4.190 & 0.239 & 0.00731 & 1847669 & 28.333 & 6.532 & 20.071 & 5.913 & 23.904 & 52.02 & \mathrm{C}\end{array}$ $\begin{array}{lllllllllllll}2123 & 3.907 & 1.021 & 0.00842 & 1782524 & 30.872 & 0.125 & 26.366 & 0.712 & 26.811 & 52.02 & C\end{array}$ $\begin{array}{llllllllllllll}3864 . & 0.207 & 0.986 & 0.00842 & 1782373 & 26.967 & 0.738 & 26.058 & 1.325 & 26.567 & 52.02 & \mathrm{C}\end{array}$ $\begin{array}{llllllllllllll}4705 . & 0.356 & 0.960 & 0.00842 & 1782298 & 26.966 & 1.352 & 25.700 & 1.634 & 26.378 & 52.02 & \mathrm{C}\end{array}$ $\begin{array}{llllllllllllll}5511 . & 0.529 & 0.931 & 0.00842 & 1782063 & 26.961 & 1.964 & 25.277 & 1.939 & 26.515 & 52.02 & \mathrm{C}\end{array}$ $\begin{array}{llllllllllll}7059 . & 0.969 & 0.857 & 0.00842 & 1781564 & 26.950 & 2.577 & 24.632 & 2.550 & 25.551 & 52.02 & C\end{array}$ $\begin{array}{llllllllllllll}8481 & 1.517 & 0.769 & 0.00842 & 1781077 & 26.940 & 3.174 & 23.878 & 3.148 & 25.960 & 52.02 & C\end{array}$ 
9344. $\quad \begin{array}{lllllllllll}1.905 & 0.702 & 0.00842 & 1779653 & 26.910 & 3.504 & 23.169 & 3.530 & 25.303 & 52.02 & \mathrm{C}\end{array}$ $\begin{array}{lllllllllllll}10517 . & 2.583 & 0.598 & 0.00842 & 1779351 . & 26.903 & 4.103 & 22.135 & 4.078 & 24.843 & 52.02 & C\end{array}$ $\begin{array}{lllllllllllll}\text { 11732. } & 3.453 & 0.469 & 0.00842 & 1779017 & 26.896 & 4.714 & 20.809 & 4.688 & 23.724 & 52.02 & \mathrm{C}\end{array}$ $\begin{array}{llllllllllll}12312 & 3.940 & 0.398 & 0.00842 & 1778847 & 26.892 & 5.325 & 19.323 & 4.997 & 22.523 & 52.02 & C\end{array}$ $\begin{array}{llllllllllll}12860 . & 4.451 & 0.325 & 0.00842 & 1778759 & 26.891 & 5.938 & 17.789 & 5.301 & 22.402 & 52.02 & C\end{array}$ $\begin{array}{lllllllllllllll}13892 . & 5.574 & 0.169 & 0.00842 & 1778594 & 26.887 & 6.532 & 16.543 & 5.913 & 21.067 & 52.02 & C\end{array}$ $\begin{array}{lllllllllllll}1237 . & 2.370 & 1.013 & 0.00668 & 1805227 & 29.876 & 0.125 & 26.894 & 0.712 & 27.355 & 52.02 & \mathrm{C}\end{array}$ $\begin{array}{lllllllllllll}2328 & 0.093 & 0.987 & 0.00668 & 1805186 & 27.449 & 0.738 & 26.615 & 1.325 & 27.188 & 52.02 & \mathrm{C}\end{array}$ $\begin{array}{lllllllllllll}2884 & 0.202 & 0.967 & 0.00668 & 1805166 . & 27.448 & 1.352 & 26.354 & 1.634 & 27.043 & 52.02 & C\end{array}$ $\begin{array}{lllllllllllll}3436 . & 0.329 & 0.944 & 0.00668 & 1805012 & 27.445 & 1.964 & 26.024 & 1.939 & 27.146 & 52.02 & \mathrm{C}\end{array}$ $\begin{array}{lllllllllllll}4555 & 0.651 & 0.885 & 0.00668 & 1804679 . & 27.438 & 2.577 & 25.518 & 2.550 & 26.469 & 52.02 & \mathrm{C}\end{array}$ $\begin{array}{lllllllllllllll}5663 & 1.051 & 0.812 & 0.00668 & 1804354 & 27.431 & 3.174 & 24.975 & 3.148 & 26.801 & 52.02 & C\end{array}$ $\begin{array}{llllllllllllll}6381 . & 1.344 & 0.755 & 0.00668 & 1803703 & 27.418 & 3.504 & 24.535 & 3.530 & 26.351 & 52.02 & C\end{array}$ $\begin{array}{lllllllllllll}7419 & 1.840 & 0.664 & 0.00668 & 1803516 & 27.414 & 4.103 & 23.662 & 4.078 & 26.064 & 52.02 & C\end{array}$ $\begin{array}{lllllllllllll}\text { 8588. } & 2.475 & 0.546 & 0.00668 & 1803308 . & 27.409 & 4.714 & 22.631 & 4.688 & 25.162 & 52.02 & C\end{array}$ $\begin{array}{llllllllllll}9187 . & 2.831 & 0.480 & 0.00668 & 1803202 & 27.407 & 5.325 & 21.250 & 4.997 & 24.265 & 52.02 & C\end{array}$ $\begin{array}{llllllllllllll}9779 . & 3.205 & 0.410 & 0.00668 & 1803133 & 27.405 & 5.938 & 19.925 & 5.301 & 24.183 & 52.02 & \mathrm{C}\end{array}$ $\begin{array}{llllllllllll}10984 & 4.024 & 0.257 & 0.00668 & 1802999 & 27.403 & 6.532 & 18.695 & 5.913 & 23.102 & 52.02 & C\end{array}$

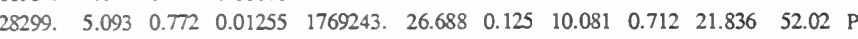
$\begin{array}{llllllllllll}22265 . & 4.432 & 0.575 & 0.01255 & 1768557 . & 26.674 & 0.738 & 14.056 & 1.325 & 21.927 & 52.02 & \mathrm{P}\end{array}$ $\begin{array}{lllllllllllll}19534 & 4.121 & 0.492 & 0.01255 & 1768211 & 26.666 & 1.352 & 16.822 & 1.634 & 22.229 & 52.02 & \mathrm{P}\end{array}$ $\begin{array}{lllllllllllll}17046 . & 3.830 & 0.421 & 0.01255 & 1767942 . & 26.660 & 1.964 & 19.050 & 1.939 & 23.582 & 52.02 & \mathrm{P}\end{array}$ $\begin{array}{llllllllllllll}12656 . & 3.292 & 0.306 & 0.01255 & 1767413 & 26.649 & 2.577 & 20.755 & 2.550 & 22.745 & 52.02 & P\end{array}$ $\begin{array}{lllllllllllll}9151 & 2.822 & 0.223 & 0.01255 & 1766897 & 26.638 & 3.174 & 21.783 & 3.148 & 23.872 & 52.02 & P\end{array}$ $\begin{array}{lllllllllllll}\text { 7325. } & 2.544 & 0.182 & 0.01255 & 1766244 & 26.624 & 3.504 & 22.416 & 3.530 & 23.920 & 52.02 & \mathrm{P}\end{array}$ $\begin{array}{llllllllllllll}5243 . & 2.201 & 0.138 & 0.01255 & 1766092 & 26.621 & 4.103 & 23.138 & 4.078 & 24.806 & 52.02 & P\end{array}$ $\begin{array}{lllllllllllll}3695 . & 1.876 & 0.104 & 0.01255 & 1765923 & 26.617 & 4.714 & 23.699 & 4.688 & 24.789 & 52.02 & \mathrm{P}\end{array}$ $\begin{array}{lllllllllllll}3218 & 1.734 & 0.090 & 0.01255 & 1765837 & 26.615 & 5.325 & 24.184 & 4.997 & 24.968 & 52.02 & P\end{array}$ $\begin{array}{llllllllllllll}2950 . & 1.608 & 0.078 & 0.01255 & 1765754 & 26.614 & 5.938 & 24.485 & 5.301 & 25.002 & 52.02 & \mathrm{P}\end{array}$ $\begin{array}{llllllllllllll}3019 & 1.399 & 0.056 & 0.01255 & 1765587 & 26.610 & 6.532 & 24.672 & 5.913 & 25.050 & 52.02 & \mathrm{P}\end{array}$ $\begin{array}{llllllllllll}24134 . & 4.685 & 0.773 & 0.01192 & 2069719 & 32.721 & 0.125 & 17.775 & 0.712 & 28.262 & 52.02 & P\end{array}$

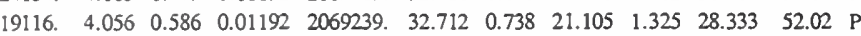
$\begin{array}{llllllllllllll}16837 . & 3.762 & 0.507 & 0.01192 & 2068997 & 32.707 & 1.352 & 23.578 & 1.634 & 28.659 & 52.02 & \mathrm{P}\end{array}$ $\begin{array}{llllllllllllll}14757 . & 3.488 & 0.439 & 0.01192 & 2068776 & 32.703 & 1.964 & 25.380 & 1.939 & 29.869 & 52.02 & P\end{array}$ $\begin{array}{llllllllllll}11072 . & 2.980 & 0.328 & 0.01192 & 2068334 & 32.694 & 2.577 & 26.838 & 2.550 & 29.234 & 52.02 & P\end{array}$ $\begin{array}{lllllllllllll}8109 . & 2.542 & 0.248 & 0.01192 & 2067903 & 32.686 & 3.174 & 27.771 & 3.148 & 30.220 & 52.02 & \mathrm{P}\end{array}$ $\begin{array}{llllllllllllll}6552 . & 2.289 & 0.207 & 0.01192 & 2067547 & 32.679 & 3.504 & 28.540 & 3.530 & 30.262 & 52.02 & \mathrm{P}\end{array}$ $\begin{array}{llllllllllllll}\text { 4755. } & 1.975 & 0.163 & 0.01192 & 2067413 & 32.677 & 4.103 & 29.004 & 4.078 & 31.049 & 52.02 & \mathrm{P}\end{array}$ $\begin{array}{llllllllllllll}3380 . & 1.681 & 0.129 & 0.01192 & 2067265 & 32.674 & 4.714 & 29.641 & 4.688 & 30.990 & 52.02 & \mathrm{P}\end{array}$ $\begin{array}{llllllllllll}2933 . & 1.554 & 0.115 & 0.01192 & 2067190 & 32.673 & 5.325 & 29.862 & 4.997 & 31.123 & 52.02 & \mathrm{P}\end{array}$ $\begin{array}{llllllllllllll}2657 & 1.445 & 0.103 & 0.01192 & 2067129 & 32.672 & 5.938 & 30.183 & 5.301 & 31.237 & 52.02 & \mathrm{P}\end{array}$ $\begin{array}{lllllllllllllll}2596 . & 1.269 & 0.081 & 0.01192 & 2067010 & 32.669 & 6.532 & 30.450 & 5.913 & 31.304 & 52.02 & \mathrm{P}\end{array}$ $\begin{array}{lllllllllllll}28506 & 5.247 & 0.772 & 0.01290 & 1832715 & 28.023 & 0.125 & 11.228 & 0.712 & 22.998 & 52.02 & P\end{array}$ $\begin{array}{llllllllllll}22432 . & 4.551 & 0.577 & 0.01290 & 1832014 & 28.009 & 0.738 & 15.223 & 1.325 & 23.150 & 52.02 & \mathrm{P}\end{array}$ $\begin{array}{lllllllllllll}19683 . & 4.225 & 0.495 & 0.01290 & 1831661 & 28.002 & 1.352 & 18.019 & 1.634 & 23.450 & 52.02 & \mathrm{P}\end{array}$ $\begin{array}{lllllllllllll}17178 . & 3.921 & 0.425 & 0.01290 & 1831388 & 27.996 & 1.964 & 20.247 & 1.939 & 24.853 & 52.02 & \mathrm{P}\end{array}$ $\begin{array}{lllllllllllll}12758 . & 3.359 & 0.310 & 0.01290 & 1830851 & 27.985 & 2.577 & 21.960 & 2.550 & 24.032 & 52.02 & P\end{array}$ $\begin{array}{lllllllllllll}9228 & 2.871 & 0.228 & 0.01290 & 1830327 & 27.974 & 3.174 & 22.999 & 3.148 & 25.156 & 52.02 & P\end{array}$ $\begin{array}{llllllllllllll}\text { 7388. } & 2.586 & 0.187 & 0.01290 & 1829760 & 27.962 & 3.504 & 23.670 & 3.530 & 25.222 & 52.02 & \mathrm{P}\end{array}$ $\begin{array}{lllllllllllll}5291 . & 2.235 & 0.144 & 0.01290 & 1829592 & 27.959 & 4.103 & 24.370 & 4.078 & 26.099 & 52.02 & P\end{array}$ $\begin{array}{llllllllllllll}3731 . & 1.904 & 0.110 & 0.01290 & 1829405 & 27.955 & 4.714 & 24.951 & 4.688 & 26.061 & 52.02 & P\end{array}$ $\begin{array}{llllllllllllll}3250 . & 1.761 & 0.097 & 0.01290 & 1829310 & 27.953 & 5.325 & 25.411 & 4.997 & 26.291 & 52.02 & P\end{array}$ $\begin{array}{llllllllllllll}2980 & 1.636 & 0.085 & 0.01290 & 1829224 & 27.951 & 5.938 & 25.722 & 5.301 & 26.296 & 52.02 & \mathrm{P}\end{array}$ $\begin{array}{lllllllllllllll}3047 . & 1.433 & 0.062 & 0.01290 & 1829052 & 27.947 & 6.532 & 25.929 & 5.913 & 26.377 & 52.02 & P\end{array}$ $\begin{array}{lllllllllllll}20936 . & 3.887 & 0.777 & 0.01205 & 2222794 & 35.542 & 0.125 & 23.181 & 0.712 & 31.884 & 52.02 & \mathrm{P}\end{array}$ $\begin{array}{lllllllllllll}16460 & 3.315 & 0.613 & 0.01205 & 22222246 . & 35.533 & 0.738 & 26.122 & 1.325 & 31.913 & 52.02 & \mathrm{P}\end{array}$

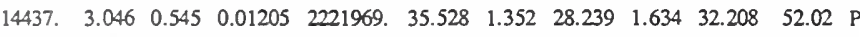
$\begin{array}{llllllllllll}12598 . & 2.795 & 0.486 & 0.01205 & 2221723 & 35.523 & 1.964 & 29.833 & 1.939 & 33.240 & 52.02 & \mathrm{P}\end{array}$ $\begin{array}{lllllllllllll}9364 . & 2.331 & 0.389 & 0.01205 & 2221234 & 35.514 & 2.577 & 31.086 & 2.550 & 32.789 & 52.02 & \mathrm{P}\end{array}$ $\begin{array}{lllllllllllll}6797 . & 1.928 & 0.321 & 0.01205 & 2220756 & 35.506 & 3.174 & 31.883 & 3.148 & 33.714 & 52.02 & \mathrm{P}\end{array}$ $\begin{array}{lllllllllllll}5471 . & 1.691 & 0.286 & 0.01205 & 2220151 . & 35.495 & 3.504 & 32.464 & 3.530 & 33.760 & 52.02 & \mathrm{P}\end{array}$

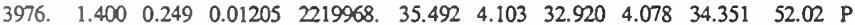
$\begin{array}{lllllllllllll}2893 & 1.126 & 0.219 & 0.01205 & 2219764 & 35.488 & 4.714 & 33.407 & 4.688 & 34.336 & 52.02 & P\end{array}$ $\begin{array}{lllllllllllll}2577 . & 1.007 & 0.207 & 0.01205 & 2219661 & 35.486 & 5.325 & 33.682 & 4.997 & 34.432 & 52.02 & \mathrm{P}\end{array}$ $\begin{array}{llllllllllllllll}2419 & 0.902 & 0.196 & 0.01205 & 2219545 & 35.484 & 5.938 & 33.966 & 5.301 & 34.571 & 52.02 & \mathrm{P}\end{array}$ $\begin{array}{llllllllllllll}2562 . & 0.732 & 0.175 & 0.01205 & 2219309 & 35.480 & 6.532 & 34.221 & 5.913 & 34.712 & 52.02 & \mathrm{P}\end{array}$ $\begin{array}{llllllllllll}20432 . & 4.075 & 0.735 & 0.00828 & 1620458 & 23.410 & 0.125 & 9.402 & 0.712 & 19.770 & 52.02 & P\end{array}$ $\begin{array}{lllllllllllll}15957 . & 3.491 & 0.524 & 0.00828 & 1620272 & 23.406 & 0.738 & 12.889 & 1.325 & 19.482 & 52.02 & \mathrm{P}\end{array}$ $\begin{array}{llllllllllll}13921 . & 3.232 & 0.437 & 0.00828 & 1620178 & 23.404 & 1.352 & 15.295 & 1.634 & 19.839 & 52.02 & \mathrm{P}\end{array}$ $\begin{array}{lllllllllllll}12058 . & 3.000 & 0.362 & 0.00828 & 1620064 & 23.401 & 1.964 & 17.225 & 1.939 & 20.795 & 52.02 & \mathrm{P}\end{array}$ $\begin{array}{lllllllllllll}\text { 8745. } & 2.602 & 0.243 & 0.00828 & 1619832 . & 23.396 & 2.57 & 18.658 & 2.550 & 20.237 & 52.02 & \mathrm{P}\end{array}$ $\begin{array}{lllllllllllll}6061 . & 2.304 & 0.160 & 0.00828 & 1619606 & 23.391 & 3.174 & 19.479 & 3.148 & 21.353 & 52.02 & \mathrm{P}\end{array}$ $\begin{array}{llllllllllllll}4635 . & 2.161 & 0.120 & 0.00828 & 1619553 & 23.390 & 3.504 & 20.027 & 3.530 & 21.274 & 52.02 & P\end{array}$ 2973. $\begin{array}{lllllllllllll}1.989 & 0.081 & 0.00828 & 1618115 & 23.357 & 4.103 & 20.599 & 4.078 & 21.783 & 52.02 & \mathrm{P}\end{array}$ $\begin{array}{lllllllllllll}1665 . & 1.885 & 0.055 & 0.00828 & 1616516 & 23.320 & 4.714 & 20.917 & 4.688 & 21.407 & 52.02 & \mathrm{P}\end{array}$ $\begin{array}{lllllllllllll}\text { 1219. } & 1.868 & 0.047 & 0.00828 & 1615706 . & 23.302 & 5.325 & 21.150 & 4.997 & 21.360 & 52.02 & P\end{array}$ 923. $\begin{array}{llllllllllll}1.895 & 0.041 & 0.00828 & 1615818 & 23.305 & 5.938 & 21.212 & 5.301 & 21.349 & 52.02 & \mathrm{P}\end{array}$ $\begin{array}{llllllllllllll}\text { 756. } & 2.022 & 0.032 & 0.00828 & 1616185 & 23.313 & 6.532 & 21.235 & 5.913 & 21.224 & 52.02 & \mathrm{P}\end{array}$

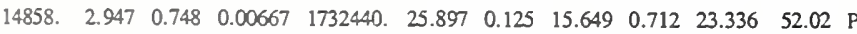
$\begin{array}{llllllllllllll}11660 . & 2.542 & 0.554 & 0.00667 & 1732408 & 25.897 & 0.738 & 18.179 & 1.325 & 22.999 & 52.02 & \mathrm{P}\end{array}$ $\begin{array}{lllllllllllll}10210 . & 2.355 & 0.473 & 0.00667 & 1732391 & 25.896 & 1.352 & 19.953 & 1.634 & 23.244 & 52.02 & P\end{array}$ $\begin{array}{llllllllllllll}\text { 8888. } & 2.178 & 0.404 & 0.00667 & 1732301 & 25.894 & 1.964 & 21.321 & 1.939 & 23.988 & 52.02 & \mathrm{P}\end{array}$ $\begin{array}{llllllllllllll}6552 . & 1.855 & 0.291 & 0.00667 & 1732106 . & 25.890 & 2.577 & 22.386 & 2.550 & 23.646 & 52.02 & \mathrm{P}\end{array}$ $\begin{array}{lllllllllllllll}4680 . & 1.580 & 0.212 & 0.00667 & 1731916 & 25.886 & 3.174 & 23.020 & 3.148 & 24.507 & 52.02 & P\end{array}$ $\begin{array}{lllllllllllll}3702 . & 1.435 & 0.172 & 0.00667 & 1732240 & 25.893 & 3.504 & 23.466 & 3.530 & 24.440 & 52.02 & \mathrm{P}\end{array}$ $\begin{array}{lllllllllllllll}2581 . & 1.244 & 0.132 & 0.00667 & 1732207 & 25.892 & 4.103 & 23.887 & 4.078 & 25.000 & 52.02 & P\end{array}$ 1737. $\begin{array}{llllllllllll}1.071 & 0.101 & 0.00667 & 1732171 & 25.891 & 4.714 & 24.222 & 4.688 & 24.779 & 52.02 & P\end{array}$

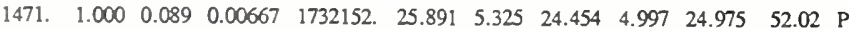

$\begin{array}{llllllllllll}1315 & 0.939 & 0.079 & 0.00667 & 1732103 & 25.890 & 5.938 & 24.616 & 5.301 & 24.850 & 52.02 & \mathrm{P}\end{array}$ $\begin{array}{lllllllllllll}\text { 1321. } & 0.848 & 0.060 & 0.00667 & 1732000 & 25.888 & 6.532 & 24.729 & 5.913 & 24.947 & 52.02 & P\end{array}$ $\begin{array}{lllllllllllll}16606 . & 3.399 & 0.758 & 0.00745 & 1832874 & 28.027 & 0.125 & 15.564 & 0.712 & 24.993 & 52.02 & P\end{array}$ $\begin{array}{llllllllllllll}12916 . & 2.893 & 0.561 & 0.00745 & 1832750 & 28.024 & 0.738 & 18.732 & 1.325 & 24.759 & 52.02 & \mathrm{P}\end{array}$ $\begin{array}{lllllllllllll}11249 . & 2.659 & 0.480 & 0.00745 & 1832688 & 28.023 & 1.352 & 20.967 & 1.634 & 25.109 & 52.02 & \mathrm{P}\end{array}$ $\begin{array}{lllllllllllll}9733 . & 2.441 & 0.411 & 0.00745 & 1832588 & 28.021 & 1.964 & 22.635 & 1.939 & 25.904 & 52.02 & P\end{array}$ $\begin{array}{llllllllllllll}7066 . & 2.044 & 0.299 & 0.00745 & 1832381 & 28.017 & 2.577 & 23.913 & 2.550 & 25.544 & 52.02 & P\end{array}$

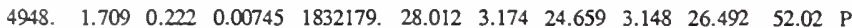
3849. $\begin{array}{llllllllllllll}1.530 & 0.185 & 0.00745 & 1832420 & 28.017 & 3.504 & 25.221 & 3.530 & 26.415 & 52.02 & \mathrm{P}\end{array}$ 2612. $\begin{array}{lllllllllllll}1.303 & 0.146 & 0.00745 & 1832399 & 28.017 & 4.103 & 25.675 & 4.078 & 27.096 & 52.02 & P\end{array}$ $\begin{array}{llllllllllllll}1715 . & 1.102 & 0.118 & 0.00745 & 1832376 & 28.016 & 4.714 & 26.095 & 4.688 & 26.979 & 52.02 & \mathrm{P}\end{array}$ $\begin{array}{lllllllllllll}\text { 1451. } & 1.021 & 0.108 & 0.00745 & 1832364 & 28.016 & 5.325 & 26.325 & 4.997 & 26.934 & 52.02 & \mathrm{P}\end{array}$ $\begin{array}{lllllllllllll}1318 . & 0.954 & 0.099 & 0.00745 & 1832316 & 28.015 & 5.938 & 26.516 & 5.301 & 27.023 & 52.02 & \mathrm{P}\end{array}$ $\begin{array}{llllllllllllll}1428 & 0.860 & 0.081 & 0.00745 & 1832212 & 28.013 & 6.532 & 26.659 & 5.913 & 27.055 & 52.02 & \mathrm{P}\end{array}$ $\begin{array}{lllllllllllll}\text { 14453. } & 3.020 & 0.748 & 0.00671 & 2006084 & 31.501 & 0.125 & 20.715 & 0.712 & 28.886 & 52.02 & \mathrm{P}\end{array}$ $\begin{array}{lllllllllllll}11240 . & 2.583 & 0.553 & 0.00671 & 2006020 & 31.500 & 0.738 & 23.458 & 1.325 & 28.531 & 52.02 & \mathrm{P}\end{array}$ $\begin{array}{llllllllllllll}9788 & 2.380 & 0.472 & 0.00671 & 2005988 & 31.499 & 1.352 & 25.401 & 1.634 & 28.780 & 52.02 & \mathrm{P}\end{array}$ $\begin{array}{llllllllllllll}8468 . & 2.189 & 0.403 & 0.00671 & 2005903 & 31.498 & 1.964 & 26.851 & 1.939 & 29.597 & 52.02 & P\end{array}$ $\begin{array}{lllllllllllllll}6148 . & 1.839 & 0.293 & 0.00671 & 2005724 & 31.494 & 2.577 & 27.970 & 2.550 & 29.318 & 52.02 & P\end{array}$ $\begin{array}{llllllllllllll}4307 . & 1.539 & 0.216 & 0.00671 & 2005549 & 31.491 & 3.174 & 28.630 & 3.148 & 30.177 & 52.02 & \mathrm{P}\end{array}$ $\begin{array}{lllllllllllll}\text { 3353. } & 1.377 & 0.178 & 0.00671 & 2005868 & 31.497 & 3.504 & 29.114 & 3.530 & 30.091 & 52.02 & P\end{array}$ $\begin{array}{llllllllllllll}2281 . & 1.166 & 0.140 & 0.00671 & 2005844 & 31.496 & 4.103 & 29.506 & 4.078 & 30.657 & 52.02 & \mathrm{P}\end{array}$ $\begin{array}{lllllllllllllll}1506 . & 0.973 & 0.112 & 0.00671 & 2005817 & 31.496 & 4.714 & 29.861 & 4.688 & 30.525 & 52.02 & \mathrm{P}\end{array}$ $\begin{array}{lllllllllllll}1280 & 0.891 & 0.102 & 0.00671 & 2005804 & 31.496 & 5.325 & 30.065 & 4.997 & 30.589 & 52.02 & \mathrm{P}\end{array}$ $\begin{array}{llllllllllllll}1168 . & 0.821 & 0.092 & 0.00671 & 2005763 & 31.495 & 5.938 & 30.240 & 5.301 & 30.604 & 52.02 & P\end{array}$ $\begin{array}{llllllllllll}1273 & 0.713 & 0.074 & 0.00671 & 2005676 . & 31.493 & 6.532 & 30.379 & 5.913 & 30.707 & 52.02 & P\end{array}$ 13801. $\begin{array}{llllllllllll}2.898 & 0.757 & 0.00670 & 2218118 & 35.458 & 0.125 & 25.016 & 0.712 & 32.981 & 52.02 & \mathrm{P}\end{array}$ $\begin{array}{lllllllllllll}10728 . & 2.479 & 0.563 & 0.00670 & 2218019 & 35.457 & 0.738 & 27.627 & 1.325 & 32.568 & 52.02 & P\end{array}$ $\begin{array}{lllllllllllll}9341 . & 2.283 & 0.483 & 0.00670 & 2217969 & 35.456 & 1.352 & 29.516 & 1.634 & 32.837 & 52.02 & P\end{array}$ $\begin{array}{lllllllllllll}8079 . & 2.100 & 0.415 & 0.00670 & 2217884 & 35.454 & 1.964 & 30.886 & 1.939 & 33.630 & 52.02 & P\end{array}$ $\begin{array}{llllllllllll}5863 . & 1.762 & 0.305 & 0.00670 & 2217708 & 35.451 & 2.577 & 31.955 & 2.550 & 33.387 & 52.02 & P\end{array}$ $\begin{array}{lllllllllllll}4107 . & 1.471 & 0.229 & 0.00670 & 2217536 & 35.448 & 3.174 & 32.592 & 3.148 & 34.184 & 52.02 & \mathrm{P}\end{array}$ $\begin{array}{lllllllllllll}3199 . & 1.313 & 0.192 & 0.00670 & 2217857 & 35.454 & 3.504 & 33.092 & 3.530 & 34.113 & 52.02 & \mathrm{P}\end{array}$ $\begin{array}{llllllllllllll}2181 . & 1.106 & 0.154 & 0.00670 & 2217835 & 35.453 & 4.103 & 33.428 & 4.078 & 34.671 & 52.02 & P\end{array}$ $\begin{array}{lllllllllllll}1449 . & 0.914 & 0.126 & 0.00670 & 2217810 & 35.453 & 4.714 & 33.802 & 4.688 & 34.522 & 52.02 & P\end{array}$ $\begin{array}{llllllllllllll}\text { 1239. } & 0.832 & 0.116 & 0.00670 & 2217797 & 35.453 & 5.325 & 33.956 & 4.997 & 34.644 & 52.02 & \mathrm{P}\end{array}$ $\begin{array}{llllllllllllll}1138 . & 0.761 & 0.107 & 0.00670 & 2217761 & 35.452 & 5.938 & 34.138 & 5.301 & 34.617 & 52.02 & \mathrm{P}\end{array}$

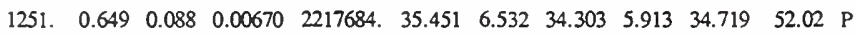
$\begin{array}{llllllllllllll}14319 . & 3.022 & 0.749 & 0.00658 & 2073059 & 32.784 & 0.125 & 22.253 & 0.712 & 30.154 & 52.02 & \mathrm{P}\end{array}$ $\begin{array}{lllllllllllll}11103 . & 2.580 & 0.549 & 0.00658 & 2072982 & 32.782 & 0.738 & 24.990 & 1.325 & 29.817 & 52.02 & \mathrm{P}\end{array}$ $\begin{array}{llllllllllll}9652 . & 2.374 & 0.467 & 0.00658 & 2072943 & 32.782 & 1.352 & 26.892 & 1.634 & 30.066 & 52.02 & \mathrm{P}\end{array}$ $\begin{array}{lllllllllllllll}8334 & 2.182 & 0.397 & 0.00658 & 2072858 & 32.780 & 1.964 & 28.354 & 1.939 & 30.904 & 52.02 & \mathrm{P}\end{array}$ $\begin{array}{llllllllllllll}6022 & 1.829 & 0.285 & 0.00658 & 2072680 & 32.777 & 2.577 & 29.459 & 2.550 & 30.631 & 52.02 & \mathrm{P}\end{array}$ $\begin{array}{llllllllllllll}4194 & 1.528 & 0.207 & 0.00658 & 2072506 & 32.773 & 3.174 & 30.097 & 3.148 & 31.448 & 52.02 & \mathrm{P}\end{array}$ $\begin{array}{lllllllllllll}3252 . & 1.366 & 0.170 & 0.00658 & 2072833 & 32.780 & 3.504 & 30.552 & 3.530 & 31.405 & 52.02 & \mathrm{P}\end{array}$ $\begin{array}{lllllllllllll}2200 & 1.155 & 0.132 & 0.00658 & 2072817 & 32.779 & 4.103 & 30.955 & 4.078 & 31.937 & 52.02 & \mathrm{P}\end{array}$ $\begin{array}{llllllllllll}\text { 1453. } & 0.963 & 0.104 & 0.00658 & 2072800 & 32.779 & 4.714 & 31.275 & 4.688 & 31.786 & 52.02 & P\end{array}$ $\begin{array}{llllllllllllll}\text { 1244. } & 0.882 & 0.094 & 0.00658 & 2072792 & 32.779 & 5.325 & 31.506 & 4.997 & 31.912 & 52.02 & P\end{array}$ $\begin{array}{llllllllllllll}1150 . & 0.814 & 0.084 & 0.00658 & 2072749 & 32.778 & 5.938 & 31.668 & 5.301 & 31.876 & 52.02 & \mathrm{P}\end{array}$ $\begin{array}{llllllllllll}\text { 1294. } & 0.710 & 0.066 & 0.00658 & 2072658 & 32.776 & 6.532 & 31.812 & 5.913 & 32.008 & 52.02 & P\end{array}$ 


\title{
Convective condensation of
}

\author{
R125 within a micro-fin tube
}

(file: taraw.tbl)

$\begin{array}{ccccccccccc}q^{\prime \prime} & \Delta T_{c} & x_{q} & m_{s} & P_{r} & T_{c} & z_{c} & T_{r} & z_{w} & T_{w} & M \text { flow } \\ \left(\mathrm{W} / \mathrm{m}^{2}\right) & (\mathrm{K}) & & (\mathrm{kg} / \mathrm{s}) & (\mathrm{Pa}) & \left({ }^{\circ} \mathrm{C}\right) & (\mathrm{m}) & \left({ }^{\circ} \mathrm{C}\right) & (\mathrm{m}) & \left({ }^{\circ} \mathrm{C}\right) & (\mathrm{g} / \text { mole })\end{array}$ $\begin{array}{llllllllllll}858 . & 6.763 & 1.073 & 0.01379 & 1553765 & 36.522 & 0.125 & 29.346 & 0.712 & 29.555 & 120.02 & \mathrm{C}\end{array}$ $\begin{array}{lllllllllllllll}1665 . & 4.788 & 1.050 & 0.01379 & 1553473 & 34.316 & 0.738 & 28.882 & 1.325 & 29.328 & 120.02 & \mathrm{C}\end{array}$ $\begin{array}{llllllllllllll}2096 & 3.320 & 1.033 & 0.01379 & 1553326 & 32.679 & 1.352 & 28.553 & 1.634 & 29.120 & 120.02 & \mathrm{C}\end{array}$ $\begin{array}{llllllllllll}2536 & 1.564 & 1.012 & 0.01379 & 1553025 & 30.722 & 1.964 & 28.142 & 1.939 & 29.132 & 120.02 & \mathrm{C}\end{array}$ $\begin{array}{llllllllllll}3466 . & 0.988 & 0.957 & 0.01379 & 1552393 & 29.638 & 2.577 & 27.556 & 2.550 & 28.358 & 120.02 & \mathrm{C}\end{array}$ $\begin{array}{lllllllllllll}4433 . & 1.602 & 0.887 & 0.01379 & 1551776 & 29.623 & 3.174 & 26.759 & 3.148 & 28.486 & 120.02 & \mathrm{C}\end{array}$ $\begin{array}{lllllllllllllll}5085 & 2.022 & 0.833 & 0.01379 & 1549710 & 29.571 & 3.504 & 26.016 & 3.530 & 27.925 & 120.02 & \mathrm{C}\end{array}$ $\begin{array}{llllllllllll}6059 . & 2.783 & 0.743 & 0.01379 & 1549262 & 29.559 & 4.103 & 24.875 & 4.078 & 27.256 & 120.02 & \text { C }\end{array}$ $\begin{array}{llllllllllllllll}7201 & 3.758 & 0.623 & 0.01379 & 1548765 & 29.547 & 4.714 & 23.310 & 4.688 & 26.147 & 120.02 & C\end{array}$ $\begin{array}{llllllllllllll}7803 . & 4.305 & 0.554 & 0.01379 & 1548513 & 29.540 & 5.325 & 21.321 & 4.997 & 24.939 & 120.02 & \mathrm{C}\end{array}$ $\begin{array}{lllllllllllll}8411 . & 4.880 & 0.481 & 0.01379 & 1548344 & 29.536 & 5.938 & 19.144 & 5.301 & 24.650 & 120.02 & \mathrm{C}\end{array}$ $\begin{array}{lllllllllllll}9681 . & 6.141 & 0.317 & 0.01379 & 1548018 & 29.528 & 6.532 & 17.176 & 5.913 & 22.972 & 120.02 & C\end{array}$ $\begin{array}{llllllllllll}724 & 6.471 & 1.080 & 0.01379 & 1798569 & 42.071 & 0.125 & 35.248 & 0.712 & 35.442 & 120.02 & \mathrm{C}\end{array}$ $\begin{array}{lllllllllllllll}1426 & 4.4917 & 1.059 & 0.01379 & 1798280 & 40.306 & 0.738 & 34.730 & 1.325 & 35.209 & 120.02 & \mathrm{C}\end{array}$ $\begin{array}{lllllllllllll}1807 & 3.751 & 1.043 & 0.01379 & 1798134 & 38.985 & 1.352 & 34.430 & 1.634 & 35.008 & 120.02 & C\end{array}$ $\begin{array}{llllllllllllll}2199 & 2.350 & 1.024 & 0.01379 & 1797870 & 37.400 & 1.964 & 34.058 & 1.939 & 35.011 & 120.02 & \mathrm{C}\end{array}$ $\begin{array}{lllllllllllllll}3039 & 0.901 & 0.973 & 0.01379 & 1797319 & 35.486 & 2.577 & 33.523 & 2.550 & 34.319 & 120.02 & \mathrm{C}\end{array}$ $\begin{array}{lllllllllllll}3927 . & 1.464 & 0.907 & 0.01379 & 1796781 & 35.474 & 3.174 & 32.826 & 3.148 & 34.396 & 120.02 & \mathrm{C}\end{array}$ $\begin{array}{lllllllllllllll}4532 & 1.861 & 0.855 & 0.01379 & 1795221 & 35.439 & 3.504 & 32.255 & 3.530 & 33.916 & 120.02 & \mathrm{C}\end{array}$ $\begin{array}{lllllllllllllll}5445 & 2.559 & 0.768 & 0.01379 & 1794868 & 35.431 & 4.103 & 31.199 & 4.078 & 33.334 & 120.02 & C\end{array}$ $\begin{array}{llllllllllll}6526 . & 3.454 & 0.653 & 0.01379 & 1794476 & 35.422 & 4.714 & 29.809 & 4.688 & 32.290 & 120.02 & C\end{array}$ $\begin{array}{lllllllllllllll}7100 . & 3.955 & 0.586 & 0.01379 & 1794277 & 35.417 & 5.325 & 27.978 & 4.997 & 31.227 & 120.02 & C\end{array}$ $\begin{array}{llllllllllllllll}7683 & 4.481 & 0.514 & 0.01379 & 1794125 & 35.414 & 5.938 & 25.980 & 5.301 & 30.924 & 120.02 & \mathrm{C}\end{array}$ $\begin{array}{lllllllllllll}8908 . & 5.635 & 0.353 & 0.01379 & 1793826 & 35.407 & 6.532 & 24.070 & 5.913 & 29.378 & 120.02 & C\end{array}$ $\begin{array}{lllllllllllllll}1810 & 1.447 & 1.007 & 0.01818 & 1729308 & 34.517 & 0.125 & 32.588 & 0.712 & 33.124 & 120.02 & \mathrm{C}\end{array}$ $\begin{array}{llllllllllllll}3215 & 1.043 & 0.971 & 0.01818 & 1728603 & 33.910 & 0.738 & 32.079 & 1.325 & 32.821 & 120.02 & \mathrm{C}\end{array}$ $\begin{array}{llllllllllll}3866 . & 1.183 & 0.945 & 0.01818 & 1728247 & 33.901 & 1.352 & 31.641 & 1.634 & 32.496 & 120.02 & \mathrm{C}\end{array}$ $\begin{array}{llllllllllll}4469 . & 1.349 & 0.915 & 0.01818 & 1727786 & 33.891 & 1.964 & 31.124 & 1.939 & 32.653 & 120.02 & C\end{array}$ $\begin{array}{llllllllllllll}5566 . & 1.773 & 0.842 & 0.01818 & 1726841 & 33.869 & 2.577 & 30.456 & 2.550 & 31.636 & 120.02 & \mathrm{C}\end{array}$ $\begin{array}{lllllllllllllll}6492 & 2.304 & 0.757 & 0.01818 & 1725917 & 33.847 & 3.174 & 29.736 & 3.148 & 31.893 & 120.02 & \mathrm{C}\end{array}$ $\begin{array}{llllllllllll}7013 . & 2.652 & 0.695 & 0.01818 & 1723023 & 33.780 & 3.504 & 29.309 & 3.530 & 31.300 & 120.02 & \mathrm{C}\end{array}$ $\begin{array}{lllllllllllllllll}7650 & 3.317 & 0.600 & 0.01818 & 1722484 & 33.767 & 4.103 & 28.330 & 4.078 & 30.731 & 120.02 & \mathrm{C}\end{array}$ $\begin{array}{llllllllllllll}8214 & 4.171 & 0.486 & 0.01818 & 1721884 & 33.753 & 4.714 & 27.294 & 4.688 & 29.742 & 120.02 & \mathrm{C}\end{array}$ $\begin{array}{llllllllllllll}8442 . & 4.650 & 0.425 & 0.01818 & 1721580 & 33.746 & 5.325 & 25.952 & 4.997 & 28.705 & 120.02 & C\end{array}$ $\begin{array}{lllllllllllll}8629 . & 5.154 & 0.364 & 0.01818 & 1721380 & 33.741 & 5.938 & 24.708 & 5.301 & 28.647 & 120.02 & \mathrm{C}\end{array}$ $\begin{array}{lllllllllllllll}8891 . & 6.260 & 0.237 & 0.01818 & 1720994 & 33.732 & 6.532 & 23.659 & 5.913 & 27.399 & 120.02 & \mathrm{C}\end{array}$ $\begin{array}{lllllllllllll}2541 . & 2.966 & 1.026 & 0.02847 & 1981893 & 41.303 & 0.125 & 37.712 & 0.712 & 38.323 & 120.02 & \mathrm{C}\end{array}$ $\begin{array}{lllllllllllll}4582 . & 1.450 & 0.990 & 0.02847 & 1980581 & 39.478 & 0.738 & 37.038 & 1.325 & 37.880 & 120.02 & \mathrm{C}\end{array}$ $\begin{array}{lllllllllllllll}5556 . & 1.662 & 0.964 & 0.02847 & 1979919 & 39.464 & 1.352 & 36.448 & 1.634 & 37.438 & 120.02 & C\end{array}$ $\begin{array}{lllllllllllll}6481 . & 1.915 & 0.935 & 0.02847 & 1979148 & 39.448 & 1.964 & 35.743 & 1.939 & 37.673 & 120.02 & \mathrm{C}\end{array}$ $\begin{array}{llllllllllllll}82230 & 2.561 & 0.861 & 0.02847 & 1977578 & 39.415 & 2.577 & 34.809 & 2.550 & 36.172 & 120.02 & \mathrm{C}\end{array}$ $\begin{array}{lllllllllllllll}9802 . & 3.371 & 0.774 & 0.02847 & 1976044 & 39.382 & 3.174 & 33.816 & 3.148 & 36.658 & 120.02 & \mathrm{C}\end{array}$

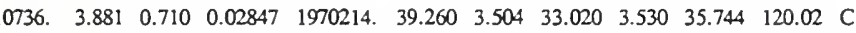
$\begin{array}{lllllllllllllll}11979 . & 4.893 & 0.609 & 0.02847 & 1969244 & 39.239 & 4.103 & 31.550 & 4.078 & 34.804 & 120.02 & \mathrm{C}\end{array}$ $\begin{array}{llllllllllll}13225 & 6.192 & 0.485 & 0.02847 & 1968165 & 39.216 & 4.714 & 29.823 & 4.688 & 33.363 & 120.02 & \mathrm{C}\end{array}$ $\begin{array}{llllllllllllll}13803 & 6.921 & 0.417 & 0.02847 & 1967618 & 39.205 & 5.325 & 27.803 & 4.997 & 31.721 & 120.02 & \mathrm{C}\end{array}$ $\begin{array}{llllllllllll}14336 . & 7.688 & 0.348 & 0.02847 & 1967271 & 39.197 & 5.938 & 25.722 & 5.301 & 31.580 & 120.02 & C\end{array}$ $\begin{array}{lllllllllllll}15301 & 9.372 & 0.201 & 0.02847 & 1966604 & 39.183 & 6.532 & 23.925 & 5.913 & 29.560 & 120.02 & \mathrm{C}\end{array}$ $\begin{array}{llllllllllll}2266 & 2.235 & 1.020 & 0.02787 & 2193984 & 44.949 & 0.125 & 42.112 & 0.712 & 42.776 & 120.02 & \mathrm{C}\end{array}$ $\begin{array}{llllllllllll}4088 & 1.328 & 0.985 & 0.02787 & 2192884 & 43.762 & 0.738 & 41.462 & 1.325 & 42.282 & 120.02 & C\end{array}$ $\begin{array}{llllllllllllll}4960 . & 1.523 & 0.960 & 0.02787 & 2192329 & 43.751 & 1.352 & 40.933 & 1.634 & 41.874 & 120.02 & \mathrm{C}\end{array}$ $\begin{array}{lllllllllllll}5787 . & 1.755 & 0.931 & 0.02787 & 2191684 & 43.739 & 1.964 & 40.284 & 1.939 & 42.072 & 120.02 & C\end{array}$ $\begin{array}{lllllllllllll}\text { 7356. } & 2.346 & 0.859 & 0.02787 & 2190370 & 43.713 & 2.577 & 39.434 & 2.550 & 40.755 & 120.02 & C\end{array}$ $\begin{array}{lllllllllllllll}8768 & 3.087 & 0.773 & 0.02787 & 2189086 & 43.688 & 3.174 & 38.552 & 3.148 & 41.189 & 120.02 & \mathrm{C}\end{array}$ $\begin{array}{llllllllllll}9609 . & 3.568 & 0.711 & 0.02787 & 2184337 & 43.596 & 3.504 & 37.890 & 3.530 & 40.385 & 120.02 & \mathrm{C}\end{array}$ $\begin{array}{lllllllllllll}10731 . & 4.491 & 0.612 & 0.02787 & 2183552 & 43.581 & 4.103 & 36.517 & 4.078 & 39.478 & 120.02 & C\end{array}$ $\begin{array}{lllllllllllllllll}11859 . & 5.675 & 0.489 & 0.02787 & 2182680 & 43.564 & 4.714 & 34.987 & 4.688 & 38.172 & 120.02 & \mathrm{C}\end{array}$ $\begin{array}{lllllllllllllll}12384 & 6.340 & 0.423 & 0.02787 & 2182238 & 43.555 & 5.325 & 33.111 & 4.997 & 36.715 & 120.02 & \mathrm{C}\end{array}$ $\begin{array}{llllllllllllll}12870 & 7.037 & 0.355 & 0.02787 & 2181918 & 43.549 & 5.938 & 31.227 & 5.301 & 36.576 & 120.02 & C\end{array}$ $\begin{array}{lllllllllllllll}13753 & 8.568 & 0.210 & 0.02787 & 2181291 & 43.537 & 6.532 & 29.621 & 5.913 & 34.755 & 120.02 & \mathrm{C}\end{array}$ $\begin{array}{lllllllllllllll}3242 . & 2.806 & 1.016 & 0.02649 & 1442728 & 28.353 & 0.125 & 25.006 & 0.712 & 25.531 & 120.02 & C\end{array}$ $\begin{array}{llllllllllllll}5760 . & 1.550 & 0.974 & 0.02649 & 1440944 & 26.745 & 0.738 & 24.400 & 1.325 & 25.043 & 120.02 & \mathrm{C}\end{array}$

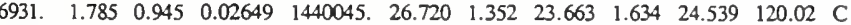
$\begin{array}{lllllllllllllll}8019 . & 2.064 & 0.911 & 0.02649 & 1438986 & 26.692 & 1.964 & 22.857 & 1.939 & 24.857 & 120.02 & \mathrm{C}\end{array}$ $\begin{array}{llllllllllllll}10011 . & 2.782 & 0.828 & 0.02649 & 1436828 & 26.635 & 2.577 & 21.778 & 2.550 & 22.985 & 120.02 & \mathrm{C}\end{array}$ $\begin{array}{llllllllllll}1707 . & 3.688 & 0.731 & 0.02649 & 1434720 & 26.578 & 3.174 & 20.548 & 3.148 & 23.655 & 120.02 & \mathrm{C}\end{array}$ $\begin{array}{llllllllllllll}12662 . & 4.194 & 0.662 & 0.02649 & 1426665 & 26.362 & 3.504 & 19.309 & 3.530 & 22.539 & 120.02 & \mathrm{C}\end{array}$ $\begin{array}{llllllllllllll}13856 & 5.340 & 0.554 & 0.02649 & 1425401 & 26.328 & 4.103 & 17.734 & 4.078 & 21.521 & 120.02 & C\end{array}$ $\begin{array}{llllllllllllll}\text { 14937. } & 6.812 & 0.422 & 0.02649 & 1423997 & 26.290 & 4.714 & 15.699 & 4.688 & 19.870 & 120.02 & \mathrm{C}\end{array}$ $\begin{array}{lllllllllllll}15387 & 7.639 & 0.353 & 0.02649 & 1423285 & 26.271 & 5.325 & 13.534 & 4.997 & 17.948 & 120.02 & \mathrm{C}\end{array}$ $\begin{array}{llllllllllll}15766 . & 8.516 & 0.282 & 0.02649 & 1422959 & 26.262 & 5.938 & 11.287 & 5.301 & 17.806 & 120.02 & \mathrm{C}\end{array}$ $\begin{array}{lllllllllllll}\text { 16335. } & 10.440 & 0.135 & 0.02649 & 1422364 & 26.246 & 6.532 & 9.460 & 5.913 & 15.572 & 120.02 & C\end{array}$ $\begin{array}{llllllllllllll}2858 & 3.418 & 1.025 & 0.02702 & 1643882 & 34.104 & 0.125 & 29.920 & 0.712 & 30.704 & 120.02 & \mathrm{C}\end{array}$ $\begin{array}{llllllllllllll}5109 . & 1.497 & 0.987 & 0.02702 & 1642290 & 31.861 & 0.738 & 29.230 & 1.325 & 30.226 & 120.02 & \mathrm{C}\end{array}$ $\begin{array}{llllllllllllll}6167 & 1.713 & 0.960 & 0.02702 & 1641488 & 31.841 & 1.352 & 28.572 & 1.634 & 29.765 & 120.02 & \text { C }\end{array}$ $\begin{array}{lllllllllllll}7160 & 1.972 & 0.929 & 0.02702 & 1640563 & 31.819 & 1.964 & 27.799 & 1.939 & 30.037 & 120.02 & \mathrm{C}\end{array}$ $\begin{array}{llllllllllll}9004 . & 2.634 & 0.852 & 0.02702 & 1638683 & 31.773 & 2.577 & 26.771 & 2.550 & 28.367 & 120.02 & \mathrm{C}\end{array}$ $\begin{array}{lllllllllllll}10612 . & 3.467 & 0.761 & 0.02702 & 1636846 & 31.729 & 3.174 & 25.694 & 3.148 & 28.931 & 120.02 & \mathrm{C}\end{array}$ $\begin{array}{lllllllllllll}11541 . & 3.954 & 0.696 & 0.02702 & 1629794 & 31.557 & 3.504 & 24.815 & 3.530 & 27.942 & 120.02 & C\end{array}$ $\begin{array}{lllllllllllll}\text { 12736. } & 5.003 & 0.593 & 0.02702 & 1628670 & 31.530 & 4.103 & 23.273 & 4.078 & 27.001 & 120.02 & C\end{array}$ 13876. $\begin{array}{llllllllllllll}6.350 & 0.467 & 0.02702 & 1627420 & 31.500 & 4.714 & 21.471 & 4.688 & 25.481 & 120.02 & C\end{array}$ $\begin{array}{llllllllllll}14377 . & 7.106 & 0.400 & 0.02702 & 1626786 & 31.484 & 5.325 & 19.314 & 4.997 & 23.765 & 120.02 & C\end{array}$ $\begin{array}{lllllllllllll}14821 . & 7.905 & 0.331 & 0.02702 & 1626444 & 31.476 & 5.938 & 17.205 & 5.301 & 23.643 & 120.02 & C\end{array}$ $\begin{array}{llllllllllll}15564 & 9.659 & 0.187 & 0.02702 & 1625799 & 31.460 & 6.532 & 15.458 & 5.913 & 21.595 & 120.02 & \mathrm{C}\end{array}$ $\begin{array}{llllllllllllll}2525 & 3.028 & 1.024 & 0.02726 & 1769504 & 36.770 & 0.125 & 33.087 & 0.712 & 33.752 & 120.02 & C\end{array}$ $\begin{array}{llllllllllllll}4547 . & 1.374 & 0.989 & 0.02726 & 1768033 & 34.820 & 0.738 & 32.446 & 1.325 & 33.319 & 120.02 & \mathrm{C}\end{array}$ $\begin{array}{llllllllllllll}5511 . & 1.574 & 0.964 & 0.02726 & 1767292 & 34.803 & 1.352 & 31.855 & 1.634 & 32.895 & 120.02 & C\end{array}$ $\begin{array}{lllllllllllll}6424 & 1.812 & 0.935 & 0.02726 & 1766438 & 34.784 & 1.964 & 31.156 & 1.939 & 33.132 & 120.02 & \mathrm{C}\end{array}$ $\begin{array}{llllllllllll}8148 . & 2.423 & 0.864 & 0.02726 & 1764702 & 34.744 & 2.577 & 30.222 & 2.550 & 31.633 & 120.02 & \mathrm{C}\end{array}$ $\begin{array}{llllllllllllll}9691 . & 3.191 & 0.779 & 0.02726 & 1763005 & 34.705 & 3.174 & 29.237 & 3.148 & 32.137 & 120.02 & \mathrm{C}\end{array}$ $\begin{array}{llllllllllllll}10604 & 3.648 & 0.718 & 0.02726 & 1756545 & 34.556 & 3.504 & 28.444 & 3.530 & 31.208 & 120.02 & C\end{array}$ $\begin{array}{lllllllllllll}11815 . & 4.612 & 0.620 & 0.02726 & 1755478 & 34.532 & 4.103 & 27.017 & 4.078 & 30.361 & 120.02 & C\end{array}$ $\begin{array}{lllllllllllll}13022 . & 5.851 & 0.500 & 0.02726 & 1754292 & 34.505 & 4.714 & 25.336 & 4.688 & 28.956 & 120.02 & \mathrm{C}\end{array}$ $\begin{array}{lllllllllllll}13578 . & 6.547 & 0.435 & 0.02726 & 1753691 & 34.491 & 5.325 & 23.335 & 4.997 & 27.379 & 120.02 & C\end{array}$ $\begin{array}{llllllllllllll}14089 & 7.280 & 0.368 & 0.02726 & 1753316 & 34.482 & 5.938 & 21.322 & 5.301 & 27.269 & 120.02 & C\end{array}$

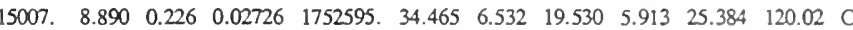
$\begin{array}{llllllllllllll}1031 & 1.599 & 1.016 & 0.01180 & 1909153 & 39.122 & 0.125 & 37.090 & 0.712 & 37.483 & 120.02 & \mathrm{C}\end{array}$ $\begin{array}{llllllllllllllll}1859 & 0.597 & 0.982 & 0.01180 & 1908909 & 37.952 & 0.738 & 36.690 & 1.325 & 37.258 & 120.02 & \mathrm{C}\end{array}$ $\begin{array}{llllllllllllll}2253 & 0.718 & 0.957 & 0.01180 & 1908786 & 37.949 & 1.352 & 36.389 & 1.634 & 37.001 & 120.02 & \text { C }\end{array}$ $\begin{array}{lllllllllllll}2628 & 0.861 & 0.929 & 0.01180 & 1908586 & 37.945 & 1.964 & 36.025 & 1.939 & 37.094 & 120.02 & C\end{array}$ $\begin{array}{lllllllllllll}3335 & 1.222 & 0.859 & 0.01180 & 1908170 & 37.936 & 2.577 & 35.528 & 2.550 & 36.393 & 120.02 & \mathrm{C}\end{array}$ $\begin{array}{lllllllllllllll}3969 & 1.673 & 0.775 & 0.01180 & 1907764 & 37.927 & 3.174 & 35.022 & 3.148 & 36.627 & 120.02 & \mathrm{C}\end{array}$ $\begin{array}{llllllllllllll}\text { 4346. } & 2.004 & 0.713 & 0.01180 & 1907116 & 37.913 & 3.504 & 34.683 & 3.530 & 36.129 & 120.02 & \mathrm{C}\end{array}$ $\begin{array}{lllllllllllll}4845 & 2.564 & 0.616 & 0.01180 & 1906931 & 37.909 & 4.103 & 33.862 & 4.078 & 35.719 & 120.02 & \mathrm{C}\end{array}$ $\begin{array}{lllllllllllllll}\text { 5345. } & 3.282 & 0.497 & 0.01180 & 1906726 & 37.904 & 4.714 & 32.954 & 4.688 & 34.797 & 120.02 & \mathrm{C}\end{array}$ $\begin{array}{llllllllllllll}5576 . & 3.684 & 0.432 & 0.01180 & 1906622 & 37.902 & 5.325 & 31.817 & 4.997 & 33.951 & 120.02 & \mathrm{C}\end{array}$ $\begin{array}{lllllllllllll}5788 . & 4.105 & 0.365 & 0.01180 & 1906539 & 37.900 & 5.938 & 30.742 & 5.301 & 33.799 & 120.02 & \text { C }\end{array}$ $\begin{array}{lllllllllllll}6172 & 5.028 & 0.225 & 0.01180 & 1906376 & 37.897 & 6.532 & 29.915 & 5.913 & 32.667 & 120.02 & \mathrm{C}\end{array}$ $\begin{array}{lllllllllllllll}425 & 0.555 & 1.007 & 0.00900 & 2141500 & 43.208 & 0.125 & 41.974 & 0.712 & 42.622 & 120.02 & \mathrm{C}\end{array}$ $\begin{array}{llllllllllllll}814 & 0.223 & 0.987 & 0.00900 & 2141391 & 42.753 & 0.738 & 41.599 & 1.325 & 42.374 & 120.02 & C\end{array}$ $\begin{array}{lllllllllllllll}1017 & 0.311 & 0.971 & 0.00900 & 2141336 & 42.752 & 1.352 & 41.406 & 1.634 & 42.243 & 120.02 & C\end{array}$ $\begin{array}{llllllllllll}1223 & 0.416 & 0.953 & 0.00900 & 2141234 & 42.750 & 1.964 & 41.154 & 1.939 & 42.300 & 120.02 & \mathrm{C}\end{array}$ $\begin{array}{lllllllllllll}1652 & 0.680 & 0.905 & 0.00900 & 2141020 & 42.746 & 2.577 & 40.735 & 2.550 & 41.854 & 120.02 & \mathrm{C}\end{array}$ $\begin{array}{lllllllllllll}2090 & 1.010 & 0.845 & 0.00900 & 2140810 & 42.742 & 3.174 & 40.392 & 3.148 & 42.000 & 120.02 & C\end{array}$ $\begin{array}{llllllllllllll}2380 & 1.258 & 0.798 & 0.00900 & 2140691 & 42.739 & 3.504 & 40.391 & 3.530 & 41.728 & 120.02 & \mathrm{C}\end{array}$ $\begin{array}{llllllllllllll}2810 . & 1.666 & 0.721 & 0.00900 & 2140647 & 42.739 & 4.103 & 39.678 & 4.078 & 41.436 & 120.02 & \mathrm{C}\end{array}$ $\begin{array}{lllllllllllllll}\text { 3308. } & 2.189 & 0.620 & 0.00900 & 2140598 & 42.738 & 4.714 & 38.969 & 4.688 & 40.731 & 120.02 & C\end{array}$ $\begin{array}{llllllllllll}3567 . & 2.482 & 0.563 & 0.00900 & 2140573 & 42.737 & 5.325 & 37.880 & 4.997 & 40.100 & 120.02 & C\end{array}$ $\begin{array}{llllllllllllll}3828 . & 2.787 & 0.502 & 0.00900 & 2140493 & 42.735 & 5.938 & 36.860 & 5.301 & 39.952 & 120.02 & \mathrm{C}\end{array}$ $\begin{array}{llllllllllllll}\text { 4367. } & 3.456 & 0.366 & 0.00900 & 2140321 & 42.732 & 6.532 & 36.006 & 5.913 & 38.999 & 120.02 & C\end{array}$ $\begin{array}{llllllllllllll}565 & 0.751 & 1.012 & 0.00955 & 2252887 & 45.588 & 0.125 & 44.219 & 0.712 & 44.753 & 120.02 & \mathrm{C}\end{array}$ $\begin{array}{lllllllllllllll}1065 . & 0.242 & 0.985 & 0.00955 & 2252772 & 44.912 & 0.738 & 43.849 & 1.325 & 44.468 & 120.02 & C\end{array}$ $\begin{array}{llllllllllll}\text { 1321. } & 0.362 & 0.966 & 0.00955 & 2252713 & 44.911 & 1.352 & 43.633 & 1.634 & 44.305 & 120.02 & \mathrm{C}\end{array}$ $\begin{array}{lllllllllllll}1576 & 0.505 & 0.942 & 0.00955 & 2252589 & 44.909 & 1.964 & 43.347 & 1.939 & 44.366 & 120.02 & C\end{array}$ $\begin{array}{lllllllllllll}2096 & 0.865 & 0.883 & 0.00955 & 2252329 & 44.904 & 2.577 & 42.901 & 2.550 & 43.805 & 120.02 & \mathrm{C}\end{array}$ $\begin{array}{llllllllllllll}2615 & 1.312 & 0.808 & 0.00955 & 2252075 & 44.899 & 3.174 & 42.461 & 3.148 & 43.954 & 120.02 & \text { C }\end{array}$ $\begin{array}{lllllllllllllll}2953 . & 1.650 & 0.751 & 0.00955 & 2251971 . & 44.897 & 3.504 & 42.224 & 3.530 & 43.553 & 120.02 & \mathrm{C}\end{array}$ $\begin{array}{lllllllllllllll}3445 & 2.204 & 0.658 & 0.00955 & 2251930 & 44.896 & 4.103 & 41.387 & 4.078 & 43.152 & 120.02 & \mathrm{C}\end{array}$ $\begin{array}{llllllllllllll}4002 & 2.913 & 0.538 & 0.00955 & 2251884 & 44.895 & 4.714 & 40.437 & 4.688 & 42.209 & 120.02 & \mathrm{C}\end{array}$ $\begin{array}{lllllllllllll}4288 . & 3.310 & 0.470 & 0.00955 & 2251861 . & 44.895 & 5.325 & 39.189 & 4.997 & 41.376 & 120.02 & \mathrm{C}\end{array}$ $\begin{array}{llllllllllllllll}4573 & 3.725 & 0.398 & 0.00955 & 2251807 & 44.894 & 5.938 & 37.952 & 5.301 & 41.163 & 120.02 & \mathrm{C}\end{array}$ $\begin{array}{lllllllllllll}5154 & 4.635 & 0.240 & 0.00955 & 2251693 & 44.892 & 6.532 & 37.046 & 5.913 & 39.914 & 120.02 & \mathrm{C}\end{array}$ $\begin{array}{llllllllllll}899 . & 2.803 & 1.029 & 0.01360 & 1393465 & 28.359 & 0.125 & 24.897 & 0.712 & 25.225 & 120.02 & \mathrm{C}\end{array}$ $\begin{array}{llllllllllll}1765 & 0.690 & 1.005 & 0.01360 & 1393118 & 25.975 & 0.738 & 24.573 & 1.325 & 24.994 & 120.02 & \mathrm{C}\end{array}$ $\begin{array}{llllllllllllll}2233 & 0.359 & 0.987 & 0.01360 & 1392944 & 25.446 & 1.352 & 24.203 & 1.634 & 24.750 & 120.02 & \mathrm{C}\end{array}$ $\begin{array}{llllllllllllll}2716 . & 0.586 & 0.966 & 0.01360 & 1392600 & 25.437 & 1.964 & 23.701 & 1.939 & 24.824 & 120.02 & \mathrm{C}\end{array}$ $\begin{array}{lllllllllllllll}\text { 3748. } & 1.160 & 0.909 & 0.01360 & 1391881 & 25.417 & 2.577 & 22.964 & 2.550 & 23.905 & 120.02 & \mathrm{C}\end{array}$ $\begin{array}{llllllllllllll}4837 & 1.879 & 0.835 & 0.01360 & 1391177 & 25.398 & 3.174 & 22.140 & 3.148 & 24.173 & 120.02 & \mathrm{C}\end{array}$ $\begin{array}{lllllllllllll}5576 . & 2.368 & 0.778 & 0.01360 & 1388845 & 25.334 & 3.504 & 21.115 & 3.530 & 23.499 & 120.02 & \mathrm{C}\end{array}$ $\begin{array}{llllllllllllll}6693 . & 3.259 & 0.681 & 0.01360 & 1388362 & 25.321 & 4.103 & 19.709 & 4.078 & 22.666 & 120.02 & C\end{array}$ $\begin{array}{llllllllllllll}8014 . & 4.402 & 0.552 & 0.01360 & 1387826 & 25.306 & 4.714 & 17.728 & 4.688 & 21.398 & 120.02 & C\end{array}$ $\begin{array}{llllllllllllll}8716 . & 5.042 & 0.477 & 0.01360 & 1387554 & 25.298 & 5.325 & 15.233 & 4.997 & 19.942 & 120.02 & C\end{array}$ 
$\begin{array}{lllllllllllll}9427 & 5.717 & 0.398 & 0.01360 & 1387402 & 25.294 & 5.938 & 12.574 & 5.301 & 19.590 & 120.02 & \mathrm{C}\end{array}$ $\begin{array}{lllllllllllll}10924 . & 7.195 & 0.219 & 0.01360 & 1387115 & 25.286 & 6.532 & 10.219 & 5.913 & 17.445 & 120.02 & C\end{array}$ $\begin{array}{llllllllllll}\text { 1479. } & 4.159 & 1.040 & 0.02132 & 1459475 & 31.127 & 0.125 & 26.304 & 0.712 & 26.816 & 120.02 & \mathrm{C}\end{array}$ $\begin{array}{llllllllllllll}2821 . & 2.019 & 1.015 & 0.02132 & 1458480 & 28.679 & 0.738 & 25.812 & 1.325 & 26.415 & 120.02 & \mathrm{C}\end{array}$ $\begin{array}{lllllllllllll}3521 . & 0.763 & 0.997 & 0.02132 & 1457978 & 27.197 & 1.352 & 25.282 & 1.634 & 26.070 & 120.02 & \mathrm{C}\end{array}$ $\begin{array}{lllllllllllll}\text { 4227. } & 1.011 & 0.975 & 0.02132 & 1457255 & 27.178 & 1.964 & 24.650 & 1.939 & 26.198 & 120.02 & \mathrm{C}\end{array}$ $\begin{array}{lllllllllllll}5689 & 1.648 & 0.918 & 0.02132 & 1455764 & 27.139 & 2.577 & 23.753 & 2.550 & 24.937 & 120.02 & C\end{array}$ 7177. $\begin{array}{llllllllllll}2.448 & 0.846 & 0.02132 & 1454306 & 27.100 & 3.174 & 22.717 & 3.148 & 25.384 & 120.02 & \mathrm{C}\end{array}$ $\begin{array}{llllllllllllll}8161 . & 2.925 & 0.792 & 0.02132 & 1448540 & 26.947 & 3.504 & 21.593 & 3.530 & 24.560 & 120.02 & \mathrm{C}\end{array}$ $\begin{array}{llllllllllllll}9614 & 3.923 & 0.701 & 0.02132 & 1447406 & 26.917 & 4.103 & 20.029 & 4.078 & 23.484 & 120.02 & \mathrm{C}\end{array}$ $\begin{array}{lllllllllllll}11286 & 5.206 & 0.583 & 0.02132 & 1446145 & 26.883 & 4.714 & 17.898 & 4.688 & 22.098 & 120.02 & C\end{array}$ $\begin{array}{llllllllllllll}12158 . & 5.926 & 0.515 & 0.02132 & 1445506 & 26.866 & 5.325 & 15.265 & 4.997 & 20.455 & 120.02 & C\end{array}$ $\begin{array}{lllllllllllll}13031 & 6.688 & 0.444 & 0.02132 & 1445153 & 26.857 & 5.938 & 12.408 & 5.301 & 20.154 & 120.02 & C\end{array}$ $\begin{array}{llllllllllll}14837 & 8.362 & 0.285 & 0.02132 & 1444486 & 26.839 & 6.532 & 9.689 & 5.913 & 18.081 & 120.02 & C\end{array}$ $\begin{array}{lllllllllllll}\text { 1773. } & 4.667 & 1.047 & 0.02178 & 1591035 & 34.940 & 0.125 & 29.489 & 0.712 & 30.112 & 120.02 & C\end{array}$ $\begin{array}{llllllllllllll}3322 . & 2.275 & 1.018 & 0.02178 & 1590072 . & 32.192 & 0.738 & 28.880 & 1.325 & 29.630 & 120.02 & \mathrm{C}\end{array}$ $\begin{array}{lllllllllllll}4110 & 0.912 & 0.996 & 0.02178 & 1589587 & 30.569 & 1.352 & 28.275 & 1.634 & 29.224 & 120.02 & C\end{array}$ $\begin{array}{lllllllllllll}4891 & 1.205 & 0.970 & 0.02178 & 1588897 & 30.552 & 1.964 & 27.560 & 1.939 & 29.397 & 120.02 & \mathrm{C}\end{array}$ $\begin{array}{llllllllllll}6473 . & 1.952 & 0.904 & 0.02178 & 1587472 . & 30.517 & 2.577 & 26.535 & 2.550 & 27.943 & 120.02 & \mathrm{C}\end{array}$ $\begin{array}{lllllllllllll}8038 & 2.887 & 0.822 & 0.02178 & 1586081 & 30.482 & 3.174 & 25.372 & 3.148 & 28.369 & 120.02 & C\end{array}$ $\begin{array}{llllllllllllll}9048 . & 3.486 & 0.761 & 0.02178 & 1580881 & 30.353 & 3.504 & 24.205 & 3.530 & 27.449 & 120.02 & \mathrm{C}\end{array}$ $\begin{array}{lllllllllllllll}10511 . & 4.652 & 0.659 & 0.02178 & 1579937 & 30.329 & 4.103 & 22.437 & 4.078 & 26.302 & 120.02 & C\end{array}$ $\begin{array}{llllllllllllll}12153 . & 6.149 & 0.528 & 0.02178 & 1578887 & 30.303 & 4.714 & 20.097 & 4.688 & 24.657 & 120.02 & C\end{array}$

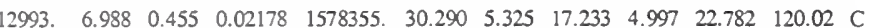
13824. $\begin{array}{llllllllllll}7.874 & 0.378 & 0.02178 & 1578076 & 30.283 & 5.938 & 14.221 & 5.301 & 22.410 & 120.02 & \mathrm{C}\end{array}$ $\begin{array}{llllllllllllll}\text { 15513. } & 9.816 & 0.208 & 0.02178 & 1577551 . & 30.270 & 6.532 & 11.597 & 5.913 & 19.944 & 120.02 & \mathrm{C}\end{array}$ $\begin{array}{lllllllllll}925 & 0.312 & 1.000 & 0.01803 & 1867675 & 37.054 & 0.125 & 36.192 & 0.712 & 36.601 & 120.02\end{array}$ $\begin{array}{lllllllllllll}1793 & 0.519 & 0.979 & 0.01803 & 1867062 & 37.040 & 0.738 & 35.778 & 1.325 & 36.344 & 120.02 & C\end{array}$

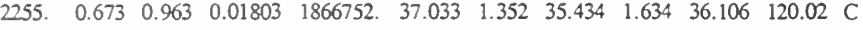
$\begin{array}{llllllllllllll}2726 . & 0.856 & 0.945 & 0.01803 & 1866325 & 37.024 & 1.964 & 35.001 & 1.939 & 36.212 & 120.02 & \mathrm{C}\end{array}$ $\begin{array}{llllllllllllllll}\text { 3720. } & 1.321 & 0.896 & 0.01803 & 1865446 . & 37.005 & 2.577 & 34.371 & 2.550 & 35.337 & 120.02 & \mathrm{C}\end{array}$ $\begin{array}{llllllllllllll}4752 . & 1.902 & 0.833 & 0.01803 & 1864587 & 36.986 & 3.174 & 33.681 & 3.148 & 35.510 & 120.02 & \mathrm{C}\end{array}$ $\begin{array}{lllllllllllll}5445 . & 2.294 & 0.785 & 0.01803 & 1861922 & 36.927 & 3.504 & 33.112 & 3.530 & 34.986 & 120.02 & \mathrm{C}\end{array}$ $\begin{array}{llllllllllllll}6483 . & 3.018 & 0.704 & 0.01803 & 1861369 & 36.915 & 4.103 & 31.992 & 4.078 & 34.332 & 120.02 & C\end{array}$ $\begin{array}{llllllllllllll}\text { 7696. } & 3.947 & 0.597 & 0.01803 & 1860755 & 36.902 & 4.714 & 30.580 & 4.688 & 33.259 & 120.02 & \mathrm{C}\end{array}$ $\begin{array}{lllllllllllllll}8335 . & 4.468 & 0.536 & 0.01803 & 1860443 & 36.895 & 5.325 & 28.736 & 4.997 & 32.115 & 120.02 & \mathrm{C}\end{array}$ $\begin{array}{llllllllllllll}8980 & 5.015 & 0.470 & 0.01803 & 1860227 & 36.890 & 5.938 & 26.743 & 5.301 & 31.892 & 120.02 & C\end{array}$ $\begin{array}{lllllllllllllll}\text { 10326. } & 6.216 & 0.324 & 0.01803 & 1859807 & 36.881 & 6.532 & 24.806 & 5.913 & 30.320 & 120.02 & \mathrm{C}\end{array}$ $\begin{array}{llllllllllllll}1401 . & 4.737 & 1.064 & 0.02019 & 2048019 & 45.288 & 0.125 & 39.963 & 0.712 & 40.477 & 120.02 & C\end{array}$ $\begin{array}{llllllllllll}2633 . & 2.988 & 1.035 & 0.02019 & 2047430 & 43.234 & 0.738 & 39.323 & 1.325 & 40.010 & 120.02 & C\end{array}$ $\begin{array}{llllllllllllll}3262 . & 1.747 & 1.014 & 0.02019 & 2047132 & 41.769 & 1.352 & 38.819 & 1.634 & 39.643 & 120.02 & \mathrm{C}\end{array}$ $\begin{array}{lllllllllllllll}3887 & 1.092 & 0.988 & 0.02019 & 2046696 & 40.848 & 1.964 & 38.233 & 1.939 & 39.764 & 120.02 & \mathrm{C}\end{array}$ 5157. $\begin{array}{llllllllllll}1.744 & 0.923 & 0.02019 & 2045794 & 40.830 & 2.577 & 37.396 & 2.550 & 38.594 & 120.02 & C\end{array}$ $\begin{array}{llllllllllllll}6419 . & 2.557 & 0.842 & 0.02019 & 2044913 & 40.812 & 3.174 & 36.467 & 3.148 & 38.817 & 120.02 & \mathrm{C}\end{array}$ $\begin{array}{lllllllllllll}7238 . & 3.120 & 0.781 & 0.02019 & 2041956 & 40.751 & 3.504 & 35.672 & 3.530 & 38.098 & 120.02 & \mathrm{C}\end{array}$ $\begin{array}{llllllllllllll}8425 & 4.129 & 0.681 & 0.02019 & 2041441 . & 40.741 & 4.103 & 34.186 & 4.078 & 37.184 & 120.02 & C\end{array}$ $\begin{array}{lllllllllllllll}9765 . & 5.422 & 0.551 & 0.02019 & 2040868 . & 40.729 & 4.714 & 32.341 & 4.688 & 35.706 & 120.02 & \mathrm{C}\end{array}$ $\begin{array}{lllllllllllllll}0452 . & 6.147 & 0.478 & 0.02019 & 2040578 . & 40.723 & 5.325 & 30.012 & 4.997 & 34.166 & 120.02 & \mathrm{C}\end{array}$ $\begin{array}{llllllllllllll}1134 & 6.908 & 0.402 & 0.02019 & 2040380 & 40.719 & 5.938 & 27.557 & 5.301 & 33.840 & 120.02 & \mathrm{C}\end{array}$ $\begin{array}{lllllllllllllll}12521 . & 8.576 & 0.232 & 0.02019 & 2039994 & 40.711 & 6.532 & 25.375 & 5.913 & 31.687 & 120.02 & \mathrm{C}\end{array}$ $\begin{array}{lllllllllllll}1110 . & 3.034 & 1.046 & 0.02021 & 2216140 & 46.953 & 0.125 & 43.283 & 0.712 & 43.924 & 120.02 & \mathrm{C}\end{array}$ 2117. $\begin{array}{llllllllllll}1.791 & 1.021 & 0.02021 & 2215617 & 45.451 & 0.738 & 42.710 & 1.325 & 43.441 & 120.02 & \mathrm{C}\end{array}$ $\begin{array}{lllllllllllll}2642 . & 0.902 & 1.003 & 0.02021 & 2215353 & 44.372 & 1.352 & 42.284 & 1.634 & 43.130 & 120.02 & \mathrm{C}\end{array}$ $\begin{array}{llllllllllllll}3171 . & 0.943 & 0.981 & 0.02021 & 2214966 & 44.189 & 1.964 & 41.778 & 1.939 & 43.245 & 120.02 & \mathrm{C}\end{array}$ $\begin{array}{llllllllllllll}4266 . & 1.496 & 0.925 & 0.02021 & 2214166 . & 44.174 & 2.577 & 41.047 & 2.550 & 42.257 & 120.02 & \mathrm{C}\end{array}$ $\begin{array}{lllllllllllll}5380 . & 2.186 & 0.854 & 0.02021 & 2213385 & 44.158 & 3.174 & 40.269 & 3.148 & 42.436 & 120.02 & C\end{array}$ $\begin{array}{lllllllllllllll}6116 . & 2.665 & 0.799 & 0.02021 & 2210818 & 44.109 & 3.504 & 39.701 & 3.530 & 41.829 & 120.02 & \mathrm{C}\end{array}$ $\begin{array}{lllllllllllllll}7202 . & 3.519 & 0.709 & 0.02021 & 2210335 & 44.100 & 4.103 & 38.389 & 4.078 & 41.055 & 120.02 & \mathrm{C}\end{array}$ $\begin{array}{llllllllllllll}8452 . & 4.615 & 0.591 & 0.02021 & 2209799 & 44.089 & 4.714 & 36.837 & 4.688 & 39.812 & 120.02 & C\end{array}$ $\begin{array}{llllllllllllllll}9102 . & 5.229 & 0.524 & 0.02021 & 2209527 . & 44.084 & 5.325 & 34.767 & 4.997 & 38.510 & 120.02 & \mathrm{C}\end{array}$ 9754. $\begin{array}{llllllllllllll}5.873 & 0.453 & 0.02021 & 2209311 . & 44.080 & 5.938 & 32.582 & 5.301 & 38.237 & 120.02 & \mathrm{C}\end{array}$ $\begin{array}{llllllllllllllll}1099 . & 7.285 & 0.296 & 0.02021 & 2208886 & 44.072 & 6.532 & 30.556 & 5.913 & 36.417 & 120.02 & C\end{array}$ $\begin{array}{lllllllllllll}\text { 12802. } & 5.298 & 0.786 & 0.01725 & 2037196 . & 40.653 & 0.125 & 29.316 & 0.712 & 35.461 & 120.02 & P\end{array}$ 10438. $\begin{array}{lllllllllllll}4.606 & 0.592 & 0.01725 & 2036813 & 40.646 & 0.738 & 31.367 & 1.325 & 35.791 & 120.02 & P\end{array}$ $\begin{array}{llllllllllllll}9346 . & 4.292 & 0.508 & 0.01725 & 2036619 & 40.642 & 1.352 & 33.015 & 1.634 & 36.174 & 120.02 & \mathrm{P}\end{array}$ $\begin{array}{llllllllllllll}\text { 8334. } 4.004 & 0.434 & 0.01725 & 2036386 & 40.637 & 1.964 & 34.305 & 1.939 & 37.168 & 120.02 & P\end{array}$ $\begin{array}{lllllllllllll}6498 . & 3.492 & 0.311 & 0.01725 & 2035911 . & 40.627 & 2.577 & 35.383 & 2.550 & 36.882 & 120.02 & P\end{array}$ $\begin{array}{llllllllllll}\text { 4956. } & 3.079 & 0.218 & 0.01725 & 2035447 . & 40.618 & 3.174 & 36.053 & 3.148 & 37.512 & 120.02\end{array}$ $\begin{array}{lllllllllllll}4104 . & 2.856 & 0.170 & 0.01725 & 2034947 . & 40.607 & 3.504 & 36.583 & 3.530 & 37.647 & 120.02 & P\end{array}$ $\begin{array}{llllllllllllll}3054 & 2.610 & 0.116 & 0.01725 & 2034889 & 40.606 & 4.103 & 36.992 & 4.078 & 38.258 & 120.02 & P\end{array}$ $\begin{array}{lllllllllllll}2132 . & 2.422 & 0.073 & 0.01725 & 2034824 & 40.605 & 4.714 & 37.420 & 4.688 & 38.162 & 120.02\end{array}$ $\begin{array}{llllllllllllll}\text { 1763. } & 2.360 & 0.057 & 0.01725 & 2034791 . & 40.604 & 5.325 & 37.603 & 4.997 & 38.182 & 120.02 & P\end{array}$ $\begin{array}{llllllllllllll}1466 . & 2.322 & 0.044 & 0.01725 & 2034758 . & 40.603 & 5.938 & 37.791 & 5.301 & 38.263 & 120.02 & P\end{array}$ $\begin{array}{llllllllllll}1062 . & 2.313 & 0.023 & 0.01725 & 2034691 . & 40.602 & 6.532 & 37.936 & 5.913 & 38.295 & 120.02\end{array}$ $\begin{array}{lllllllllllll}0267 & 4.366 & 0.806 & 0.01693 & 2256267 & 44.979 & 0.125 & 35.584 & 0.712 & 40.719 & 120.02 & \mathrm{P}\end{array}$ $\begin{array}{lllllllllllll}8404 & 3.797 & 0.634 & 0.01693 & 2255921 & 44.972 & 0.738 & 37.234 & 1.325 & 40.954 & 120.02 & P\end{array}$ $\begin{array}{lllllllllllll}7548 . & 3.533 & 0.561 & 0.01693 & 2255747 & 44.969 & 1.352 & 38.620 & 1.634 & 41.277 & 120.02 & P\end{array}$ $\begin{array}{lllllllllllll}6760 & 3.288 & 0.495 & 0.01693 & 2255548 & 44.965 & 1.964 & 39.674 & 1.939 & 42.099 & 120.02\end{array}$ $\begin{array}{lllllllllllllll}5342 . & 2.839 & 0.385 & 0.01693 & 2255145 & 44.957 & 2.577 & 40.584 & 2.550 & 41.930 & 120.02 & \mathrm{P}\end{array}$ $\begin{array}{lllllllllllll}\text { 4168. } & 2.458 & 0.300 & 0.01693 & 2254750 . & 44.950 & 3.174 & 41.173 & 3.148 & 42.499 & 120.02 & \mathrm{P}\end{array}$ $\begin{array}{llllllllllll}3531 . & 2.242 & 0.255 & 0.01693 & 2254430 & 44.944 & 3.504 & 41.647 & 3.530 & 42.610 & 120.02 & P\end{array}$ $\begin{array}{lllllllllllll}2761 . & 1.980 & 0.204 & 0.01693 & 2254263 & 44.941 & 4.103 & 41.988 & 4.078 & 43.179 & 120.02 & P\end{array}$ $\begin{array}{llllllllllllll}2112 . & 1.744 & 0.160 & 0.01693 & 2254077 & 44.937 & 4.714 & 42.412 & 4.688 & 43.157 & 120.02 & P\end{array}$ $\begin{array}{llllllllllllll}1867 . & 1.647 & 0.141 & 0.01693 & 2253982 . & 44.935 & 5.325 & 42.597 & 4.997 & 43.240 & 120.02 & P\end{array}$ $\begin{array}{llllllllllllll}1680 & 1.567 & 0.125 & 0.01693 & 2253892 . & 44.934 & 5.938 & 42.835 & 5.301 & 43.347 & 120.02 & P\end{array}$ $\begin{array}{llllllllllllll}1468 . & 1.450 & 0.097 & 0.01693 & 2253712 & 44.930 & 6.532 & 43.092 & 5.913 & 43.492 & 120.02 & P\end{array}$ $\begin{array}{llllllllllll}16679 . & 6.071 & 0.784 & 0.02035 & 1550752 & 29.597 & 0.125 & 14.308 & 0.712 & 23.463 & 120.02\end{array}$ $\begin{array}{llllllllllll}13384 & 5.135 & 0.599 & 0.02035 & 1549854 & 29.574 & 0.738 & 17.540 & 1.325 & 24.219 & 120.02 & P\end{array}$ $\begin{array}{lllllllllllll}1879 & 4.705 & 0.521 & 0.02035 & 1549401 & 29.563 & 1.352 & 19.986 & 1.634 & 24.832 & 120.02 & \mathrm{P}\end{array}$ $\begin{array}{llllllllllllll}0499 . & 4.312 & 0.452 & 0.02035 & 1549042 & 29.554 & 1.964 & 21.974 & 1.939 & 25.989 & 120.02 & P\end{array}$ $\begin{array}{lllllllllllll}8034 & 3.607 & 0.339 & 0.02035 & 1548336 & 29.536 & 2.577 & 23.554 & 2.550 & 25.539 & 120.02 & \mathrm{P}\end{array}$ $\begin{array}{llllllllllllll}6022 & 3.025 & 0.255 & 0.02035 & 1547646 & 29.518 & 3.174 & 24.511 & 3.148 & 26.477 & 120.02 & \mathrm{P}\end{array}$ $\begin{array}{lllllllllllll}\text { 4942. } & 2.691 & 0.213 & 0.02035 & 1546513 & 29.490 & 3.504 & 25.181 & 3.530 & 26.579 & 120.02 & \mathrm{P}\end{array}$ $\begin{array}{lllllllllllll}3669 . & 2.322 & 0.166 & 0.02035 & 1546237 & 29.483 & 4.103 & 25.830 & 4.078 & 27.444 & 120.02 & P\end{array}$ $\begin{array}{lllllllllllllll}2641 . & 2.018 & 0.128 & 0.02035 & 1545931 . & 29.475 & 4.714 & 26.396 & 4.688 & 27.367 & 120.02 & P\end{array}$ $\begin{array}{llllllllllllll}2276 . & 1.906 & 0.112 & 0.02035 & 1545776 . & 29.471 & 5.325 & 26.805 & 4.997 & 27.507 & 120.02 & P\end{array}$ 2018. $\begin{array}{lllllllllllll}1.824 & 0.099 & 0.02035 & 1545637 & 29.467 & 5.938 & 27.105 & 5.301 & 27.637 & 120.02 & P\end{array}$ $\begin{array}{llllllllllll}\text { 1806. } & 1.743 & 0.076 & 0.02035 & 1545360 & 29.460 & 6.532 & 27.343 & 5.913 & 27.774 & 120.02 & P\end{array}$ $\begin{array}{lllllllllllll}18462 . & 6.486 & 0.791 & 0.02110 & 1410543 & 25.926 & 0.125 & 9.019 & 0.712 & 19.369 & 120.02 & \mathrm{P}\end{array}$ $\begin{array}{lllllllllllll}14826 . & 5.505 & 0.601 & 0.02110 & 1409530 & 25.899 & 0.738 & 12.588 & 1.325 & 20.146 & 120.02 & \mathrm{P}\end{array}$ $\begin{array}{lllllllllllll}13164 & 5.056 & 0.520 & 0.02110 & 1409019 & 25.885 & 1.352 & 15.289 & 1.634 & 20.821 & 120.02 & \mathrm{P}\end{array}$ $\begin{array}{lllllllllllll}11638 & 4.645 & 0.450 & 0.02110 & 1408623 & 25.874 & 1.964 & 17.534 & 1.939 & 22.058 & 120.02 & P\end{array}$ $\begin{array}{lllllllllllll}8907 . & 3.910 & 0.333 & 0.02110 & 1407848 & 25.853 & 2.577 & 19.289 & 2.550 & 21.476 & 120.02 & P\end{array}$ $\begin{array}{lllllllllllll}6668 . & 3.305 & 0.247 & 0.02110 & 1407091 & 25.832 & 3.174 & 20.342 & 3.148 & 22.529 & 120.02 & P\end{array}$ $\begin{array}{lllllllllllll}5462 & 2.954 & 0.203 & 0.02110 & 1405757 & 25.796 & 3.504 & 21.051 & 3.530 & 22.585 & 120.02 & \mathrm{P}\end{array}$ $\begin{array}{lllllllllllll}4030 . & 2.576 & 0.155 & 0.02110 & 1405470 & 25.788 & 4.103 & 21.802 & 4.078 & 23.540 & 120.02 & P\end{array}$ $\begin{array}{lllllllllllll}2858 . & 2.267 & 0.116 & 0.02110 & 1405150 & 25.780 & 4.714 & 22.397 & 4.688 & 23.416 & 120.02 & P\end{array}$ $\begin{array}{llllllllllllll}2433 . & 2.155 & 0.101 & 0.02110 & 1404988 & 25.775 & 5.325 & 22.865 & 4.997 & 23.563 & 120.02 & \mathrm{P}\end{array}$ $\begin{array}{lllllllllllll}2126 . & 2.074 & 0.088 & 0.02110 & 1404845 & 25.771 & 5.938 & 23.169 & 5.301 & 23.682 & 120.02 & P\end{array}$ $\begin{array}{lllllllllllll}\text { 1840. } & 2.001 & 0.066 & 0.02110 & 1404559 . & 25.763 & 6.532 & 23.390 & 5.913 & 23.824 & 120.02 & P\end{array}$ $\begin{array}{llllllllllllll}\text { 14810. } & 5.629 & 0.785 & 0.02076 & 1822186 & 36.046 & 0.125 & 22.157 & 0.712 & 30.393 & 120.02 & P\end{array}$ $\begin{array}{llllllllllll}1917 . & 4.775 & 0.611 & 0.02076 & 1821414 & 36.028 & 0.738 & 24.991 & 1.325 & 31.045 & 120.02 & \mathrm{P}\end{array}$ $\begin{array}{llllllllllll}10595 . & 4.382 & 0.537 & 0.02076 & 1821025 & 36.020 & 1.352 & 27.226 & 1.634 & 31.554 & 120.02 & P\end{array}$ $\begin{array}{lllllllllllll}\text { 9383. } 4.020 & 0.473 & 0.02076 & 1820698 & 36.012 & 1.964 & 28.930 & 1.939 & 32.659 & 120.02 & \mathrm{P}\end{array}$ $\begin{array}{lllllllllllll}7216 . & 3.367 & 0.365 & 0.02076 & 1820049 & 35.998 & 2.577 & 30.337 & 2.550 & 32.331 & 120.02 & P\end{array}$ 5446. $\begin{array}{llllllllllll}2.822 & 0.285 & 0.02076 & 1819416 & 35.984 & 3.174 & 31.222 & 3.148 & 33.127 & 120.02 & \mathrm{P}\end{array}$ 4496. $\begin{array}{lllllllllllll}2.507 & 0.245 & 0.02076 & 1818324 & 35.959 & 3.504 & 31.886 & 3.530 & 33.276 & 120.02 & \mathrm{P}\end{array}$ $\begin{array}{llllllllllllll}3374 & 2.152 & 0.199 & 0.02076 & 1818068 & 35.953 & 4.103 & 32.415 & 4.078 & 34.044 & 120.02 & P\end{array}$ $\begin{array}{llllllllllllll}2466 . & 1.849 & 0.162 & 0.02076 & 1817783 & 35.947 & 4.714 & 32.991 & 4.688 & 34.026 & 120.02 & P\end{array}$ $\begin{array}{llllllllllllll}2142 . & 1.732 & 0.147 & 0.02076 & 1817639 & 35.944 & 5.325 & 33.289 & 4.997 & 34.165 & 120.02 & \mathrm{P}\end{array}$ $\begin{array}{lllllllllllllll}1913 & 1.641 & 0.134 & 0.02076 & 1817482 & 35.940 & 5.938 & 33.607 & 5.301 & 34.287 & 120.02 & \mathrm{P}\end{array}$ $\begin{array}{lllllllllllll}\text { 1720. } & 1.532 & 0.110 & 0.02076 & 1817164 & 35.933 & 6.532 & 33.865 & 5.913 & 34.448 & 120.02 & \mathrm{P}\end{array}$ $\begin{array}{llllllllllllll}19624 & 6.569 & 0.788 & 0.02773 & 1820884 & 36.016 & 0.125 & 20.351 & 0.712 & 29.267 & 120.02 & P\end{array}$ $\begin{array}{llllllllllllll}15803 & 5.604 & 0.615 & 0.02773 & 1819567 & 35.987 & 0.738 & 23.534 & 1.325 & 30.289 & 120.02 & P\end{array}$ $\begin{array}{lllllllllllll}14060 \text {. } & 5.157 & 0.542 & 0.02773 & 1818903 & 35.972 & 1.352 & 25.977 & 1.634 & 30.755 & 120.02 & \mathrm{P}\end{array}$ $\begin{array}{lllllllllllll}12464 . & 4.747 & 0.478 & 0.02773 & 1818384 & 35.960 & 1.964 & 27.974 & 1.939 & 32.112 & 120.02 & P\end{array}$ $\begin{array}{llllllllllllll}9618 . & 4.003 & 0.371 & 0.02773 & 1817367 & 35.938 & 2.577 & 29.529 & 2.550 & 31.526 & 120.02 & \mathrm{P}\end{array}$ $\begin{array}{llllllllllll}7303 . & 3.378 & 0.291 & 0.02773 & 1816373 & 35.915 & 3.174 & 30.522 & 3.148 & 32.383 & 120.02 & P\end{array}$ $\begin{array}{lllllllllllll}6068 \text {. } & 2.989 & 0.250 & 0.02773 & 1813899 & 35.860 & 3.504 & 31.205 & 3.530 & 32.676 & 120.02 & P\end{array}$ $\begin{array}{lllllllllllll}4619 . & 2.573 & 0.204 & 0.02773 & 1813395 & 35.848 & 4.103 & 31.883 & 4.078 & 33.520 & 120.02 & \mathrm{P}\end{array}$ $\begin{array}{lllllllllllll}3463 . & 2.209 & 0.166 & 0.02773 & 1812835 & 35.836 & 4.714 & 32.504 & 4.688 & 33.556 & 120.02 & P\end{array}$ $\begin{array}{llllllllllllll}3059 \text {. } & 2.065 & 0.150 & 0.02773 & 1812552 & 35.830 & 5.325 & 32.945 & 4.997 & 33.720 & 120.02 & \mathrm{P}\end{array}$ $\begin{array}{lllllllllllllll}2783 . & 1.951 & 0.136 & 0.02773 & 1812299 & 35.824 & 5.938 & 33.310 & 5.301 & 33.874 & 120.02 & \mathrm{P}\end{array}$ $\begin{array}{llllllllllllll}2587 . & 1.799 & 0.110 & 0.02773 & 1811793 & 35.813 & 6.532 & 33.620 & 5.913 & 34.077 & 120.02 & \mathrm{P}\end{array}$

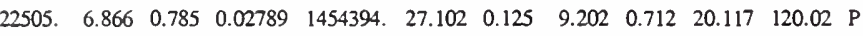
$\begin{array}{lllllllllllll}18173 . & 5.932 & 0.608 & 0.02789 & 1452699 & 27.058 & 0.738 & 12.830 & 1.325 & 20.939 & 120.02 & P\end{array}$ $\begin{array}{llllllllllllll}16195 & 5.496 & 0.532 & 0.02789 & 1451845 & 27.035 & 1.352 & 15.588 & 1.634 & 21.495 & 120.02 & P\end{array}$ $\begin{array}{lllllllllllll}14378 . & 5.095 & 0.465 & 0.02789 & 1451201 & 27.018 & 1.964 & 17.916 & 1.939 & 22.899 & 120.02 & \mathrm{P}\end{array}$ $\begin{array}{llllllllllll}11130 . & 4.361 & 0.355 & 0.02789 & 1449942 & 26.984 & 2.577 & 19.715 & 2.550 & 22.072 & 120.02 & P\end{array}$ $\begin{array}{llllllllllll}8472 . & 3.733 & 0.272 & 0.02789 & 1448711 . & 26.952 & 3.174 & 20.848 & 3.148 & 23.095 & 120.02 & P\end{array}$ $\begin{array}{lllllllllllll}7042 . & 3.315 & 0.230 & 0.02789 & 1445589 & 26.868 & 3.504 & 21.590 & 3.530 & 23.322 & 120.02 & P\end{array}$ $\begin{array}{llllllllllllll}5348 . & 2.884 & 0.182 & 0.02789 & 1444994 & 26.853 & 4.103 & 22.406 & 4.078 & 24.316 & 120.02 & \mathrm{P}\end{array}$ $\begin{array}{lllllllllllllll}3968 . & 2.492 & 0.142 & 0.02789 & 1444332 & 26.835 & 4.714 & 23.088 & 4.688 & 24.285 & 120.02 & P\end{array}$ $\begin{array}{llllllllllllll}3470 . & 2.329 & 0.125 & 0.02789 & 1443997 & 26.826 & 5.325 & 23.634 & 4.997 & 24.460 & 120.02 & \mathrm{P}\end{array}$

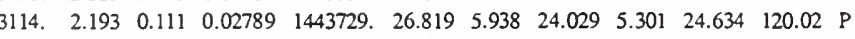

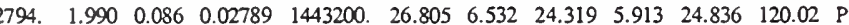
$\begin{array}{lllllllllllll}11694 . & 4.551 & 0.759 & 0.01300 & 1395225 & 25.509 & 0.125 & 12.117 & 0.712 & 21.026 & 120.02 & P\end{array}$ $\begin{array}{llllllllllll}9260 . & 3.825 & 0.565 & 0.01300 & 1394965 & 25.502 & 0.738 & 15.094 & 1.325 & 21.324 & 120.02 & \mathrm{P}\end{array}$ $\begin{array}{lllllllllllll}8151 . & 3.493 & 0.484 & 0.01300 & 1394835 & 25.498 & 1.352 & 17.428 & 1.634 & 21.952 & 120.02 & \mathrm{P}\end{array}$ $\begin{array}{llllllllllllll}7136 . & 3.189 & 0.414 & 0.01300 & 1394696 & 25.494 & 1.964 & 19.342 & 1.939 & 22.903 & 120.02 & \mathrm{P}\end{array}$ $\begin{array}{lllllllllllll}5330 . & 2.647 & 0.299 & 0.01300 & 1394414 & 25.486 & 2.577 & 20.735 & 2.550 & 22.603 & 120.02 & \mathrm{P}\end{array}$ $\begin{array}{llllllllllll}3866 . & 2.206 & 0.217 & 0.01300 & 1394139 & 25.479 & 3.174 & 21.535 & 3.148 & 23.323 & 120.02 & P\end{array}$ $\begin{array}{llllllllllllllll}3084 & 1.969 & 0.177 & 0.01300 & 1393977 & 25.475 & 3.504 & 22.121 & 3.530 & 23.276 & 120.02 & \mathrm{P}\end{array}$ 2174. $\begin{array}{lllllllllllll}1.703 & 0.133 & 0.01300 & 1394101 & 25.478 & 4.103 & 22.657 & 4.078 & 24.021 & 120.02 & \mathrm{P}\end{array}$ 1454. $\begin{array}{llllllllllll}1.492 & 0.100 & 0.01300 & 1394238 & 25.482 & 4.714 & 23.091 & 4.688 & 23.925 & 120.02 & \mathrm{P}\end{array}$ $\begin{array}{llllllllllllll}1207 & 1.421 & 0.088 & 0.01300 & 1394307 & 25.484 & 5.325 & 23.410 & 4.997 & 24.020 & 120.02 & \mathrm{P}\end{array}$ 1041. $\begin{array}{lllllllllllll}1.368 & 0.078 & 0.01300 & 1394205 & 25.481 & 5.938 & 23.615 & 5.301 & 24.084 & 120.02 & P\end{array}$ $\begin{array}{lllllllllllll}\text { 939. } & 1.330 & 0.060 & 0.01300 & 1393973 & 25.474 & 6.532 & 23.762 & 5.913 & 24.191 & 120.02 & P\end{array}$ $\begin{array}{lllllllllllll}12623 & 4.988 & 0.764 & 0.01336 & 1442236 & 26.779 & 0.125 & 12.294 & 0.712 & 21.868 & 120.02 & P\end{array}$ $\begin{array}{llllllllllll}9984 . & 4.188 & 0.558 & 0.01336 & 1441982 & 26.772 & 0.738 & 15.496 & 1.325 & 22.192 & 120.02 & \mathrm{P}\end{array}$ $\begin{array}{lllllllllllll}8779 . & 3.828 & 0.472 & 0.01336 & 1441855 & 26.769 & 1.352 & 18.018 & 1.634 & 22.904 & 120.02 & P\end{array}$ $\begin{array}{lllllllllllll}\text { 7673. } & 3.501 & 0.397 & 0.01336 & 1441706 . & 26.765 & 1.964 & 20.060 & 1.939 & 23.893 & 120.02 & \mathrm{P}\end{array}$ $\begin{array}{lllllllllllll}5697 & 2.929 & 0.276 & 0.01336 & 1441403 & 26.757 & 2.577 & 21.553 & 2.550 & 23.587 & 120.02 & \mathrm{P}\end{array}$ $\begin{array}{lllllllllllll}\text { 4082. } & 2.480 & 0.190 & 0.01336 & 1441107 & 26.749 & 3.174 & 22.402 & 3.148 & 24.307 & 120.02 & \mathrm{P}\end{array}$ $\begin{array}{llllllllllllll}3214 & 2.250 & 0.148 & 0.01336 & 1440929 & 26.744 & 3.504 & 23.016 & 3.530 & 24.258 & 120.02 & \mathrm{P}\end{array}$ $\begin{array}{llllllllllllll}2191 . & 1.998 & 0.104 & 0.01336 & 1440694 & 26.738 & 4.103 & 23.566 & 4.078 & 24.991 & 120.02 & \mathrm{P}\end{array}$ $\begin{array}{llllllllllllll}\text { 1362. } & 1.825 & 0.073 & 0.01336 & 1440433 & 26.731 & 4.714 & 23.993 & 4.688 & 24.847 & 120.02 & \mathrm{P}\end{array}$ 1066. $\begin{array}{lllllllllllll}1.780 & 0.062 & 0.01336 & 1440301 . & 26.727 & 5.325 & 24.272 & 4.997 & 24.880 & 120.02 & \mathrm{P}\end{array}$ $\begin{array}{lllllllllllllll}856 . & 1.765 & 0.053 & 0.01336 & 1440178 & 26.724 & 5.938 & 24.436 & 5.301 & 24.963 & 120.02 & \mathrm{P}\end{array}$ $\begin{array}{lllllllllllll}679 . & 1.820 & 0.040 & 0.01336 & 1439932 . & 26.717 & 6.532 & 24.505 & 5.913 & 24.933 & 120.02 & P\end{array}$ $\begin{array}{llllllllllll}\text { 10511. } & 4.369 & 0.780 & 0.01328 & 1902336 & 37.809 & 0.125 & 25.800 & 0.712 & 33.519 & 120.02 & P\end{array}$ $\begin{array}{llllllllllllll}8247 & 3.662 & 0.585 & 0.01328 & 1902083 & 37.804 & 0.738 & 28.545 & 1.325 & 33.819 & 120.02 & \mathrm{P}\end{array}$ $\begin{array}{llllllllllllll}\text { 7222. } & 3.338 & 0.503 & 0.01328 & 1901956 & 37.801 & 1.352 & 30.671 & 1.634 & 34.414 & 120.02 & P\end{array}$ $\begin{array}{lllllllllllll}6288 . & 3.042 & 0.433 & 0.01328 & 1901804 & 37.798 & 1.964 & 32.242 & 1.939 & 35.231 & 120.02 & P\end{array}$ $\begin{array}{llllllllllllll}\text { 4639. } & 2.511 & 0.320 & 0.01328 & 1901494 & 37.791 & 2.577 & 33.479 & 2.550 & 35.115 & 120.02 & \mathrm{P}\end{array}$ $\begin{array}{lllllllllllll}3323 & 2.078 & 0.239 & 0.01328 & 1901191 . & 37.785 & 3.174 & 34.184 & 3.148 & 35.759 & 120.02 & P\end{array}$ 
$\begin{array}{llllllllllll}2633 . & 1.844 & 0.200 & 0.01328 & 1900960 & 37.780 & 3.504 & 34.749 & 3.530 & 35.768 & 120.02 & \mathrm{P}\end{array}$ $\begin{array}{llllllllllllll}1851 . & 1.571 & 0.159 & 0.01328 & 1900713 & 37.774 & 4.103 & 35.161 & 4.078 & 36.402 & 120.02 & P\end{array}$ $\begin{array}{lllllllllllll}1268 . & 1.350 & 0.127 & 0.01328 & 1900439 & 37.768 & 4.714 & 35.588 & 4.688 & 36.362 & 120.02 & P\end{array}$ $\begin{array}{lllllllllllll}1088 & 1.272 & 0.115 & 0.01328 & 1900300 & 37.765 & 5.325 & 35.805 & 4.997 & 36.414 & 120.02 & \mathrm{P}\end{array}$ $\begin{array}{llllllllllllll}987 . & 1.217 & 0.104 & 0.01328 & 1900177 . & 37.763 & 5.938 & 36.025 & 5.301 & 36.526 & 120.02 & P\end{array}$ $\begin{array}{lllllllllllll}\text { 1011. } & 1.172 & 0.084 & 0.01328 & 1899932 & 37.757 & 6.532 & 36.230 & 5.913 & 36.643 & 120.02 & \mathrm{P}\end{array}$ $\begin{array}{lllllllllllll}6031 . & 2.696 & 0.782 & 0.01042 & 2256177 & 44.977 & 0.125 & 37.498 & 0.712 & 42.412 & 120.02 & P\end{array}$ $\begin{array}{llllllllllll}\text { 4761. } & 2.278 & 0.621 & 0.01042 & 2256055 & 44.975 & 0.738 & 39.072 & 1.325 & 42.453 & 120.02 & P\end{array}$ $\begin{array}{lllllllllllll}4187 . & 2.085 & 0.554 & 0.01042 & 2255994 & 44.973 & 1.352 & 40.388 & 1.634 & 42.786 & 120.02 & P\end{array}$ $\begin{array}{lllllllllllll}\text { 3664. } & 1.905 & 0.496 & 0.01042 & 2255884 & 44.971 & 1.964 & 41.275 & 1.939 & 43.317 & 120.02 & P\end{array}$ $\begin{array}{lllllllllllll}2743 & 1.577 & 0.401 & 0.01042 & 2255655 & 44.967 & 2.577 & 42.025 & 2.550 & 43.312 & 120.02 & P\end{array}$ $\begin{array}{lllllllllllll}2008 . & 1.300 & 0.332 & 0.01042 & 2255431 . & 44.963 & 3.174 & 42.483 & 3.148 & 43.742 & 120.02 & P\end{array}$ $\begin{array}{lllllllllllll}1625 . & 1.151 & 0.298 & 0.01042 & 2255569 & 44.965 & 3.504 & 42.885 & 3.530 & 43.705 & 120.02 & \mathrm{P}\end{array}$ $\begin{array}{lllllllllllll}1192 . & 0.960 & 0.261 & 0.01042 & 2255398 & 44.962 & 4.103 & 43.079 & 4.078 & 44.143 & 120.02 & P\end{array}$ $\begin{array}{llllllllllllll}873 & 0.791 & 0.230 & 0.01042 & 2255207 & 44.958 & 4.714 & 43.431 & 4.688 & 44.148 & 120.02 & P\end{array}$ $\begin{array}{llllllllllllll}776 . & 0.723 & 0.218 & 0.01042 & 2255111 & 44.957 & 5.325 & 43.508 & 4.997 & 44.191 & 120.02 & P\end{array}$ $\begin{array}{llllllllllllll}724 . & 0.666 & 0.207 & 0.01042 & 2255003 & 44.955 & 5.938 & 43.693 & 5.301 & 44.270 & 120.02 & P\end{array}$ $\begin{array}{lllllllllllll}748 . & 0.586 & 0.186 & 0.01042 & 2254783 & 44.950 & 6.532 & 43.905 & 5.913 & 44.378 & 120.02 & \mathrm{P}\end{array}$ $\begin{array}{llllllllllllll}\text { 18113. } & 7.062 & 0.800 & 0.02401 & 2138906 & 42.704 & 0.125 & 24.651 & 0.712 & 35.569 & 120.02 & \mathrm{P}\end{array}$ $\begin{array}{lllllllllllll}14253 . & 5.883 & 0.599 & 0.02401 & 2138146 & 42.689 & 0.738 & 28.836 & 1.325 & 36.535 & 120.02 & \mathrm{P}\end{array}$ $\begin{array}{llllllllllll}12504 . & 5.344 & 0.515 & 0.02401 & 2137763 & 42.682 & 1.352 & 31.984 & 1.634 & 37.279 & 120.02\end{array}$ $\begin{array}{llllllllllllll}10909 . & 4.851 & 0.443 & 0.02401 & 2137477 . & 42.676 & 1.964 & 34.440 & 1.939 & 38.659 & 120.02 & P\end{array}$ $\begin{array}{lllllllllllll}8093 . & 3.971 & 0.326 & 0.02401 & 2136920 & 42.665 & 2.577 & 36.310 & 2.550 & 38.433 & 120.02 & P\end{array}$ $\begin{array}{lllllllllllll}5839 . & 3.252 & 0.242 & 0.02401 & 2136375 & 42.654 & 3.174 & 37.409 & 3.148 & 39.261 & 120.02 & \mathrm{P}\end{array}$ $\begin{array}{lllllllllllll}4657 . & 2.852 & 0.201 & 0.02401 & 2135379 & 42.634 & 3.504 & 38.174 & 3.530 & 39.605 & 120.02 & P\end{array}$ $\begin{array}{lllllllllllll}3312 . & 2.399 & 0.158 & 0.02401 & 2135019 & 42.627 & 4.103 & 38.913 & 4.078 & 40.475 & 120.02 & P\end{array}$ $\begin{array}{lllllllllllll}2303 . & 2.033 & 0.123 & 0.02401 & 2134620 & 42.619 & 4.714 & 39.518 & 4.688 & 40.497 & 120.02 & P\end{array}$ $\begin{array}{llllllllllllll}1988 & 1.903 & 0.110 & 0.02401 & 2134417 & 42.615 & 5.325 & 39.965 & 4.997 & 40.618 & 120.02 & \mathrm{P}\end{array}$ $\begin{array}{lllllllllllll}\text { 1806. } & 1.814 & 0.099 & 0.02401 & 2134294 & 42.613 & 5.938 & 40.276 & 5.301 & 40.788 & 120.02 & P\end{array}$ $\begin{array}{llllllllllllll}1826 . & 1.742 & 0.077 & 0.02401 & 2134059 & 42.608 & 6.532 & 40.566 & 5.913 & 40.963 & 120.02 & P\end{array}$ $\begin{array}{llllllllllllll}18333 . & 6.720 & 0.820 & 0.02573 & 2177632 & 43.465 & 0.125 & 27.629 & 0.712 & 36.643 & 120.02 & P\end{array}$ $\begin{array}{llllllllllllll}14741 . & 5.715 & 0.626 & 0.02573 & 2176777 & 43.448 & 0.738 & 30.820 & 1.325 & 37.556 & 120.02 & P\end{array}$ $\begin{array}{llllllllllllll}13102 . & 5.253 & 0.544 & 0.02573 & 2176345 & 43.440 & 1.352 & 33.367 & 1.634 & 38.094 & 120.02 & P\end{array}$ $\begin{array}{lllllllllllll}11599 . & 4.830 & 0.472 & 0.02573 & 2175993 & 43.433 & 1.964 & 35.303 & 1.939 & 39.428 & 120.02 & P\end{array}$ $\begin{array}{llllllllllllll}8916 . & 4.067 & 0.352 & 0.02573 & 2175298 & 43.420 & 2.577 & 36.863 & 2.550 & 39.064 & 120.02 & P\end{array}$ $\begin{array}{lllllllllllll}6728 . & 3.436 & 0.263 & 0.02573 & 2174619 & 43.406 & 3.174 & 37.862 & 3.148 & 39.809 & 120.02 & P\end{array}$ $\begin{array}{llllllllllllll}5556 & 3.068 & 0.218 & 0.02573 & 2173025 & 43.375 & 3.504 & 38.604 & 3.530 & 40.133 & 120.02 & P\end{array}$ $\begin{array}{lllllllllllllll}4176 . & 2.657 & 0.167 & 0.02573 & 2172575 & 43.366 & 4.103 & 39.232 & 4.078 & 40.951 & 120.02 & P\end{array}$ $\begin{array}{llllllllllllll}3066 . & 2.313 & 0.125 & 0.02573 & 2172075 & 43.357 & 4.714 & 39.871 & 4.688 & 40.970 & 120.02 & P\end{array}$ $\begin{array}{llllllllllllll}2674 . & 2.183 & 0.108 & 0.02573 & 2171821 & 43.352 & 5.325 & 40.224 & 4.997 & 41.106 & 120.02 & P\end{array}$ $\begin{array}{lllllllllllll}2399 . & 2.087 & 0.094 & 0.02573 & 2171706 & 43.349 & 5.938 & 40.564 & 5.301 & 41.264 & 120.02 & P\end{array}$ $\begin{array}{llllllllllll}2184 . & 1.983 & 0.067 & 0.02573 & 2171494 & 43.345 & 6.532 & 40.882 & 5.913 & 41.428 & 120.02 & P\end{array}$ 
APPENDIX C3

\title{
Convective condensation of
}

R410A within a micro-fin tube

\author{
(file: taraw.tbl)
}

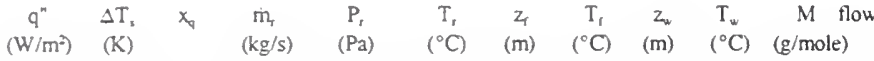
$\begin{array}{lllllllllllll}26759 & 6.719 & 0.775 & 0.01858 & 1836296 & 28.862 & 0.125 & 10.385 & 0.712 & 22.162 & 72.59 & P\end{array}$ $\begin{array}{llllllllllll}21346 & 5.751 & 0.590 & 0.01858 & 1836294 & 28.833 & 0.738 & 14.428 & 1.325 & 22.814 & 72.59 & \mathrm{P}\end{array}$ $\begin{array}{llllllllllllll}18881 . & 5.308 & 0.513 & 0.01858 & 1836293 & 28.821 & 1.352 & 17.386 & 1.634 & 23.342 & 72.59 & \mathrm{P}\end{array}$ $\begin{array}{lllllllllllll}16623 . & 4.898 & 0.446 & 0.01858 & 1836242 & 28.810 & 1.964 & 19.788 & 1.939 & 24.854 & 72.59 & \mathrm{P}\end{array}$ $\begin{array}{llllllllllllll}12606 . & 4.162 & 0.336 & 0.01858 & 1836133 & 28.792 & 2.577 & 21.681 & 2.550 & 24.013 & 72.59 & \mathrm{P}\end{array}$ $\begin{array}{lllllllllllll}9344 . & 3.551 & 0.255 & 0.01858 & 1836026 & 28.778 & 3.174 & 22.821 & 3.148 & 25.278 & 72.59 & \mathrm{P}\end{array}$ $\begin{array}{llllllllllllll}7607 . & 3.233 & 0.215 & 0.01858 & 1836761 & 28.788 & 3.504 & 23.606 & 3.530 & 25.376 & 72.59 & \mathrm{P}\end{array}$ $\begin{array}{lllllllllllllll}5576 & 2.831 & 0.171 & 0.01858 & 1836753 & 28.781 & 4.103 & 24.402 & 4.078 & 26.299 & 72.59 & P\end{array}$ $\begin{array}{llllllllllllll}3968 & 2.490 & 0.136 & 0.01858 & 1836743 & 28.776 & 4.714 & 25.044 & 4.688 & 26.212 & 72.59 & P\end{array}$ $\begin{array}{lllllllllllllll}3414 & 2.358 & 0.122 & 0.01858 & 1836738 & 28.774 & 5.325 & 25.528 & 4.997 & 26.344 & 72.59 & P\end{array}$ $\begin{array}{lllllllllllllll}3039 & 2.255 & 0.110 & 0.01858 & 1836709 & 28.772 & 5.938 & 25.876 & 5.301 & 26.516 & 72.59 & \mathrm{P}\end{array}$ $\begin{array}{lllllllllllllll}2801 . & 2.131 & 0.089 & 0.01858 & 1836646 & 28.768 & 6.532 & 26.137 & 5.913 & 26.659 & 72.59 & \mathrm{P}\end{array}$

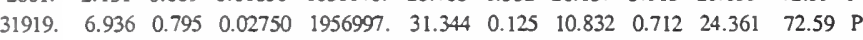
$\begin{array}{llllllllllllll}25481 . & 5.891 & 0.642 & 0.02750 & 1956996 & 31.320 & 0.738 & 15.389 & 1.325 & 25.136 & 72.59 & \mathrm{P}\end{array}$ $\begin{array}{lllllllllllll}22553 & 5.407 & 0.578 & 0.02750 & 1956995 & 31.310 & 1.352 & 18.797 & 1.634 & 25.764 & 72.59 & \mathrm{P}\end{array}$ $\begin{array}{lllllllllllll}19875 . & 4.958 & 0.522 & 0.02750 & 1956945 . & 31.300 & 1.964 & 21.586 & 1.939 & 27.509 & 72.59 & P\end{array}$ $\begin{array}{lllllllllllll}15117 & 4.138 & 0.431 & 0.02750 & 1956834 & 31.284 & 2.577 & 23.717 & 2.550 & 26.411 & 72.59 & \mathrm{P}\end{array}$ $\begin{array}{llllllllllll}11269 . & 3.444 & 0.364 & 0.02750 & 1956725 & 31.272 & 3.174 & 25.071 & 3.148 & 27.831 & 72.59 & \mathrm{P}\end{array}$ $\begin{array}{lllllllllllll}9228 . & 3.070 & 0.330 & 0.02750 & 1957471 . & 31.282 & 3.504 & 25.960 & 3.530 & 28.043 & 72.59 & \mathrm{P}\end{array}$ $\begin{array}{llllllllllllll}6857 . & 2.589 & 0.292 & 0.02750 & 1957466 . & 31.277 & 4.103 & 26.864 & 4.078 & 28.959 & 72.59 & P\end{array}$ $\begin{array}{lllllllllllll}5003 & 2.156 & 0.262 & 0.02750 & 1957461 & 31.272 & 4.714 & 27.645 & 4.688 & 29.060 & 72.59 & P\end{array}$ $\begin{array}{lllllllllllll}\text { 4378. } & 1.977 & 0.250 & 0.02750 & 1957459 & 31.270 & 5.325 & 28.228 & 4.997 & 29.283 & 72.59 & P\end{array}$ $\begin{array}{llllllllllllll}3969 . & 1.828 & 0.239 & 0.02750 & 1957429 & 31.268 & 5.938 & 28.683 & 5.301 & 29.436 & 72.59 & \mathrm{P}\end{array}$ $\begin{array}{llllllllllllll}3766 & 1.608 & 0.219 & 0.02750 & 1957364 & 31.264 & 6.532 & 29.043 & 5.913 & 29.670 & 72.59 & P\end{array}$ $\begin{array}{lllllllllllll}26134 . & 5.551 & 0.830 & 0.02744 & 2179801 & 35.640 & 0.125 & 19.199 & 0.712 & 30.076 & 72.59 & P\end{array}$ $\begin{array}{lllllllllllll}20753 . & 4.644 & 0.699 & 0.02744 & 2179802 & 35.619 & 0.738 & 22.960 & 1.325 & 30.745 & 72.59 & \mathrm{P}\end{array}$ $\begin{array}{lllllllllllllll}18308 & 4.227 & 0.645 & 0.02744 & 2179802 & 35.610 & 1.352 & 25.786 & 1.634 & 31.221 & 72.59 & \mathrm{P}\end{array}$ $\begin{array}{lllllllllllllll}16074 & 3.840 & 0.597 & 0.02744 & 2179751 & 35.601 & 1.964 & 28.006 & 1.939 & 32.663 & 72.59 & P\end{array}$ $\begin{array}{lllllllllllll}\text { 12115. } & 3.141 & 0.520 & 0.02744 & 2179638 & 35.587 & 2.577 & 29.725 & 2.550 & 31.889 & 72.59 & \mathrm{P}\end{array}$ $\begin{array}{lllllllllllll}8926 . & 2.557 & 0.464 & 0.02744 & 2179529 & 35.577 & 3.174 & 30.833 & 3.148 & 33.052 & 72.59 & \mathrm{P}\end{array}$ $\begin{array}{lllllllllllll}7243 . & 2.248 & 0.435 & 0.02744 & 2180286 & 35.586 & 3.504 & 31.553 & 3.530 & 33.231 & 72.59 & \mathrm{P}\end{array}$ $\begin{array}{lllllllllllll}5300 & 1.856 & 0.405 & 0.02744 & 2180283 & 35.582 & 4.103 & 32.238 & 4.078 & 33.908 & 72.59 & \mathrm{P}\end{array}$ $\begin{array}{llllllllllllll}3805 . & 1.514 & 0.380 & 0.02744 & 2180281 & 35.578 & 4.714 & 32.867 & 4.688 & 34.010 & 72.59 & \mathrm{P}\end{array}$ $\begin{array}{llllllllllll}3313 . & 1.379 & 0.370 & 0.02744 & 2180280 & 35.576 & 5.325 & 33.283 & 4.997 & 34.195 & 72.59 & \mathrm{P}\end{array}$ $\begin{array}{llllllllllllll}3004 & 1.270 & 0.362 & 0.02744 & 2180251 & 35.575 & 5.938 & 33.636 & 5.301 & 34.294 & 72.59 & \mathrm{P}\end{array}$ $\begin{array}{lllllllllllllll}2910 . & 1.126 & 0.346 & 0.02744 & 2180188 & 35.571 & 6.532 & 33.928 & 5.913 & 34.462 & 72.59 & \mathrm{P}\end{array}$ $\begin{array}{llllllllllllll}25786 & 6.163 & 0.821 & 0.02209 & 2219802 & 36.373 & 0.125 & 19.519 & 0.712 & 30.197 & 72.59 & \mathrm{P}\end{array}$ $\begin{array}{llllllllllll}20550 . & 5.219 & 0.659 & 0.02209 & 2219803 & 36.347 & 0.738 & 23.270 & 1.325 & 30.922 & 72.59 & \mathrm{P}\end{array}$ $\begin{array}{lllllllllllllll}18173 . & 4.783 & 0.591 & 0.02209 & 2219803 & 36.336 & 1.352 & 26.053 & 1.634 & 31.382 & 72.59 & \mathrm{P}\end{array}$ $\begin{array}{llllllllllllll}16001 & 4.377 & 0.532 & 0.02209 & 2219752 & 36.326 & 1.964 & 28.254 & 1.939 & 32.830 & 72.59 & \mathrm{P}\end{array}$ $\begin{array}{llllllllllllll}12152 . & 3.638 & 0.436 & 0.02209 & 2219639 & 36.309 & 2.577 & 29.983 & 2.550 & 32.139 & 72.59 & \mathrm{P}\end{array}$ $\begin{array}{lllllllllllll}9055 . & 3.013 & 0.365 & 0.02209 & 2219529 & 36.297 & 3.174 & 31.078 & 3.148 & 33.286 & 72.59 & \mathrm{P}\end{array}$ $\begin{array}{llllllllllllll}\text { 7422. } & 2.677 & 0.330 & 0.02209 & 2220288 & 36.305 & 3.504 & 31.838 & 3.530 & 33.478 & 72.59 & \mathrm{P}\end{array}$ $\begin{array}{llllllllllllll}5539 . & 2.245 & 0.290 & 0.02209 & 2220287 & 36.299 & 4.103 & 32.549 & 4.078 & 34.313 & 72.59 & P\end{array}$ 4093. $\begin{array}{llllllllllllll}1.856 & 0.258 & 0.02209 & 2220287 & 36.294 & 4.714 & 33.214 & 4.688 & 34.388 & 72.59 & \mathrm{P}\end{array}$ $\begin{array}{llllllllllllll}3620 . & 1.697 & 0.245 & 0.02209 & 2220287 & 36.293 & 5.325 & 33.672 & 4.997 & 34.577 & 72.59 & \mathrm{P}\end{array}$ $\begin{array}{lllllllllllll}3326 . & 1.563 & 0.233 & 0.02209 & 2220258 & 36.290 & 5.938 & 34.058 & 5.301 & 34.717 & 72.59 & \mathrm{P}\end{array}$ $\begin{array}{llllllllllllll}3247 . & 1.367 & 0.211 & 0.02209 & 2220196 . & 36.286 & 6.532 & 34.390 & 5.913 & 34.930 & 72.59 & \mathrm{P}\end{array}$ $\begin{array}{lllllllllllllll}26872 . & 6.794 & 0.796 & 0.01971 & 2082562 . & 33.805 & 0.125 & 15.934 & 0.712 & 27.020 & 72.59 & \mathrm{P}\end{array}$ $\begin{array}{lllllllllllll}21466 . & 5.766 & 0.612 & 0.01971 & 2081559 & 33.756 & 0.738 & 19.829 & 1.325 & 27.740 & 72.59 & P\end{array}$ $\begin{array}{lllllllllllll}19005 . & 5.294 & 0.535 & 0.01971 & 2081054 & 33.734 & 1.352 & 22.692 & 1.634 & 28.270 & 72.59 & \mathrm{P}\end{array}$ $\begin{array}{lllllllllllllll}16752 . & 4.860 & 0.469 & 0.01971 & 2080653 & 33.716 & 1.964 & 24.982 & 1.939 & 29.774 & 72.59 & \mathrm{P}\end{array}$ $\begin{array}{llllllllllll}12744 . & 4.078 & 0.359 & 0.01971 & 2079867 & 33.685 & 2.577 & 26.803 & 2.550 & 29.026 & 72.59 & \mathrm{P}\end{array}$ $\begin{array}{lllllllllllll}9495 . & 3.426 & 0.279 & 0.01971 & 2079098 & 33.658 & 3.174 & 27.925 & 3.148 & 30.260 & 72.59 & \mathrm{P}\end{array}$ $\begin{array}{lllllllllllll}\text { 7767. } & 3.052 & 0.238 & 0.01971 & 2077812 & 33.628 & 3.504 & 28.710 & 3.530 & 30.398 & 72.59 & \mathrm{P}\end{array}$ $\begin{array}{llllllllllllll}5752 . & 2.624 & 0.194 & 0.01971 & 2077521 & 33.616 & 4.103 & 29.471 & 4.078 & 31.330 & 72.59 & \mathrm{P}\end{array}$ $\begin{array}{llllllllllllll}\text { 4163. } & 2.257 & 0.158 & 0.01971 & 2077198 & 33.604 & 4.714 & 30.130 & 4.688 & 31.287 & 72.59 & P\end{array}$ $\begin{array}{llllllllllllll}3619 & 2.114 & 0.143 & 0.01971 & 2077034 & 33.599 & 5.325 & 30.594 & 4.997 & 31.423 & 72.59 & \mathrm{P}\end{array}$ $\begin{array}{llllllllllllll}\text { 3256. } & 2.003 & 0.131 & 0.01971 & 2076891 & 33.594 & 5.938 & 30.957 & 5.301 & 31.566 & 72.59 & \mathrm{P}\end{array}$ $\begin{array}{llllllllllllll}3041 . & 1.862 & 0.108 & 0.01971 & 2076605 & 33.586 & 6.532 & 31.250 & 5.913 & 31.755 & 72.59 & \mathrm{P}\end{array}$ $\begin{array}{llllllllllll}27335 . & 5.221 & 0.831 & 0.02695 & 1718574 & 26.333 & 0.125 & 9.385 & 0.712 & 21.050 & 72.59 & P\end{array}$ $\begin{array}{lllllllllllll}21750 . & 4.374 & 0.703 & 0.02695 & 1715942 & 26.254 & 0.738 & 13.228 & 1.325 & 21.738 & 72.59 & \mathrm{P}\end{array}$ $\begin{array}{llllllllllllll}19209 & 3.980 & 0.650 & 0.02695 & 1714615 & 26.217 & 1.352 & 16.131 & 1.634 & 22.171 & 72.59 & \mathrm{P}\end{array}$ $\begin{array}{lllllllllllllll}16886 . & 3.616 & 0.603 & 0.02695 & 1713451 & 26.184 & 1.964 & 18.457 & 1.939 & 23.401 & 72.59 & P\end{array}$ $\begin{array}{llllllllllll}\text { 12759. } & 2.950 & 0.528 & 0.02695 & 1711140 & 26.121 & 2.577 & 20.216 & 2.550 & 22.487 & 72.59 & \mathrm{P}\end{array}$ $\begin{array}{lllllllllllll}9421 . & 2.381 & 0.473 & 0.02695 & 1708881 & 26.063 & 3.174 & 21.364 & 3.148 & 23.737 & 72.59 & \mathrm{P}\end{array}$ $\begin{array}{lllllllllllllll}7651 . & 1.916 & 0.446 & 0.02695 & 1701009 & 25.884 & 3.504 & 22.081 & 3.530 & 23.850 & 72.59 & \mathrm{P}\end{array}$ $\begin{array}{llllllllllllll}5592 . & 1.524 & 0.416 & 0.02695 & 1699535 & 25.847 & 4.103 & 22.819 & 4.078 & 24.543 & 72.59 & \mathrm{P}\end{array}$ $\begin{array}{lllllllllllllll}3981 . & 1.167 & 0.392 & 0.02695 & 1697898 & 25.807 & 4.714 & 23.439 & 4.688 & 24.609 & 72.59 & P\end{array}$ $\begin{array}{lllllllllllll}3437 . & 1.017 & 0.383 & 0.02695 & 1697068 & 25.787 & 5.325 & 23.906 & 4.997 & 24.784 & 72.59 & \mathrm{P}\end{array}$ $\begin{array}{lllllllllllll}3081 . & 0.879 & 0.375 & 0.02695 & 1695721 & 25.756 & 5.938 & 24.249 & 5.301 & 24.872 & 72.59 & P\end{array}$
\end{abstract}

2901. $\quad \begin{array}{lllllllllllll}0.659 & 0.360 & 0.02695 & 1692927 & 25.691 & 6.532 & 24.504 & 5.913 & 25.018 & 72.59 & \mathrm{P}\end{array}$ $\begin{array}{lllllllllllll}30763 . & 5.956 & 0.854 & 0.02767 & 1849486 & 29.152 & 0.125 & 9.977 & 0.712 & 23.155 & 72.59 & \mathrm{P}\end{array}$ $\begin{array}{llllllllllllll}24458 . & 4.994 & 0.711 & 0.02767 & 1847024 & 29.077 & 0.738 & 14.353 & 1.325 & 23.884 & 72.59 & \mathrm{P}\end{array}$ $\begin{array}{llllllllllll}21592 . & 4.546 & 0.651 & 0.02767 & 1845783 & 29.042 & 1.352 & 17.666 & 1.634 & 24.416 & 72.59 & P\end{array}$ $\begin{array}{lllllllllllllll}18973 . & 4.132 & 0.599 & 0.02767 & 1844715 & 29.011 & 1.964 & 20.294 & 1.939 & 25.805 & 72.59 & \mathrm{P}\end{array}$ $\begin{array}{llllllllllllll}14324 & 3.374 & 0.514 & 0.02767 & 1842599 & 28.954 & 2.577 & 22.303 & 2.550 & 24.852 & 72.59 & \mathrm{P}\end{array}$ $\begin{array}{lllllllllllll}10571 . & 2.725 & 0.452 & 0.02767 & 1840530 . & 28.901 & 3.174 & 23.603 & 3.148 & 26.208 & 72.59 & P\end{array}$ $\begin{array}{lllllllllllll}8585 . & 2.235 & 0.422 & 0.02767 & 1833406 & 28.747 & 3.504 & 24.414 & 3.530 & 26.393 & 72.59 & \mathrm{P}\end{array}$ $\begin{array}{lllllllllllllll}6283 & 1.781 & 0.389 & 0.02767 & 1832004 & 28.713 & 4.103 & 25.257 & 4.078 & 27.184 & 72.59 & P\end{array}$ $\begin{array}{lllllllllllllll}4495 . & 1.365 & 0.362 & 0.02767 & 1830446 & 28.676 & 4.714 & 25.964 & 4.688 & 27.270 & 72.59 & P\end{array}$ $\begin{array}{lllllllllllll}\text { 3898. } & 1.190 & 0.351 & 0.02767 & 1829656 & 28.658 & 5.325 & 26.494 & 4.997 & 27.480 & 72.59 & P\end{array}$ $\begin{array}{lllllllllllll}3514 & 1.041 & 0.342 & 0.02767 & 1828926 & 28.641 & 5.938 & 26.894 & 5.301 & 27.614 & 72.59 & P\end{array}$ $\begin{array}{llllllllllllll}3353 & 0.811 & 0.325 & 0.02767 & 1827462 & 28.608 & 6.532 & 27.199 & 5.913 & 27.768 & 72.59 & P\end{array}$ $\begin{array}{lllllllllllll}26619 . & 5.467 & 0.830 & 0.02253 & 1728048 & 26.541 & 0.125 & 9.648 & 0.712 & 21.078 & 72.59 & P\end{array}$ $\begin{array}{llllllllllll}21230 . & 4.634 & 0.680 & 0.02253 & 1726275 & 26.479 & 0.738 & 13.439 & 1.325 & 21.625 & 72.59 & P\end{array}$ $\begin{array}{lllllllllllll}18782 . & 4.244 & 0.618 & 0.02253 & 1725381 & 26.450 & 1.352 & 16.274 & 1.634 & 22.052 & 72.59 & \mathrm{P}\end{array}$ $\begin{array}{llllllllllllll}16543 . & 3.882 & 0.564 & 0.02253 & 1724606 & 26.424 & 1.964 & 18.564 & 1.939 & 23.457 & 72.59 & P\end{array}$ $\begin{array}{llllllllllll}\text { 12571. } & 3.213 & 0.474 & 0.02253 & 1723070 & 26.377 & 2.577 & 20.327 & 2.550 & 22.523 & 72.59 & P\end{array}$ $\begin{array}{llllllllllll}9365 . & 2.634 & 0.409 & 0.02253 & 1721569 & 26.334 & 3.174 & 21.442 & 3.148 & 23.703 & 72.59 & \mathrm{P}\end{array}$ $\begin{array}{llllllllllll}7669 & 2.225 & 0.377 & 0.02253 & 1717169 & 26.232 & 3.504 & 22.163 & 3.530 & 23.870 & 72.59 & \mathrm{P}\end{array}$ $\begin{array}{llllllllllllll}5703 . & 1.811 & 0.341 & 0.02253 & 1716296 . & 26.208 & 4.103 & 22.929 & 4.078 & 24.667 & 72.59 & P\end{array}$ $\begin{array}{lllllllllllllll}4177 . & 1.421 & 0.311 & 0.02253 & 1715327 & 26.182 & 4.714 & 23.566 & 4.688 & 24.732 & 72.59 & P\end{array}$ $\begin{array}{llllllllllllll}3667 . & 1.252 & 0.299 & 0.02253 & 1714835 & 26.170 & 5.325 & 24.079 & 4.997 & 24.930 & 72.59 & P\end{array}$ $\begin{array}{llllllllllllll}3340 . & 1.105 & 0.289 & 0.02253 & 1714403 & 26.159 & 5.938 & 24.451 & 5.301 & 25.073 & 72.59 & \mathrm{P}\end{array}$

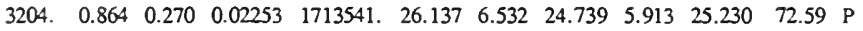
$\begin{array}{llllllllllllll}12607 & 3.378 & 0.776 & 0.00837 & 1695396 & 25.809 & 0.125 & 14.640 & 0.712 & 22.611 & 72.59 & P\end{array}$ $\begin{array}{lllllllllllll}9862 . & 2.840 & 0.590 & 0.00837 & 1695285 & 25.777 & 0.738 & 17.398 & 1.325 & 22.607 & 72.59 & \mathrm{P}\end{array}$ $\begin{array}{llllllllllllll}8621 . & 2.591 & 0.513 & 0.00837 & 1695229 & 25.765 & 1.352 & 19.396 & 1.634 & 23.105 & 72.59 & P\end{array}$ $\begin{array}{llllllllllllll}7492 & 2.359 & 0.447 & 0.00837 & 1695121 & 25.753 & 1.964 & 20.936 & 1.939 & 23.785 & 72.59 & \mathrm{P}\end{array}$ $\begin{array}{llllllllllllll}5504 . & 1.936 & 0.341 & 0.00837 & 1694894 & 25.732 & 2.577 & 22.133 & 2.550 & 23.470 & 72.59 & \mathrm{P}\end{array}$ $\begin{array}{llllllllllllll}3924 . & 1.575 & 0.267 & 0.00837 & 1694672 & 25.717 & 3.174 & 22.803 & 3.148 & 24.235 & 72.59 & P\end{array}$ $\begin{array}{lllllllllllllll}3103 & 1.380 & 0.230 & 0.00837 & 1694912 & 25.717 & 3.504 & 23.283 & 3.530 & 24.218 & 72.59 & P\end{array}$ $\begin{array}{llllllllllllll}2178 . & 1.128 & 0.192 & 0.00837 & 1694853 & 25.711 & 4.103 & 23.737 & 4.078 & 24.824 & 72.59 & \mathrm{P}\end{array}$ $\begin{array}{llllllllllllll}1503 . & 0.897 & 0.163 & 0.00837 & 1694786 . & 25.705 & 4.714 & 24.112 & 4.688 & 24.800 & 72.59 & P\end{array}$ $\begin{array}{llllllllllllll}1303 . & 0.800 & 0.152 & 0.00837 & 1694752 & 25.703 & 5.325 & 24.404 & 4.997 & 24.914 & 72.59 & \mathrm{P}\end{array}$ $\begin{array}{llllllllllllll}1200 . & 0.718 & 0.142 & 0.00837 & 1694731 & 25.701 & 5.938 & 24.602 & 5.301 & 24.978 & 72.59 & P\end{array}$

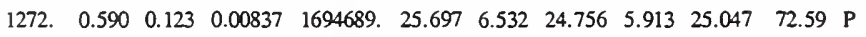
$\begin{array}{lllllllllllll}12477 . & 3.316 & 0.808 & 0.00837 & 1847804 & 29.109 & 0.125 & 18.080 & 0.712 & 25.973 & 72.59 & P\end{array}$ $\begin{array}{lllllllllllll}9729 . & 2.785 & 0.618 & 0.00837 & 1847698 & 29.077 & 0.738 & 20.826 & 1.325 & 25.971 & 72.59 & \mathrm{P}\end{array}$ $\begin{array}{lllllllllllll}\text { 8489. } & 2.538 & 0.540 & 0.00837 & 1847644 & 29.064 & 1.352 & 22.816 & 1.634 & 26.418 & 72.59 & \mathrm{P}\end{array}$ $\begin{array}{llllllllllllll}\text { 7361. } & 2.309 & 0.473 & 0.00837 & 1847534 & 29.051 & 1.964 & 24.306 & 1.939 & 27.149 & 72.59 & \mathrm{P}\end{array}$ $\begin{array}{llllllllllllllll}5382 . & 1.888 & 0.366 & 0.00837 & 1847304 & 29.031 & 2.577 & 25.479 & 2.550 & 26.838 & 72.59 & P\end{array}$ $\begin{array}{llllllllllllll}3816 . & 1.528 & 0.292 & 0.00837 & 1847079 & 29.015 & 3.174 & 26.143 & 3.148 & 27.584 & 72.59 & \mathrm{P}\end{array}$ $\begin{array}{lllllllllllllll}3006 . & 1.333 & 0.255 & 0.00837 & 1847350 & 29.016 & 3.504 & 26.633 & 3.530 & 27.576 & 72.59 & \mathrm{P}\end{array}$ $\begin{array}{llllllllllllll}2100 . & 1.080 & 0.218 & 0.00837 & 1847284 & 29.009 & 4.103 & 27.048 & 4.078 & 28.156 & 72.59 & \mathrm{P}\end{array}$ $\begin{array}{llllllllllllll}\text { 1453. } & 0.846 & 0.189 & 0.00837 & 1847211 . & 29.003 & 4.714 & 27.433 & 4.688 & 28.137 & 72.59 & \mathrm{P}\end{array}$ $\begin{array}{lllllllllllllll}\text { 1268. } & 0.746 & 0.177 & 0.00837 & 1847173 & 29.001 & 5.325 & 27.691 & 4.997 & 28.245 & 72.59 & \mathrm{P}\end{array}$ $\begin{array}{llllllllllllll}1182 & 0.660 & 0.167 & 0.00837 & 1847116 & 28.998 & 5.938 & 27.900 & 5.301 & 28.346 & 72.59 & \mathrm{P}\end{array}$ $\begin{array}{llllllllllllll}1293 . & 0.524 & 0.147 & 0.00837 & 1846998 & 28.993 & 6.532 & 28.069 & 5.913 & 28.419 & 72.59 & \mathrm{P}\end{array}$ $\begin{array}{lllllllllllllll}9864 . & 2.602 & 0.819 & 0.00838 & 2197672 . & 35.968 & 0.125 & 27.174 & 0.712 & 33.568 & 72.59 & \mathrm{P}\end{array}$ $\begin{array}{llllllllllllll}7681 . & 2.160 & 0.659 & 0.00838 & 2197548 . & 35.939 & 0.738 & 29.351 & 1.325 & 33.479 & 72.59 & \mathrm{P}\end{array}$ $\begin{array}{lllllllllllll}6696 . & 1.956 & 0.593 & 0.00838 & 2197485 & 35.928 & 1.352 & 30.975 & 1.634 & 33.835 & 72.59 & P\end{array}$ $\begin{array}{llllllllllllll}5801 . & 1.765 & 0.536 & 0.00838 & 2197377 & 35.917 & 1.964 & 32.145 & 1.939 & 34.452 & 72.59 & \mathrm{P}\end{array}$ $\begin{array}{lllllllllllllll}4232 . & 1.416 & 0.446 & 0.00838 & 2197150 & 35.899 & 2.577 & 33.078 & 2.550 & 34.267 & 72.59 & \mathrm{P}\end{array}$ $\begin{array}{lllllllllllll}2991 . & 1.119 & 0.383 & 0.00838 & 2196929 & 35.885 & 3.174 & 33.623 & 3.148 & 34.877 & 72.59 & \mathrm{P}\end{array}$ $\begin{array}{lllllllllllllll}2351 . & 0.958 & 0.353 & 0.00838 & 2197171 & 35.885 & 3.504 & 34.054 & 3.530 & 34.871 & 72.59 & \mathrm{P}\end{array}$ $\begin{array}{llllllllllllll}1638 & 0.749 & 0.321 & 0.00838 & 2197098 & 35.879 & 4.103 & 34.334 & 4.078 & 35.297 & 72.59 & \mathrm{P}\end{array}$ $\begin{array}{lllllllllllllll}\text { 1131. } & 0.557 & 0.297 & 0.00838 & 2197017 & 35.874 & 4.714 & 34.670 & 4.688 & 35.305 & 72.59 & \mathrm{P}\end{array}$ $\begin{array}{lllllllllllll}989 . & 0.476 & 0.287 & 0.00838 & 2196976 & 35.872 & 5.325 & 34.824 & 4.997 & 35.378 & 72.59 & \mathrm{P}\end{array}$ $\begin{array}{llllllllllllll}925 . & 0.406 & 0.279 & 0.00838 & 2196910 & 35.869 & 5.938 & 35.000 & 5.301 & 35.460 & 72.59 & P\end{array}$ $\begin{array}{llllllllllllll}1024 & 0.295 & 0.262 & 0.00838 & 2196772 & 35.864 & 6.532 & 35.177 & 5.913 & 35.533 & 72.59 & \mathrm{P}\end{array}$ $\begin{array}{lllllllllllll}14360 . & 3.826 & 0.814 & 0.01053 & 2018906 & 32.575 & 0.125 & 20.034 & 0.712 & 28.875 & 72.59 & P\end{array}$ $\begin{array}{llllllllllll}\text { 11145. } & 3.162 & 0.635 & 0.01053 & 2018692 & 32.542 & 0.738 & 23.228 & 1.325 & 29.072 & 72.59 & P\end{array}$ $\begin{array}{lllllllllllll}9697 . & 2.856 & 0.561 & 0.01053 & 2018584 & 32.528 & 1.352 & 25.531 & 1.634 & 29.575 & 72.59 & P\end{array}$ $\begin{array}{lllllllllllll}\text { 8383. } & 2.574 & 0.499 & 0.01053 & 2018430 & 32.516 & 1.964 & 27.257 & 1.939 & 30.433 & 72.59 & \mathrm{P}\end{array}$ $\begin{array}{llllllllllllll}6080 . & 2.061 & 0.399 & 0.01053 & 2018114 & 32.494 & 2.577 & 28.589 & 2.550 & 30.112 & 72.59 & P\end{array}$ $\begin{array}{llllllllllllll}\text { 4267. } & 1.632 & 0.330 & 0.01053 & 2017805 & 32.478 & 3.174 & 29.353 & 3.148 & 30.945 & 72.59 & \mathrm{P}\end{array}$ $\begin{array}{lllllllllllll}\text { 3334. } & 1.397 & 0.297 & 0.01053 & 2017791 & 32.473 & 3.504 & 29.912 & 3.530 & 30.965 & 72.59 & \mathrm{P}\end{array}$ $\begin{array}{lllllllllllllll}2300 & 1.107 & 0.262 & 0.01053 & 2017678 & 32.466 & 4.103 & 30.364 & 4.078 & 31.557 & 72.59 & \mathrm{P}\end{array}$ $\begin{array}{llllllllllllll}\text { 1578. } & 0.853 & 0.237 & 0.01053 & 2017554 & 32.459 & 4.714 & 30.792 & 4.688 & 31.566 & 72.59 & \mathrm{P}\end{array}$ $\begin{array}{lllllllllllll}\text { 1383. } & 0.750 & 0.226 & 0.01053 & 2017491 & 32.457 & 5.325 & 31.075 & 4.997 & 31.675 & 72.59 & \mathrm{P}\end{array}$ $\begin{array}{lllllllllllllll}\text { 1303. } & 0.666 & 0.217 & 0.01053 & 2017407 & 32.454 & 5.938 & 31.307 & 5.301 & 31.791 & 72.59 & \mathrm{P}\end{array}$ $\begin{array}{llllllllllll}1480 & 0.548 & 0.199 & 0.01053 & 2017235 & 32.448 & 6.532 & 31.521 & 5.913 & 31.890 & 72.59 & \mathrm{P}\end{array}$ 
$\begin{array}{llllllllllll}2460 . & 9.619 & 1.064 & 0.01607 & 1670956 & 34.849 & 0.125 & 24.629 & 0.712 & 25.123 & 72.59 & \mathrm{C}\end{array}$ $\begin{array}{lllllllllllll}4528 & 5.085 & 1.032 & 0.01607 & 1670168 & 30.021 & 0.738 & 24.112 & 1.325 & 24.748 & 72.59 & C\end{array}$ $\begin{array}{llllllllllllll}5548 . & 1.910 & 1.009 & 0.01607 & 1669771 & 26.630 & 1.352 & 23.579 & 1.634 & 24.441 & 72.59 & \mathrm{C}\end{array}$ $\begin{array}{llllllllllllll}6538 . & 0.788 & 0.983 & 0.01607 & 1669164 & 25.253 & 1.964 & 22.981 & 1.939 & 24.530 & 72.59 & \mathrm{C}\end{array}$

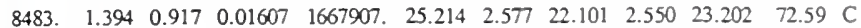
$\begin{array}{llllllllllllll}10327 . & 2.154 & 0.837 & 0.01607 & 1666680 & 25.173 & 3.174 & 20.940 & 3.148 & 23.731 & 72.59 & \mathrm{C}\end{array}$ $\begin{array}{llllllllllllllll}11483 & 2.636 & 0.777 & 0.01607 & 1661847 & 25.054 & 3.504 & 19.851 & 3.530 & 22.863 & 72.59 & \mathrm{C}\end{array}$ $\begin{array}{llllllllllllll}13094 . & 3.581 & 0.682 & 0.01607 & 1660864 & 25.017 & 4.103 & 18.411 & 4.078 & 21.893 & 72.59 & \mathrm{C}\end{array}$ $\begin{array}{lllllllllllllll}14828 . & 4.795 & 0.563 & 0.01607 & 1659771 . & 24.974 & 4.714 & 16.508 & 4.688 & 20.507 & 72.59 & C\end{array}$ $\begin{array}{llllllllllllll}15686 . & 5.476 & 0.497 & 0.01607 & 1659217 & 24.952 & 5.325 & 14.311 & 4.997 & 18.863 & 72.59 & \mathrm{C}\end{array}$ $\begin{array}{lllllllllllll}16515 . & 6.201 & 0.429 & 0.01607 & 1659135 & 24.940 & 5.938 & 11.915 & 5.301 & 18.750 & 72.59 & \mathrm{C}\end{array}$ $\begin{array}{llllllllllllll}18143 & 7.792 & 0.283 & 0.01607 & 1659042 & 24.917 & 6.532 & 9.713 & 5.913 & 16.910 & 72.59 & \mathrm{C}\end{array}$ $\begin{array}{llllllllllllll}1830 & 0.518 & 1.003 & 0.01648 & 2079270 & 34.117 & 0.125 & 32.949 & 0.712 & 33.481 & 72.59 & \mathrm{C}\end{array}$ $\begin{array}{llllllllllllll}3425 & 0.397 & 0.978 & 0.01648 & 2078545 & 33.758 & 0.738 & 32.502 & 1.325 & 33.194 & 72.59 & \mathrm{C}\end{array}$ $\begin{array}{llllllllllll}4231 & 0.562 & 0.961 & 0.01648 & 2078180 & 33.748 & 1.352 & 32.095 & 1.634 & 32.946 & 72.59 & \mathrm{C}\end{array}$ $\begin{array}{llllllllllllll}5028 . & 0.757 & 0.939 & 0.01648 & 2077663 & 33.734 & 1.964 & 31.590 & 1.939 & 33.086 & 72.59 & \mathrm{C}\end{array}$ $\begin{array}{llllllllllllll}6634 . & 1.252 & 0.886 & 0.01648 & 2076598 & 33.705 & 2.577 & 30.863 & 2.550 & 32.018 & 72.59 & \mathrm{C}\end{array}$ $\begin{array}{llllllllllllll}8210 . & 1.872 & 0.819 & 0.01648 & 2075557 & 33.674 & 3.174 & 29.968 & 3.148 & 32.331 & 72.59 & \mathrm{C}\end{array}$ $\begin{array}{lllllllllllllll}9226 . & 2.281 & 0.769 & 0.01648 & 2071817 & 33.594 & 3.504 & 29.287 & 3.530 & 31.600 & 72.59 & \mathrm{C}\end{array}$ $\begin{array}{lllllllllllllll}10683 . & 3.051 & 0.688 & 0.01648 & 2071073 & 33.567 & 4.103 & 28.059 & 4.078 & 30.917 & 72.59 & \mathrm{C}\end{array}$ $\begin{array}{lllllllllllll}12308 . & 4.041 & 0.584 & 0.01648 & 2070245 & 33.534 & 4.714 & 26.553 & 4.688 & 29.769 & 72.59 & C\end{array}$ $\begin{array}{llllllllllllll}13135 . & 4.596 & 0.527 & 0.01648 & 2069826 & 33.517 & 5.325 & 24.645 & 4.997 & 28.461 & 72.59 & \mathrm{C}\end{array}$ $\begin{array}{llllllllllllll}13951 . & 5.180 & 0.466 & 0.01648 & 2069559 & 33.503 & 5.938 & 22.618 & 5.301 & 28.369 & 72.59 & C\end{array}$

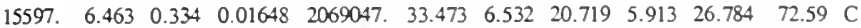
$\begin{array}{llllllllllll}2831 & 1.307 & 1.006 & 0.02640 & 1853786 & 30.101 & 0.125 & 28.146 & 0.712 & 28.695 & 72.59 & \mathrm{C}\end{array}$ $\begin{array}{lllllllllllll}5309 . & 0.724 & 0.984 & 0.02640 & 1851543 & 29.216 & 0.738 & 27.623 & 1.325 & 28.300 & 72.59 & \mathrm{C}\end{array}$ $\begin{array}{lllllllllllll}6566 & 0.919 & 0.967 & 0.02640 & 1850412 & 29.190 & 1.352 & 27.033 & 1.634 & 27.944 & 72.59 & \mathrm{C}\end{array}$ $\begin{array}{llllllllllllll}7811 & 1.151 & 0.947 & 0.02640 & 1849044 & 29.158 & 1.964 & 26.360 & 1.939 & 28.167 & 72.59 & \mathrm{C}\end{array}$ $\begin{array}{lllllllllllllll}\text { 10326. } & 1.748 & 0.897 & 0.02640 & 1846252 & 29.091 & 2.577 & 25.394 & 2.550 & 26.630 & 72.59 & \mathrm{C}\end{array}$ $\begin{array}{llllllllllllll}12806 . & 2.505 & 0.836 & 0.02640 & 1843524 & 29.024 & 3.174 & 24.111 & 3.148 & 27.284 & 72.59 & C\end{array}$

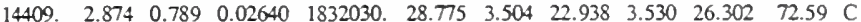
$\begin{array}{llllllllllllll}16716 . & 3.827 & 0.713 & 0.02640 & 1829891 & 28.717 & 4.103 & 21.308 & 4.078 & 25.415 & 72.59 & \mathrm{C}\end{array}$ $\begin{array}{llllllllllllll}19301 & 5.055 & 0.617 & 0.02640 & 1827515 & 28.652 & 4.714 & 19.175 & 4.688 & 23.882 & 72.59 & C\end{array}$ $\begin{array}{lllllllllllll}20620 . & 5.746 & 0.563 & 0.02640 & 1826311 & 28.618 & 5.325 & 16.613 & 4.997 & 22.245 & 72.59 & C\end{array}$ $\begin{array}{lllllllllllll}21923 . & 6.480 & 0.506 & 0.02640 & 1825583 & 28.595 & 5.938 & 13.720 & 5.301 & 22.169 & 72.59 & \mathrm{C}\end{array}$ $\begin{array}{llllllllllllll}24566 . & 8.093 & 0.382 & 0.02640 & 1824191 & 28.547 & 6.532 & 10.902 & 5.913 & 20.222 & 72.59 & \mathrm{C}\end{array}$ $\begin{array}{llllllllllllll}3501 . & 14.431 & 1.099 & 0.02052 & 1823306 & 42.767 & 0.125 & 27.826 & 0.712 & 28.416 & 72.59 & \mathrm{C}\end{array}$ $\begin{array}{llllllllllllll}6351 . & 9.442 & 1.063 & 0.02052 & 1822109 & 37.429 & 0.738 & 27.011 & 1.325 & 27.793 & 72.59 & \mathrm{C}\end{array}$ $\begin{array}{llllllllllllll}7724 & 5.991 & 1.037 & 0.02052 & 1821506 & 33.721 & 1.352 & 26.295 & 1.634 & 27.360 & 72.59 & \mathrm{C}\end{array}$

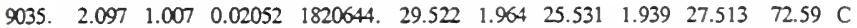
$\begin{array}{lllllllllllllll}11544 . & 1.865 & 0.934 & 0.02052 & 1818865 & 28.520 & 2.577 & 24.478 & 2.550 & 25.822 & 72.59 & \mathrm{C}\end{array}$ $\begin{array}{lllllllllllllll}13835 & 2.768 & 0.847 & 0.02052 & 1817126 & 28.469 & 3.174 & 23.094 & 3.148 & 26.497 & 72.59 & C\end{array}$ $\begin{array}{lllllllllllll}15223 . & 3.324 & 0.783 & 0.02052 & 1810066 . & 28.309 & 3.504 & 21.859 & 3.530 & 25.419 & 72.59 & C\end{array}$ $\begin{array}{lllllllllllll}17092 . & 4.449 & 0.682 & 0.02052 & 1808742 & 28.265 & 4.103 & 20.166 & 4.078 & 24.286 & 72.59 & \mathrm{C}\end{array}$ $\begin{array}{llllllllllllll}19012 & 5.897 & 0.558 & 0.02052 & 1807271 & 28.214 & 4.714 & 17.979 & 4.688 & 22.698 & 72.59 & \mathrm{C}\end{array}$ $\begin{array}{lllllllllllll}19923 . & 6.710 & 0.490 & 0.02052 & 1806525 & 28.188 & 5.325 & 15.475 & 4.997 & 20.766 & 72.59 & \mathrm{C}\end{array}$ $\begin{array}{llllllllllllll}20778 . & 7.569 & 0.421 & 0.02052 & 1806152 & 28.170 & 5.938 & 12.804 & 5.301 & 20.664 & 72.59 & \mathrm{C}\end{array}$ $\begin{array}{lllllllllllll}22376 . & 9.457 & 0.275 & 0.02052 & 1805459 & 28.134 & 6.532 & 10.363 & 5.913 & 18.474 & 72.59 & \mathrm{C}\end{array}$ $\begin{array}{lllllllllllll}2957 . & 5.125 & 1.038 & 0.02781 & 2035292 & 37.651 & 0.125 & 31.768 & 0.712 & 32.520 & 72.59 & C\end{array}$ $\begin{array}{lllllllllllll}5552 . & 2.413 & 1.014 & 0.02781 & 2033324 & 34.621 & 0.738 & 31.110 & 1.325 & 32.001 & 72.59 & C\end{array}$ $\begin{array}{lllllllllllll}6872 & 0.892 & 0.997 & 0.02781 & 2032332 & 32.868 & 1.352 & 30.479 & 1.634 & 31.613 & 72.59 & C\end{array}$ $\begin{array}{llllllllllllll}8181 . & 1.141 & 0.976 & 0.02781 & 2031030 & 32.839 & 1.964 & 29.749 & 1.939 & 31.805 & 72.59 & C\end{array}$ $\begin{array}{llllllllllllll}10831 . & 1.779 & 0.925 & 0.02781 & 2028356 . & 32.778 & 2.577 & 28.724 & 2.550 & 30.254 & 72.59 & \mathrm{C}\end{array}$ $\begin{array}{llllllllllllll}13451 . & 2.585 & 0.861 & 0.02781 & 2025744 & 32.716 & 3.174 & 27.410 & 3.148 & 30.920 & 72.59 & \mathrm{C}\end{array}$ $\begin{array}{llllllllllllll}\text { 15148. } 3.006 & 0.813 & 0.02781 & 2014419 & 32.487 & 3.504 & 26.310 & 3.530 & 29.887 & 72.59 & \mathrm{C}\end{array}$ $\begin{array}{llllllllllllll}17596 . & 4.017 & 0.734 & 0.02781 & 2012357 & 32.433 & 4.103 & 24.547 & 4.078 & 28.957 & 72.59 & \mathrm{C}\end{array}$ $\begin{array}{llllllllllllll}20345 . & 5.317 & 0.634 & 0.02781 & 2010066 & 32.372 & 4.714 & 22.350 & 4.688 & 27.362 & 72.59 & \mathrm{C}\end{array}$ $\begin{array}{lllllllllllll}21750 . & 6.048 & 0.578 & 0.02781 & 2008904 & 32.341 & 5.325 & 19.535 & 4.997 & 25.603 & 72.59 & \mathrm{C}\end{array}$ $\begin{array}{lllllllllllll}23141 . & 6.823 & 0.519 & 0.02781 & 2008193 & 32.318 & 5.938 & 16.486 & 5.301 & 25.602 & 72.59 & \mathrm{C}\end{array}$ $\begin{array}{llllllllllllll}25965 . & 8.525 & 0.391 & 0.02781 & 2006828 & 32.271 & 6.532 & 13.588 & 5.913 & 23.500 & 72.59 & \mathrm{C}\end{array}$ $\begin{array}{llllllllllllll}3651 . & 9.102 & 1.073 & 0.02847 & 2196802 & 44.706 & 0.125 & 34.808 & 0.712 & 35.763 & 72.59 & \mathrm{C}\end{array}$ $\begin{array}{lllllllllllll}6766 . & 5.857 & 1.044 & 0.02847 & 2194949 & 41.074 & 0.738 & 33.900 & 1.325 & 34.940 & 72.59 & C\end{array}$ $\begin{array}{lllllllllllll}8322 & 3.591 & 1.023 & 0.02847 & 2194015 & 38.525 & 1.352 & 33.133 & 1.634 & 34.459 & 72.59 & C\end{array}$ $\begin{array}{llllllllllllllll}9845 . & 1.310 & 0.997 & 0.02847 & 2192796 & 35.908 & 1.964 & 32.268 & 1.939 & 34.661 & 72.59 & \mathrm{C}\end{array}$ $\begin{array}{llllllllllllll}12874 . & 2.103 & 0.935 & 0.02847 & 2190294 & 35.851 & 2.577 & 31.067 & 2.550 & 32.840 & 72.59 & \mathrm{C}\end{array}$ $\begin{array}{lllllllllllllll}15795 . & 3.099 & 0.859 & 0.02847 & 2187850 & 35.793 & 3.174 & 29.541 & 3.148 & 33.594 & 72.59 & \mathrm{C}\end{array}$ $\begin{array}{llllllllllllll}17650 . & 3.687 & 0.802 & 0.02847 & 2177428 & 35.591 & 3.504 & 28.268 & 3.530 & 32.332 & 72.59 & \mathrm{C}\end{array}$ $\begin{array}{llllllllllllll}20276 . & 4.931 & 0.711 & 0.02847 & 2175644 & 35.543 & 4.103 & 26.225 & 4.078 & 31.181 & 72.59 & \mathrm{C}\end{array}$ $\begin{array}{llllllllllllll}23156 . & 6.529 & 0.595 & 0.02847 & 2173662 & 35.488 & 4.714 & 23.670 & 4.688 & 29.486 & 72.59 & \mathrm{C}\end{array}$ $\begin{array}{llllllllllllllll}24602 . & 7.427 & 0.531 & 0.02847 & 2172656 & 35.460 & 5.325 & 20.451 & 4.997 & 27.308 & 72.59 & \mathrm{C}\end{array}$ $\begin{array}{llllllllllllll}26014 & 8.375 & 0.465 & 0.02847 & 2172101 & 35.439 & 5.938 & 17.049 & 5.301 & 27.174 & 72.59 & \mathrm{C}\end{array}$ $\begin{array}{lllllllllllll}28829 & 10.458 & 0.320 & 0.02847 & 2171050 & 35.398 & 6.532 & 13.942 & 5.913 & 24.570 & 72.59 & \mathrm{C}\end{array}$ $\begin{array}{lllllllllllll}2696 . & 2.048 & 1.012 & 0.02289 & 1741411 & 28.519 & 0.125 & 25.784 & 0.712 & 26.384 & 72.59 & C\end{array}$ $\begin{array}{lllllllllllllll}5018 . & 0.633 & 0.987 & 0.02289 & 1739618 & 26.821 & 0.738 & 25.286 & 1.325 & 26.005 & 72.59 & C\end{array}$ $\begin{array}{llllllllllllll}6182 & 0.819 & 0.970 & 0.02289 & 1738714 & 26.799 & 1.352 & 24.724 & 1.634 & 25.672 & 72.59 & \mathrm{C}\end{array}$ $\begin{array}{lllllllllllll}\text { 7327. } & 1.037 & 0.949 & 0.02289 & 1737576 & 26.770 & 1.964 & 24.090 & 1.939 & 25.869 & 72.59 & C\end{array}$ $\begin{array}{llllllllllllll}9614 . & 1.601 & 0.896 & 0.02289 & 1735246 & 26.710 & 2.577 & 23.186 & 2.550 & 24.433 & 72.59 & C\end{array}$ $\begin{array}{llllllllllllll}\text { 11834. } & 2.314 & 0.831 & 0.02289 & 1732971 . & 26.650 & 3.174 & 21.999 & 3.148 & 25.054 & 72.59 & \mathrm{C}\end{array}$ $\begin{array}{lllllllllllllll}13254 & 2.679 & 0.783 & 0.02289 & 1723514 & 26.434 & 3.504 & 20.944 & 3.530 & 24.148 & 72.59 & \mathrm{C}\end{array}$ $\begin{array}{llllllllllllll}15273 & 3.575 & 0.704 & 0.02289 & 1721714 & 26.382 & 4.103 & 19.440 & 4.078 & 23.304 & 72.59 & \mathrm{C}\end{array}$ $\begin{array}{lllllllllllll}17503 & 4.730 & 0.605 & 0.02289 & 1719714 & 26.322 & 4.714 & 17.512 & 4.688 & 21.930 & 72.59 & \mathrm{C}\end{array}$ $\begin{array}{llllllllllllll}18628 . & 5.379 & 0.549 & 0.02289 & 1718700 & 26.292 & 5.325 & 15.166 & 4.997 & 20.269 & 72.59 & \mathrm{C}\end{array}$ $\begin{array}{llllllllllllll}\text { 19732. } & 6.069 & 0.492 & 0.02289 & 1718093 & 26.270 & 5.938 & 12.611 & 5.301 & 20.226 & 72.59 & \mathrm{C}\end{array}$ $\begin{array}{lllllllllllll}21945 . & 7.584 & 0.366 & 0.02289 & 1716935 & 26.226 & 6.532 & 10.104 & 5.913 & 18.434 & 72.59 & \mathrm{C}\end{array}$ $\begin{array}{llllllllllll}\text { 1304. } & 7.601 & 1.056 & 0.01002 & 1817819 & 36.380 & 0.125 & 28.248 & 0.712 & 28.493 & 72.59 & \mathrm{C}\end{array}$ $\begin{array}{lllllllllllllll}2470 . & 3.860 & 1.028 & 0.01002 & 1817611 . & 32.391 & 0.738 & 27.846 & 1.325 & 28.271 & 72.59 & \mathrm{C}\end{array}$ $\begin{array}{llllllllllllll}3072 & 1.181 & 1.008 & 0.01002 & 1817506 & 29.530 & 1.352 & 27.497 & 1.634 & 28.038 & 72.59 & \mathrm{C}\end{array}$ $\begin{array}{lllllllllllllll}3674 . & 0.361 & 0.983 & 0.01002 & 1817282 & 28.495 & 1.964 & 27.074 & 1.939 & 28.121 & 72.59 & C\end{array}$

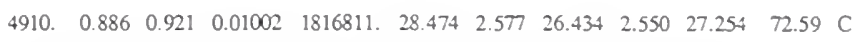
$\begin{array}{llllllllllll}6152 . & 1.540 & 0.843 & 0.01002 & 1816352 & 28.452 & 3.174 & 25.529 & 3.148 & 27.499 & 72.59 & \mathrm{C}\end{array}$ $\begin{array}{llllllllllllll}6967 . & 2.011 & 0.784 & 0.01002 & 1815083 & 28.415 & 3.504 & 24.675 & 3.530 & 26.837 & 72.59 & \mathrm{C}\end{array}$ $\begin{array}{llllllllllllll}8158 . & 2.817 & 0.688 & 0.01002 & 1814755 & 28.393 & 4.103 & 23.446 & 4.078 & 26.225 & 72.59 & \mathrm{C}\end{array}$ $\begin{array}{llllllllllllll}9515 . & 3.851 & 0.563 & 0.01002 & 1814389 & 28.366 & 4.714 & 21.765 & 4.088 & 24.917 & 72.59 & \mathrm{C}\end{array}$ $\begin{array}{lllllllllllll}10217 . & 4.431 & 0.493 & 0.01002 & 1814204 & 28.352 & 5.325 & 19.751 & 4.997 & 23.619 & 72.59 & C\end{array}$ $\begin{array}{llllllllllllll}10917 . & 5.040 & 0.419 & 0.01002 & 1814096 & 28.338 & 5.938 & 17.640 & 5.301 & 23.284 & 72.59 & C\end{array}$ $\begin{array}{llllllllllll}12352 & 6.375 & 0.257 & 0.01002 & 1813891 & 28.311 & 6.532 & 15.748 & 5.913 & 21.385 & 72.59\end{array}$

$\begin{array}{llllllllllll}1152 . & 9.119 & 1.079 & 0.01014 & 2202960 & 45.525 & 0.125 & 35.862 & 0.712 & 36.236 & 72.59 & \mathrm{C}\end{array}$ $\begin{array}{lllllllllllllll}2198 . & 6.084 & 1.053 & 0.01014 & 2202775 . & 42.256 & 0.738 & 35.299 & 1.325 & 35.928 & 72.59 & \mathrm{C}\end{array}$ $\begin{array}{lllllllllllll}2742 . & 3.899 & 1.033 & 0.01014 & 2202682 & 39.900 & 1.352 & 34.976 & 1.634 & 35.708 & 72.59 & \mathrm{C}\end{array}$ $\begin{array}{lllllllllllll}3290 . & 1.362 & 1.009 & 0.01014 & 2202491 & 37.159 & 1.964 & 34.573 & 1.939 & 35.744 & 72.59 & \mathrm{C}\end{array}$ $\begin{array}{llllllllllllll}4425 . & 0.787 & 0.950 & 0.01014 & 2202090 & 36.070 & 2.577 & 33.964 & 2.550 & 34 & 965 & 72.59 & \mathrm{C}\end{array}$ $\begin{array}{lllllllllllll}5577 . & 1.405 & 0.875 & 0.01014 & 2201699 & 36.051 & 3.174 & 33.168 & 3.148 & 35.148 & 72.59 & \mathrm{C}\end{array}$ $\begin{array}{lllllllllllll}6339 . & 1.857 & 0.818 & 0.01014 & 2200781 & 36.024 & 3.504 & 32.544 & 3.530 & 34.526 & 72.59 & C\end{array}$ $\begin{array}{llllllllllllll}7461 . & 2.619 & 0.724 & 0.01014 & 2200558 & 36.005 & 4.103 & 31.369 & 4.078 & 33.991 & 72.59 & \mathrm{C}\end{array}$ $\begin{array}{llllllllllllll}8750 . & 3.596 & 0.602 & 0.01014 & 2200310 & 35.981 & 4.714 & 29.862 & 4.688 & 32.748 & 72.59 & \mathrm{C}\end{array}$ $\begin{array}{lllllllllllll}9421 . & 4.143 & 0.533 & 0.01014 & 2200184 & 35.968 & 5.325 & 27.937 & 4.997 & 31.576 & 72.59 & \mathrm{C}\end{array}$ $\begin{array}{llllllllllllll}10092 . & 4.717 & 0.461 & 0.01014 & 2200091 & 35.955 & 5.938 & 25.961 & 5.301 & 31.261 & 72.59 & \mathrm{C}\end{array}$ $\begin{array}{lllllllllllllll}11478 . & 5.975 & 0.301 & 0.01014 & 2199907 & 35.928 & 6.532 & 24.162 & 5.913 & 29.429 & 72.59 & C\end{array}$ $\begin{array}{lllllllllllll}2696 . & 7.737 & 1.066 & 0.02120 & 2299161 & 45.318 & 0.125 & 36.955 & 0.712 & 37.685 & 72.59 & C\end{array}$ $\begin{array}{llllllllllll}4971 . & 4.595 & 1.037 & 0.02120 & 2298158 & 41.872 & 0.738 & 36.259 & 1.325 & 37.084 & 72.59 & \mathrm{C}\end{array}$ $\begin{array}{llllllllllllll}6097 . & 2.415 & 1.015 & 0.02120 & 2297652 & 39470 & 1.352 & 35.683 & 1.634 & 36.709 & 72.59 & \mathrm{C}\end{array}$ $\begin{array}{llllllllllll}7192 . & 1.000 & 0.990 & 0.02120 & 2296963 & 37.791 & 1.964 & 35.025 & 1.939 & 36.840 & 72.59 & \mathrm{C}\end{array}$

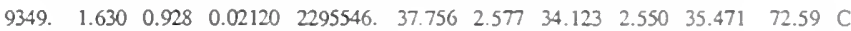
$\begin{array}{lllllllllllll}11401 . & 2.419 & 0.853 & 0.02120 & 2294161 & 37.718 & 3.174 & 33.011 & 3.148 & 35.993 & 72.59 & \mathrm{C}\end{array}$ 12693. $\begin{array}{llllllllllll}2.935 & 0.796 & 0.02120 & 2288837 & 37.614 & 3.504 & 32.142 & 3.530 & 34.969 & 72.59 & \mathrm{C}\end{array}$ $\begin{array}{lllllllllllll}14498 . & 3.915 & 0.706 & 0.02120 & 2287825 & 37.582 & 4.103 & 30.638 & 4.078 & 34.095 & 72.59 & C\end{array}$ $\begin{array}{llllllllllllll}16450 . & 5.174 & 0.593 & 0.02120 & 2286700 & 37.544 & 4.714 & 28.797 & 4.688 & 32.715 & 72.59 & C\end{array}$ $\begin{array}{lllllllllllll}17418 & 5.880 & 0.531 & 0.02120 & 2286130 & 37.524 & 5.325 & 26.558 & 4.997 & 31.072 & 72.59 & \mathrm{C}\end{array}$ $\begin{array}{llllllllllllll}18357 & 6.623 & 0.466 & 0.02120 & 2285783 & 37.508 & 5.938 & 24.136 & 5.301 & 30.965 & 72.59 & \mathrm{C}\end{array}$ $\begin{array}{lllllllllllll}20204 . & 8.256 & 0.327 & 0.02120 & 2285118 & 37.475 & 6.532 & 21.874 & 5.913 & 28.992 & 72.59 & \mathrm{C}\end{array}$ $\begin{array}{lllllllllllll}3696 & 13.183 & 1.095 & 0.02402 & 1937727 & 43.852 & 0.125 & 30.052 & 0.712 & 30.786 & 72.59 & \mathrm{C}\end{array}$ $\begin{array}{llllllllllllll}6757 . & 8.852 & 1.061 & 0.02402 & 1936215 & 39.148 & 0.738 & 29.137 & 1.325 & 30.071 & 72.59 & \mathrm{C}\end{array}$ $\begin{array}{lllllllllllllll}\text { 8252. } & 5.841 & 1.037 & 0.02402 & 1935453 & 35.863 & 1.352 & 28.365 & 1.634 & 29.590 & 72.59 & \mathrm{C}\end{array}$ $\begin{array}{llllllllllllll}9693 . & 2.429 & 1.009 & 0.02402 & 1934405 & 32.125 & 1.964 & 27.533 & 1.939 & 29.767 & 72.59 & \mathrm{C}\end{array}$ $\begin{array}{lllllllllllllll}\text { 12491. } & 1.995 & 0.941 & 0.02402 & 1932249 & 30.870 & 2.577 & 26.387 & 2.550 & 27.964 & 72.59 & \mathrm{C}\end{array}$ $\begin{array}{llllllllllllll}15103 . & 2.957 & 0.858 & 0.02402 & 1930141 . & 30.813 & 3.174 & 24.901 & 3.148 & 28.724 & 72.59 & C\end{array}$ $\begin{array}{llllllllllllll}16717 & 3.531 & 0.797 & 0.02402 & 1921249 & 30.623 & 3.504 & 23.610 & 3.530 & 27.536 & 72.59 & C\end{array}$ $\begin{array}{lllllllllllll}18937 . & 4.732 & 0.700 & 0.02402 & 1919670 & 30.575 & 4.103 & 21.718 & 4.078 & 26.386 & 72.59 & C\end{array}$ $\begin{array}{lllllllllllll}21284 & 6.276 & 0.579 & 0.02402 & 1917915 & 30.520 & 4.714 & 19.309 & 4.688 & 24.741 & 72.59 & C\end{array}$ $\begin{array}{llllllllllllll}22425 & 7.144 & 0.513 & 0.02402 & 1917026 & 30.492 & 5.325 & 16.437 & 4.997 & 22.596 & 72.59 & C\end{array}$ $\begin{array}{llllllllllllll}23517 . & 8.061 & 0.445 & 0.02402 & 1916556 & 30.472 & 5.938 & 13.398 & 5.301 & 22.477 & 72.59 & C\end{array}$ $\begin{array}{llllllllllll}25623 & 10.075 & 0.299 & 0.02402 & 1915674 & 30.433 & 6.532 & 10.600 & 5.913 & 20.072 & 72.59 & \mathrm{C}\end{array}$ $\begin{array}{lllllllllllll}3521 . & 11.526 & 1.089 & 0.02447 & 2106292 & 45.474 & 0.125 & 33.301 & 0.712 & 34.062 & 72.59 & C\end{array}$ $\begin{array}{llllllllllllll}6458 . & 7.681 & 1.057 & 0.02447 & 2104870 & 41.264 & 0.738 & 32.396 & 1.325 & 33.355 & 72.59 & \mathrm{C}\end{array}$ $\begin{array}{lllllllllllll}\text { 7898. } & 5.012 & 1.034 & 0.02447 & 2104153 & 38.326 & 1.352 & 31.660 & 1.634 & 32.887 & 72.59 & \mathrm{C}\end{array}$ $\begin{array}{llllllllllllll}9292 . & 1.991 & 1.006 & 0.02447 & 2103162 & 34.987 & 1.964 & 30.851 & 1.939 & 33.054 & 72.59 & \mathrm{C}\end{array}$ $\begin{array}{llllllllllllll}\text { 12013. } & 1.990 & 0.940 & 0.02447 & 2101121 & 34.182 & 2.577 & 29.734 & 2.550 & 31.340 & 72.59 & \mathrm{C}\end{array}$ $\begin{array}{llllllllllllll}14571 . & 2.936 & 0.859 & 0.02447 & 2099128 & 34.131 & 3.174 & 28.318 & 3.148 & 32.051 & 72.59 & \mathrm{C}\end{array}$ $\begin{array}{llllllllllllll}16164 & 3.518 & 0.799 & 0.02447 & 2090905 & 33.964 & 3.504 & 27.142 & 3.530 & 30.858 & 72.59 & C\end{array}$ $\begin{array}{lllllllllllll}\text { 18367. } & 4.697 & 0.704 & 0.02447 & 2089445 & 33.921 & 4.103 & 25.292 & 4.078 & 29.726 & 72.59 & C\end{array}$ $\begin{array}{llllllllllllll}20718 & 6.213 & 0.585 & 0.02447 & 2087822 & 33.871 & 4.714 & 22.955 & 4.688 & 28.159 & 72.59 & \mathrm{C}\end{array}$ $\begin{array}{llllllllllllll}21870 . & 7.063 & 0.519 & 0.02447 & 2087000 & 33.846 & 5.325 & 20.127 & 4.997 & 26.076 & 72.59 & \mathrm{C}\end{array}$ $\begin{array}{lllllllllllll}22978 . & 7.962 & 0.452 & 0.02447 & 2086552 & 33.827 & 5.938 & 17.138 & 5.301 & 25.943 & 72.59 & \mathrm{C}\end{array}$ $\begin{array}{llllllllllllll}25132 . & 9.934 & 0.307 & 0.02447 & 2085708 & 33.789 & 6.532 & 14.444 & 5.913 & 23.548 & 72.59 & \mathrm{C}\end{array}$ $\begin{array}{llllllllllllll}3315 . & 9.016 & 1.075 & 0.02488 & 2266968 & 45.951 & 0.125 & 36.228 & 0.712 & 37.068 & 72.59 & C\end{array}$ $\begin{array}{lllllllllllll}6111 . & 5.684 & 1.044 & 0.02488 & 2265587 & 42.260 & 0.738 & 35.393 & 1.325 & 36.323 & 72.59 & C\end{array}$ $\begin{array}{lllllllllllll}7494 & 3.373 & 1.022 & 0.02488 & 2264890 & 39.686 & 1.352 & 34.696 & 1.634 & 35.872 & 72.59 & \mathrm{C}\end{array}$ $\begin{array}{lllllllllllll}8839 & 1.202 & 0.996 & 0.02488 & 2263960 & 37.202 & 1.964 & 33.914 & 1.939 & 36.045 & 72.59 & \mathrm{C}\end{array}$ $\begin{array}{llllllllllllll}11490 & 1.945 & 0.932 & 0.02488 & 2262050 & 37.157 & 2.577 & 32.832 & 2.550 & 34.403 & 72.59 & \mathrm{C}\end{array}$ $\begin{array}{lllllllllllll}14013 . & 2.877 & 0.853 & 0.02488 & 2260183 & 37.110 & 3.174 & 31.481 & 3.148 & 35.086 & 72.59 & \mathrm{C}\end{array}$ $\begin{array}{llllllllllllll}15600 & 3.465 & 0.794 & 0.02488 & 2252609 & 36.964 & 3.504 & 30.377 & 3.530 & 33.884 & 72.59 & \mathrm{C}\end{array}$ $\begin{array}{lllllllllllll}17820 & 4.625 & 0.701 & 0.02488 & 2251273 & 36.925 & 4.103 & 28.572 & 4.078 & 32.804 & 72.59 & C\end{array}$ $\begin{array}{lllllllllllllll}20221 . & 6.115 & 0.583 & 0.02488 & 2249789 & 36.879 & 4.714 & 26.286 & 4.688 & 31.266 & 72.59 & C\end{array}$ $\begin{array}{lllllllllllll}21413 . & 6.952 & 0.518 & 0.02488 & 2249036 & 36.856 & 5.325 & 23.523 & 4.997 & 29.234 & 72.59 & \mathrm{C}\end{array}$ $\begin{array}{lllllllllllllll}22568 & 7.834 & 0.451 & 0.02488 & 2248604 & 36.838 & 5.938 & 20.581 & 5.301 & 29.087 & 72.59 & C\end{array}$ $\begin{array}{llllllllllllll}24841 . & 9.769 & 0.306 & 0.02488 & 2247784 & 36.801 & 6.532 & 17.896 & 5.913 & 26.699 & 72.59 & C\end{array}$ $\begin{array}{lllllllllllll}24755 . & 6.539 & 0.756 & 0.01557 & 1772932 & 27.509 & 0.125 & 10.654 & 0.712 & 21.073 & 72.59 & \mathrm{P}\end{array}$ $\begin{array}{lllllllllllll}19732 . & 5.629 & 0.554 & 0.01557 & 1772240 & 27.462 & 0.738 & 14.202 & 1.325 & 21.513 & 72.59 & \mathrm{P}\end{array}$ $\begin{array}{lllllllllllll}17429 & 5.223 & 0.470 & 0.01557 & 1771891 & 27.442 & 1.352 & 16.782 & 1.634 & 22.013 & 72.59 & P\end{array}$ $\begin{array}{lllllllllllll}15309 . & 4.860 & 0.398 & 0.01557 & 1771621 & 27.426 & 1.964 & 18.901 & 1.939 & 23.417 & 72.59 & \mathrm{P}\end{array}$ $\begin{array}{lllllllllllll}11497 . & 4.234 & 0.279 & 0.01557 & 1771090 & 27.397 & 2.577 & 20.557 & 2.550 & 22.604 & 72.59 & \mathrm{P}\end{array}$ $\begin{array}{llllllllllll}8350 . & 3.755 & 0.194 & 0.01557 & 1770571 & 27.374 & 3.174 & 21.531 & 3.148 & 23.676 & 72.59 & \mathrm{P}\end{array}$ $\begin{array}{lllllllllllllll}6641 . & 3.518 & 0.152 & 0.01557 & 1770296 & 27.362 & 3.504 & 22.169 & 3.530 & 23.661 & 72.59 & P\end{array}$ $\begin{array}{lllllllllllll}4587 . & 3.274 & 0.108 & 0.01557 & 1770099 & 27.352 & 4.103 & 22.845 & 4.078 & 24.484 & 72.59 & \mathrm{P}\end{array}$ $\begin{array}{llllllllllllll}2868 . & 3.131 & 0.076 & 0.01557 & 1769880 & 27.343 & 4.714 & 23.312 & 4.688 & 24.126 & 72.59 & \mathrm{P}\end{array}$ $\begin{array}{lllllllllllll}2222 . & 3.109 & 0.065 & 0.01557 & 1769769 & 27.339 & 5.325 & 23.611 & 4.997 & 24.211 & 72.59 & \mathrm{P}\end{array}$ $\begin{array}{llllllllllllll}1735 . & 3.122 & 0.056 & 0.01557 & 1769703 & 27.336 & 5.938 & 23.762 & 5.301 & 24.162 & 72.59 & P\end{array}$ $\begin{array}{lllllllllllllll}1203 . & 3.248 & 0.043 & 0.01557 & 1769578 & 27.332 & 6.532 & 23.823 & 5.913 & 24.092 & 72.59 & \mathrm{P}\end{array}$ $\begin{array}{llllllllllllll}27262 . & 6.800 & 0.775 & 0.01946 & 1862214 & 29.405 & 0.125 & 11.056 & 0.712 & 22.681 & 72.59 & P\end{array}$ $\begin{array}{llllllllllll}21830 . & 5.833 & 0.594 & 0.01946 & 1861131 & 29.354 & 0.738 & 14.950 & 1.325 & 23.220 & 72.59 & \mathrm{P}\end{array}$ $\begin{array}{llllllllllllll}19350 . & 5.388 & 0.518 & 0.01946 & 1860585 & 29.331 & 1.352 & 17.834 & 1.634 & 23.735 & 72.59 & \mathrm{P}\end{array}$ $\begin{array}{llllllllllll}17077 . & 4.980 & 0.452 & 0.01946 & 1860171 . & 29.312 & 1.964 & 20.184 & 1.939 & 25.260 & 72.59 & \mathrm{P}\end{array}$ $\begin{array}{lllllllllllll}13018 . & 4.246 & 0.343 & 0.01946 & 1859363 & 29.279 & 2.577 & 22.013 & 2.550 & 24.396 & 72.59 & \mathrm{P}\end{array}$ $\begin{array}{llllllllllllllll}9707 . & 3.634 & 0.263 & 0.01946 & 1858573 & 29.251 & 3.174 & 23.151 & 3.148 & 25.678 & 72.59 & \mathrm{P}\end{array}$

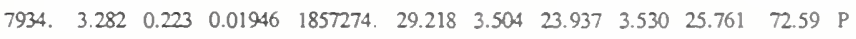

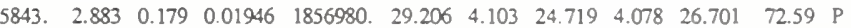


$\begin{array}{lllllllllllll}\text { 4159. } & 2.541 & 0.144 & 0.01946 & 1856654 & 29.194 & 4.714 & 25.374 & 4.688 & 26.590 & 72.59 & \mathrm{P}\end{array}$ $\begin{array}{lllllllllllll}3562 . & 2.409 & 0.130 & 0.01946 & 1856489 & 29.189 & 5.325 & 25.820 & 4.997 & 26.731 & 72.59 & \mathrm{P}\end{array}$

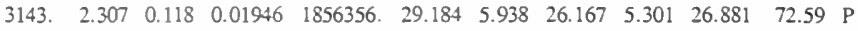

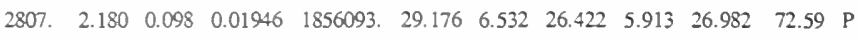
$\begin{array}{lllllllllll}30791 . & 7.536 & 0.770 & 0.02279 & 1995640 & 32.110 & 0.125 & 11.583 & 0.712 & 24.555 & 72.59\end{array}$ $\begin{array}{lllllllllllll}24651 . & 6.444 & 0.591 & 0.02279 & 1994244 & 32.054 & 0.738 & 15.968 & 1.325 & 25.341 & 72.59 & \mathrm{P}\end{array}$ $\begin{array}{lllllllllllll}21850 & 5.941 & 0.516 & 0.02279 & 1993540 & 32.028 & 1.352 & 19.249 & 1.634 & 25.919 & 72.59 & \mathrm{P}\end{array}$ $\begin{array}{llllllllllll}19281 . & 5.480 & 0.450 & 0.02279 & 1993030 & 32.008 & 1.964 & 21.928 & 1.939 & 27.619 & 72.59 & \mathrm{P}\end{array}$ $\begin{array}{lllllllllllll}14700 . & 4.646 & 0.343 & 0.02279 & 1992039 & 31.973 & 2.577 & 23.979 & 2.550 & 26.624 & 72.59 & \mathrm{P}\end{array}$ $\begin{array}{lllllllllllll}10965 . & 3.948 & 0.264 & 0.02279 & 1991071 & 31.942 & 3.174 & 25.272 & 3.148 & 27.988 & 72.59 & \mathrm{P}\end{array}$ $\begin{array}{lllllllllllll}8967 . & 3.540 & 0.225 & 0.02279 & 1989310 . & 31.901 & 3.504 & 26.153 & 3.530 & 28.160 & 72.59 & P\end{array}$ $\begin{array}{llllllllllllll}6615 . & 3.078 & 0.181 & 0.02279 & 1988916 & 31.887 & 4.103 & 27.036 & 4.078 & 29.191 & 72.59 & \mathrm{P}\end{array}$ $\begin{array}{llllllllllllll}\text { 4727. } & 2.677 & 0.146 & 0.02279 & 1988478 . & 31.874 & 4.714 & 27.788 & 4.688 & 29.142 & 72.59 & \mathrm{P}\end{array}$ $\begin{array}{lllllllllllll}4062 . & 2.519 & 0.133 & 0.02279 & 1988256 & 31.867 & 5.325 & 28.308 & 4.997 & 29.280 & 72.59 & \mathrm{P}\end{array}$ $\begin{array}{lllllllllllllll}3598 . & 2.394 & 0.121 & 0.02279 & 1988100 & 31.862 & 5.938 & 28.701 & 5.301 & 29.473 & 72.59 & \mathrm{P}\end{array}$ $\begin{array}{lllllllllllll}3241 . & 2.232 & 0.100 & 0.02279 & 1987796 & 31.853 & 6.532 & 28.995 & 5.913 & 29.633 & 72.59 & P\end{array}$ 


\title{
APPENDIX C4
}

\author{
Convective condensation of \\ R134a within a micro-fin tube
}

(file: taraw.tbl)

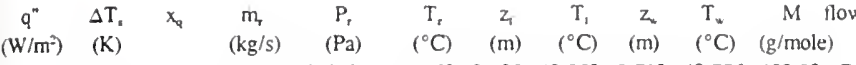
$\begin{array}{llllllllllll}373 . & 1.250 & 1.014 & 0.01685 & 1038738 & 42.769 & 0.125 & 40.352 & 0.712 & 40.776 & 102.03 & \mathrm{C}\end{array}$ $\begin{array}{lllllllllllll}1176 . & 0.544 & 1.007 & 0.01685 & 1037505 & 41.709 & 0.738 & 39.969 & 1.325 & 40.482 & 102.03 & \mathrm{C}\end{array}$ $\begin{array}{llllllllllll}\text { 1749. } & -0.162 & 1.000 & 0.01685 & 1036883 & 40.744 & 1.352 & 39.603 & 1.634 & 40.315 & 102.03 & C\end{array}$ $\begin{array}{lllllllllllll}2426 & 0.121 & 0.990 & 0.01685 & 1036183 & 40.719 & 1.964 & 39.072 & 1.939 & 40.323 & 102.03 & \mathrm{C}\end{array}$

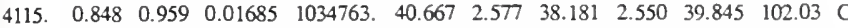
$\begin{array}{lllllllllllllll}6194 & 1.763 & 0.911 & 0.01685 & 1033374 & 40.617 & 3.174 & 37.134 & 3.148 & 39.734 & 102.03 & C\end{array}$ $\begin{array}{llllllllllllll}7747 . & 2.196 & 0.870 & 0.01685 & 1025371 . & 40.326 & 3.504 & 36.101 & 3.530 & 39.054 & 102.03 & \mathrm{C}\end{array}$ $\begin{array}{llllllllllllll}10278 . & 3.322 & 0.792 & 0.01685 & 1023824 & 40.270 & 4.103 & 33.912 & 4.078 & 38.186 & 102.03 & C\end{array}$ $\begin{array}{llllllllllll}13508 . & 4.774 & 0.679 & 0.01685 & 1022106 & 40.207 & 4.714 & 30.665 & 4.688 & 36.482 & 102.03 & C\end{array}$ $\begin{array}{lllllllllllllll}15314 . & 5.591 & 0.609 & 0.01685 & 1021235 & 40.175 & 5.325 & 26.047 & 4.997 & 34.299 & 102.03 & C\end{array}$ $\begin{array}{llllllllllll}\text { 17202. } & 6.461 & 0.532 & 0.01685 & 1020770 & 40.158 & 5.938 & 20.532 & 5.301 & 33.532 & 102.03 & \mathrm{C}\end{array}$ $\begin{array}{llllllllllll}21335 & 8.375 & 0.347 & 0.01685 & 1019895 & 40.126 & 6.532 & 15.082 & 5.913 & 30.376 & 102.03 & C\end{array}$ $\begin{array}{llllllllllll}632 & 2.157 & 1.022 & 0.01887 & 1221001 & 49.934 & 0.125 & 46.003 & 0.712 & 46.932 & 102.03 & \mathrm{C}\end{array}$ $\begin{array}{llllllllllllll}1657 & 1.234 & 1.012 & 0.01887 & 1219748 & 48.582 & 0.738 & 45.370 & 1.325 & 46.546 & 102.03 & C\end{array}$ $\begin{array}{lllllllllllll}2342 & 0.342 & 1.003 & 0.01887 & 1219116 & 47.375 & 1.352 & 44.833 & 1.634 & 46.304 & 102.03 & C\end{array}$ $\begin{array}{lllllllllllll}3127 & 0.254 & 0.991 & 0.01887 & 1218369 & 46.912 & 1.964 & 44.092 & 1.939 & 46.337 & 102.03 & \mathrm{C}\end{array}$ $\begin{array}{llllllllllllll}5035 & 1.151 & 0.955 & 0.01887 & 1216847 & 46.863 & 2.577 & 42.821 & 2.550 & 45.681 & 102.03 & \mathrm{C}\end{array}$ $\begin{array}{lllllllllllllll}7326 & 2.275 & 0.901 & 0.01887 & 1215361 & 46.815 & 3.174 & 41.451 & 3.148 & 45.585 & 102.03 & C\end{array}$ $\begin{array}{lllllllllllll}\text { 9014. } & 2.890 & 0.856 & 0.01887 & 1207126 & 46.551 & 3.504 & 41.013 & 3.530 & 44.855 & 102.03 & \mathrm{C}\end{array}$ $\begin{array}{llllllllllllll}11736 & 4.278 & 0.773 & 0.01887 & 1205612 & 46.502 & 4.103 & 38.323 & 4.078 & 43.644 & 102.03 & C\end{array}$ $\begin{array}{llllllllllll}15179 . & 6.064 & 0.654 & 0.01887 & 1203930 & 46.447 & 4.714 & 34.682 & 4.688 & 41.534 & 102.03 & C\end{array}$ $\begin{array}{llllllllllll}17092 . & 7.067 & 0.581 & 0.01887 & 1203078 . & 46.420 & 5.325 & 29.280 & 4.997 & 39.071 & 102.03 & C\end{array}$ $\begin{array}{lllllllllllll}19085 & 8.133 & 0.501 & 0.01887 & 1202692 & 46.407 & 5.938 & 23.189 & 5.301 & 38.121 & 102.03 & C\end{array}$ $\begin{array}{lllllllllllllll}23429 & 10.473 & 0.311 & 0.01887 & 1201986 & 46.385 & 6.532 & 17.648 & 5.913 & 34.264 & 102.03 & C\end{array}$ $\begin{array}{lllllllllllll}789 . & 2.135 & 1.023 & 0.01918 & 1219517 & 50.049 & 0.125 & 46.226 & 0.712 & 46.835 & 102.03 & C\end{array}$ $\begin{array}{llllllllllllll}2005 . & 1.022 & 1.011 & 0.01918 & 1218218 & 48.423 & 0.738 & 45.640 & 1.325 & 46.455 & 102.03 & C\end{array}$

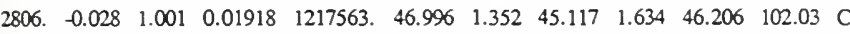
$\begin{array}{lllllllllllll}3719 . & 0.284 & 0.987 & 0.01918 & 1216772 & 46.861 & 1.964 & 44.369 & 1.939 & 46.228 & 102.03 & C\end{array}$ $\begin{array}{lllllllllllllll}5922 . & 1.361 & 0.945 & 0.01918 & 1215158 & 46.809 & 2.577 & 43.107 & 2.550 & 45.153 & 102.03 & C\end{array}$ $\begin{array}{lllllllllllllll}8551 . & 2.711 & 0.883 & 0.01918 & 1213580 & 46.758 & 3.174 & 41.644 & 3.148 & 45.466 & 102.03 & C\end{array}$ $\begin{array}{lllllllllllllll}10481 & 3.488 & 0.831 & 0.01918 & 1205067 . & 46.484 & 3.504 & 40.313 & 3.530 & 44.452 & 102.03 & C\end{array}$ $\begin{array}{llllllllllllll}13586 . & 5.156 & 0.736 & 0.01918 & 1203541 . & 46.435 & 4.103 & 37.200 & 4.078 & 43.007 & 102.03 & C\end{array}$ $\begin{array}{lllllllllllll}17503 . & 7.299 & 0.601 & 0.01918 & 1201845 & 46.380 & 4.714 & 32.801 & 4.688 & 40.496 & 102.03 & C\end{array}$ $\begin{array}{lllllllllllllll}19677 . & 8.503 & 0.518 & 0.01918 & 1200986 & 46.352 & 5.325 & 26.564 & 4.997 & 37.573 & 102.03 & C\end{array}$ $\begin{array}{llllllllllllll}21939 & 9.781 & 0.427 & 0.01918 & 1200674 & 46.342 & 5.938 & 19.440 & 5.301 & 36.373 & 102.03 & C\end{array}$ $\begin{array}{lllllllllllll}26863 & 12.587 & 0.213 & 0.01918 & 1200130 & 46.325 & 6.532 & 13.349 & 5.913 & 31.672 & 102.03 & C\end{array}$ 415. $2.639 \begin{array}{lllllllllll}1.027 & 0.02366 & 1318917 & 53.490 & 0.125 & 49.343 & 0.712 & 50.006 & 102.03 & \mathrm{C}\end{array}$ $\begin{array}{llllllllllllll}\text { 1448. } & 2.249 & 1.021 & 0.02366 & 1317181 & 52.632 & 0.738 & 48.678 & 1.325 & 49.584 & 102.03 & C\end{array}$ $\begin{array}{llllllllllllll}2207 . & 1.721 & 1.014 & 0.02366 & 1316306 & 51.762 & 1.352 & 48.144 & 1.634 & 49.322 & 102.03 & C\end{array}$ $\begin{array}{lllllllllllll}3109 & 0.889 & 1.005 & 0.02366 & 1315229 & 50.522 & 1.964 & 47.404 & 1.939 & 49.333 & 102.03 & \mathrm{C}\end{array}$ $\begin{array}{llllllllllllll}5387 . & 1.253 & 0.974 & 0.02366 & 1313028 & 49.859 & 2.577 & 46.147 & 2.550 & 48.206 & 102.03 & C\end{array}$ $\begin{array}{lllllllllllllll}8214 & 2.463 & 0.926 & 0.02366 & 1310878 . & 49.794 & 3.174 & 44.723 & 3.148 & 48.551 & 102.03 & C\end{array}$ $\begin{array}{lllllllllllllll}10336 & 3.062 & 0.885 & 0.02366 & 1299158 & 49.437 & 3.504 & 43.758 & 3.530 & 47.589 & 102.03 & C\end{array}$ $\begin{array}{llllllllllllll}13803 . & 4.559 & 0.806 & 0.02366 & 1296982 & 49.371 & 4.103 & 40.837 & 4.078 & 46.393 & 102.03 & C\end{array}$ $\begin{array}{lllllllllllllll}18245 & 6.485 & 0.690 & 0.02366 & 1294565 & 49.297 & 4.714 & 36.559 & 4.688 & 44.135 & 102.03 & C\end{array}$ $\begin{array}{lllllllllllllll}20733 & 7.568 & 0.619 & 0.02366 & 1293339 & 49.259 & 5.325 & 30.310 & 4.997 & 41.349 & 102.03 & C\end{array}$ $\begin{array}{lllllllllllll}23336 . & 8.722 & 0.539 & 0.02366 & 1292746 & 49.241 & 5.938 & 22.789 & 5.301 & 40.368 & 102.03 & C\end{array}$ $\begin{array}{llllllllllllll}29045 . & 11.258 & 0.348 & 0.02366 & 1291649 & 49.207 & 6.532 & 15.364 & 5.913 & 36.166 & 102.03 & \mathrm{C}\end{array}$ $\begin{array}{llllllllllll}519 . & 2.937 & 1.030 & 0.02381 & 1282874 & 52.796 & 0.125 & 48.321 & 0.712 & 48.902 & 102.03 & C\end{array}$ $\begin{array}{lllllllllllllll}1670 . & 2.428 & 1.023 & 0.02381 & 1281061 & 51.781 & 0.738 & 47.645 & 1.325 & 48.476 & 102.03 & \mathrm{C}\end{array}$ $\begin{array}{lllllllllllll}\text { 2497. } & 1.804 & 1.015 & 0.02381 & 1280146 . & 50.786 & 1.352 & 47.096 & 1.634 & 48.210 & 102.03 & \mathrm{C}\end{array}$ $\begin{array}{llllllllllllll}3474 . & 0.851 & 1.004 & 0.02381 & 1279022 & 49.391 & 1.964 & 46.321 & 1.939 & 48.212 & 102.03 & C\end{array}$ $\begin{array}{lllllllllllllll}5919 . & 1.322 & 0.971 & 0.02381 & 1276723 & 48.748 & 2.577 & 45.025 & 2.550 & 47.012 & 102.03 & \mathrm{C}\end{array}$ $\begin{array}{llllllllllll}\text { 8934. } & 2.634 & 0.919 & 0.02381 & 1274478 & 48.679 & 3.174 & 43.530 & 3.148 & 47.408 & 102.03 & C\end{array}$ $\begin{array}{llllllllllllll}11188 . & 3.284 & 0.875 & 0.02381 & 1262047 & 48.292 & 3.504 & 42.180 & 3.530 & 46.326 & 102.03 & C\end{array}$ $\begin{array}{llllllllllllllll}14863 & 4.908 & 0.792 & 0.02381 & 1259791 . & 48.222 & 4.103 & 38.982 & 4.078 & 45.025 & 102.03 & C\end{array}$ $\begin{array}{lllllllllllll}19559 . & 6.998 & 0.669 & 0.02381 & 1257284 & 48.143 & 4.714 & 34.280 & 4.688 & 42.577 & 102.03 & C\end{array}$ $\begin{array}{llllllllllllllll}22186 . & 8.173 & 0.594 & 0.02381 & 1256013 & 48.104 & 5.325 & 27.427 & 4.997 & 39.570 & 102.03 & \mathrm{C}\end{array}$ $\begin{array}{lllllllllllll}24932 & 9.425 & 0.510 & 0.02381 & 1255423 & 48.085 & 5.938 & 19.278 & 5.301 & 38.494 & 102.03 & C\end{array}$ $\begin{array}{lllllllllllll}30947 . & 12.177 & 0.310 & 0.02381 & 1254340 & 48.051 & 6.532 & 11.599 & 5.913 & 33.925 & 102.03 & \mathrm{C}\end{array}$ $\begin{array}{lllllllllllll}2776 & 3.977 & 1.023 & 0.02135 & 990435 . & 42.386 & 0.125 & 37.554 & 0.712 & 38.440 & 102.03 & \mathrm{C}\end{array}$ $\begin{array}{lllllllllllllll}5089 & 0.865 & 0.993 & 0.02135 & 988158 & 38.951 & 0.738 & 36.834 & 1.325 & 37.852 & 102.03 & C\end{array}$ $\begin{array}{lllllllllllll}6224 & 1.058 & 0.972 & 0.02135 & 987010 & 38.908 & 1.352 & 36.198 & 1.634 & 37.473 & 102.03 & \mathrm{C}\end{array}$ $\begin{array}{lllllllllllllll}7323 . & 1.289 & 0.946 & 0.02135 & 985684 & 38.858 & 1.964 & 35.425 & 1.939 & 37.657 & 102.03 & \mathrm{C}\end{array}$ $\begin{array}{llllllllllllllll}9466 . & 1.897 & 0.884 & 0.02135 & 982985 & 38.757 & 2.577 & 34.343 & 2.550 & 36.127 & 102.03 & C\end{array}$ $\begin{array}{lllllllllllll}11482 . & 2.678 & 0.807 & 0.02135 & 980348 & 38.658 & 3.174 & 33.212 & 3.148 & 36.680 & 102.03 & C\end{array}$ $\begin{array}{lllllllllllll}\text { 12726. } & 2.850 & 0.752 & 0.02135 & 967480 & 38.170 & 3.504 & 32.525 & 3.530 & 35.732 & 102.03 & C\end{array}$ $\begin{array}{lllllllllllllll}\text { 14462. } & 3.846 & 0.661 & 0.02135 & 965315 & 38.088 & 4.103 & 30.850 & 4.078 & 34.748 & 102.03 & C\end{array}$ $\begin{array}{llllllllllllll}16313 & 5.135 & 0.546 & 0.02135 & 962909 & 37.996 & 4.714 & 28.941 & 4.688 & 33.312 & 102.03 & C\end{array}$ $\begin{array}{lllllllllllllll}17220 & 5.861 & 0.483 & 0.02135 & 961689 & 37.949 & 5.325 & 26.566 & 4.997 & 31.541 & 102.03 & \mathrm{C}\end{array}$ $\begin{array}{llllllllllllll}\text { 18092. } & 6.650 & 0.418 & 0.02135 & 961152 . & 37.929 & 5.938 & 24.003 & 5.301 & 31.358 & 102.03 & \mathrm{C}\end{array}$ $\begin{array}{llllllllllll}\text { 19787. } & 8.386 & 0.276 & 0.02135 & 960176 & 37.891 & 6.532 & 21.741 & 5.913 & 29.130 & 102.03 & \mathrm{C}\end{array}$ $\begin{array}{llllllllllll}2911 . & 3.575 & 1.019 & 0.02122 & 945809 & 40.287 & 0.125 & 35.968 & 0.712 & 36.739 & 102.03 & \mathrm{C}\end{array}$ $\begin{array}{llllllllllll}5324 & 0.858 & 0.989 & 0.02122 & 943420 & 37.246 & 0.738 & 35.299 & 1.325 & 36.160 & 102.03 & \mathrm{C}\end{array}$ $\begin{array}{lllllllllllll}6503 & 1.050 & 0.966 & 0.02122 & 942215 & 37.199 & 1.352 & 34.672 & 1.634 & 35.772 & 102.03 & C\end{array}$ $\begin{array}{llllllllllll}7640 . & 1.279 & 0.940 & 0.02122 & 940820 & 37.145 & 1.964 & 33.924 & 1.939 & 35.963 & 102.03 & \mathrm{C}\end{array}$ $\begin{array}{lllllllllllll}9851 . & 1.883 & 0.875 & 0.02122 & 937983 & 37.034 & 2.577 & 32.879 & 2.550 & 34.451 & 102.03 & C\end{array}$ $\begin{array}{llllllllllll}11916 . & 2.662 & 0.796 & 0.02122 & 935210 & 36.926 & 3.174 & 31.761 & 3.148 & 34.998 & 102.03 & \mathrm{C}\end{array}$ $\begin{array}{llllllllllll}13184 & 2.799 & 0.739 & 0.02122 & 921750 & 36.398 & 3.504 & 30.837 & 3.530 & 33.956 & 102.03 & \mathrm{C}\end{array}$ $\begin{array}{lllllllllllll}14942 & 3.796 & 0.645 & 0.02122 & 919486 & 36.308 & 4.103 & 29.179 & 4.078 & 33.000 & 102.03 & C\end{array}$ $\begin{array}{llllllllllll}\text { 16803. } & 5.088 & 0.528 & 0.02122 & 916971 & 36.209 & 4.714 & 27.242 & 4.688 & 31.539 & 102.03 & \mathrm{C}\end{array}$ $\begin{array}{lllllllllllll}17710 & 5.817 & 0.464 & 0.02122 & 915696 & 36.158 & 5.325 & 24.915 & 4.997 & 29.785 & 102.03 & \mathrm{C}\end{array}$

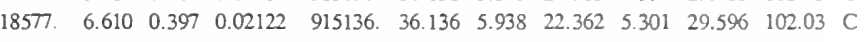
$\begin{array}{lllllllllllll}20249 & 8.358 & 0.252 & 0.02122 & 914116 & 36.095 & 6.532 & 20.105 & 5.913 & 27.407 & 102.03 & C\end{array}$ $\begin{array}{llllllllllll}2913 & 6.692 & 1.047 & 0.02856 & 1290112 & 55.245 & 0.125 & 47.832 & 0.712 & 48.611 & 102.03 & C\end{array}$ $\begin{array}{llllllllllll}5493 . & 3.708 & 1.022 & 0.02856 & 1287336 & 51.884 & 0.738 & 47.011 & 1.325 & 47.890 & 102.03 & C\end{array}$ $\begin{array}{lllllllllllll}6817 . & 1.550 & 1.003 & 0.02856 & 1285938 & 49.450 & 1.352 & 46.312 & 1.634 & 47.464 & 102.03 & C\end{array}$ $\begin{array}{llllllllllll}8136 . & 1.403 & 0.981 & 0.02856 & 1284098 & 48.976 & 1.964 & 45.469 & 1.939 & 47.611 & 102.03 & \mathrm{C}\end{array}$ $\begin{array}{llllllllllllll}10829 . & 2.114 & 0.924 & 0.02856 & 1280321 & 48.859 & 2.577 & 44.285 & 2.550 & 45.955 & 102.03 & \mathrm{C}\end{array}$ $\begin{array}{llllllllllllll}13518 & 3.026 & 0.853 & 0.02856 & 1276631 & 48.745 & 3.174 & 43.017 & 3.148 & 46.567 & 102.03 & \mathrm{C}\end{array}$ $\begin{array}{llllllllllll}15268 . & 3.298 & 0.800 & 0.02856 & 1260599 & 48.247 & 3.504 & 42.019 & 3.530 & 45.371 & 102.03 & C\end{array}$ $\begin{array}{lllllllllllll}17820 & 4.467 & 0.712 & 0.02856 & 1257739 & 48.158 & 4.103 & 39.983 & 4.078 & 44.287 & 102.03 & C\end{array}$ $\begin{array}{llllllllllllll}20712 & 5.978 & 0.597 & 0.02856 & 1254561 & 48.058 & 4.714 & 37.567 & 4.688 & 42.547 & 102.03 & C\end{array}$ $\begin{array}{llllllllllllll}22201 . & 6.830 & 0.533 & 0.02856 & 1252950 & 48.008 & 5.325 & 34.523 & 4.997 & 40.590 & 102.03 & C\end{array}$ $\begin{array}{llllllllllll}23681 . & 7.747 & 0.465 & 0.02856 & 1252113 & 47.982 & 5.938 & 31.086 & 5.301 & 40.386 & 102.03 & C\end{array}$ $\begin{array}{lllllllllllll}26704 & 9.767 & 0.314 & 0.02856 & 1250546 & 47.932 & 6.532 & 27.917 & 5.913 & 37.685 & 102.03 & \mathrm{C}\end{array}$ $\begin{array}{llllllllllll}2516 . & 1.956 & 1.009 & 0.01642 & 991594 & 40.443 & 0.125 & 37.903 & 0.712 & 38.531 & 102.03 & C\end{array}$ $\begin{array}{lllllllllllll}4535 & 0.803 & 0.975 & 0.01642 & 990157 & 39.026 & 0.738 & 37.367 & 1.325 & 38.089 & 102.03 & \mathrm{C}\end{array}$ $\begin{array}{lllllllllllll}5497 . & 0.970 & 0.950 & 0.01642 & 989432 & 38.999 & 1.352 & 36.879 & 1.634 & 37.773 & 102.03 & \mathrm{C}\end{array}$ $\begin{array}{llllllllllll}6409 . & 1.166 & 0.921 & 0.01642 & 988516 & 38.965 & 1.964 & 36.288 & 1.939 & 37.931 & 102.03 & \mathrm{C}\end{array}$ $\begin{array}{lllllllllllll}8131 . & 1.676 & 0.850 & 0.01642 & 986641 . & 38.894 & 2.577 & 35.472 & 2.550 & 36.686 & 102.03 & \mathrm{C}\end{array}$ $\begin{array}{llllllllllllll}9674 & 2.328 & 0.765 & 0.01642 & 984809 & 38.826 & 3.174 & 34.610 & 3.148 & 36.978 & 102.03 & C\end{array}$ $\begin{array}{llllllllllllll}\text { 10586. } & 2.611 & 0.704 & 0.01642 & 977987 & 38.569 & 3.504 & 33.900 & 3.530 & 36.224 & 102.03 & C\end{array}$ $\begin{array}{lllllllllllll}11798 . & 3.445 & 0.606 & 0.01642 & 976676 & 38.519 & 4.103 & 32.650 & 4.078 & 35.352 & 102.03 & C\end{array}$ $\begin{array}{llllllllllll}13008 . & 4.521 & 0.486 & 0.01642 & 975221 & 38.464 & 4.714 & 31.209 & 4.688 & 34.215 & 102.03 & \mathrm{C}\end{array}$ $\begin{array}{llllllllllll}\text { 13566. } & 5.126 & 0.420 & 0.01642 & 974482 & 38.436 & 5.325 & 29.528 & 4.997 & 32.838 & 102.03 & \mathrm{C}\end{array}$ $\begin{array}{lllllllllllll}14079 & 5.776 & 0.354 & 0.01642 & 974147 & 38.423 & 5.938 & 27.713 & 5.301 & 32.684 & 102.03 & \mathrm{C}\end{array}$ $\begin{array}{llllllllllll}15002 & 7.205 & 0.212 & 0.01642 & 973532 & 38.400 & 6.532 & 26.202 & 5.913 & 31.079 & 102.03 & \mathrm{C}\end{array}$ $\begin{array}{lllllllllllll}2407 . & 1.306 & 1.006 & 0.01765 & 1374852 & 52.422 & 0.125 & 50.433 & 0.712 & 51.161 & 102.03 & C\end{array}$ $\begin{array}{llllllllllll}4345 & 0.819 & 0.972 & 0.01765 & 1373735 & 51.666 & 0.738 & 49.804 & 1.325 & 50.720 & 102.03 & C\end{array}$ $\begin{array}{llllllllllll}5271 . & 1.000 & 0.948 & 0.01765 & 1373172 & 51.649 & 1.352 & 49.324 & 1.634 & 50.398 & 102.03 & \mathrm{C}\end{array}$ $\begin{array}{lllllllllllll}6150 . & 1.213 & 0.920 & 0.01765 & 1372440 & 51.628 & 1.964 & 48.688 & 1.939 & 50.544 & 102.03 & \mathrm{C}\end{array}$ $\begin{array}{llllllllllll}\text { 7815. } & 1.761 & 0.850 & 0.01765 & 1370937 & 51.584 & 2.577 & 47.850 & 2.550 & 49.331 & 102.03 & C\end{array}$ $\begin{array}{llllllllllll}9314 & 2.452 & 0.767 & 0.01765 & 1369470 & 51.541 & 3.174 & 47.035 & 3.148 & 49.445 & 102.03 & C\end{array}$ $\begin{array}{lllllllllllll}\text { 10205. } & 2.851 & 0.706 & 0.01765 & 1364336 & 51.390 & 3.504 & 46.522 & 3.530 & 48.764 & 102.03 & C\end{array}$ $\begin{array}{lllllllllllll}11393 . & 3.723 & 0.610 & 0.01765 & 1363385 & 51.362 & 4.103 & 45.192 & 4.078 & 47.988 & 102.03 & C\end{array}$ $\begin{array}{llllllllllllll}12588 . & 4.844 & 0.491 & 0.01765 & 1362330 & 51.331 & 4.714 & 43.786 & 4.688 & 46.791 & 102.03 & C\end{array}$ $\begin{array}{lllllllllllll}13143 . & 5.473 & 0.427 & 0.01765 & 1361795 & 51.315 & 5.325 & 41.962 & 4.997 & 45.435 & 102.03 & C\end{array}$ $\begin{array}{lllllllllllll}13656 & 6.140 & 0.361 & 0.01765 & 1361523 & 51.307 & 5.938 & 40.125 & 5.301 & 45.227 & 102.03 & \mathrm{C}\end{array}$ $\begin{array}{lllllllllllll}14589 . & 7.605 & 0.220 & 0.01765 & 1361016 & 51.292 & 6.532 & 38.760 & 5.913 & 43.494 & 102.03 & C\end{array}$ $\begin{array}{llllllllllll}\text { 3246. } & 1.276 & 1.004 & 0.01414 & 999938 & 40.002 & 0.125 & 38.095 & 0.712 & 38.777 & 102.03 & \mathrm{C}\end{array}$ $\begin{array}{lllllllllllll}5642 & 0.926 & 0.953 & 0.01414 & 998923 & 39.353 & 0.738 & 37.521 & 1.325 & 38.318 & 102.03 & \mathrm{C}\end{array}$ $\begin{array}{llllllllllll}6703 . & 1.126 & 0.918 & 0.01414 & 998411 & 39.334 & 1.352 & 36.994 & 1.634 & 37.970 & 102.03 & \mathrm{C}\end{array}$ $\begin{array}{llllllllllll}\text { 7653. } & 1.361 & 0.877 & 0.01414 & 997729 & 39.309 & 1.964 & 36.341 & 1.939 & 38.141 & 102.03 & \mathrm{C}\end{array}$ $\begin{array}{llllllllllllll}9274 & 1.966 & 0.781 & 0.01414 & 996326 & 39.256 & 2.577 & 35.455 & 2.550 & 36.758 & 102.03 & \mathrm{C}\end{array}$ $\begin{array}{llllllllllll}10488 . & 2.730 & 0.671 & 0.01414 & 994955 & 39.205 & 3.174 & 34.419 & 3.148 & 36.793 & 102.03 & C\end{array}$ $\begin{array}{llllllllllllll}11078 . & 3.180 & 0.593 & 0.01414 & 990618 & 39.043 & 3.504 & 33.691 & 3.530 & 36.040 & 102.03 & C\end{array}$ $\begin{array}{lllllllllllllll}11654 . & 4.152 & 0.477 & 0.01414 & 989901 & 39.016 & 4.103 & 32.390 & 4.078 & 35.241 & 102.03 & C\end{array}$ $\begin{array}{llllllllllll}11932 . & 5.402 & 0.344 & 0.01414 & 989104 & 38.987 & 4.714 & 30.923 & 4.688 & 33.888 & 102.03 & C\end{array}$ $\begin{array}{llllllllllllll}11927 . & 6.103 & 0.275 & 0.01414 & 988700 & 38.972 & 5.325 & 29.311 & 4.997 & 32.441 & 102.03 & C\end{array}$ $\begin{array}{lllllllllllllll}11827 . & 6.848 & 0.208 & 0.01414 & 988549 & 38.966 & 5.938 & 27.818 & 5.301 & 32.147 & 102.03 & C\end{array}$ $\begin{array}{lllllllllllll}11338 . & 8.481 & 0.076 & 0.01414 & 988284 & 38.956 & 6.532 & 27.246 & 5.913 & 30.335 & 102.03 & C\end{array}$

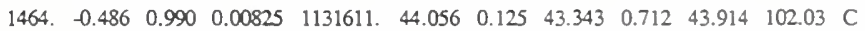
$\begin{array}{llllllllllllll}\text { 2619. } & -0.162 & 0.948 & 0.00825 & 1131345 & 44.047 & 0.738 & 42.924 & 1.325 & 43.637 & 102.03 & C\end{array}$ $\begin{array}{llllllllllll}3164 & 0.077 & 0.919 & 0.00825 & 1131210 & 44.043 & 1.352 & 42.596 & 1.634 & 43.454 & 102.03 & C\end{array}$ $\begin{array}{lllllllllllll}3676 . & 0.360 & 0.885 & 0.00825 & 1130992 & 44.035 & 1.964 & 42.131 & 1.939 & 43.535 & 102.03 & C\end{array}$ $\begin{array}{lllllllllllll}4631 & 1.07 & 0.801 & 0.00825 & 1130539 & 44.020 & 2.577 & 41.358 & 2.550 & 42.663 & 102.03 & C\end{array}$ $\begin{array}{llllllllllllll}5468 & 1.970 & 0.702 & 0.00825 & 1130096 & 44.005 & 3.174 & 40.368 & 3.148 & 42.752 & 102.03 & C\end{array}$ $\begin{array}{lllllllllllllll}5956 & 2.625 & 0.629 & 0.00825 & 1129339 & 43.979 & 3.504 & 39.677 & 3.530 & 42.109 & 102.03 & C\end{array}$ $\begin{array}{llllllllllllll}\text { 6586. } & 3.731 & 0.516 & 0.00825 & 1129115 & 43.972 & 4.103 & 38.032 & 4.078 & 41.387 & 102.03 & \mathrm{C}\end{array}$ $\begin{array}{lllllllllllll}7193 & 5.147 & 0.378 & 0.00825 & 1128868 & 43.963 & 4.714 & 36.081 & 4.688 & 39.669 & 102.03 & C\end{array}$ $\begin{array}{lllllllllllll}7464 . & 5.941 & 0.304 & 0.00825 & 1128742 & 43.959 & 5.325 & 33.619 & 4.997 & 38.006 & 102.03 & C\end{array}$ $\begin{array}{llllllllllllll}7705 & 6.774 & 0.228 & 0.00825 & 1128672 & 43.957 & 5.938 & 31.847 & 5.301 & 37.556 & 102.03 & C\end{array}$ $\begin{array}{lllllllllllll}8117 . & 8.599 & 0.068 & 0.00825 & 1128541 & 43.952 & 6.532 & 31.610 & 5.913 & 33.652 & 102.03 & C\end{array}$ 
$\begin{array}{llllllllllll}113 & 4.950 & 1.036 & 0.00734 & 1027047 & 45.698 & 0.125 & 40.152 & 0.712 & 40.402 & 102.03 & \mathrm{C}\end{array}$ $\begin{array}{lllllllllllll}\text { 433. } 4.277 & 1.031 & 0.00734 & 1026800 & 44.855 & 0.738 & 39.844 & 1.325 & 40.233 & 102.03 & C\end{array}$ $\begin{array}{llllllllllllll}673 . & 3.497 & 1.024 & 0.00734 & 1026675 & 43.949 & 1.352 & 39.714 & 1.634 & 40.166 & 102.03 & \mathrm{C}\end{array}$ $\begin{array}{llllllllllllll}960 & 2.330 & 1.016 & 0.00734 & 1026511 & 42.634 & 1.964 & 39.522 & 1.939 & 40.157 & 102.03 & \mathrm{C}\end{array}$ $\begin{array}{lllllllllllll}1690 & 0.426 & 0.987 & 0.00734 & 1026172 & 40.356 & 2.577 & 39.183 & 2.550 & 39.809 & 102.03 & C\end{array}$

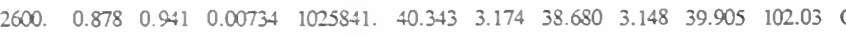

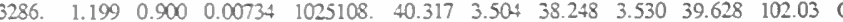
$\begin{array}{llllllllllll}4409 & 1.758 & 0.824 & 0.00734 & 1024811 . & 40.306 & 4.103 & 37.235 & 4.078 & 39.201 & 102.03\end{array}$

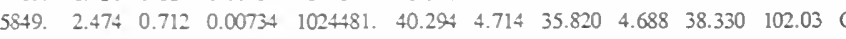

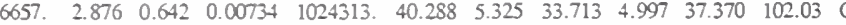
$\begin{array}{llllllllllll}7503 & 3.299 & 0.564 & 0.00734 & 1024186 & 40.283 & 5.938 & 31.230 & 5.301 & 36.970 & 102.03 & C\end{array}$ $\begin{array}{llllllllllll}9360 . & 4.226 & 0.379 & 0.00734 & 1023937 & 40.274 & 6.532 & 28.621 & 5.913 & 35.233 & 102.03 & C\end{array}$ $\begin{array}{llllllllllllll}140 . & 5.092 & 1.037 & 0.00659 & 1015681 & 45.425 & 0.125 & 39.749 & 0.712 & 39.995 & 102.03 & \mathrm{C}\end{array}$ $\begin{array}{lllllllllllll}471 . & 4.247 & 1.030 & 0.00689 & 1015506 & 44.410 & 0.738 & 39.448 & 1.325 & 39.829 & 102.03 & \mathrm{C}\end{array}$ $\begin{array}{lllllllllllll}712 . & 3.339 & 1.023 & 0.00689 & 1015419 & 43.376 & 1.352 & 39.313 & 1.634 & 39.753 & 102.03 & \mathrm{C}\end{array}$

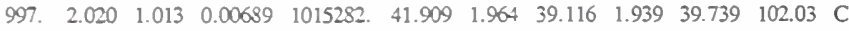
$\begin{array}{lllllllllllll}1714 & 0.433 & 0.982 & 0.00689 & 1015000 & 39.947 & 2.577 & 38.771 & 2.550 & 39.396 & 102.03 & C\end{array}$ $\begin{array}{llllllllllll}2602 & 0.888 & 0.933 & 0.00659 & 1014724 & 39.937 & 3.174 & 38.257 & 3.148 & 39.483 & 102.03 & C\end{array}$

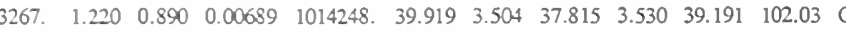
$\begin{array}{lllllllllllll}1.781 & 0.810 & 0.00689 & 1014009 & 39.910 & 4.103 & 36.807 & 4.078 & 38.776 & 102.03 & C\end{array}$ $\begin{array}{lllllllllllll}2.502 & 0.693 & 0.00689 & 1013742 & 39.901 & 4.714 & 35.398 & 4.688 & 37.901 & 102.03 & C\end{array}$

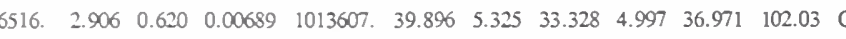
$\begin{array}{lllllllllllll}7328 & 3.330 & 0.539 & 0.00689 & 1013520 & 39.893 & 5.938 & 30.891 & 5.301 & 36.541 & 102.03 & C\end{array}$

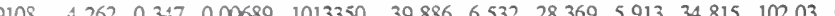
$\begin{array}{llllllllllll}5.375 & 1.031 & 0.02666 & 1042554 & 45.414 & 0.125 & 39.248 & 0.712 & 40.111 & 102.03 & C\end{array}$ $\begin{array}{llllllllllll}7477 . & 1.202 & 0.995 & 0.02666 & 1039257 & 40.830 & 0.738 & 38.431 & 1.325 & 39.401 & 102.03\end{array}$ $\begin{array}{llllllllllllll}9081 & 1.443 & 0.970 & 0.02666 & 1037595 & 40.770 & 1.352 & 37.660 & 1.634 & 38.892 & 102.03 & C\end{array}$ 10610. $\begin{array}{llllllllllll}1.726 & 0.940 & 0.02666 & 1035530 & 40.695 & 1.964 & 36.740 & 1.939 & 39.142 & 102.03 & C\end{array}$ $\begin{array}{llllllllllll}13520 . & 2.477 & 0.867 & 0.02666 & 1031310 & 40.542 & 2.577 & 35.452 & 2.550 & 37.258 & 102.03 & C\end{array}$ $\begin{array}{lllllllllllll}16159 & 3.449 & 0.780 & 0.02666 & 1027185 & 40.392 & 3.174 & 33.841 & 3.148 & 37.667 & 102.03 & C\end{array}$ $\begin{array}{llllllllllll}\text { 17747. } & 3.650 & 0.717 & 0.02666 & 1009719 & 39.753 & 3.504 & 32.597 & 3.530 & 36.457 & 102.03 & \mathrm{C}\end{array}$ $\begin{array}{lllllllllllll}19872 . & 4.918 & 0.616 & 0.02666 & 1006810 & 39.645 & 4.103 & 30.529 & 4.078 & 35.262 & 102.03 & C\end{array}$ $\begin{array}{lllllllllllll}-6.558 & 0.490 & 0.02666 & 1003578 & 39.526 & 4.714 & 28.129 & 4.688 & 33.413 & 102.03 & C\end{array}$ $\begin{array}{lllllllllllll}23047 & 7.483 & 0.422 & 0.02666 & 1001940 & 39.465 & 5.325 & 25.217 & 4.997 & 31.396 & 102.03 & C\end{array}$ $\begin{array}{lllllllllllll}23992 & 8.487 & 0.351 & 0.02666 & 1001205 & 39.438 & 5.938 & 22.168 & 5.301 & 31.083 & 102.03 & C\end{array}$ $\begin{array}{llllllllllll}25736 & 10.697 & 0.201 & 0.02666 & 999865 & 39.388 & 6.532 & 19.533 & 5.913 & 28.313 & 102.03 & C\end{array}$ $\begin{array}{llllllllllll}4015 & 4.608 & 1.005 & 0.02791 & 991962 & 42.758 & 0.125 & 37.362 & 0.712 & 38.259 & 102.03 & \mathrm{C}\end{array}$ $\begin{array}{lllllllllllll}7297 . & 1.198 & 0.992 & 0.02791 & 988179 & 38.952 & 0.738 & 36.548 & 1.325 & 37.569 & 102.03 & C\end{array}$ $\begin{array}{lllllllllllll}8884 & 1.417 & 0.969 & 0.02791 & 986271 & 38.880 & 1.352 & 35.778 & 1.634 & 37.056 & 102.03 & C\end{array}$ $\begin{array}{llllllllllllll}10404 & 1.674 & 0.942 & 0.02791 & 983895 & 38.791 & 1.964 & 34.863 & 1.939 & 37.323 & 102.03 & \mathrm{C}\end{array}$ $\begin{array}{lllllllllllll}13324 & 2.364 & 0.874 & 0.02791 & 979035 & 38.608 & 2.577 & 33.588 & 2.550 & 35.421 & 102.03 & C\end{array}$ 16007. $\begin{array}{llllllllllll}3.266 & 0.792 & 0.02791 & 974287 & 38.429 & 3.174 & 31.986 & 3.148 & 35.884 & 102.03 & \mathrm{C}\end{array}$ $\begin{array}{llllllllllllll}17641 . & 3.296 & 0.735 & 0.02791 & 953783 & 37.646 & 3.504 & 30.758 & 3.530 & 34.619 & 102.03 & C\end{array}$ $\begin{array}{lllllllllllll}19857 & 4.489 & 0.639 & 0.02791 & 950308 & 37.512 & 4.103 & 28.728 & 4.078 & 33.517 & 102.03 & C\end{array}$ $\begin{array}{lllllllllllll}22152 & 6.038 & 0.521 & 0.02791 & 946448 & 37.363 & 4.714 & 26.332 & 4.688 & 31.710 & 102.03 & C\end{array}$ $\begin{array}{llllllllllllll}23249 & 6.914 & 0.456 & 0.02791 & 944490 & 37.287 & 5.325 & 23.441 & 4.997 & 29.708 & 102.03 & C\end{array}$ $\begin{array}{lllllllllllll}24284 & 7.874 & 0.389 & 0.02791 & 943582 & 37.252 & 5.938 & 20.319 & 5.301 & 29.535 & 102.03 & C\end{array}$ $\begin{array}{lllllllllllllll}26235 & 9.991 & 0.245 & 0.02791 & 941914 & 37.187 & 6.532 & 17.536 & 5.913 & 26.938 & 102.03 & C\end{array}$ $\begin{array}{lllllllllllllll}4322 & 5.046 & 1.031 & 0.02797 & 1208013 & 50.780 & 0.125 & 44.807 & 0.712 & 45.733 & 102.03 & C\end{array}$ $\begin{array}{lllllllllllll}7819 . & 1.191 & 0.995 & 0.02797 & 1204966 & 46.481 & 0.738 & 43.923 & 1.325 & 45.021 & 102.03 & C\end{array}$

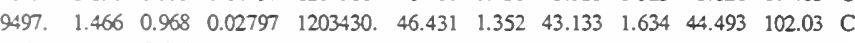
$\begin{array}{lllllllllllllll}11094 & 1.791 & 0.938 & 0.02797 & 1201525 & 46.370 & 1.964 & 42.167 & 1.939 & 44.757 & 102.03 & C\end{array}$ $\begin{array}{lllllllllllll}14136 . & 2.641 & 0.862 & 0.02797 & 1197632 & 46.244 & 2.577 & 40.822 & 2.550 & 42.788 & 102.03 & C\end{array}$ $\begin{array}{llllllllllllll}16894 . & 3.728 & 0.771 & 0.02797 & 1193828 & 46.120 & 3.174 & 39.169 & 3.148 & 43.147 & 102.03 & C\end{array}$ $\begin{array}{lllllllllllll}\text { 18553. } 4.125 & 0.706 & 0.02797 & 1178191.45 .609 & 3.504 & 37.932 & 3.530 & 41.908 & 102.03 & C\end{array}$ $\begin{array}{lllllllllllll}20772 & 5.527 & 0.600 & 0.02797 & 1175714 & 45.528 & 4.103 & 35.751 & 4.078 & 40.564 & 102.03 & C\end{array}$ $\begin{array}{llllllllllllll}23029 & 7.336 & 0.470 & 0.02797 & 1172962 & 45.437 & 4.714 & 33.206 & 4.688 & 38.749 & 102.03 & C\end{array}$ $\begin{array}{lllllllllllll}24089 & 8.354 & 0.399 & 0.02797 & 1171567 & 45.391 & 5.325 & 30.135 & 4.997 & 36.412 & 102.03 & C\end{array}$ $\begin{array}{lllllllllllllll}25076 . & 9.448 & 0.325 & 0.02797 & 1171010 & 45.373 & 5.938 & 26.958 & 5.301 & 36.054 & 102.03 & C\end{array}$

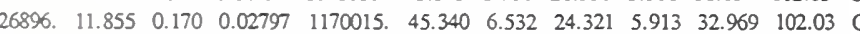
$\begin{array}{lllllllllllll}3487 & 4.279 & 1.028 & 0.02829 & 1313358 & 53.427 & 0.125 & 48.409 & 0.712 & 49.179 & 102.03 & C\end{array}$ $\begin{array}{llllllllllllll}6421 . & 1.020 & 0.998 & 0.02829 & 1310553 & 49.784 & 0.738 & 47.633 & 1.325 & 48.526 & 102.03 & C\end{array}$ $\begin{array}{lllllllllllll}7871 & 1.259 & 0.976 & 0.02829 & 1309140 & 49.741 & 1.352 & 46.954 & 1.634 & 48.067 & 102.03 & C\end{array}$ $\begin{array}{llllllllllllll}9280 . & 1.540 & 0.950 & 0.02829 & 1307383 & 49.688 & 1.964 & 46.121 & 1.939 & 48.269 & 102.03 & C\end{array}$ $\begin{array}{llllllllllll}12051 . & 2.276 & 0.885 & 0.02829 & 1303790 & 49.578 & 2.577 & 44.957 & 2.550 & 46.625 & 102.03\end{array}$ $\begin{array}{lllllllllllll}14684 & 3.217 & 0.805 & 0.02829 & 1300280 & 49.471 & 3.174 & 43.532 & 3.148 & 46.915 & 102.03 & C\end{array}$ $\begin{array}{lllllllllllllll}16336 & 3.543 & 0.747 & 0.02829 & 1285270 & 49.012 & 3.504 & 42.467 & 3.530 & 45.831 & 102.03 & C\end{array}$ $\begin{array}{lllllllllllll}18644 . & 4.750 & 0.652 & 0.02829 & 1282756 & 48.934 & 4.103 & 40.510 & 4.078 & 44.682 & 102.03 & C\end{array}$ $\begin{array}{lllllllllllll}21136 . & 6.309 & 0.532 & 0.02829 & 1279964 & 48.848 & 4.714 & 38.249 & 4.688 & 43.008 & 102.03 & C\end{array}$ $\begin{array}{lllllllllllll}22370 . & 7.186 & 0.466 & 0.02829 & 1278548 & 48.804 & 5.325 & 35.401 & 4.997 & 41.106 & 102.03 & C\end{array}$ $\begin{array}{lllllllllllll}23565 . & 8.129 & 0.396 & 0.02829 & 1277868 & 48.783 & 5.938 & 32.340 & 5.301 & 40.795 & 102.03 & C\end{array}$ $\begin{array}{lllllllllllll}25913 & 10.203 & 0.246 & 0.02829 & 1276610 & 48.744 & 6.532 & 29.594 & 5.913 & 38.102 & 102.03\end{array}$ $\begin{array}{llllllllllll}2999 . & 7.067 & 1.051 & 0.02389 & 1304709 & 56.088 & 0.125 & 48.279 & 0.712 & 49.102 & 102.03 & C\end{array}$ $\begin{array}{lllllllllllllll}5511 . & 3.373 & 1.020 & 0.02389 & 1302751 . & 52.052 & 0.738 & 47.512 & 1.325 & 48.465 & 102.03 & C\end{array}$ $\begin{array}{lllllllllllll}6747 & 1.087 & 0.997 & 0.02389 & 1301764 & 49.517 & 1.352 & 46.900 & 1.634 & 48.049 & 102.03 & C\end{array}$ $\begin{array}{llllllllllll}7946 . & 1.345 & 0.971 & 0.02389 & 1300436 . & 49.476 & 1.964 & 46.163 & 1.939 & 48.199 & 102.03 & C\end{array}$

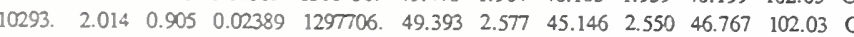
$\begin{array}{lllllllllllllll}12511 . & 2.865 & 0.825 & 0.02389 & 1295038 & 49.311 & 3.174 & 43.921 & 3.148 & 47.032 & 102.03 & C\end{array}$ $\begin{array}{lllllllllllll}13897 . & 3.233 & 0.766 & 0.02389 & 1284218 & 48.979 & 3.504 & 43.074 & 3.530 & 46.100 & 102.03 & C\end{array}$ $\begin{array}{lllllllllllll}15823 & 4.318 & 0.670 & 0.02389 & 1282296 & 48.920 & 4.103 & 41.355 & 4.078 & 45.027 & 102.03 & C\end{array}$ $\begin{array}{lllllllllllllll}17888 . & 5.716 & 0.549 & 0.02389 & 1280160 & 48.854 & 4.714 & 39.403 & 4.688 & 43.601 & 102.03 & C\end{array}$ $\begin{array}{lllllllllllll}18906 . & 6.503 & 0.483 & 0.02389 & 1279078 & 48.821 & 5.325 & 36.934 & 4.997 & 41.806 & 102.03 & C\end{array}$ 19887. $\begin{array}{llllllllllll}7.344 & 0.413 & 0.02389 & 1278544 & 48.804 & 5.938 & 34.316 & 5.301 & 41.552 & 102.03 & C\end{array}$ $\begin{array}{lllllllllllll}21804 & 9.195 & 0.263 & 0.02389 & 1277552 & 48.774 & 6.532 & 31.953 & 5.913 & 39.230 & 102.03 & C\end{array}$ $\begin{array}{lllllllllllll}3261 . & 6.740 & 1.044 & 0.02347 & 1139352 & 50.415 & 0.125 & 43.037 & 0.712 & 43.751 & 102.03 & C\end{array}$ $\begin{array}{llllllllllllll}5952 & 2.470 & 1.011 & 0.02347 & 1137089 & 45.794 & 0.738 & 42.319 & 1.325 & 43.113 & 102.03 & C\end{array}$

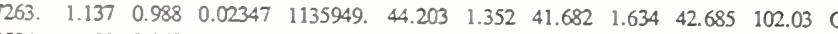
$\begin{array}{lllllllllllll}8524 & 1.393 & 0.960 & 0.02347 & 1134476 & 44.153 & 1.964 & 40.932 & 1.939 & 42.856 & 102.03 & C\end{array}$ $\begin{array}{llllllllllll}10965 . & 2.064 & 0.891 & 0.02347 & 1131457 & 44.051 & 2.577 & 39.885 & 2.550 & 41.333 & 102.03 & \mathrm{C}\end{array}$

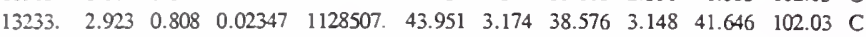
$\begin{array}{lllllllllllll}\text { 14629. } & 3.222 & 0.747 & 0.02347 & 1116187 & 43.531 & 3.504 & 37.564 & 3.530 & 40.651 & 102.03 & C\end{array}$ $\begin{array}{lllllllllllllll}16541 . & 4.321 & 0.649 & 0.02347 & 1113969 & 43.456 & 4.103 & 35.824 & 4.078 & 39.575 & 102.03 & \mathrm{C}\end{array}$ $\begin{array}{llllllllllllll}18550 . & 5.741 & 0.526 & 0.02347 & 1111504 & 43.371 & 4.714 & 33.794 & 4.688 & 38.084 & 102.03 & \mathrm{C}\end{array}$ $\begin{array}{lllllllllllll}19523 & 6.541 & 0.459 & 0.02347 & 1110255 & 43.328 & 5.325 & 31.345 & 4.997 & 36.237 & 102.03 & \mathrm{C}\end{array}$ $\begin{array}{llllllllllllll}20450 . & 7.402 & 0.389 & 0.02347 & 1109661 & 43.308 & 5.938 & 28.700 & 5.301 & 35.998 & 102.03 & C\end{array}$ $\begin{array}{lllllllllllll}22225 . & 9.296 & 0.239 & 0.02347 & 1108565 & 43.270 & 6.532 & 26.315 & 5.913 & 33.650 & 102.03 & C\end{array}$

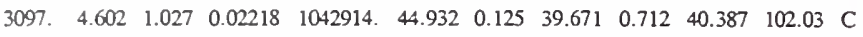
$\begin{array}{llllllllllllll}5645 . & 0.877 & 0.995 & 0.02218 & 1040631 & 40.879 & 0.738 & 39.015 & 1.325 & 39.817 & 102.03 & \mathrm{C}\end{array}$ $\begin{array}{llllllllllllll}6881 & 1.075 & 0.972 & 0.02218 & 1039480 & 40.838 & 1.352 & 38.413 & 1.634 & 39.415 & 102.03 & \mathrm{C}\end{array}$ $\begin{array}{llllllllllllll}8069 . & 1.308 & 0.945 & 0.02218 & 1038015 & 40.785 & 1.964 & 37.700 & 1.939 & 39.589 & 102.03 & C\end{array}$

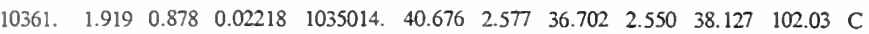
$\begin{array}{lllllllllllll}12480 & 2.706 & 0.797 & 0.02218 & 1032083 & 40.570 & 3.174 & 35.464 & 3.148 & 38.471 & 102.03 & \mathrm{C}\end{array}$ $\begin{array}{llllllllllllll}13778 . & 2.931 & 0.738 & 0.02218 & 1019893 & 40.126 & 3.504 & 34.510 & 3.530 & 37.508 & 102.03 & C\end{array}$ $\begin{array}{lllllllllllll}\text { 15549. } 3.944 & 0.642 & 0.02218 & 1017657 & 40.044 & 4.103 & 32.882 & 4.078 & 36.509 & 102.03 & C\end{array}$ $\begin{array}{lllllllllllllll}17400 . & 5.254 & 0.523 & 0.02218 & 1015172 & 39.953 & 4.714 & 30.995 & 4.688 & 35.087 & 102.03 & \mathrm{C}\end{array}$ $\begin{array}{lllllllllllll}18291 & 5.993 & 0.457 & 0.02218 & 1013912 & 39.907 & 5.325 & 28.699 & 4.997 & 33.375 & 102.03 & C\end{array}$ $\begin{array}{lllllllllllll}\text { 19136. } & 6.792 & 0.390 & 0.02218 & 1013309 & 39.885 & 5.938 & 26.240 & 5.301 & 33.166 & 102.03 & \mathrm{C}\end{array}$ $\begin{array}{lllllllllllllll}20745 . & 8.551 & 0.245 & 0.02218 & 1012195 & 39.844 & 6.532 & 24.003 & 5.913 & 31.037 & 102.03 & C\end{array}$ $\begin{array}{lllllllllllll}2144 . & 1.199 & 1.005 & 0.01543 & 1065609 & 42.532 & 0.125 & 40.853 & 0.712 & 41.357 & 102.03 & C\end{array}$

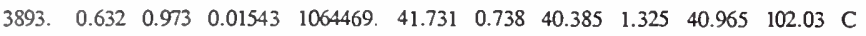
$\begin{array}{llllllllllllll}4736 . & 0.782 & 0.950 & 0.01543 & 1063894 & 41.711 & 1.352 & 39.976 & 1.634 & 40.694 & 102.03 & \mathrm{C}\end{array}$ $\begin{array}{llllllllllllll}5540 . & 0.960 & 0.923 & 0.01543 & 1063149 & 41.684 & 1.964 & 39.476 & 1.939 & 40.822 & 102.03 & \mathrm{C}\end{array}$ 7079. $\begin{array}{llllllllllll}1.419 & 0.856 & 0.01543 & 1061623 & 41.630 & 2.577 & 38.769 & 2.550 & 39.804 & 102.03 & \mathrm{C}\end{array}$ $\begin{array}{llllllllllllll}8482 & 2.003 & 0.776 & 0.01543 & 1060131 . & 41.577 & 3.174 & 37.915 & 3.148 & 40.036 & 102.03 & \mathrm{C}\end{array}$ $\begin{array}{lllllllllllll}9334 . & 2.286 & 0.717 & 0.01543 & 1054651 . & 41.382 & 3.504 & 37.306 & 3.530 & 39.300 & 102.03 & \mathrm{C}\end{array}$ $\begin{array}{llllllllllllll}10477 . & 3.029 & 0.623 & 0.01543 & 1053570 & 41.344 & 4.103 & 36.171 & 4.078 & 38.553 & 102.03 & C\end{array}$ $\begin{array}{lllllllllllllll}11651 . & 3.986 & 0.507 & 0.01543 & 1052369 & 41.301 & 4.714 & 34.879 & 4.688 & 37.547 & 102.03 & C\end{array}$ $\begin{array}{lllllllllllllll}12207 & 4.524 & 0.443 & 0.01543 & 1051759 & 41.279 & 5.325 & 33.321 & 4.997 & 36.351 & 102.03 & C\end{array}$ $\begin{array}{llllllllllll}12728 . & 5.099 & 0.378 & 0.01543 & 1051444 . & 41.268 & 5.938 & 31.646 & 5.301 & 36.210 & 102.03 & C\end{array}$

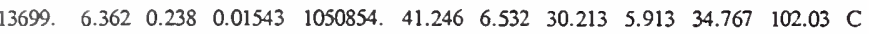
$\begin{array}{lllllllllllll}426 . & 0.584 & 1.006 & 0.00994 & 1090640 & 43.554 & 0.125 & 42.137 & 0.712 & 42.628 & 102.03 & \mathrm{C}\end{array}$ $\begin{array}{llllllllllll}\text { 1010. } & -0.127 & 0.995 & 0.00994 & 1090226 & 42.636 & 0.738 & 41.829 & 1.325 & 42.418 & 102.03 & C\end{array}$ $\begin{array}{lllllllllllll}\text { 1380. } & 0.017 & 0.985 & 0.00994 & 1090017 & 42.629 & 1.352 & 41.622 & 1.634 & 42.294 & 102.03 & C\end{array}$ $\begin{array}{lllllllllllllll}1795 & 0.188 & 0.972 & 0.00994 & 1089727 & 42.618 & 1.964 & 41.343 & 1.939 & 42.316 & 102.03 & \mathrm{C}\end{array}$ $\begin{array}{lllllllllllll}2775 . & 0.622 & 0.934 & 0.00994 & 1089128 & 42.598 & 2.577 & 40.852 & 2.550 & 41.813 & 102.03 & C\end{array}$ $\begin{array}{lllllllllllll}3925 & 1.166 & 0.881 & 0.00994 & 1088543 & 42.577 & 3.174 & 40.174 & 3.148 & 41.949 & 102.03 & C\end{array}$ $\begin{array}{lllllllllllll}\text { 4761. } & 1.525 & 0.836 & 0.00994 & 1086681 & 42.512 & 3.504 & 39.688 & 3.530 & 41.529 & 102.03 & C\end{array}$ $\begin{array}{lllllllllllll}6094 & 2.200 & 0.755 & 0.00994 & 1086174 & 42.495 & 4.103 & 38.453 & 4.078 & 40.919 & 102.03 & C\end{array}$ $\begin{array}{llllllllllllll}\text { 7763. } & 3.067 & 0.641 & 0.00994 & 1085611 . & 42.475 & 4.714 & 36.897 & 4.688 & 39.891 & 102.03 & \mathrm{C}\end{array}$ $\begin{array}{llllllllllllll}8685 & 3.553 & 0.573 & 0.00994 & 1085326 & 42.465 & 5.325 & 34.609 & 4.997 & 38.739 & 102.03 & C\end{array}$ $\begin{array}{llllllllllllll}9641 . & 4.067 & 0.498 & 0.00994 & 1085158 . & 42.459 & 5.938 & 32.036 & 5.301 & 38.394 & 102.03 & C\end{array}$ $\begin{array}{lllllllllllll}11714 . & 5.194 & 0.321 & 0.00994 & 1084839 & 42.448 & 6.532 & 29.592 & 5.913 & 36.518 & 102.03 & C\end{array}$ $\begin{array}{llllllllllllll}1128 & -0.061 & 1.000 & 0.00912 & 1266153 & 48.452 & 0.125 & 47.715 & 0.712 & 48.259 & 102.03 & \mathrm{C}\end{array}$ $\begin{array}{llllllllllllll}2103 . & 0.136 & 0.970 & 0.00912 & 1265854 & 48.411 & 0.738 & 47.310 & 1.325 & 47.985 & 102.03 & \mathrm{C}\end{array}$ $\begin{array}{llllllllllllll}2595 . & 0.305 & 0.948 & 0.00912 & 1265704 & 48.406 & 1.352 & 47.048 & 1.634 & 47.815 & 102.03 & C\end{array}$ $\begin{array}{lllllllllllll}3079 . & 0.505 & 0.921 & 0.00912 & 1265469 & 48.399 & 1.964 & 46.674 & 1.939 & 47.884 & 102.03 & C\end{array}$ $\begin{array}{lllllllllllllll}\text { 4051. } & 1.013 & 0.854 & 0.00912 & 1264983 & 48.384 & 2.577 & 46.085 & 2.550 & 47.127 & 102.03 & C\end{array}$ $\begin{array}{lllllllllllll}5000 . & 1.646 & 0.771 & 0.00912 & 1264507 & 48.369 & 3.174 & 45.375 & 3.148 & 47.219 & 102.03 & C\end{array}$ $\begin{array}{lllllllllllll}5609 . & 2.103 & 0.708 & 0.00912 & 1263554 & 48.339 & 3.504 & 44.888 & 3.530 & 46.625 & 102.03 & C\end{array}$ $\begin{array}{lllllllllllll}6480 . & 2.890 & 0.606 & 0.00912 & 1263298 . & 48.331 & 4.103 & 43.701 & 4.078 & 46.074 & 102.03 & C\end{array}$ $\begin{array}{llllllllllllll}7448 . & 3.899 & 0.476 & 0.00912 & 1263012 & 48.322 & 4.714 & 42.295 & 4.688 & 44.823 & 102.03 & C\end{array}$ $\begin{array}{llllllllllllll}\text { 7938. } & 4.464 & 0.403 & 0.00912 & 1262868 . & 48.318 & 5.325 & 40.539 & 4.997 & 43.582 & 102.03 & C\end{array}$ $\begin{array}{llllllllllllll}8421 . & 5.058 & 0.326 & 0.00912 & 1262787 & 48.315 & 5.938 & 38.697 & 5.301 & 43.207 & 102.03 & C\end{array}$ $\begin{array}{llllllllllllll}9392 & 6.361 & 0.159 & 0.00912 & 1262635 & 48.311 & 6.532 & 37.761 & 5.913 & 41.439 & 102.03 & C\end{array}$ $\begin{array}{llllllllllll}34034 & 8.189 & 0.760 & 0.02482 & 1073678 & 42.057 & 0.125 & 17.475 & 0.712 & 33.927 & 102.03 & \mathrm{P}\end{array}$ $\begin{array}{llllllllllllll}26428 . & 6.953 & 0.562 & 0.02482 & 1071102 & 41.966 & 0.738 & 23.484 & 1.325 & 34.620 & 102.03 & \mathrm{P}\end{array}$

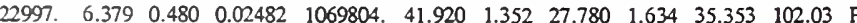
$\begin{array}{llllllllllllll}19879 . & 5.861 & 0.409 & 0.02482 & 1069027 & 41.893 & 1.964 & 31.063 & 1.939 & 37.194 & 102.03 & \mathrm{P}\end{array}$ $\begin{array}{llllllllllll}14409 . & 4.920 & 0.297 & 0.02482 & 1067556 & 41.841 & 2.577 & 33.457 & 2.550 & 36.241 & 102.03 & \mathrm{P}\end{array}$ $\begin{array}{llllllllllll}10083 . & 4.121 & 0.219 & 0.02482 & 1066118 & 41.790 & 3.174 & 34.923 & 3.148 & 37.499 & 102.03 & \mathrm{P}\end{array}$

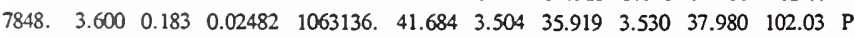
$\begin{array}{llllllllllllll}5357 . & 3.071 & 0.145 & 0.02482 & 1062598 . & 41.665 & 4.103 & 36.816 & 4.078 & 39.013 & 102.03 & \mathrm{P}\end{array}$ $\begin{array}{lllllllllllllll}3584 . & 2.602 & 0.116 & 0.02482 & 1061999 & 41.644 & 4.714 & 37.607 & 4.688 & 39.021 & 102.03 & P\end{array}$ $\begin{array}{lllllllllllll}3086 . & 2.412 & 0.105 & 0.02482 & 1061696 & 41.633 & 5.325 & 38.101 & 4.997 & 39.179 & 102.03 & P\end{array}$ $\begin{array}{llllllllllllll}2859 . & 2.259 & 0.095 & 0.02482 & 1061479 & 41.625 & 5.938 & 38.490 & 5.301 & 39.332 & 102.03 & P\end{array}$ $\begin{array}{lllllllllllllll}3192 . & 2.047 & 0.076 & 0.02482 & 1061056 . & 41.610 & 6.532 & 38.811 & 5.913 & 39.554 & 102.03 & \mathrm{P}\end{array}$ $\begin{array}{lllllllllllll}34745 . & 8.338 & 0.762 & 0.02507 & 1079976 & 42.278 & 0.125 & 17.134 & 0.712 & 34.029 & 102.03 & P\end{array}$

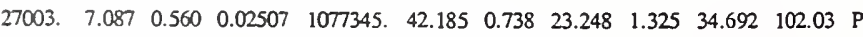
$\begin{array}{lllllllllllll}23507 . & 6.505 & 0.477 & 0.02507 & 1076019 & 42.139 & 1.352 & 27.648 & 1.634 & 35.408 & 102.03 & P\end{array}$ $\begin{array}{llllllllllllll}20329 . & 5.982 & 0.406 & 0.02507 & 1075239 & 42.111 & 1.964 & 30.985 & 1.939 & 37.308 & 102.03 & \mathrm{P}\end{array}$ $\begin{array}{llllllllllllll}14748 . & 5.031 & 0.292 & 0.02507 & 1073765 & 42.060 & 2.577 & 33.432 & 2.550 & 36.334 & 102.03 & \mathrm{P}\end{array}$ $\begin{array}{llllllllllllll}10327 . & 4.226 & 0.213 & 0.02507 & 1072324 & 42.009 & 3.174 & 34.936 & 3.148 & 37.620 & 102.03 & \mathrm{P}\end{array}$ $\begin{array}{llllllllllllll}\text { 8036. } & 3.703 & 0.176 & 0.02507 & 1069367 . & 41.904 & 3.504 & 35.970 & 3.530 & 38.091 & 102.03 & \mathrm{P}\end{array}$ $\begin{array}{llllllllllllll}5474 . & 3.172 & 0.137 & 0.02507 & 1068849 . & 41.886 & 4.103 & 36.872 & 4.078 & 39.148 & 102.03 & \mathrm{P}\end{array}$ $\begin{array}{llllllllllllll}3634 . & 2.704 & 0.108 & 0.02507 & 1068274 & 41.866 & 4.714 & 37.684 & 4.688 & 39.144 & 102.03 & P\end{array}$ $\begin{array}{llllllllllllll}3107 . & 2.515 & 0.097 & 0.02507 & 1067982 & 41.856 & 5.325 & 38.166 & 4.997 & 39.311 & 102.03 & \mathrm{P}\end{array}$ $\begin{array}{lllllllllllll}2854 . & 2.363 & 0.088 & 0.02507 & 1067767 & 41.848 & 5.938 & 38.553 & 5.301 & 39.466 & 102.03 & P\end{array}$ $\begin{array}{lllllllllllllll}3145 . & 2.155 & 0.069 & 0.02507 & 1067343 & 41.833 & 6.532 & 38.871 & 5.913 & 39.643 & 102.03 & P\end{array}$ $\begin{array}{llllllllllllll}16808 . & 4.909 & 0.760 & 0.01338 & 1204892 & 46.479 & 0.125 & 33.503 & 0.712 & 41.717 & 102.03 & \mathrm{P}\end{array}$ $\begin{array}{llllllllllllll}\text { 13144. } & 4.159 & 0.571 & 0.01338 & 1204233 & 46.457 & 0.738 & 36.535 & 1.325 & 42.031 & 102.03 & \mathrm{P}\end{array}$ $\begin{array}{llllllllllllll}\text { 11487. } & 3.813 & 0.493 & 0.01338 & 1203901 & 46.447 & 1.352 & 38.725 & 1.634 & 42.423 & 102.03 & P\end{array}$ $\begin{array}{llllllllllll}9979 . & 3.497 & 0.426 & 0.01338 & 1203667 & 46.439 & 1.964 & 40.314 & 1.939 & 43.503 & 102.03 & \mathrm{P}\end{array}$ $\begin{array}{llllllllllllll}7324 & 2.926 & 0.317 & 0.01338 & 1203214 & 46.424 & 2.577 & 41.575 & 2.550 & 43.198 & 102.03 & \mathrm{P}\end{array}$ $\begin{array}{lllllllllllll}5212 . & 2.451 & 0.240 & 0.01338 & 1202772 . & 46.410 & 3.174 & 42.363 & 3.148 & 43.938 & 102.03 & \mathrm{P}\end{array}$ $\begin{array}{llllllllllllll}4114 . & 2.194 & 0.203 & 0.01338 & 1202613 & 46.405 & 3.504 & 42.938 & 3.530 & 44.089 & 102.03 & \mathrm{P}\end{array}$ $\begin{array}{lllllllllllll}2875 . & 1.887 & 0.164 & 0.01338 & 1202503 . & 46.401 & 4.103 & 43.353 & 4.078 & 44.774 & 102.03 & P\end{array}$ 
1968. $\quad \begin{array}{llllllllllll} & 1.626 & 0.134 & 0.01338 & 1202380 & 46.397 & 4.714 & 43.848 & 4.688 & 44.793 & 102.03 & \mathrm{P}\end{array}$ $\begin{array}{llllllllllllll}1698 . & 1.527 & 0.122 & 0.01338 & 1202318 & 46.395 & 5.325 & 44.042 & 4.997 & 44.879 & 102.03 & P\end{array}$ $\begin{array}{llllllllllll}1556 . & 1.450 & 0.112 & 0.01338 & 1202246 & 46.393 & 5.938 & 44.260 & 5.301 & 44.913 & 102.03 & \mathrm{P}\end{array}$ $\begin{array}{llllllllllllll}1646 . & 1.358 & 0.092 & 0.01338 & 1202098 & 46.388 & 6.532 & 44.476 & 5.913 & 44.982 & 102.03 & P\end{array}$

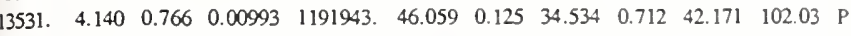
$\begin{array}{lllllllllllll}10534 . & 3.478 & 0.562 & 0.00993 & 1191639 & 46.049 & 0.738 & 37.268 & 1.325 & 42.259 & 102.03 & \mathrm{P}\end{array}$

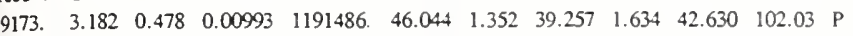

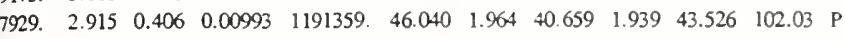
$\begin{array}{lllllllllllll}5723 . & 2.453 & 0.291 & 0.00993 & 1191111 & 46.032 & 2.577 & 41.753 & 2.550 & 43.263 & 102.03 & \mathrm{P}\end{array}$ $\begin{array}{llllllllllllll}3944 . & 2.095 & 0.211 & 0.00993 & 1190868 & 46.024 & 3.174 & 42.437 & 3.148 & 43.975 & 102.03 & \mathrm{P}\end{array}$ $\begin{array}{llllllllllllll}3004 & 1.932 & 0.174 & 0.00993 & 1191254 & 46.036 & 3.504 & 42.967 & 3.530 & 44.076 & 102.03 & P\end{array}$ 1919. $\begin{array}{lllllllllllll}1.746 & 0.137 & 0.00993 & 1191225 & 46.035 & 4.103 & 43.284 & 4.078 & 44.609 & 102.03 & P\end{array}$ $\begin{array}{lllllllllllll}1082 & 1.632 & 0.112 & 0.00993 & 1191192 & 46.034 & 4.714 & 43.663 & 4.688 & 44.395 & 102.03 & \mathrm{P}\end{array}$ $\begin{array}{llllllllllllll}805 . & 1.610 & 0.104 & 0.00993 & 1191176 . & 46.034 & 5.325 & 43.685 & 4.997 & 44.379 & 102.03 & P\end{array}$ $\begin{array}{lllllllllllllll}631 . & 1.612 & 0.098 & 0.00993 & 1191098 & 46.031 & 5.938 & 43.764 & 5.301 & 44.383 & 102.03 & P\end{array}$ $\begin{array}{llllllllllllll}572 & 1.688 & 0.088 & 0.00993 & 1190933 & 46.026 & 6.532 & 43.877 & 5.913 & 44.293 & 102.03 & P\end{array}$ $\begin{array}{llllllllllll}11786 . & 3.475 & 0.751 & 0.00971 & 1142013 & 44.407 & 0.125 & 34.490 & 0.712 & 41.111 & 102.03 & P\end{array}$ $\begin{array}{llllllllllllll}9185 . & 2.950 & 0.572 & 0.00971 & 1141638 & 44.394 & 0.738 & 36.854 & 1.325 & 41.180 & 102.03 & \mathrm{P}\end{array}$ $\begin{array}{lllllllllllll}8012 . & 2.706 & 0.497 & 0.00971 & 1141449 & 44.388 & 1.352 & 38.577 & 1.634 & 41.517 & 102.03 & \mathrm{P}\end{array}$ $\begin{array}{lllllllllllll}\text { 6947. } & 2.480 & 0.434 & 0.00971 & 1141310 & 44.383 & 1.964 & 39.812 & 1.939 & 42.285 & 102.03 & P\end{array}$ $\begin{array}{lllllllllllll}5079 . & 2.065 & 0.331 & 0.00971 & 1141040 & 44.374 & 2.577 & 40.778 & 2.550 & 42.062 & 102.03 & \mathrm{P}\end{array}$ $\begin{array}{llllllllllllll}3605 . & 1.711 & 0.259 & 0.00971 & 1140775 . & 44.366 & 3.174 & 41.382 & 3.148 & 42.664 & 102.03 & \mathrm{P}\end{array}$ $\begin{array}{llllllllllllll}2846 . & 1.525 & 0.224 & 0.00971 & 1141018 & 44.374 & 3.504 & 41.847 & 3.530 & 42.800 & 102.03 & P\end{array}$ $\begin{array}{llllllllllllll}2002 . & 1.278 & 0.187 & 0.00971 & 1140941 . & 44.371 & 4.103 & 42.152 & 4.078 & 43.314 & 102.03 & \mathrm{P}\end{array}$ $\begin{array}{llllllllllllll}1405 & 1.055 & 0.158 & 0.00971 & 1140855 & 44.368 & 4.714 & 42.554 & 4.688 & 43.313 & 102.03 & \mathrm{P}\end{array}$ $\begin{array}{llllllllllllll}1241 . & 0.961 & 0.147 & 0.00971 & 1140811 & 44.367 & 5.325 & 42.704 & 4.997 & 43.404 & 102.03 & \mathrm{P}\end{array}$ $\begin{array}{llllllllllll}1169 . & 0.881 & 0.137 & 0.00971 & 1140739 & 44.364 & 5.938 & 42.888 & 5.301 & 43.471 & 102.03 & P\end{array}$

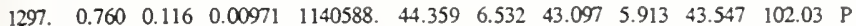
$\begin{array}{llllllllllll}22997 . & 6.428 & 0.768 & 0.01605 & 1041801 . & 40.921 & 0.125 & 23.410 & 0.712 & 34.655 & 102.03 & \mathrm{P}\end{array}$ $\begin{array}{lllllllllllll}18023 & 5.427 & 0.561 & 0.01605 & 1040656 & 40.880 & 0.738 & 27.493 & 1.325 & 35.176 & 102.03 & P\end{array}$ $\begin{array}{lllllllllllll}15758 . & 4.975 & 0.474 & 0.01605 & 1040078 & 40.859 & 1.352 & 30.402 & 1.634 & 35.631 & 102.03 & \mathrm{P}\end{array}$ $\begin{array}{llllllllllllll}13685 & 4.572 & 0.400 & 0.01605 & 1039740 & 40.847 & 1.964 & 32.527 & 1.939 & 37.001 & 102.03 & \mathrm{P}\end{array}$ $\begin{array}{lllllllllllll}10000 & 3.870 & 0.281 & 0.01605 & 1039101 . & 40.824 & 2.577 & 34.195 & 2.550 & 36.480 & 102.03 & \mathrm{P}\end{array}$ $\begin{array}{llllllllllll}\text { 7015. } & 3.317 & 0.198 & 0.01605 & 1038476 . & 40.802 & 3.174 & 35.235 & 3.148 & 37.392 & 102.03 & \mathrm{P}\end{array}$ $\begin{array}{lllllllllllll}5427 . & 3.027 & 0.158 & 0.01605 & 1037921 & 40.782 & 3.504 & 36.007 & 3.530 & 37.612 & 102.03 & \mathrm{P}\end{array}$ $\begin{array}{lllllllllllll}3580 & 2.727 & 0.118 & 0.01605 & 1037698 & 40.773 & 4.103 & 36.553 & 4.078 & 38.488 & 102.03 & P\end{array}$ $\begin{array}{llllllllllllll}2128 . & 2.524 & 0.089 & 0.01605 & 1037451 . & 40.765 & 4.714 & 37.136 & 4.688 & 38.288 & 102.03 & P\end{array}$

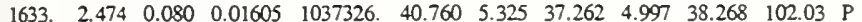
$\begin{array}{lllllllllllllll}1305 . & 2.462 & 0.073 & 0.01605 & 1037267 & 40.758 & 5.938 & 37.406 & 5.301 & 38.249 & 102.03 & P\end{array}$

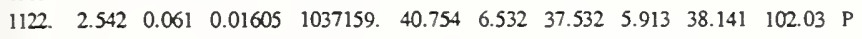

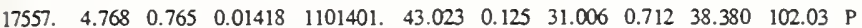
$\begin{array}{lllllllllllll}13840 . & 4.092 & 0.582 & 0.01418 & 1100530 & 42.993 & 0.738 & 33.689 & 1.325 & 38.683 & 102.03 & P\end{array}$ $\begin{array}{lllllllllllll}12158 . & 3.77 & 0.506 & 0.01418 & 1100090 & 42.978 & 1.352 & 35.611 & 1.634 & 38.989 & 102.03 & P\end{array}$ $\begin{array}{llllllllllllll}10628 . & 3.488 & 0.440 & 0.01418 & 1099774 & 42.967 & 1.964 & 37.041 & 1.939 & 39.989 & 102.03 & P\end{array}$ $\begin{array}{lllllllllllll}\text { 7933. } & 2.958 & 0.333 & 0.01418 & 1099159 . & 42.946 & 2.577 & 38.189 & 2.550 & 39.687 & 102.03 & \mathrm{P}\end{array}$ $\begin{array}{llllllllllllll}5789 . & 2.506 & 0.256 & 0.01418 & 1098559 & 42.925 & 3.174 & 38.932 & 3.148 & 40.456 & 102.03 & P\end{array}$ $\begin{array}{llllllllllll}4676 . & 2.242 & 0.217 & 0.01418 & 1097932 . & 42.903 & 3.504 & 39.469 & 3.530 & 40.577 & 102.03\end{array}$ $\begin{array}{lllllllllllll}3417 . & 1.935 & 0.175 & 0.01418 & 1097728 . & 42.896 & 4.103 & 39.883 & 4.078 & 41.166 & 102.03 & P\end{array}$ $\begin{array}{llllllllllllll}2494 . & 1.656 & 0.141 & 0.01418 & 1097500 & 42.888 & 4.714 & 40.344 & 4.688 & 41.209 & 102.03 & P\end{array}$ $\begin{array}{llllllllllll}2219 . & 1.541 & 0.128 & 0.01418 & 1097385 & 42.884 & 5.325 & 40.598 & 4.997 & 41.347 & 102.03 & P\end{array}$ $\begin{array}{lllllllllllll}2073 . & 1.445 & 0.115 & 0.01418 & 1097300 & 42.881 & 5.938 & 40.853 & 5.301 & 41.426 & 102.03 & P\end{array}$ $\begin{array}{lllllllllllll}2160 & 1.304 & 0.091 & 0.01418 & 1097134 & 42.876 & 6.532 & 41.086 & 5.913 & 41.540 & 102.03 & P\end{array}$ 34831. $\begin{array}{llllllllllllll}7.978 & 0.790 & 0.02502 & 1089600 . & 42.614 & 0.125 & 20.060 & 0.712 & 34.759 & 102.03 & P\end{array}$ $\begin{array}{llllllllllllll}27243 . & 6.858 & 0.586 & 0.02502 & 1086928 . & 42.521 & 0.738 & 25.334 & 1.325 & 35.258 & 102.03 & \mathrm{P}\end{array}$ $\begin{array}{lllllllllllll}23814 & 6.334 & 0.502 & 0.02502 & 1085580 & 42.474 & 1.352 & 29.130 & 1.634 & 35.904 & 102.03 & \mathrm{P}\end{array}$ $\begin{array}{lllllllllllll}20694 & 5.862 & 0.429 & 0.02502 & 1084763 & 42.445 & 1.964 & 32.030 & 1.939 & 37.676 & 102.03 & P\end{array}$ $\begin{array}{llllllllllllll}15206 . & 4.998 & 0.312 & 0.02502 & 1083211 . & 42.391 & 2.577 & 34.191 & 2.550 & 36.730 & 102.03 & \mathrm{P}\end{array}$ $\begin{array}{lllllllllllll}10848 . & 4.256 & 0.230 & 0.02502 & 1081694 & 42.338 & 3.174 & 35.551 & 3.148 & 37.943 & 102.03 & P\end{array}$ $\begin{array}{llllllllllllll}8584 . & 3.753 & 0.190 & 0.02502 & 1078399 & 42.222 & 3.504 & 36.466 & 3.530 & 38.361 & 102.03 & \mathrm{P}\end{array}$ $\begin{array}{llllllllllll}6038 . & 3.250 & 0.148 & 0.02502 & 1077800 & 42.201 & 4.103 & 37.317 & 4.078 & 39.386 & 102.03\end{array}$ $\begin{array}{llllllllllll}\text { 4187. } & 2.792 & 0.115 & 0.02502 & 1077135 . & 42.178 & 4.714 & 38.083 & 4.688 & 39.375 & 102.03 & \mathrm{P}\end{array}$ $\begin{array}{lllllllllllll}3643 . & 2.600 & 0.103 & 0.02502 & 1076797 & 42.166 & 5.325 & 38.578 & 4.997 & 39.550 & 102.03 & P\end{array}$ $\begin{array}{llllllllllllll}3366 . & 2.440 & 0.091 & 0.02502 & 1076547 & 42.157 & 5.938 & 38.961 & 5.301 & 39.694 & 102.03\end{array}$ 3587. $\quad \begin{array}{lllllllllll}2.200 & 0.069 & 0.02502 & 1076054 & 42.140 & 6.532 & 39.281 & 5.913 & 39.890 & 102.03 & P\end{array}$ $\begin{array}{llllllllllllll}31135 . & 7.550 & 0.778 & 0.02452 & 1213463 & 46.755 & 0.125 & 25.982 & 0.712 & 39.287 & 102.03 & P\end{array}$ $\begin{array}{lllllllllllll}24416 . & 6.447 & 0.586 & 0.02452 & 1211207 & 46.682 & 0.738 & 30.754 & 1.325 & 39.893 & 102.03 & \mathrm{P}\end{array}$ $\begin{array}{llllllllllllll}21379 & 5.933 & 0.507 & 0.02452 & 1210070 & 46.645 & 1.352 & 34.234 & 1.634 & 40.496 & 102.03 & \mathrm{P}\end{array}$ $\begin{array}{lllllllllllll}18615 . & 5.467 & 0.438 & 0.02452 & 1209344 & 46.622 & 1.964 & 36.813 & 1.939 & 42.127 & 102.03 & P\end{array}$ $\begin{array}{lllllllllllll}13751 . & 4.616 & 0.328 & 0.02452 & 1207956 & 46.577 & 2.577 & 38.791 & 2.550 & 41.418 & 102.03 & \mathrm{P}\end{array}$ $\begin{array}{llllllllllll}9883 . & 3.891 & 0.249 & 0.02452 & 1206599 & 46.534 & 3.174 & 40.066 & 3.148 & 42.568 & 102.03 & P\end{array}$ $\begin{array}{lllllllllllllll}\text { 7873. } & 3.413 & 0.211 & 0.02452 & 1203614 & 46.437 & 3.504 & 40.980 & 3.530 & 42.891 & 102.03 & P\end{array}$ $\begin{array}{lllllllllllll}5608 . & 2.925 & 0.170 & 0.02452 & 1203058 & 46.419 & 4.103 & 41.705 & 4.078 & 43.833 & 102.03 & P\end{array}$ $\begin{array}{lllllllllllllll}3955 & 2.486 & 0.138 & 0.02452 & 1202441 & 46.399 & 4.714 & 42.490 & 4.688 & 43.896 & 102.03\end{array}$ $\begin{array}{lllllllllllllll}3465 & 2.306 & 0.125 & 0.02452 & 1202127 & 46.389 & 5.325 & 42.864 & 4.997 & 44.058 & 102.03 & P\end{array}$ $\begin{array}{lllllllllllll}\text { 3211. } & 2.158 & 0.113 & 0.02452 & 1201886 & 46.381 & 5.938 & 43.259 & 5.301 & 44.200 & 102.03 & P\end{array}$

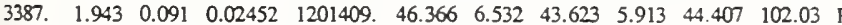
$\begin{array}{llllllllllllll}33558 . & 7.910 & 0.761 & 0.02802 & 1278386 & 48.799 & 0.125 & 26.710 & 0.712 & 40.929 & 102.03 & P\end{array}$ $\begin{array}{lllllllllllll}26194 . & 6.701 & 0.578 & 0.02802 & 1275706 & 48.717 & 0.738 & 31.943 & 1.325 & 41.635 & 102.03 & P\end{array}$ $\begin{array}{llllllllllllll}22872 . & 6.138 & 0.502 & 0.02802 & 1274355 & 48.675 & 1.352 & 35.720 & 1.634 & 42.360 & 102.03 & \mathrm{P}\end{array}$ $\begin{array}{llllllllllllll}19856 . & 5.627 & 0.437 & 0.02802 & 1273477 & 48.648 & 1.964 & 38.574 & 1.939 & 44.095 & 102.03 & \mathrm{P}\end{array}$ $\begin{array}{llllllllllllll}14565 . & 4.693 & 0.333 & 0.02802 & 1271793 & 48.595 & 2.57 & 40.718 & 2.550 & 43.343 & 102.03 & P\end{array}$ $\begin{array}{llllllllllllll}10386 . & 3.896 & 0.259 & 0.02802 & 1270148 & 48.544 & 3.174 & 42.088 & 3.148 & 44.577 & 102.03 & P\end{array}$ $\begin{array}{llllllllllllll}8231 . & 3.346 & 0.224 & 0.02802 & 1265864 & 48.411 & 3.504 & 43.018 & 3.530 & 44.933 & 102.03 & P\end{array}$ $\begin{array}{lllllllllllll}5831 . & 2.805 & 0.186 & 0.02802 & 1265071 . & 48.386 & 4.103 & 43.809 & 4.078 & 45.852 & 102.03 & P\end{array}$ $\begin{array}{lllllllllllll}4131 . & 2.318 & 0.156 & 0.02802 & 1264189 & 48.359 & 4.714 & 44.610 & 4.688 & 45.992 & 102.03 & P\end{array}$ $\begin{array}{llllllllllllll}3658 . & 2.117 & 0.144 & 0.02802 & 1263742 & 48.345 & 5.325 & 45.074 & 4.997 & 46.215 & 102.03 & P\end{array}$ $\begin{array}{llllllllllll}3448 . & 1.951 & 0.134 & 0.02802 & 1263391 & 48.334 & 5.938 & 45.507 & 5.301 & 46.370 & 102.03 & P\end{array}$ $\begin{array}{llllllllllll}3793 . & 1.708 & 0.112 & 0.02802 & 1262700 & 48.313 & 6.532 & 45.910 & 5.913 & 46.614 & 102.03 & P\end{array}$ $\begin{array}{lllllllllllll}37990 & 8.435 & 0.779 & 0.02841 & 1152326 & 44.753 & 0.125 & 20.305 & 0.712 & 36.412 & 102.03 & P\end{array}$ $\begin{array}{llllllllllll}29669 & 7.224 & 0.580 & 0.02841 & 1149232 & 44.649 & 0.738 & 26.069 & 1.325 & 37.010 & 102.03 & \mathrm{P}\end{array}$ $\begin{array}{lllllllllllllll}25912 . & 6.657 & 0.498 & 0.02841 & 1147672 & 44.597 & 1.352 & 30.247 & 1.634 & 37.694 & 102.03 & P\end{array}$ $\begin{array}{llllllllllllll}22495 . & 6.147 & 0.428 & 0.02841 & 1146720 & 44.565 & 1.964 & 33.414 & 1.939 & 39.619 & 102.03 & P\end{array}$ $\begin{array}{lllllllllllll}16493 . & 5.209 & 0.315 & 0.02841 & 1144911 . & 44.504 & 2.577 & 35.774 & 2.550 & 38.612 & 102.03 & P\end{array}$ $\begin{array}{llllllllllllll}11734 & 4.403 & 0.235 & 0.02841 & 1143143 & 44.445 & 3.174 & 37.271 & 3.148 & 39.881 & 102.03 & \mathrm{P}\end{array}$ $\begin{array}{lllllllllllll}9268 . & 3.835 & 0.197 & 0.02841 & 1138798 & 44.299 & 3.504 & 38.271 & 3.530 & 40.355 & 102.03 & P\end{array}$ $\begin{array}{llllllllllllll}6506 . & 3.284 & 0.156 & 0.02841 & 1138071 . & 44.274 & 4.103 & 39.166 & 4.078 & 41.381 & 102.03 & P\end{array}$ $\begin{array}{llllllllllllll}4516 . & 2.778 & 0.125 & 0.02841 & 1137263 & 44.247 & 4714 & 40.010 & 4.688 & 41.457 & 102.03 & P\end{array}$ $\begin{array}{lllllllllllll}3942 & 2.565 & 0.112 & 0.02841 & 1136854 & 44.233 & 5.325 & 40.541 & 4.997 & 41.673 & 102.03 & P\end{array}$ $\begin{array}{lllllllllllllll}3663 & 2.387 & 0.101 & 0.02841 & 1136536 & 44.223 & 5.938 & 40.969 & 5.301 & 41.817 & 102.03 & P\end{array}$ $\begin{array}{lllllllllllll}3959 . & 2.112 & 0.080 & 0.02841 & 1135910 & 44.202 & 6.532 & 41.338 & 5.913 & 42.043 & 102.03 & \mathrm{P}\end{array}$ $\begin{array}{llllllllllll}38870 . & 8.344 & 0.768 & 0.02809 & 1006642 & 39.639 & 0.125 & 14.857 & 0.712 & 31.349 & 102.03 & P\end{array}$ $\begin{array}{lllllllllllll}30403 . & 7.185 & 0.570 & 0.02809 & 1003120 & 39.509 & 0.738 & 20.631 & 1.325 & 31.955 & 102.03 & P\end{array}$ $\begin{array}{lllllllllllll}26576 & 6.640 & 0.488 & 0.02809 & 1001345 & 39.443 & 1.352 & 24.841 & 1.634 & 32.576 & 102.03 & P\end{array}$ $\begin{array}{llllllllllllll}23095 . & 6.153 & 0.417 & 0.02809 & 1000276 & 39.403 & 1.964 & 28.082 & 1.939 & 34.498 & 102.03 & P\end{array}$ $\begin{array}{llllllllllllll}16971 . & 5.258 & 0.304 & 0.02809 & 998250 & 39.328 & 2.577 & 30.473 & 2.550 & 33.305 & 102.03 & \mathrm{P}\end{array}$ $\begin{array}{lllllllllllll}12105 . & 4.483 & 0.223 & 0.02809 & 996270 & 39.254 & 3.174 & 31.974 & 3.148 & 34.613 & 102.03 & P\end{array}$ $\begin{array}{lllllllllllll}9578 . & 3.908 & 0.186 & 0.02809 & 991513 & 39.077 & 3.504 & 32.950 & 3.530 & 35.025 & 102.03 & \mathrm{P}\end{array}$ $\begin{array}{lllllllllllll}6733 . & 3.380 & 0.144 & 0.02809 & 990756 & 39.048 & 4.103 & 33.901 & 4.078 & 36.093 & 102.03 & P\end{array}$ $\begin{array}{lllllllllllllll}\text { 4664. } & 2.890 & 0.113 & 0.02809 & 989914 & 39.017 & 4.714 & 34.722 & 4.688 & 36.131 & 102.03 & \mathrm{P}\end{array}$ $\begin{array}{llllllllllllll}\text { 4054. } & 2.681 & 0.100 & 0.02809 & 989487 & 39.001 & 5.325 & 35.312 & 4.997 & 36.329 & 102.03 & \mathrm{P}\end{array}$ $\begin{array}{llllllllllllll}3743 . & 2.505 & 0.089 & 0.02809 & 989178 & 38.989 & 5.938 & 35.728 & 5.301 & 36.461 & 102.03 & \mathrm{P}\end{array}$ $\begin{array}{llllllllllllllll}3983 . & 2.228 & 0.068 & 0.02809 & 988573 & 38.967 & 6.532 & 36.064 & 5.913 & 36.686 & 102.03 & P\end{array}$ $\begin{array}{llllllllllllll}25929 . & 6.284 & 0.769 & 0.01959 & 1035781 & 40.704 & 0.125 & 23.490 & 0.712 & 34.579 & 102.03 & \mathrm{P}\end{array}$ $\begin{array}{lllllllllllll}20416 . & 5.433 & 0.578 & 0.01959 & 1034025 & 40.641 & 0.738 & 27.364 & 1.325 & 34.891 & 102.03 & \mathrm{P}\end{array}$ $\begin{array}{llllllllllll}17921 . & 5.033 & 0.498 & 0.01959 & 1033141 & 40.609 & 1.352 & 30.185 & 1.634 & 35.305 & 102.03 & P\end{array}$ $\begin{array}{llllllllllllll}15647 . & 4.669 & 0.429 & 0.01959 & 1032561 & 40.588 & 1.964 & 32.275 & 1.939 & 36.696 & 102.03 & \mathrm{P}\end{array}$ $\begin{array}{lllllllllllll}11637 . & 4.000 & 0.316 & 0.01959 & 1031449 & 40.547 & 2.577 & 33.904 & 2.550 & 36.066 & 102.03 & \mathrm{P}\end{array}$ $\begin{array}{lllllllllllll}8437 . & 3.420 & 0.236 & 0.01959 & 1030362 . & 40.508 & 3.174 & 34.960 & 3.148 & 37.061 & 102.03 & P\end{array}$ $\begin{array}{lllllllllllll}6768 . & 3.045 & 0.196 & 0.01959 & 1028468 & 40.439 & 3.504 & 35.725 & 3.530 & 37.321 & 102.03 & \mathrm{P}\end{array}$ $\begin{array}{llllllllllll}4871 . & 2.644 & 0.154 & 0.01959 & 1028066 & 40.424 & 4.103 & 36.342 & 4.078 & 38.132 & 102.03 & \mathrm{P}\end{array}$ $\begin{array}{llllllllllllll}3465 . & 2.273 & 0.120 & 0.01959 & 1027620 & 40.408 & 4.714 & 36.975 & 4.688 & 38.104 & 102.03 & P\end{array}$ $\begin{array}{lllllllllllll}\text { 3033. } & 2.114 & 0.106 & 0.01959 & 1027394 & 40.400 & 5.325 & 37.314 & 4.997 & 38.243 & 102.03 & \mathrm{P}\end{array}$ $\begin{array}{llllllllllllll}2794 & 1.980 & 0.095 & 0.01959 & 1027241 . & 40.394 & 5.938 & 37.652 & 5.301 & 38.401 & 102.03 & \mathrm{P}\end{array}$ $\begin{array}{lllllllllllll}2870 . & 1.768 & 0.072 & 0.01959 & 1026946 & 40.384 & 6.532 & 37.933 & 5.913 & 38.584 & 102.03 & P\end{array}$ $\begin{array}{llllllllllllll}27802 . & 6.617 & 0.772 & 0.01976 & 938041 & 37.037 & 0.125 & 18.901 & 0.712 & 30.567 & 102.03 & P\end{array}$ $\begin{array}{lllllllllllll}21862 & 5.732 & 0.573 & 0.01976 & 936046 & 36.959 & 0.738 & 23.030 & 1.325 & 30.920 & 102.03 & P\end{array}$ $\begin{array}{lllllllllllll}\text { 19172. } & 5.317 & 0.490 & 0.01976 & 935040 . & 36.920 & 1.352 & 25.978 & 1.634 & 31.352 & 102.03 & P\end{array}$ $\begin{array}{lllllllllllll}\text { 16720. } & 4.943 & 0.418 & 0.01976 & 934411 & 36.895 & 1.964 & 28.254 & 1.939 & 32.796 & 102.03 & \mathrm{P}\end{array}$ $\begin{array}{llllllllllllll}12392 . & 4.256 & 0.303 & 0.01976 & 933211 & 36.848 & 2.577 & 29.968 & 2.550 & 32.033 & 102.03 & P\end{array}$ $\begin{array}{llllllllllll}8932 . & 3.665 & 0.220 & 0.01976 & 932038 & 36.802 & 3.174 & 31.055 & 3.148 & 33.043 & 102.03 & P\end{array}$ $\begin{array}{lllllllllllll}7123 & 3.280 & 0.179 & 0.01976 & 930060 & 36.725 & 3.504 & 31.798 & 3.530 & 33.363 & 102.03 & P\end{array}$ $\begin{array}{llllllllllllll}5063 . & 2.879 & 0.136 & 0.01976 & 929651 & 36.709 & 4.103 & 32.496 & 4.078 & 34.207 & 102.03 & \mathrm{P}\end{array}$ $\begin{array}{llllllllllllll}3525 & 2.512 & 0.102 & 0.01976 & 929195 & 36.691 & 4.714 & 33.100 & 4.688 & 34.157 & 102.03 & \mathrm{P}\end{array}$ $\begin{array}{llllllllllllll}3048 . & 2.357 & 0.089 & 0.01976 & 928964 & 36.682 & 5.325 & 33.523 & 4.997 & 34.345 & 102.03 & P\end{array}$ $\begin{array}{llllllllllllll}2776 . & 2.229 & 0.078 & 0.01976 & 928833 & 36.676 & 5.938 & 33.823 & 5.301 & 34.471 & 102.03 & P\end{array}$ $\begin{array}{llllllllllllll}2826 & 2.034 & 0.056 & 0.01976 & 928584 & 36.667 & 6.532 & 34.046 & 5.913 & 34.534 & 102.03 & \mathrm{P}\end{array}$ $\begin{array}{lllllllllllll}22647 . & 5.908 & 0.768 & 0.01974 & 1216992 & 46.868 & 0.125 & 31.436 & 0.712 & 41.065 & 102.03 & P\end{array}$ $\begin{array}{lllllllllllll}17839 . & 5.041 & 0.595 & 0.01974 & 1215461 . & 46.819 & 0.738 & 34.894 & 1.325 & 41.523 & 102.03 & P\end{array}$ $\begin{array}{lllllllllllllll}15666, & 4.637 & 0.522 & 0.01974 & 1214689 & 46.794 & 1.352 & 37.430 & 1.634 & 41.936 & 102.03 & P\end{array}$ $\begin{array}{lllllllllllll}13687 . & 4.266 & 0.460 & 0.01974 & 1214150 & 46.777 & 1.964 & 39.280 & 1.939 & 43.181 & 102.03 & P\end{array}$ $\begin{array}{lllllllllllll}10205 . & 3.587 & 0.358 & 0.01974 & 1213105 & 46.743 & 2.577 & 40.751 & 2.550 & 42.767 & 102.03 & P\end{array}$ $\begin{array}{llllllllllllll}7436 & 3.006 & 0.285 & 0.01974 & 1212084 & 46.710 & 3.174 & 41.720 & 3.148 & 43.715 & 102.03 & \mathrm{P}\end{array}$ $\begin{array}{lllllllllllll}5997 . & 2.631 & 0.249 & 0.01974 & 1210035 & 46.644 & 3.504 & 42.424 & 3.530 & 43.915 & 102.03 & P\end{array}$ $\begin{array}{llllllllllllll}4373 . & 2.232 & 0.209 & 0.01974 & 1209574 & 46.629 & 4.103 & 42.955 & 4.078 & 44.669 & 102.03 & P\end{array}$ $\begin{array}{llllllllllllll}3186 & 1.868 & 0.178 & 0.01974 & 1209061 . & 46.613 & 4.714 & 43.581 & 4.688 & 44.706 & 102.03 & P\end{array}$ $\begin{array}{lllllllllllll}2833 & 1.716 & 0.165 & 0.01974 & 1208801 & 46.605 & 5.325 & 43.857 & 4.997 & 44.865 & 102.03 & P\end{array}$ $\begin{array}{lllllllllllll}2649 . & 1.589 & 0.153 & 0.01974 & 1208594 & 46.598 & 5.938 & 44.186 & 5.301 & 44.977 & 102.03 & P\end{array}$ $\begin{array}{llllllllllllll}2769 . & 1.398 & 0.130 & 0.01974 & 1208185 & 46.585 & 6.532 & 44.520 & 5.913 & 45.185 & 102.03 & P\end{array}$ $\begin{array}{llllllllllll}23440 . & 6.331 & 0.778 & 0.02032 & 1372091 & 51.617 & 0.125 & 35.451 & 0.712 & 45.357 & 102.03 & \mathrm{P}\end{array}$ $\begin{array}{lllllllllllll}18367 . & 5.361 & 0.597 & 0.02032 & 1370668 & 51.576 & 0.738 & 39.201 & 1.325 & 45.969 & 102.03 & P\end{array}$ $\begin{array}{lllllllllllll}16078 . & 4.911 & 0.522 & 0.02032 & 1369950 & 51.555 & 1.352 & 41.902 & 1.634 & 46.437 & 102.03 & P\end{array}$ $\begin{array}{llllllllllllll}13999 . & 4.498 & 0.458 & 0.02032 & 1369435 & 51.540 & 1.964 & 43.907 & 1.939 & 47.746 & 102.03 & P\end{array}$ $\begin{array}{llllllllllllll}10349 . & 3.746 & 0.353 & 0.02032 & 1368434 & 51.510 & 2.577 & 45.474 & 2.550 & 47.397 & 102.03 & P\end{array}$ $\begin{array}{lllllllllllll}7463 . & 3.108 & 0.279 & 0.02032 & 1367455 & 51.481 & 3.174 & 46.482 & 3.148 & 48.359 & 102.03 & P\end{array}$ $\begin{array}{llllllllllllll}5973 & 2.713 & 0.242 & 0.02032 & 1365551 & 51.426 & 3.504 & 47.187 & 3.530 & 48.615 & 102.03 & P\end{array}$ $\begin{array}{llllllllllllll}4309 . & 2.284 & 0.203 & 0.02032 & 1365139 & 51.413 & 4.103 & 47.763 & 4.078 & 49.379 & 102.03 & P\end{array}$ $\begin{array}{llllllllllllll}3125 . & 1.903 & 0.172 & 0.02032 & 1364682 & 51.400 & 4.714 & 48.383 & 4.688 & 49.450 & 102.03 & P\end{array}$ $\begin{array}{llllllllllll}2791 . & 1.748 & 0.159 & 0.02032 & 1364450 & 51.393 & 5.325 & 48.712 & 4.997 & 49.610 & 102.03 & P\end{array}$ $\begin{array}{lllllllllllll}2638 & 1.621 & 0.147 & 0.02032 & 1364240 & 51.387 & 5.938 & 49.046 & 5.301 & 49.734 & 102.03 & \mathrm{P}\end{array}$ $\begin{array}{lllllllllllll}2856 . & 1.442 & 0.124 & 0.02032 & 1363822 & 51.375 & 6.532 & 49.389 & 5.913 & 49.953 & 102.03 & P\end{array}$ $\begin{array}{lllllllllllll}28540 & 6.887 & 0.787 & 0.02109 & 1083268 & 42.393 & 0.125 & 23.792 & 0.712 & 35.666 & 102.03 & \mathrm{P}\end{array}$ $\begin{array}{lllllllllllll}22380 . & 5.934 & 0.590 & 0.02109 & 1081287 & 42.324 & 0.738 & 28.098 & 1.325 & 36.047 & 102.03 & P\end{array}$ 19596. $\begin{array}{lllllllllllll}5.488 & 0.507 & 0.02109 & 1080289 & 42.289 & 1.352 & 31.167 & 1.634 & 36.541 & 102.03 & P\end{array}$ $\begin{array}{lllllllllllll}17063 . & 5.082 & 0.437 & 0.02109 & 1079647 & 42.266 & 1.964 & 33.519 & 1.939 & 38.026 & 102.03 & \mathrm{P}\end{array}$

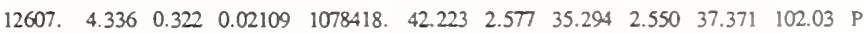

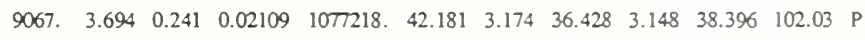
$\begin{array}{llllllllllllll}7229 & 3.269 & 0.201 & 0.02109 & 1074781 & 42.095 & 3.504 & 37.204 & 3.530 & 38.763 & 102.03 & \mathrm{P}\end{array}$ $\begin{array}{lllllllllllll}5160 . & 2.827 & 0.158 & 0.02109 & 1074322 & 42.079 & 4.103 & 37.910 & 4.078 & 39.643 & 102.03 & \mathrm{P}\end{array}$ $\begin{array}{lllllllllllllll}3655 . & 2.420 & 0.125 & 0.02109 & 1073812 & 42.061 & 4.714 & 38.551 & 4.688 & 39.614 & 102.03 & P\end{array}$ $\begin{array}{lllllllllllll}3211 . & 2.248 & 0.112 & 0.02109 & 1073554 . & 42.052 & 5.325 & 38.976 & 4.997 & 39.77 & 102.03 & \mathrm{P}\end{array}$ $\begin{array}{lllllllllllll}2985 & 2.102 & 0.100 & 0.02109 & 1073355 . & 42.045 & 5.938 & 39.314 & 5.301 & 39.925 & 102.03 & \mathrm{P}\end{array}$ $\begin{array}{llllllllllllll}3160 & 1.877 & 0.076 & 0.02109 & 1072963 & 42.031 & 6.532 & 39.615 & 5.913 & 40.109 & 102.03 & \mathrm{P}\end{array}$ $\begin{array}{lllllllllllll}\text { 13851. } & 3.932 & 0.768 & 0.00985 & 934773 & 36.909 & 0.125 & 26.093 & 0.712 & 33.191 & 102.03 & P\end{array}$ $\begin{array}{lllllllllllll}10876 . & 3.322 & 0.569 & 0.00985 & 934350 & 36.893 & 0.738 & 28.657 & 1.325 & 33.286 & 102.03 & P\end{array}$ $\begin{array}{lllllllllllll}9525 . & 3.045 & 0.486 & 0.00985 & 934137 & 36.884 & 1.352 & 30.459 & 1.634 & 33.656 & 102.03 & P\end{array}$ $\begin{array}{llllllllllllll}8290 & 2.793 & 0.415 & 0.00985 & 933977 & 36.878 & 1.964 & 31.810 & 1.939 & 34.509 & 102.03 & P\end{array}$ 
$\begin{array}{lllllllllllll}6102 . & 2.345 & 0.300 & 0.00985 & 933666 & 36.866 & 2.577 & 32.857 & 2.550 & 34.159 & 102.03 & P\end{array}$ $\begin{array}{lllllllllllllll}4341 . & 1.984 & 0.219 & 0.00985 & 933361 & 36.854 & 3.174 & 33.492 & 3.148 & 34.916 & 102.03 & \mathrm{P}\end{array}$ $\begin{array}{llllllllllllll}3412 & 1.806 & 0.179 & 0.00985 & 933536 & 36.861 & 3.504 & 33.973 & 3.530 & 34.959 & 102.03 & P\end{array}$ $\begin{array}{lllllllllllllll}2340 & 1.593 & 0.138 & 0.00985 & 933497 & 36.859 & 4.103 & 34.361 & 4.078 & 35.599 & 102.03 & P\end{array}$ $\begin{array}{lllllllllllll}\text { 1516. } & 1.430 & 0.107 & 0.00985 & 933454 & 36.857 & 4.714 & 34.746 & 4.688 & 35.470 & 102.03 & P\end{array}$ $\begin{array}{llllllllllllll}\text { 1246. } & 1.377 & 0.096 & 0.00985 & 933432 & 36.857 & 5.325 & 34.906 & 4.997 & 35.460 & 102.03 & P\end{array}$ $\begin{array}{lllllllllllll}1078 & 1.342 & 0.087 & 0.00985 & 933345 & 36.853 & 5.938 & 35.036 & 5.301 & 35.460 & 102.03 & P\end{array}$ $\begin{array}{llllllllllllll}1030 & 1.329 & 0.070 & 0.00985 & 933159 & 36.846 & 6.532 & 35.133 & 5.913 & 35.457 & 102.03 & P\end{array}$ $\begin{array}{llllllllllllll}12593 . & 3.555 & 0.759 & 0.00976 & 1030591 . & 40.516 & 0.125 & 30.715 & 0.712 & 37.163 & 102.03 & \mathrm{P}\end{array}$ $\begin{array}{lllllllllllll}9852 . & 3.025 & 0.573 & 0.00976 & 1030204 & 40.502 & 0.738 & 33.054 & 1.325 & 37.198 & 102.03 & \mathrm{P}\end{array}$ $\begin{array}{llllllllllll}8615 . & 2.779 & 0.496 & 0.00976 & 1030009 & 40.495 & 1.352 & 34.715 & 1.634 & 37.540 & 102.03 & p\end{array}$ $\begin{array}{llllllllllllll}7489 & 2.550 & 0.429 & 0.00976 & 1029842 & 40.489 & 1.964 & 35.959 & 1.939 & 38.337 & 102.03 & P\end{array}$ $\begin{array}{llllllllllllll}\text { 5513. } & 2.133 & 0.322 & 0.00976 & 1029511 . & 40.477 & 2.577 & 36.920 & 2.550 & 38.061 & 102.03 & \mathrm{P}\end{array}$ $\begin{array}{lllllllllllll}3947 . & 1.777 & 0.246 & 0.00976 & 1029188 & 40.465 & 3.174 & 37.515 & 3.148 & 38.708 & 102.03 & P\end{array}$ $\begin{array}{lllllllllllllll}3138 . & 1.591 & 0.208 & 0.00976 & 1029381 & 40.472 & 3.504 & 37.949 & 3.530 & 38.797 & 102.03 & \mathrm{P}\end{array}$ $\begin{array}{lllllllllllll}2231 . & 1.349 & 0.168 & 0.00976 & 1029300 & 40.469 & 4.103 & 38.299 & 4.078 & 39.376 & 102.03 & P\end{array}$ $\begin{array}{lllllllllllllll}1580 & 1.131 & 0.137 & 0.00976 & 1029211 & 40.466 & 4.714 & 38.675 & 4.688 & 39.323 & 102.03 & P\end{array}$ $\begin{array}{llllllllllllll}1393 . & 1.041 & 0.125 & 0.00976 & 1029166 & 40.464 & 5.325 & 38.878 & 4.997 & 39.510 & 102.03 & \mathrm{P}\end{array}$ $\begin{array}{lllllllllllllll}1303 . & 0.965 & 0.114 & 0.00976 & 1029095 & 40.462 & 5.938 & 39.057 & 5.301 & 39.448 & 102.03 & \mathrm{P}\end{array}$ $\begin{array}{llllllllllllll}1407 . & 0.852 & 0.092 & 0.00976 & 1028950 & 40.457 & 6.532 & 39.231 & 5.913 & 39.526 & 102.03 & P\end{array}$ $\begin{array}{llllllllllll}12474 & 3.730 & 0.792 & 0.01002 & 1321063 & 50.101 & 0.125 & 39.878 & 0.712 & 46.576 & 102.03 & P\end{array}$ $\begin{array}{llllllllllllll}9728 & 3.175 & 0.600 & 0.01002 & 1320770 & 50.093 & 0.738 & 42.255 & 1.325 & 46.634 & 102.03 & \mathrm{P}\end{array}$ $\begin{array}{llllllllllllll}8489 & 2.918 & 0.520 & 0.01002 & 1320623 & 50.088 & 1.352 & 44.012 & 1.634 & 46.981 & 102.03 & \mathrm{P}\end{array}$ $\begin{array}{lllllllllllll}7362 . & 2.680 & 0.452 & 0.01002 & 1320479 & 50.084 & 1.964 & 45.247 & 1.939 & 47.774 & 102.03 & P\end{array}$ $\begin{array}{lllllllllllllll}5385 & 2.248 & 0.342 & 0.01002 & 1320190 & 50.075 & 2.577 & 46.222 & 2.550 & 47.593 & 102.03 & P\end{array}$ $\begin{array}{lllllllllllll}3820 & 1.886 & 0.265 & 0.01002 & 1319908 & 50.067 & 3.174 & 46.848 & 3.148 & 48.238 & 102.03 & \mathrm{P}\end{array}$ $\begin{array}{llllllllllllll}3012 & 1.696 & 0.227 & 0.01002 & 1320124 & 50.073 & 3.504 & 47.320 & 3.530 & 48.285 & 102.03 & P\end{array}$ $\begin{array}{llllllllllllll}2108 & 1.454 & 0.187 & 0.01002 & 1320060 & 50.071 & 4.103 & 47.618 & 4.078 & 48.850 & 102.03 & \mathrm{P}\end{array}$ $\begin{array}{llllllllllllll}\text { 1461. } & 1.244 & 0.157 & 0.01002 & 1319989 & 50.069 & 4.714 & 48.041 & 4.688 & 48.837 & 102.03 & P\end{array}$ $\begin{array}{llllllllllllll}\text { 1278. } & 1.160 & 0.145 & 0.01002 & 1319953 & 50.068 & 5.325 & 48.153 & 4.997 & 48.902 & 102.03 & P\end{array}$ 1192. $\quad \begin{array}{lllllllllllll}1.093 & 0.135 & 0.01002 & 1319895 & 50.066 & 5.938 & 48.336 & 5.301 & 48.953 & 102.03 & \mathrm{P}\end{array}$ 1304. $1.002 \quad 0.114 \quad 0.01002 \quad 1319775 . \quad 50.063 \quad 6.532 \quad 48.554 \quad 5.913 \quad 49.008 \quad 102.03 \quad P$ $\begin{array}{llllllllllllll}21402 . & 6.076 & 0.782 & 0.01633 & 1235476 & 47.457 & 0.125 & 30.855 & 0.712 & 41.454 & 102.03 & P\end{array}$

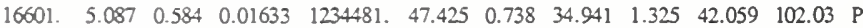

$\begin{array}{lllllllllllll}14438 & 4.633 & 0.502 & 0.01633 & 1233979 & 47.409 & 1.352 & 37.825 & 1.634 & 42.586 & 102.03 & P\end{array}$ $\begin{array}{llllllllllllll}\text { 12475. } & 4.218 & 0.432 & 0.01633 & 1233628 & 47.398 & 1.964 & 39.934 & 1.939 & 43.898 & 102.03 & P\end{array}$ $\begin{array}{lllllllllllll}9038 & 3.473 & 0.321 & 0.01633 & 1232949 & 47.377 & 2.577 & 41.563 & 2.550 & 43.528 & 102.03 & P\end{array}$ $\begin{array}{llllllllllllll}6331 . & 2.855 & 0.244 & 0.01633 & 1232286 & 47.356 & 3.174 & 42.573 & 3.148 & 44.563 & 102.03 & P\end{array}$ $\begin{array}{lllllllllllll}4940 . & 2.509 & 0.207 & 0.01633 & 1231604 & 47.334 & 3.504 & 43.287 & 3.530 & 44.688 & 102.03 & P\end{array}$ $\begin{array}{lllllllllllll}3401 . & 2.116 & 0.168 & 0.01633 & 1231379 . & 47.327 & 4.103 & 43.833 & 4.078 & 45.432 & 102.03 & P\end{array}$ $\begin{array}{lllllllllllll}2327 . & 1.787 & 0.139 & 0.01633 & 1231129 & 47.319 & 4.714 & 44.416 & 4.688 & 45.454 & 102.03 & P\end{array}$ $\begin{array}{llllllllllllll}2038 . & 1.664 & 0.128 & 0.01633 & 1231003 & 47.315 & 5.325 & 44.709 & 4.997 & 45.627 & 102.03 & P\end{array}$ $\begin{array}{llllllllllllll}1922 & 1.572 & 0.118 & 0.01633 & 1230918 & 47.312 & 5.938 & 45.000 & 5.301 & 45.728 & 102.03 & \mathrm{P}\end{array}$ $\begin{array}{llllllllllllll}2194 . & 1.475 & 0.097 & 0.01633 & 1230754 & 47.307 & 6.532 & 45.281 & 5.913 & 45.854 & 102.03 & P\end{array}$ $\begin{array}{lllllllllllll}21433 . & 6.081 & 0.781 & 0.01632 & 1233976 . & 47.409 & 0.125 & 30.800 & 0.712 & 41.408 & 102.03 & P\end{array}$ $\begin{array}{llllllllllllll}16624 & 5.095 & 0.583 & 0.01632 & 1232982 & 47.378 & 0.738 & 34.889 & 1.325 & 41.999 & 102.03 & P\end{array}$ $\begin{array}{llllllllllllll}14458 . & 4.641 & 0.502 & 0.01632 & 1232480 & 47.362 & 1.352 & 37.775 & 1.634 & 42.536 & 102.03 & P\end{array}$ $\begin{array}{llllllllllllll}12492 . & 4.227 & 0.432 & 0.01632 & 1232134 & 47.351 & 1.964 & 39.889 & 1.939 & 43.831 & 102.03 & \mathrm{P}\end{array}$ $\begin{array}{lllllllllllll}9050 . & 3.482 & 0.320 & 0.01632 & 1231464 & 47.330 & 2.57 & 41.520 & 2.550 & 43.472 & 102.03 & P\end{array}$ $\begin{array}{llllllllllllll}6339 & 2.864 & 0.243 & 0.01632 & 1230809 & 47.309 & 3.174 & 42.532 & 3.148 & 44.506 & 102.03 & P\end{array}$ $\begin{array}{llllllllllllll}\text { 4946. } & 2.515 & 0.206 & 0.01632 & 1230059 & 47.285 & 3.504 & 43.242 & 3.530 & 44.633 & 102.03 & \mathrm{P}\end{array}$ $\begin{array}{llllllllllllll}3404 . & 2.120 & 0.167 & 0.01632 & 1229829 & 47.278 & 4.103 & 43.792 & 4.078 & 45.369 & 102.03 & P\end{array}$ $\begin{array}{llllllllllllll}2329 . & 1.788 & 0.138 & 0.01632 & 1229574 & 47.270 & 4.714 & 44.370 & 4.688 & 45.403 & 102.03 & P\end{array}$ $\begin{array}{lllllllllllllll}2040 . & 1.663 & 0.127 & 0.01632 & 1229444 & 47.265 & 5.325 & 44.669 & 4.997 & 45.592 & 102.03 & \mathrm{P}\end{array}$ $\begin{array}{lllllllllllll}1924 & 1.568 & 0.116 & 0.01632 & 1229330 & 47.262 & 5.938 & 44.960 & 5.301 & 45.700 & 102.03 & P\end{array}$ $\begin{array}{llllllllllll}2197 . & 1.464 & 0.096 & 0.01632 & 1229101 & 47.255 & 6.532 & 45.239 & 5.913 & 45.793 & 102.03 & P\end{array}$ 

